Derivative Classifier DBprome

D.B. Moore, Section Manager

Authorized Derivative Classifier
WSRC-RP- -90-1031

DE91 007205

\title{
GEOCHEMICAL AND PHYSICAL PROPERTIES OF SOILS AND SHALLOW SEDIMENTS AT THE SAVANNAH RIVER SITE (U)
}

B.B. Looney ${ }^{1}$, C.A. Eddy' ${ }^{1}$, M. Ramdeen 1 , J. Pickett ${ }^{2}$,V. Rogers ${ }^{3}, 1$, M.T. Scott ${ }^{4}$, and P.A. Shirley ${ }^{4}$

August 31,1990

Authentication: B.B. Loong.

Approved by:

D.B. Moore, Manager

Environmental Sciences Section

Savannah River Laboratory

\footnotetext{
1 Westinghouse Savannah River Company, Savannah River Laboratory, Environmental Sciences Section, Aiken, South Carolina, 29808

2 Westinghouse Savannah River Company, Raw Materials Engineering and Technology, Aiken, South Carolina, 29808

3 U.S. Department of Agriculture, Soil Conservation Service, Savannah River Site, Aiken, South Carolina, 29808

${ }^{4}$ Sirrine Environmental Consultants, P. O. Box 24000, Greenville, South Carolina 29616
}

\section{WESTTINGHOUSE SAVANNAH RIVER COMPANY SAVANNAH RIVER SITE \\ Aiken South Carolina 29808}

Prepared For the U.S. Department of Energy under Contract No. DE-AC09-89SR18035

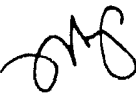




\section{GEOCHEMICAL AND PHYSICAL PROPERTIES OF SOILS AND SHALLOW SEDIMENTS AT THE SAVANNAH RIVER SITE}

\section{Table of Contents}

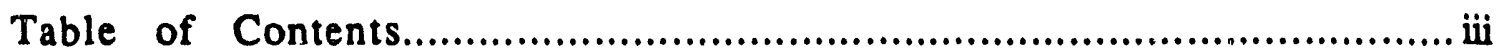

List of Tables ..............................................................................

List of Figures ...................................................................... vii

List of Appendices ................................................................... ix

1.0 Executive Sumnary .......................................................

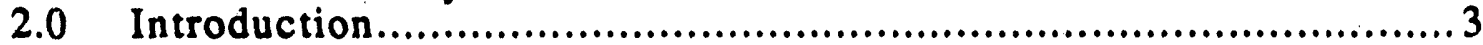

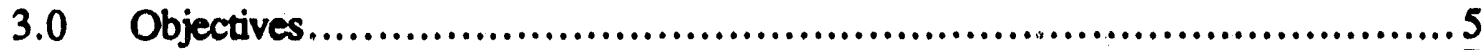

4.0 Background..................................................................

4.1 Review of Soil Formation and Classification ............................... 7

5.0 Methods................................................................... 17

5.1 Study Design.............................................................. 17

5.2 Sample Collection ..................................................... 18

5.2.1 Split Spoon Samples.............................................. 19

5.2 .2 Grab Samples................................................ 20

5.2.3 Drilling Equipment Decontamination...................................20

5.3 Sample Analysis................................................. 21

5.4 Statistical Methods ........................................................... 22

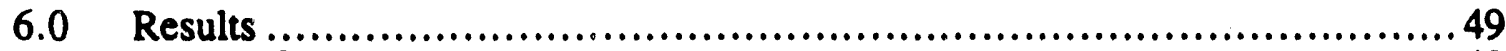

6.1 Current Study ....................................................... 49

6.1.1 Metals..................................................... 49

6.1.2 Other Inorganic Parameters ......................................55

6.1.3 Organic Compounds ............................................ 54

6.1.4 Radiological Parameters ......................................55

6.1.5 Agricultural Parameters.......................................55

6.1.6 Mineralogical Analysis...........................................5 56

6.1.7 Carbon Steel/Stainless Steel Comparison Data .....................56

6.1.8 Laboratory Comparison Data ....................................56

6.2 Past Studies at SRS ...............................................56

6.3 Regional, National, and Global Studies ................................57

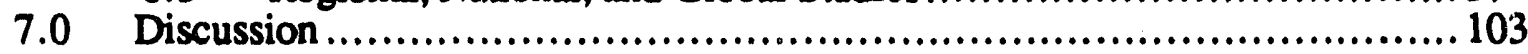

7.1 Summary of Data ................................................... 103

7.1.1 Metals, Radionuclides and Bulk Geochemistry...................... 103

7.1.2 Organics............................................................ 103

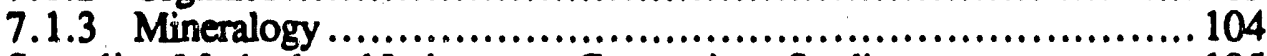

7.2 Sampling Method and Laboratory Comparison Studies ........................ 105

7.2.1 Carbon Steel And Stainless Steel Comparison.......................... 105

7.2.2 Laboratory Comparison ........................................ 106

8.0 References ............................................................ 


\section{GEOCHEMICAL AND PHYSICAL PROPERTIES OF SOILS AND SHALLOW SEDIMENTS AT THE SAVANNAH RIVER SITE}

\section{List of Tables}

Table 4.1

Table 5.1.

Table 5.2.

Table 5.3.

Table 5.4

Table 5.5

Table 5.6

Table 5.7

Table 5.8

Table 5.9

Table 5.10

Table 5.11

Table 6.1.

Table 6.2.

Table 6.3.

Table 6.4.

Table 6.5.

Table 6.6.

Table 6.7.

Table 6.8.

Table 6.9.

Table 6.10.

Table 6.11.

Table 6.12.

Table 6.13.

Table 6.14.

Table 6.15.

Table 6.16.

Table 6.17.

Table 6.18.

Table 6.19.

Table 6.20.

Table 6.21.

Table 6.22.

Table 6.23.

Table 6.24.

Table 6.25.

Table 6.26.

Table 6.27.

Table 6.28.

Table 6.29.

Table 6.30.

Table 6.31.

Table 6.32

Table 6.33

Table 6.34.

Table 6.35

Table 6.36
Summary of Taxonomic Terms Applicable to Soils

SRS Soil Study Taxonomic Name for Soil Series Found on SRS ......... 25

General Soil Horizon Descriptions........................................... 27

Soil Boring Coordinates................................................... 33

Analytical Methods - metaTRACE Labs ................................. 34

Analytical Methods - Weston Labs...................................... 35

Sample Specifications For Blanton Soil Series ............................ 36

Sample Specifications For Orangeburg Soil Series ...................... 37

Sample Specifications For Fuquay Soil Series ......................... 38

Sample Specifications For Vaucluse Soil Series................................ 39

Sample Specifications For Lakeland Soil Series.............................. 40

Sample Specifications For Udorthent Soil Series .......................41

Summary Statistics for Metals Measured (all samples) ...................60

Summary Statistics for Aluminum (by series and layer)....................61

Summary Statistics for Arsenic (by series and layer) ....................62

Summary Statistics for Barium (by series and layer) ....................63

Summary Statistics for Cadmilum (by series and layer).....................6 64

Summary Statistics for Chromium (by series and layer) ................65

Summary Statistics for Copper (by series and layer) ....................66

Summary Statistics for Iron (by series and layer) .......................67

Summary Statistics for Lead (by series and layer) .........................68

Summary Statistics for Magnesium (by series and layer) ................69

Summary Statistics for Manganese (by series and layer) ................70

Summary Statistics for Mercury (by series and layer) ................... 71

Summary Statistics for Nickel (by series and layer) ....................72

Summary Statistics for Potassiurn (by series and layer) ....................73

Summary Statistics for Selenium (by series and layer) $\ldots \ldots \ldots \ldots \ldots \ldots \ldots \ldots 74$

Summary Statistics for Silver (by series and layer) ....................... 75

Summary Statistics for Sodium (by series and layer) ...................... 76

Summary Statistics for Zinc (by series and layer) ........................77

Summary Statistics for Other Inorganic Parameters Measured .............78

Summary Statistics for Chloride (by series and layer)....................79

Summary Statistics for Nitrate (by series and layer) .......................8 80

Summary Statistics for Sulfate (by series and layer) $\ldots \ldots \ldots \ldots \ldots \ldots \ldots \ldots \ldots 81$

Summary Statistics for Bulk Organic Constituents (all sásnples)........ 82

Summary Statistics for Total Organic Carbon (by series and layer)........83

SRS Soil Study Appendix VIII Organic Compounds Detected............ 84

Summary Statistics for Radionuclides (all samples) ....................85

Summary Statistics for Gross Alpha (by series and layer) ................86

Summary Statistics for Nonvolatile Beta (by series and layer) ...........87

Summary Statistics for Uranium (by series and layer).......................88

SRS Soil Study X-Ray Diffraction Analyses Of Whole Soil Samples...... 89

SRS Soil Study X-Ray Diffraction Analyses Of Clay Mineral Fraction... 90

Summary of Carbon Steel vs Stairless Steel Sampler Results.............. 91

Summary of Interlaboratory Comparison Results..........................92

Summary of Control/Background Data for Various Constituents from

Sewage Sludge Application Test Sites .................................... 95

Radionuclide Data for SRS Soils........................................ 96

Regional, National, and Global Soil Concentrations....................... 97 


\title{
GEOCHEMICAL AND PHYSICAL PROPERTIES OF SOILS AND SHALLOW SEDIMENTS AT THE SAVANNAH RIVER SITE
}

\author{
List of Figures
}

Figure 4.1 Recent Changes in Master Horizon Designations ........................ 14

Figure 4.2 Hypothetical Soil Profile Showing The Principal Horizons And

Potential Occurrence On The Savannah River Site......................... 15

Figure 5.1 Sample Locations for Grouping One (Blanton) Soils.....................42

Figure 5.2 Sample Locations for Grouping Two (Orangeburg) Soils ....................43

Figure 5.3 Sample Locations for Grouping Three (Fuquay) Soils ...................44

Figure 5.4 Sample Locations for Grouping Four (Vaucluse) Soils ....................45

Figure 5.5 Sample Locations for Grouping Five (Lakeland) Soils....................4 46

Figure 5.6 Sample Locations for Grouping Six (Udorthent) Soils....................47

Figure 6.1 Frequency of Occurrence of Organic Compounds in Appendix VIII Analy ses.....................................................................59 59

Figure 7.1 X-ray diffraction pattern of typical sample of chloritized-vermiculite showing collapse to $10 \AA$ structure on heating to $350^{\circ} \mathrm{C}$.................... 107

Figure 7.2 Depth profile showing distribution of minerals in the clay fraction of the Blanton Soil Series from four horizons at locations BSS 33 and

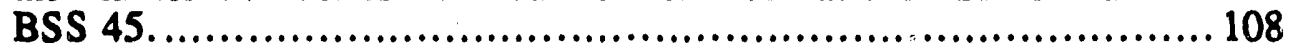

Figure 7.3 Depth profile showing the distribution of minerals in the clay fraction of the Fuquay Soil Series from four horizons at locations BSS 42 and BSS 44........................................................ 109

Figure 7.4 Depth profile showing the distribution of minerals in the clay fraction of the Lakeland Soil Series from three horizons at locations BSS 41 and BSS 51 .

Figure 7.5 Depth profile showing the distribution of minerals in the clay fraction of the Orangeburg Soil Series from four horizons at locations BSS 34 and BSS 46 .

\section{DISCLAIMER}

\begin{abstract}
This report was prepared as an account of work sponsored by an agency of the United States Government. Neither the United States Government nor any agency thereof, nor any of their employees, makes any warranty, express or implied, or assumes any legal liability or responsibility for the accuracy, completeness, or usefulness of any information, apparatus, product, or process disclosed, or represents that its use would not infringe privately owned rights. Reference herein to any specific commercial product, process, or service by trade name, trademark, manufacturer, or otherwise does not necessarily constitute or imply its endorsement, recommendation, or favoring by the United States Government or any agency thereof. The views and opinions of authors expressed herein co not necessarily state or reflect those of the United States Government or any agency thereof.
\end{abstract}




\section{GEOCHEMICAL AND PHYSICAL PROPERTIES OF SOILS AND SHALLOW SEDIMENTS AT THE SAVANNAH RIVER SITE}

\section{List of Appendices}

Appendix A Appendix B.I Appendix B.2 Appendix B.3 Appendix C.1 Appendix C.2 Appendix C.3 Appendix C.4 Appendix C.5 Appendix C.6 Appendix C.7
Background Soil Study Quality Control and Sampling and Analysis Plan Background Soil Sample Locations

ASTM Classification of Background Soil Samples

Modified Wentworth Classification of Background Soil Samples

Metal Data Summary

Other Inorganic Data Summary

Organic Data Summary

Radiological Data Summary

Particle Size Data Summary

Carbon Steel/Stainless Steel Data Summary

metaTRACE/Weston Laboratory Comparison Data Summary 


\section{Geochemical and Physical Properties of Soils and Shallow Sediments at the Savannah River Plant}

\subsection{Executive Summary}

A program to characterize the geochemical and physical properties of the unimpacted soils and shallow sediments at the Savannah River Site (SRS) has been completed. The maximum, minimum, median, standard deviation, and mean values for metals, radionuclides, inorganic anions, organic compounds, and agricultural indicator parameters are summarized for six soil series that were identified as representative of the 29 soil series at SRS.

The soils from unimpacted areas of SRS are typical of soils thound in moderately aggressive weathering environments, including the southeastern United Staies. The temperate climate and relatively high rainfall in the region result in leached soils that have low concentrations of metals. In general, metal concentrations increase writh depth and in proportion to the clay content of the soil. The upper soil horizons are generally more highly leached, and exhibit lower concentrations, than the lower horizons and the parent material. The mineralogy of the soils is dominated by quartz and the primary clay mineral found in SRS soils is kaolinite. A similar age, climate and moisture regime governed the formation of most of the various soils at SRS. The similarity of the conditions gover, iing soil formation minimizes the various geochensical delineations. A few notable exceptions were found (e.g., $\mathrm{Mn}$ in the Orangeburg soil series).

Appendix VIII organic compounds were detected in all samples. Since these constituents are not generally present in soil, this portion of the investigation was intended to assess possible laboratory artifacts. A total of six different compounds were detected in the samples, and not all of these parameters were detected in all samples. Of the six compounds deteited, four are commonly occurring laboratory artifacts and two may result from usage at SRS, or may be analytical artifacts.

An additional objective of the SRS Soil Study was to determine if the composition of the split spoon sampler biased chemical analy is of the the soils. Two adjacent sample borings were collected using split spoon samplers constructed of carbon steel and stainless steel. Only two constituents, $\mathrm{Cd}$ and $\mathrm{Pb}$, were found to be significantly different. In both cases, the absolute value of the variation was relatively small and would be unlikely to impact screening decisions based on the data.

Twenty-five duplicate samples were analyzed for a number of metals, radiological and agricultural parameters, and organics by two laboratories currently contracted with to analyze samples during waste site characterization. In all cases, the absolute values of the average differences are relatively small compared to the overall variability in the population. Nonetheless, the possibility of differences resulting from variation in analyzing laboratory should be considered if detailed statistical comparisons are made using the SRS Soil Study. 


\subsection{Introduction}

The Savannah River Site (SRS) is a nuclear production facility operated for the U. S. Department of Energy by Westinghouse Savannah River Company. A program to characterize the geochemical and physical properties of the soils and shallow sediments at SRS has been completed. This program provides baseline data to support regulatory decision making as well as to support a large number of research programs ranging from studies of ecology to studies of innovative soil and groundwater remediation technologies. The design of this SRS Soil Study was developed to meet a wide range of objectives in a most cost effective manner. The twenty-nine different taxonomic series of soils at SRS were organized into six groupings based on the descriptive properties of each series. Several samples of one soil series from each grouping were analyzed to determine the chemical, physical and mineralogical properties for the grouping.

The resulting data will be used to investigate the qualitative and quantitative impacts of SRS operations on the environment. and will assist in regulatory decision making related to waste site remediation. The results will also be used to evaluate natural or ecological processes that are governed by soil or shallow sediment properties. 


\subsection{Objectives}

The SRS Soil Study was designed to meet several specific objectives. These objectives were:

1) Determine the characteristics of SRS soils. Specific characteristics evaluated were trace element concentrations, major element concentrations, bulk chemical properties that may affect migration of chemicals through the soil (e.g. cation exchange capacity), indicator parameters (e.g., exchangeable base metals), physical properties (e.g., particle size distribution), and mineralogy. Specific constituents measured are listed below'.

Metals

Aluminum
Arsenic
Barium
Cadmium
Chromium
Copper
Iron
Lead
Lithium
Magnesium
Manganese
Mercury
Nickel
Potassium
Selenium
Silver
Sodium
Zinc
Organics
Total Organic Carbon
Total Organic Halogens

\section{Radiological Parameters}

\author{
Gross Alpha \\ Gross Beta \\ Strontium-90 \\ Uranium
}

Other Inorganic Constituents

Chloride
Cyanide
Fluoride
Nitrate
Nitrite
Phosphate
Sulfate

Agricultural Parameters

Cation Exchange Capacity (Looney et al.,1990) Exchangeable Acidity (Looney et al.,1990). Exchangeable Base Metals (Looney et al.,1990) pH (Looney et al.,1990)

2) Compare the chemical data for soils collected using split spoon samplers constructed of carbon steel vs. stainless steel to determine if the composition of the split spoon sampler results in a bias in the chemical analysis of the the soils.

3) Compare the chemical data for duplicate samples analyzed by the laboratories currently contracted to analyze samples during waste site characterization.

The analytical results from the SRS Soils Study were evaluated using multivariate statistical techniques to address three additional objectives that are discussed in a separate report (Looney et al., 1990). First, the raw data were analyzed to determine the statistical distribution of each consituent in order to use the appropriate methods to properly characterize censored data and to determine the most reasonable underlying distribution. Second, paradigms were developed to allow application of the baseline data to regulatory decision making. Two specific cases were addressed, (1) "is a constituent in a potential waste disposal area statistically different than background," and (2) "what is a reasonable 
background level to set as a goal for waste site excavation remedial actions that are based on background values rather than health based standards?". The final objective of the SRS Soils Study was to perform a mreliminary evaluation of the soil series most likely to be present at or near the sites currently identified in the Resource Conservation and Recovery Act (RCRA) Facility Investigation (RFI) program. These results along with statistically defensible screening levels are presented in Looney et al., 1990.

It is clear from the above list that both fundamental and pragmatic objectives were addressed in the SRS Soil Study. In a few cases, compromises were made to address the wide range of objectives in the nost efficient and justifiable overall manner. For example, to meet regulatory objectives, standard EPA protocols for sample extrartion ard handling were employed. Standard EPA protocols for soil samples require dissolution by nitric acid alone which may result in incomplete dissolution of soil samples especially mineral constituents. Soils generally are composed at least partially of mineral constituents: accessory minerals such as zircon, monazite, magnetite, etc., may constitute only a minor percentage of the bulk soil but may contribute significant concentrations of trace elements to the bulk analysis. These accessory minerals are particularly resistant to acid attack and would likely constitute part of the insoluble resicue. The ranges in measured concentrations of major, minor and trace elements reported in this study should be more variable than total concentrations generated by completely dissolving the soil. It should be recognized that samples prepared by other dissolution protocols may not be directly compared to the results of the SRS Soil Survey. Additionally, the number and type of samples and sample analyses were determined in such a way that each of the listed objectives was addressed in a cost effective manner. The results of the SRS Soil Study in terms of each of the listed objeztives are documented in the following sections. 


\subsection{Background}

\subsection{Review of Soil Formation and Classification}

While there are a wide number of possible definitions of the term soil, soil scientists and geologists define soil as (Rose et al., 1979):

"a natural body of mineral and organic constituents, differentiated into horizons, of variable depth, which differs from the material below in morphology, physical make-up, chemical properties and composition, and hiological characteristics."

Thus, the unique characteristic of soil is its organization into identifiable layers that are related to the present day land surface and change with depth. The term sediment is generally used to describe unconsolidated material beneath the soil; the sediment directly beneath the soil zone is often the parent material of the soil. Five interrelated soil forming factors determine the thickness and characteristics of the various layers in a soil profile. The five factors are parent material, climate, biologic activity, topography, and time. The thickness and characteristics of the resulting soil layers in turn determine the classification of the subject soil. Each of the soil forming factors and the aspects of soil taxonomy pertinent to SRS soils are reviewed below.

The parent material is the source of the bulk raw materials that form the soil. The influence of parent material on overlying soil properties is strongest in young soils, in the deeper horizons that grade into the parent materials, and in climates where chemical weathering is slow. In well developed soils, the influence of the parent material on a soil is subordinate to other factors. Even in these soils, however, the rnineralogical and trace element assemblages, as well as the rate of soil development, will be related to the chemistry and stability of the original parent material.

Climate is the most significant soil forming factor. In well developed soils, similar parent materials may form vastly different soils depending on climate. Similarly, different parent materials will form very similar soils when influenced by the same climate for sufficient time. In general, the soils in humid climates, such as SRS, tend to be leached and to posses iron rich lower horizons while soils in arid climates tend to be less leached in the surface layers. Temperature combined with the downward and upward movements of water, as influenced by precipitation and evaporation, will determine the dissolution and movement of soil components and deposition of dissolved salts. Climate is a key factor in the $\mathrm{pH}$, clay minerals, calcium, iron, organic matter, and trace metal composition of soils.

Biological factors in soil formation include the type of vegetation and decomposi: : on of plant debris carried out by soil microorganisms. The accumulation of humus is favored by humid climates (i.e., more vegetation) and cool temperatures (i.e., less decomposition). Warm climates restrict the accumulation of humus because of increased decomposition, but accelerate the action of the resulting organic acids on the lower soil layers. Different types of vegetation may have different root depths and may yield different decay products.

Topographic position and relief influence soil formation through interaction between drainage, erosion and ground water level. For example, soil horizons are most strongly differentiated in regions of moderate to high rainfall where there is free drainage and effective leaching. Such profiles develop in interstream areas at SRS. In low-lying areas, such as wetlands near streams and Carolina bays, the terrain may be saturated with water resulting in a significantly different soil profile. Under such circumstances, the profile often consists of an organic rich surface layer underlain by a pallid or mottled subsoil in which reducing conditions are present. If water stands at the surface, peat may develop. Soils on steep slopes tend to be thinner, show less distinct layer 
development and have a higher content of stony material than those on gentler slopes. In some areas, erosion may truncate a profile and move material downslope.

Accumulation of parent material and differentiation into soil horizons are slow processes. Soil development is a a time or rate dependent process, and generally, development of horizons progresses from development of a faint upper zone in time periods as short as decades to development of full profiles in time periods ranging from centuries to tens of thousands of years. Soils are sometimes classified as juvenile or mature by comparing their development to completely developed soil horizons under present-day surface conditions. Where erosion is active, soils will remain in a juvenile condition irrespective of time. If erosion proceeds no faster than soil formation, a mature profile will eventually result.

The soils that are formed by the five factors discussed above are characteristically organized into layers that differ from each other and the underlying parent material in properties and composition. Apart from differences in color and texture, the layers can differ in pH, organic matter, clay minerals, and inorganic chemical composition. Comparison of a given soil sample to a background soil sample is only reasonable if equivalent horizons within the soil profile are compared and the factors responsible for the formation of the two samples are similar. A typical soil profile is described below, along with the soil classification scheme that may be used to logically divide soils into groupings expected to have similar characteristics.

A soil profile is comprised of individual layers that are referred to as soil horizons and may range from a few centimeters to a meter or more in thickness. Profile development is primarily the result of vertical (upward and downward) movement of material in solution and suspension, accompanied by a complex series of chemical reactions. Water is the essential medium in which this transfer and reconstitution takes place.

Soil profiles vary within wide limits according to their genetic and geographic environment. Most well-developed profiles, however, can be divided into principal or master horizons. From the surface downward these are identified by the letters $A, E, B, C$, and $R$ `. The A, E, and B horizons constitute the solum, or "true soil", while the $C$ horizon is the partly weathered parent material from which the solum has been derived by soil-forming processes, and the $R$ horizon is the underlying rock material. A horizon of nearly pure organic matter $(0)$ may lie above the $A$ horizon. A hypothetical soil profile is shown diagrammatically in Figure 4.1. Master soil horizons are shown in Figure 4.2.

the entire soil sequence is not always represented. For instance, immature soils frequently lack a B horizon, or erosion may lead to truncated profiles, sometimes to the extent of exposing the $C$ horizon. When studied in detail, each of the principal horizons may be further subdivided. These subdivisions are identified by combined letters and numbers, e.g., A, E, B1, B2, B3, etc (Figure 4.1). Recognition of these subdivisions, apart from the A and E horizons, is usually unnecessary in a geochemical studies. The distribution of metals may vary markedly with major changes down the profile. It is therefore important to distinguish the master horizons and to recognize immature and truncated profiles when these are encountered (U.S.D.A., 1981).

The $\mathrm{A}$ and $\mathrm{E}$ horizons are characterized by a process of leaching known as eluviation, meaning "to wash out", with maximum eluviation taking place in the E horizon. Eluviation is accomplished by the downward percolation of water through the soil. Some constituents are removed as ions or molecules in solution in the downward-moving water, others are removed as dispersed colloidal particies. The leaching of the A horizon may be accelerated by organic acids.

Under moist conditions and free drainage, soluble constituents will be carried to the water table. Some suspended matter may follow the same path. More of ten, however, colloidal silicates, oxides and organic matter, as well as some dissolved constituents will be deposited in the $\mathrm{Bt}$ 
horizon (t signifying the accumulation of clay), a zone of accumulation or illuviation (meaning "to wash in"). Thus, the Bt horizon tends to be enriched in clay and oxides and, in well aerated upland soils, assurnes a red or yellow-brown color.

The level at which iliuviation takes place is dependent on acidity, infiltration rate, hydrologic conductivity of the soil, rainfall, and climate; in fact, all of the soil forming factors influence illuviation either directly or indirectly.

The $\mathrm{C}$ horizon consist; of variably weathered parent material and lacks evidence of biological activity. Material compriling the $C$ horizon on the SPS has been deposited by marine and/or fluvial activity. There is evidence ihat only a few million years ago a large stream, perhaps the Savannah River, cut across the site and flowed into or close to where the Salkehatchic River presently enters what is known as St. Helena Sound. The soils at the SRS have been weathered, formed, and moved many times and represent the summation of an active history of soil formation. As a rule, inorganic decomposition extends below the depth that is routinely described in the classification of soils. In most areas, the $C$ horizon could be subdivided further into weathering zones that decrease with depth. The $C$ horizon has little or no organic matter, no structural development, little or no evidence of illuvial clay, and often has stratified sands and clays indicating sediment deposition by varied velocities of water flow. As a rule, inorganic decomposition extends deeper than soil formation, and the $\mathrm{C}$ horizon can often be subdivided into zones of weathering that decrease in intensity with depth. Organic matter is at a minimum in the C horizon, which usually contains less clay.

Soils are divided into categories based on characteristics related to the factors discussed above. The prefixes and suffixes in the taxonomic names provide information about the five soil forming factors: climate, topography, parent material, time, and biological activity. At the broadest level of classification in soil taxonomy, soils are divided into ten Orders determined largely by the presence of one or more of the diagnostic horizons. The ten Orders are listed in Table 4.1, along with their diagnostic features. Diagnostic horizons are the key to separation and classification of Orders, and are potentially important in recognizing the nature of trace-metal concentrations. For example, oxisols are highly leached soils, and altisols are less leached than ultisols. Ultisols are old mineral soils having an illuvial horizon $(\mathrm{Bt})$ of silicate clays, low base saturation, and subject to rapid leaching. Entisols are young mineral soils with weak or no pedogenic horizons or an old soil that has mostly inert parent material such as quartz. The five Orders that are found at the SRS are identified in Table 4.1.

Orders are subdivided into Suborders on the basis of climate, drainage, or other distinctive features or properties. Names for Suborders are formed from a prefix denoting the "formative element" (Table 4.1) and a suffix denoting the Order. For example, an aquod is a water-saturated (aqu-) spodosol (-od). About 50 Suborders are recognized. Successively more detailed subdivisions are Great groups, Subgroups, Families, and Series. Great groups are formed by adding a prefix to denote diagnostic horizons or variants of horizons. The names of Subgroups are formed by an adjective preceding the name of the Great group to indicate its gradational position in relation to the neighboring Great groups. Family names add more adjectives to indicate properties important to plant growth. For example, a Family name for a given soil might be fine-loamy, siliceous, thermic family of aquic Kandiudults. This is a soil having 18 to 35 percent clay content, more than 90 percent silica minerals, and a mean temperature of $15^{\circ}$ to $22^{\circ} \mathrm{C}$ at $50 \mathrm{~cm}$ below the surface. This soil is moderately well drained or water may perch in the profile at 50 to $75 \mathrm{~cm}$ below the surface for the duration of a few months in most years, has a CEC below $16 \mathrm{meg} / 100 \mathrm{~g}$, has a clay rich (Bt) horizon, is an old well developed soil (ult), formed in a humid region, and has few remaining mineral phases. From the SRS perspective, this soil has typically a low capacity to hold complex cations within the soil. 
The soil series is the most detailed and most specific part of the caxonomic system. A series must come from a particular type of parent material and must have similar horizon development. The soil surface may differ so series are further divided into soil type by adding the surface texture, for example, Orangeburg loamy sand. There are about 29 series correlated in the SRS soils report, and approximately 30 more series of such limited areal extent that they were not identified in this report. There are about 14,000 different soil series in the U.S. 
Table 4.1 Summary of Taxonomic Terms Applicable to Soils SOIL OR DERS AND SUFFIXES

\begin{tabular}{|c|c|c|}
\hline $\begin{array}{l}\text { SOIL ORDER AND } \\
\text { SUFFIX TO FORM } \\
\text { NAME OF } \\
\text { SUBORDER } \\
\end{array}$ & $\begin{array}{l}\text { LATIN OR OTHER } \\
\text { ROOT }\end{array}$ & DESCRIPTION \\
\hline \multicolumn{3}{|c|}{ SOIL ORDERS FOUND AT SRS } \\
\hline Entisols(-ent) & Recent & $\begin{array}{l}\text { Mineral soils without significant profile development, with little } \\
\text { modified parent material, and no deep wide cracks most years. }\end{array}$ \\
\hline Inceptisols(-ept) & $\begin{array}{l}\text { L. inceptum, } \\
\text { beginning }\end{array}$ & $\begin{array}{l}\text { Mineral soils, some pedogenic horizons and some weatherable } \\
\text { minerals, moisture availabie to mature a crop most years, no } \\
\text { horizon of illuvial clay, relatively low in either organic matter or } \\
\text { base saturation, or both. }\end{array}$ \\
\hline Spodosils(od) & $\begin{array}{c}\text { L. spodos, wood } \\
\text { ash }\end{array}$ & $\begin{array}{l}\text { Mineral soils, an illuvial horizon of amorphous aluminum and } \\
\text { o ganic matter, with or without amorphous iron. Only small } \\
\text { areas on SRS are in this vider. }\end{array}$ \\
\hline Ultisols(ult) & L. ultimus, last & $\begin{array}{l}\text { Mineral soils, an illuvial argillic or kandic horizon of silicate } \\
\text { clays, low base saturation, old well developed profile. }\end{array}$ \\
\hline Histosol (-ist) & L. histos, & $\begin{array}{l}\text { Soils with organic material comprising more than half of the } \\
\text { upper eighty centimeters. }\end{array}$ \\
\hline \multicolumn{3}{|c|}{ SOIL ORDERS NOT FOUND AT SRS } \\
\hline Alfiso! & Al-Fe & $\begin{array}{l}\text { Soils with an argillic, kandic or natric B horizon, base saturation } \\
\text { >35\%; moist part of the time; lack calcic, gypsic, or mollic } \\
\text { horizons. }\end{array}$ \\
\hline Oxisol (-ox) & Oxide & Soils with an oxic B horizon. \\
\hline Aridosol (-id) & L. aridus, dry & $\begin{array}{l}\text { Soils with an ochric } A \text { horizon and high base saturation,may } \\
\text { have calcic, gypsic, argillic, kandic, or natric B horizons. }\end{array}$ \\
\hline Mollisol (-oll) & L. mollis, soft & Soils with a mollic $\mathrm{A}$ horizon and high base horizon. \\
\hline Vertisol (-ert) & L. berto, change & $\begin{array}{l}\text { Soils with a high content of sweiii ig clay,forming wide cracks } \\
\text { when dry, into which surface soil falls or is washed. }\end{array}$ \\
\hline
\end{tabular}


Table 4.1 (Cont.)

Summary of Taxonomic Terms Applicable to Soils SRS Soil Study

\section{PREFIXES USED IN NAMING SUBORDERS AND GREAT GROUPS PRESENT AT SRS}

Aqu-

Arg-

Dys-, Dystr-

Fluv-

Kan-

Hapl-

Hum-

Med-

Ochr-

Pale-

Psamm-

Quartz

Sapr-

Ud-

aeric

aquic

arenic

cumulic

fluventhic grossarenic plinthic

typic
Soils that are wet for long period (Suborder)

Soils having an illuvial horizon of silicate clay accumulation (Great group)

Soils with low base saturation (Great group)

Recent soil material deposited by streams (Suborder)

Soils with CEC $<16 \mathrm{meq} / \mathrm{g}$ clay and ECEC $<12 \mathrm{meq} / \mathrm{g}$ clay (Great group)

The simplest set of horizons (Great group)

Soils with appreciable amounts of humus (Suborder or Great group)

A soil of midlatitudes (Great group)

Snils with little organic matter, light colored or both.(Suborder)

A soil having more horizon development than normal (Gieat group)

Sandy soils to a depth of 1 meter or more (Suborder or Great group)

Soils with more than ninety-five percent quartz (Great group)

Soils with highly decomposed plant fibers (Suborder)

Soils of humid climates where the soil is moist but not wet and dry for short periods or not at all (Suborder or Great group)

\section{OTHER MODIFIERS USED TO DESCRIBE SOILS AT SRS}

Less gray and better aerated than is typical of subgroup -- used to show variation from the typical subgroup

More water in soil than is typical of the subgroup -- used to show variation from the typical subgroup

Sandy eluvial horizon (sand or loamy sand), mostly between fifty and one hundred centimeters thick

An epipedon with a greater accumulation of humus than is typical of the humic epipedon.

Sandy eluvial horizon (sand or loamy sand) $>1 \mathrm{~m}$ thick

Presence of diagnostic amounts of plinthite, an iron-rich material that hardens irreversibly upon exposure to wet and dry conditions

Characteristic typical of the specific Great group 


\section{Table 4.1 (Cont.)}

Summary of Taxonomic Terms Applicable to Classification of Soils into Taxonomic Families for the SRS Soil Study

\section{PARTICLE SIZE MODIFIERS}

Sandy The texture of the fine earth is sand or loamy sand and rock fragments comprise less than $35 \%$ by volume.

Loamy The texture of the fine earth is loamy, very fine sand or finer, the amount of clay is $<35 \%$; rock fragments comprise $<35 \%$.

Coarse loamy Fifteen percent or more of the particles are fine sand or coarser, including fragments up to $7.5 \mathrm{~cm}$. in. diameter

Fine-loamy Fifteen percent or more of the particles are fine sand or coarser, including fragments up to $7.5 \mathrm{~cm}$. in. diameter, 18 through 35 percent clay in the fine earth fraction.

Coarse-silty Fifteen percent of the particles are fine sand or coarser, including fragments up to $7.5 \mathrm{~cm}$. in. diameter, $<18$ percent clay in the fine earth fraction.

Fine-silty Fifieen percent of the particles are fine sand or coarser, including fragments up to $7.5 \mathrm{~cm}$. in. diameter, 18 - 34 percent clay in the fine earth fraction.

Clayey The fine earth contains $\geq 35$ percent clay, and rock fragments are $<35$ percent .

Fine A clayey particle-size class for soils having 35 through 59 percent clay in the fine earth fraction.

\section{MINERAL CONTENT MODIFIERS}

Siliceous More than ninety percent by weight of silica minerals and other durable minerals that are resistant to weathering.

Mixed Soils that have $<40$ percent of any one mineral other than quartz or feldspar

Kaolinitic More than half kaolinite, tabular halloysite, dickite, nacrite by weight and smaller amounts of 1:1 or minor nonexpanding 2:1 layer minerals

\section{OTHER MODIFIERS}

Acid

Dysic

Nonacid

Coated

Soil with a pH $<5.0$ (in $\mathrm{CaCl}$ ) throughout the control section

Soil with a pH $<4.5$ (in $\mathrm{CaCl} 2$ ) in parts of organic material in the control section

Soil with a $\mathrm{pH}>5.0$ (in $\mathrm{CaCl} 2$ ) in a part of the control section

Soil in which sand grains are coated with a sufficient amount of clay to increase the moisture equivalent to two percert

Thermic Soil with a mean annual soil temperature form $15^{\circ}$ to $22^{\circ} \mathrm{C}$ at a depth of $50 \mathrm{~cm}$. 
SOIL SURVEY MANUAL, 1951

MAY 1962 SUPPLEMENT
岂

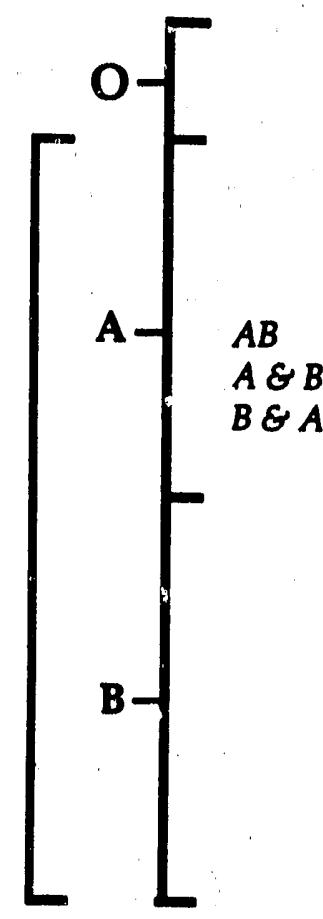

SOIL SURVEY MANUAL

JUNE 1981

MINERAL ORGANIC

O1 Oi, Oe

$\mathrm{O} 2 \mathrm{Oa}, \mathrm{Oe}$

A

A1

$\mathrm{A} 2$

A2

A3

B1

B1

B2

B3

C

$\mathbf{R}$

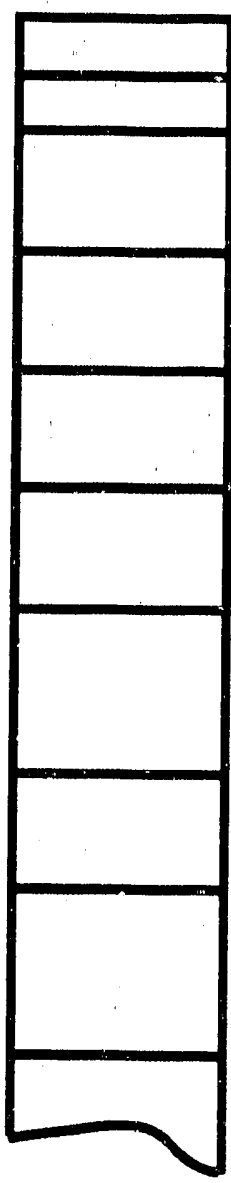

$\sum_{0}^{5}$

$\mathrm{B}$ or $\mathrm{Bw}$

$\mathrm{BC}$ or $\mathrm{CB}$

C

$\mathbf{R}$

Figure 4.1 Recent Changes in Master Horizon Designations (after: U.S.D.A. Agency for International Development, Department of Agronomy, Cornell University, October, 1986) 


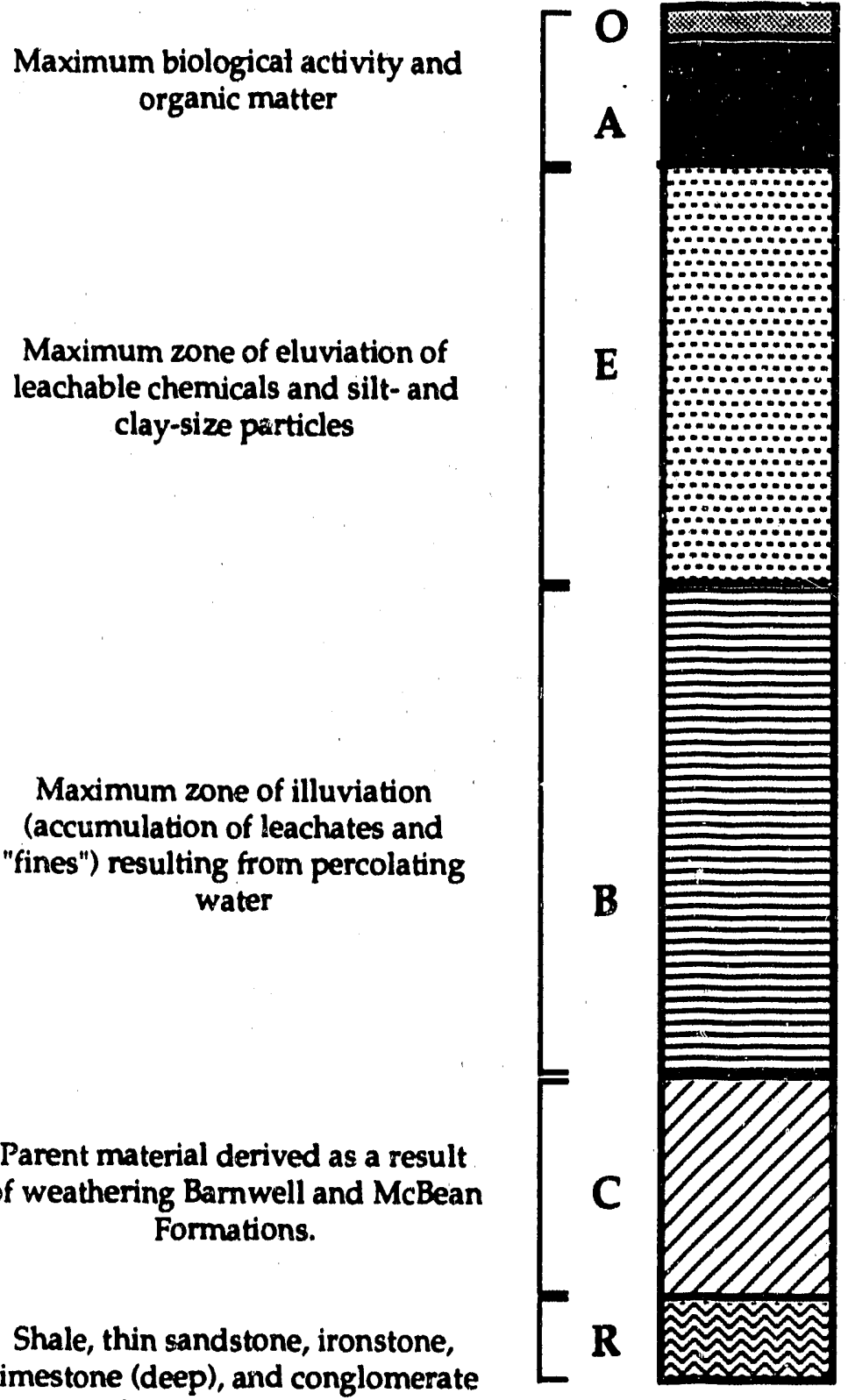

Organic debris, partially decomposed.

Mineral soil with traces or stains of humus size organic matter

Light-colored horizon of maximum eluviation. Prominent in some soils, faint or absent in others. Generally loose structure.
Horizons are in shades of red to brown, may have mottles in shades of yellow, gray, red or brown; accumulation of silicate clays; structure is subangular, blocky to massive, cemented and brittle in some soils

Lacks structure development and is relatively void of biological activity.

Bedrock of varying hardness

Figure 4.2 Hypothetical Soil Profile Showing The Principal Horizons And Potential Occurrence On The Savannah River Site

(Modified from U.S.D.A. National Soil Survey Manual, 1981). 


\subsection{Methods}

Several categories of activities were integrated to meet the overall objectives of the SRS Soil Study. These activities included:

1) Review existing data and define archetype soil series.

2) Collect and analyze samples using protocol methods. Compare sampling methods and laboratories using duplicate samples.

3) Summarize the data for the various series, as well as for areas with disturbed, missing, or filled soil profiles.

\subsection{Study Design}

To meet the fundamental and pragmatic objectives on the SRS Soil Study, logical groupings of soils were developed based on taxonomic similarities. The number of analyses was determined based on a balance of statistical requirements and cost. Thus, the goal of the study design was to describe as much of the plant area as possible within a reasonable budget and tinve.

As shown on Table 5.1, there are twenty-nine soil series identified at SRS (Soil Conservation Service, 1989). Since testing all twenty-nine series was not feasible, some of the soil series were eliminated and the remaining series were grouped so that the data would be as representative as possible. For example, it is unlikely that a waste site would be located on wetland soils such as Fluvaquents and Pickney. Restricting the study to the upland series reduced the number of series to be tested from twenty-nine to fifteen. By using the taxonomic class for these fifteen soil series, we were able to come up with six broad soil groupings. These six groupings cover approximately 75.4 percent of the site (Table 5.1). A single representative, soil series from each of the six groupings was sampled. The data were analyzed and presented for the reference series in each grouping; the characteristics of the other soil series in the various groupings are expected to be similar to the pertinent reference series. A summary of taxonomic groupings applicable to upland SRS soils is presented in Table 4.1.

Blanton sand is the predominant soil series on SRS. It covers 21 percent of the plant site. The Blanton soil series was chosen as the central soil series in grouping one as the remaining soils in grouping one had the same or similar properties of Blanton. The taxonomic name for the Blanton soil series (Table 4.1) indicates that the soil is composed almost entirely of silica or other weather resistant minerals. Also, the sandy layer is greater than one meter thick, the soil is very old and well developed with a very low base saturation. The Troup soil series, Albany soil series, Wagram soil series, and Lucy soil series were also placed into grouping one. The Albany and Troup soil series are nearly a perfect match for Blanton soil series although both the Albany and Troup have a higher water table than Blanton. ' he Lucy soil series was placed in this grouping because large areas of Troup are intermixed with Lucy. Also, the Lucy soil series shares most of the same characteristics except that it is arenic rather than grossarenic indicating that the sandy layer is not quite as thick. The Wagram soil series shares the same properties as Lucy soil series.

The central soil series in grouping two is the Orangeburg series. This soil type covers 3.4 percent of SRS. The taxonomic name for Orangeburg (Table 5.1) suggests that this soil consists of at least 15 percent sand and approximately 18 to 35 percent clay. Like the Blanton soil series, it is composed almost entirely of silica or other weather resistant minerals. This seri $s$ is characterized by a well developed mineral soil with an illuvial horizon that is loamy and siliceous. The Orangeburg soil series has a low base saturation. The Norfolk soil series (Table 5.1) was 
placed into grouping two because Norfolk and Orangeburg share the same taxonomic name. The grouping two series covers about 5 percent of the SRS.

The second most common soil type on SRS is Fuquay soil series. This series encompasses roughly 11.3 percent of the site, therefore, it was made the central series of grouping three. The taxonomic name for Fuquay (Table 5.1) indicates that it is composed of less than 35 percent clay material and is almost entirely silica or other weather resistant minerals. The sandy layer is between 20 and 40 inches deep. The Fuquay soil series contains more than 5 percent plinthite, an iron-rich matcrial that hardens irreversibly upon wetting and drying. The series is very well formed and has a low base saturation. The other soil series that was grouped with Fuquay was Dothan. The Dothan and Fuquay soil series differ only in particle size and in the thickness of the sandy surface and subsurface horizons. The most important characteristic of both soils series is that they are the only plinthic soils found at SRS. Grouping three soils cover 19.2 percent of SRS.

The central series in grouping four is the Vaucluse soil series. This soil series includes the Vaucluse-Ailey complex. This series covers 6.9 percent of SRS. The taxonomic name for Vaucluse (Table 5.1) indicates that the soil was very well developed with a simple set of horizons. It is at least 15 percent sand and approximately 18 to 35 percent clay. Over 90 percent of the soil is either silica minerals or other weather resistant minerals. The Ailey soil series has similar characteristics to Vaucluse. The differences between Ailey and Vaucluse is the thickness of the sandy surface and subsurface layers. Since Vaucluse and Ailey are very often intermixed, it is very hard to find an area that is predominantly one or the other.

Lakeland sand is the only soil series at SRS that is classified as a quartzipsamment (deep sand) and therefore is the only soil series in grouping five. The taxonomic name for Lakeland (Table 5.1 ) indicates that the soil is composed of coated sands to depths of 2 meters or more. It is an old soil but has very weak or no pedogenic development due to the slow weathering of the quartz parent material. Lakeland covers 4.3 percent of SRS.

The sixth and final grouping is the Udorthents which include firm and friable substratum along with paved streets, buildings, etc. This grouping is difficult to define because the diagnostic horizons used to classify soils have been destroyed or have been rearranged with heavy equipment to the extent that the horizons cannot be identified. Udorthent areas may be found at the bottom of a borrow pit or a spoil of soil layers from a borrow pit that may be adjacent to or up to a few miles from the area in which the material had formed pedogenic horizons. Within a few feet, the properties of Udorthent soil can change dramatically. Thus, wide variability is expected within the Udorthents classification. Udorthents cover 3.9 percent of SRS.

The groupings of SRS series are summarized in Table 5.1. Within these groupings, the selection of horizons for sample collection was based on major descriptive variations within the specific soil series. When correlating the data to a grouping series, the correct layer should be used. A summary of the layers sampled is presented in Table 5.2. The samples came from the central soil series of each grouping with a wide distribution in the location of the sample sites. The sample locations and descriptions are shown in Figures 5.1 through 5.6 and Table 5.3. Approximately ten percent of the samples were randomly replicated at an alternate laboratory for $\mathrm{QA}$ purposes. Approximately ten percent of the total sample intervals were tested for the carbon steel vs. stainless steel comparison.

\subsection{Sample Collection}

Soil samples were collected from target intervals at each soil boring location. These target intervals varied for each soil grouping and at each boring location. Five of the six archetypal soil groupings were comprised of a identifiable set of soil horizons (Table 5.2). The sixth archetypal soil grouping (Udorthents) did not exhibit a consistent soil horizon profile, therefore target 
intervals were located at pre-determined intervals below the surface. When the target interval corresponded to a specific soil horizon, the horizon was confirmed based on a visual comparison with the written soil grouping description.

Soil samples for non-volatile organic analysis were collected by homogenizing the soil from the arget interval in a stainless steel mixing bowl with stainless steel utensils. The soil in the mixing bowl was then divided into quadrants. One aliquot of sample from each quadrant was collected using a stainless steel spoon and placed in the appropriate containers. Following collection of the subsample from each of the four quadrants, the remaining sample was rehomogenized and the process repeated until all of the containers were filled. The containers were then sealed, labeled, and placed in a pre-cooled ice chest. Residual soil from the target interval was placed in core boxes for storage.

Soil samples collected for volatile organic compound analysis were not homogenized in order to minimize the loss of any volatile compounds present. Following confirmation of the proper sample horizon, a sample aliquot was immediately collected from the midpoint of the target interval. The sample was then placed in the containers such that no headspace remained. The containers were sealed immediately, labeled, and placed in a pre-cooled ice chest. Residual soil from the target interval was placed in core boxes for storage.

\subsubsection{Split Spoon Samples}

Samples for chemical analysis were collected with a split spoon at all soil boring sites. Samples were collected with either 2 inch outside diameter or 3 inch outside diameter stainless steel or carbon steel split spoon samplers depending on the volume of sample required to fill the containers. Each split spoon sample was collected according to the following procedure:

1) The sampler was decontaminated as specified in Section 5.2.3.

2) The split spoon was driven into the subcurface with a 140 pound hammer falling 30 inches.

3) Sample aliquots were removed from the split spoon sampler using a decontaminated stainless steel spatula or spoon and placed in the appropriate prelabeled containers or mixing bowls.

4) The samples were immediately stored in a pre-cooled ice chest at $4^{\circ} \mathrm{C}$.

5) If the sample volume collected from the split spoon was insufficient for the required analyses, additional samples were collected immediately adjacent to the original location at the same depth interval(s).

6) The sample logbook and chain of custody form were then completed.

At eight locations, two borings were advanced side by side, approximately 2 to 5 feet apart. Carbon steel split spoons were used to collect samples in one boring while stainless steel split spoons were used to collect samples from the same target intervals in the adjacent boring. These samples were analyzed for various metals in order to determine if the composition of the splitspoon sampler biased the results of analyses of the soils. 


\subsubsection{Grab Samples}

If the upper soil horizon was between 0 and 18 inches in depth, the sample was collected with a stainless steel hand trowel. Near surface soil samples collected in this manner were considered grab samples. Procedures for collection of these near surface soil samples were as follows:

1) The sampling tool (stainless steel hand trowel) was decontaminated as specified in Section 5.2.3.

2) The soil was excavated to a maximum of 6 inches with the hand trowel.

3) The sampling tool was decontaminated again according to the procedures outlined in Section 5.2.3.

4) Sample aliquots were placed in the appropriate pre-labeled containers or a stainless steel mixing bowl. The sample location was extended radially from the sample origin in order to obtain the necessary volume.

5) The sample logbook and chain of custody were completed.

Shallow grab samples that were tested for volatile organic compounds were collected by excavating the soil to the midpoint of the upper horizon. Soil from above the midpoint was placed in the stainless steel mixing bowl for homogenization. Suil from immediately below the mid-point was placed in a sample containter for volatile organic compounc analysis. The container was filled such that no headspace remained. The cortainer was then sealed, labeled, and placed in a precooled ice chest.

\subsubsection{Drilling Equipment Decontamination}

The drill rig and equipment were cleaned to remove excess grease, oils, or caked-on soils from previous work, upon arrival at each site. The work area of the drill rig, all downhole tools, splitspoon sampler, and subsampling instruments were decontaminated before each sample was taken in accordance with the following procedures:

1) Clean with tap water and phosphate-free laboratory detergent using a brush if necessary to remove particulate matter and surface films.

2) Rinse thoroughly with tap water.

3) Rinse thoroughly with distilled or deionized water.

4) Rinse two times with pesticide grade isopropanol solvent and allow to air dry for as long as possible.

5) Rinse thoroughly with distilled or deionized water and allow to air dry for as long as possible.

6) Wrap with aluminum foil, if appropriate, to prevent contamination if equipment is going to be stored or transported.

An alternate decontamination procedure consisted of steam cleaning instead of Steps 1 through 3 above. Decontamination continued with Steps 4 through 6 . Personnel involved with the decontamination procedures wore surgical inner gloves with nitrile outer gloves which were washed with soapy water and rinsed with distilled or deionized water immediately prior to handling 
any equipment used in sampling. After steam cleaning, tools and equipment were stored on plastic sheeting to avoid contamination.

\subsection{Sample Analysis}

Chemical and physical analyses were performed on soil samples collected during the SRS Soil Study. The analyses performed included metals and inorganic ions, volatile and semi-volatile organic compounds, radionuclides, cation exchange, and mineralogy. These parameters are listed on Tables 5.4 and 5.5 for metaTRACE and Weston (quality control laboratory) laboratories, with corresponding reference methods. The parameters selected were intended to characterize the levels of naturally occurring trace elements and to determine physical, chemical, and mineralogical parameters for use in calculating the attenuation of contaminants.

Agricultural parameters were measured in an attempt to assess the bulk geochemical character of the soils. The agricultura' parameters inciucie cation exchange capacity (CEC), exchangeable base metals, exchangeable acidity, and pH. Cation exchange capacity (CEC) is a measure of the capacity of the soil to retain elements on the ion exchange sites associated with particle surfaces. The CEC is largely a function of the clay content and the organic matter content of the soil. Therefore, small changes in clay or organic matter content of the soil may have significant influences on the chemistry of the soil profile. The mineralogy of the clay fraction alsu has a significant affect on CEC; specifically, kaolinitic soils have much lower CECs than illitic or smectitic. The relative dominance of acidic or basic cations in the soil is expressed by the base saturation percentage as a proportion of the CEC. Base saturation is defined as the sum of the exchangeable basic cations, calcium, magnesium, potassium, and socium. Exchangeable acidity is defined as the sum of the acidic cations, i.e., aluminum and hydrogen. The cation exchange capacity then represents the sum of the exchangeable base metals and exchangeable acidity of a soil. The parameter $\mathrm{pH}$ is another important property of soil in that it affects the availability and transport of the various elements. At a low $\mathrm{pH}$, for example, the acid cations are predominant and basic cations are relatively less available.

The majority of the analytical procedures follow reference methods specified in EPA document SW-846, entitled "Test Methods For Evaluating Solid Wastes," Third Edition. Reference methods for the agricultural and radiological parameters were taken from other standard procedure manuals, as specified on the table. With two exceptions, all analyses were conducted by metaTRACE Laboratories. Weston Analytics performed analyses of split samples collected for quality control purposes and X-ray diffraction analysis was performed by Conoco. Quality control samples were denoted by $a$ " $C$ " in the sample identification number field of the data summary in Appendix D.

The thirty-two samples from several horizons in representative soil series were characterized by $X$-ray diffraction analysis at Conoco Research. $X$-ray diffraction analyses of whole soils and clay separates were used along with modeling of the diffraction patterns to identify the minerals present and to quantify their abundances. Samples were prepared and analyzed using the procedure outlined below.

An aliquot of approximately 3 grams of sample was oven dried and then ground to less than 32 micrometers in a uniform particle size grinder. The powder was side packed into sample holder for $X$-ray diffraction analysis on a Siemens D-500 diffractometer. The analysis was done be step scanning in $0.02^{\circ}$ iririements over the range of 2 to $52^{\circ} 20$ with 0.6 seconds counting time at each step. The diffracton eter was equipped with a $1^{\circ}$ divergence slit and $0.15^{\circ}$ receiving silts. Quantification was done by matching peak intensities against files of standard minerals for each phase identified in the sample. The quantification of quartz with this system is very good, however, the clay fraction of the whole-soil samples is relatively poor with an estimated error of \pm 20 percent of the value given. A normalization procedure at the end of the quantification routine was used to spread this error over other values in the sample. 
For clay mineral analysis approximately ten grams of sample were dispersed in distilleddeionized water in a $250 \mathrm{ml}$ centrifuge bottle, Samples which could not be completely dispersed were washed by centrifuging and re-dispersed. The fraction of the sample less than 2 micrometers in diameter was then separated by centrifugation at a fixed speed for a known length of time. Immediately after the separation, the supernatant was filtered through a 0.45 micrometer Millipore filter. The clay film from the filter was transierred to a glass slide by pressing the filter on the slide and peeling off the filter. The sample was allow to air-dry prior to the first $x$-ray diffraction analysis. All of the samples were subjected to four hours of exposure to ethylene glycol vapors at $70^{\circ} \mathrm{C}$ prior to the second $x$-ray diffraction analysis. Heat treatment of some of the samples at $350^{\circ} \mathrm{C}$ for one hour was required to identify the vermiculite by collapsing the interlayer structure. The $x$-ray diffraction was carried out as above except that only the range from 2 to $32^{\circ}$ was scanned. Several samples were run using 4 second/step counting times to enhance the signal to noise ratio and provide more definitive peak profiles for quantitative analysis.

A comparison of stainless steei and carbon steel split spoon samplers was also performed during this study. Samples were collected using each type of split spoon from identical soil intervals at adjacent boring locations. All samples collected with a carbon steel split spoon sampler were denoted by an "A" suffix in Appendix D, and those collected with a stainless steel split spoon were denoted with a "B" suffix. Soil samples collected for comparison were only tested for the presence of metals.

A summary of parameters tested, and a list of field, duplicate and stainless/carbon steel samples collected is specified in Tables 5.6 through 5.10 for each soil grouping.

\subsection{Statistical Methods}

Statistical interpretation of environmental data has been addressed by investigators in a variety of disciplines. As a result, a large number of useful techniques have been developed to answer specific questions. A description of the various statistical methods used to answer specific questions about the soils at SRS is presented below.

The procedures for comparing two statistical populations are of two types: those for paired data, and those for independent data sets. Examples of paired data are (i) measurements of two pollutants on each of $n$ field samples, (ii) measurements of a pollutant on air filters collected at two adjacent locations for $n$ time periods, and (iii) measurements of a pollutant on both leaves and roots of the same $n$ plants. Independent data sets are those for which there is no natural way to pair the data. For example, if $n$ soil samples are collected at each of two hazardous waste sites, there may be no rational way to pair a pollution measurement for one site with a pollution measurement for the other site.

Within the SRS Soil Study, two sampling investigations were conducted where inferences about the differences between two sampled populations are required. In the first investigation, soil cores were taken adjacent to each other using stainless steel and carbon steel samplers. The objective was to determine whether or not either of the two samplers contribute to any of the measured constituents and/or parameters. The second investigation was to compare chemical data from duplicate (split) samples analyzed by two separate laboratories currently under contract at SRS. Both scenarios discussed above represent "dependent sampling" in that paired samples were taken at randomly selected locations. This type of sampling is a requirement for a paired difference experiment which is often advantageous for comparison of independent populations. The objective of the comparison study is to determine if a difference existed between the true means of the sampled populations. 
A paired difference experiment can provide more information about the difference between population means than an independent sample experiment. The paired difference procedure removes the variability due to the dimension on which the observations are paired (e.g., the natural variability of concentrations across soil types and layers for the carbon/stainless steel test). The removal of the variability due to this extra dimension is called blocking, and the paired difference experiment is a simple example of a randomized block experimint. However, half the degrees of freedom are sacrificed in the $t$ statistic when a paired difference design is used instead of an independent sample design. This is a loss of information, and unless this loss is more than compensated for by the reduction in variability obtained by biocking (pairing), the paired difference experiment will result in a net loss of information about the difference in the true means. In the background soil study, the pairing significantly reduces the variability, thus blocking was used.

To use the paired difference $t$-test, the differences known as residuals must follow the normal distribution. The differerices between the paired measureinents are used to make inferences about the mean of the population of differences, which is equal to the difference between the true means of the two sampled populations. For example, the mean of the sample population of differences equals the difference between the population means. Thus, the null hypothesis is that the difference in the true means is zero. The alternate hypothesis being that a difference does exist betwee/, the two means. The test statistic is a one-sample $t$, since we are now analyzing a single sample of differences. The test statistic, $t$, is found by the following

$$
t=\frac{\overline{x_{d}}}{s_{d}} \sqrt{n_{d}}
$$
where

$\begin{array}{lll}\overline{x_{d}} & = & \text { Sample Mean Difference } \\ S_{d} & = & \text { Sample Standard Deviation Of Differences } \\ \text { nd } & = & \text { Number of Differences }\end{array}$

To find the rejection region, the probability of rejecting the null hypothesis, a, must be chosen. Then, the null hypothesis is rejected if $t<-t_{a} / 2$ or $t>t_{2} / 2$ where $t_{a} / 2$ is based on (nd - 1) degrees of freedom. The assumptions for this test are 1) the population of differences is approximately normally distributed and 2) the sample differences are randomly selected from a population of differences.

When the assumption of a normal distribution for the population of differences is not satisfied as required for use of the t-test, the Wilcoxon Signed Rank Test or another of the paired difference tests may be appropriate. Some of these are distribution-free or non-parametric statistical tests (i.e, they do not require the assumption that the observations themselves or their averages have a normal distribution). When data are paired, we can compare the two populations by looking at the sign or the magnitude of the set of $n$ differences $D_{i}=x_{2} i-x 1 i, i=1,2,3 \ldots, n$. The sign test uses the signs and the Wilcoxon signed rank test uses the magnitudes. The sign test can be used no matter what the underlying distribution may be. The test is simple to iompute and can also accommodate a few ND (not detected) concentrations. It is more versatile than the Wilcoxon signed rank test since the latter test requires that the underlying distribution be symmetric (though not necessarily normal) and that no NDs be present. The sign test statistic, $B$, is the number of pairs $\left(x_{1 i}, x_{2 i}\right)$ for which $x_{1 i}<x_{2 i}$, that is, the number of positive differences $D_{i}$. The magnitudes of the $D_{i}$ are not considered; only their signs are. If any $D_{i}$ is zero so that $a+$ or - sign cannot be assigned, this data pair is dropped from the data set and $\mathrm{n}$ is reduced by 1 . The statistic $\mathrm{B}$ is used to test the null hypothesis. The null hypothesis, Ho, for this test is that the median of the population of all possible differences is zero, that is, $x_{1 i}$ is as likely to be larger than $x_{2 i}$ as $x_{2 i}$ is likely to be larger 
than $x_{1 i}$. If the number of + and - signs are about equal, there is little reason to reject Ho. Friedman's test is an extension of the sign test from two paired populations to one or a number of related populations. The underlying distribution need not be normal or even symmetric. Also, a moderate number of ND values can be accommodated without seriously affecting the test conclusions. However, no missing values are allowed. The null hypothesis is that there is no tendency for one population to have larger or smaller values than any other of the other populations. The usual alternate hypothesis is that at least one population tends to have larger values than one or more of the other populations. However, the Wilcoxon Rank Sign Test will usually have more power than the sign test to detect differences between the two populations and was thus chosen for the background soil study.

The Wilcoxon signed rank test can be used instead of the sign test if the underlying distribution is symmetric, though it need not be a normal distribution. The Wilcoxon test (not to be confused with the Wilcoxon rank sum test) is more complicated to compute than the sign test because it requires computing and ranking of the paired differences. In most situations it should have greater power to find differences in two populations than does the sign test. Since this is a paired difference experiment, the differences between the measurements are analyzed. However, the nonparametric approach requires that the ranks of the absolute values of the differences between the measurements be calculated. The tied absolute differences are assigned the average of the ranks they would receive if they were unequal but successive measurements. After the absolute differences are ranked, the sum of the ranks of the positive differences and the sum of the negative differences are computed. The nonparametric hypothesis can then be tested. The null hypothesis for this test is that the probability distributions for the two sampled populations are identical. The alternate hypothesis is that the probability distribution for sampled population 1 is shifted to the right or left of the probability distribution of the sampled population 2 . The test statistic is equal to the smaller of the positive and negative rank sums. The stmaller the value of the test statistic, the greater will be the evidence to indicate that the two probability distributions differ in location. The rejection region for the test statistic can be determined by consulting standard references tables. The assumptions for this test are 1) a random sample of pairs of observations has been taken and 2) the absolute differences in the paired observations can be ranked. No assumptions have to be made about the form of the population probability distributions. However, the effectiveness of the Wilcoxon test will, like the Student's t-test, be reduced if other sources of contamination are introduced. 


\section{Table 5.1. SRS Snil Study Taxonomic Name for Soil Series Found on}

SRS

\section{Series Name Taxonomic Name}

$\begin{array}{ll}\text { Ailey } & \text { Loamy, siliceous, thermic Arenic Kanhapludults } \\ \text { Albany } & \text { Loamy, siliceous, thermic Grossarenic Paleudults } \\ \text { Blanton } & \text { Loamy, siliceous, thermic Grossarenic Paleudults** } \\ \text { Chastain } & \text { Fine, mixed, acid, thermic Typic Fluvaquents } \\ \text { Dorovan } & \text { Dysic, thermic Typic Medisaprists } \\ \text { Dothan } & \text { Fine-loamy, siliceous, thermic Plinthic Kandiudults } \\ \text { Eunola } & \text { Fine-loamy, siliceous, thermic Aquic Hapludults } \\ \text { Fluvaquents } & \text { Fluvaquents } \\ \text { Fuquay } & \text { Loamy, siliceous, thermic Arenic Plinthic Kandiudults } \\ \text { Hornsville } & \text { Clayey, kaclinitic, thermic Aquic Hapludilts } \\ \text { Kinston } & \text { Fine-loamy, mixed, nonacid, thermic Typic Fluvaquents } \\ \text { Lakeland } & \text { Thermic, coated Typic Quartzipsamments } \\ \text { Lucy } & \text { Loamy, siliceous, thermic Arenic Kandiudults } \\ \text { Neeses } & \text { Clayey, kaolinitic, thermic Typic Hapludults } \\ \text { Norfolk } & \text { Fine-loamy, siliceous, thermic Typic Paleudults** } \\ \text { Ochlockonee } & \text { Coarse-loamy, siliceous, acid, thermic Typic Udifluvents } \\ \text { Ocilla } & \text { Loamy, siliceous, thermic Aquic Arenic Paleudults } \\ \text { Ogeechee } & \text { Fine-loamy, siliceous, thermic Typic Ochraquults } \\ \text { Orangeburg } & \text { Fine-loamy, siliceous, thermic Typic Kandiudults } \\ \text { Pickney } & \text { Sandy, siliceous, thermic Cumulic Humaquepts } \\ \text { Rembert } & \text { Clayey, kaolinitic, thermic Typic Ochraquults } \\ \text { Shellbluff } & \text { Fine-silty, mixed, thermic Fluventic Dystrochrepts } \\ \text { Smithboro } & \text { Clayey, kaolinitic, thermic Aeric Paleaquults } \\ \text { Tawcaw } & \text { Fine, kaolinitic, thermic Fluventic Dystrochrepts } \\ \text { Toccoa } & \text { Coarse-loamy, siliceous/acid, thermic Typic Udifluvents } \\ \text { Troup } & \text { Loamy, siliceous, thermic Grossarenic Kandiudults } \\ \text { Udorthents } & \text { Udorthents } \\ \text { Vaucluse } & \text { Fine-loamy, siliceous, thermic Typic Kanhapludults* } \\ \text { Wagram } & \text { Loamy, siliceous, thermic Arenic Kandiudults } \\ \text { Williman } & \text { Loamy, siliceous, thermic Arenic Ochraquults } \\ & \text { * Some are Hapludults } \\ & \text { ** Some are Kar tiudults }\end{array}$


Table 5.1 (Continued)

$$
\begin{aligned}
& \text { SRS Soil Study Groupings (Summary) } \\
& \text { Percent of Plant Site }
\end{aligned}
$$

Grouping 1:

$\begin{array}{ll}\text { Blanton } & 21 \\ \text { Troup } & 10.3 \\ \text { Albany } & 0.5 \\ \text { Wagram } & 2.9 \\ \text { Lucy } & 1.2 \\ \text { Total for Grouping 1 } & 35.9\end{array}$

Grouping 2:

Orangeburg

Norfolk

3.4

1.6

Total for Grouping $2 \quad 5.0$

Grouping 3:

Fuquay

11.3

Dothan

7.9

Total for Grouping 3

19.2

Grouping 4:
Ailey
Vaucluse
1.2
6.9
Total for Grouping 4
8.1

Grouping 5:

Lakeland

4.3

Grouping 6:

$\begin{array}{ll}\text { Udorthent } & 3.9 \\ \text { Total for All Groupings } & \mathbf{7 6 . 4}\end{array}$


The following are general descriptions of the soil horizons found in the six major soil groupings during the SRS Soils Study at the Savannah River Plant. Although variability was found to exist among soil borings of the same series several distinguishing features were apparent in the horizons unique to each. Each description reflects the major characteristics and properties of the soil horizons. Any significant compositional variability found in a particular soil horizon is noted and discussed. The layer thicknesses for the other series within a grouping should be
adjusted as appropriate.

\section{Blanton (Grouping 1)}

Soil Horizon

First (AP)

Second (E1,E2,E3)

Third (Bt1, Bi2)

Fourth (Parent)
Depth Interval (inches)

0.6

$6-65$

$65-90$

$120-132$
Description

fine-grained, olive gray to olive black, well sorted, subangular, trace silt, trace organic material, moist.

fine-grauned, grayish orange to dark yellowish orange, well sorted, angular to subangular, trace silt/clay, trace opaques, moist.

fine to medium-grained, light brown to moderate reddish brown, moderately well sorted, angular to subangular, with clay (5-20\%), mottled, moist.

fine-grained, pale yellowish orange to moderate reddish brown, well to moderately sorted, angular to subangular, with clay $(5-10 \%)$, moist. 
Table 5.2 (Cont.)

\section{General Soil Horizon Descriptions}

\section{Orangeburg (Grouping 2)}

Soil Horizon

First (AP)

Second (Bt1,Bt2)

Third (Bt3,Bt4)

Fourth (Bt5)

Fifth (Parent)
Depth Interval (inches)

$0-6$

$6-26$

$26-34$

$34-75$

$120-132$
Description

fine-grained, olive black, well sorted, angular to subangular, trace silt, trace organic material, moist.

sandy (40-45\%), light brown to moderate reddish brown, hard; fine to medium grained, moderately well sorted, angular to suban Ellar, moist.

sandy (40-45\%), light brown, hard; fine to coarse-grained, poorly sorted, angular to subangular, trace pebbles, moist.

sandy (40-45\%), light brown, hard; fine to coarse-grained, poorly sorted, angular to subangular, trace pebbles, moist, acidic.

sandy (35-40\%), light brown to grayish orange to grayish purple, firm to hard, mottled; fine to coarse-grained, poorly sorted, angular to subangular, trace muscovite, moist. 
Table 5.2 (Cont.)

\section{General Soil Horizon Descriptions}

\section{Euquay (Grouping 3)}

Soil Horizon

First (AP)

Second (E1,E2)

Third (Btv1, Btv2)

Fourth (Parent)
Depth Intervals

(inches)

$0-6$

$6-40$

$40-84$

$120-132$
Description

fine-grained, olive black to dark yellowish brown, well sorted, angular to subangular, trace silt, trace organic material, moist.

fine-grained, grayish orange to yellowish gray, well sorted, angular to subangular, trace silt, moist.

sandy (40-45\%), light brown to moderate reddish brown, hard, mottled; fine to medium-grained, moderately well sorted, angular to subangular, trace plinthite nodules, moist.

This interval tended to be composed of varying amounts of sand and clay. In some borings this horizon was a sandy clay and in others a clayey sand

sandy (25-30\%), moderate reddish brown, hard, sometimes mottled; fine to medium-grained, moderately sorted, suljangular, moist. 
Table 5.2 (Cont.)

\section{General Soil Horizon Descriptions}

\section{Vaucluse (Grouping 4)}

Soil Horizon

First (A,E,Bt1)

Second $(\mathrm{Bt} 2, \mathrm{Bt} 3)$

Third (C)

Fourth (Parent)
Depth Intervals (inches)

$0-16$

$16-48$

$48-79$

$120-132$
Description

fine to medium-grained, olive black to light brown to grayish orange, moderately sorted, angular to subangular, trace silt, moist.

Trace organic material in upper 9 inches. High percentage of clay sometimes found in lower 6 inches of this horizon.

sandy (30-35\%), light brown, firm; fine to medium-grained, moderately sorted, angular to subangular, moist.

The sand/clay ratio is variable within this horizon. This horizon consists mostly of sand in one boring. Gravel and pebbles sometimes encountered.

fine to coarse-grained, light brown to very pale orange to grayish orange, moderately poorly sorted, angular to subangular, with clay/silt (5-10\%), moist.

fine to coarse-grained, grayish orange, moderately to poorly sorted, angular to subangular, with clay/silt (5-10\%), moist. 
Table 5.2 (Cont.)

\section{General Soil Horizon Descriptions}

\section{Lakeland (Grouping 5)}

Soil Horizon

Depth Intervals

(inches)

Description

First (A1,A2)

0.6

fine-grained, olive black to pale brown, well sorted, angular to subangular, trace silt, trace organic material, moist.

Second $(C 1, C 2, C 3) \quad 6-80$

fine-grained, dark yellowish orange to grayish orange, well sorted, angular to subangular, trace silt, moist.

In a majority of these borings the color changed to very pale orange between 60 and 72 inches.

Third (Parent)

$120-132$

fine to coarse-grained, light brown to pale red purple, poorly sorted, angular to subangular, with clay (5-10\%), moist. 


\section{Table 5.2 (Cont.)}

\section{General Soil Horizon Descriptions}

\section{Udorthent (Grouping 6)}

Udorthent areas are generally the result of construction work and pit filling. There are no uniform characteristics for this soil grouping. The eight soil borings tested in the SRS Soils Study showed a wide compositional variability. Varying amounts of gravels, sands, and clays were encountered in these borings and no two borings were similar in lithology. Thus, a general soil description can not be formulated for the Udorthent soil grouping. Depth intervals sampled were: First ( 0 - 12 inches), Seccnd (12 - 36 inches), and Third (48 - 60 inches). 
Table 5.3. Soil Boring Coordinates

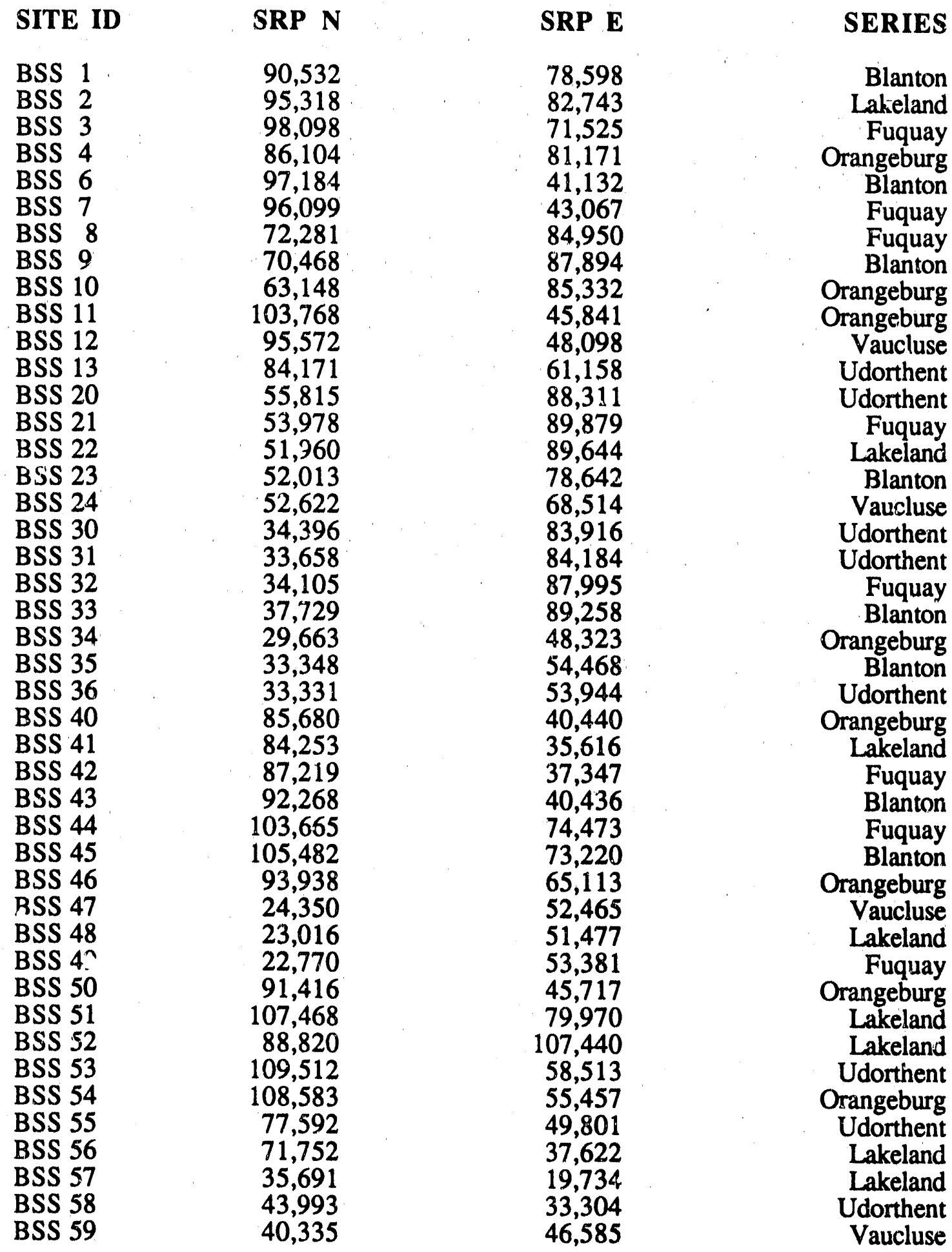


Table 5.4 Analytical Methods - metaTRACE Labs

\begin{tabular}{|c|c|c|c|}
\hline Parameter & Method & Procedure & $\begin{array}{l}\text { Reference } \\
\text { Document }\end{array}$ \\
\hline Aluminum & 6010 & ICP & EPA SW846 \\
\hline Arsenic & 7060 & AA, Furnace & EPA SW846 \\
\hline Barium & 6010 & ICP & EPA SW846 \\
\hline Cadmium & 6010 & ICP & EPA SW846 \\
\hline Chromium & 6010 & ICP & EPA SW846 \\
\hline Copper & 6010 & ICP & EPA SW846 \\
\hline Iron & 6010 & ICP & EPA SW846 \\
\hline Lead & 7421 & AA, Furnace & EPA SW846 \\
\hline Mercury & 7471 & Manual Cold-Vapor & EPA SW846 \\
\hline Nickel & 6010 & ICP & EPA SW846 \\
\hline Potassium & 6010 & ICP & EPA SW846 \\
\hline Silver & 6010 & ICP & EPA SW846 \\
\hline Sodium & 6010 & $\mathrm{ICP}$ & EPA SW846 \\
\hline Zinc & 6010 & ICP & EPA SW846 \\
\hline Lithium & 6010 & ICP & EPA SW846 \\
\hline Magnesium & 6010 & ICP & EPA SW846 \\
\hline Manganese & 6010 & ICP & EPA SW846 \\
\hline Selenium & 7740 & AA, Furnace & EPA SW846 \\
\hline Cyanide & 9010 & Colorimetric & EPA SW846 \\
\hline Chloride & S300 & IC & EPA $600 / 4-79-020$ \\
\hline Fluoride & S300 & IC & EPA $600 / 4-79-020$ \\
\hline Nitrate & S300 & IC & EPA $600 / 4-79-020$ \\
\hline Nitrite & S300 & IC. & EPA $600 / 4-79-020$ \\
\hline Phosphorous & S300 & IC & EPA $600 / 4-79-020$ \\
\hline Sulfate & S300 & IC & EPA $600 / 4-79-020$ \\
\hline pH & 9045 & Electrometric & EPA SW846 \\
\hline Total Organic Carbon & 9060 & Carbonaceous Analyzer & EPA SW846 \\
\hline Total Organic Halogens & 9020 & Carbon Adsorption & EPA SW846 \\
\hline Cation Exchange Capacity & 9080 & Ammonium Acetate & EPA SW846 \\
\hline Exchangeable Äcidity & $6 \mathrm{H} 2 \mathrm{a}$ & & USDA, 1967 \\
\hline Exchangeable Base Metals & $5 \mathrm{~B} 1$ & & USDA,1967 \\
\hline Gross Alpha & 900.0 & & EPA $600 / 4-80-032$ \\
\hline Gross Beta & 900.0 & & EPA $600 / 4-80-032$ \\
\hline Uranium & 900.0 & & EPA $600 / 4-80-032$ \\
\hline Strontium & 900.0 & & HASL-300 \\
\hline Particle Size Dist. & D421, D422 & & ASTM Vol. 408 \\
\hline Appendix VIII Semi-Volati & jiles 8270 & EPA SW846 & \\
\hline Appendix VIII Volatiles & 8240 & EPA SW846 & \\
\hline Appendix VIII Metals & $\begin{array}{c}6010 / 760 / \\
7421 / 7470 / \\
7741 / 7841\end{array}$ & $\begin{array}{l}\text { EPA SW846 } \\
\text { EPA SW846 } \\
\text { EPA SW846 }\end{array}$ & \\
\hline Appendix VIII Pesticides & $8080 / 8140$ & EPA SW846 & \\
\hline Appendix VIII Herbicides & 8150 & EPA SW846 & \\
\hline Appendix VIII Dioxins/Fu & Irans8280 & EPA SW846 & \\
\hline
\end{tabular}


Table 5.5

\section{Parameter}

Aluminum

Arsenic

Barium

Cadmium

Chromium

Copper

Iron

Lead

Mercury

Nickel

Potassium

Silver

Sodium

Zinc

Lithium

Magnesium

Manganese

Selenium

Cyanide

Sulfate

$\mathrm{pH}$

Total Organic Carbon

Total Organic Halogens

Cation Exchange Capacity

Exchangeable Acidity

Exchangeable Base Metals

Gross Alpha

Gross Beta

Uranium

Strontium

Particle Size Dist.

Appendix IX Semi-Volatiles

Appendix IX Volatiles

Appendix IX Metals

$\begin{array}{lc} & 7421 / 7470 / \\ & 7741 / 7841 \\ \text { Appendix IX Pesticides } & 8080 / 8140 \\ \text { Appendix IX Herbicides } & 8150 \\ \text { Appendix IX Dioxins/Furans } 8280\end{array}$

6010

7741

9010

9038

9045

9060

9020

711B
Analytical Methods - Weston Labs
Document

EPA SW846

EPA SW846

EPA SW846

EPA SW846

EPA SW846

EPA SW846

EPA SW846

AA, Furnace EPA SW846

Manual Cold-Vapor EPA SW846

ICP EPA SW846

ICP EPA SW846

ICP EPA SW846

ICP EPA SW846

ICP

ICP

ICP

ICP

Gaseous Hydride

Colormetric

Turbidometric

Electrometric

Carbonaceous Analyzer

Carbon Adsorption

*SCS

*SCS

*SCS

HASL-300

HASL-300

EPA Standard Methods

HASL-300

EPA SW846

EPA SW846

EPA SW846

EPA SW846

EPA SW846

EPA SW846

EPA SW846

EPA SW846

SCS - Soil Conservation Service technical notes document supplied by SRP. 
Table 5.6 Sample Specifications For Blanton Soil Series

PARAMETER

$\begin{array}{cccc}\text { FIELD } & \text { DUPLICATE } & \text { STAINLESS/ } & \text { TOTAL } \\ \text { MARBON } & \text { SAMPLES }\end{array}$

Metals and inorganic ions Soil

Soil pH

Soil

Total Organic Carbon Soil

Total Organic Halogens Soil

Cation Exchange Capacity Soil

Exchangeable Acidity Soil

Exchangeable Base Metals Soil

Gross Alpha

Gross Beta

Soil

Soil

Soil

32

Uranium

Soil

Particle Size Distribution Soil

Appendix VIII Soil

$\mathrm{X}$-Ray Diffraction

Soil

3

$-36$

- 36

- $\quad 36$

-
$-\quad 36$

$-36$

- $\quad 36$

- 36

- 36

- $\quad 36$

- $\quad 36$

-
$-\quad 5$

- 3

$\begin{array}{lll} & - & 3\end{array}$ 
Table 5.7 Sample Specifications For Orangeburg Soil Series

$\begin{array}{llcll}\text { PARAMETER } & \text { FIELD } & \text { DUPLICATE } & \text { STAINLESS/ } & \text { TOTAL } \\ \text { SATRIX } & \text { SAMPLES } & \text { SAMPLES } & \text { CARBON } & \text { SAMPLES }\end{array}$

\begin{tabular}{llllll} 
Metals and inorganic ions & Soil & 40 & 5 & 12 & 57 \\
Soil pH & Soil & 40 & 5 & - & 45 \\
Total Organic Carbon & Soil & 40 & 5 & - & 45 \\
Total Organic Halogens & Soil & 40 & 5 & - & 45 \\
\multicolumn{2}{l}{ Cation Exchange Capacity Soil } & 40 & 5 & - & 45 \\
Exchangeable Acidity & Soil & 40 & 5 & - & 45 \\
Exchangeable Base Metals Soil & 40 & 5 & - & 45 \\
Gross Alpha & Soil & 40 & 5 & - & 45 \\
Gross Beta & Soil & 40 & 5 & - & 45 \\
Uranium & Soil & 40 & 5 & - & 45 \\
Strontium & Soil & 40 & 5 & - & 45 \\
Particle Size Distribution & Soil & 4 & 1 & - & 5 \\
Appendix VIII & Soil & 3 & 0 & - & 3 \\
X-Ray Diffraction & Soil & 10 & 0 & - & 10
\end{tabular}


Table 5.8 Sample Specifications For Fuquay Soil Series

\begin{tabular}{|c|c|c|c|c|}
\hline PAR & MATRIX & $\begin{array}{l}\text { FIELD } \\
\text { SAMPLES }\end{array}$ & $\begin{array}{l}\text { DUPLICATE } \\
\text { SAMPLES }\end{array}$ & $\begin{array}{c}\text { STAINLESS/ TOTAL } \\
\text { CARBON }\end{array}$ \\
\hline
\end{tabular}

Metals and inorganic ions Soil

Soil pH Soil

Total Organic Carbon Soil

Total Organic Halogens Soil

Cation Exchange Capacity Soil

Exchangeable Ácidity Soil

Exchangeable Base Metals Soil

Gross Alpha

Soil

Gross Beta Soil

Uranium Soil

Strontium Soil

Particle Size Distribution Soil

Appendix VIII

Soil

X-Ray Diffraction

32
32
32
32
32
32
32
32
32
32
32
5
3
8

4
4
4
4
4
4
4
4
4
4
4
1
1
0

42

36

36

36

36

36

36

36

36

36

36

6

4
8 
Table 5.9 Sample Specifications For Vaucluse Soil Series

\begin{tabular}{|c|c|c|c|c|}
\hline PARAMETER & MATRIX & $\begin{array}{l}\text { FIELD } \\
\text { SAMPLES }\end{array}$ & $\begin{array}{l}\text { DUPLICATE } \\
\text { SAMPLES }\end{array}$ & $\begin{array}{l}\text { STAINLESS/ } \\
\text { CARBON }\end{array}$ \\
\hline
\end{tabular}

\begin{tabular}{|c|c|c|c|c|}
\hline $\begin{array}{l}\text { Metals and inorganic ions } \\
\text { Soil pH } \\
\text { Total Organic Carton } \\
\text { Total Organic Halogens } \\
\text { Cation Exchange Capacity } \\
\text { Exchangeable Acidity } \\
\text { Exchangeable Base Metals } \\
\text { Gri: ; Alpha } \\
\text { Gross Beta } \\
\text { Uranium } \\
\text { Strontium } \\
\text { Particle Size Distribution } \\
\text { Appendix VII }\end{array}$ & $\begin{array}{l}\text { Soil } \\
\text { Soil } \\
\text { Soil } \\
\text { Soil } \\
\text { Soil } \\
\text { Soil } \\
\text { Soil } \\
\text { S Soil } \\
\text { Soil } \\
\text { Soil } \\
\text { Soil } \\
\text { Soil } \\
\text { Soil } \\
\text { Soil }\end{array}$ & $\begin{array}{l}16 \\
16 \\
16 \\
16 \\
16 \\
16 \\
16 \\
16 \\
16 \\
16 \\
16 \\
16 \\
2 \\
2\end{array}$ & $\begin{array}{l}4 \\
4 \\
4 \\
4 \\
4 \\
4 \\
4 \\
4 \\
4 \\
4 \\
4 \\
1 \\
1\end{array}$ & $\begin{array}{l}- \\
- \\
- \\
- \\
-\end{array}$ \\
\hline
\end{tabular}


Table 5.10 Sample Specifications For Lakeland Soil Series

PARAMETER

Metals and inorganic ions Soil

Soil pH

Total Organic Carbon

Soil

Total Organic Halogens Soil

Cation Exchange Capacity Soil

Exchangeable Acidity

Soil

Exchangeable Base Metals Soil

Gross Alpha

Gross Beta

Uranium

Strontium

Particle Size Distribution

Appendix VIII

$X$-Ray Diffraction
Soil

Soil

Soil

Soil

Soil

Soil

Soil
FIELD SAMPLES
DUPLICATE SAMPLES

24

24

24

24

24

24

24

24

24

24

24

4

3

6
STAINLESS/ TOTAL CARBON SAMPLES

$\begin{array}{lc}2 & 30 \\ - & 28 \\ - & 28 \\ - & 28 \\ - & 28 \\ - & 28 \\ - & 28 \\ - & 28 \\ - & 28 \\ - & 28 \\ - & 28 \\ - & 5 \\ - & 5 \\ - & 6\end{array}$


Table 5.11 Sample Specifications For Udorthent Soil Series

$\begin{array}{lccc}\text { PARAMETER } & \text { MATRLX } & \text { FIELD } & \text { DUPLICATE } \\ \text { SAMPLES } & \begin{array}{c}\text { STAINLESS/ } \\ \text { CARBON }\end{array} & \begin{array}{c}\text { TOTAL } \\ \text { SAMPLES }\end{array}\end{array}$

$\begin{array}{lr}\text { Metals and inorganic ions } & \text { Soil } \\ \text { Soil pH } & \text { Soil } \\ \text { Total Organic Carbon } & \text { Soil } \\ \text { Total Organic Halogens } & \text { Soil } \\ \text { Cation Exchange Capacity } & \text { Soil } \\ \text { Exchangeable Acidity } & \text { Soil } \\ \text { Exchangeable Base Metals Soil } \\ \text { Gross Alpha } & \text { Soil } \\ \text { Gross Beta } & \text { Soil } \\ \text { Uranium } & \text { Soil } \\ \text { Strontium } & \text { Soil } \\ \text { Particle Size Distribution } & \text { Soil } \\ \text { Appendix VIII } & \text { Soil }\end{array}$

$\begin{array}{cccc}24 & 4 & 3 & 31 \\ 24 & 4 & - & 28 \\ 24 & 4 & - & 28 \\ 24 & 4 & - & 28 \\ 24 & 4 & - & 28 \\ 24 & 4 & - & 28 \\ 24 & 4 & - & 28 \\ 24 & 4 & - & 28 \\ 24 & 4 & - & 28 \\ 24 & 4 & - & 28 \\ 24 & 4 & - & 28 \\ 5 & 2 & - & 7 \\ 3 & 0 & - & 3\end{array}$




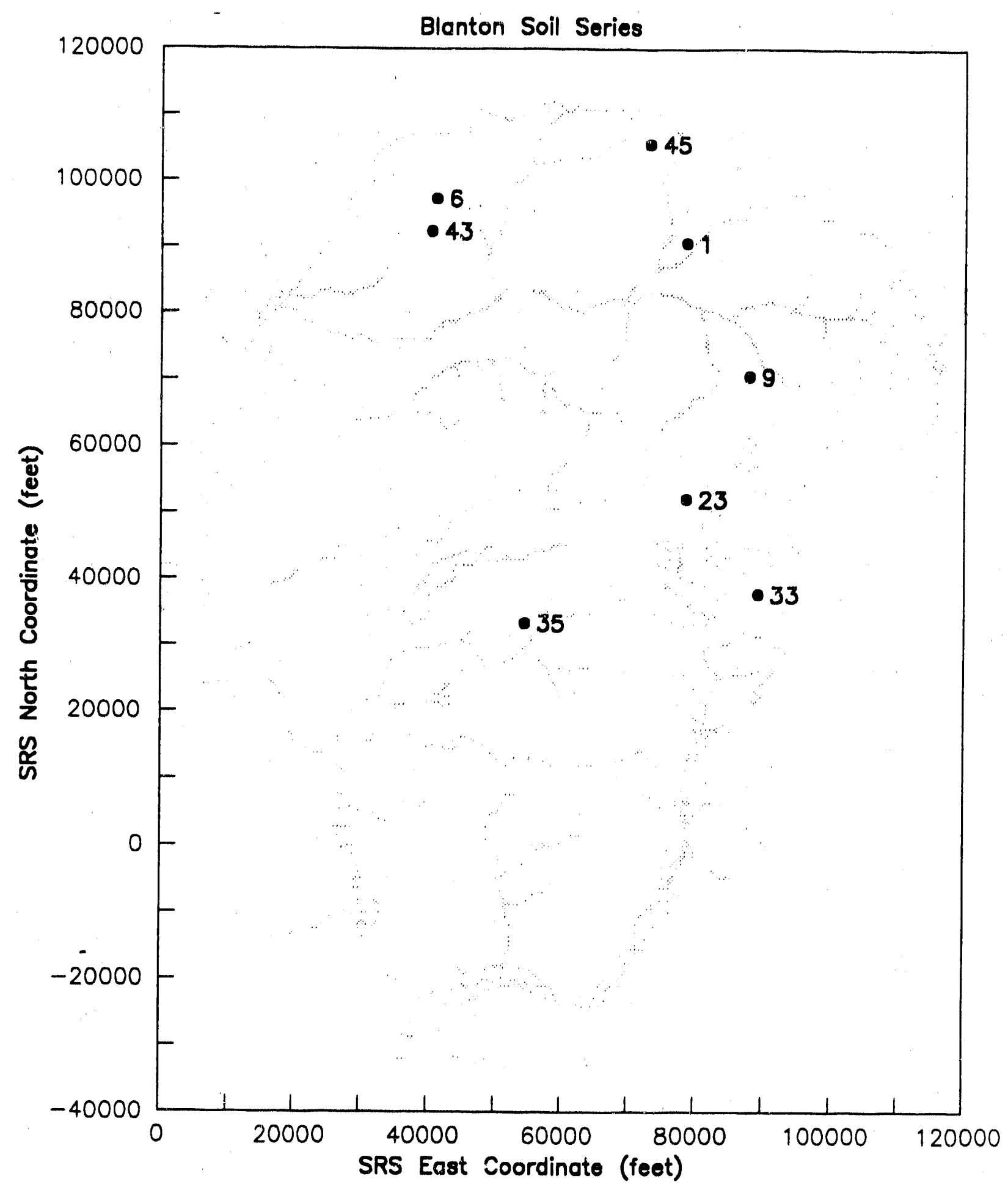

Figure 5.1 Sample Locations for Grouping One (Blanton) Soils 


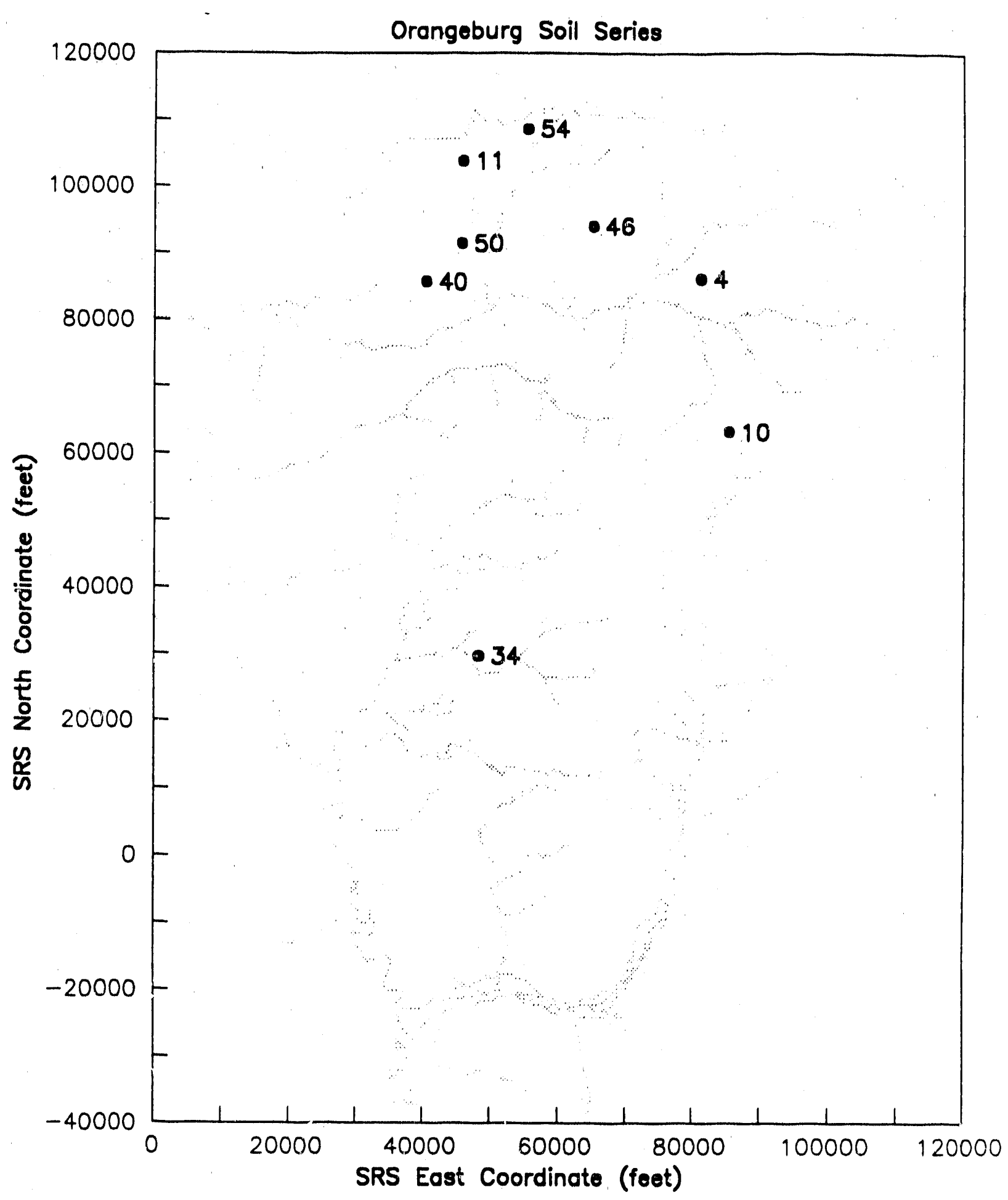

Figure 5.2 Sample Locations for Grouping Two (Orangeburg) Soils 


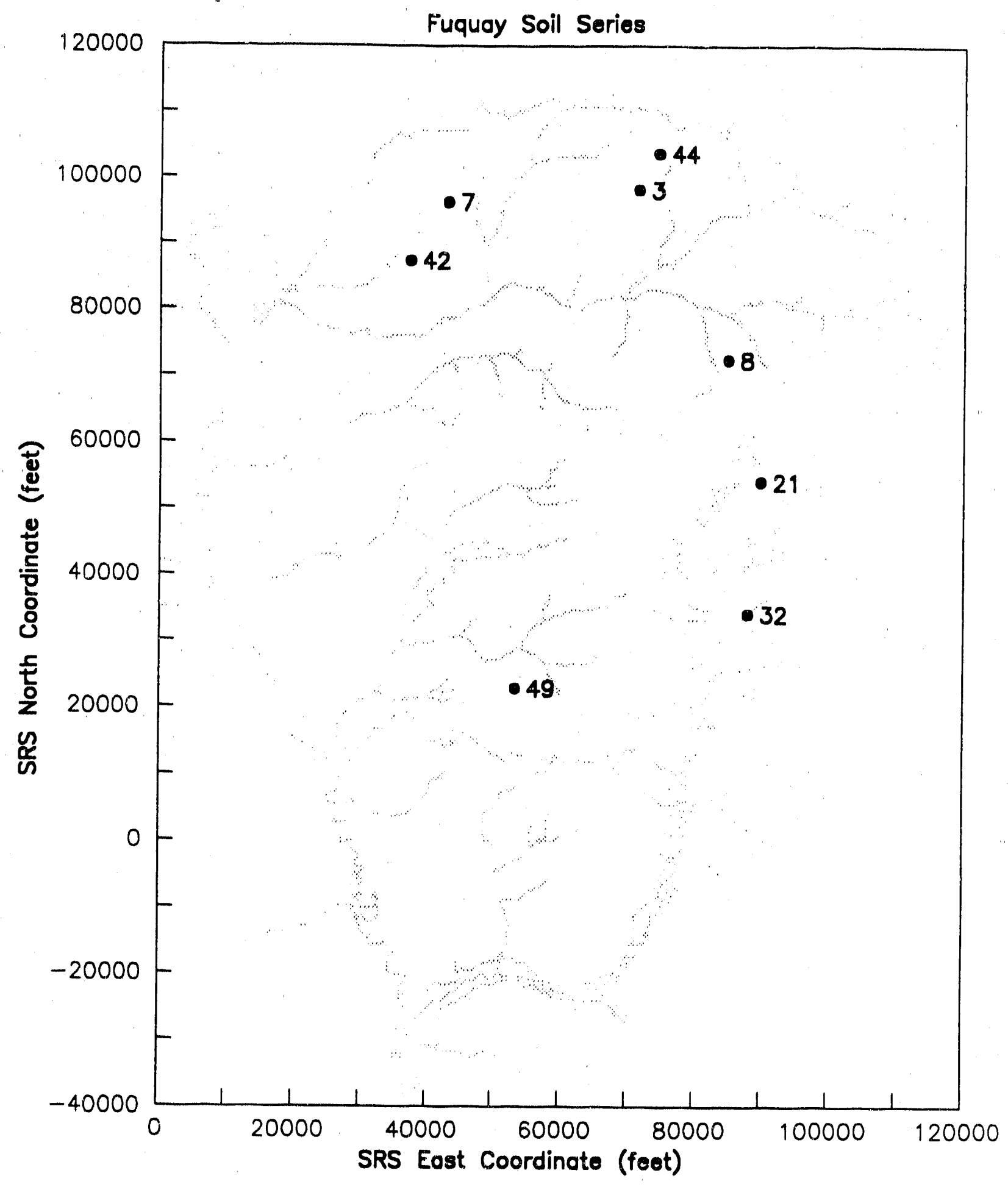

Figure 5.3 Sample Locations for Grouping Three (Fuquay) Soils 


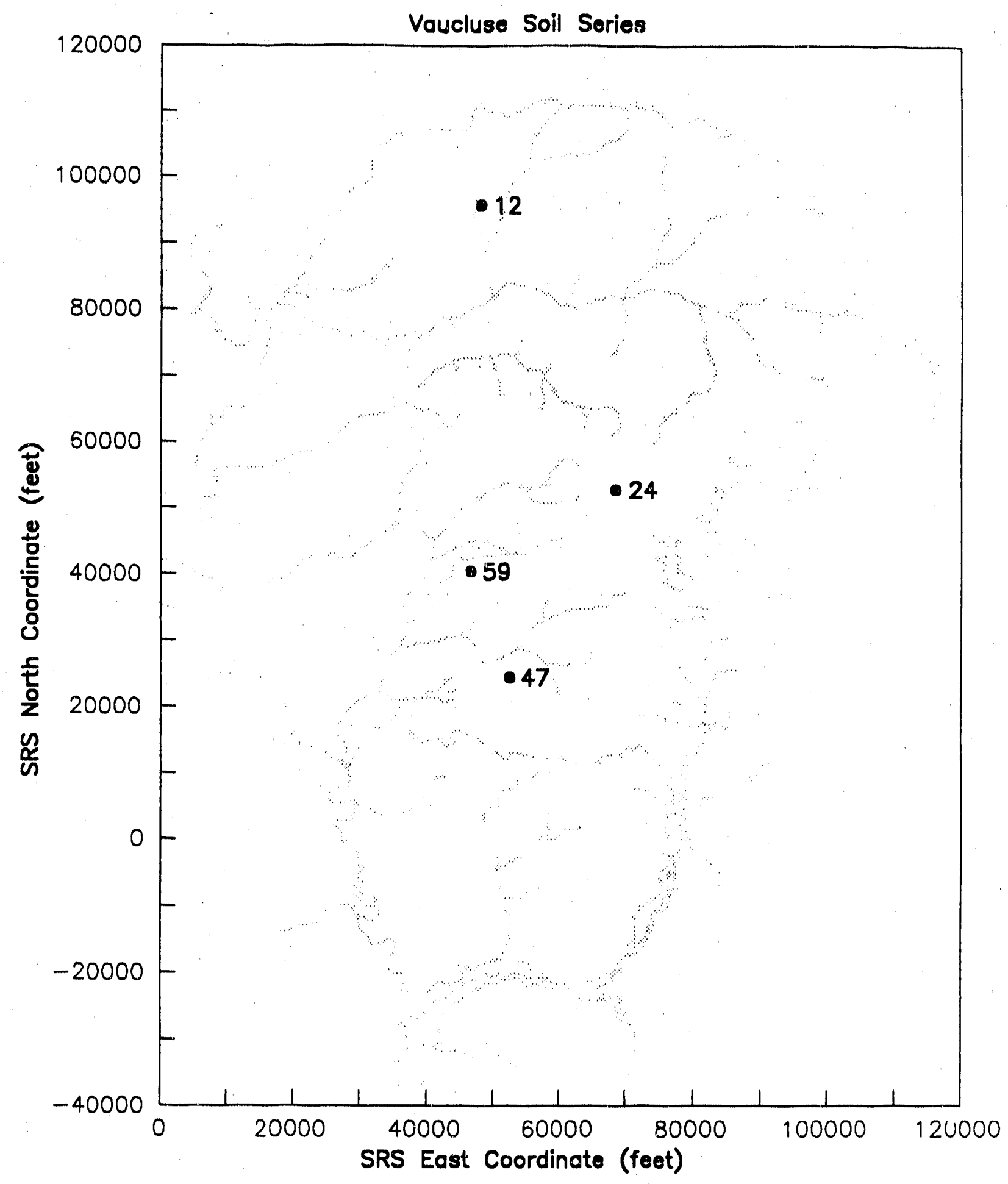

Figure 5.4 Sample Locations for Grouping Four (Vaucluse) Soils 


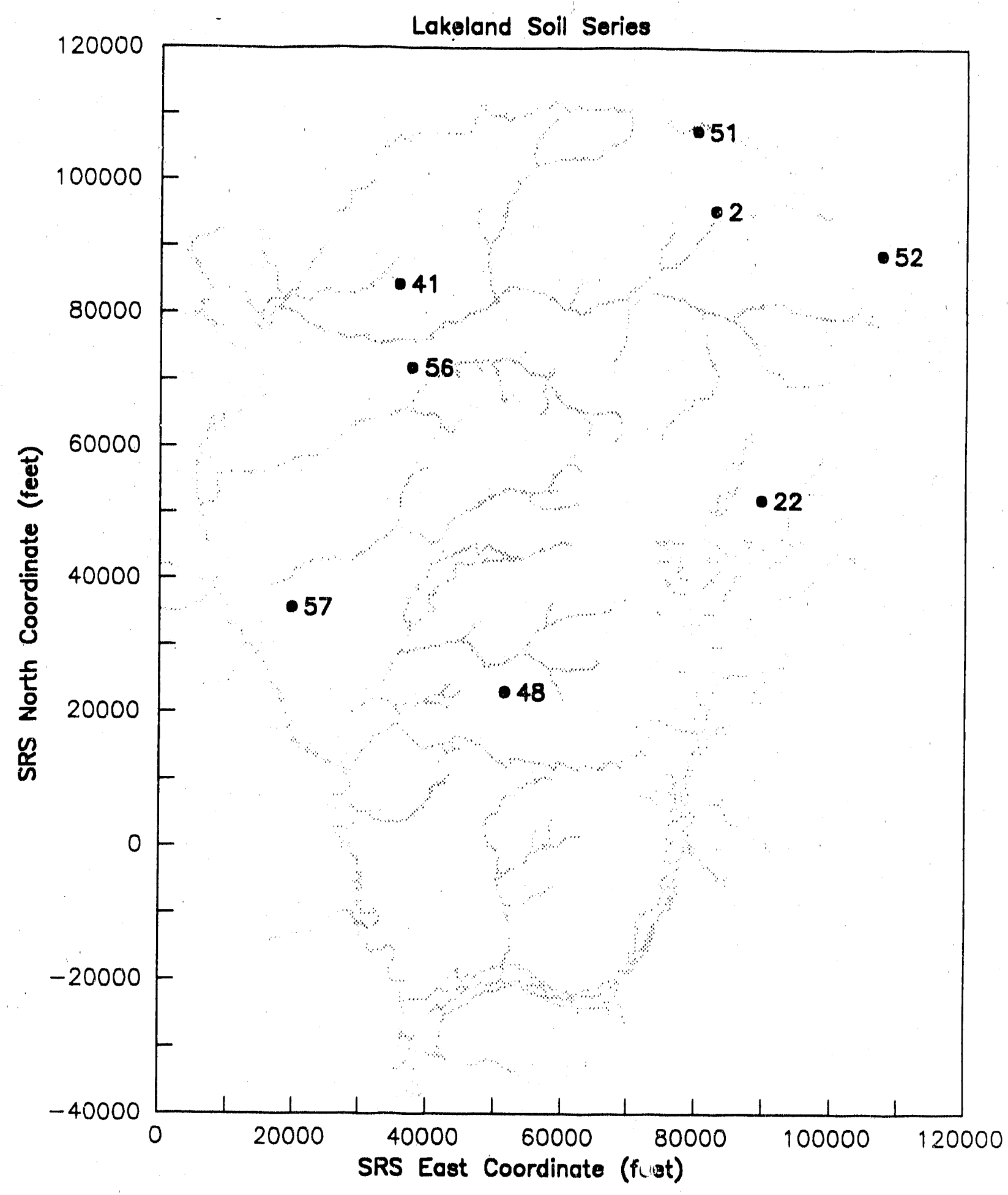

Figure 5.5 Sample Locations for Grouping Five (Lakeland) Soils 


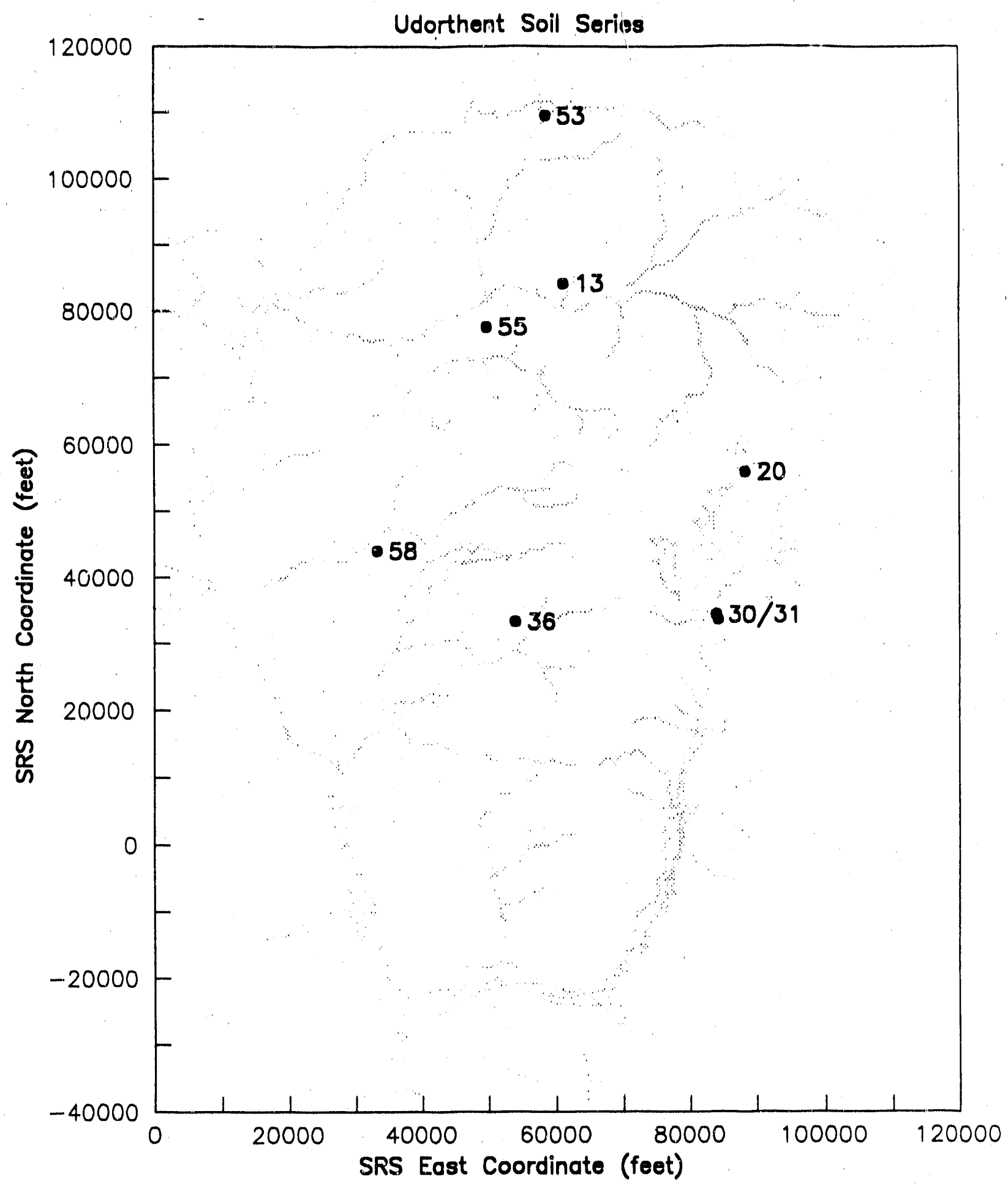

Figure 5.6 Sample Locations for Grouping Six (Udorthent) Soils

$$
47 / 48
$$




\subsection{Results}

\subsection{Current Study}

The soils from unimpacted areas of SRS are typical of soils found in moderately aggressive weathering environments such as the southeast United States. The temperate climate and relatively high rainfall result in leached soils that have low concentrations of metals. The primary clay mineral is kaolinite A similar age, climate and moisture regime governed the formation of most of the various soils at SRS. Thus, the various geochemical delineations tend to be more similar than different . A few notable exceptions were found; these are discussed below. The data for metals, inorganic anions, organic compounds, radionuclides, and indicator parameters are summarized for the six soil series that were identified as representative of the 29 soil series at SRS.

Geochemical results are summarized for each parameter, first for all samples, and then by soil series and layer. The median, minimum, maximum, mean, and standard deviation of values are presented in the tables and significant trends within soil groupings and between soil horizons are noted in the text. For some parameters, a large percentage of values were censored as they were present in concentrations below the detection limit of the instrument. This censoring affects the mean values and standard deviation values presented. If only a portion of the data are censored, the mean is based on substitution of a random uniform deviate between zero and the detection limit for each censored result. This has the effect of minimizing the bias on the mean and standard deviation. In many cases, the data are censored at several different detection limits. In these cases, the modal detection limit (the most common value) was used as a nominal or reference value. For distributions that are heavily censored (i.e., the calculated mian is below the modal detection limit) or entirely censored, a value of less than the modal detection limit is presented for the mean and no standard deviation is calculated. Note that the median value tends to be a more representative measure of central tendency than the arithmetic mean if the underlying distribution is unknown. The raw data are presented in Appendix D by constiuent, soil series, and layer.

\subsubsection{Metals}

In general, metal concentrations increase with depth and in proportion to the clay content of the soil. The upper scil horizons are generally more highly leached, and exhibit lower concentrations, than the lower horizons and the parent material.

\section{Aluminum - Tables 6.1 and 6.2}

Aluminum was detected in all 168 samples. The data range from approximately $715 \mathrm{ug} / \mathrm{g}$ to approximately $53,530 \mathrm{ug} / \mathrm{g}$ with a median of $8060 \mathrm{ug} / \mathrm{g}$. The median concentrations for the various depth intervals range from approximately $2050 \mathrm{ug} / \mathrm{g}$ to $28500 \mathrm{ug} / \mathrm{g}$. The medians of the Blanton, Fuquay and Orangeburg series tend to increase in the lower horizons that contain fine grained materials concentrated from above. The concentrations are relatively higher in the Orangeburg series in all depth intervals than in any other series. The clay content of this series is greater than any of the others investigated. In the Vaucluse series, the concentration decreases ir the lower intervals.

\section{Arsenic - Tables 6.1 and 6.3}

Arsenic was detected in approximately one half of the 168 samples. The data range from $<0.5$ $\mathrm{ug} / \mathrm{g}$ to $15.2 \mathrm{ug} / \mathrm{g}$ with a median of $<2 \mathrm{ug} / \mathrm{g}$. Eighteen of the 80 detected values are below the nominal detection limit of $2 \mathrm{ug} / \mathrm{g}$. No clear trends are evident within intervals or series. Note that arsenic was used as a component of agricultural chemicals in the period before SRS existed. Thus, a few of the detected values may be a result of farming activities in the 1930's through 1950. 
Antimony - Table 6.1

Antimony was measured in the Appendix VIII samples; only 3 of the 17 samples were reported as detected. Two of these samples $(11.4 \mathrm{ug} / \mathrm{g}$ and $15.2 \mathrm{ug} / \mathrm{g}$ ) were above the nominal detection limit of $10.6 \mathrm{ug} / \mathrm{g}$. The remaining detected value was $5.5 \mathrm{ug} / \mathrm{g}$.

Barium - Tables 6.1 and 6.4

Barium was detected in all but one of the 168 samples. The data range from approximately 1 $\mathrm{ug} / \mathrm{g}$ to approximately $77 \mathrm{ug} / \mathrm{g}$ with a median of $11 \mathrm{ug} / \mathrm{g}$. The median concentrations for the various depth intervals range from approximately $2.4 \mathrm{ug} / \mathrm{g}$ to $59 \mathrm{ug} / \mathrm{g}$. The medians of the Blanton, Fuquay and Orangeburg series tend to increase in the lower horizons that contain fine grained materials concentrated from above. In the Vaucluse series, the concentration decreases in the lower intervals. This pattem is similar to the results for gluminum.

Beryllium - Table 6.1

Beryllium was measured in the Appendix VIII samples; only 6 of the 17 samples were reported as detected. One of these samples $(0.43 \mathrm{ug} / \mathrm{g})$ was above the nominal detection limit of $0.4 \mathrm{ug} / \mathrm{g}$. The remaining detected values ranged from 0.12 to $0.4 \mathrm{ug} / \mathrm{g}$.

Cadmium - Tables 6.1 and 6.5

Cadmium was not detected in over $75 \%$ of the 168 samples. The data range from $0.12 \mathrm{ug} / \mathrm{g}$ to $1.19 \mathrm{ug} / \mathrm{g}$ with a median of $<0.6 \mathrm{ug} / \mathrm{g}$. Twenty-two of the 34 detected values are below the nominal detection limit of $0.6 \mathrm{ug} / \mathrm{g}$. No clear trends are evident within intervals or series.

Chromium - Tables 6.1 and 6.6

All 168 sample results for chromium are reported as detected. The data range from approximately $1.3 \mathrm{ug} / \mathrm{g}$ to approximately $105 \mathrm{ug} / \mathrm{g}$ with a median of $13.6 \mathrm{ug} / \mathrm{g}$. The median concentrations for the various depth intervals range from approximately $3.5 \mathrm{ug} / \mathrm{g}$ to $33.3 \mathrm{ug} / \mathrm{g}$. The medians of the Blanton, Fuquay and Orangeburg series tend to increase in the lower horizons that contain fine grained materials concentrated from above. The concentrations are relatively lower in the Lakeland series with all depth intervals considered than any other series. The clay content of this series is less than any of the others investigated.

Cobalt - Table 6.1

Cobalt was measured in the Appendix VII samples; only 7 of the 17 samples were reported as detected. Two of these samples $(2.2 \mathrm{ug} / \mathrm{g}$ and $5.3 \mathrm{ug} / \mathrm{g})$ were above the nominal detection limit of $2 \mathrm{ug} / \mathrm{g}$. The remaining detected values ranged from 0.46 to $1.8 \mathrm{ug} / \mathrm{g}$.

\section{Copper - Tables 6.1 and 6.7}

Copper was detected in over $99 \%$ of the 168 samples. The data range from approximately $0.36 \mathrm{ug} / \mathrm{g}$ to approximately $14 \mathrm{ug} / \mathrm{g}$ with a median of $3.1 \mathrm{ug} / \mathrm{g}$. The median concentrations for the various depth intervals range from approximately $1.45 \mathrm{ug} / \mathrm{g}$ to $7.45 \mathrm{ug} / \mathrm{g}$. The medians of the Blanton, Fuquay and Orangeburg series tend to increase in the lower horizons that contain fine grained materials concentrated from above. The concentrations are relatively higher in the Orangeburg series within all depth intervals considered than in any other series. The clay content of this series is greater than any of the others investigated. In the Vaucluse series, the 
concentration decreases in the lower intervals. This pattern is similar to the results reported for aluminum.

Iron - Tables 6.1 and 6.8

Iron was detected in all 168 samples. The data range from approximately $886 \mathrm{ug} / \mathrm{g}$ to approximately $79,600 \mathrm{ug} / \mathrm{g}$ with a median of $8551 \mathrm{ug} / \mathrm{g}$. The median concentrations for the various depth intervals range from approximately $1416 \mathrm{ug} / \mathrm{g}$ to $26,475 \mathrm{ug} / \mathrm{g}$. The medians of the Blanton, Fuquay and Orangeburg series tend to increase in the lower horizons that contain fine grained materials concentrated from above. Lakeland exhibited low iron concentrations.

Lead - Tables 6.1 and 6.9

Lead was detected in over $90 \%$ of the 168 samples. The data range from approximately <1 $\mathrm{ug} / \mathrm{g}$ to approximately $16.7 \mathrm{ug} / \mathrm{g}$ with a median of $5.1 \mathrm{ug} / \mathrm{g}$. The median concentrations for the various depth intervals range from approximately $1.6 \mathrm{ug} / \mathrm{g}$ to $8.6 \mathrm{ug} / \mathrm{g}$. The medians of the Blanton, Fuquay and Orangeburg series tend to increase in the lower horizons that contain fine grained materials concentrated from above. The concentrations are relatively higher in the Orangeburg series within all depth intervals considered than in any other series. The clay content of this series is greater than any of the others investigated. This pattern is similar to the results reported for aluminum.

\section{Lithium - Table 6.1}

Lithium was not detected in over $95 \%$ of the 168 samples. The data range from $<10 \mathrm{ug} / \mathrm{g}$ to 19 $\mathrm{ug} / \mathrm{g}$ with a median of $<10 \mathrm{ug} / \mathrm{g}$. None of the 5 detected values are below the nominal detection limit of $10 \mathrm{ug} / \mathrm{g}$. All of the detected values are in the lower intervals of the Orangeburg series and the Fuquay series.

\section{Magnesium - Tables 6.1 and 6.10}

Magnesium was detected in all 168 samples. The data range from approximately $12.9 \mathrm{ug} / \mathrm{g}$ to approximately $759 \mathrm{ug} / \mathrm{g}$ with a median of $100 \mathrm{ug} / \mathrm{g}$. The median concentrations for the various depth intervals range from approximately $28.6 \mathrm{ug} / \mathrm{g}$ to $278 \mathrm{ug} / \mathrm{g}$. The medians of the Orangeburg series tend to increase in the lower horizons that contain fine grained materials concentrated from above. The concentrations are relatively higher in the Orangeburg series within all depth intervals considered than in any other series. The clay content of this series is greater than any of the others investigated. This pattem is similar to the results reported for aluminum.

Manganése - Tables 6.1 and 6.11

Manganese was detected in approximately $80 \%$ of the 168 samples. The data range from approximately $<1.6 \mathrm{ug} / \mathrm{g}$ to approximately $498 \mathrm{ug} / \mathrm{g}$ with a median of $7.9 \mathrm{ug} / \mathrm{g}$. The median concentrations for the various depth intervals range from approximately $<2.3 \mathrm{ug} / \mathrm{g}$ to $102 \mathrm{ug} / \mathrm{g}$. The medians of the various series tend to increase in the parent material.

Mercury - Tables 6.1 and 6.12

Approximately $80 \%$ of the 168 sample results for mercury are reported as not detected. The data range from $r 0.01 \mathrm{ug} / \mathrm{g}$ to $0.89 \mathrm{ug} / \mathrm{g}$ with a median of $<0.1 \mathrm{ug} / \mathrm{g}$. None of the 24 detected values are below the nominal detection limit of $10 \mathrm{ug} / \mathrm{g}$. The concentrations do not exhibit a clear pattern in terms of soil series or layer.

Nickel - Tables 6.1 and 6.13 
Nickel was detected in approximateiy $70 \%$ of the 168 samples. The data range from approximately $0.11 \mathrm{ug} / \mathrm{g}$ to approximately $17.9 \mathrm{ug} / \mathrm{g}$ with a median of $3.8 \mathrm{ug} / \mathrm{g}$. The median concentrations for the various depth intervals range from approximately $<2 \mathrm{ug} / \mathrm{g}$ to $8.8 \mathrm{ug} / \mathrm{g}$. The medians of the Orangeburg and Fuquay series tend to increase in the lower horizons that contain fine grained materials concentrated from above. The concentrations are relatively higher in the Orangeburg series within all depth intervals considered in than any other series. The clay content of this series is greater than any of the others investigated. This pattern is similar to the results reported for aluminum.

\section{Potassium - Tables 6.1 and 6.14}

Potassium was not detected in approximately $60 \%$ of the 168 samples. The data range from $28.6 \mathrm{ug} / \mathrm{g}$ to $1118 \mathrm{ug} / \mathrm{g}$ with a median of $<180 \mathrm{ug} / \mathrm{g}$. The concentrations do not exhibit a clear pattern in terms of soil series or layer, also, the laboratory provided widely varying detection limits making further interpretation difficult.

Selenium - Tables 6.1 and 6.15

Selenium was not detected in over $70 \%$ of the 168 samples. The data range from $<0.2 \mathrm{ug} / \mathrm{g}$ to $<4 \mathrm{ug} / \mathrm{g}$ (the highest actual value measured was $1.66 \mathrm{ug} / \mathrm{g}$ ) with a median of $<0.4 \mathrm{ug} / \mathrm{g}$. Five of the 47 detected values are below the nominal detection limit of $0.4 \mathrm{ug} / \mathrm{g}$. Layers with detected values tend to be in the lower intervals of the various series.

Silver - Tables 6.1 and 6.16

Silver was not detected in over $90 \%$ of the 168 samples. The data range from $0.01 \mathrm{ug} / \mathrm{g}$ to 1.8 $\mathrm{ug} / \mathrm{g}$ with a median of $<0.65 \mathrm{ug} / \mathrm{g}$. Six of the 10 detected values are below the nominal detection limit of $0.6 \mathrm{ug} / \mathrm{g}$. The concentrations exhibit no clear pattern in terms of soil series or layer.

Sodium - Tables 6.1 and 6.17

Sodium was detected in over $98 \%$ of the 168 samples. The data range from approximately $<7$ $\mathrm{ug} / \mathrm{g}$ to approximately $760 \mathrm{ug} / \mathrm{g}$ with a median of $42.5 \mathrm{ug} / \mathrm{g}$. The median concentrations for the various depth intervals range from approximately $28 \mathrm{ug} / \mathrm{g}$ to $85 \mathrm{ug} / \mathrm{g}$. The concentrations are relatively higher in the Orangeburg series with all depth intervals considered than any other series.

\section{Thallium - Table 6.1}

Thalfium was measured in the Appendix VIII samples; None of the 17 samples were reported as detected above the nominal detection limit of $1.5 \mathrm{ug} / \mathrm{g}$.

Vanadium - Table 6.1

Vanadium was measured in the Appendix VIII samples; Over $90 \%$ of the 17 sample results were reported as detected. The data range from approximately $3.6 \mathrm{ug} / \mathrm{g}$ to approximately $72.1 \mathrm{ug} / \mathrm{g}$ with a median of $21.2 \mathrm{ug} / \mathrm{g}$.

Zinc - Tables 6.1 and 6.18

Zinc was detected in all of the 168 samples. The data range from approximately $1.8 \mathrm{ug} / \mathrm{g}$ to approximately $267 \mathrm{ug} / \mathrm{g}$ with a median of $10.3 \mathrm{ug} / \mathrm{g}$. The median concentrations for the various depth intervals range from approximately $5.3 \mathrm{ug} / \mathrm{g}$ to $17.9 \mathrm{ug} / \mathrm{g}$. The medians of the Orangeburg, Fuquay and Blanton series tend to increase in the lower horizons that contain fine grained materials 
concentrated from above. The concentrations are relatively higher in the Orangeburg series with all depth intervals considered than any other series. The clay content of this series is greater than any of the others investigated. This pattern is similar to the results reported for aluminum.

\subsubsection{Other Inorganic Parameters}

Soil samples were collected from six distinct soil series (Blanton, Fuquay, Lakeland, Orangeburg, Udorthent, and Vaucluse) for the measurement of following inorganic constituents: chloride, cyanide, fluoride, phosphate, sulfate, nitrate, and nitrite. The data for these constituents is summarized across all soil types and layers in Table 6.19. One ohjective of the SRS Soils Study was to develop background information to assess areas of potential release of hazardous constituents from solid waste management units. A list of "hazardous" constituents is defined for this purpose by EPA in 40 CFR part 261. Cyanide is the only inorganic constituent included in the SRS Soil Study that is included on this list. Although the additional inorganic constituents are not listed as hazardous constituents in 40 CFR 261, elevated levels of these ions may serve as indicators of potential impact and might form the basis for further investigation based on site specific conditions. A discussion of the results for each of the "other inorganic parameters" tested follows:

Bromide - Table 6.19

Bromide was detected in only $10 \%$ of the 168 samples. The data range from $0.25 \mathrm{ug} / \mathrm{g}$ to 3.7 $\mathrm{ug} / \mathrm{g}$ with a median of $<1.25 \mathrm{ug} / \mathrm{g}$. One of the 12 detected values is below the nominal detection limit of $1.25 \mathrm{ug} / \mathrm{g}$.

Chloride - Tables 6.19 and 6.20

Chloride was detected in approximately $90 \%$ of the 168 samples. The ciata range from approximately $0.7 \mathrm{ug} / \mathrm{g}$ to approximately $118 \mathrm{ug} / \mathrm{g}$ with a median of $2.6 \mathrm{ug} / \mathrm{g}$. The median concentrations for the various depth intervals range from approximately $<1.5 \mathrm{ug} / \mathrm{g}$ to $4.95 \mathrm{ug} / \mathrm{g}$. There is no clear pattern of concentrations as a function of layer or series.

Cyanide - Table 6.19

None of the 168 sample results for cyanicie are reported above the nominal detection limit of $0.25 \mathrm{ug} / \mathrm{g}$.

Fluoride - Table 6.19

Approximately $90 \%$ of the 168 sample results for fluoride are reported as not detected. The data range from $<0.25 \mathrm{ug} / \mathrm{g}$ to $4.3 \mathrm{ug} / \mathrm{g}$ with a median of $<1.25 \mathrm{ug} / \mathrm{g}$. None of the 19 detected values are below the nominal detection limit of $1.25 \mathrm{ug} / \mathrm{g}$.

Nitrate - Tables 6.19 and 6.21

Nitrate was detected in approximately $70 \%$ of the 168 samples. The data range from approximately $<0.1 \mathrm{ug} / \mathrm{g}$ to approximately $44.4 \mathrm{ug} / \mathrm{g}$ with a median of $1.0 \mathrm{ug} / \mathrm{g}$. The median concentrations for the various depth intervals range from approximately $<0.5 \mathrm{ug} / \mathrm{g}$ to $1.7 \mathrm{ug} / \mathrm{g}$. There is no clear pattern of concentrations as a function of layer or series.

Nitrite - Table 6.19 $\mathrm{ug} / \mathrm{g}$.

None of the 168 sample results for nitrite are reported above the nominal detection limit of 1.25 
Phosphate - Table 6.19

Phosphate was not detected in over $90 \%$ of the 168 samples. The data range from $<0.25 \mathrm{ug} / \mathrm{g}$ to $13.7 \mathrm{ug} / \mathrm{g}$ with a median of $<5 \mathrm{ug} / \mathrm{g}$. None of the 10 detected values are below the nominal detection limit of $5 \mathrm{ug} / \mathrm{g}$.

Sulfate - Tables 6.19 and 6.22

Sulfate was detected in approximately $70 \%$ of the 168 samples. The data range from approximately $<5 \mathrm{ug} / \mathrm{g}$ to approximately $25.1 \mathrm{ug} / \mathrm{g}$ with a median of $8.05 \mathrm{ug} / \mathrm{g}$. The median concentrations for the various depth intervals range from approximately $<5 \mathrm{ug} / \mathrm{g}$ to $18.0 \mathrm{ug} / \mathrm{g}$. There is no clear pattern of concentratiois as a function of layer or series.

\subsubsection{Organic Compounds}

Organic constituents were measured in soil samples from six distinct soil series (Blanton, Fuquay, Lakeland, Orangeburg, Udorthent, and Vaucluse). The parameters investigated are total organic carton and total organic halogens. The data for these constituents is summarized across all soil types and layers in Table 6.23. The total organic carbon content of a soil is an indicator of the concentration of natural soil organic matter, such as humic and fulvic acids, as well as organic scil material that was introduced as a contaminant. The total organic halogen content is comprised of the halogenated hydrocarbons, specifically a hydrocarbon containing fluorine, chlorine, bromine, and iodine. Since organic halogens do not occur naturally in significant quantities, the presence of halogenated hydrocarbons indicates either contamination at the sampling location or an induced laboratory artifact. The total organic halogen data, combined with the Appendix VIII analyses discussed below, were intended to assess possible labos atory artifacts. A discussion of the results for each the organic constituents follows:

\section{Total Organic Carbon - Tables 6.23 and 6.24}

Total organic carbon was detected in all of the 168 samples. The data range from approximately $32 \mathrm{ug} / \mathrm{g}$ to approximately $14493 \mathrm{ug} / \mathrm{g}$ with a median of $644 \mathrm{ug} / \mathrm{g}$. The median values tend to decrease from the top depth intervals to the bottom intervals with similar variability throughout the series. This decrease in concentration is explained by the accumulation of natural organic compounds from plant and animal decay in the topsoil interval. These constituents are biologically recycled in the topsoil zone and therefore are not as concentrated in the subsurface horizons.

\section{Total Organic Halogens - Table 6.23}

Total organic halogens were not detected in over $98 \%$ of the 168 samples. The two reported values are $10 \mathrm{ug} / \mathrm{g}$ and $17.4 \mathrm{ug} / \mathrm{g}$ (neither of the 2 detected values are below the nominal detection limit of $10 \mathrm{ug} / \mathrm{g}$ ). While the two positive results are likely artifacts, the data suggest that there was little systematic problem with artifacts of total organic halogens in the SRS Soil Study. 
Appendix VIII Analyses

Seventeen samples were tested for the presence of the Appendix VIII organic constituents consisting of volatiles, semivolatiles, pesticides, and PCBs. Organic compounds were detected in all 17 samples tested. A total of six different compounds were detected, however, not all the parameters were detected in all 17 samples (Figure 6.1). The parameters detected include: bis (2ethylhexyl) phthalate, acetone, methylene chloride, dieldrin, endosulfan sulfate, and butylbenzyl phthalate. Bis (2-ethylhexyl) phthalate occurred in 10 samples of a concentration range of 10 to $4,800 \mathrm{ppb}$, acetone occurred in 13 samples with a range of 42 to $1,600 \mathrm{ppb}$, methylene chloride occurred in 15 samples with a range of 4 to $70 \mathrm{ppb}$, dieldrin occurred in 1 sample at a concentration of $9.6 \mathrm{ppb}$, endosulfan sulfate occurred in 5 samples with a range of 28 to $3940 \mathrm{ppb}$, and butylbenzyl phthalate occurred in 1 sample at a concentration of $10 \mathrm{ppb}$.

\subsubsection{Radiological Parameters}

Soil samples were collected from six distinct soil series (Blanton, Fuquay, Lakeland, Orangeburg, Udorthent, and Vaucluse) for the measurement of background radiation. The parameters measured are gross alpha, gross beta, uranium, and $90 \mathrm{Sr}$. The data for these constituents is summarized across all soil types and layers in Table 6.26. Additional radioactive species have been measured in previous studies of background soils at SRS; these results are presented in section 6.2. A discussion of the results from the current study follows:

Gross Alpha - Tables 6.26 and 6.27

Gross alpha was detected in approximately $60 \%$ of the 168 samples. The data range from approximately $<4 \mathrm{pCi} / \mathrm{g}$ to approximately $20 \mathrm{pCi} / \mathrm{g}$ with a median of $5.4 \mathrm{pCi} / \mathrm{g}$. The median concentrations for the various depth intervals range from approximately $<4 \mathrm{pCi} / \mathrm{g}$ to $7.45 \mathrm{pCi} / \mathrm{g}$. The concentrations are higher in the lower zones of Blanton, Fuquay, Lakeland and Orangeburg. These zones are accumulating fine grained materials from above.

Nonvolatile Beta - Tables 6.26 and 6.28

Nonvolatile beta nas detected in approrimately $60 \%$ of the 168 samples. The data range from approximately $<5 \mathrm{pCi} / \mathrm{g}$ to approximately $23 \mathrm{pCi} / \mathrm{g}$ with a median of $8.1 \mathrm{pCi} / \mathrm{g}$. The median concentrations for the various depth intervals range from approximately $<5 \mathrm{pCi} / \mathrm{g}$ to $12 \mathrm{pCi} / \mathrm{g}$. The concentrations are higher in the lower zones of Blanton, Fuquay and Orangeburg. These zones are accumulating fine grained materials from above.

Strontium 90 - Table 6.26

None of the 168 sample results for $90 \mathrm{Sr}$ are reported above the nominal detection limit of 1.0 $\mathrm{pCi} / \mathrm{g}$.

Uranium - Tables 6.26 and 6.29

Uranium was detected in approximately $50 \%$ of the 168 samples. The data range from approximately $<1 \mathrm{pCi} / \mathrm{g}$ to approximately $4.7 \mathrm{pCi} / \mathrm{g}$ with a median of $1.0 \mathrm{pCi} / \mathrm{g}$. The median concentrations for the various depth intervals range from approximately $<1 \mathrm{pCi} / \mathrm{g}$ to $1.45 \mathrm{pCi} / \mathrm{g}$. The concentrations are higher in the lower zones of Blanton, Fuquay and Orangeburg. These zones are accumulating fine grained materials from above.

\subsubsection{Agricultural Parameters}


Soil samples were collected from six distinct soil series (Blanton, Fuquay, Lakeland, Orangeburg, Udorthent, and Vaucluse) for the measurement of background agricultural parameters. The parameters investigated were cation exchange capacity, exchangeable acidity, exchangeable base metals, and pH. The data for these constituents is summarized across all soil types and layers in Table 6.30. Many of the parameters measured are closely related to clay content of the soil. This results in higher concentrations in the series that characteristically have argillic horizons (zones of subsoil clay enrichment due to illuviation), including the Fuquay and Orangeburg. Below the zone of weathering, clay content and CEC are generally lower than the weathered intervals above; the background data reflect this trend. Of the soil series investigated, the CEC for the Orangeburg series is highest. This observation was anticipated as the Orangeburg series has the highest concentration of clay of any series studied. The trends for the cation exchange capacity are paralleled by the trends in exchangeable acidity and exchangeable base metals as expected. Final results for the agricultural parameters are still being prepared by the laboratory and will be presented in a separate report (Looney et al., 1990).

\subsubsection{Mineralogical Analysis}

$\mathrm{X}$-ray diffraction analysis was performed on thirty two samples collected from several horizons in representative soil groupings to determine the mineralogy of the whole soil, and size-fractionated whole soil samples were examined for the mineralogy of clay-sized material. Table 6.30 lists the results of the X-ray diffraction analyses of whole soil samples. Table 6.31 lists the results of the $X$-ray diffraction analyses of the clay mineral fraction.

\subsubsection{Carbon Steel/Stainless Steel Comparison Data}

In the carbon and stainless steel comparison study, a total of 26 comparison samples were taken. The carbon steel sampler served as the primary sampler and the stainless steel sampler was used only for comparison purposes so that potential contamination from either sampler could be determined. The comparison samples taken with the stainless steel sampler were tested for a number of metals. The raw data are presented in Appendix $C$ with the paired samples adjacent to each other and for each metal tested. A statistical summary of the carbon steel/stainless steel comparison for a number of metals is presented in Table 6.32. The table includes results for the normality test, paired difference t-test, and the Wilcoxon Signed Rank Test.

\subsubsection{Laboratory Comparison Data}

In the metaTRACE and Weston laboratory comparison study, a total of 25 comparison or duplicate samples were tested. The duplicate samples were tested for a number of metals, radiological and agricultural parameters, and organics. A statistical summary of the metaTRACE and Weston comparisons for a number of metals is presented in Table 6.33. The table includes results for the normality test, paired difference t-test, and the Wilcoxon Signed Rank Test.

\subsection{Past Studies at SRS}

Several geochemical/radiochemical studies of shallow soils have been performed at SRS. Background data were collected as part of a tes: of sewage sludge fertilization of pine forest (Davis and Corey, 1989, and unpublished data). Radionuclides were measured by Fay and Pickett (1987); limited radionuclide data are presented each year in the annual environmental report for SRS (e.g., Mikol, 1988; Davis et al., 1989). The data are summarized in Tables 6.34 and 6.35.

Sites for the Sewage Sludge Study were selected to represent 'sandy' and 'clayey' textured soils typical of SRS and the southeastern Coastal Plain. The study sites were used for agriculture, and were reforested when operation began in the early 1950 's. As defined by Davis and Corey (1989), the clayey soils were Orangeburg and Fuquay and the sandy soils were Lucy, Wagram, 
and Fuquay. The samples were a composite from the surface to 30 inches deep. The soil chemistry data from this study are summarized in Table 6.34. The concentrations of the various elements are similar in magnitude and distribution to the results from this study presented in Table 6.1 through 6.29 .

Radionuclides in surface soils were measured by Fay and Pickett (1987). Nineteen sites were tested using gamma counting and neutron activation analysis. Two recent annual reports (Mikol, 1988; Davis et al., 1989) contain representative data for a few constituents. The data from these sources are summarized in Table 6.35. Sites are grouped according to proximity to sources where appropriate, and sites directly impacted by discharges to surface water (e.g. swamps) are not included in the table. As expected potassium, uranium and thorium are the parent radionuclides that contribute most of the natural radiation in background soils. Cesium, contributed by weapons testing and other atmospheric sources, is also present. Most of the background potassium, uranium, and thorium is contributed by minerals found in soil. Potassium is associated with clay minerals (correlating with other clay mineral elements such as sodium and aluminum). Most of the uranium and thorium in SRS soils is associated with the heavy mineral monazite. Monazite is a lanthanide phosphate that contains 0.5 to 1 percent uranium and 4 to 5 percent thorium. Thus the concentrations of uranium and thorium correlated with each other and with other lathanide elements. Uranium and thorium produce a series of daughters that are also present in background soils.

\subsection{Regional, National, and Global Studies}

Table 6.36 provides summary statistics and ranges in background soil values for metals from previous regional, national, and global studies. Generally, several reported values are available for most metals. In some cases, however, only limited data are available (e.g., antimony). If provided in the referenced studies, the number of samples and the range of analytical values, the various means and standand deviations are included in the Table 6.36. A brief discussion of some of the major studies is provided below.

Connor and Shacklette (1975) conducted a national geochemical survey to determine background levels of various elements in rocks, soils, and plants. Their study includes a regional assessment of nine central and southern Georgia counties. In this study, three soil horizons were sampled at thirty sites and analyzed. Summary statistics and sampling locations were compiled from other field and laboratory studies since 1958. Background values are included along with factors indicating the observed variation, the analytical variation, the observed range of values, and total number of element analyses. The purpose of their study was to assess the regional background variations so data known or suspected to reflect epigenetic mineralization, or pollution and other man-induced effects were intentionally excluded. Data collection was designed to insure that estimates of the variation were unbiased and represent uncontaminated values. Nearly all of the sample suites were analyzed in a randomized sequence to circumvent effects of systematic laboratory error.

Anderson et al. (in press) report values for the distribution of Be in soils of the Piedmont and Coastal Plain physiographic regions of the Southeastern United States. Soils were collected near Athens, Ga., and on the Savannah River Site. Total Be for soils ranged from 0.3 to $30.5 \mathrm{ppb}$ and was consistently higher in Piedmont soils than in Coastal Plain soils.

Additional soil geochemistry studies by Robinson (1914) and Robinson (1917) stressed the variation in soil of agricultural factors including texture, province, and condition of fertility. A comparatively small number of soil types were examined. Robinson et al. (1917) discuss the chemical variation in yarious soil types and provinces. Shacklette et al. (1971) conducted a soil sampling program that included 863 analyses from across the United States. Samples were collected at a depth of 8 inches. The arithmetic and geometric mean, the geometric deviation, and a 
histogram showing frequencies of analytical are given for 30 elements. Prior to this study, few data were available on the abundance of elements in surficial materials of the United States. Most of the early publications discuss only elements of economic importance to mining or agriculture. For the most part, the data cannot be evaluated with reference to average, or "normal," amounts in undisturbed materials because sampling was biased toward anomalous localities.

In general, Shacklette et al , (1971) found that surficial materials of the western half of the United States generally contain more calcium, magnesium, strontium, potassium, sodium, aluminum, and barium, but contain less titanium and zirconium than do those of the eastern half. Surficial material in the Atlantic Coastal Plain tends to have lower concentrations of most metals relative to the rest of the U.S. Materials in the Basin and Range province, in parts of the Rocky Mountains, and in Maine and adjacent states generally have high metal concentrations. Shacklette and Boerngen (1984) reports values for an additional 355 samples. Samples of both studies were analyzed for $\mathbf{5 0}$ elements with results plotted on maps and statistically analyzed.

Global estimates on soil geochemistry are discussed in Bear (1964), Siegel (1974), Lindsay (1979), Waldron (1980), Nriagu (1980a, b, c), and Hutchinson and Meema (1987). The crust of the earth is composed principally of igneous and metamorphic rocks of granitic and basaltic composition. Since 1890 many geologists have computed averages for the major element composition of the crust based on the following general approaches: (1) averages of available analyses, (2) averages weighted in proportion to occurrence, (3) computations of an indirect nature based on the composition of sediments and various combinations of mafic and felsic rocks, and (4) abundances of elements based on crustal models. Most of these averages fall within a small range of composition. Less at 'ntion has been given by geologists to the computation of abundance of minor elements in the $\mathrm{cn} t \mathrm{t}$ and in various types of rocks. The abundance of some minor elements still is poorly known, and $L$ e estimation of their abundance is hampered by lack of available analyses, inadequate sampling, inaccurate results of some analytical procedures, and imprecise methods of estimation. 


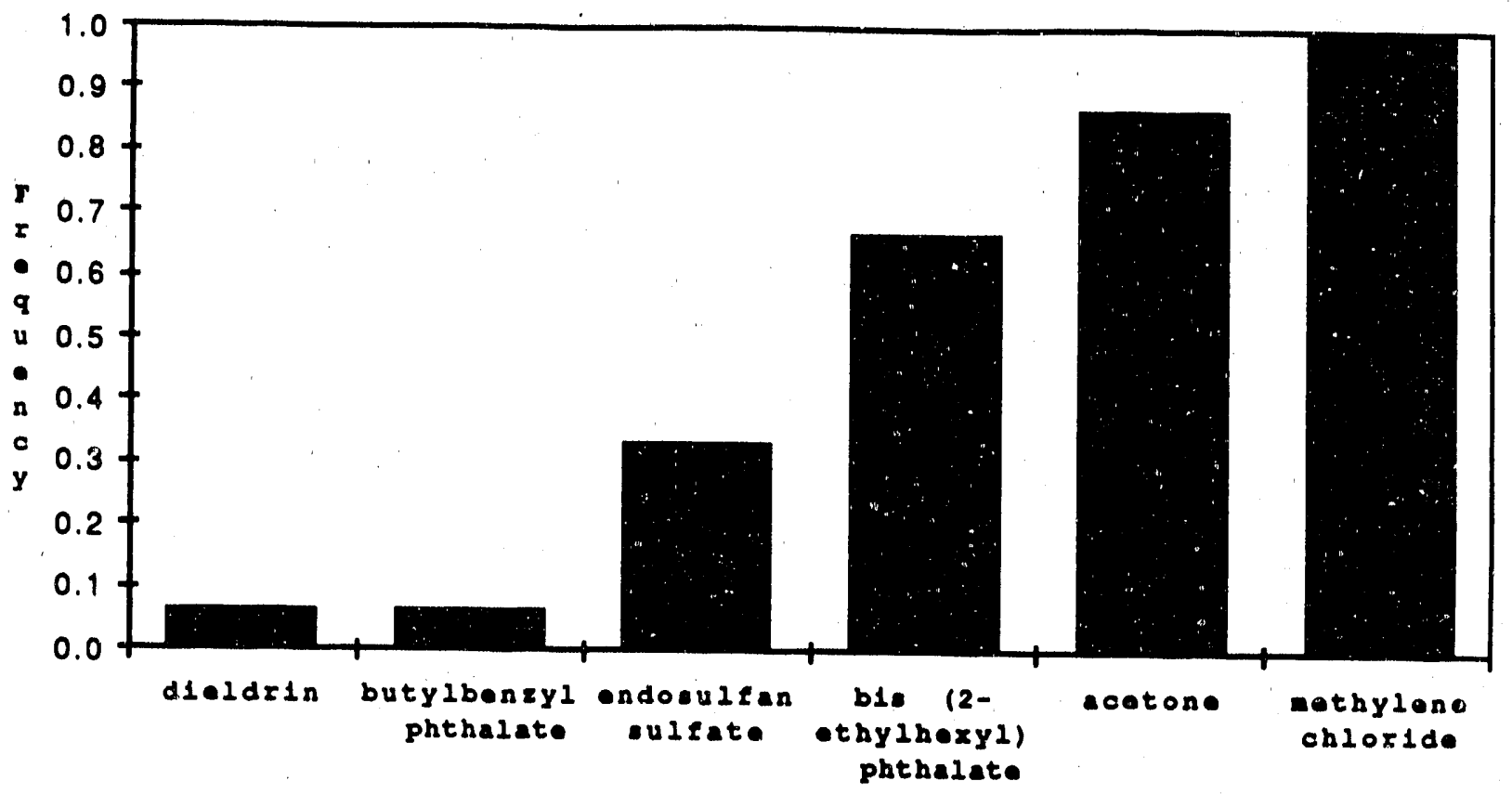

Figure 6.1 Frequency of Occurrence of Organic Compounds in Appendix VIII Analyses. 
Table 6.1. Summary Statistics for Metals Measured (all samples)

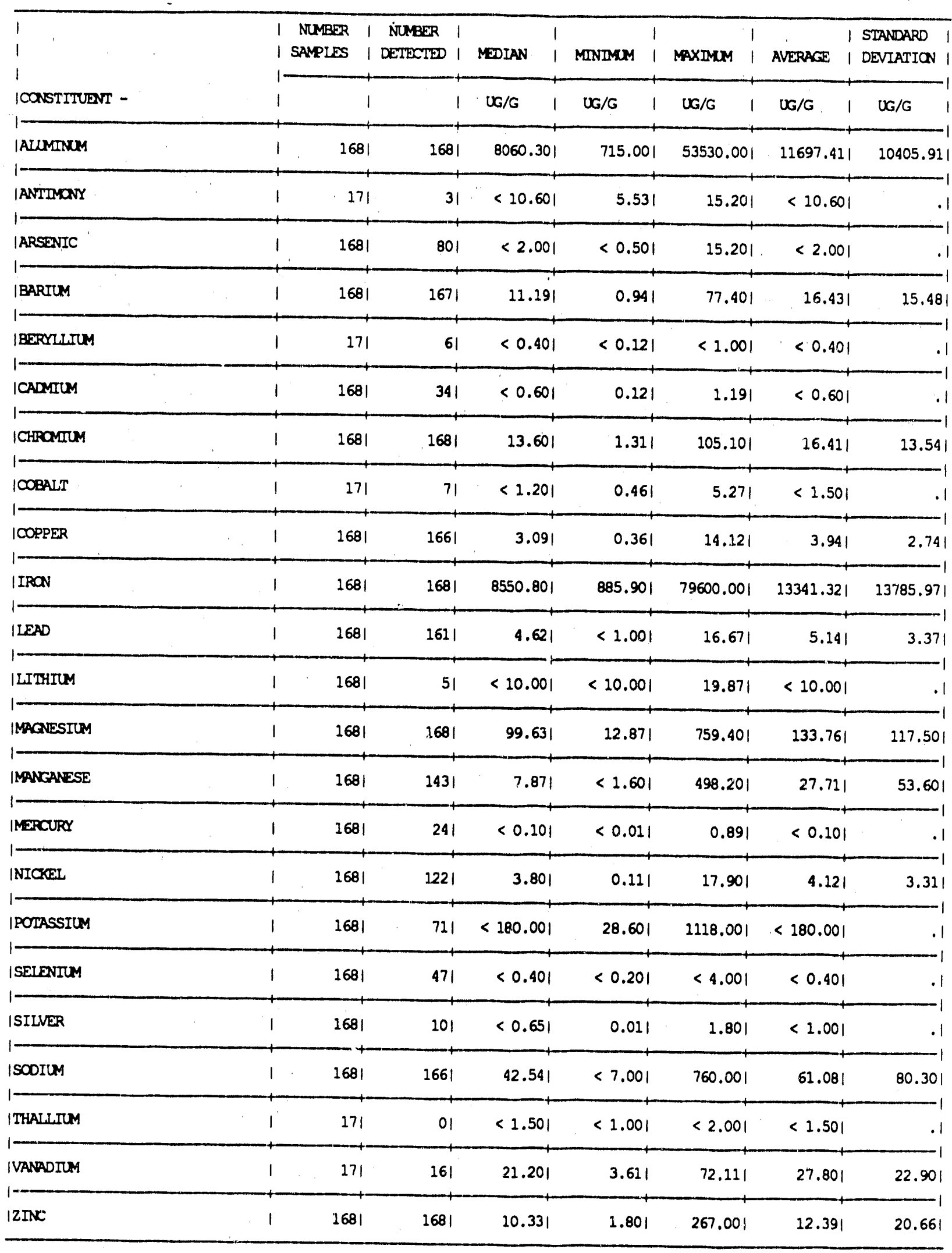


Table 6.2. Summary Statistics for Aluminum (by series and layer)

CONSTITUENT - ALUMDNUM

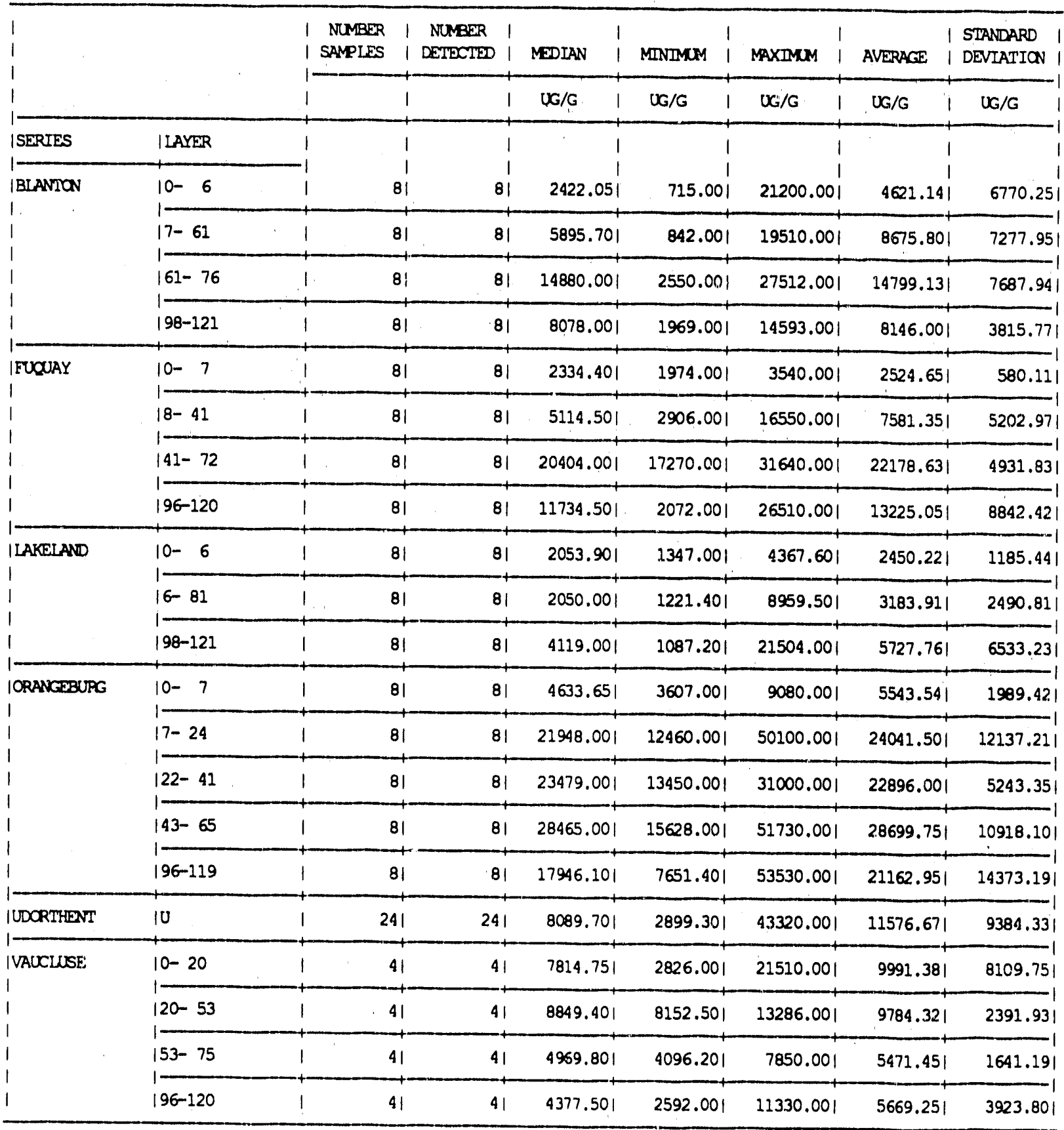


Table 6.3. Summary Statistics for Arsenic (by series and layer)

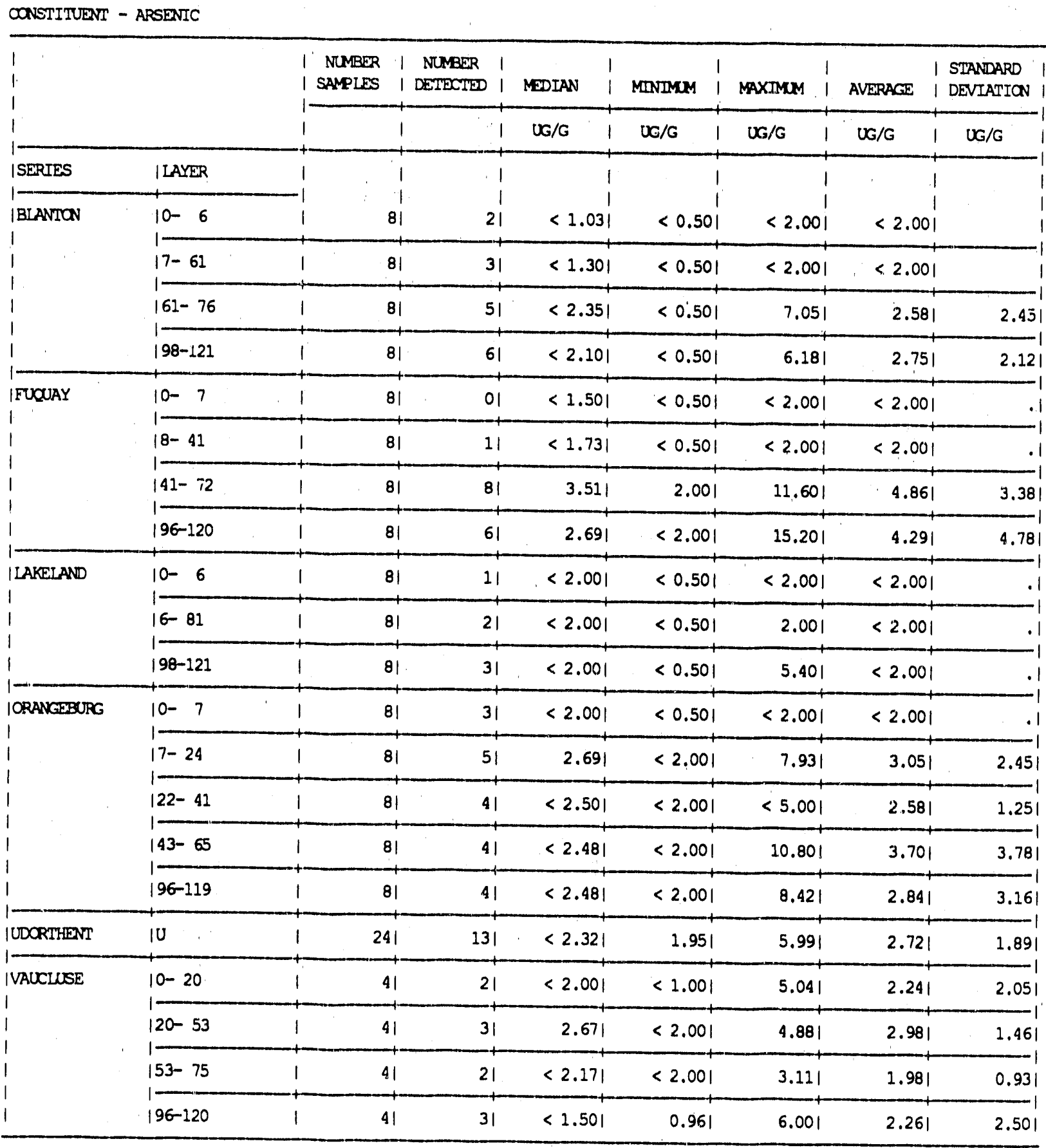


Table 6.4. Summary Statistics for Barium (by series and layer)

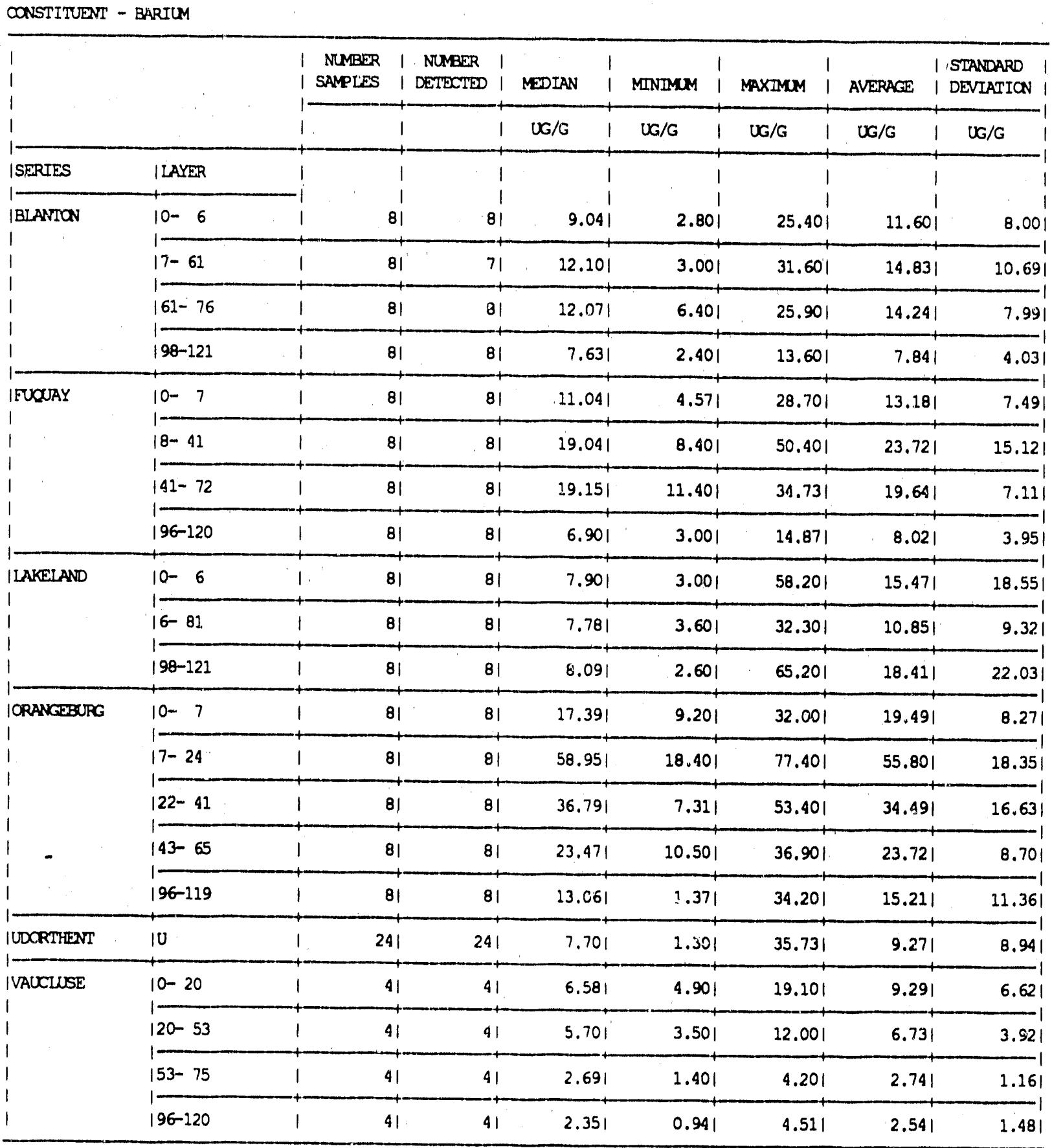


Table 6.5. Summary Statistics for Cadmium (by series and layer)

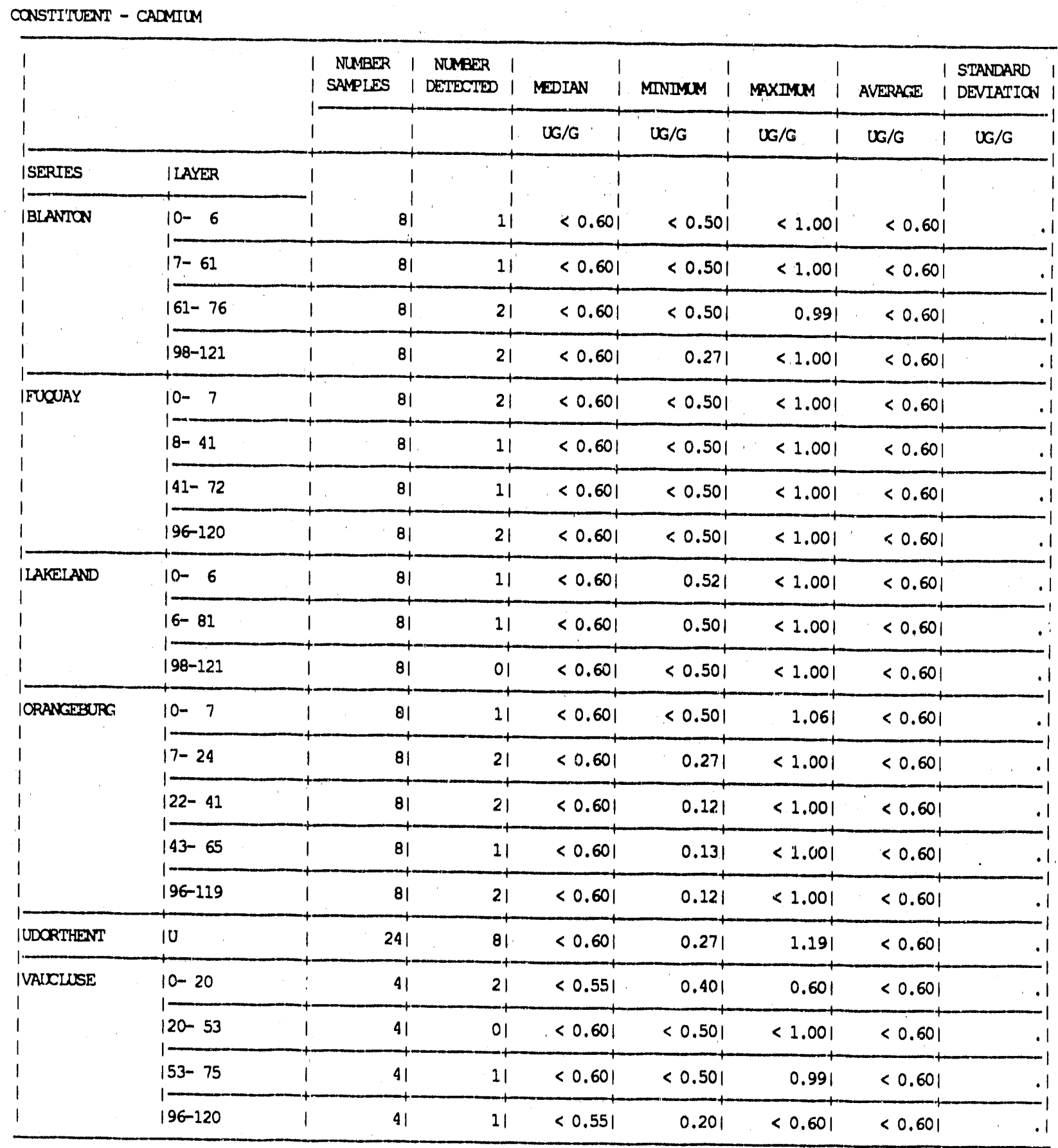


Table 6.6. Summary Statistics for Chromium (by series and layer)

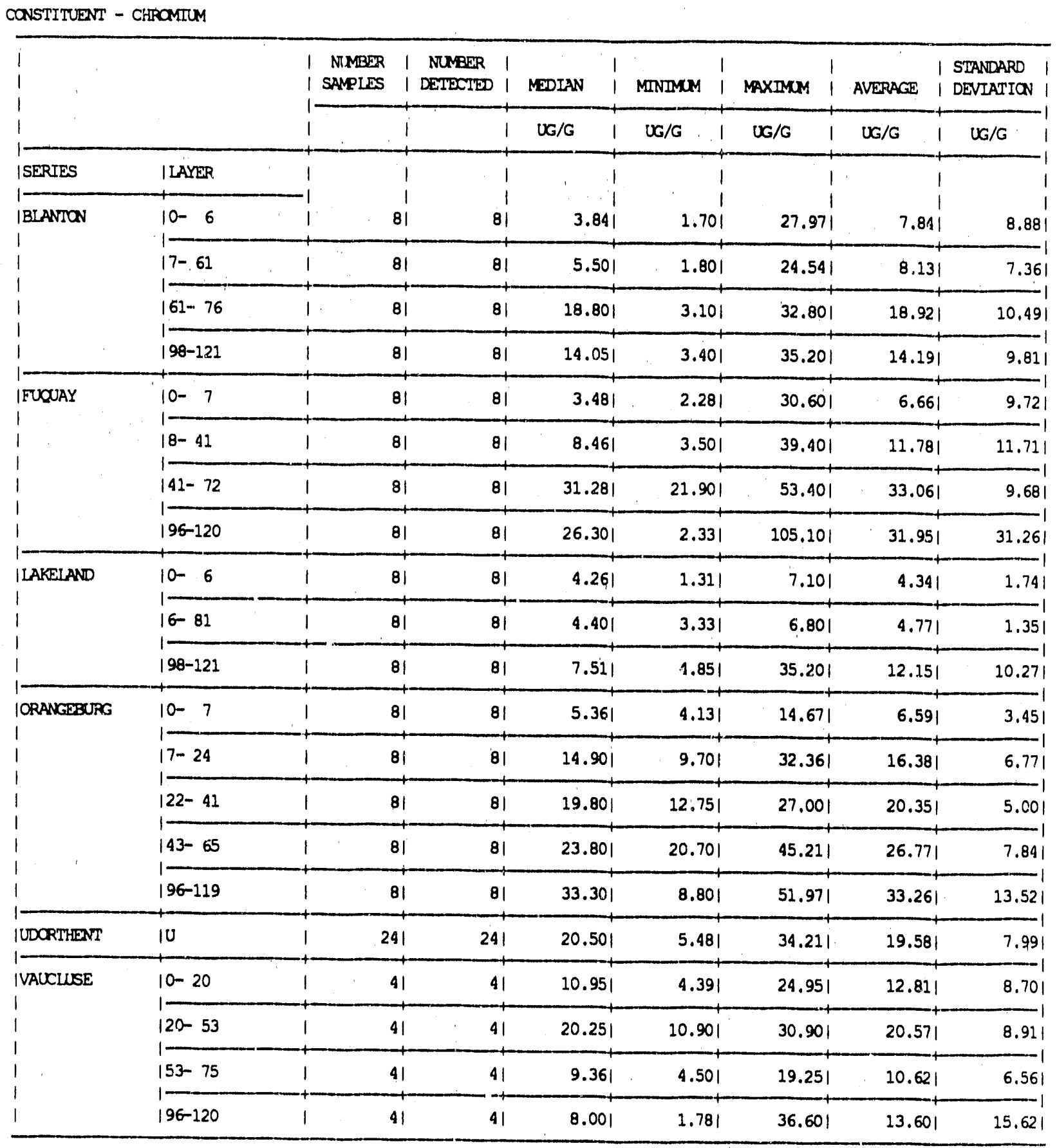


Table 6.7. Summary Statistics for Copper (by series and layer)

CONSTITUENT - COPPER

\begin{tabular}{|c|c|c|c|c|c|c|c|c|c|}
\hline $\begin{array}{l}1 \\
1\end{array}$ & & $\begin{array}{l}\text { NUMBER } \\
\text { I SAMPLES }\end{array}$ & $\begin{array}{l}1 \text { NUMBER } \\
1 \text { DETECTED }\end{array}$ & & MEDIAN 1 & MINIMMM & MxIMUM & AVIRAGE & $\begin{array}{l}\text { SIANDARD } \\
\text { DEVIATION }\end{array}$ \\
\hline 1 & & $i$ & 1 & 1 & $U G / G$ & UG/G & UG/G & $U G / G$ & $U G / G$ \\
\hline ISERIES & IIAYER & 1 & 1 & 1 & 1 & 1 & 1 & 1 & 1 \\
\hline & + & -1 & 1 & 1 & 1 & 1 & 1 & 1 & 1 \\
\hline IBIANTON & $10-6$ & 1 & 81. & 81 & 1.991 & 0.361 & 7.011 & 2.241 & 2.071 \\
\hline i & $17-61$ & 1 & 81 & 71 & 1.911 & $<0.601$ & 6.151 & 2.171 & 1.82 \\
\hline 1 & $161-76$ & 1 & 81 & 81. & $5.501^{\prime}$ & 0.851 & 8.201 & 4.961 & 2.581 \\
\hline i & $98-121$ & 1 & 81 & 81 & 2.721 & 1.001 & 5.401 & 3.241 & 1.621 \\
\hline IFUQUAY & $10-7$ & 1 & 81 & 81 & 1.611 & 0.711 & 2.301 & 1.621 & 0.611 \\
\hline 1 & $18-41$ & 1 & 81 & 81 & 1.451 & 0.851 & 5.001 & 2.351 & 1.571 \\
\hline 1 & $141-72$ & 1 & 81 & 81 & 5.711 & 0.781 & 13.001 & 6.101 & 3.711 \\
\hline . & $196-120$ & 1 & 81 & 81 & 4.581 & 0.641 & 11.101 & 5.251 & 3.401 \\
\hline IILAKELAND & $10-6$ & 1 & 81 & 81 & 1.901 & 1.001 & 2.701 & 1.851 & 0.521 \\
\hline i & $16-81$ & 1 & 81 & 81 & 2.121 & 1.281 & 5.501 & 2.381 & 1.301 \\
\hline $\begin{array}{l}1 \\
1\end{array}$ & $198-121$ & 1 & 81 & 81 & 2.651 & 1.281 & 9.601 & 3.631 & 2.641 \\
\hline IORANGEBURG & $10-7$ & 1 & 81 & 81 & 2.151 & 0.701 & 10.901 & 3.301 & 3.211 \\
\hline 1 & $17-24$ & 1 & 81 & 81 & 5.08 & 3.591 & 11.431 & 6.131 & 2.521 \\
\hline $\begin{array}{l}1 \\
1\end{array}$ & $122-41$ & 1 & 81 & 81 & 5.601 & 3.201 & 7.201 & 5.331 & 1.341 \\
\hline $\begin{array}{l}1 \\
1\end{array}$ & $1-73-65$ & 1 & 81 & 81 & 7.451 & 3.231 & 14.121 & 7.601 & 3.121 \\
\hline $\begin{array}{l}1 \\
1\end{array}$ & $196-119$ & 1 & 81 & 81 & 7.001 & 3.001 & 9.901 & $6.64 \mid$ & 2.301 \\
\hline IUDORTHENT & IU & 1 & 241 & 241 & 3.901 & 1.301 & 10.691 & 4.181 & 2.461 \\
\hline IVAUCLUSE & $10-20$ & 1 & 41 & 41 & 2.471 & 1.011 & 4.801 & 2.691 & 1.571 \\
\hline $\begin{array}{l}1 \\
1\end{array}$ & $120-53$ & 1 & 41 & 31 & 3.321 & $<1.001$ & 5.201 & 3.011 & 2.291 \\
\hline $\begin{array}{l}1 \\
1\end{array}$ & $153-75$ & 1 & 41 & 41 & 2.521 & 2.101 & 3.801 & 2.741 & 0.781 \\
\hline $\begin{array}{l}1 \\
1\end{array}$ & $196-120$ & 1 & 41 & 41 & 2.761 & 1.331 & 4.301 & 2.791 & 1.221 \\
\hline
\end{tabular}




\section{Table 6.8. Summary Statistics for Iron (by series and layer)}

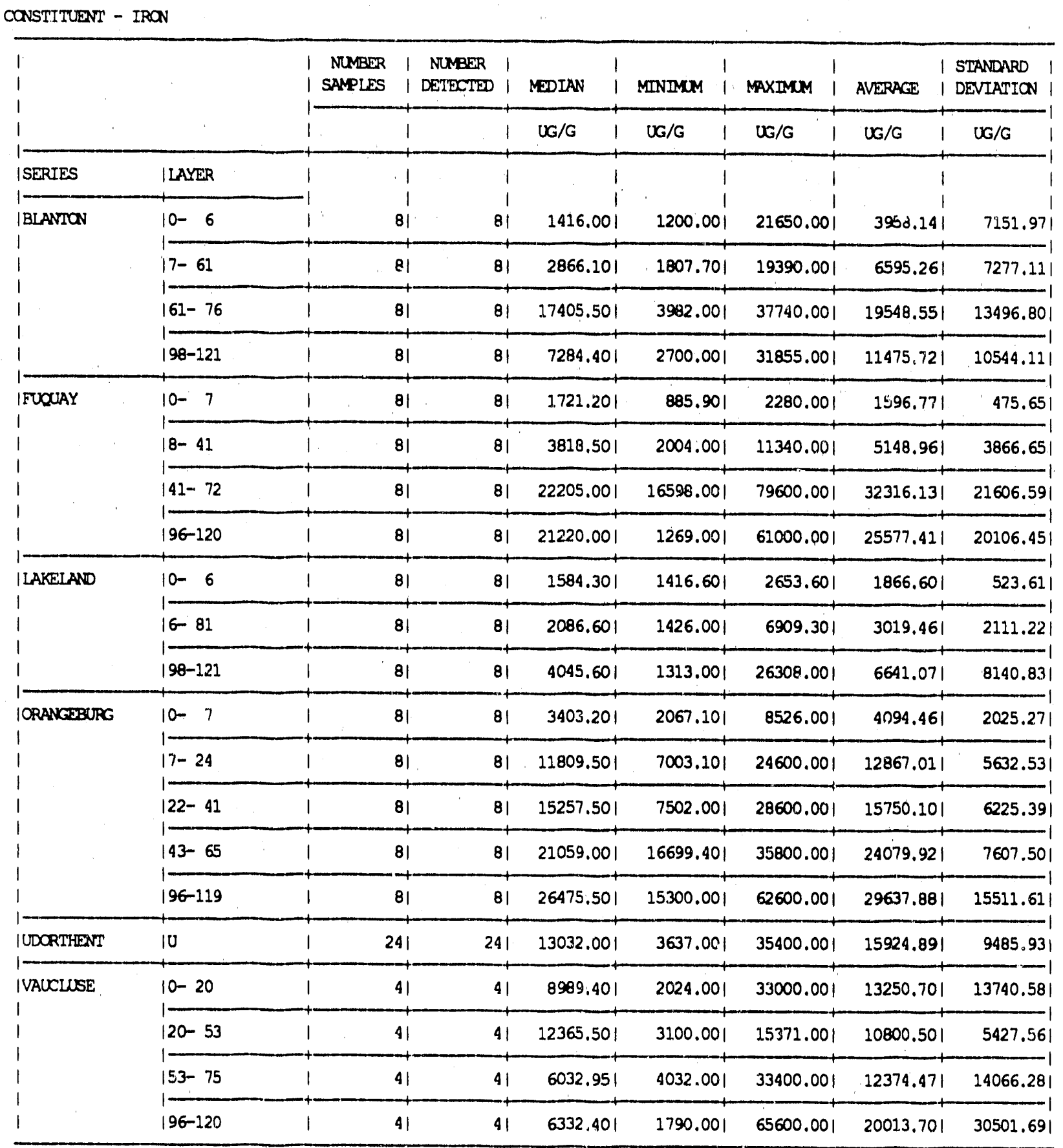


Table 6.9. Summary Statistics for Lead (by series and layer)

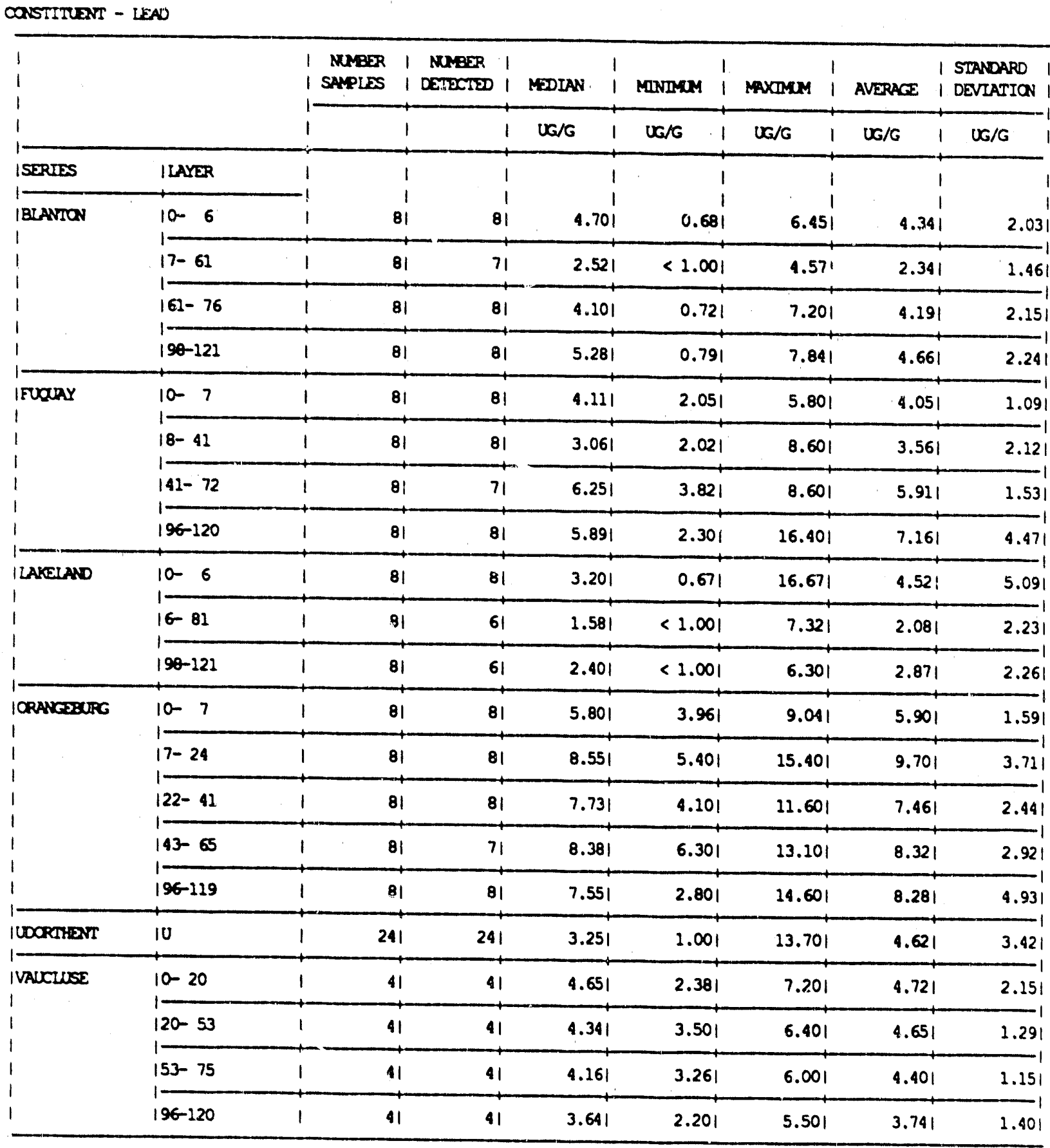


Table 6.10. Summary Statistics for Magnesium (by series and layer)

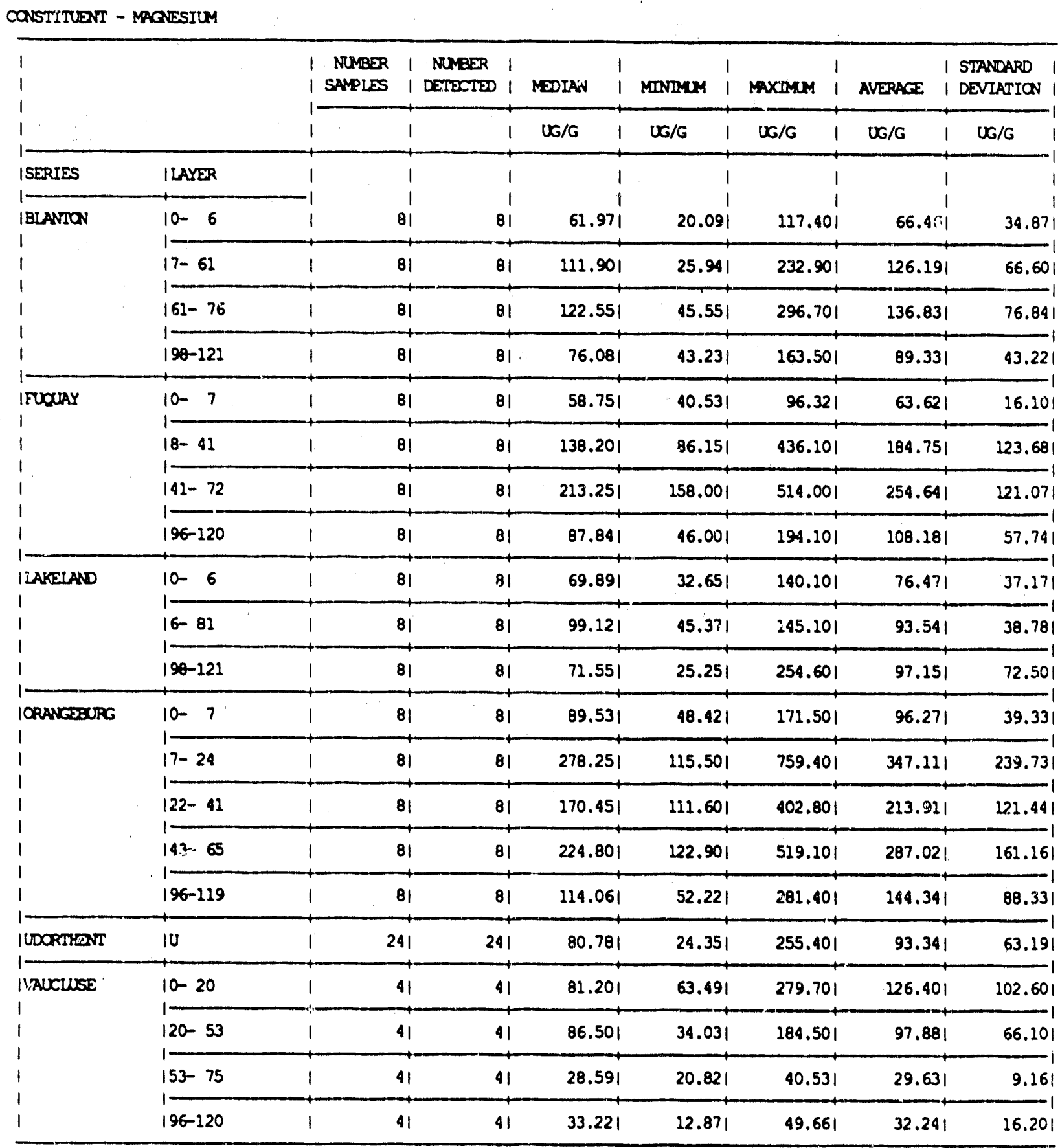


Table 6.11. Summary Statistics for Manganese (by series and layer)

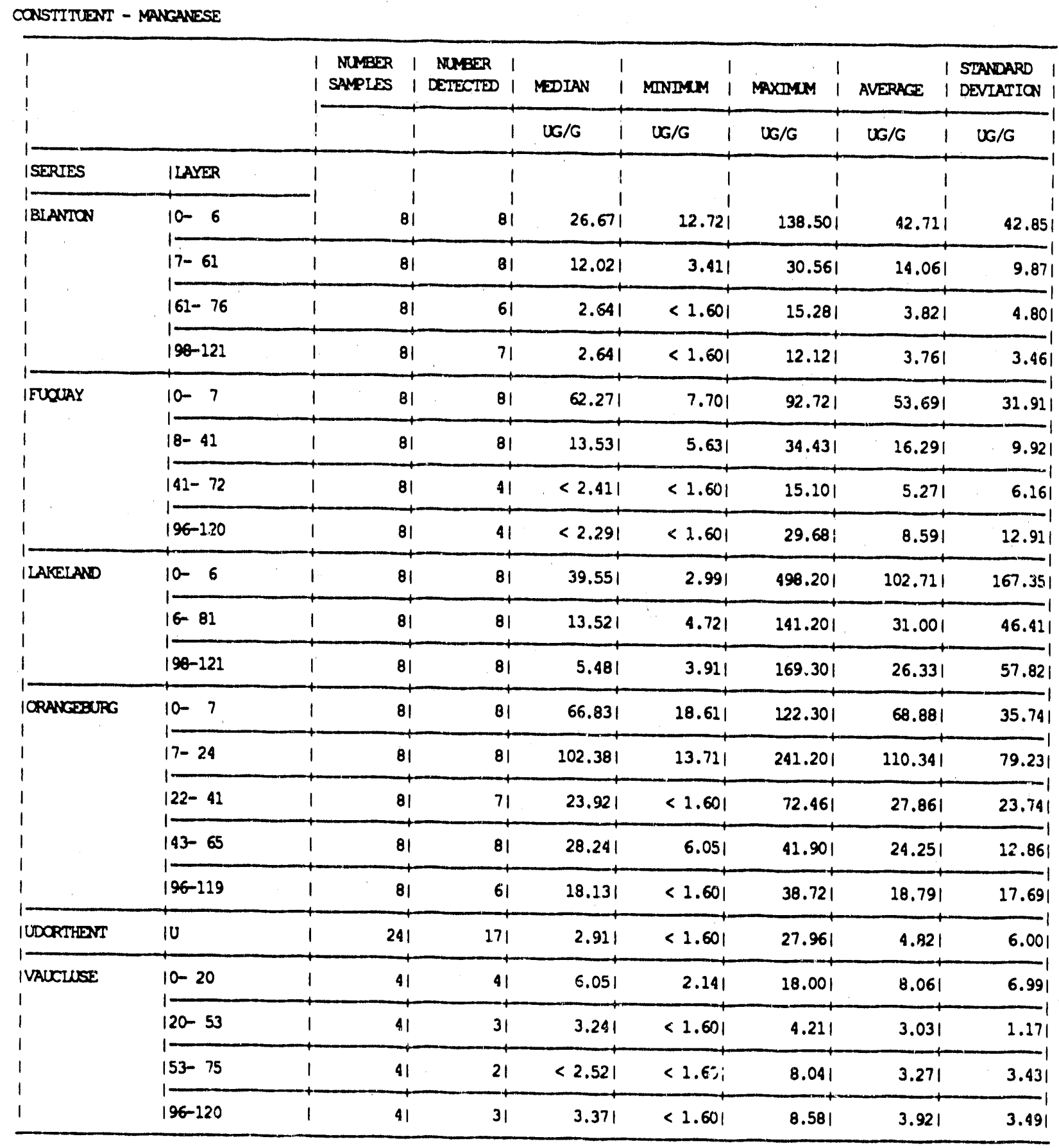


Table 6.12. Summary Statistics for Mercury (by series and layer)

\begin{tabular}{|c|c|c|c|c|c|c|c|c|c|}
\hline $\begin{array}{l}1 \\
1\end{array}$ & & $\begin{array}{l}\text { I NUMBER } \\
\text { I SAMPLES }\end{array}$ & $\begin{array}{l}\text { NIXBER } \\
\text { DETECIED }\end{array}$ & 1 & MEDIAN & MINIMUM & MaximuM & AVERAGE & $\begin{array}{l}\text { STANDARD } \\
\text { DEVTATION }\end{array}$ \\
\hline 1 & & 1 & 1 & 1 & $u g / G$ & UG/G & $U G / G$ & UG/G & $U G / G$ \\
\hline ISERTES & | LAYYRR & 1 & 1 & 1 & 1 & 1 & I & 1 & \\
\hline BLANTON & $10-6$ & -1 & 1 & 1 & 1 & 1 & 1 & 1 & \\
\hline 1 & 1 & 1 & & 11 & $<0.101$ & $<0.101$ & 0.191 & $<0.101$ & 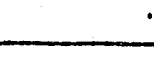 \\
\hline 1 & $17-61$ & 1 & 81 & 31 & $<0.101$ & $<0.101$ & 0.151 & $<0.101$ & 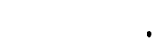 \\
\hline i & $161-76$ & 1 & 81 & 11 & $<0.101$ & $<0.101$ & 0.251 & $<0.101$ & 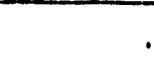 \\
\hline 1 & $190-121$ & 1 & 81 & 31 & $<0.101$ & $<0.101$ & 0.191 & $<0.101$ & 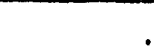 \\
\hline IFUQUAY & $10-7$ & 1 & 81 & 11 & $<0.101$ & $<0.101$ & 0.891 & $<0.101$ & 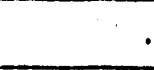 \\
\hline 1 & $18-41$ & 1 & 81 & 2.1 & $<0.101$ & $<0.101$ & 0.101 & $<0.101$ & 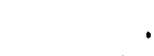 \\
\hline 1 & $141-72$ & 1 & 81 & 31 & $<0.101$ & $<0.101$ & 0.321 & $<0.101$ & 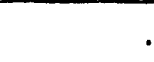 \\
\hline i & $196-120$ & 1 & 81 & 11 & $<0.101$ & $<0.101$ & 0.221 & $<0.101$ & 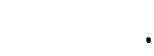 \\
\hline ILAKELAND & $10-6$ & 1 & 81 & 01 & $<0.101$ & $<0.101$ & $<0.101$ & $<0.101$ & 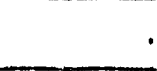 \\
\hline 1 & $18-81$ & 1 & 81 & 11 & $<0.101$ & $<0.201$ & 0.101 & $<0.101$ & 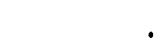 \\
\hline 1 & $190-121$ & 1 & 81 & 11 & $<0.101$ & $<0.101$ & 0.141 & $<0.101$ & 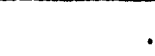 \\
\hline IORANGERURG & $10-7$ & 1 & 81 & 01 & $<0.101$ & $<0.101$ & $<0.101$ & $<0.101$ & . \\
\hline 1 & $17-24$ & 1 & 81 & 01 & $<0.101$ & $<0.101$ & $<0.101$ & $<0.101$ & 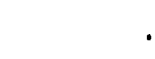 \\
\hline i & $122-41$ & 1 & 81 & 21 & $<0.101$ & $<0.101$ & 0.111 & $<0.101$ & 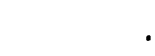 \\
\hline i & $143-65$ & 1 & 81 & 21 & $<0.101$ & $<0.011$ & $0.19 !$ & $<0.101$ & . \\
\hline i & $196-119$ & 1 & 81 & 11 & $<0.101$ & $<0.101$ & 0.171 & $<0.101$ & 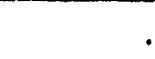 \\
\hline IUDORTHENT & 10 & 1 & 241 & 21 & $<0.101$ & $<0.101$ & 0.151 & $<0.101$ & 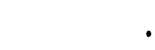 \\
\hline IVALCLUSE & $10-20$ & 1 & 41 & 01 & $<0.101$ & $<0.011$ & $<0.101$ & $<0.101$ & \\
\hline i & $120-53$ & 1 & 41 & 01 & $<0.101$ & $<0.101$ & $<0.101$ & $<0.101$ & \\
\hline 1 & $153-75$ & 1 & $4 i$ & 01 & $<0.101$ & $<0.101$ & $<0.101$ & $<0.101$ & \\
\hline 1 & $196-120$ & 1 & 41 & 01 & $<0.101$ & $<0.101$ & $<0.101$ & $<0.101$ & \\
\hline
\end{tabular}


Table 6.13. Summary Statistics for Nickel (by series and layer)

CONSTITUENT - NICKEL

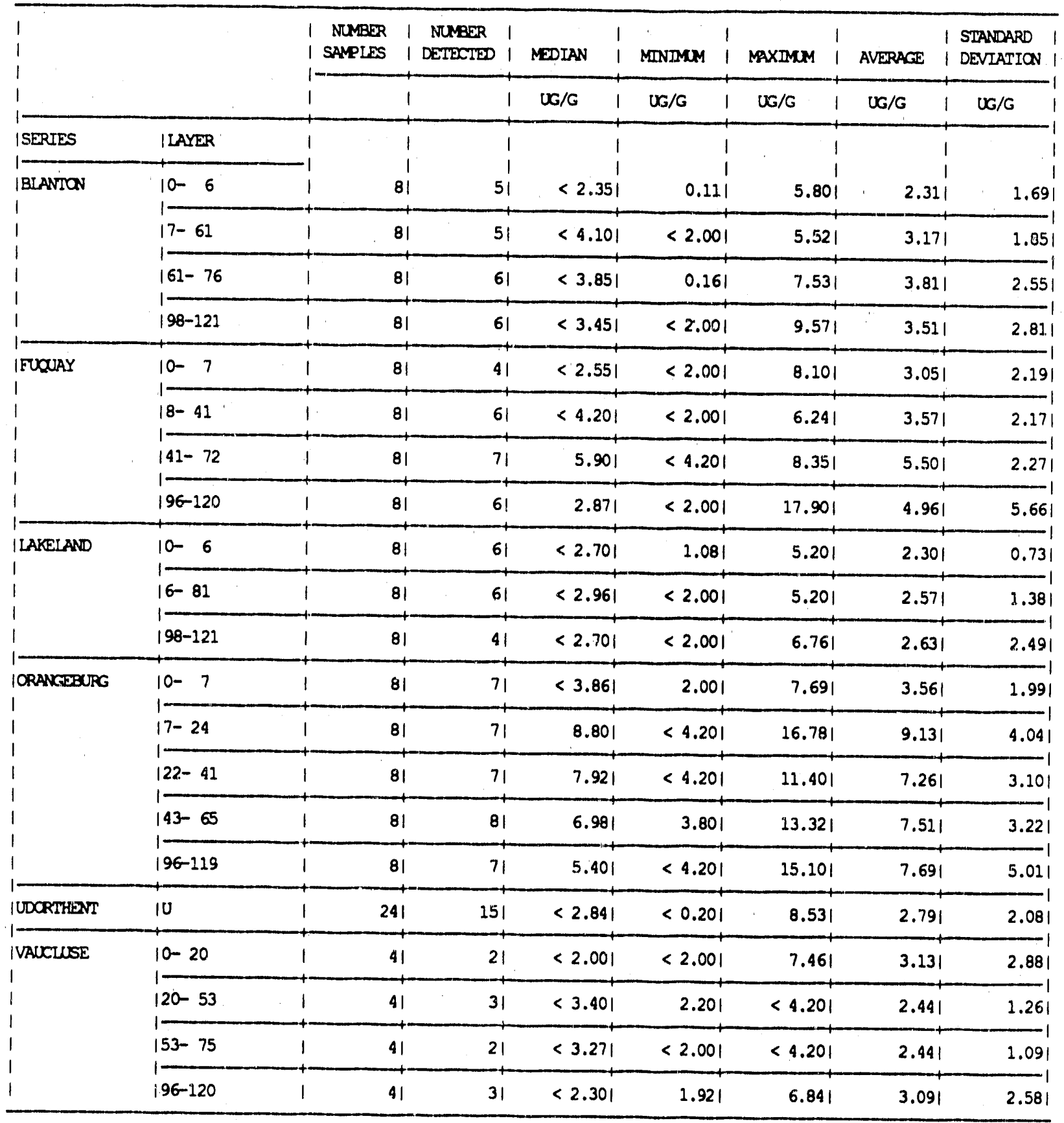


Table 6.14. Summary Statistics for Potassium (by series and layer)

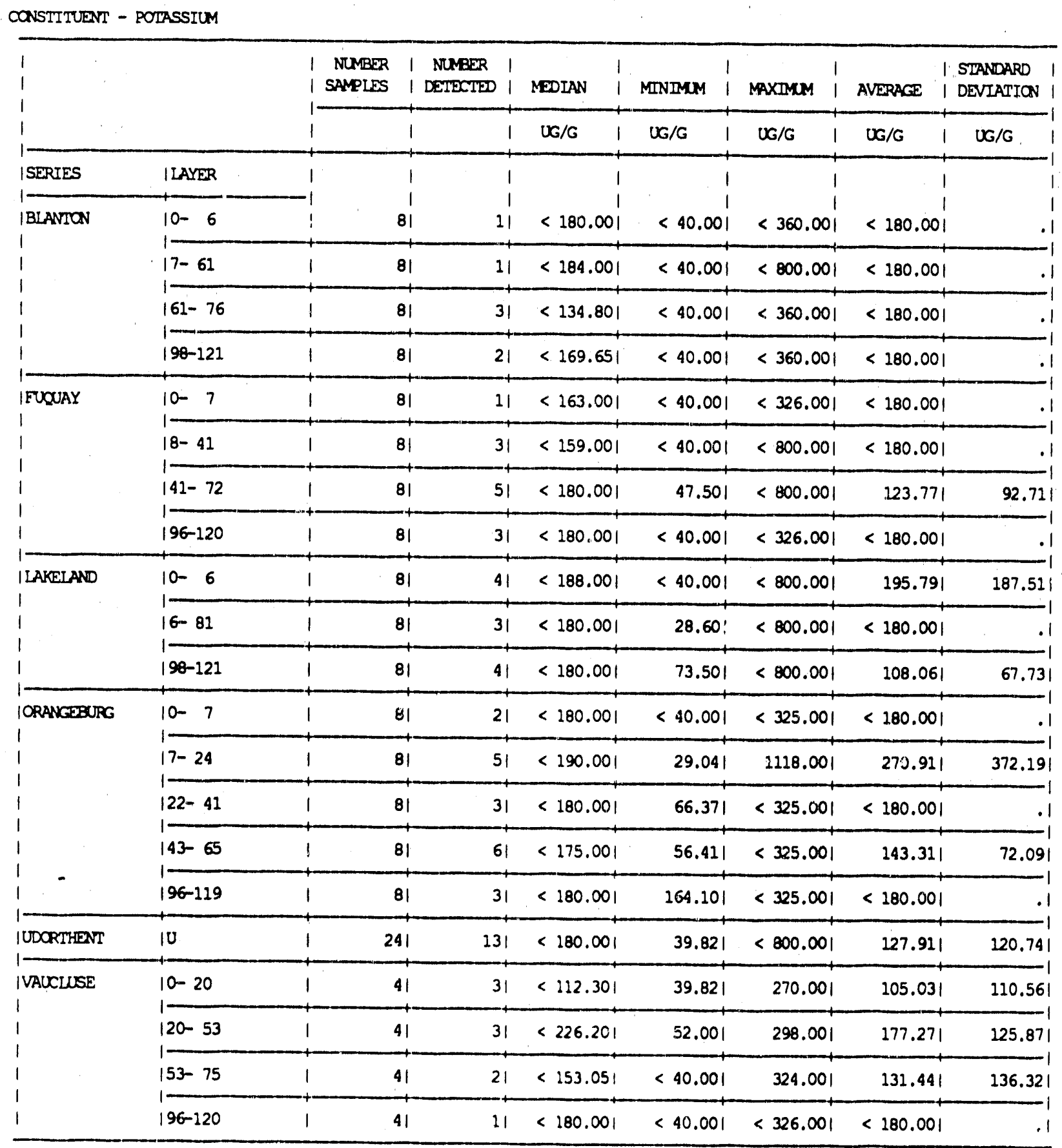


Table 6.15. Summary Statistics for Selenium (by series and layer)

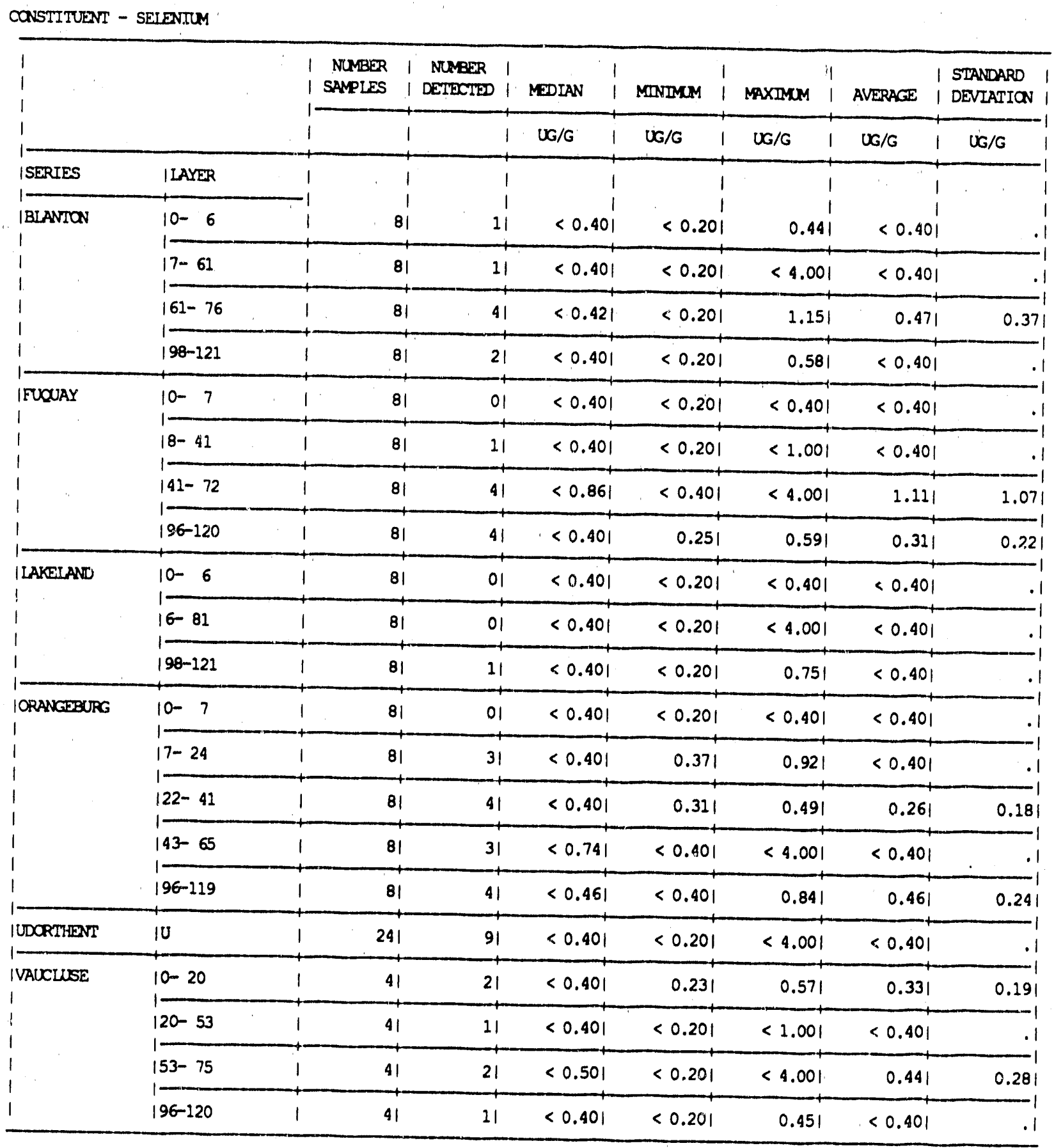


Table 6.16. Summary Statistics for Silver (by series and layer)

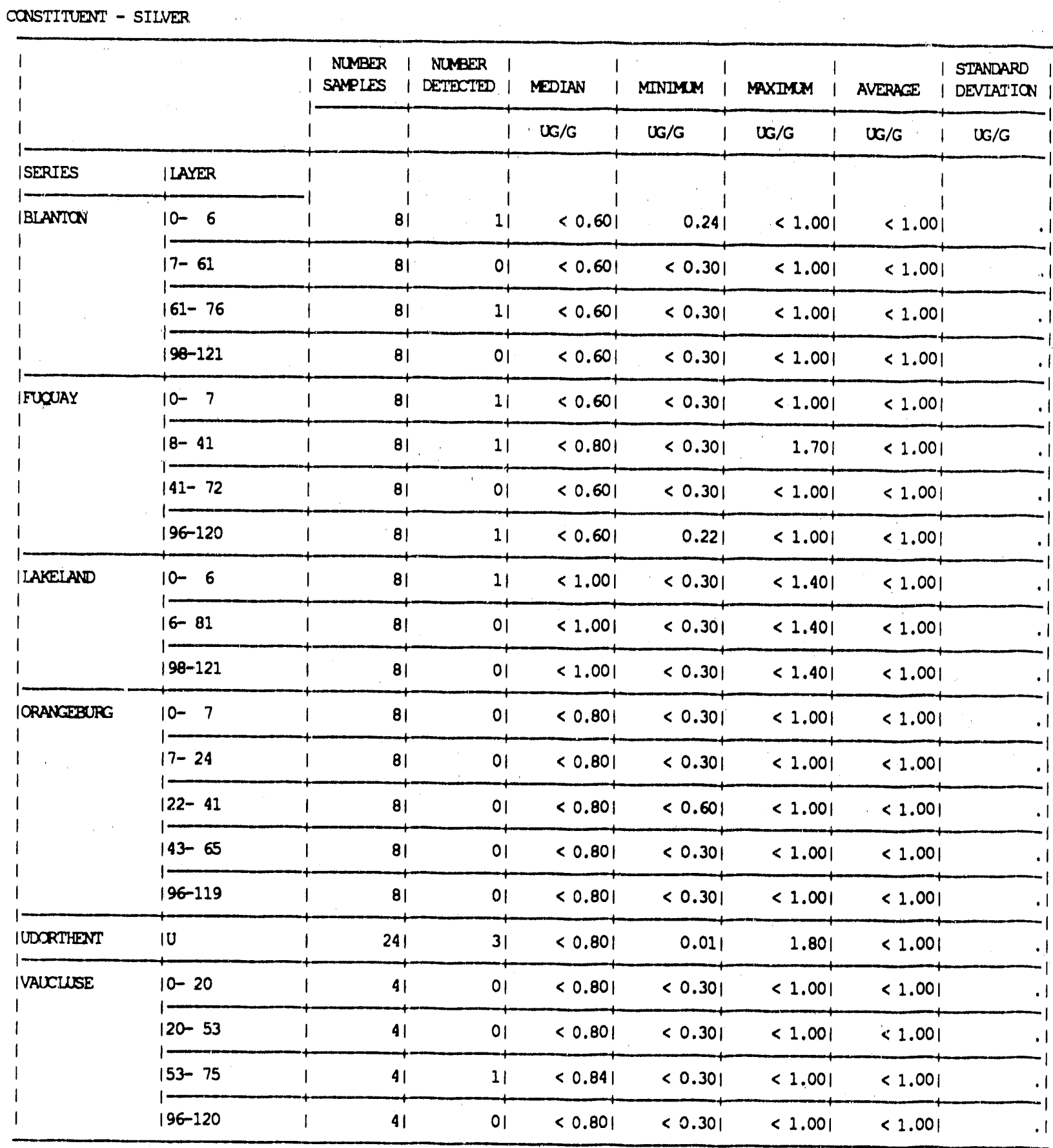


Table 6.17. Summary Statistics for Sodium (by series and layer)

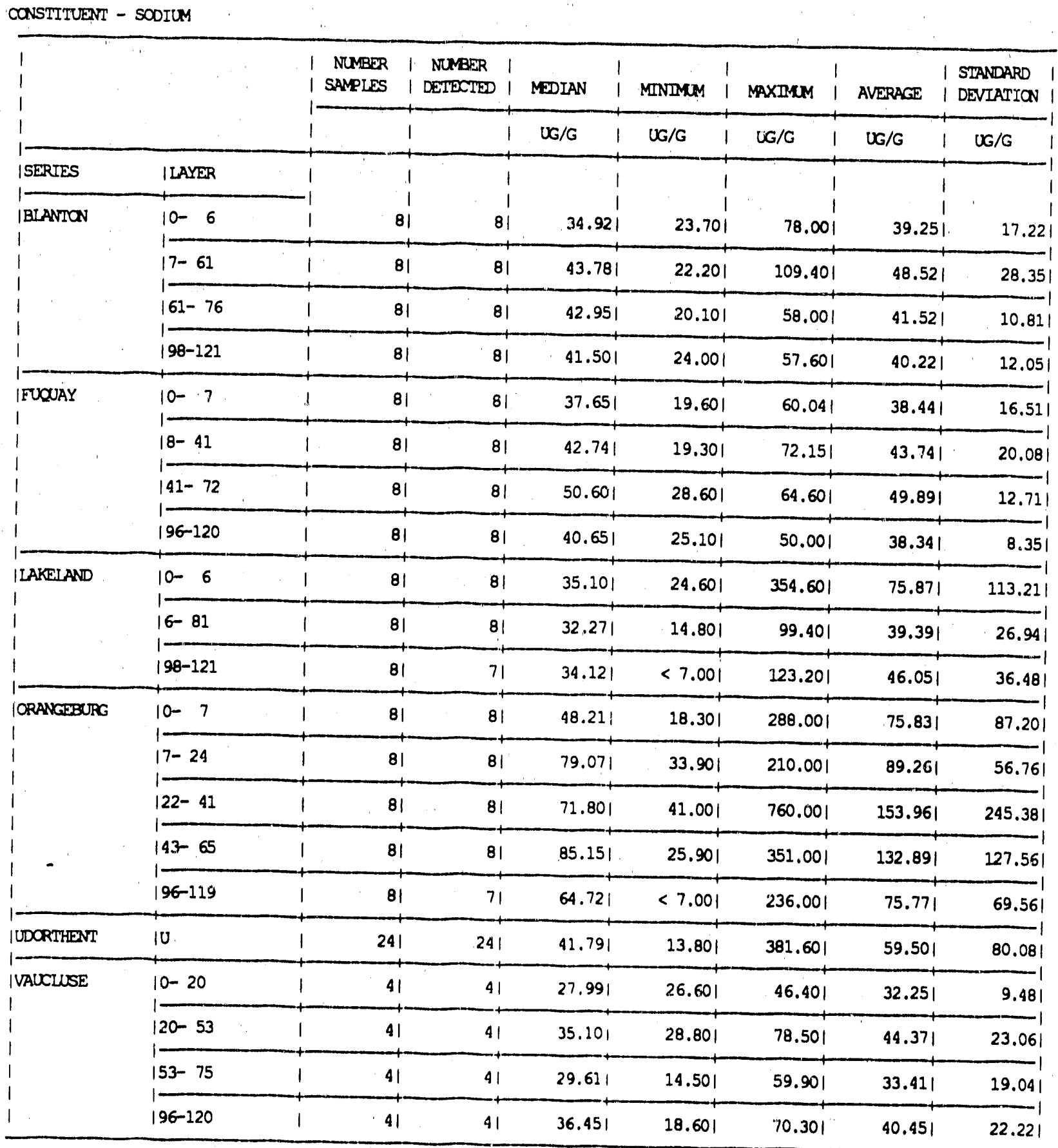


Table 6.18. Summary Statistics for Zinc (by series and layer)

\begin{tabular}{|c|c|c|c|c|c|c|c|c|c|}
\hline \multirow{3}{*}{$\begin{array}{l}1 \\
1 \\
1 \\
1\end{array}$} & & \multirow{2}{*}{$\begin{array}{l}\text { NUMBER } \\
\text { SAMPLIES } \\
\end{array}$} & \multirow{2}{*}{$\begin{array}{r}\text { N NUMBER } \\
\text { I DETECTED } \\
\end{array}$} & \multirow[t]{2}{*}{1} & \multirow[t]{2}{*}{ MEDIAN } & $\therefore \quad 1$ & $\operatorname{MxIMM}$ & AVERACEE & $\begin{array}{l}\text { STANDARD } \\
\text { DEVIATION }\end{array}$ \\
\hline & & & & & & & & & \\
\hline & & 1 & 1 & 1 & $x \in / G$ & UG/G & $U G / G$ & UG/G & $U G / G$ \\
\hline ISERTES & I LAYER & 1 & 1 & 1 & 1 & 1 & 1 & $\cdot .1$ & I \\
\hline & & -1 & 1 & $i$ & & & 1 & i & \\
\hline IBIANTION & $10-6$ & 1 & 81 & 81 & 5.321 & 3.201 & 13.591 & 7.011 & 3.791 \\
\hline $\begin{array}{l}1 \\
1\end{array}$ & $17-61$ & -1 & 81 & 81 & 8.581 & 3.001 & 14.501 & 8.521 & 4.28 \\
\hline 1 & 1 & 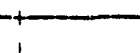 & & & & & & & \\
\hline 1. & $161-76$ & 1 & 81 & 81 & 9.881 & 5.801 & 13.601 & 9.761 & 2.791 \\
\hline 1 & $198-121$ & 1 & 81 & 81 & 10.331 & 3.401 & 24.601 & 11.331 & 6.201 \\
\hline IFUQUAY & $10-7$ & 1 & 81 & 81 & 6.851 & 4.691 & 12.951 & 7.621 & 2.951 \\
\hline $\begin{array}{l}1 \\
1\end{array}$ & $18-41$ & $i$ & 81. & 81 & 6.611 & 2.201 & 16.801 & 7.701 & 4.661 \\
\hline 1 & & & & & & & & & \\
\hline 1 & $141-72$ & 1 & 81 & 81 & 13.351 & 4.201 & 19.601 & 12.181 & 5.281 \\
\hline $\begin{array}{l}1 \\
1\end{array}$ & $196-120$ & 1 & 81 & 81 & 9.021 & 3.401 & 14.401 & 9.301 & 4.181 \\
\hline ILAKELAND & $10-6$ & 1 & 81 & 81 & 7.231 & 3.601 & 11.801 & 7.191 & 2.681 \\
\hline 1 & 1 & & & - & & & & & \\
\hline i & $16-81$ & 1 & 81 & 81 & 8.651 & 9.801 & 17.201 & 10.151 & 4.821 \\
\hline 1 & $198-121$ & $\begin{array}{r}+- \\
1\end{array}$ & (1) & 81 & 8.961 & 4.201 & 12.101 & 8.881 & 2.821 \\
\hline IORANGEBURG & $10-7$ & 1 & 81 & 81 & 8.261 & 5.201 & 24.601 & 10.231 & 6.231 \\
\hline 1 & $7-24$ & + & 81 & 81 & 17.901 & 12.301 & 28.461 & 18.75 & 5.201 \\
\hline 1 & 1 & - & - & + & & & & & \\
\hline$i$ & |22- 41 & 1 & 81 & 81 & 14.761 & 9.001 & 23.941 & $15.28 !$ & 4.451 \\
\hline i & $143-65$ & I & 81 & 81 & 13.401 & 9.001 & 33.421 & $15.53 \mid$ & 7.811 \\
\hline 1 & $96-119$ & 1 & 81 & 81 & 11.601 & 7.001 & 28.601 & 14.241 & 7.511 \\
\hline IUDORTHENT & |ט & 1 & 291 & 241 & 9.651 & 1.801 & 30.141 & 10.601 & 7.221 \\
\hline IVAUCLUSE & $10-20$ & 1 & 41 & 41 & 11.701 & 6.331 & 18.001 & 11.931 & 4.781 \\
\hline 1 & $120-53$ & 1 & 41 & 41 & 10.701 & 6.201 & 32.701 & 15.071 & 11.951 \\
\hline$i$ & & & & & & & & & \\
\hline 1 & $153-75$ & 1 & 41 & 41 & 9.251 & 7.181 & 267.001 & 73.171 & $129.23 \mid$ \\
\hline $\begin{array}{l}1 \\
1\end{array}$ & $196-120$ & 1 & 41 & 41 & 8.831 & 5.001 & 13.901 & 9.141 & 4.131 \\
\hline
\end{tabular}


Table 6.19. Summary Statistics for Other Inorganic Parameters Measured (all samples)

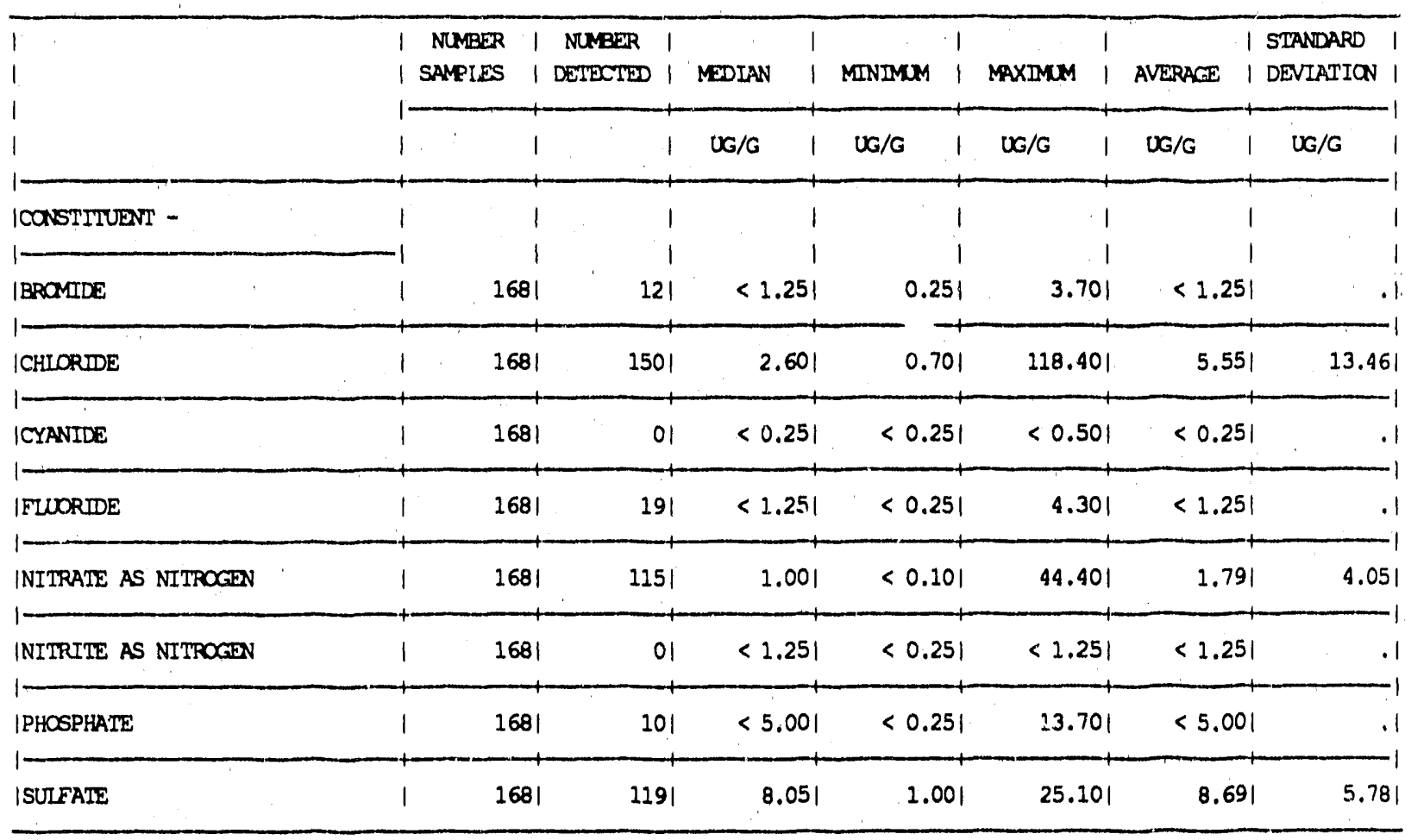


Table 6.20. Summary Statistics for Chloride (by series and layer)

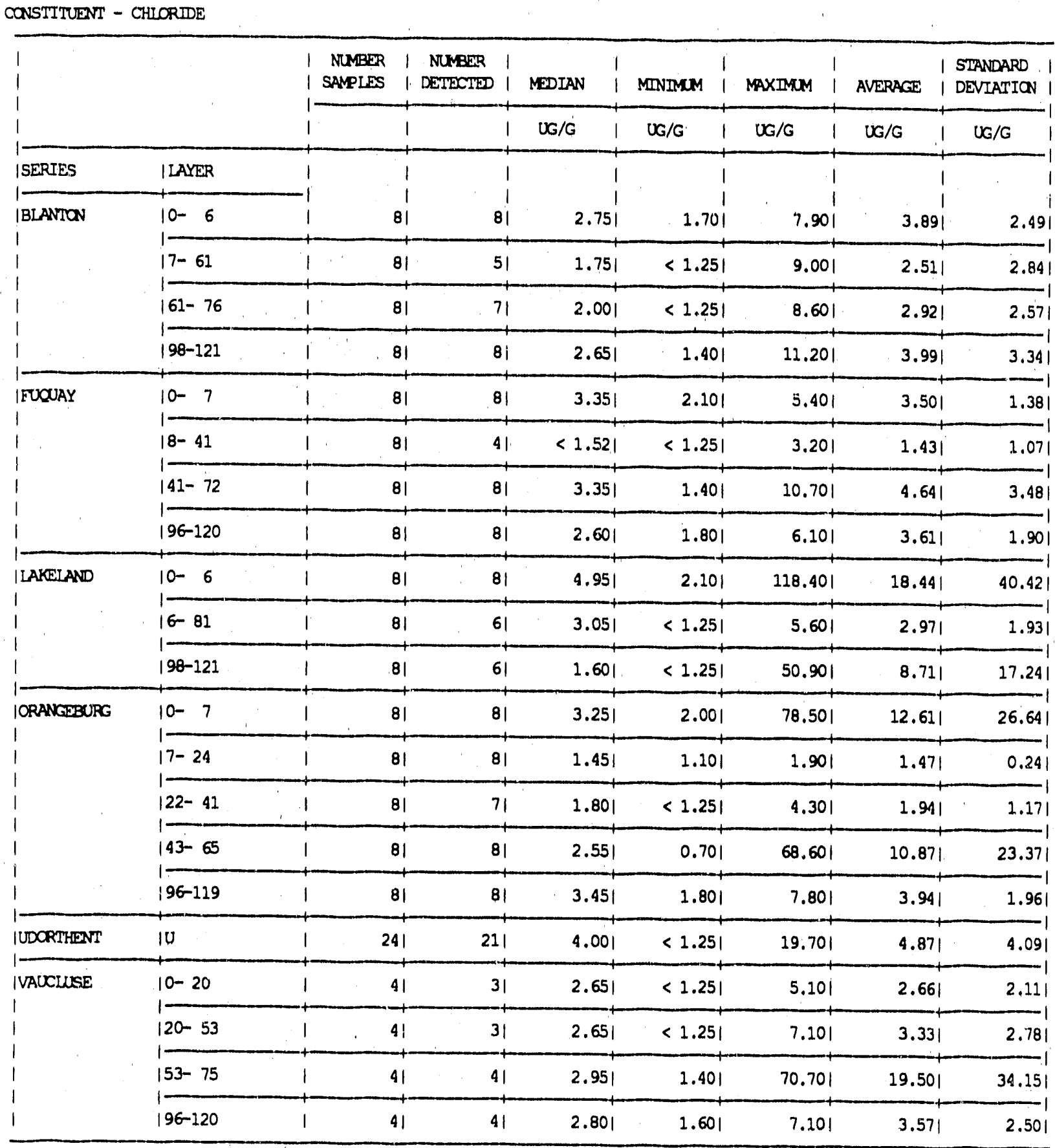


Table 6.21. Summary Statistics for Nitrate (by series and layer)

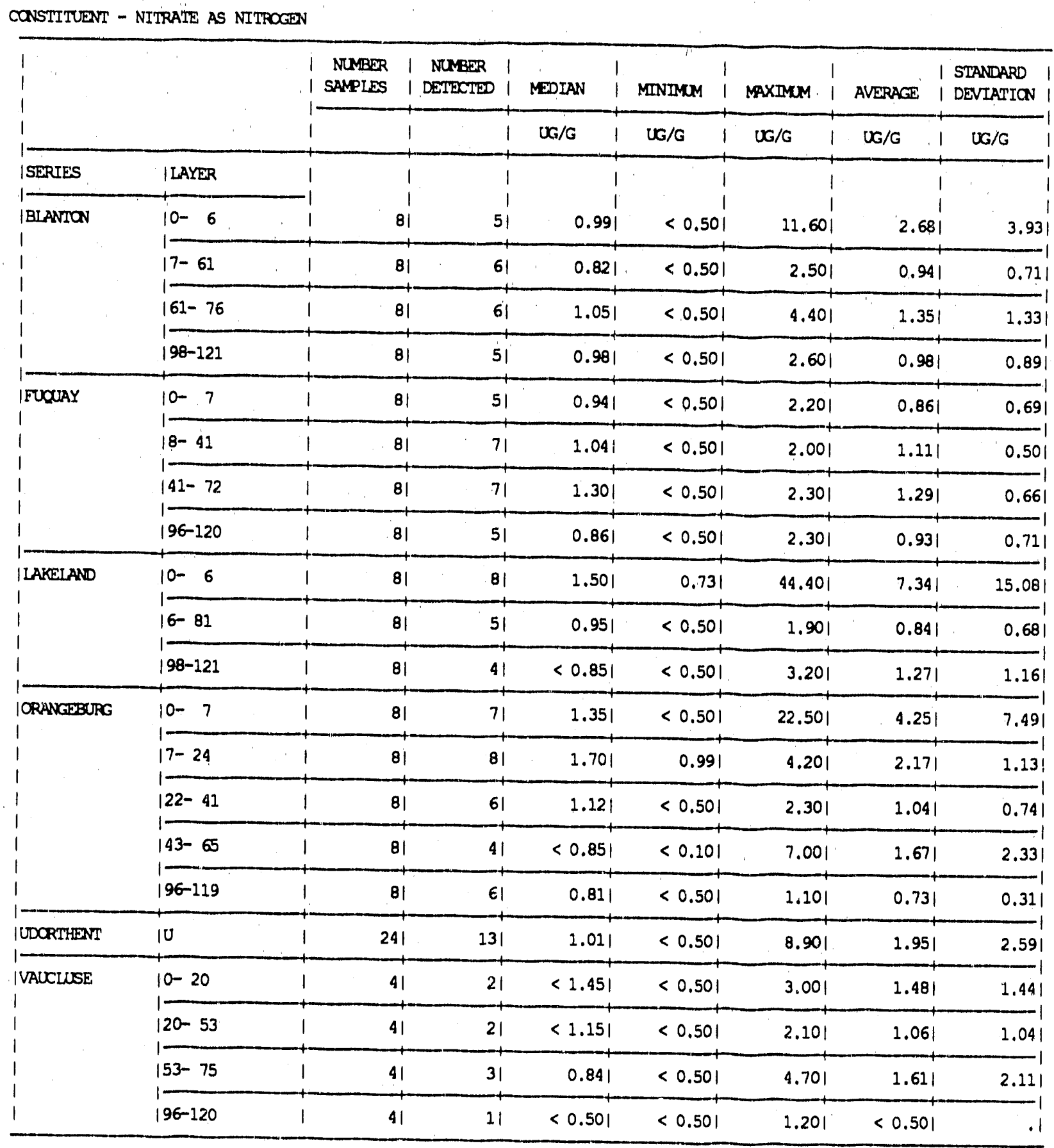


-Table 6.22. Summary Statistics for Sulfate (by series and layer)

\begin{tabular}{|c|c|c|c|c|c|c|c|c|c|}
\hline 1 & & $\begin{array}{l}\text { NUMBER } \\
\text { SAMPLES }\end{array}$ & \begin{tabular}{l|c} 
I NUMBER \\
I DETECTED
\end{tabular} & & $\begin{array}{ll}1 \\
\text { MEDIAN } 1\end{array}$ & $\begin{array}{l}1 \\
\text { MINDMUM }\end{array}$ & MAXIMCM & AVERAGE ! & $\begin{array}{l}\text { STANDARD } \\
\text { DEVIATIION }\end{array}$ \\
\hline 1 & & 1 & 1 & 1 & $U G / G$ & $U G / G$ & $U G / G$ & $U_{G / G}$ & UG/G \\
\hline | SERTES & | LAYERR & 1 & 1 & 1 & 1 & 1 & 1 & 1 & 1 \\
\hline BLANTION & $10-6$ & $\begin{array}{r}-1 \\
1\end{array}$ & o1 & $8 !$ & $\begin{array}{r}1 \\
10.851\end{array}$ & 6.501 & 18.201 & $\begin{array}{r}1 \\
10.991\end{array}$ & 4.151 \\
\hline . & $17-61$ & 1 & 81 & 81 & 8.901 & 7.701 & 16.901 & 11.271 & 3.851 \\
\hline . & $161-76$ & 1. & B) & 71 & 9.501 & $<5.001$ & 14.301 & 8.751 & 3.501 \\
\hline 1 & $198-121$ & 1 & 81 & 31 & $<5.001$ & $<5.001$ & 14.301 & $<5.001$ & .1 \\
\hline IFUQUAY & $10-7$ & 1 & 8) & 81 & 10.151 & 6.401 & 19.501 & 11.111 & 4.281 \\
\hline 1 & $18-41$ & 1 & 81 & 81 & 11.101 & 7.301 & 19.201 & 12.111 & 3.841 \\
\hline $\begin{array}{l}1 \\
1\end{array}$ & $141-72$ & 1 & 81 & 61 & 6.001 & 4.401 & 10.501 & 6.251 & 1.99 \\
\hline $\begin{array}{l}1 \\
1\end{array}$ & $96-120$ & + & 81 & 11 & $<5.001$ & $<5.001$ & 10.501 & $<5.001$ & -1 \\
\hline ILAKEIAND & $10-6$ & 1 & 81 & 81 & 11.201 & 7.101 & 19.901 & 12.091 & 4.811 \\
\hline 1 & $1 \overline{6-81}$ & + & 81 & 81 & 8.501 & 7.401 & 13.301 & 9.071 & 1.381 \\
\hline 1 & $190-121$ & -+ & $-1-$ & 71 & 11.351 & $<5.001$ & 17.801 & 10.301 & 4.651 \\
\hline IORANGEBURG & $10-7$ & 1 & 81 & 81 & 10.601 & 5.801 & 14.301 & 9.901 & 3.121 \\
\hline 1 & 1 & + & & a) & $1<051$ & & & & \\
\hline 1 & $17-24$ & 1 & 81 & 81 & 16.951 & 14.101 & 20.701 & 17.411 & 2.761 \\
\hline 1 & $122-41$ & 1 & 81 & 71 & .14 .901 & $<5.001$ & 18.501 & 1.3 .741 & 5.721 \\
\hline 1 & $143-65$ & 1 & 81 & 61 & $<5.901$ & 1.001 & 21.401 & 8.221 & 7.891 \\
\hline $\begin{array}{l}1 \\
1\end{array}$ & $196-119$ & -+ & 81 & 21 & $<5.001$ & 4.901 & 6.801 & $<5.001$ & .1 \\
\hline IUDCRTHEAT & |U & 1 & 241 & 101 & $<5.001$ & 2.601 & 21.201 & $<5.001$ & .1 \\
\hline IVAUCLUSE & $10-20$ & 1 & 41 & 41 & 18.001 & $9.50 !$ & 25.101 & 17.651 & 6.531 \\
\hline 1 & $120-53$ & 1 & 41 & 11 & $<5.001$ & $<5.001$ & 5.301 & $<5.001$ & .1 \\
\hline $\begin{array}{l}1 \\
1\end{array}$ & 15 & $\begin{array}{c}-+- \\
1\end{array}$ & 41 & 11 & $<5.001$ & $<5.001$ & 21.401 & $<5.001$ & .1 \\
\hline 1 & $196-120$ & + & 41 & 01 & $<5.001$ & $<5.001$ & $<5.001$ & $<5.001$ & 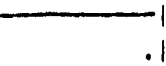 \\
\hline
\end{tabular}


Table 6.23. Summary Statistics for Bulk Organic Constituents (all samples)

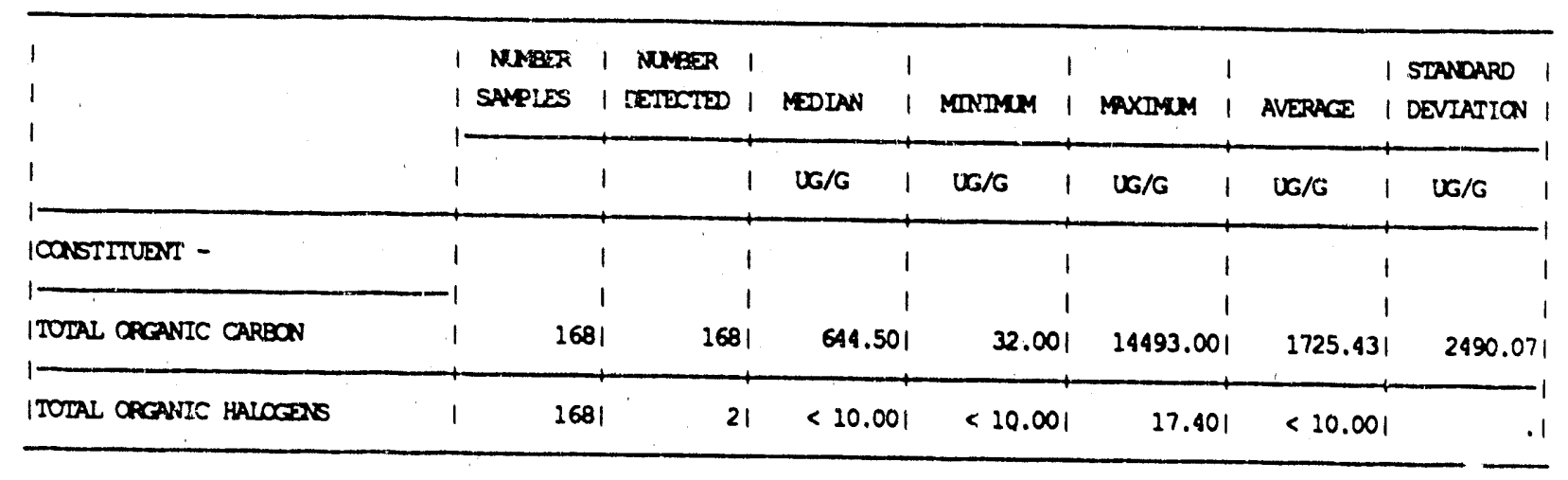


Table 6.24. Summary Statistics for Total Organic Carbor (by series and layer)

CONSTITUENT - TOTAL ORCANIC CARBON

\begin{tabular}{|c|c|c|c|c|c|c|c|c|c|}
\hline $\begin{array}{l}3 \\
1 \\
1\end{array}$ & & $\begin{array}{l}\text { MMPER } \\
\text { I SAMPLES } \\
\end{array}$ & $\begin{array}{r}1 \text { NUMBER } \\
1 \text { DETECTED } \\
\end{array}$ & & $\begin{array}{l}1 \\
\text { MEDIAN } 1 \\
\end{array}$ & $\begin{array}{r}1 \\
\text { MnNaMaM } 1 \\
\end{array}$ & maxim 1 & AVERACE & $\begin{array}{l}\text { STANDARD . I } \\
\text { DEVIATION }\end{array}$ \\
\hline i & & 1 & 1 & 1 & $U G / G$ & $U G / G \quad \mid$ & $\cup \mathbf{G} / \mathbf{G}$ & 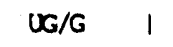 & $U G / G$ \\
\hline ISERIES & I LAYER & $i$ & 1 & 1 & 1 & 1 & 1 & 1 & 1 \\
\hline & & -1 & 1 & 1 & 1 & 1 & 1 & 1 & 1 \\
\hline IBLANTON & $10-6$ & 1 & 81 & 81 & 4234.501 & 3080.001 & 8842.001 & 4754.631 & 1884.251 \\
\hline 1 & $17-61$ & 1 & 81 & 81 & 1108.501 & 300.001 & 4838.001 & 1516.251 & 1483.401 \\
\hline 1 & $161-76$ & 1 & 81 & 81 & 614.501 & 290.001 & 1429.001 & $668.88 \mid$ & 387.351 \\
\hline 1 & $198-121$ & 1 & 81 & 81 & 357.001 & 270.001 & 860.001 & 441.131 & 200.151 \\
\hline $\begin{array}{l}\text { IFUQUAY } \\
1\end{array}$ & $10-7$ & 1 & 81 & 81 & 4424.001 & 2957.001 & 13146.001 & 6068.381 & 3846.251 \\
\hline 1 & $18-41$ & 1 & 81 & 81 & 577.001 & 301.001 & 1270.001 & 730.501 & 351.371 \\
\hline 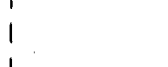 & $141-72$ & 1 & 81 & 81 & 595.501 & 394.001 & 1330.001 & 704.131 & 354.201 \\
\hline 1 & $196-120$ & 1 & 81 & 81 & 312.501 & 221.001 & 220.001 & 378.381 & 254.461 \\
\hline $\begin{array}{l}\text { | LAKELAND } \\
\text { | }\end{array}$ & $10-6$ & 1 & 81 & 81 & 7265.001 & 908.001 & 14493.001 & 7183.131 & 4678.501 \\
\hline $\begin{array}{l}1 \\
1\end{array}$ & $16-81$ & 1 & 81 & 81 & 441.001 & 220.001 & 4555.001 & 930.881 & 1468.861 \\
\hline 1 & $190-121$ & 1 & 81 & 81 & 672.001 & 202.001 & 1646.001 & 676.751 & 449.101 \\
\hline $\begin{array}{l}\text { IORANCEBURG } \\
1\end{array}$ & $10-7$ & 1 & 81 & 81 & 3324.501 & 2499.001 & 5480.001 & 3442.131 & 932.901 \\
\hline 1 & $17-24$ & 1 & 81 & 81 & 1576.501 & 619.001 & 2678.001 & 1576.251 & 664.761 \\
\hline 1 & $122-41$ & 1 & 81 & 81 & 933.501 & 625.001 & 2615.001 & 1139.631 & 617.401 \\
\hline 1 & $143-65$ & 1 & 81 & 81 & 719.501 & 399.001 & 1272.001 & 765.381 & 340.731 \\
\hline 1 & $196-119$ & 1 & 81 & 81 & 479.001 & 257.001 & 1176.001 & 522.251 & 281.421 \\
\hline $\begin{array}{l}\text { |UDCRT.ADVI } \\
\text { | }\end{array}$ & 10 & 1 & 241 & 291 & 340.501 & 32.001 & 5260.001 & 572.171 & 1033.121 \\
\hline IVAUK LUSE & $10-20$ & 1 & 41 & 91 & 3930.001 & 971.001 & 5703.001 & 3633.501 & 2317.171 \\
\hline i & $120-53$ & 1 & 41 & 91 & 662.501 & 263.001 & 2236.001 & 1956.001 & 2860.071 \\
\hline 1 & $153-75$ & 1 & 41 & 41 & 261.501 & 90.001 & 487.001 & 275.001 & 163.521 \\
\hline$i$ & $196-120$ & 1 & 41 & 41 & 190.501 & 79.001 & 233.001 & 173.251 & 65.961 \\
\hline
\end{tabular}


Table 6.25. SRS Soil Study Appendix VIII Organic Compounds Detected

Organic Compound

bis (2-ethylhexyl) phthalate
acetone
methylene chloride
dieldrin
endosulfan sulfate
butylbenzyl phthalate

bis (2-ethylhexyl) phthalate dieldrin butylbenzyl phthalate

\section{Frequency of} Occurrence

10

13

15

1

5

1
Concentration

Range (ppb)

$10-4,800$

$42-1,600$

4. 70

9.6

28.2 - 3940

10 
Table 6.26. Summary Statistics for Radionuclides (all samples)

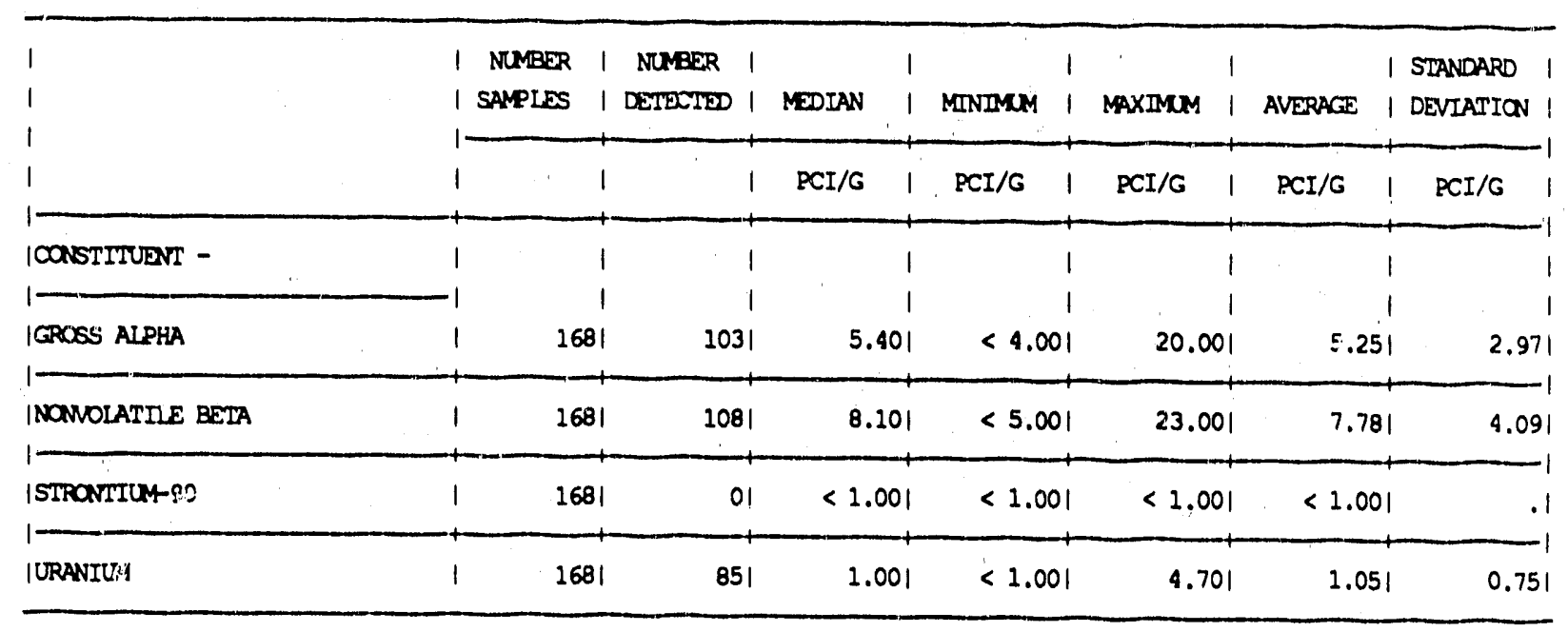


Table 6.27. Summary Statistics for Gross Alpha (by series and layer)

\begin{tabular}{|c|c|c|c|c|c|c|c|c|c|}
\hline 1 & & $\begin{array}{l}\text { NUMBER } \\
\text { I SAMPLES }\end{array}$ & . I NUMBER & $\begin{array}{ll}1 \\
0 & 1\end{array}$ & $\begin{array}{ll} & 1 \\
\text { MEDIAN } & 1\end{array}$ & MINDMUM & MAXIMUM & AVERACE 1 & $\begin{array}{l}\text { STANDARD I } \\
\text { DEVIATION । }\end{array}$ \\
\hline 1 & & i & 1 & 1 & $P C I / G$ & $\mathrm{PCI} / \mathrm{G}$ & $\mathrm{PCI} / \mathrm{G}$ & PCI/G I & $\mathrm{PCI} / \mathrm{G}$ \\
\hline ISERIES & ILAYER & 1 & 1 & 1 & 1 & . 1 & 1 & 1 & 1 \\
\hline IBLANTON & $10-6$ & $\begin{array}{r}-1 \\
1\end{array}$ & $\begin{array}{r}1 \\
81\end{array}$ & 21 & $<4.001$ & 2.901 & 9.401 & $<4.001$ & $\begin{array}{r}1 \\
.1\end{array}$ \\
\hline 1 & $17-61$ & -+ & 81 & 31 & $<4.001$ & 3.801 & 9.901 & $<4.001$ & .1 \\
\hline $\begin{array}{l}1 \\
1\end{array}$ & $161-76$ & 1 & 81 & 71 & 6.101 & 5.201 & 8.501 & 6.421 & 1.381 \\
\hline 1 & $198-121$ & 1 & 81 & 51 & $<5.801$ & $<4.001$ & 10.001 & 5.441 & 2.371 \\
\hline IFUQUAY & $10-7$ & 1 & 81 & $3 !$ & $<4.951$ & $<4.001$ & 6.901 & $<4.001$ & .1 \\
\hline$\frac{1}{1}$ & $18-41$ & 1 & 81 & 51 & $<5.591$ & $<4.001$ & 10.001 & 4.651 & 3.131 \\
\hline 1 & $141-72$ & 1 & 81 & 61 & 7.051 & $<4.001$ & 9.001 & 6.311 & 2.431 \\
\hline 1 & $196-120$ & 1 & 81 & 71 & 7.001 & 3.901 & 20.001 & 8.461 & 5.721 \\
\hline ILAKELAND & $10-6$ & 1 & 81 & 31 & $<4.451$ & 3.801 & 6.801 & $<4.001$ & .1 \\
\hline 1 & $16-81$ & 1 & 81 & $\overline{11}$ & $<4.101$ & $<4.001$ & $<5.001$ & $<4.001$ & .1 \\
\hline 1 & $198-121$ & -+ & 81 & 21 & 5.001 & $<4.001$ & 6.101 & $<4.001$ & -1 \\
\hline IORANGEBURG & $10-7$ & 1 & 81 & 31 & $<4.251$ & $<4.001$ & 10.001 & $<4.001$ & .1 \\
\hline $\begin{array}{l}1 \\
1\end{array}$ & $17-24$ & $\begin{array}{c}-+- \\
1\end{array}$ & 81 & 51 & $<6.401$ & $<4.001$ & 7.201 & 5.281 & 2.10 \\
\hline 1 & $122-41$ & I & 81 & 71 & 6.851 & 9.701 & 8.701 & 6.491 & 1.86 \\
\hline $\begin{array}{l}1 \\
1\end{array}$ & $143-65$ & 1 & 81 & 61 & 6.051 & $<4.001$ & 11.001 & 6.181 & 3.52 \\
\hline 1 & $196-119$ & 1 & 81 & 71 & 5.751 & 4.901 & 8.101 & 5.911 & 1.79 \\
\hline IUDORTHENT & 10 & 1 & 241 & 211 & 7.251 & 3.901 & 21.001 & 7.141 & 2.65 \\
\hline IVAUCLUSE & $10-20$ & 1 & 41 & 41 & 7.451 & 4.401 & 7.801 & 6.771 & 1.59 \\
\hline $\begin{array}{l}1 \\
1\end{array}$ & $120-53$ & 1 & 4) & 31 & $<5.901$ & 4.101 & 7.801 & 5.721 & 1.87 \\
\hline $\begin{array}{l}1 \\
1\end{array}$ & $153-75$ & 1 & 41 & 21 & $<4.651$ & $<4.001$ & 5.001 & 3.681 & 1.48 \\
\hline $\begin{array}{l}1 \\
1\end{array}$ & $196-120$ & + & 41 & 11 & $<4.501$ & $<4.001$ & 5.901 & $<4.001$ & . \\
\hline
\end{tabular}


Table 6:28. Summary Statistics for Nonvolatile Beta (by series and layer)

CONSTITUENT TOMOUATILE BETA

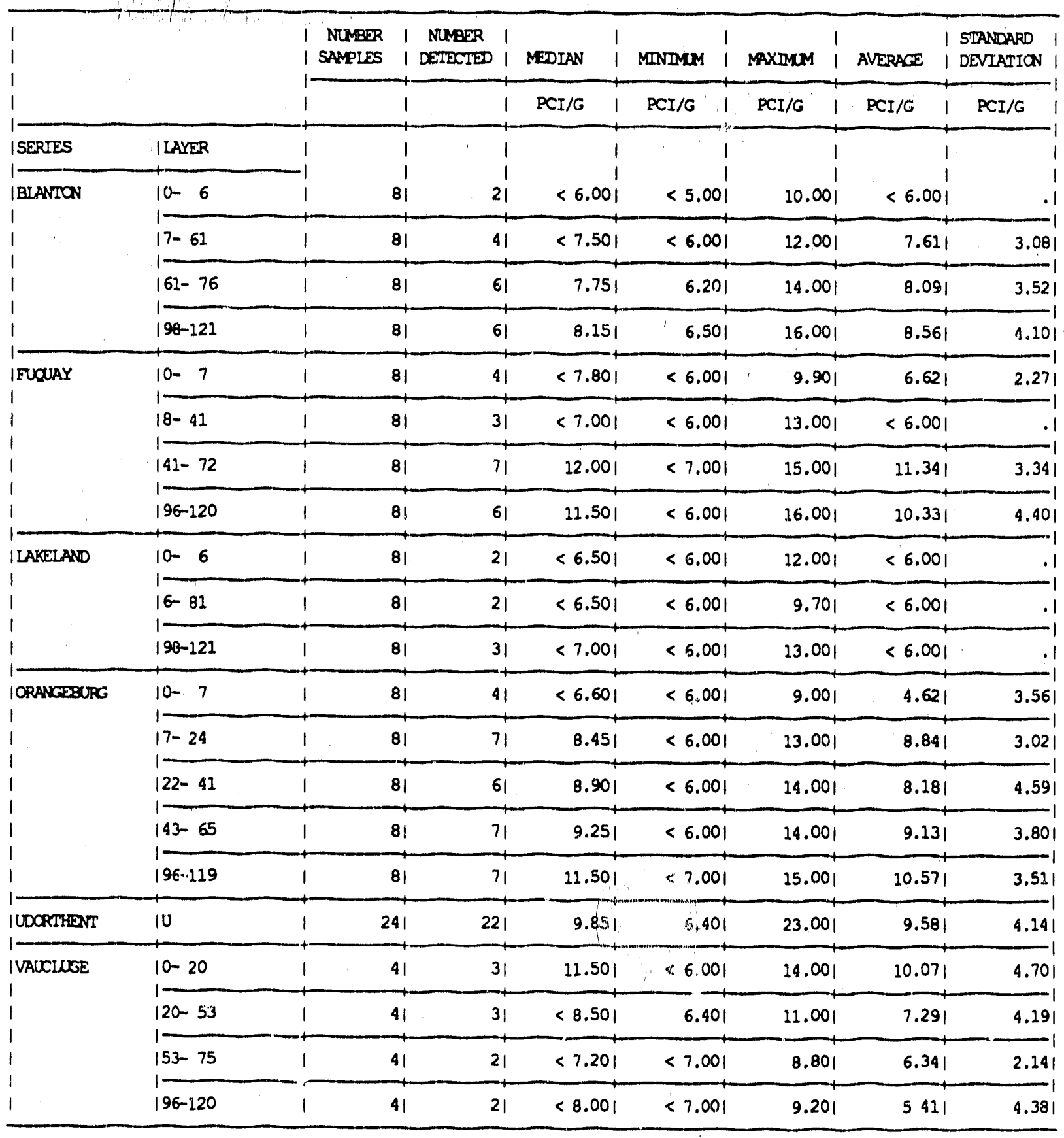


Table 6.29. Summary Statistics for Uranium (by series and layer)

CONSTITUENT - URANTUM

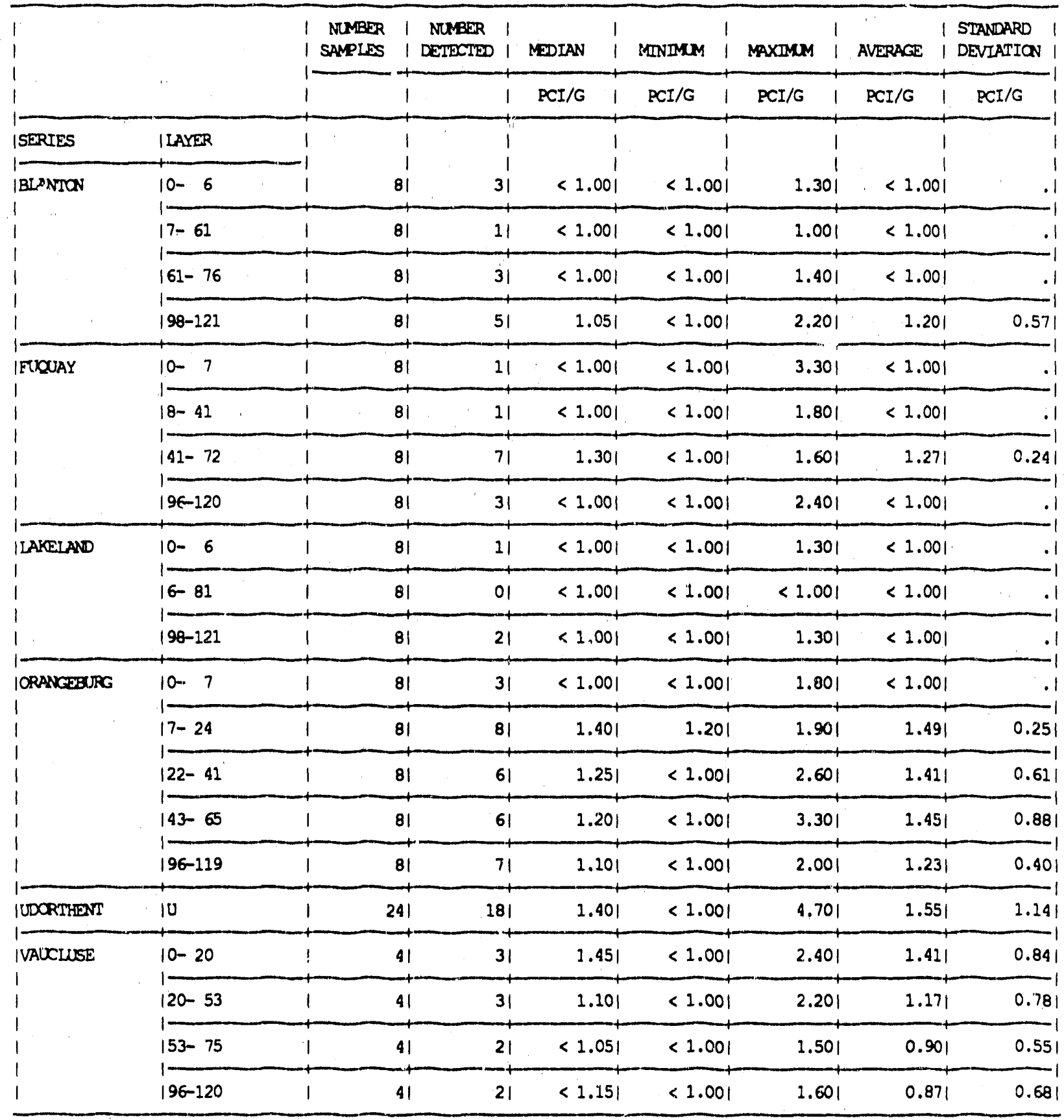


Table 6.30. SRS Soil Study X-Ray Diffraction Analyses Of Whole Soil Samples

\begin{tabular}{ccc} 
Sample Number & \% Ouartz & \% Clay \\
\cline { 2 - 3 } BSS-331 & 96 & 3 \\
BSS-332 & 96 & 3 \\
BSS-333 & 94 & 5 \\
BSS-334 & 88 & 1 \\
BSS-341 & 96 & 3 \\
BSS-342 & 93 & 6 \\
BSS-343 & 88 & 1 \\
BSS-344 & 88 & 1 \\
BSS-345 & 93 & 6 \\
BSS-411 & 97 & 2 \\
BSS-412 & 97 & 2 \\
BSS-413 & 95 & 4 \\
BSS-421 & 100 & 0 \\
BSS-422 & 94 & 5 \\
BSS-423 & 90 & 9 \\
BSS-424 & 87 & 2 \\
BSS-441 & 97 \\
BSS-442 & 97 & 10 \\
BSS-443 & 97 & 13 \\
BSS-444 & 88 & 3 \\
BSS-451 & 86 & 3 \\
BSS-452 & 96 & 10 \\
BSS-453 & 96 & 8 \\
BSS-454 & 89 & 3 \\
BSS-461 & 91 & 5 \\
BSS-462 & 96 & 6 \\
BSS-463 & 96 & 9 \\
BSS-464 & 94 & 17 \\
BSS-465 & 93 & 0 \\
BSS-511 & 82 & 0 \\
BSS-512 & 100 & 8 \\
BSS-513 & 100 & \\
& 91 &
\end{tabular}


Table 6.31. SRS Soil Study X-Ray Diffraction Analyses of Clay Mineral Fraction

\begin{tabular}{cccc} 
Sample Number & Vermiculite & Ilite & Kaolinite \\
\cline { 2 - 4 } BSS-331 & 28.1 & 0.9 & 71.0 \\
BSS-332 & 24.0 & 2.4 & 73.6 \\
BSS-333 & 9.6 & 0.0 & 90.4 \\
BSS-334 & 6.8 & 0.0 & 93.2 \\
BSS-341 & 24.1 & 0.0 & 75.9 \\
BSS-342 & 16.8 & 0.0 & 83.2 \\
BSS-343 & 11.8 & 0.0 & 88.2 \\
BSS-344 & 14.8 & 0.0 & 85.2 \\
BSS-345 & 0.7 & 1.4 & 97.9 \\
BSS-411 & 17.4 & 0.3 & 82.3 \\
BSS-412 & 15.2 & 2.9 & 81.9 \\
BSS-413 & 2.7 & 1.0 & 96.3 \\
BSS-421 & 25.7 & 5.0 & 69.3 \\
BSS-422 & 18.5 & 2.6 & 78.9 \\
BSS-423 & 11.3 & 1.0 & 87.6 \\
BSS-424 & 1.5 & 3.6 & 94.9 \\
BSS-441 & 19.9 & 4.8 & 75.3 \\
BSS-442 & 16.5 & 1.7 & 81.8 \\
BSS-443 & 6.4 & 0.0 & 93.6 \\
BSS-444 & 0.8 & 0.4 & 98.8 \\
BSS-451 & 27.7 & 1.6 & 70.7 \\
BSS-452 & 22.5 & 0.0 & 77.5 \\
BSS-453 & 14.0 & 0.0 & 86.0 \\
BSS-454 & 1.4 & 6.7 & 91.8 \\
BSS-461 & 16.6 & 0.5 & 82.9 \\
BSS-462 & 16.4 & 0.9 & 82.8 \\
BSS-463 & 16.0 & 1.1 & 82.9 \\
BSS-464 & 13.8 & 0.5 & 85.6 \\
BSS-465 & 2.5 & 2.0 & 95.6 \\
BSS-511 & 34.3 & 0.0 & 62.6 \\
BSS-512 & 30.3 & 7.1 & 90.8 \\
BSS-513 & 9.2 & 0.0 & \\
B. & & &
\end{tabular}



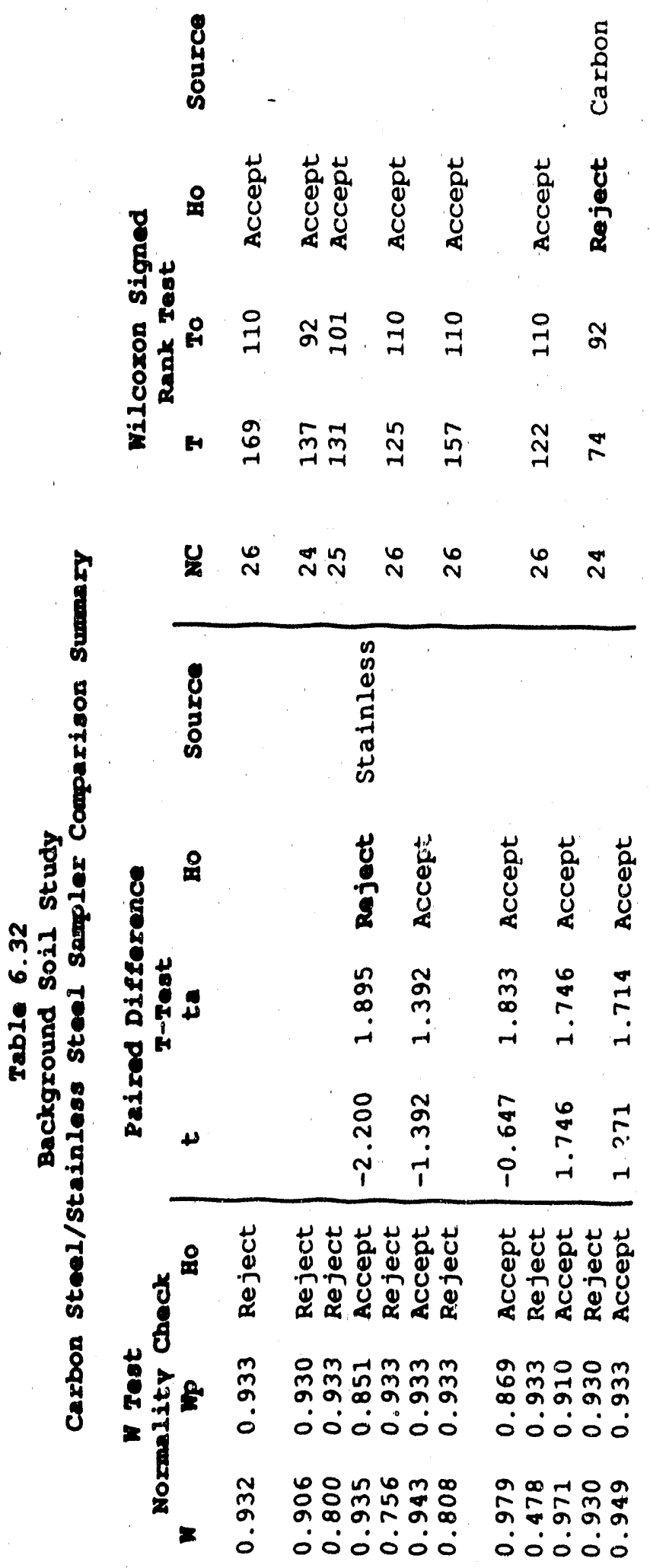

-

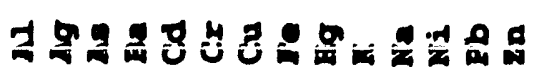

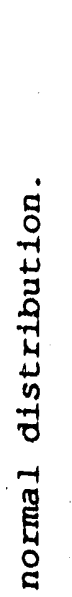

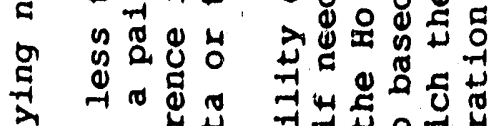

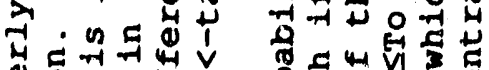

ข

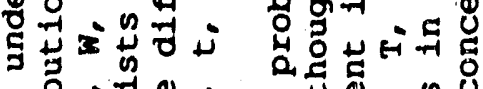

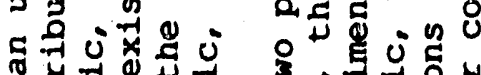

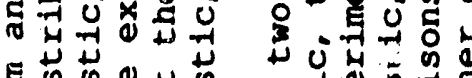

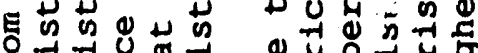

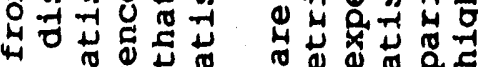

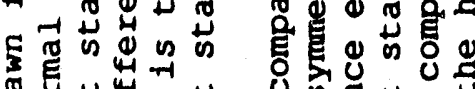

है E

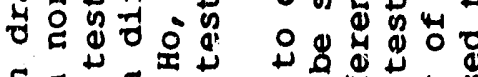

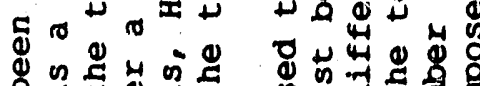

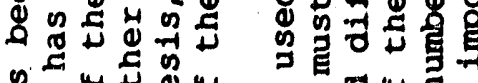

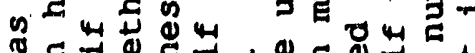
త

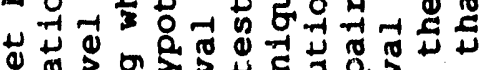

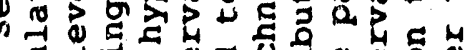

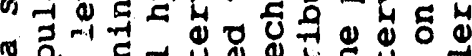

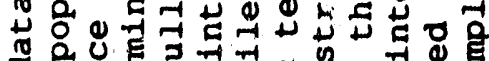
2 ๙

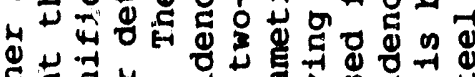

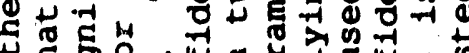
( )

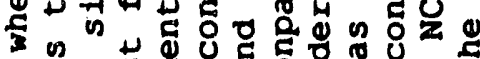

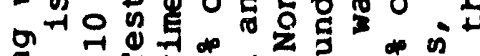

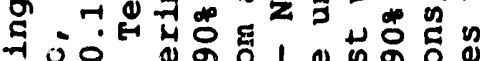
క E

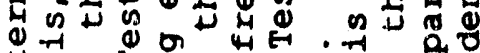

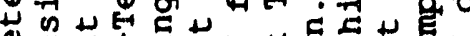

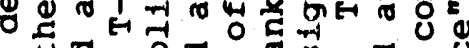

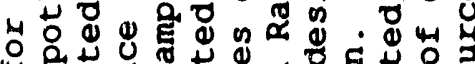
廿

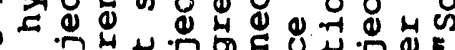

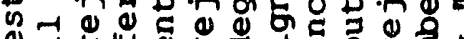

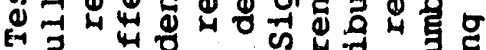

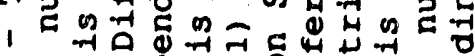

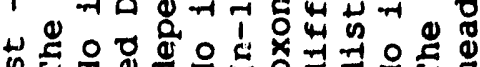

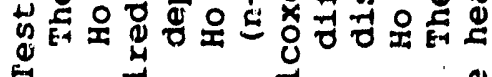

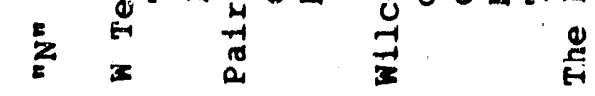



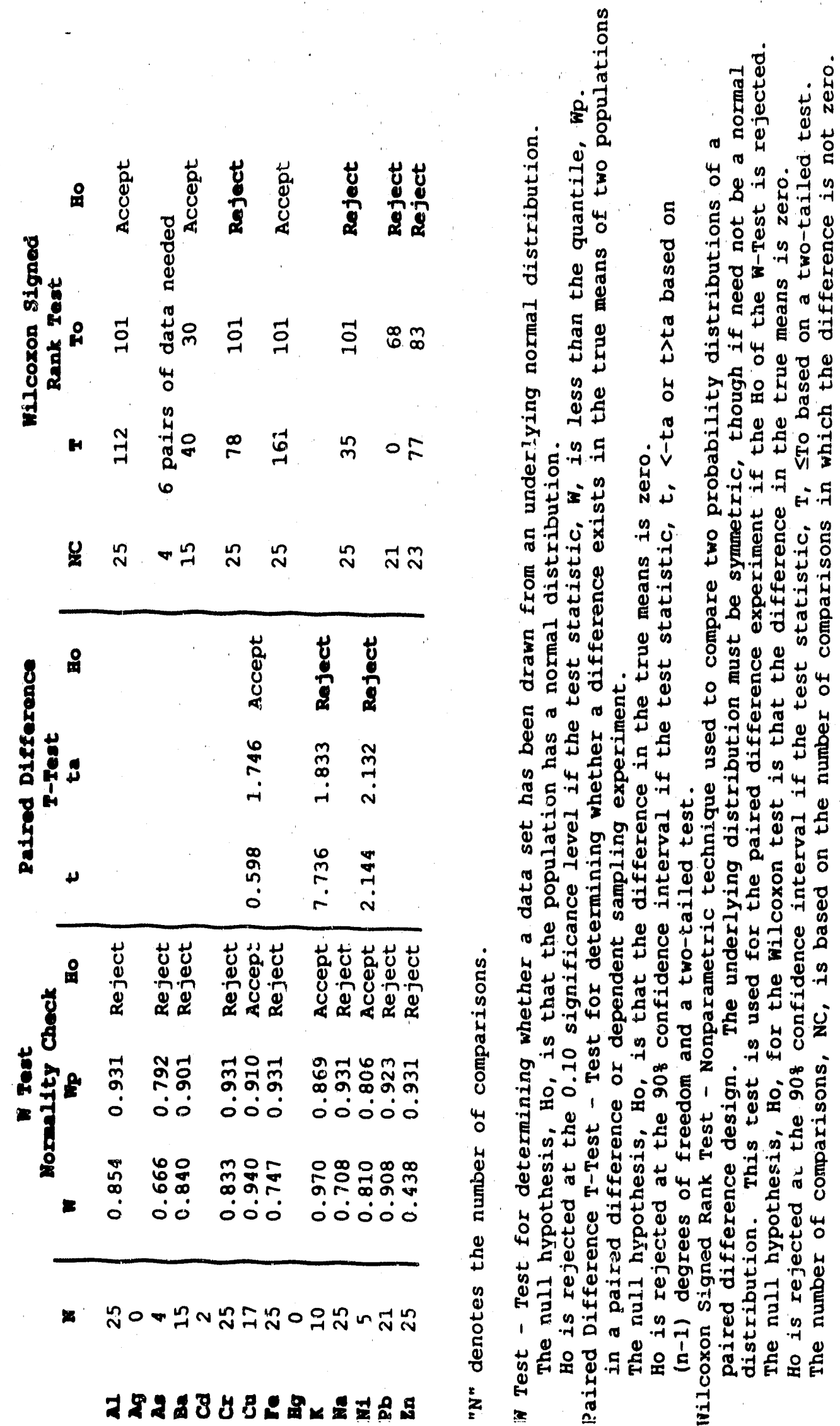

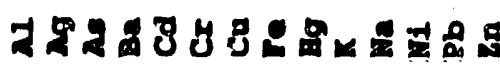




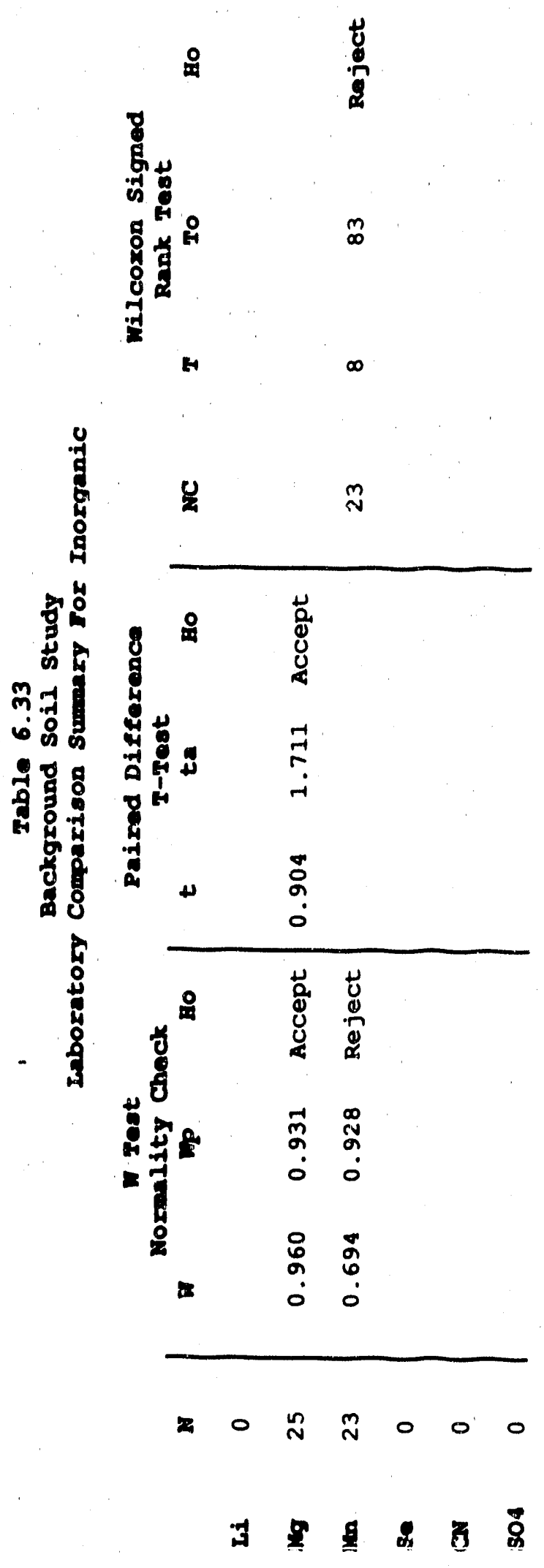

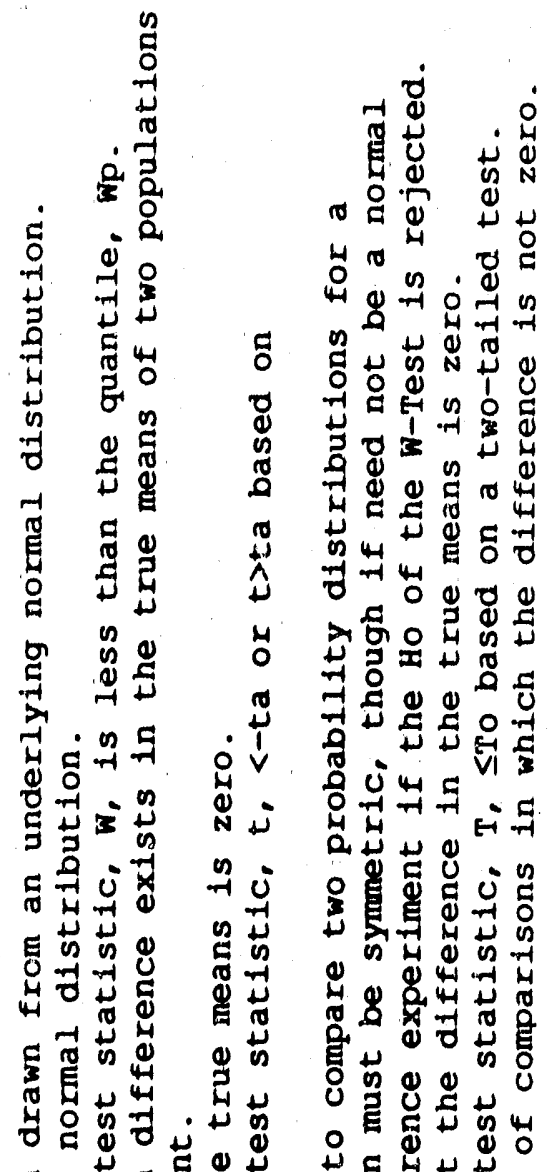

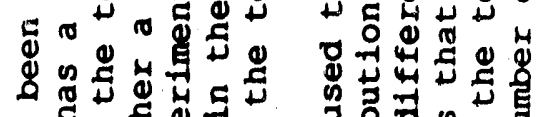

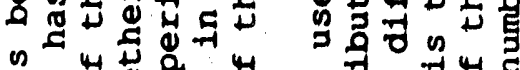
0

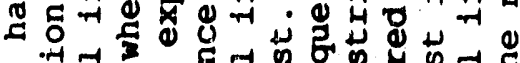
出

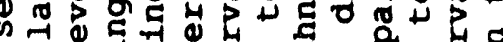

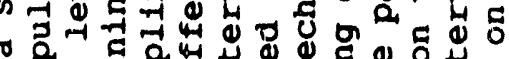

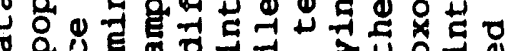

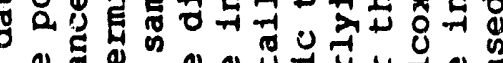

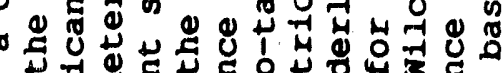

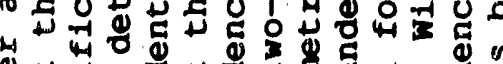
幽

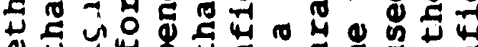

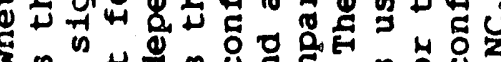
के 둥 동 1 |

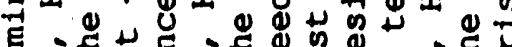

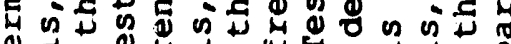

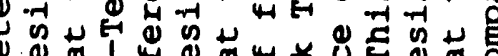
d मे

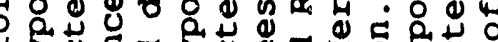

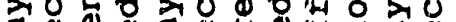

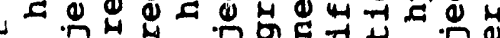

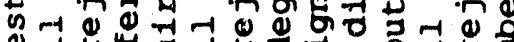

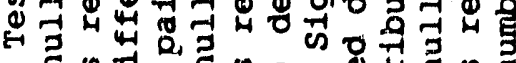

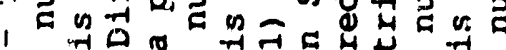

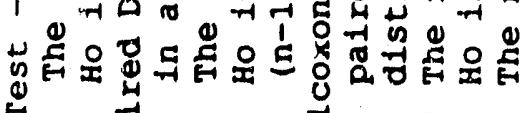

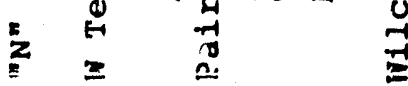




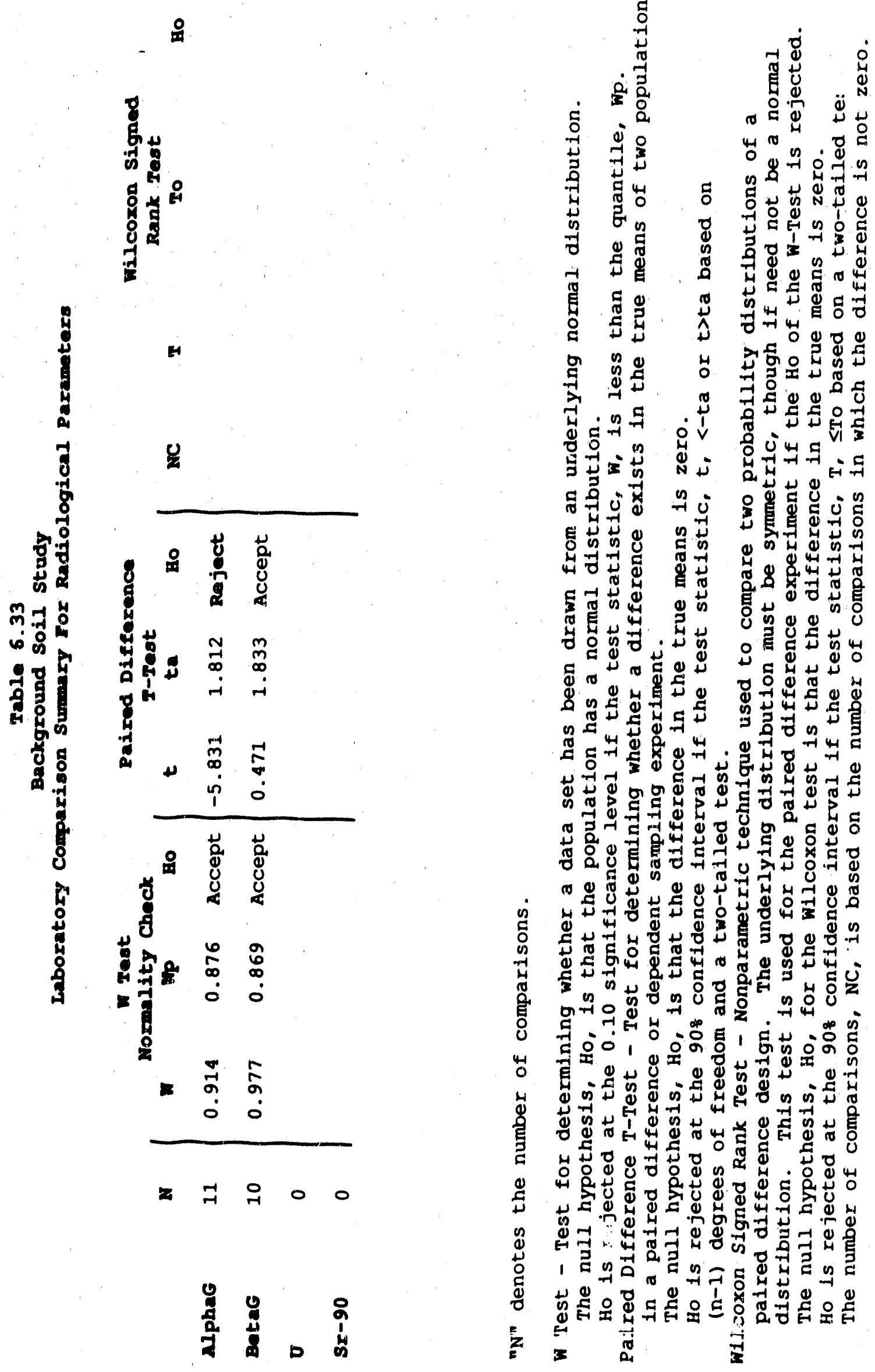


Table 6.34. Summary of Control/Background Data for Various Constituents from Sewage Sludge Application Test Sites (Davis and Corey, 1989, and unpublished data)

\begin{tabular}{|c|c|c|c|c|c|c|}
\hline & & All Sample & $(n=287$ & & & \\
\hline & Minimum & Maximum & Mean & Median & Std. Dev. & units \\
\hline pH & 4.09 & 7.17 & 5.18 & 5.20 & 0.29 & $\mathrm{pH}$ \\
\hline total nitrogen & 80.00 & 760.00 & 219.30 & 190.00 & 117.44 & ppm \\
\hline mercury & ND & 0.51 & 0.08 & 0.07 & 0.08 & $\mathrm{ppm}$ \\
\hline copper & 1.00 & 21.00 & 5.91 & 5.00 & 4.00 & $\mathrm{ppm}$ \\
\hline potassium & 50.0 & 707.0 & 235.7 & 192.0 & 133.8 & ppm \\
\hline zinc & 1.00 & 37.00 & 12.06 & 10.00 & 6.39 & ppm \\
\hline calcium & 28 & 46110 & 946 & 154 & 5085 & ppm \\
\hline iron & 1700 & 79400 & 9788 & 5800 & 11165 & ppm \\
\hline magnesium & 30.0 & 1645.0 & 114.9 & 79.0 & 128.7 & $\mathrm{ppm}$ \\
\hline nickel & ND & 42.00 & 10.80 & 9.00 & 8.09 & $\mathrm{ppm}$ \\
\hline sodium & 4.0 & 370.0 & 15.8 & 11.0 & 28.4 & $\mathrm{ppm}$ \\
\hline manganese & 39.0 & 460.0 & 153.9 & 135.0 & 76.0 & $\mathrm{ppm}$ \\
\hline lead & ND & 27.00 & 4.20 & ND & 6.14 & ppm \\
\hline exchangeable base metals & 0.10 & 6.53 & 1.08 & 1.04 & 0.69 & $\mathrm{meq} / 100 \mathrm{~g}$ \\
\hline & & Clay Soils & $=216)$ & & & \\
\hline pH & 4.09 & 7.17 & 5.20 & 5.24 & 0.31 & $\mathrm{pH}$ \\
\hline total nitrogen & 80.00 & 760.00 & 211.99 & 170.00 & 120.80 & $\mathrm{ppm}$ \\
\hline mercury & ND & 0.31 & 0.07 & 0.06 & 0.07 & ppm \\
\hline copper & $1 . m$ & 16.00 & 5.75 & 5.00 & 3.44 & $\mathrm{ppm}$ \\
\hline potassium & 50.0 & 616.0 & 234.7 & 199.5 & 125.5 & $\mathrm{ppm}$ \\
\hline zinc & 4.00 & 27.00 & 11.55 & 10.00 & 5.61 & ppm \\
\hline calcium & 28 & 46110 & 1142 & 164 & 5784 & ppm \\
\hline iron & 1700 & 79400 & 10462 & 5850 & 12453 & ppm \\
\hline magnesium & 30.0 & 1645.0 & 116.3 & 80.0 & 140.8 & $\mathrm{ppm}$ \\
\hline nickel & ND & 40.00 & 9.72 & 9.00 & 6.64 & $\mathrm{ppm}$ \\
\hline sodium & 4.0 & 370.0 & 16.8 & 11.0 & 31.7 & ppm \\
\hline manganese & 39.0 & 365.0 & 141.1 & 129.5 & 68.1 & ppm \\
\hline lead & ND & 27.00 & 4.44 & 1.00 & 6.07 & ppm \\
\hline exchangeable base metals & 0.10 & 6.53 & 1.22 & 1.15 & 0.66 & meq/100g \\
\hline
\end{tabular}

Sandy Soils $(n=71)$

$\mathrm{pH}$

total nitrogen

mercury

copper

potassium

zinc

calcium

iron

magnesium

nickel

sodium

manganese

lead

exchangeable base metals
4.69

90.00

ND

2.00

57.0

1.00

46

2000

34.0

1.00

5.0

61.0

ND

0.14

5.68
620.00
0.51
21.00
707.0
37.00
13130
21900
651.0
42.00
116.0
460.0
25.00
2.22

5.68

0.51

21.00

37.00

13130

651.0

42.00

460.0

2.22

5.13
241.55
0.09
6.39
238.9
13.61
351
7739
110.5
14.08
12.8
192.7
3.45
0.63
5.14
220.00
0.08
4.00
186.0
11.00
133
5800
78.0
11.00
9.0
195.0
ND
0.45

$\begin{array}{rc}0.19 & \text { pH } \\ 104.22 & \text { ppm } \\ 0.10 & \text { ppm } \\ 5.35 & \text { ppm } \\ 157.8 & \text { ppm } \\ 8.19 & \text { ppm } \\ 1548 & \text { ppm } \\ 5228 & \text { ppm } \\ 81.8 & \text { ppm } \\ 10.85 & \text { ppm } \\ 13.7 & \text { ppm } \\ 85.6 & \text { ppm } \\ 6.32 & \text { ppm } \\ 0.55 & \mathrm{meq} / 100 \mathrm{~g}\end{array}$




\section{Table 6.35 Radionuclide Data for SRS Soils}

\section{Source: Fay and Pickett (1987)}

$\underset{\mathrm{p} C \mathrm{Cl} / \mathrm{g}}{\operatorname{Maximum}}$

Median
PCI/8

Potassium 40

Cesium 137

Thallium 208**

Lead 212**

Bismuth 214*

Radium 226*

Actinium 228**

Thorium 232***

Uranium 234***

Uranium 235***

Uranium 238***

0.54
0.08
0.10
0.30
0.29
0.45
0.33
0.55
0.64
0.03
0.62

1.28

0.24

0.37

1.24

0.76

1.90

1.05

2.36

1.76

0.08

1.71
Mean

S.D.

$\mathrm{PCl} / \mathrm{g}$

0.75

0.15

0.18

0.44

0.48

0.90

0.57

1.42

1.04

0.05

1.01
0.27

0.04

0.06

0.27

0.11

0.35

0.20

0.41

0.28

0.01

0.27

Source: Mikol (1988) and Davis et al. (1989)****

\begin{tabular}{|c|c|c|c|c|c|c|}
\hline & & & & ind $H$ Are & & \\
\hline & & $\underset{\mathrm{pCl} / \mathrm{g}}{\operatorname{Minimum}}$ & $\begin{array}{l}\text { Maximum } \\
\text { pCl/g }\end{array}$ & $\begin{array}{l}\text { Modian } \\
\mathrm{pCl} / \mathrm{g}\end{array}$ & $\begin{array}{l}\text { Mean } \\
\mathrm{PCl} / \mathrm{g}\end{array}$ & $\begin{array}{l}\text { S.D. } \\
\text { PCI/g }\end{array}$ \\
\hline $\begin{array}{l}\text { Stro } \\
\text { Cesi } \\
\text { Plut } \\
\text { Plut }\end{array}$ & $\begin{array}{l}90 \\
137 \\
\text { um } 238 \\
\text { im } 239\end{array}$ & $\begin{array}{c}0.01 \\
0.39 \\
0.013 \\
0.005\end{array}$ & $\begin{array}{l}0.31 \\
1.48 \\
0.15 \\
0.15\end{array}$ & $\begin{array}{l}0.02 \\
0.90 \\
0.16 \\
0.08\end{array}$ & $\begin{array}{c}0.07 \\
0.79 \\
0.047 \\
0.075\end{array}$ & $\begin{array}{c}0.11 \\
0.39 \\
0.004 \\
0.005\end{array}$ \\
\hline & & & & Boundary & & \\
\hline $\begin{array}{l}\text { Stro } \\
\text { Cesi } \\
\text { Plut } \\
\text { Plute }\end{array}$ & $\begin{array}{l}\text { Im } 90 \\
137 \\
\text { im } 238 \\
\text { im } 239\end{array}$ & $\begin{array}{c}-0.03 \\
0.58 \\
0.003 \\
0.003\end{array}$ & $\begin{array}{c}0.03 \\
0.81 \\
0.014 \\
0.020\end{array}$ & $\begin{array}{c}0.007 \\
0.75 \\
0.004 \\
0.016\end{array}$ & $\begin{array}{c}0.005 \\
0.69 \\
0.006 \\
0.016\end{array}$ & $\begin{array}{c}0.023 \\
0.10 \\
0.005 \\
0.003\end{array}$ \\
\hline & - & & & Ie Radius & & \\
\hline $\begin{array}{l}\text { Stror } \\
\text { Cesi } \\
\text { Plute } \\
\text { Plutc }\end{array}$ & $\begin{array}{l}\text { Im } 90 \\
137 \\
\text { Im } 238 \\
\text { Im } 239\end{array}$ & $\begin{array}{l}\text { n.a. } \\
\text { n.a. } \\
\text { n.a. } \\
\text { n.a. }\end{array}$ & $\begin{array}{l}\text { n.a. } \\
\text { n.a. } \\
\text { n.a. } \\
\text { n.a. }\end{array}$ & $\begin{array}{l}\text { n.a. } \\
\text { n.a. } \\
\text { n.2. } \\
\text { n.... }\end{array}$ & $\begin{array}{l}.013 \\
0.36 \\
0.002 \\
0.011\end{array}$ & $\begin{array}{c}0.032 \\
0.27 \\
0.001 \\
0.007\end{array}$ \\
\hline$*$ & $\begin{array}{l}\text { Daughte } \\
\text { Daughte }\end{array}$ & ium & & & & \\
\hline ******* & $\begin{array}{l}\text { Values } \\
\text { isotopes } \\
\text { Average }\end{array}$ & tha from eac & tion for 198 & 988 were & & $\begin{array}{l}\text { ural ratios of } \\
\text { information }\end{array}$ \\
\hline
\end{tabular}




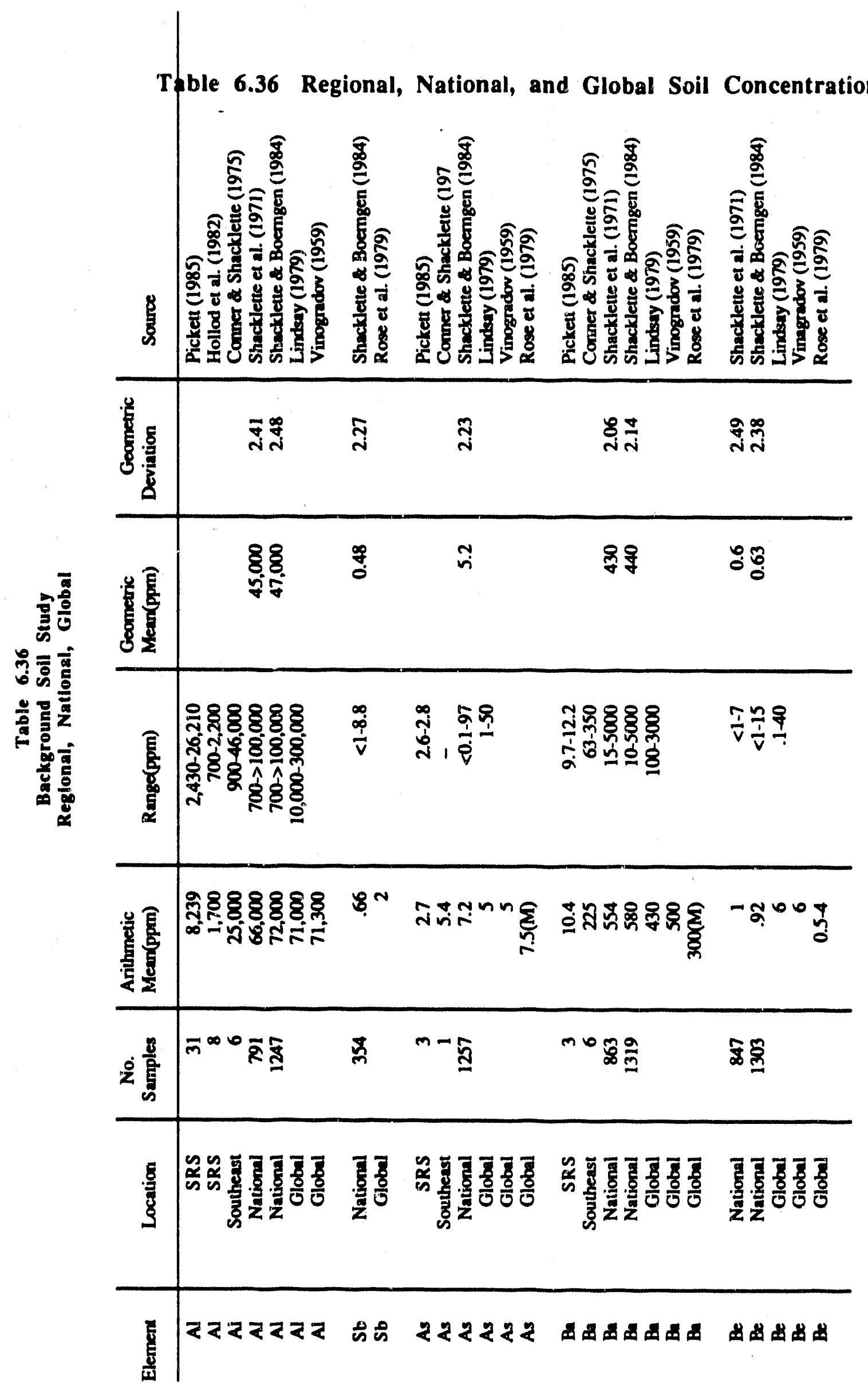




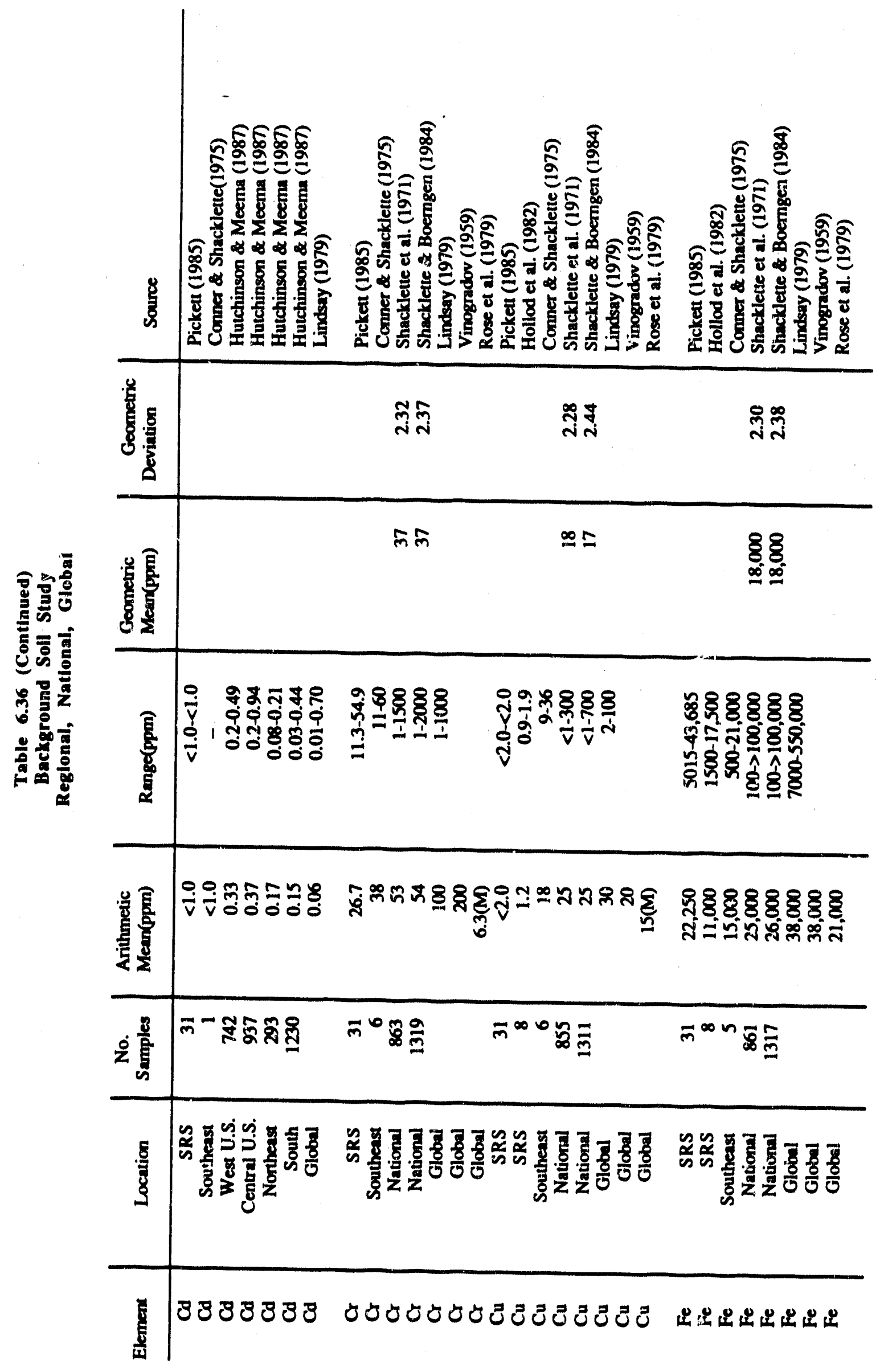




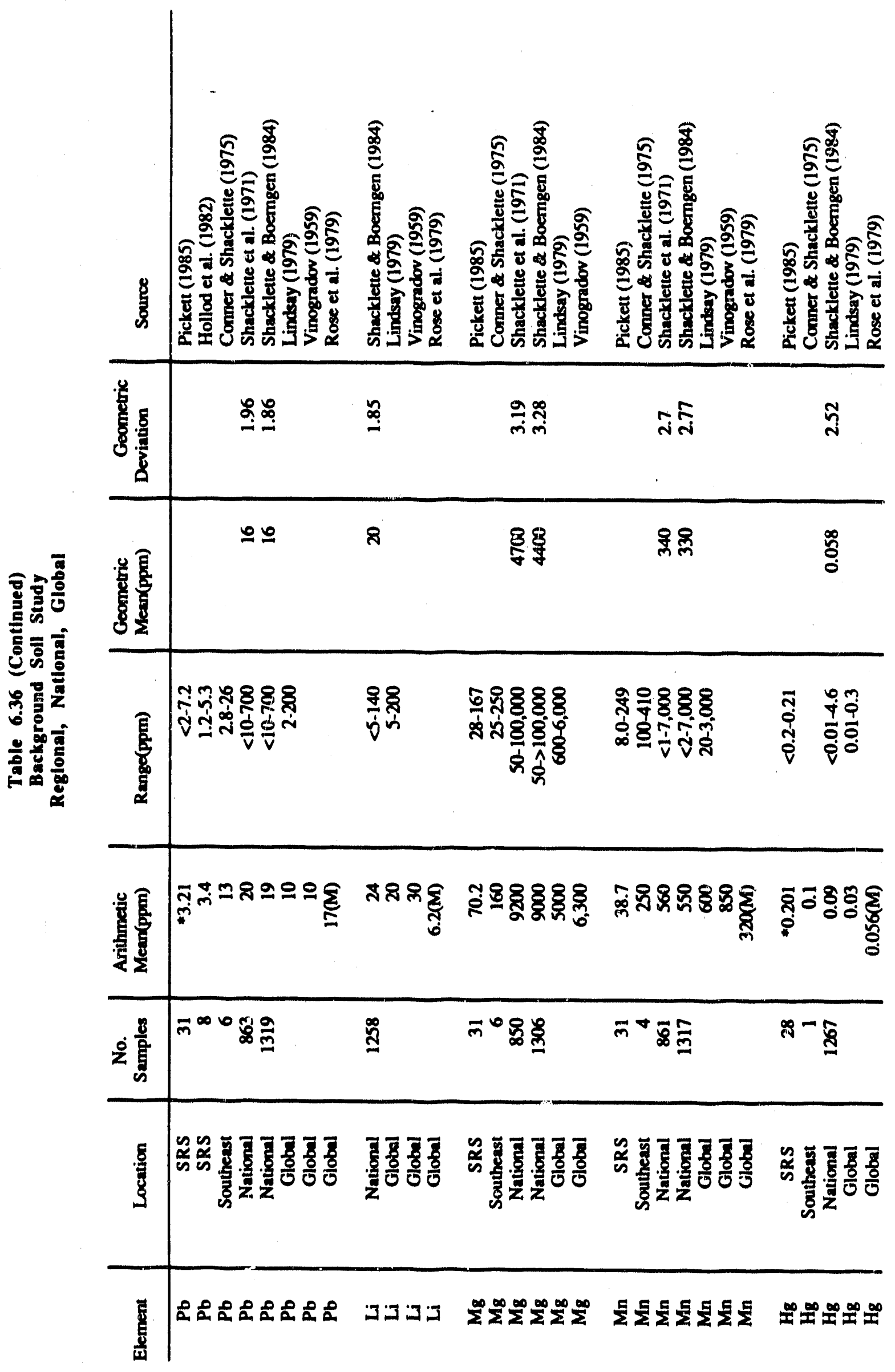




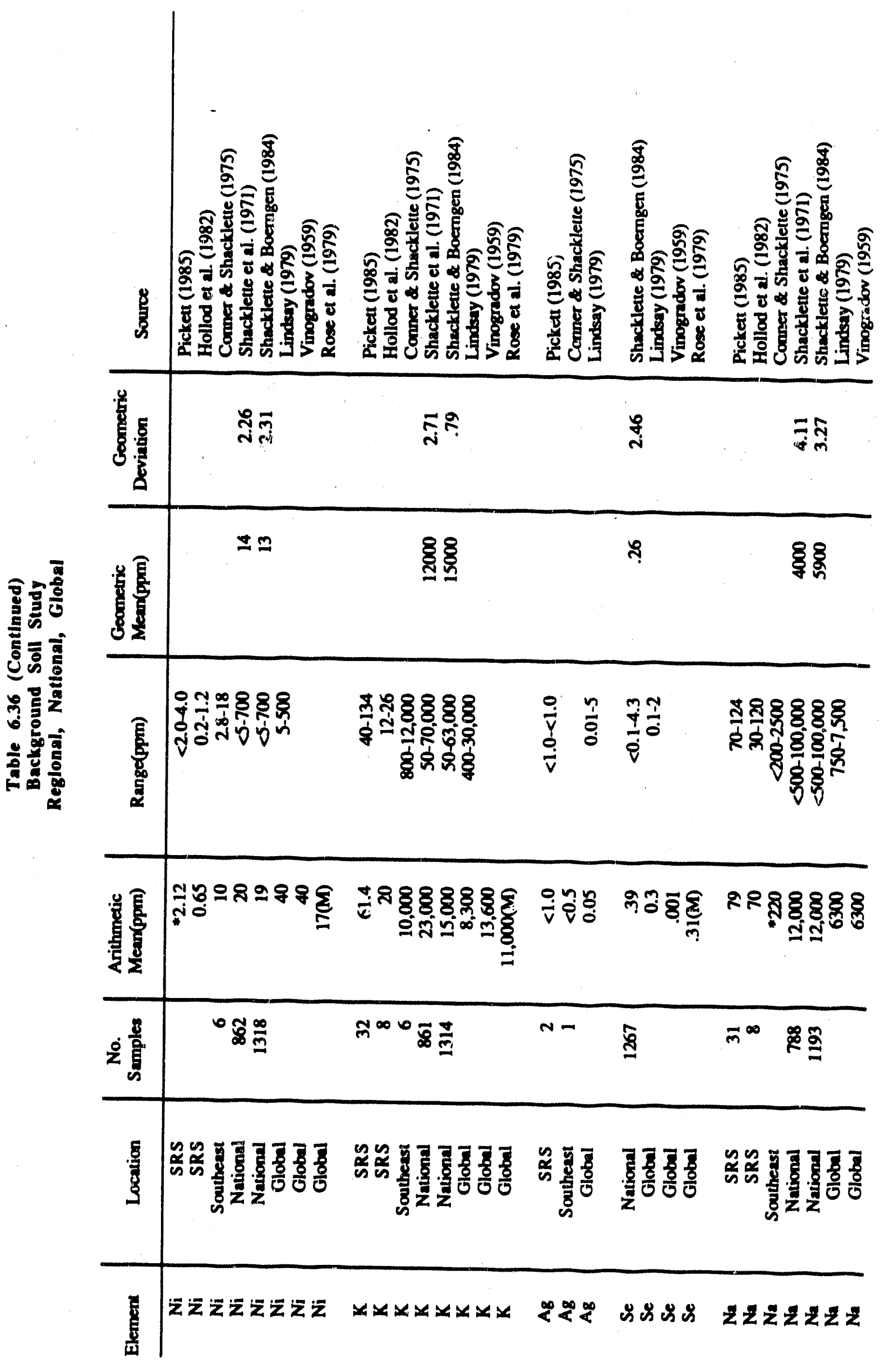




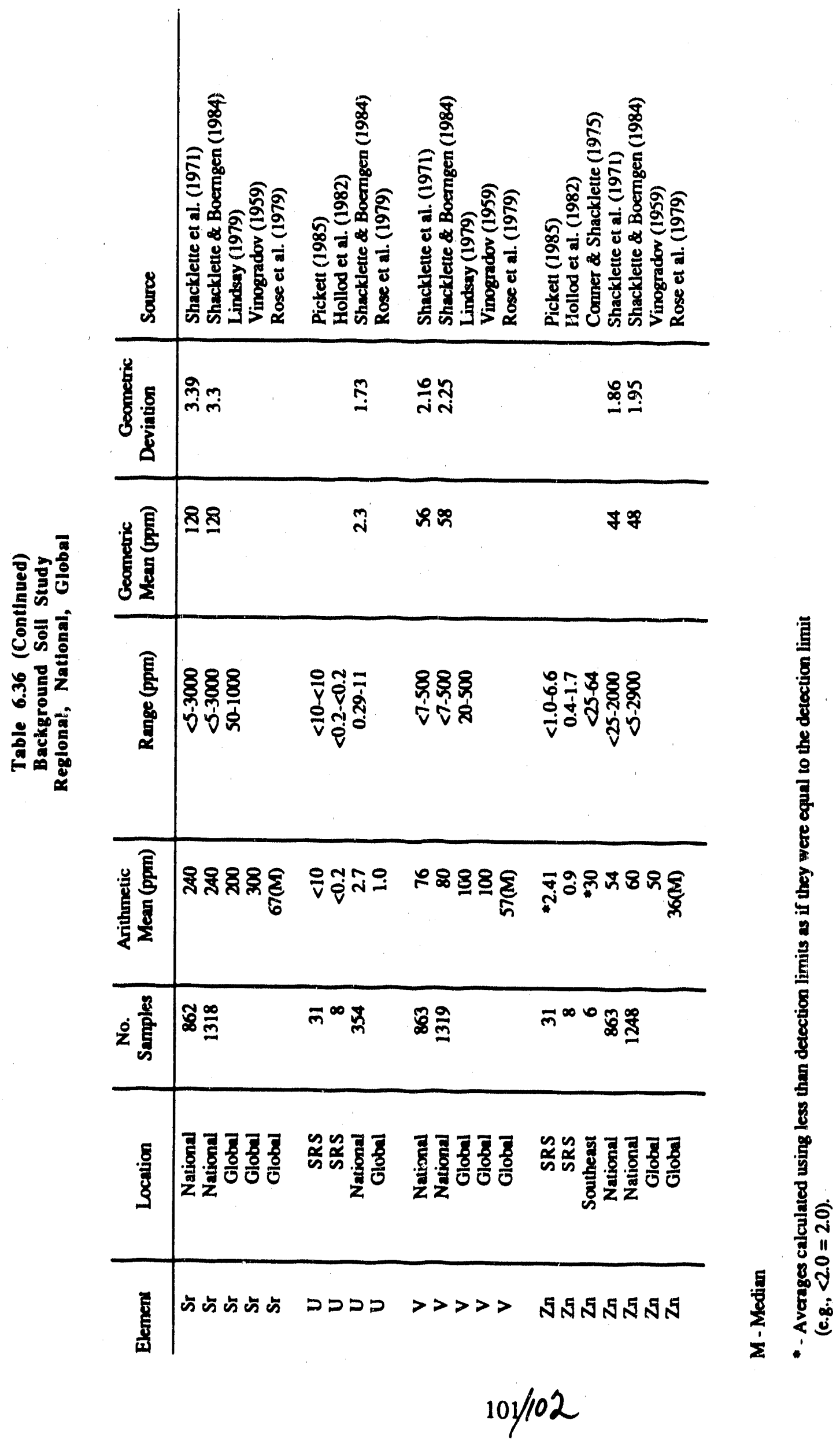




\subsection{Discussion}

\subsection{Summary of Data}

\subsubsection{Metals, Radionuclides and Bulk Geochemistry}

In general, metal concentrations increase with depth and in proportion to the clay content of the soil. The upper soil horizons are generally more highly leached, and exhibit lower concentrations, than the lower horizons and the parent material. The concentrations measured in this study were similar to those measured in previous studies of SRS and regional-national-global studies from similar highly weathered environments. The radionuclide data presented in Section 6.2 (Past Studies at SRS) are an important adjunct to the limited radionuclide data contained within this study.

When using these data the reader is reminded that compromises were made to address the wide range of project objectives. For example, to meet regulatory objectives, standard EPA protocols for sample extraction and handling were employed and these protocols for soil samples require dissolution solely by nitric acid. Since the only acid that will readily dissolve silicate material is hydrofluoric acid and generally $\mathrm{HF}$ acid is used in conjunction with either perchloric or nitric acid to increase the solubility of potassium and calcium salts (Potts, 1987), EPA sample protocols generally will result in the incomplete dissolution of soil samples. Soils generally are composed at least partially of mineral constituents including accessory minerals such as zircon, monazite, magnetite, etc, that may constitute only a minor percentage of the bulk soil yet may contribute significant concentrations of trace elements. These accessory minerals are particularly resistant to acid attack and would likely constitute part of the insoluble residue. Since standard EPA procedures lead to incomplete dissolution, the resulting ranges in concentrations of major, minor and trace elements will be more variable than total concentrations generated by completely dissolving the soil. It should be recognized that samples prepared by other dissolution protocols may not be directly compared to the results of the SRS Soil Survey. The data for these constituents are summarized across all soil types and layers in Table 6.1.

One objective of the SRS Soils Study was to develop background information to help assess areas of potential release of hazardous constituents from solid waste management units. A list of "hazardous" constituents is defined for this purpose by EPA in 40 CFR part 261. The metals included in this list were measured in the SRS Soil Study, along with a few additional metals. Although the additional metals are not listed as hazardous constituents in 40 CFR 261, elevated levels of these metals may serve as indicators of potential impacts and might form the basis for further investigation based on site specific conditions.

\subsubsection{Organics}

As noted above, organic compounds were detected in all 17 of the samples tested by metaTRACE. A total of six different compounds were detected in the samples, however, not all of these parameters were detected in all 17 samples. The parameters detected included: bis (2ethylhexyl) phthalate, acetone, methylene chloride, dieldrin, endosulfan sulfate, and butylbenzyl phthalate. No organic Appendix VIII constituents are known to occur naturally in soil, therefore, this portion of the investigation was intended to assess possible laboratory artifacts.

Of the six compounds detected, four are commonly sccurring laboratory artifacts (bis (2ethylhexyl) phthalate, acetone, methylene chloride, and butylbenzyl phthalate). The remaining two compounds, dieldrin and endosulfan sulfate, may result from usage at (or in the vicinity of SRS), or may be analytical artifacts. The first four compounds listed above are commonly reported as detected in trace level organic analyses. Bis (2-ethylhexyl) phthalate and butylbenzyl phthalate are 
commonly used as a plasticizer for polyvinyl and cellulosic resins. Acetone and methylene chloride are used in analytical laboratories for extraction solvents.

Three of the samples tested by metaTRACE for Appendix VIII constituents were split and submitted to Weston Laboratories for Appendix IX analyses. Organic compounds were detected in all three of the samples tested by Weston. A total of three compounds were detected in the three samples, however, not all of these parameters were detected in all samples. The parameters detected included: bis (2-ethylhexyl) phthalate, acetone, and methylene chloride. As noted above, all of these compounds are common laboratory artifacts.

The occurrence of these compounds emphasizes the necessity for critical review and validation of all trace level laboratory data. EPA (EPA, 1988) recognizes the potential bias that is oftentimes introduced as a result of method interferences caused by contaminants in solvents, reagents, glassware, and other sample processing hardware. These interferences often lead to discrete artifacts and/or elevated baselines in gas chromatograms. Certain common laboratory solvents are allowed to carryover into method blank samples at levels up to 5 times the contract required detection limit. In evaluating data usability, EPA uses certain general guidelines for assessing the presence of common laboratory artifacts in samples. If the sample concentration is not greater than ten times the blank level, the presence of that compound in the sample is considered suspect and is reported as not detected.

Comparison of metaTRACE method blank results to the accompanying SRS Soil Study samples shows that the common laboratory artifacts detected in the soil samples were not always detected in the accompanying blank sample. This fact demonstrates an additional bias which is likely to occur with trace level organic analyses that cannot easily be prevented, and may be difficult to resolve in a regulatory sense. Stringent quality control procedures must be followed in the laboratory and in the field to minimize the potential for these and other possible false positive detections.

\subsubsection{Mineralogy}

The mineralogy of the soils is dominated by quartz. Kaolinite dominates the clay mineral assemblage in all of the samples examined in this study. Iron oxicie minerals which give the distinctive red coloration to many of the soils are not reported as they are below the detection limits of $\mathrm{x}$-ray diffraction analysis using copper radiation.

The clay mineral fraction of the soils is usually less than ten percent by weight and is composed of the minerals kaolinite, illite, and vermiculite. The soils can be divided on the basis of clay composition into two distinct groups. The first group is almost entirely kaolinite in composition. The second group is dominated by kaolinite with lesser amounts of hydroxy-interlayered vermiculite present. Illite may or may not be present in minor amounts in both groups along with clay -sized quartz.

Kaolinite dominates the clay mineral assemblage in all of the samples examined in this study. This clay is ubiquitous in Coastal Plain soils in the southeastern U.S. and is one of the most stable phases in the weathering zone. The illite identified in the clay fraction has been called illite because of its $10 \AA$ basal refection and vecause it occurs in the clay-sized fraction. The structure of this mineral is degraded and the poor crystallinity is demonstrated by its broad diffraction profile.

Chloritized-vermiculite or hydroxy-interlayered vermiculite is present in some of the samples. This clay does not expand upon glycolation but does partially collapse to the $10 \AA$ structure on heating to $350^{\circ} \mathrm{C}$ (Figure 7.1). Chloritized-vermiculite can result from the degradation of chlorite, or more likely in the case of samples from SRS, it results from the deposition of hydroxy materials within the interlayered spaces of expansible layer silicates during the soil formation process. The 
development of hydroxy interlayered vermiculite is discussed by the Bamhisel (1977). The geographic distribution of hydroxy interlayered minerals is wide but their frequency is greatest in the Ultisols and the Alfisols (the primary soils found at SRS). Distribution of the hydroxy interlayered clays within the solum may be uniform or restricted to one horizon. More frequeritly, however, hydroxyl-interlayering is greatest in the surface horizon and decreases with depth (i.e., the weathering of layer silicate minerals to hydroxy-interlayered vermiculite is greatest in the upper layers).

Figures 7.2, 7.3, 7.4 and Figure 7.5 show the distribution with depth of kaolinite, illite and vermiculite in the soil horizons for samples from the Lakeland, Orangeburg, Blanton and Fuquay soil series, respectively. In all cases, the percentage of vermiculite in the clay fraction decreases with depth. Kaolinite is the dominant clay mineral in all samples and generally illite is not present or found in small amounts $(<2 \%)$. The raw compositional data for percentage of quartz and clay are presented in Table 6.30; sieve data for representative samples are presented in Appendix C.5. Field logs for all samples are included in Appendix B.

\subsection{Sampling Method and Laboratory Comparison Studies}

\subsubsection{Carbon Steel And Stainless Steel Comparison}

In the carbon and stainless steel comparison study, a total of 26 comparison samples were taken. The comparison data are presented in Appendix $\mathbf{C}$ with the paired samples adjacent to each other for each metal tested. The difference and relative percent difference for the paired samples is also included. The purpose of this comparison is to determine whether any bias is associated with either the carbon or stainless steel sampler. The first step in the analysis was to determine if the sampled populations follow a normal distribution (many of the statistical tests require this). In the carbon and stainless steel conparison, as well as the laboratory comparison study discussed below, the W-Test is used to determine if the sampled populations follow a normal distribution. The residuals, or differences between paired samples, represent sample distribution for this paired test.. If the W-Test reflected that the sampled distributions were normal, the Paired Difference TTest was used to determine if any difference existed between the samplers. If the W-Test reflects that the sampled distribution was not normal, the Wilcoxon Rank Signed Test was used to determine if a difference exists between the samplers.

Of the fourteen metals compared, two metals, silver and mercury, did not have an adequate number of comparisons for statistical purposes due to a large number of less than detection limit values. As presented in Table 6.37, 5 of the remaining 12 metals are described by a normal distribution and the Paired Difference T-Test was used accordingly. Overall, two metals were found to be statistically different between the carbon and stainless steel sampler comparison. At the $90 \%$ confidence interval, a statistical cadmium bias is associated with the stainless steel sampler and $\mathrm{a} \mathrm{Pb}$ bias is associated with the carbon steel sampler. If values are included for the large percentage of censored values for cadmium, the conclusion of cadmium bias might both cases, the absolute values of the average differences are relatively small compared to the overall variability in the population. The small difference statistically determined to be associated with each laboratory is unlikely to impact screening decisions based on the data. If the data were available to compare the two data sets in a manner analogous to the methods for round robin acceptance into a program such as the EPA contract lab program (i.e., qualification of labs generating results that lie in a relatively broad range besed on results from many laboratories), the results would almost certainly be qualified all constituents. 


\subsubsection{Laboratory Comparison}

In the metaTRAGE and Weston laboratory comparison study, a total of 25 comparison or duplicate samples were tested. The duplicate samples were tested for a number of metals, radiological and agricultural parameters, and organics. The raw data are presented in Appendix C. The difference and relative percent difference of the paired samples is also presented in the Appendix. The data were analyzed exactly as described above. Of tine 14 metals compared in this report; arsenic, silver, cadmium, and mercury did not have an adequate number of residuals for statistical purposes due to the number of less than detection values. A summary of the results is presented in Table 6.38. A number of metals were rejected by the Paired Difference $T$-Test and Wilcoxon Signed Rank Test.

The concentration of the metals chromium, potassium, sodium, nickel, lead, and zinc were found to be statistically different at the $90 \%$ confidence interval between the two laboratories. However, when analytical methods are perforned on matrices which contain very low concentrations of the tested analyte as is the case in the SRS Soil Stucy, many natural, experimental, and instrumental interferences may pose problems. In all cases, the absolute values of the average differences are relatively small compared to the overall variability in the population. The smill difference statistically determined to be associated with each sampler is unlikely to impact screening decision based on the data. If the data were available to compare the two data sets in a manner analogous to the methods for round robin acceptance into a program such as the EPA contract lab program (i.e., qualification of labs generating results that lie in a reistively broad range based on results from many laboratories), ine results would almost certainly be qualified for all constituents. :ionetheless, the possibility of differences resulting from variation in analyzing laboratory snould be considered if detailed statistical comparisons are made using the SRS Soil Study. 


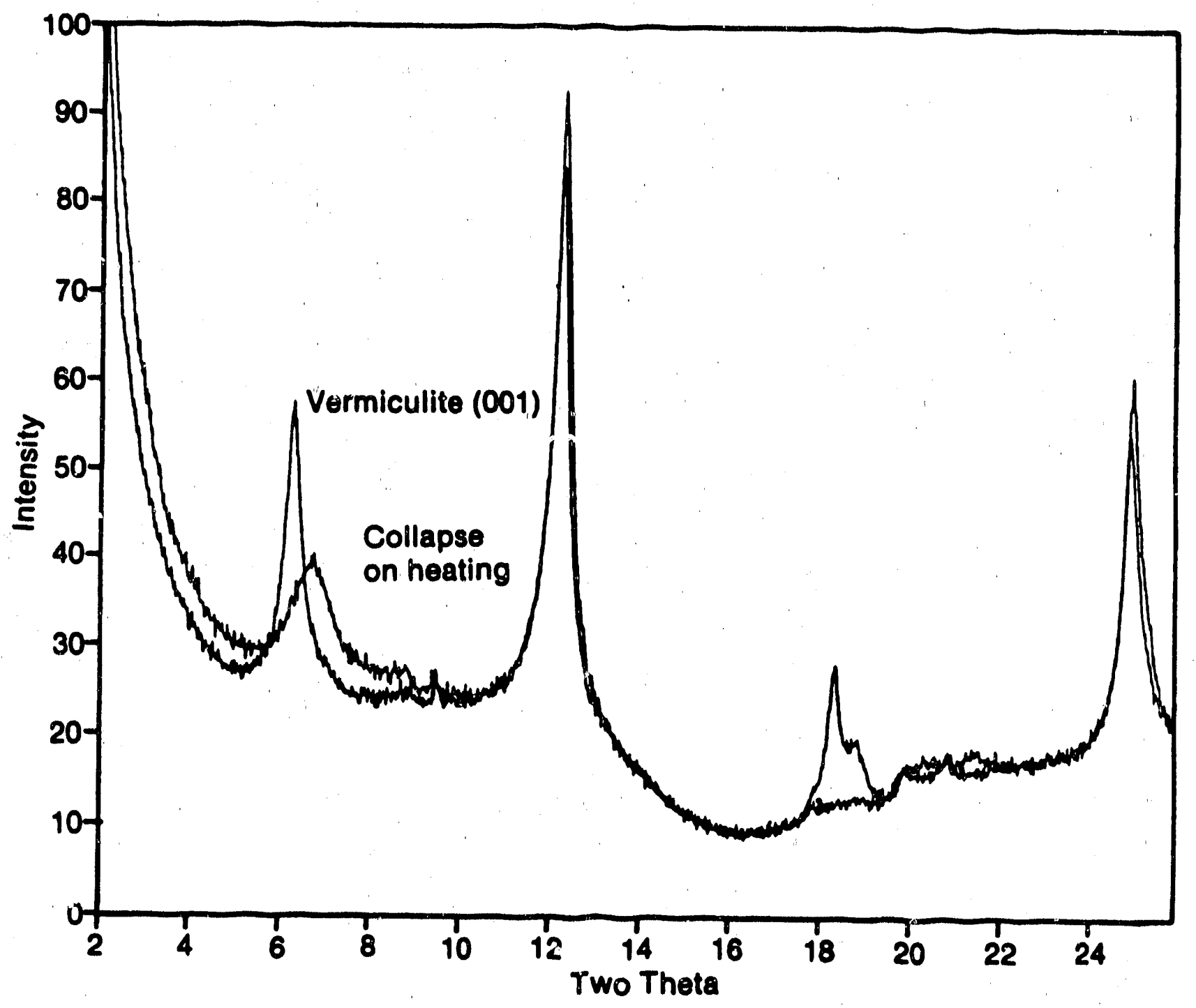

Figure 7.1 X-ray diffraction pattern of a typical sample of chloritizedvermiculite showing collapse to $10 \mathrm{~A}$ structure on heating to $350^{\circ} \mathrm{C}$. 


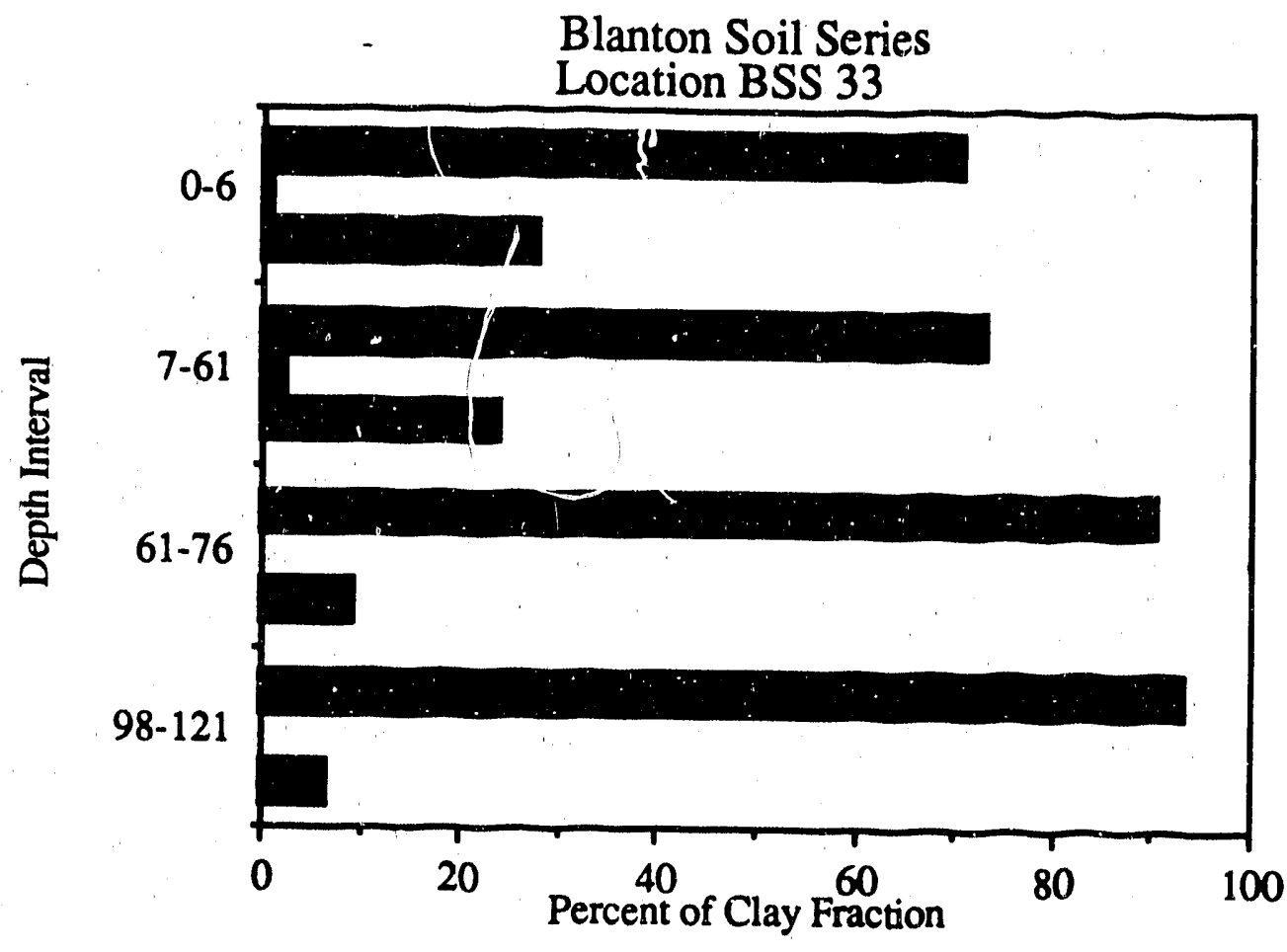

\begin{tabular}{|ll|}
\hline Kaolinite \\
Illite \\
Vermiculite \\
\hline
\end{tabular}

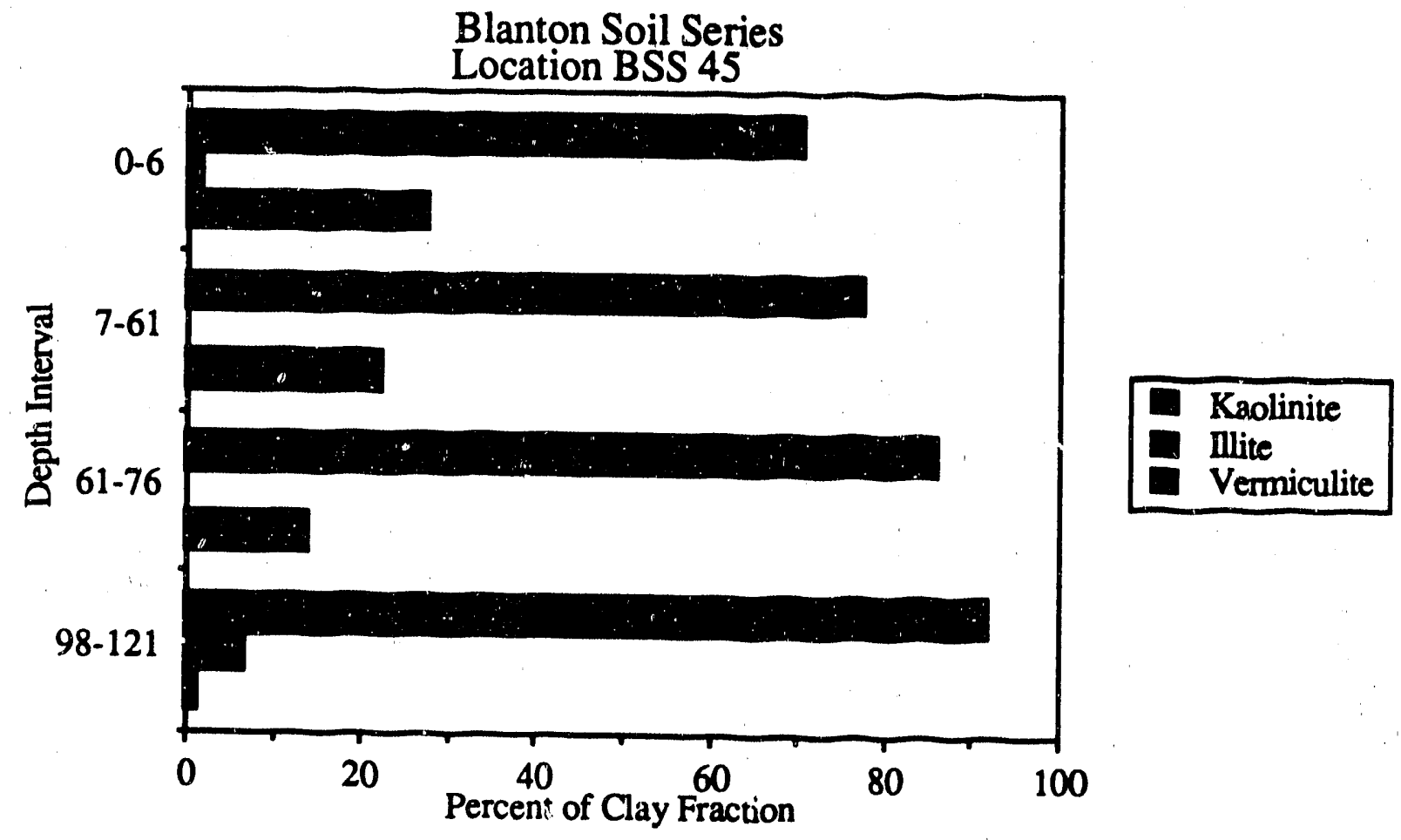

Figure 7.2 Depth profile showing distribution of minerals in the clay fraction of the Blanton Soil Series from four horizons at locations BSS 33 and BSS 45. 

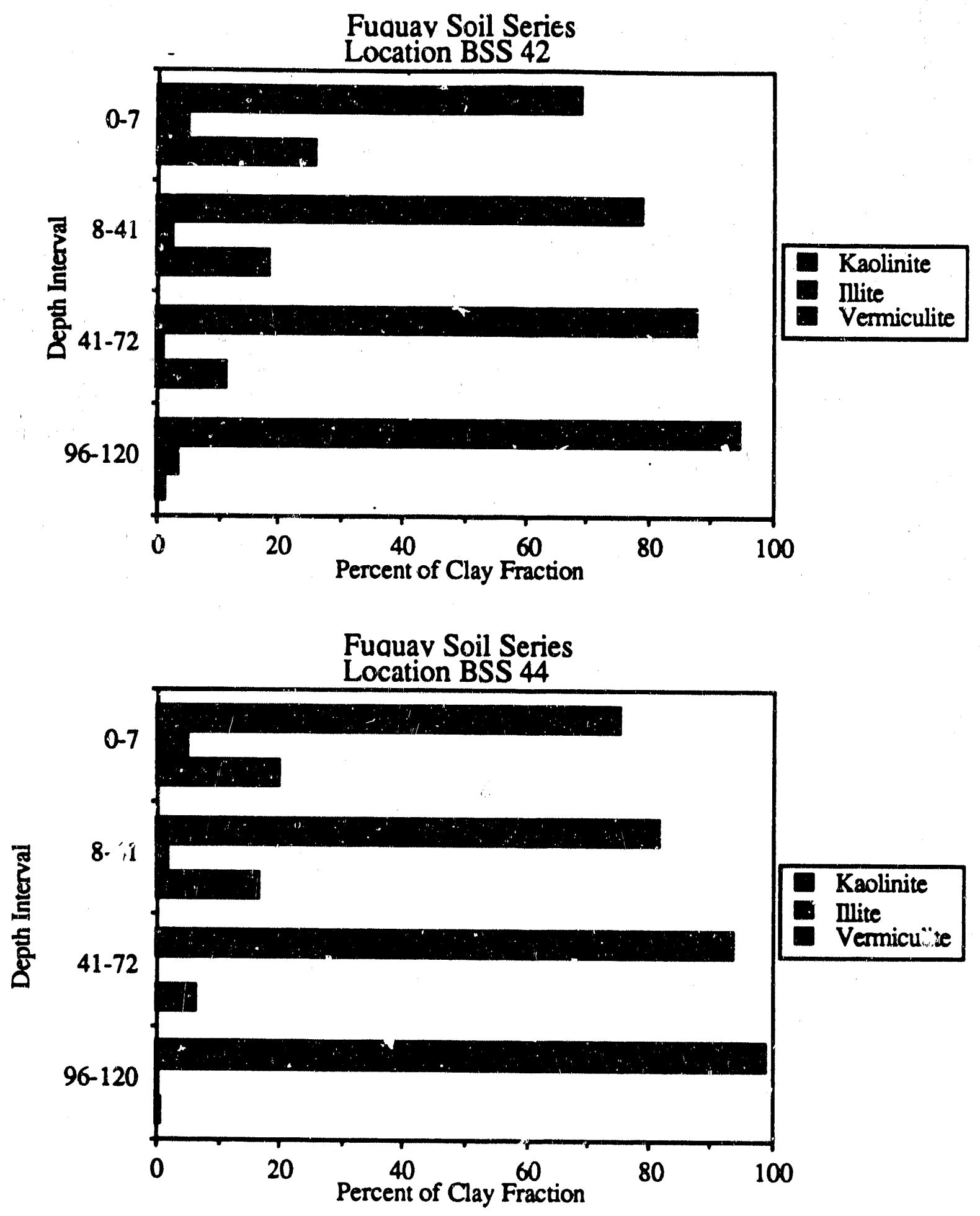

Figure 7.3 Depth profile showing the distribution of minerals in the clay fraction of the Fuquay Soil Series from four horizons at locations BSS 42 and BSS 44. 


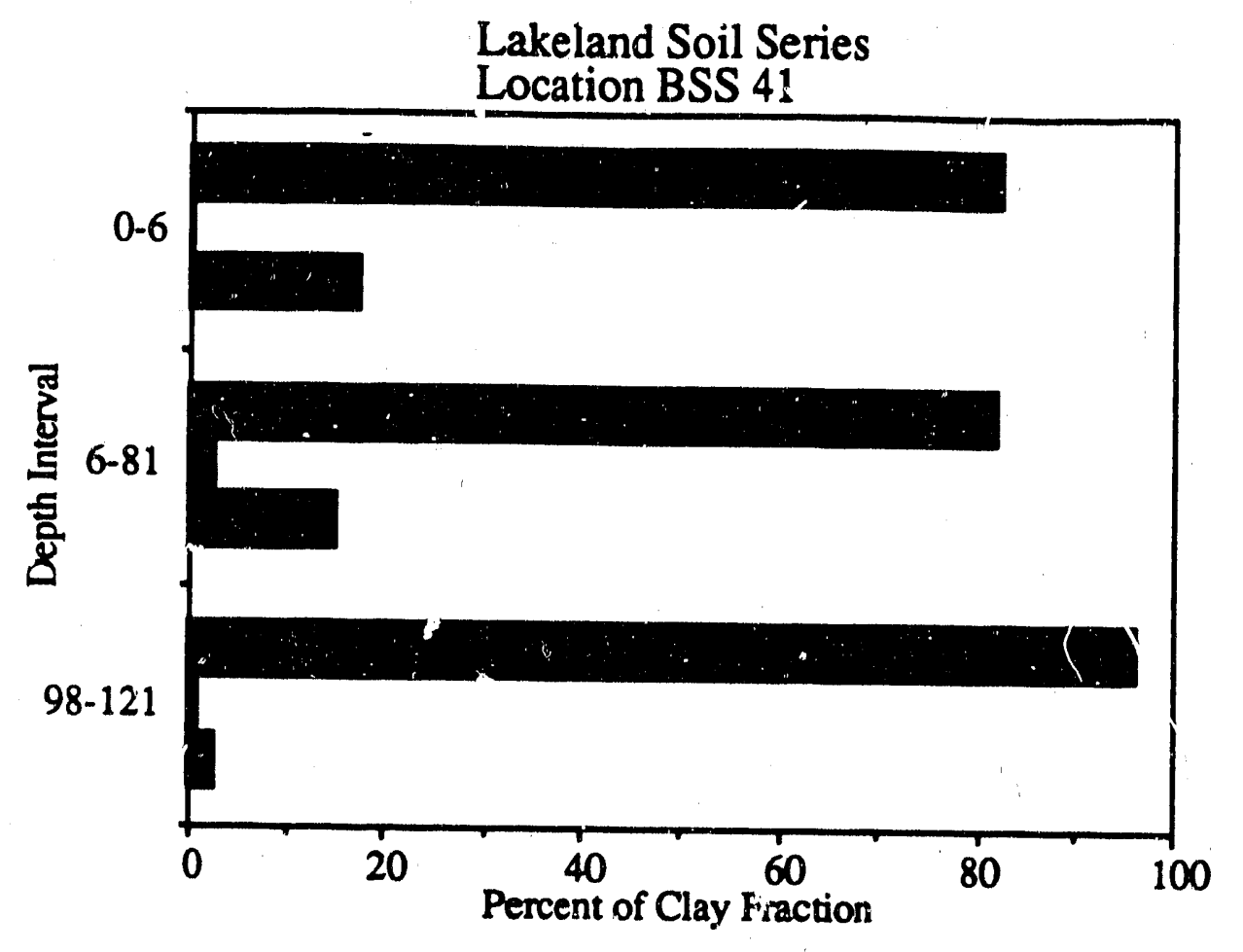

\begin{tabular}{|l|}
\hline Kaolinite \\
nlite \\
Vermiculite \\
\hline
\end{tabular}

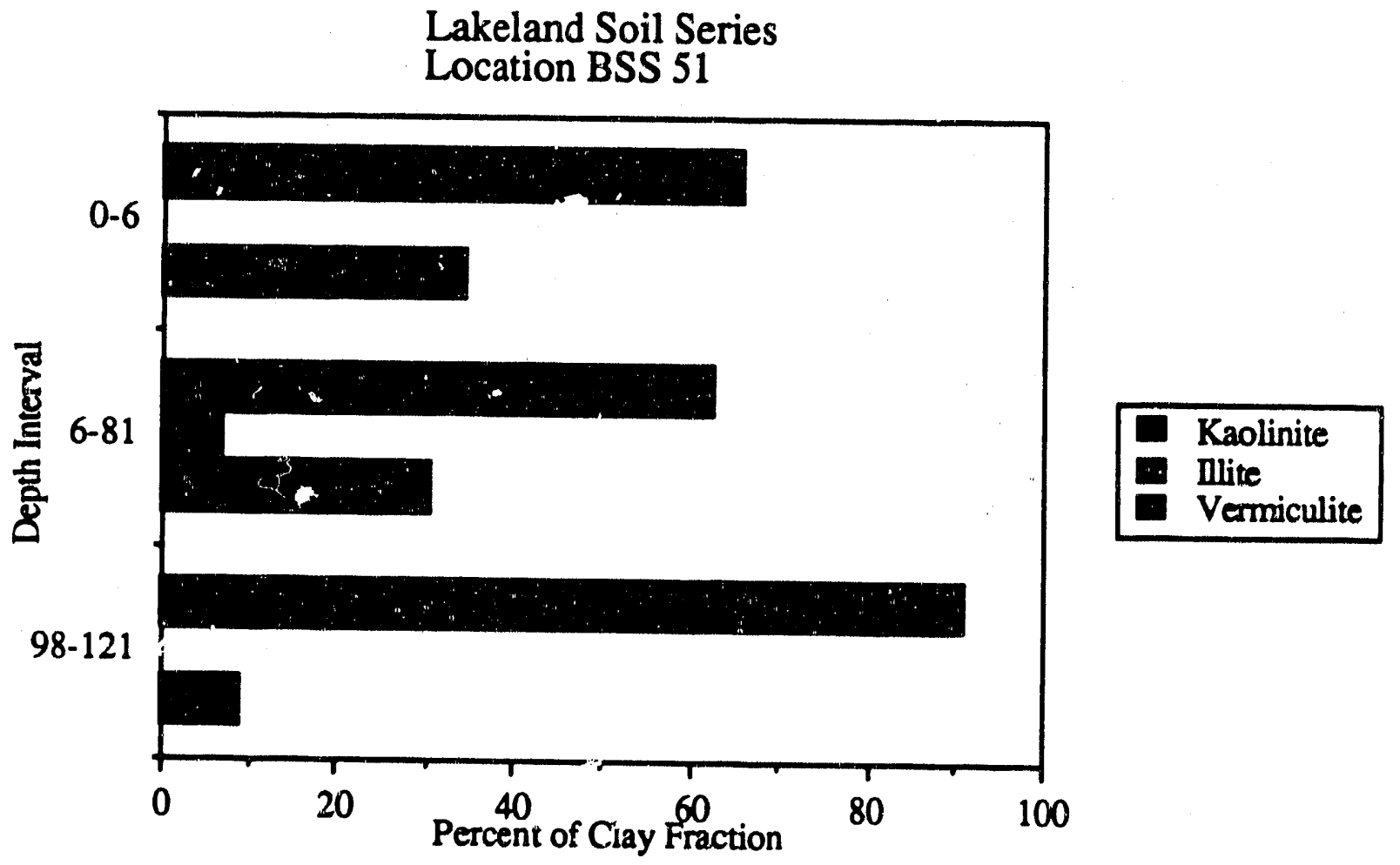

Figure 7.4 Depth profile showing the distribution of minesals in the clay fraction of the Lakeland Soil Series from three horizons at locations BSS 41 and BSS 51. 

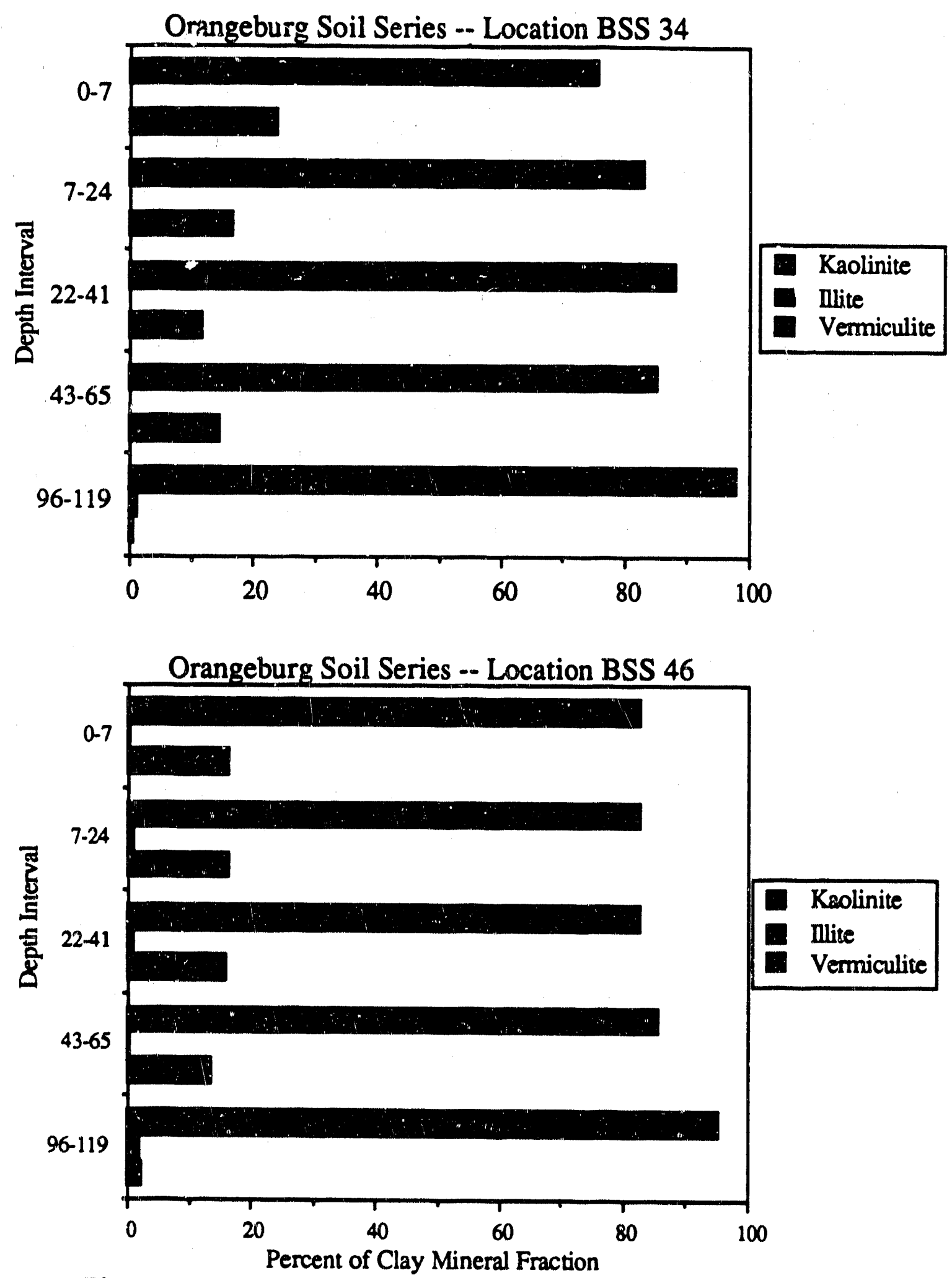

Figure 7.5 Depth profile showing the distribution of minerals in the clay fraction of the Orangeburg Soil Series from four horizons at locations BSS 34 and BSS 46. 


\subsection{References.}

Barnhisel, R. I, 1977. Chlorites and Hydroxy Interlayered Vermiculite and Smectite, in Minerals in Soil Environments, Soil Science Society of America, Madison, Wisconsin.

Bear, F. E., 1964. Chemistry of the Soil, Reinhold Publishing Corporation, New York.

Connor, J. J. and H. T. Shacklette, 1975. Backeround Geochemistry of Some Rocks, Soillis Plants, and Vegetables in the Conterminous United States, U.S. Geological Survey Professional Paper 574-F, Government Printing Office, Washington, D.C.

Davis, C. E., and J. C. Corey, 1989, Forest Land Application of Sewage Sludge on the Savannah River Plant. DP-1763, Westinghouse Savannah River Company, Aiken, South Carolina, 29808.

Davis, H. A., D. K. Martin, and J. L.Todd, 1989, Sayannah River Site Environmental Report for 1988, WSRC-RP-89-59-1, Westinghouse Savannah River Company, Aiken, South Carolina, 29808.

EPA, 1980. Prescribed Procedures for Measurement of Radioactivity in Drinking Water, EPA600/4-80-32, U.S. Environmental Protection Agency, Cincinnati, OH.

EPA, 1986. Test Methods for Evaluating Solid Waste, (SW-846), 3rd ed., Vol. II, U.S. Environmental Protection Agency, Office of Solid Waste, Washington, D.C.

EPA, 1988. Laboratory Data Validation Functional Guidelines For Evaluating Organic Analyses, Contract Laboratory Program, Office of Emergency and Remedial Response, Washington, D.C.

Fay, W. M., and J. B. Pickett, 1987, Documentation of 1982 Soil Analyses to Determine the Nanural Backpround Radioactivity in SRP Surface Soils, DPST-87-260, E.I. du Pont de Nemours and Company, Savannah River Laboratory, Aiken, S.C.

Hollod, G. J., I. W. Marine, H. W. Bledsoe, Jr., and J. P. Ryan, 1982. Metals and Organics in the Soil Beneath the M-Area Settling Basin, DPST-82-721, E.I. du Pont de Nemours and Company, Savannah River Laboratory, Aiken, S.C.

Hubbard, R. K., C. R. Berdanier, H. F. Perkins, and R. A. Leonard, 1985. Characteristics of Selected Upland Soils of the Georpia Coastal Plain, U.S. Department of Agriculture, Agriculture Research Service ARS-37.

Hutchinson, T. C. and K. M. Meema, 1987. Lead. Mercury. Cadmium and Arsenic in the Environment, Scope 31, John Wiley \& Sons, Inc. New York.

Lindsay, W. L. 1979. Chemical Equilibria in Soils, John Wiley \& Sons, Inc., New York.

Looney, B. B., C.A. Eddy. M. Ramdeen, J. Pickett, V. Rogers, P. A. Shirley, and M. T. Scott, 1990. Evaluation of Geochemical Properties of Soils ind Shallow Sediments at the Savannah River Site, WRSC-RP-90-1032, Westinghouse Savannah River Company, Savannah River Laboratory, Aiken, S.C. 
Mikol, Sue C., L. T. Burckhalter, J. L. Todd, and D. K. Martin, 1988, Savannah River Plant Environmental Report. Annual Report for 1987, E.I. du Pont de Nemours and Company, Savannah River Laboratory, Aiken, S.C.

Nriagu, J. O., 1980a. Cadmium in the Environment, John Wiley \& Sons, Inc., New York.

Nriagu, J. O., 1980b. Nickel in the Environment, John Wiley \& Sons, Inc., New York.

Nriagu, J. O., 1980c . Zinc in the Environment, John Wiley \& Sons, Inc., New York.

Pickett, J. B., 1985 Technical Data Summary. Extended Characterization of the M-Area Settling Basin and Vicinity, DPSTD-85.121, (Rev. 10/85), E.I. du Pont de Nemours and Company, Savannah River Laboratory, Aiken, S.C.

Potts, P. J., 1987, A Handbook of Silicate Rock Analysis, Blackie and Son, Limited, London.

Robinson, W. O., 1914. The Inorganic Composition of Some Important American Soils, U.S. Department of Agriculture Professional Paper 122, Government Printing Office, Washington, D.C.

Robinson, W. O., 1917. Yariation in the Chemical Composition of Soils, U.S. Department of Agriculture Professional Paper 551, Government Printing Office, Washington, D.C.

Rose, A. W., H. E. Hawkes, and J. S. Webb, 1979, Geochemisty in Mineral Exploration. Second Edition, London Academic Press, London.

Shacklette, H. T. and J. G. Boerngen, 1984. Element Concentrations in Soils and other Materials of the Conterminous United States, U.S. Geological Survey Professional Paper 1270, United States Government Printing Office, Washington, D.C.

Shacklette, H. T., J. C. Hamilton, J. G. Boerngen, and J. M. Bowles, 1971 Elemental Composition of Surficial Materials in the U.S. U. S. Geological Survey Professional Paper 574-D, United States Government Printing Office, Washington, D.C.

Siegel, F. R., 1974. Applied Geochemistry, John Wiley \& Sons, New York.

U.S.D.A., 1989. Soil Survey of the Savannah River Plant. Aiken County Area. South Carolina, United States Department of Agriculture, Soil Conservation Service, Aiken, South Carolina.

U.S.D.A., 1986, Designations for Master Horizons and Layers in Soils Agency for International Development, Department of Agronomy, Cornell University.

U.S.D.A., 1981, Soil Survey Manual. United States Department of Agriculture Handbook, Government Printing Office, Washington, D.C.

Vinogradov, A. P., 1959, The Geochemistry of Rare and Dispersed Chemical Elements in Soils, Second Edition, Consultants Bureau Enterprises, New York.

Waldron, H. A., 1980. Metais in the Environment, Academic Press, New York. 
GEOCHEMICAL AND PHYSICAL PROPERTIES OF SOILS AND SHALLOW SEDIMENTS AT THE SAVANNAH RIVER SITE

\author{
Appendix A
}

Background Soil Study Quality Control and Sampling and Analysis Plan 
QUALITY CONTROL

\title{
AND SAMPLING AND ANALYSIS PLAN \\ SAVANNAH RIVER PLANT \\ RFI BACKGROUND SOILS STUDY
}

\author{
PREPARED FOR: \\ E.I. DU PONT DE NEMOURS \& COMPANY \\ SAVANNAH RIVER PLANT \\ AIKEN, SOUTH CAROLINA
}

\author{
PREPARED BY: \\ SIRRINE ENVIRONMENTAL CONSULTANTS, INC. \\ GREENVILLE, SOUTH CAROLINA
}

SEC JOB NO. G-8163

AUGUST 18,1988 
1.0 INTRODUCTION

2.0 PROJECT DESCRIPTION

2.1 General Project Description

$2-1$

2.2 Site Description

2.3 Previous Investigations

2.4 Hazard Assessment

$2-1$

$2-1$

$2-2$

$2-2$

3.0 PROJECT ORGANIZATION

4.0 AUGER DRILLING

$4-1$

4.1 Introduction

4.2 Soil Drilling Procedures

4.3 Backfilling and Disposal of Excess Cuttings

$4-1$

$4-1$

4.4 Drilling Equipment Decontamination

$4-2$

$4-2$

5.0 SAMPLING AND CHAIN OF CUSTODY PROCEDURES

5.1 Introduction

$5-1$

5.2 Sample Site Selection

5.3 Soil Sample Locations and Descriptions

$5-1$

5.4 Soil Sampling Procedure

5.4.1. Split Spoon Samples

$5-1$

$5-5$

$5-6$

5.4.2 Grab Samples

$5-7$

5.5 Field Quality Control

$5-7$

5.6 Field Cleaning Procedures

$5-8$

5.7 Field Quality Control Samples

$5-8$

$5-8$

5.8 Sample Packaging

$5-8$

5.9 Sample Custody

5.9.1 Chain of Custody Record Form

$5-10$

5.9.2 Field Collection and Shipment

$5-11$

5.9.3 Sample Disposal

$5-13$

$5-14$

6.0 ANALYTICAL METHODS AND PROCEDURES $6-1$

7.0 PROJECT DOCUMENTATION $7-1$

7.1 Project Record Book

$7-1$

7.2 Communication Report

$7-1$

7.3 Field Geologic Log

7.4 Test Boring Report

$7-1$

$7-1$

8.0 PROJECT ACCOUNTING

$8-1$ 


\subsection{INTRODUCTION}

This document is intended to present the Quality Control Plan (QCP) and Sampling and Analysis Plan (SAP) for the Savannah River Plant RFI Background Soils study. The QCP describes the methods and approaches for providing quality control of field activities performed by sirrine. Environmental Consultants, Inc., (SEC) and the drilling and analytical subcontractors involved with this project. In addition, the SAP includes the procedures to be followed when conducting sampling and analytical activities during this project.

This investigation is being conducted under Subcontract AX-715304, Task Order 24, for E.I. du Pont de Nemours \& Company (du Pont). 


\subsection{PROJECT DESCRIPTION}

\subsection{Geileral Project Description}

This plan describes the procedures which will govern all investigative sumpling and chemical analyses activities to be performed by SEC aS part of the RCRA Facility Investigation (RFI) Background Soils Study at the Savannah River Plant (SRP). The principal objective of this study is to determine representative background concentrations for various chemical constituents in soils at the SRP. These data will be compared with data obtained from Solid Waste Management Unit (SWMU) sites a $\bar{t}$ the SRP which may have received hazardous materials to determine what, if any, clean up actions are necessary. Approximately 65 of these sites are currently being investigated in accordance with EPA RFI guidelines. Also, SEC will be performing a comparison of stainless steel and carbon steel split spoon samplers during this study. Samples will be collected using each type of split spoon from identical soil intervals at adjacent boring locations.

\subsection{Site Description}

The SRP is a U.S. Department of Energy facility located near Aiken, South Carolina, along the Savannah River. The plant is a major DOE installation engaged in the production of defense nuclear materials. The production of these materials and the operation, fabrication, separation, and support facilities result in the generation of hazardous, low-level radioactive, and mixed wastes (radioactive and hazardous). Certain waste materials generated by the SRP are regulated by RCRA, and require post-closure or operating permits. The RFI program requires that other waste sites (SWMUS) be investigated to determine if releases of hazardous cunstituents have occurred. Approximately 65 SWMU sites have been identified for investigation at the SRP. A map of the entire SRP is shown on Figure 1. 

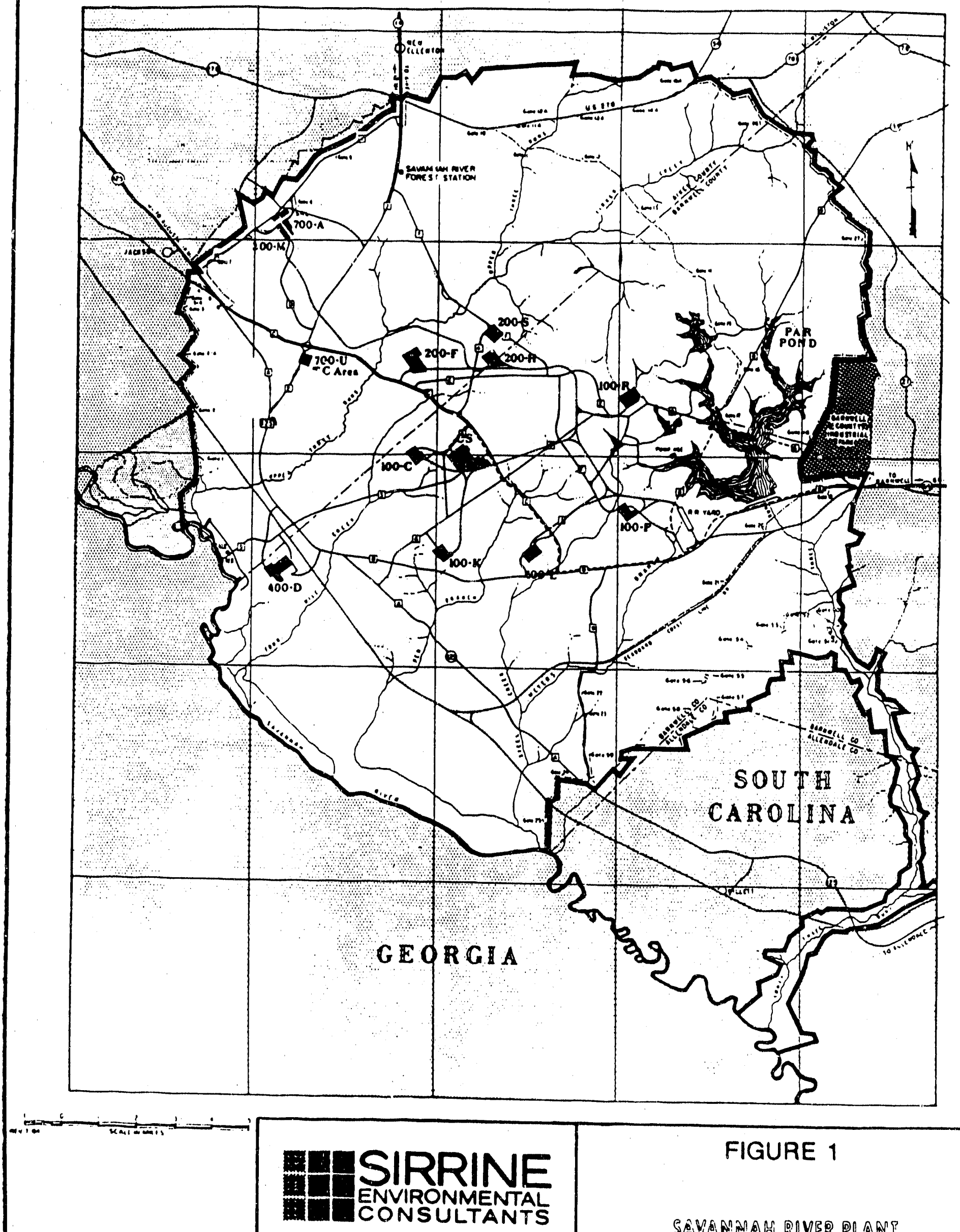

Greenvilib. South Corolino

FIGURE 1

SEVARNAW DUVER PDANP SITE MAP 


\subsection{Previous Investigations}

- Soil Survey of Aiken County Area, South Carolina, United States Denartment of Agriculture, Soil Conservation Service.

This study describes the various soil series found in Aiken county, South Carolina. Although the Savannah River Plant is excluded from this report, many of the soil series discussed in this publication are found on SRP. Of the six soil groups to be studied in the Background Soils Study, only the Blanton is not mentioned in this report.

- Floodplain Sediment Study, part of the 1985 Comprehensive Cooling Water Study, Savannah River Plant.

This report describes the analytical methods and results used to characterize the soil types from floodplains at SRP. Soil cores taken from floodplains on the SRP facility were analyzed for certain trace elements and radionuclides.

- Soil Survey of the Savannah River Plant, Aiken County Area, South Carolina, Vergil Rogers, United States Department of Agriculture, Soil Conservation Service, Vergil Rogers, in press.

This study describes the various soil series found on the Savannah River Plant.

\subsection{Hazard Assessment}

No hazardous constituents are expected to be encountered during the Background soils study. The purpose of this study is to establish background levels for certain analytical parameters found in the various Savannah River Plant soil types. The sites to be studied during this investigation are located in areas known to be nonhazardous to insure that "true" background information is obtained. 


\subsection{PROJECT ORGANIZATION}

The organization of the SRP RFI Background Soils Study is shown on Figure 2. Key project personnel include the SRP Project Manager, Brian Looney, and the SEC Project Manager, Pat Shirley. Other key project personnel and their telephone numbers are listed on Table 1. The SEC Project Manager will report directly to the SRP Project Manager. SEC will be responsible for coordination with all subcontractors involved with this project. The major subcontraciors selected for this project consist of:

- metaTRACE Laboratories, the primary analytical laboratory,

- Weston Analytics, the Quality Assurance Laboratory, and

- Monitor Testing Corporation, the drilling contractor.

Coordination with the drilling subcontractor will be conducted with the assistance of Health Protection (HP). SEC will maintain daily records for drilling pay items. These records will be submitted to HP on a routine frequency for accounting purposes. SEC will be responsible for direct coordination of the analytical laboratories, including scheduling, accounting, and data validation. 

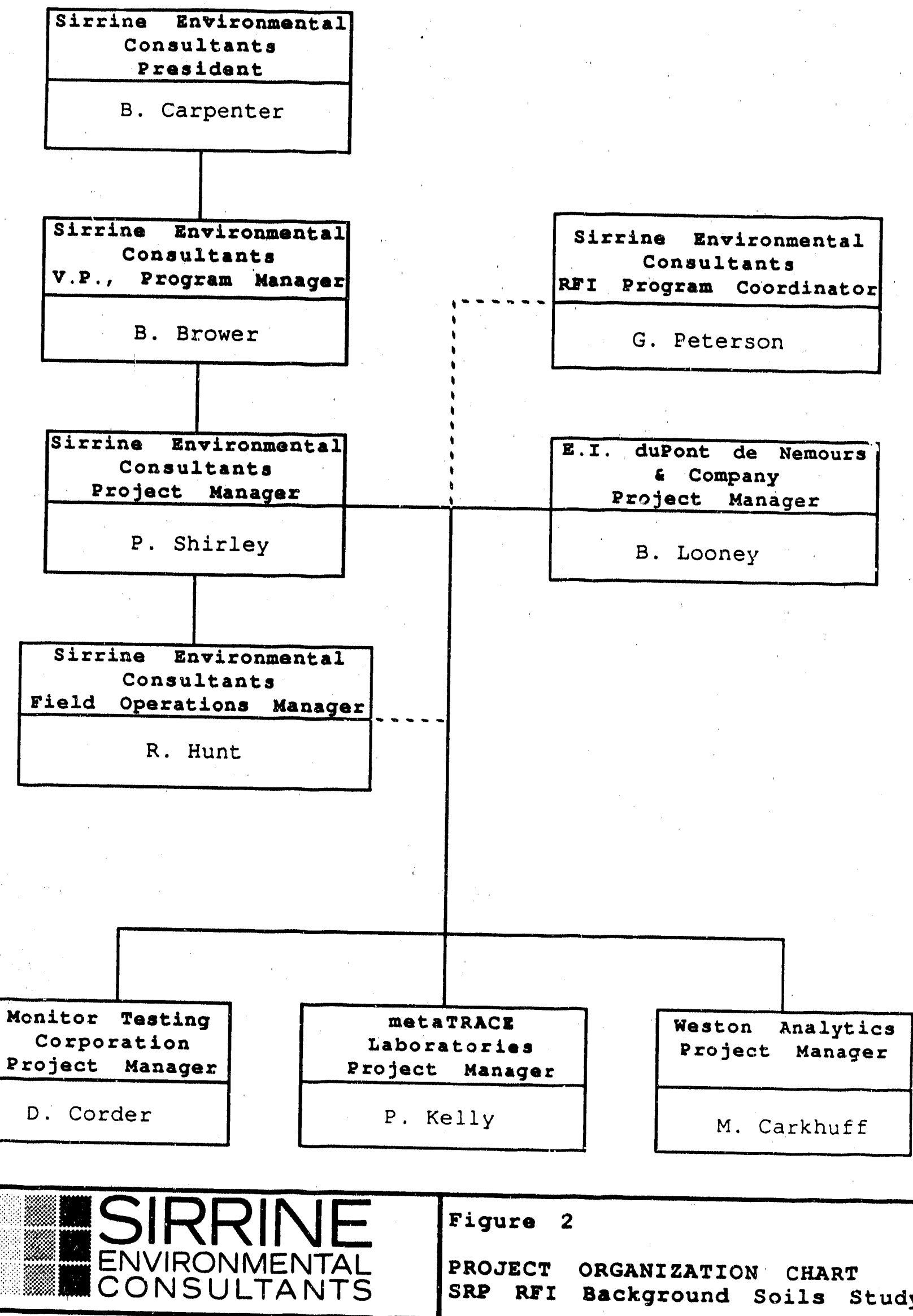

Figure 2

PROJECT ORGANIZATION CHART

SRP REI Background Soils study 
TABLE 1

SIRRINE ENVIRONMENTAL CONSULTANTS, INC.

PERSONNEL CONTACTS

RFI BACKGROUND SOILS STUDY

Letter Subcontract AX-715304, Task Order 24

SEC Job No. G-8163

Ed Zillioux, H\&S Director

$234-3061$

$232-3891$

Office

Home

Mark McCoy, H\&S Coordinator

$234-3595$

Office

Pat Shirley, Project Manager

$234-3068$

$297-1711$

office

Home

SEC Aiken Apar tment

$649-0396$

SEC Well Building Office

$557-6745$

E.I. du Pont de Nemours \& Company

Savannah River Plant

TC1 Area, Building 772-7G

Aiken, South Carolina 29808

SEC M-Area Office

$725-2350$

SRP CONTACTS

Brian Looney, Contract Administrator

$725-5189$

Office

Bonnie Ferguson

(Brian Looney's Secretary at 773-42A)

$725-5178$

Office

Bob Hunter

Norm Bryan

$557-6648$

Office

Janelle Janssen

$725-2456$

Office

Ed Campbel1

$725-2037$

Office

\section{LABORATORY CONTACTS}

metaTRACE, Inc.

13715 Rider Trail North

Earth City, Missouri 63045

SRM 7812

(314) $298-8566$

Patrick Kelly, Project Manager

Pich Mannz, Assistant Project Manager

lioston Analytics

208 Welsh Pool Road

SRM 7811

Lionville, Pennsylvania 19353 


\subsection{AUGER DRILLING}

\subsection{Introduction}

All drilling will be performed with a Mobile B-24 truck-mounted auger drilling rig. An experienced (SEC) geologist will continuously observe all drilling operations. No grease or oil shall be used on drili pipe joints.

\subsection{Soil Drilling Procedures}

Soil drilling will be accomplished using $31 / 4$ inch I.D. (6 7;8 inch 0.D.) hollow-stem augers.

All soil samples will be taken using split spoons which are either 18 or 24 inches long and either $13 / 8$ inch 1.D. (2 inch 0.D.) OR $27 / 8$ inch I.D. ( 3 inch 0.D.). The split spoons will be manufactured of carbon or stainless steel. The samples will be obtained by driving the split-spoon sampler using a 140-pound hi.nmer, free-falling for 30 inches. Representative portions of each sample will be stored in labeied glass jars until needed for testing or the contract is complete.

Soil samples for lithologic description shall be taken continuously from the land surface to the completion depth of 20 feet in each boring. All soil samples will be identified in the field by an SEC geologist using visual/manual techniques described in ASTM D-2487 and 0-2488. The soils will be classified in accordance with he Unified Soils classification system and a log of each boring will be produced. In addition, all samples will also be classified according to the SRP Field Classification System (RFI Program Plan, Appendix I).

Personnel involved in removing and preserving soil samples for chemical analysis will wear surgical inner gloves with nitrile outer gloves which will be washed with soapy water and rinsed with distilled or 
deionized water immediately prior to handling each sample.

The split-spoon sampler and subsampling instruments will be decontaminated before each sample is taken as described in Section 4.4.

\subsection{Backfilling and Disposal of Excess ruttings}

Cuttings from all drilling operations will be backfilled in the original borehole.

\subsection{Drilling Equipment Decontamination}

The drill rig and equipment will be cleaned to remove excess grease, oils, or caked-on soils from previous work, upon arrival at the site. Equipment which leaks fuel, coolant, crankcase oil, transmission fluid, hydraulic fluid or lubricants will be removed from site and repaired prior to use. The work area of the drill rig, all downhole tools, split-spoon sampler and subsampling instruments will be decontaminated before each sample is taken in accordance with the following procedures:

1. Clean with tap water and phosphate-free laboratory detergent using a brush if necessary to remove particulate matter and surface films.

2. Rinse thoroughly with tap water.

3. Rinse thoroughly with distilled or deionized water.

4. Rinse two times with pesticide grade isopropanol solvent and allow to air dry for as long as possible.

5. Rinse thoroughly with distilled or deionized water and allow to air dry for as long as possible. 
6. Wrap with aluminum foil, if appropriate, to prevent contamination if equipment is going to be stored or transported.

An alternate decontamination procedure will consist of steam cleaning instead of Steps 1 through 3 above. Decontamination will continue with Steps 4 through 6 . Personnel involved with the decontamination procedures will wear surgical inner gloves with nitrile outer gloves which will be washed with soapy water and rinsed with distilled or deionized water immediately prior to handling any equipment used in sampling.

After steam cleaning, tools and equipment shall be stored on plastic sheeting to avoid contamination. 
5.0 SAMPLING AND CHAIN OF CUSTODY PROCEDURES

\subsection{Introduction}

The following sections present requirements for numbers, general locations, and analyses of samples to be collected. These requirements are summarized in Tables $2,3,4,5,6,7$, and 8 .

Sampling procedures to be used are outlined in the respective section below. Sample containers and preservation techniques are summarized in Table 9. Since the samples to be collected are in areas known to be nonhazardous, the samples collected during this investigation will be considered low-level environmental samples.

\subsection{Sample Site Selection}

The rationale for the selection of sampling sites is to determine representative backgrourid concentrations for various shemical constituents in soils at the SRP. The sites are to be located in areas known to be nonhazardous to insure that "true" background information is obtained. The sites were predetermined during a site walkover.

\subsection{Soil Sample Locations and Descriptions}

Approximately 48 boreholes will be augered to a depth of 20 feet at predetermined locations throughout the plant site. Twenty-nine soil series types have been identified at the Savannah River Plant. Many of these series were characterized as part of the Comprehensive Cooling Water/Floodplain Study. Therefore, the soil series which occur predominantly in floodplain areas have been eliminated as candidates for further investigation. The results contained in the existing floodplain study report will, however, be incorporated into the data base for the background soils study. Table 10 lists the soil series name, location, and coordinates for the samples collected during the floodplain study. Exclusion of the floodplain soil series reduced the 


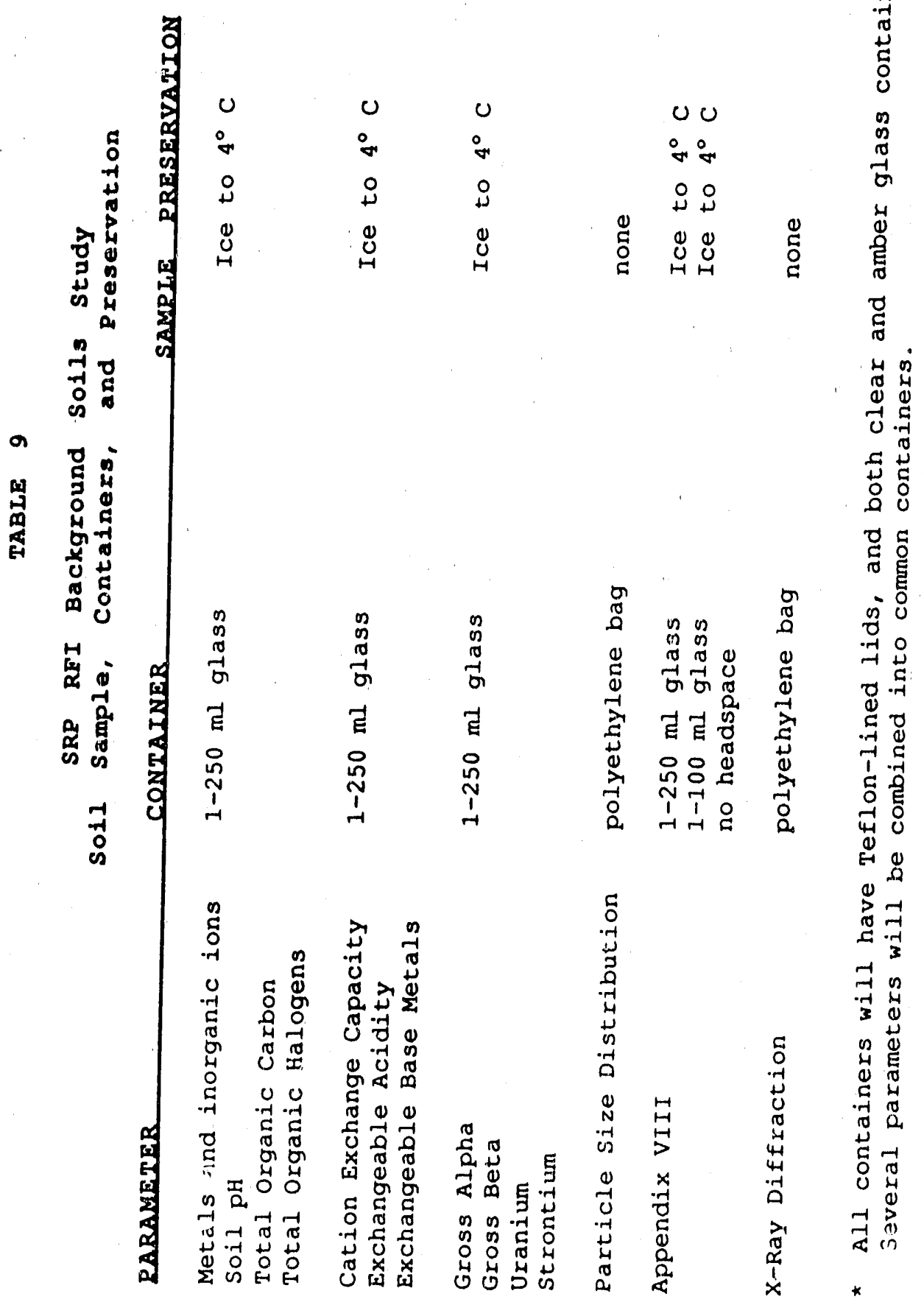


number of -soil series from 29 to 15 . The remaining soil series have been categorized by taxonomic class into six archetypal soil groups which cover approximately 75 percent of the SRP. Ten locations for each of the six soil groups have been selected by du pont.

The soil series groups and the 10 possible sample locations for each group are listed on Table 11. It is important to note that only one soil series (type) will be tested for each of the six archetypal soil groups. The remaining series within a particular group closely resemble the soil series tested.

A description of each of the six groups is provided below.

\section{Group One}

Blanton sand is the predominant soil type on SRP. It covers 21 percent of the plant area. Using Blanton as the central series in group one, the remaining soils in group one had the same or similar properties of Blanton.

The taxonomic name for Blanton indicates that the soil is almost entirely silica or other weather resistant minerals. Also, the sandy layer is greater than 1 meter thick, the soil is very old and well developed, and it is moist with a very low base saturation.

Troup, Albany, Wagram, and Lucy were placed into group one. Albany and Troup, are nearly a perfect match for Blanton. The only difference appears to be Blanton. Lucy was placed in this group because generally large areas of Troup are very intermixed with Lucy. Also, Lucy shares most of the same characteristics except that it is arenic rather than grossarenic. This means the sandy layer is not quite as thick. Wagram shares the same properties as Lucy; therefore, it was also included in group one. 
Table 11 Groupings and Possible Soil Core Locations

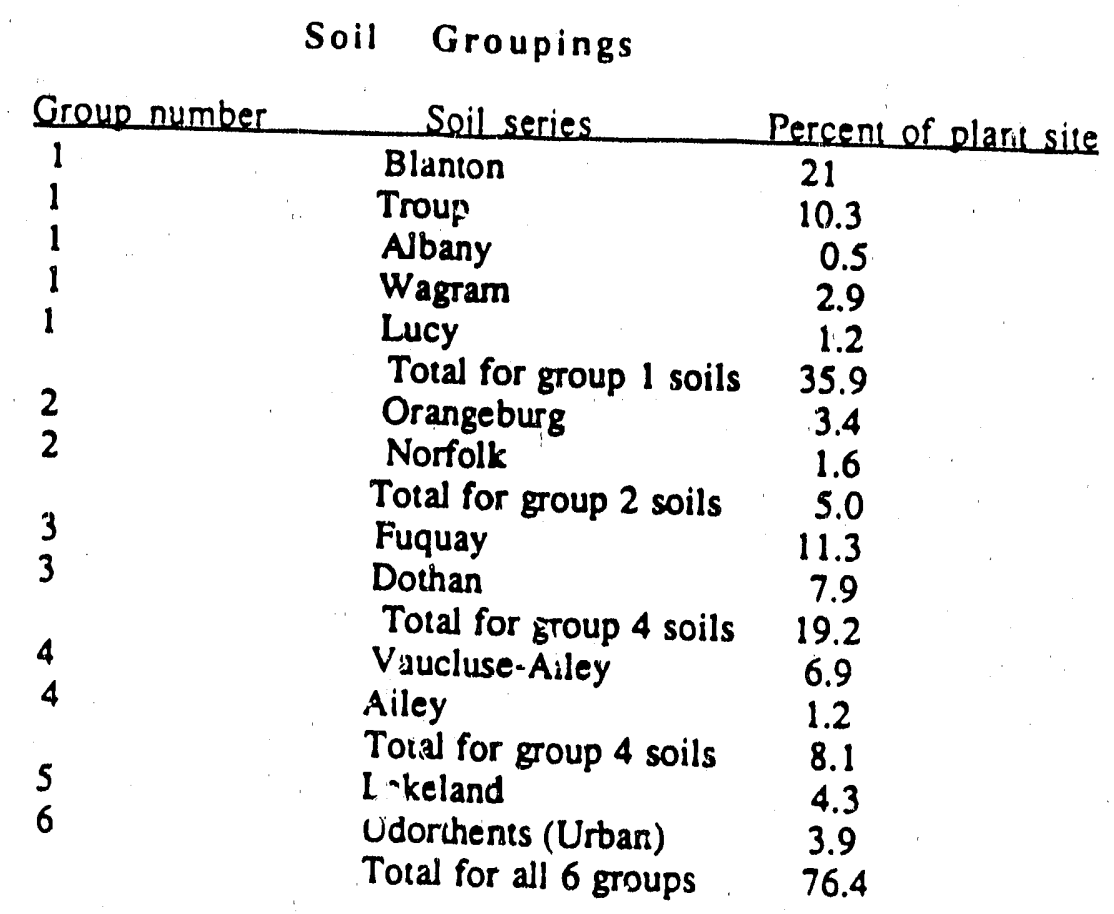

\section{Group 1 Site Locations}

Location

1) North of Road 2-1

2) Road 8

3) Juncrion Road $A$ and Cook Road

4) Junction of Roads $2 \cdot 1$ and 8-4

5) North of Road G

6) Junction of $115 \mathrm{KV}$ Trans Line and Super Control Relay Cable

7) South Junction of Road C 21

8) Seaboard Coastline South and Road $9 \quad 28$

9) Between Risher Pond Road M Line 30 and Seaboard Coastline

10) East of Junction of $115 \mathrm{KV}$ Trans

Line and Seaboard Coastline 
Table 11 Cont.

Group 2 Site Locations

Locations

Map Number

1) North of Road 2-1

2) Road A South of Seaboard Coastline

3) $M$ Line

4) Road 2-1

9

5) Northwest or D Art

6) Cato Road

7) Road B West of Pen Branch 21

8) SRP Boundary 1/4 mile from 28 Lower Three Runs Creek $\quad 30$

9) Road A 1/2 mile east of Pen Branch 32

10) SRP Boundary $1 / 4$ mile from Lower Three Runs

\section{Group 3 Site Locations}

Locations

1) North of Road 2-1

Map Numbers

2) Near Road 8

3) Cook Road just past

115KV Trans Line

6

8

9

4) Monroe Owens Road North of R Area 17

5) Junction of Roads 5 and 3

6) K line North of IISKV Trans Line 28

7) Seaboard Coastline 30

8) Seaboard Coastline beiween Meyers
Branch and 115KV Trans Line

9) Road 2-1 12

10) Road A Northwest of Pen Branch 32 
Table 11 Cont.

Group 4 Site Locations

Locarions

Map Numbers

1) North of Road 2.1 between

2-1.1 and Upper Three Runs

6

38

2) CSX South of Seaboard Cuastline

3) Unnamed Road between

$M$ Line and Road D

4) Undergtound Burial Cable between County Line and Mill Creek

5) East Bank of Pond C

6) Road A-6 West of Road A-6.1 Northj of C Area

7) Road C North of Road 7

8) Seaboard Coastline South of $M$ Line

9) Road A just East of Pen Branch

10) Seaboard Coastline North of 115KV Trans Line

\section{Group 5 Site Locations}

Locations

Map Numbers

1) Road F-1 North of Road 2-1

2) West of Road 8

6

3) Road B-6 near SRP boundary

8

5) Road 3,1/3 of at very top of map

Par Pond and $R$ Area

6) Road 3 West of Four Mile Creek . 21

7) Near SRP Boundary North of Road A 1

8) B-6.4 South of Lower Three Runs 30

9) North of unnamed Road extending from Par Pond near SRP Boundary 24

10) East of C-4 South of

Upper Three Runs 
Tabie 11 Cont.

Group 6 Site Locations

Locations

1) 700 Area

2) South of Road 2-1 just inside of Aiken County

3) $R$ Area

4) C Area

5) L Area

6) Between Road B and Par Dam

7) Seaboard Coastline between

$115 \mathrm{KV}$ Trans Line and

Meyers Branch

8) $\mathrm{H}$ Area

9) D Area

10) CS Area
Map Numbers

\section{9}

12

17

21

28

30

34

16

26

22 
Group Two

The central series in group two is Orangeburg. This soil type covers 3.4 percent of SRP. By using the taxonomic name for Orangeburg it was determined that this soil type is at least 15 percent sand and approximately 18 to 34 percent clay. Like Blanton, it is almost entirely silica or other weather resistant minerals. This series is a well developed mineral soil with an alluvial horizon of silicate clay. Orangeburg has a low base saturation and is moist.

By using the taxonomic name, Norfolk was placed into group two. Norfolk and Orangeburg seem to match perfectly, in that they share the same taxonomic name. The group two series covers about 5 percent of the plant.

\section{Group Three}

The second most common soil type on SRP is Fuquay. This series encompasses roughly 11.3 percent of the plant, therefore, it was made the central series of group three.

The taxonomis name for Fuquay indicates that it is less than 35 percent clay. It is almost entirely silica or other weather resistant minerals. The sandy layer is between 20 and 40 inches deep. Fuquay contains more than 5 percent plinthite, an iron-rich material that hardens ir reversibly upon exposure. The series is very well formed and has a low base saturation.

The only other soil series which matched Fuquay was Dothan. Dothan and Fuquay differ only in particle size and depth of the sandy layer. Dothan has finer particles while Fuquay has a deeper sandy layer. Most importantly, both soils are plinthic. These are the only two plinthic soils on SRP. Group three soils cover 19.2 percent of SRP. 


\section{Group Four}

The central series in group four is Vaucluse. This series includes the Vaucluse-Ailey complex. This complete series covers 6.9 percent of SRP. The taxonomic name for Vaucluse indicated that the soil was very well developed with a simple set of horizons. It is at least 15 percent sand and approximately 18 to 34 percent clay. Over 90 percent of the soil is either silica minerals or other weather resistant minerals.

Ailey also fits into this group. The differences between Ailey and Vaucluse are the particle sizes and the depth of the sandy layer. Ailey has finer particles and a deeper sandy layer than Vaucluse. Vaucluse and Ailey are very often intermixed, and it is very hard to find an area that is predominantly one or the other. Because of this fact, Vaucluse and Ailey were grouped together.

\section{Group Five}

Lakeland sand is group five. It is the only quartzipsamment soil and thus had to be grouped alone. The taxonomic name for Lakeland tells us the soil is very sandy and the sand grains are mostly coated. It is a recently formed mineral soil with weak or no pedogenic horizon. Lakeland covers 4.3 percent of SRP.

\section{Group Six}

The sixth and final group is the most difficult to define. This group consists of the Udorthents. Udorthents are areas that are not predominantly one soil type. Udorthent areas are generally the result of construction work and pit filling. There are no uniform characteristics for this soil type. Within a few feet, the properties of Udorthent soil can change dramatically. Thus, wide variability is expected within the Udorthents classification. To obtain our background results, we will test eight different Udorthent sites plus 
use the results from the analysis of the other soil types. Udorthents cover 3.9 percent of SRP.

Du Pont personnel will flag each sampling location in the presence of SEC, confirm the predetermined soil group and series, and label as such. The SEC Field Manager will supervise the sampling activities at each site in accordance with the sampling protocol described herein.

\subsection{Soil Sampling Procedure}

Soil samples for nonvolatile organic analysis will be collected by obtaining a sample from the target interval. The soil horizon will be confirmed based upon a visual comparison with the written soil series description. The soil sample will be placed in a stainless steel mixing bowl and homogenized using stainless steel utensils. The soil in the mixing bowl will then be divided into imaginary quadrants. One aliquot of sample from each quadrant will be collected using a stainless steel spoon and placed in the sample containers. Following collection of the subsample from each of the four quadrants, the remaining sample will be rehomogenized and the process repeated until all containers are filled. Residual soil from the target interval will be placed in core boxes for storage.

Samples collected for volatile organic compound analysis will be collected in a similar manner. Following confirmation of the proper sample horizon, a sample aliquot will be collected immediately from the mid-point of the target interval. The sample will be placed in the container such that no headspace remains; then it will be sealed, labeled, and placed in a precooled ice chest.

If the upper soil horizon is between 0 and 18 inches in depth, or any fraction thereof, the sample may be collected using a hand trowel, hand auger, or split spoon. The sample location may be extended radially from the sample origin to obtain the necessary volume. Shallow grab samples to be tested for volatile organic compounds will be collected 
by excavating soil to the mid-point of the upper horizon. Soil from above the mid-point will be placed in the mixing bowl for homogenization as described above. Soil from immediately below the mid-point will be placed in the sample container for volatile organic compound analysis. The container will be filled such that no headspace remains; then it will be sealed, labeled, and placed in a precooled ice chest.

Decontamination procedures for all downhole tools and subsampling equipment will be conducted as described in Section 4.4 .

5.4.1 Split-Spoon Samples. Split-spoon samples will be collected for chemical analyses at all soil boring sites.

Either 18 or 24 -inch long, standard $13 / 8$ inch I.D. (2 inch 0.D.), or $27 / 8$ inch 1.D. (3 $1 / 2$ inch 0.D.) stainless steel or carbon steel, split-spoon samplers will be used to collect samples. Each split-spoon sample will be taken according to the following procedure:

1. Decontaminate sampler as specified in Section 4.4 .

2. Orive split spoon with a 140 -pound hammer falling 30 inches.

3. Sample aliquots will be removed from the split-spoon sampler using a decontaminated stainless steel spatula, scoop, or teaspoon and placed in the appropriate prelabeled containers or mixing bowls.

4. Immediately store samples in precooled ice chest at $4^{\circ} \mathrm{C}$.

5. Only stainless steel or glass mixing bowls and utensils will be used for compositing soils samples.

6. If sample volume is insufficient for the required analyses, additional samples will be collected immediately below the desired sample zone or immediately adjacent to the original location and 
at the same depth interval.

7. The sample logbook and chain of custody will then be completed.

5.4.2 Grab Samples. The first soil sample at each boring will be considered a grab soil sample.

Equipment to be utilized for collection of near surface soil samples include: stainless steel trowel, stainless steel spoons, and stainless steel hand auger. Procedures for collection of near surface soil samples are as follows:

1. Use stainless steel trowel, stainless steel spoons, or stainless steel hand auger to excavate to 6 inches.

2. Decontaminate sampler as specified in Section 4.4.

3. Collect representative sample using decontaminated sampler.

4. Sample aliquots will be removed from the sampling instrument using a decontaminated stainless steel spatula, scoop, or teaspoon and placed in the appropriate prelabeled containers or stainless steel mixing bowls.

5. Only stainless steel utensils will be used for compositing soil samples.

6. Samples will then be placed into coolers and packed on ice.

7. Complete sample logbook and chain of custody.

\subsection{Field Qual ity Control}

A strict quality control program will be maintained by SEC in the field to ensure that sample integrity is maintained during sample collection 
and transfer to the laboratory. In addition, all equipment and instruments will be maintained and calibrated to prevent sample bias and ensure that field measurements are accurate. All quality control procedures followed in the field will be documented in the field Logbook. In addition to these, filed procedures, external quality control samples will be collected and analyzed to verify the results.

\subsection{Field Cleaning Procedures}

All field equipmeni will be cleaned at the SEC laboratory according to the laboratory procedures prior to travel to the site. Field equipment includes: stainless steel trowel, mixing bowels, spoons, and sounding rod.

Field equipment must be used at multiple sampling locations and will be cleaned according to the Drilling Equipment Decontamination Procedures in Section 4.4 .

\subsection{Field Quality Control Samples}

In order to verify the quality of the sample collection, transportation, and analys is of samples collected during the Background Soils Study, dupticate samples will be collected.

5.7.1 Duplicate Samples. Duplicate samples will be collected on 10 percent of the field samples collected during this investigation. These samples will be collected such that they are representative of the same depth interval. All duplicate samples will be tested by Weston Analytics.

\subsection{Sample Packaging}

The transportation of environmental samples from the time they are collected to their arrival at metaTRACE Laboratories in Earth City, Missouri, is an integral part of the Background Soils Study. The mode 
of travel -must be such that the sample is not altered physically, chemically, or biologically. The travel time to the laboratory must not interfere with the sample holding time. The chain of custody must also be maintained during the transportation process.

Samples collected at the site will be immediately placed in the sample cooler. Once the cooler is filled with samples, it is locked and securely positioned in a SEC sampling vehicle or other secure storage facility until the completion of the day's sampling activities. The following protocol will be followed for packaging of samples:

- Only waterproof metal or equivalent strength plastic ice chests and coolers will be used.

- Following the completion of the sample label and tag, the sample will be carefully placed into the container in the field. The sample tag will then be attached to the container.

0 The labels will be permanently affixed to each container. All labels will be completed with an indelible pen.

0 Inert cushioning material will be placed in the bottom of the cooler. The sample bottles will be individually wrapped with a bubble-wrap packing material.

- Additional inert packing material will be placed in the cooler to partially cover the sample bottles. Ice or freeze packs will be placed around, among, and on top of the sample bottles.

- Each cooler will be filled with additional cushioning materials to prevent movement of samples during shipment.

- The chain of custody record will be placed in a waterproof plastic bag and taped to the inside lid of the cooler. 
- The lid will be secured with strapping tape at a minimum of two locations. No labels will be covered.

- The completed shipping label will be attached tot he top of the cooler.

0 The weight limit of the shipper will be maintained.

\subsection{Sample Custody}

An overriding consideration for environmental measurement data is the ability to demonstrate that samples have been obtained from the locations stated and that they have reached the laboratory without alteration. Evidence of collection, shipment, laboratory receipt and laboratory custody untiil disposal must be documented to accomplish this objective. Documentation is accomplished through a chain of custody records each sample and the individuals responsible for sample collection, shipment, and receipt. A sample is considered in custody if it is:

- In a person's actual possession

$0 \quad$ In view after being in physical possession

- Sealed so that no one can tamper with it after having been in physical custody

- In a secured area, restricted to authorized personnel.

Sample custody will be initiated by SEC field personnel upon collection of samples. Documents similar to those included in this section will be used for recording pertinent information about the types and numbers of samples collected and shipped for analysis. Labels and $10 \mathrm{~g}$ information are checked to be sure there is no error in identification. 
The samples are then packaged to prevent breakage or leakage, and labeled according to DOT regulations or transported by private company vehicle as laboratory samples. Copies of forms will be maintained onsite; originals will be maintained at MetaTrace Laboratories.

Samples used for analysis will be held for 30 daj's following report of data before disposal. Archived samples will be stored until the end of the project. Disposal of the samples are the responsibility of the contracted laboratories.

Certain samples are designated to be sent to Weston Analytics for independent confirmation of analytical results. Such samples will contain copies of the chain of custody documentation and be shipped according to DOT regulations.

5.9.1 Chain of Custody Record Form. Figure 3 is the chain of custody form to be used by SEC personnel in collecting and shipping samples. MetaTRACE Laboratories and Weston Laboratories shall not accept samples for analysis without a correctly prepared chain of custody.

The chain of custody form shall be signed by each individual who has the samples in their possession. Preparation of the chain of custody form shall be as follows:

- The chain of custody record shall be initiated in the field by the person collecting the sample, for every sample. Every sample shall be assigned a unique identification number that is entered on the chain of custody form. Samples can be grouped for shipment and using a single form. The chain of custody form allows for tweive samples. If more than twelve samples are shipped in the same container, more than one chain of custody form is required.

- The record shall be completed in the field to indicate project, sampling team, etc. 
- If the person co?lecting the sample does not transport the samples to the laboratory or deliver the sample containers for shipment, the first block for "Relinquished By " "Received By " shall be completed in the field.

- The person transporting the samples to the laboratory or delivering them for shipment shall sign the record form as "Relinquished By ."

- If the samples are stipped to the laboratory by commercial carrier, the chain of custody form shall be sealed in a watertight container, placed in the shipping container, and the shipping container sealed prior to being given to the carrier.

- If the samples are directly transported to the laboratory, the chain of custody shall be kept in possession of the person delivering the samples.

- For samples shipped by commercial carrier, the waybill shall serve as an extension of the chain of custody record between the final field custodian and receipt in the laboratory.

- Upon receipt in the laboratory, the Sample Receiving Supervisor shall open the shipping containers, compare the contents with the chain of custody record, insure that document control information is accurate and complete, and sign and date the record. Any discrepancies shall be noted on the chain of custody form.

- If discrepancies occur, the samples in question shall be segregated from normal sample storage and the field personnel immediately notified.

- The chain of custody is completed after sample disposal. Note the blocks for this purpose on Figure 3. 


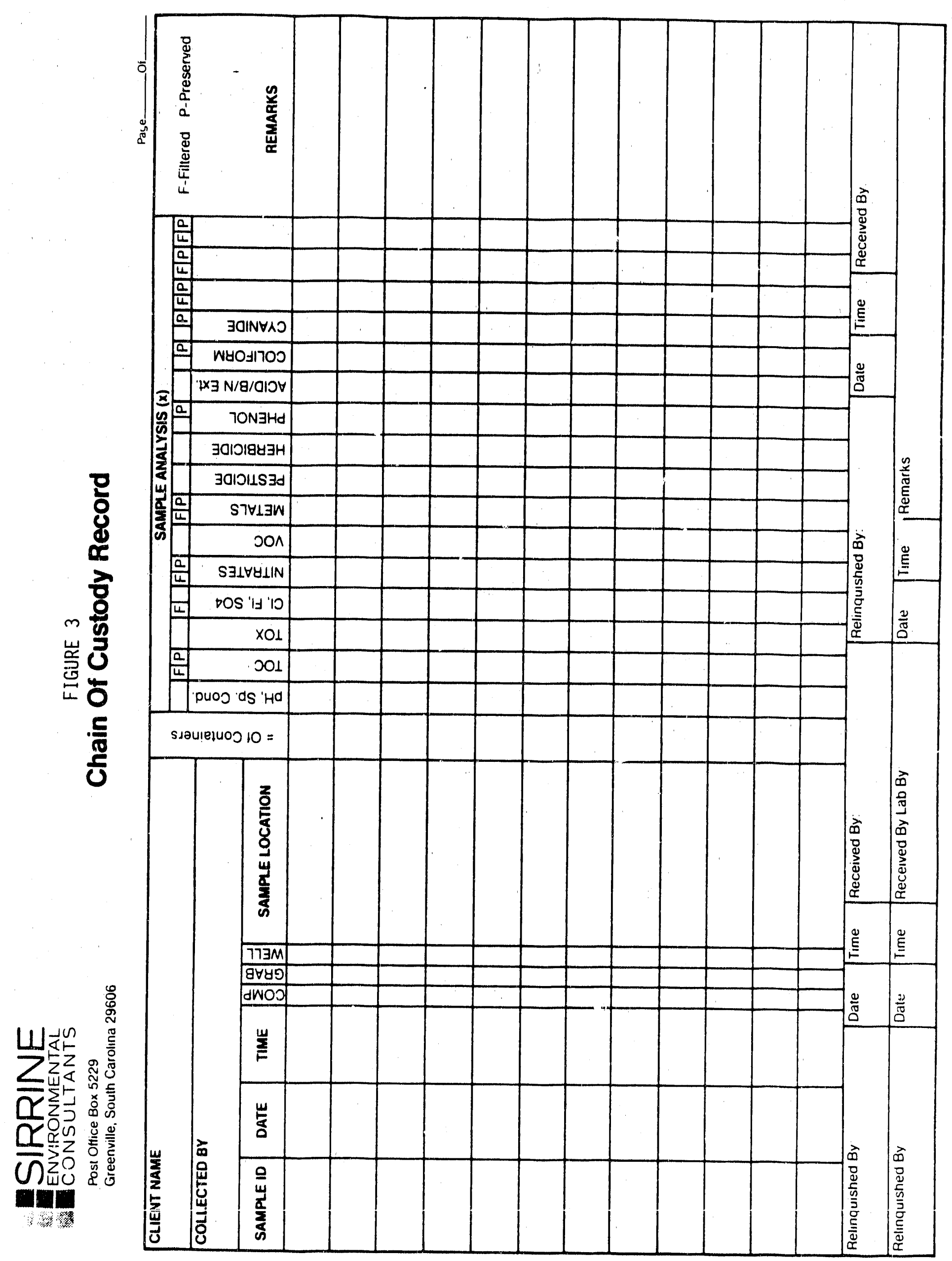


Chain- of custody records shall be maintained with the records for a specific project, becoming part of the data package.

5.9.2 Field Collection and Shipment. Samples must be placed in containers compatible with the intended analysis and properly preserved. Also, collection of samples must corisider the time interval between acquiring the sample and analysis (holding time) so that the sample is representative. Table 9 provides requirements of the analytical parameters of interest to this project with respect to the type of container arid preservation methods.

0. Package samples according to section 5.8 .

- Mark the appropriate "SRM No." on a piece of tape and place on the outside of the cooler.

- Notify John Frazier with Occupational Health Protection (OHP) to have them scan the samples/cooler for radiation. Making it clear to them that the samples are from remote background locations and that we have exerted an effort to avoid waste site areas.

- Take samples to Lizzie Overstreet with shipping/receiving in Building 713-A by 1 p.m. to have them shipped by Federal Express.

When samples are delivered to SRP shipping/Receiving SEC will relinquish custody of the samples to SRP.

Shipping containers are to be sealed prior to shipment, whether shipped by direct transport by field personnel or commercial carrier. Samples will not be retained at the site for more than 24 hours following collection.

The final step is to provide information to the laboratory shown in Figure 4. The Request for Analysis form will be completed by the field personnel and included with the chain of custody record. It is 
imperative that the Request. for Analys is form be provided so that analytical requirements are defined and sample holding times are not exceeded.

5.9.3 Sample Disposal. The chain of custody for the sample is completed as part of sample disfosal. The contract laboratories will be responsible for the disposal of any remaining samples after the analyses is complete. The samples are to be taken from non-hazardous sites so no special disposal methods are necessary. 


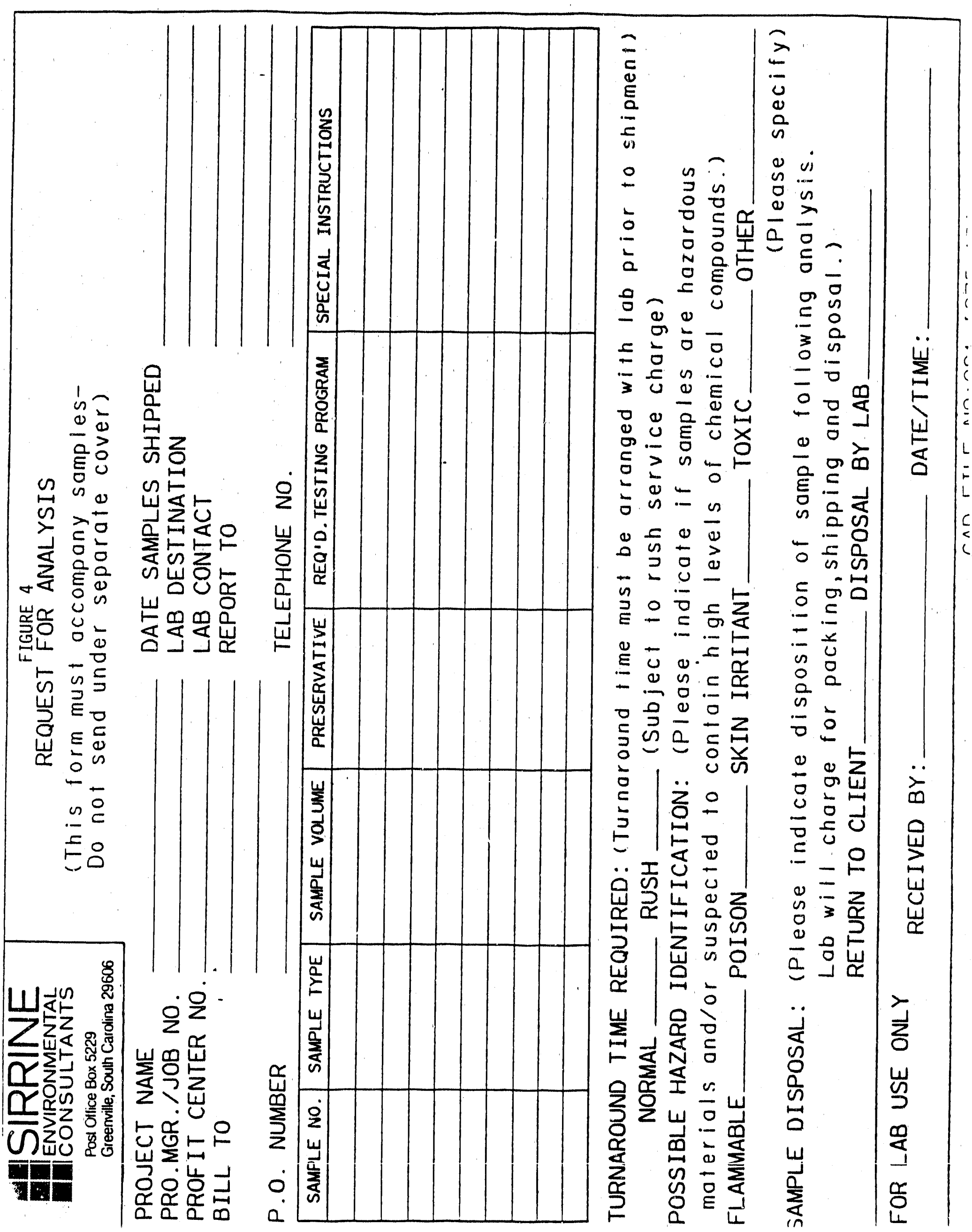




\subsection{ANALYTICAL METHODS AND PROCEDURES}

Analytical procedures and reference methods to be followed for the background solls study are listed on Table 2. The majority of the analytical procedures follow reference methods specified in E.PA document SW. 846, entitled "Test Methods for Evaluating Solids Wastes, "Third Edition. Reference methods for the agricultural and radiological parameters were taken from other procedure manuals, as specified on the table. contract required detection limits for metals and inorganic ions are specified on Table 13.

All analyses will be conducted by metaTRACE Laboratories, with two exceptions. Weston Analytics will perform analyses of split samples collected for quality control purposes. X-ray diffraction analysis of samples collected during this project will be performed by conoco. 


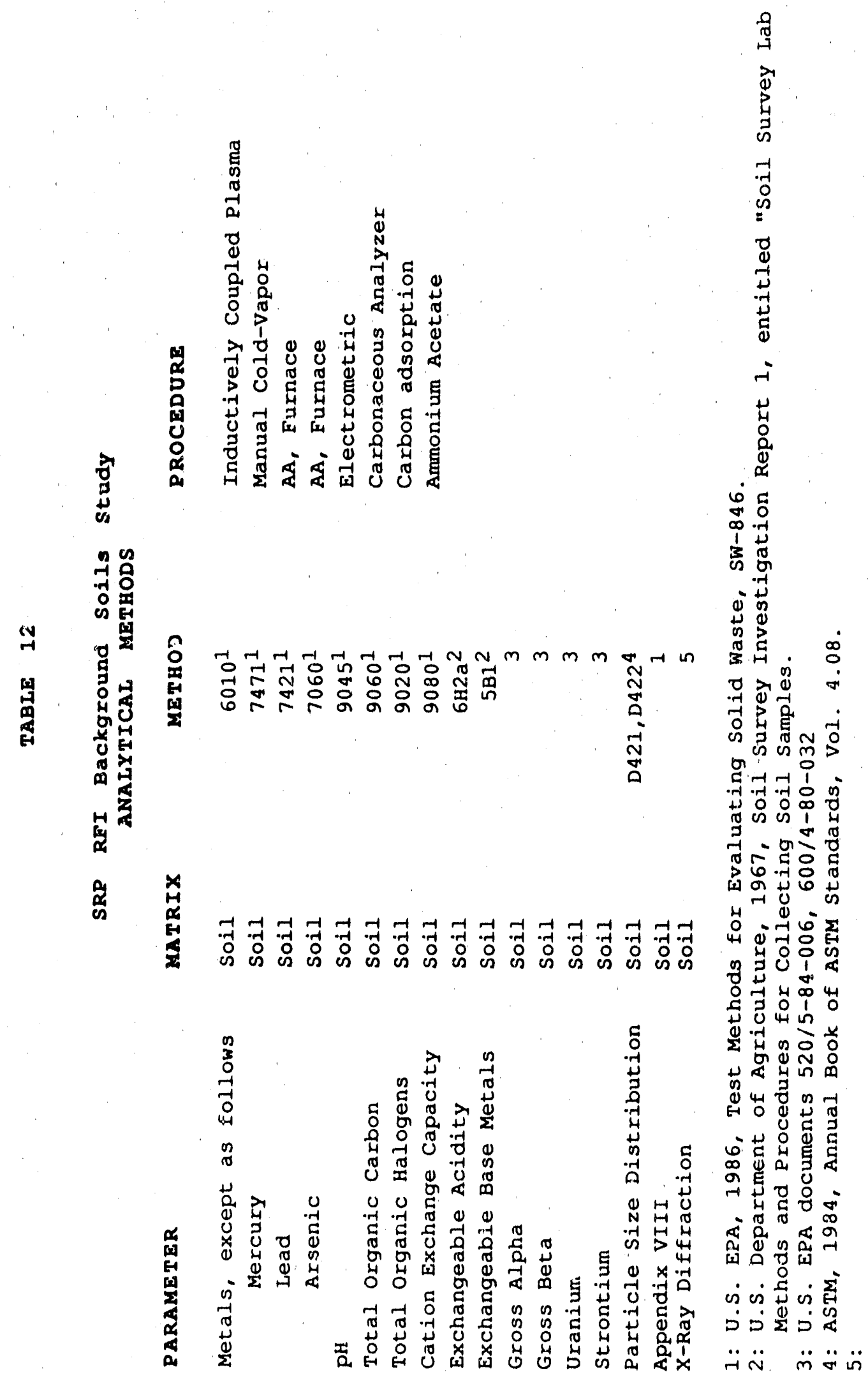


TABLE 13

\title{
SRP RFI Background Solls study \\ Specific Metals and Inorganic Ions Required \\ METAT \\ DETECTION IIMIT
}

\begin{abstract}
Aluminum
Arsenic

Barium

Cadmium

Chromium

Copper

Iron

Lead

Mercury

Nickel

Potassium

Silver

Sodium

Uranium

zinc
\end{abstract}

10
0.12
2
1
4
2
4
2
0.05
0.1
0.1
0.1
1
1
1

* Units in $\mu g / 1$ 


\subsection{PROJECT DOCUMENTATION}

\subsection{Project Record Book}

A numbered log book will be kept by SEC personnel throughout the entire project. The information entered in the logbook will consist of daily drilling activities, personnel entering and leaving specific sites, health and safety matters, and conversations with individuals regarding the project. The Project Record Log Book will serve as a single source documenting all project activities.

\subsection{Communication Report}

An SEC "Communications Report" form will be utilized to document important conversations pertaining to the Background Soils Study. This includes conversations leading to changes in the original scope of Work, notification to MetaTRACE and Weston Laboratories of sample shipments, and other important conversations. This form is shown on Figure 5.

\subsection{Field Geologic Log}

The Savannah River Plant "Field Geologic Log" will be completed for each test boring drilled during the Background Soils Study. Soils described on the Field Geologic Log will be classified according to the system jescribed in Appendix I of the RCRA Facility Investigation Program Plan. This document was prepared by Sirrine Environmental Consultants for the Savannah River Plant. This form is illustrated on Figure 6 .

\subsection{Test Boring Report}

In addition to the "Field Geologic Log", a "Test Boring Report" form will be completed for each test boring. Soils described on the "Test Boring Report" will be identified using visual/manual techniques 
FIGURE 5

COMMUNICATION REPORT

Name

Company

Address
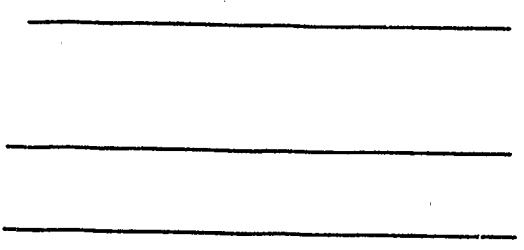

Telephone No.

Recorded By

Data:
Project No.

Date Time

$\square$ Telephone Conversation

$\square \quad$ Office Conversation

Re: 


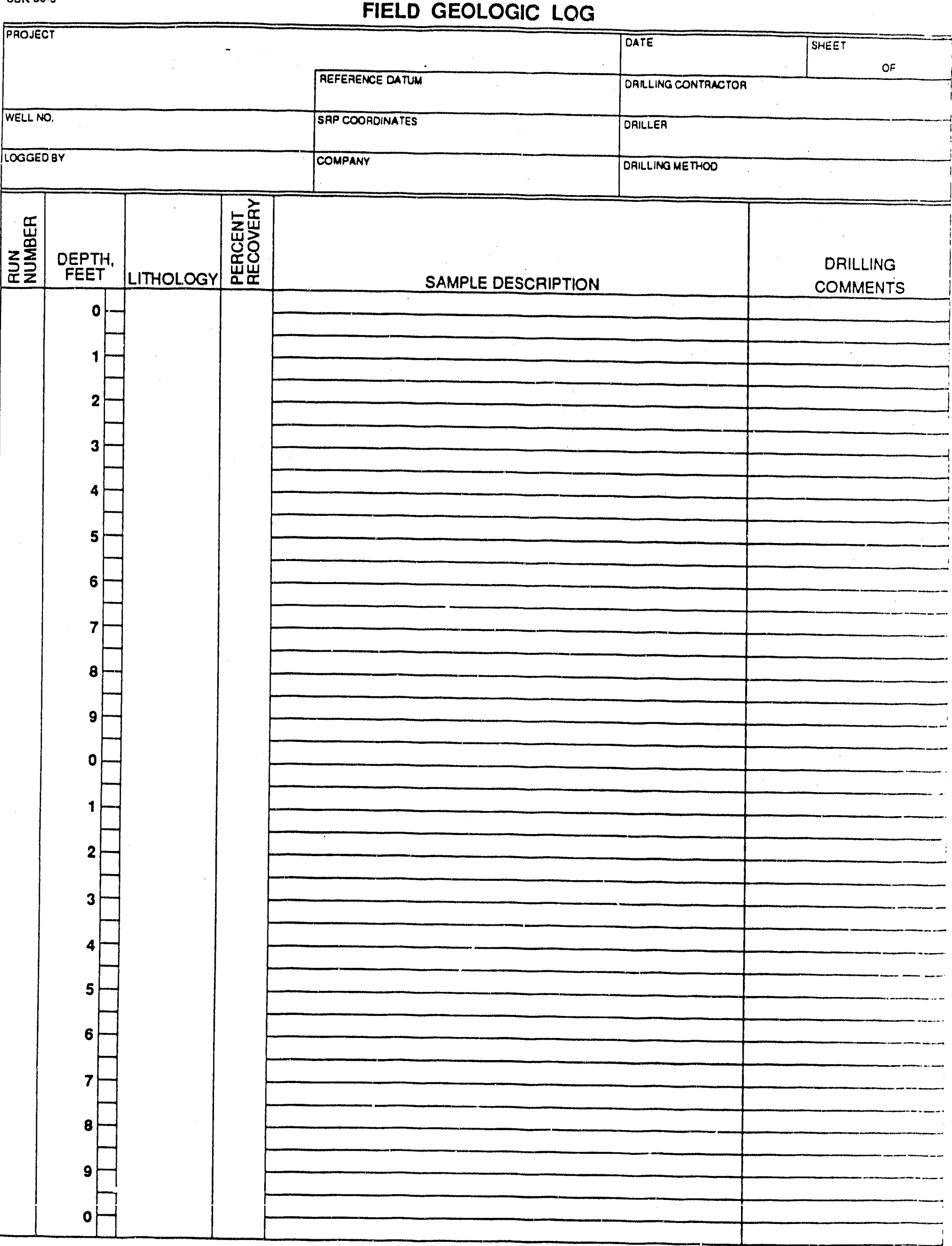


described in ASTM D-2487 and D-2488. The soils will be classified in accordance with the United Soils Classification System. This form is illustrated in Figure 7. 


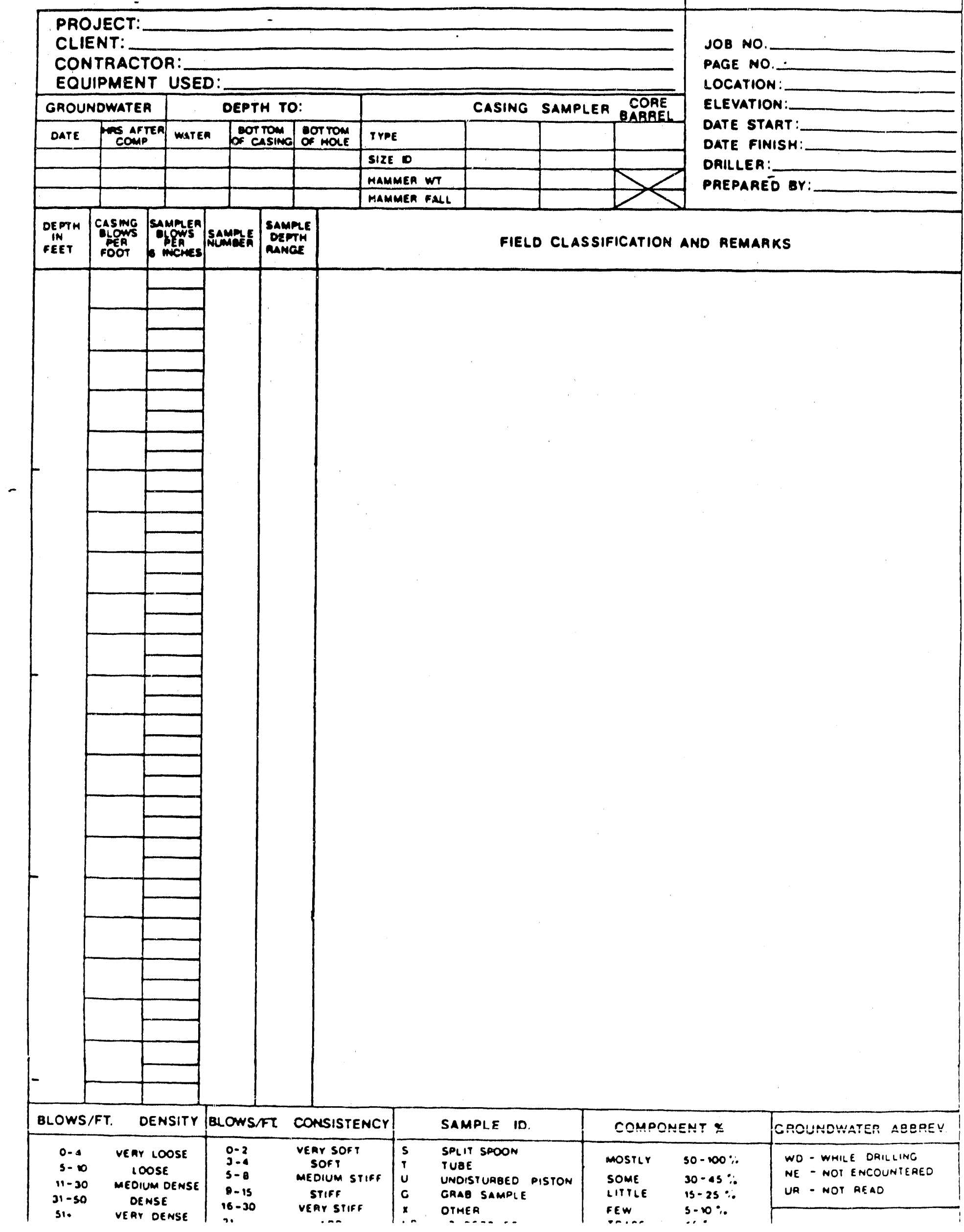




\subsection{PROJECT ACCOUNTING}

The Savannah River Plant "HP Orilling Subcontractor Expense Record" form will be utilized to keep records of billable drilling activities (Ex: Materials and hourly pay items). A separate form will be completed for each test boring drilled during the Background Soils Study. This form is shown in Figure 8. 


\section{HP DRILLING SUBCONTRACTOR EXPENSE RECORD}

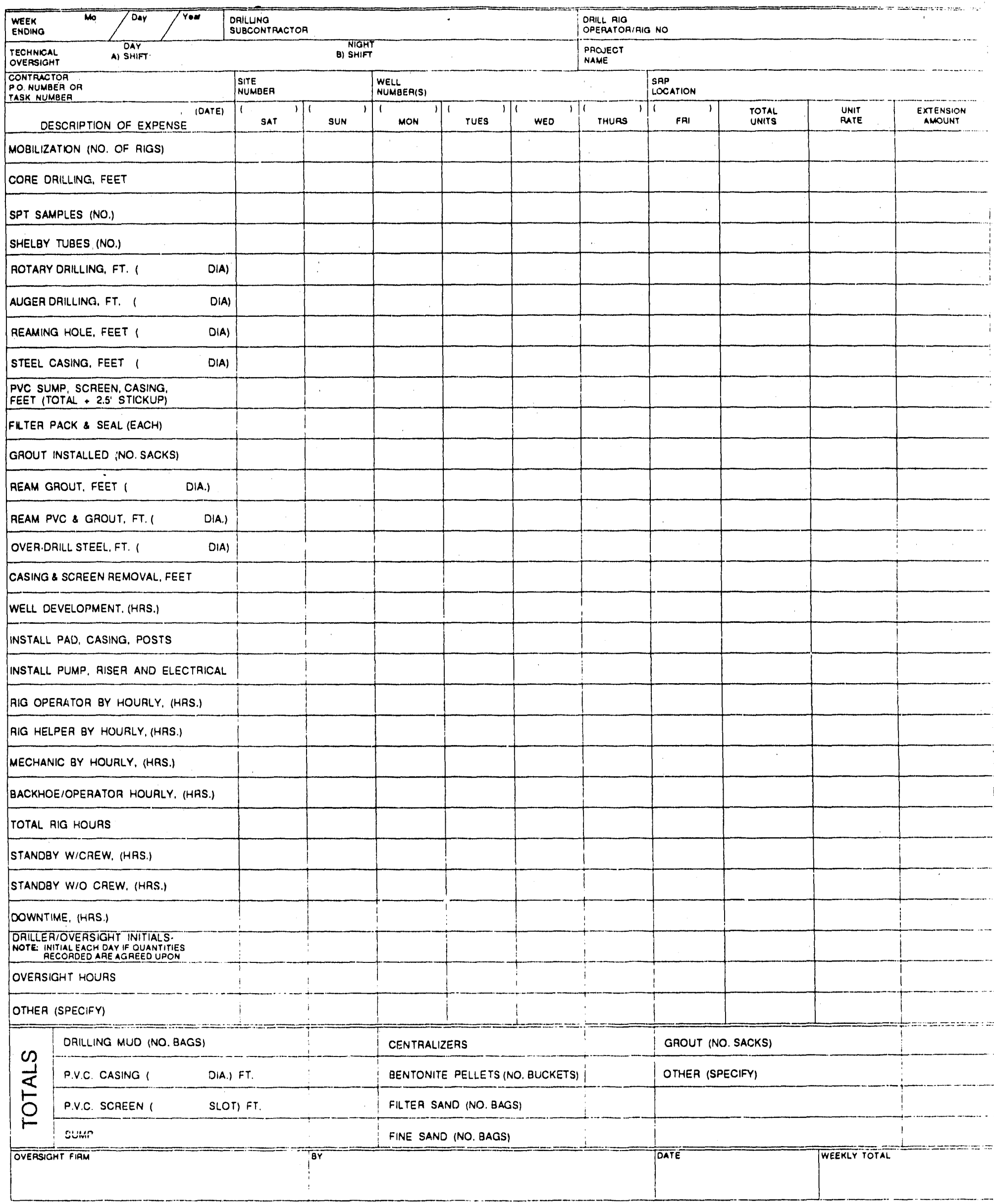




\title{
GEOCHEMICAL AND PHYSICAL PROPERTIES OF SOILS AND
}

SHALLOW SEDIMENTS AT THE SAVANNAH RIVER SITE

\author{
Appendix B.l
}

Background Soil Sample Locations 
From the intersection of Road $2-1$ and Road F-1, travel $0.7 \mathrm{mi}$. E on Road 2-1. The site is located on the left side (N), approximately 20 feet off the road among some small pine trees.

BSS-02 - Lakeland Group

Erom the intersection of Road 2-1 and Road F-1, travel $1.35 \mathrm{mi}$. N-NE on Road F-1. The site is located on the left side (NW), approximately 30 feet off the road among some tall pine trees.

BSS-03 - Fuquay Group

Located on a sandy access road off of Road 2-1, $1.1 \mathrm{mi}$. E of the Savannah River Forest Station: Turn left (N) onto access road and proceed $0.1 \mathrm{mi}$. The site is located on the right (E), approximately 30 feet off the road among some pine trees.

BSS-04 - Orangeburg Group

From the intersection of Road 2-1 and Road 2-1-1, turn right (SW) onto Road 2-1-1. Proceed down Road 2-1-1 for $0.15 \mathrm{mi}$. The site is located on the left side (SE), approximately 40-50 feet off the road among some pine and oak trees.

BSS-06 - Blanton Group

Located on a dirt access road approximately $1.3 \mathrm{mi}$. W of the intersection of Road 2 and Road $C$. Travel on the dirt road heading $N$ for $0.5 \mathrm{mi}$. Proceed on the middle dirt road (NW) at a 3 way fork for $0.3 \mathrm{mi}$. Site is located on the right, approximately 25 feet off the road among some pine trees. 
BSS-07 - Euquay Group

Located along the $\mathrm{C}-1$ power line, $0.7 \mathrm{mi}$. N of the intersection of the $C-1$ power line and Road $C$. The site is located on the $W$ side of the power line, approximately 25 feet into the treeline.

BSS-08 - Fuquay Group

From the intersection of Road $2-1$ and Road E-2, travel $1.4 \mathrm{mi}$. SE on Road 2-1. A gravel road branches off of Road 2-1 at this point. Proceed $0.3 \mathrm{mi}$. SE on this road. The site is on the right side (S), approximately 50-60 feet from the road into the treeline.

BSS-09 - Blanton Group

From the intersection of Road 2-1 and Road E-2, travel $2.0 \mathrm{mi}$. E-SE on Road 2-1. At this point, an overgrown grassy road turns off to the right $(S W)$. Proceed $S-S W$ on this road for $0.1 \mathrm{mi}$. The site is on the left (SE), approximately 40 feet into a brushy/wooded area.

BSS-10 - Orangeburg Group

From the intersection of Wood Ward Road $(A-4)$ and Road 2-1, travel 1.35 $\mathrm{mi}$. S on Wood Ward Road. The site is on the right side (W), approximately 20-30 feet into the treeline of pines.

BSS-11 - Orangeburg Group

From the intersection of Silverton Road $(C-1.1)$ and Road $D$, travel 0.15 mi. W on Silverton Road. Turn right (N-NW) onto the dirt road. Proceed $0.7 \mathrm{mi}$. NW on this dirt road. The site is on the right (NE), near the intersection with another dirt road. It is approximately 20 feet from the road among some pine trees.

BSS-12 - Vaucluse Group

From the intersection of Road 2 and Road C-1.1, turn left (NW) onto Road C-1.1. Proceed $0.4 \mathrm{mi}$. NW on Road C-1.1. Turn left (SW) onto a dirt road and proceed $0.1 \mathrm{mi}$. into a recently logged timber clear cut area. The site is located 75 feet to the right (NW) of the road in the cleared area. 
BSS-13 - Udorthent Group

From the intersection of Road $F$ and the F-4 power line road, turn onto the dirt road $(F-4)$ and proceed $S w$ for $0.5 \mathrm{mi}$. The road will open into a pit at this point. Travel along the right side of the pit for 0.15 $\mathrm{mi}$. The site is along the right side (NW) pit wall.

BSS-20 - Udorthent Group

From the pond C bridge on Road 8, travel $1.6 \mathrm{mi}$. NE on Road 8 . Turn left (NW) onto an unnamed paved road. Travel $1.1 \mathrm{mi}$. NW on the road, approaching Pond $B$. The site is on the left (S), approximately 20 feet off the road in a tall grassy clearing.

BSS-21 - Fuquay Group

From the Pond $C$ bridge on Road 8 , travel $1.6 \mathrm{mi}$. NE on Road 8 . Turn left (NW) onto an unnamed paved road. Travel $1.0 \mathrm{mi}$. along this road and turn left (S) onto a sandy access road (which doubles back and parallels the paved road). Proceed on this road $0.5 \mathrm{mi}$. SE. The site is located on the right (S), approximately 30 feet from the road among some pine trees.

BSS-22 - Lakeland Group

From the Pond C bridge on Road 8 , travel $1.6 \mathrm{mi}$. NE on Road 8 . Turn left (NW) onto an unnamed paved road. Travel $1.0 \mathrm{mi}$. along this road and turn left (S) onto a sandy access road (which doubles back and parallels the paved road). Proceed on this road $1.0 \mathrm{mi}$. SE. The site is located in a clearing approximately 40 feet from the right side (W) of the road.

BSS-23 - Blanton Group

From the Pond C bridge on Road G, travel $1.2 \mathrm{mi}$. W on Road G. Thesite is on the left (S), approximately 40 feet off the road among some pine trees. 


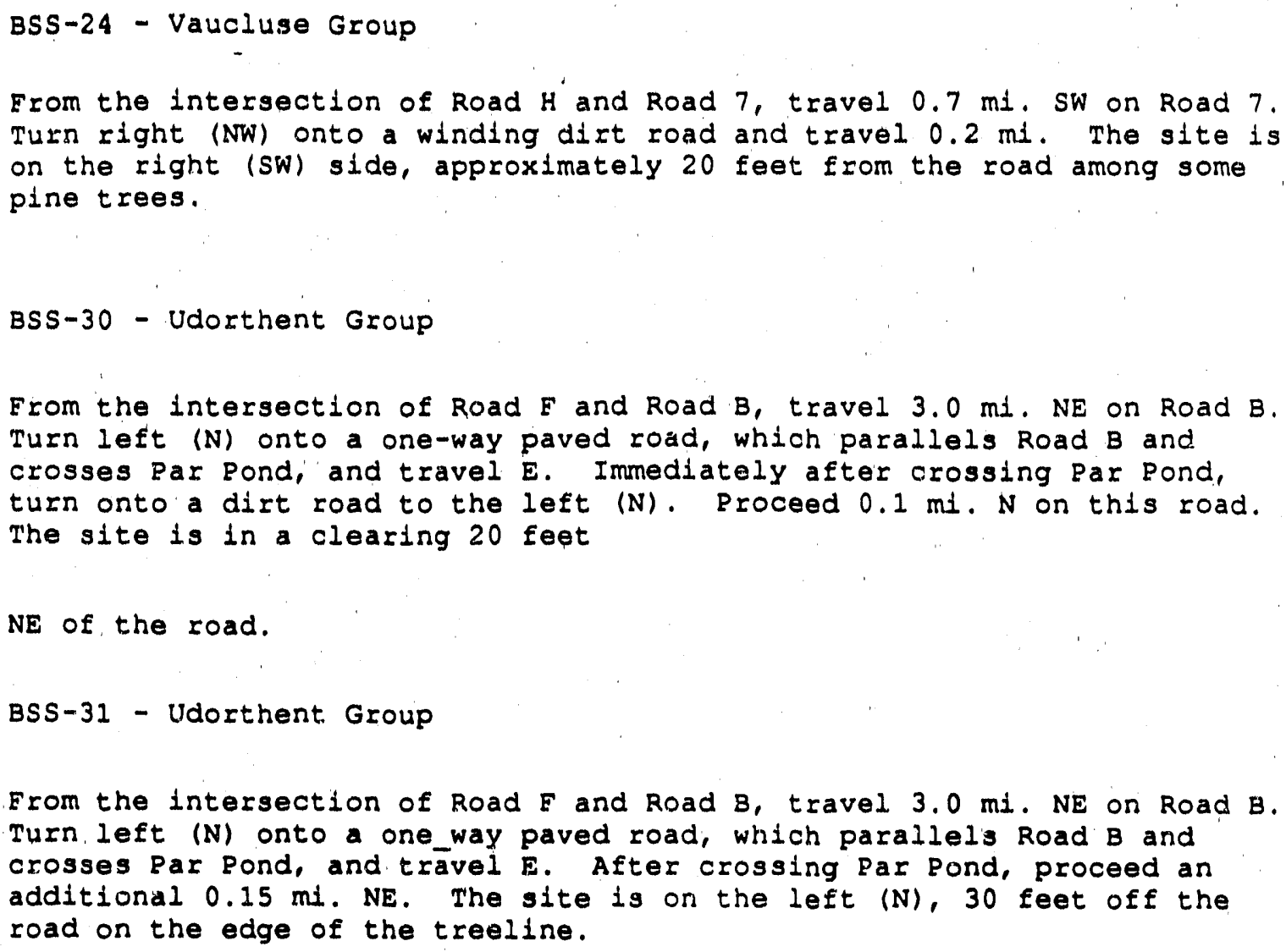

From the intersection of Road 9 and Road A-14, turn onto a small dirt road heading $\mathrm{NW}$. Proceed $0.1 \mathrm{mi}$. along this road. The site is located on the left (W), approximately 20_30 feet among pine trees. 
BSS-35 - Blanton Group

From the intersection of Road 9 and Road B-6.2, turn left (E) onto Road $B-6.2$ and travel $0.4 \mathrm{mi}$. Turn left (N) onto an overgrown grassy road and proceed $0.35 \mathrm{mi}$. The site is at the end of the road on the right side (NE).

BSS-36 - Udorthent Group

From the intersection of Road 9 and Road B-6.2, turn left (E) onto Road $B-6.2$ and travel $0.4 \mathrm{mi}$. Turn left (N) onto an overgrown grassy road and proceed $0.3 \mathrm{mi}$. The grassy road will pass underneath a power line. The site is just beyond the power line in a pit. It is on the left (W), approximately 100 feet from the road.

BSS-40 - Orangeburg Group

From the intersection of Road 2 and Road $\mathrm{C}-2$. travel $0.3 \mathrm{mt}$. SW on Road 2. Turn left $(E-S E)$ onto a dirt road and proceed $0.2 \mathrm{mi}$. The site is on the right (S), approximately 30 feet off the road among some pine and oak trees.

BSS-41 - Lakeland Group

Located on a dirt road $0.4 \mathrm{mi}$. NE of Barricade 8 on Road 2 . Travel 0.25 $\mathrm{mi}$. SE on the dirt road. The site is on the left side of che road, approximately 40 feet from the road among some pine trees.

BSS-42 - Euquay Group

From the intersection of the C-1 power line and Road 2, turn onto the C1 power line road and travel $0.8 \mathrm{mi}$. N. The site is on the right (E) of the power line, approximately 20 feet into the treeline.

BSS-43 - Blanton Group

From the intersection of the $C-1$ power line and Road $C$, turn onto the $C$ 1 power line road and travel $0.2 \mathrm{mi}$. S. The site is on the right (W) of the power line, approximately 25 feet into the treeline. 
BSS-44 - Fuquay Group

Turn onto a small, sandy access road adjacent to Barricade 2 (New Ellenton Wackenhut gate). Proceed $E$. This road forks immediately. Bear to the right at this fork and travel $0.7 \mathrm{mi}$. SE. The site is approximately $0.1 \mathrm{mi}$. from the road (N) in mature pine woods.

BSS-45 - Blanton Group

Turn onto a small, sandy access road adjacent to Barricade 2 (New Ellenton wackenhut gate). This road forks immediately. Bear to the right at this fork and proceed $0.3 \mathrm{mi}$. SE. The site is on the left side (N) of the road, among several pine trees, next to a large trench

BSS-46 - Orangeburg Group

Turn onto the power line road off of Road $\mathrm{F}, 1.3 \mathrm{mi}$. SE of the intersection of Road $E$ and Road 2. Take a right onto the power line road. Immediately turn left and proceed $0.2 \mathrm{mi}$. S-SE on an overgrown grassy road. The site is in a wooded

area near an old trench.

BSS-47 - Vaucluse Group

Go to the intersection of Road 9 and Tennessee Road (locked gate). Take a left (E) onto Tennessee Road. Proceed $1.4 \mathrm{mi}$. E on Tennessee Road and turn onto Kirkland Road (left turn going $\mathrm{N}$ ). After $0.2 \mathrm{mi}$. the road forks. Bear to the right and proceed an additional $0.6 \mathrm{mi}$. Location is on a hill to the right (NE), approximately 100 feet into a wooded area.

\section{BSS-48 - Lakeland Group}

Go to the intersection of Road 9 and Tennessee Road (locked gate). Take a left (E) onto Tennessee Road. Proceed $1.4 \mathrm{mi}$. E on Tennessee Road and turn onto Kirkland Road (left turn going $N$ ). After $0.2 \mathrm{mi}$. the road forks. Bear to the right and proceed an additional $0.2 \mathrm{mi}$. The site is on the left $(N)$, approximately 30 feet off the road in a clearing with limited vegetation. 
BSS-49 - Euquay Group

Travel so the intersection of Road 9 and Tennessee Road (iocked gate). Take a left $(E)$ onto Tennessee Roud. Proceed $2.0 \mathrm{mi}$. E on Tennessee Road and turn left onto an unnamed road. Proceed $0.5 \mathrm{mi}$. on this road. Site is on the left (SW) approximately 100 feet up a slope in a densely wooded arez.

BSS-50 - Orangeburg Group

From the intersection of Road $D$ and Road 2, travel $N$ approximately 100 yards and turn left (W) onto dirt access road. Proceed approximately 50 yards and site is 50 feet on the right side $(N)$ of dirt road in mature pine woods.

BSS-51 - Lakeland Group

From the intersection of Highway 278 and Highway 19, turn onto Highway 278 and travel E. Go $0.7 \mathrm{mi}$. and turn onto a small sandy road to the left (N). Travel $0.1 \mathrm{mi}$. and turn ont a small sandy road to the $E$. Proceed $0.7 \mathrm{mi}$. (around a curve) and tile site is approximately 30 feet off che road to the right in a grassy clearing.

BSS-52 - Lakeland Group

From the intersection of Highway 278 and Highway 19, turn onto Highway 278 and travel $E$. Travel $7.6 \mathrm{mi}$. and bear left at the fork in the road (NE). Proceed $0.1 \mathrm{mi}$. and the site is approximately 50 feet off the road to the left (NW) in a wooded area.

BSS-53 - Udorthent Group

Erom the intersection of Road 1 and Road A-1, turn onto Road 1 and travel NE. Go $0.2 \mathrm{mi}$. and turn left (NW) onto a road paralleling the power line. The site is to the immediate right amongst pine trees in an overgrown grassy area. 


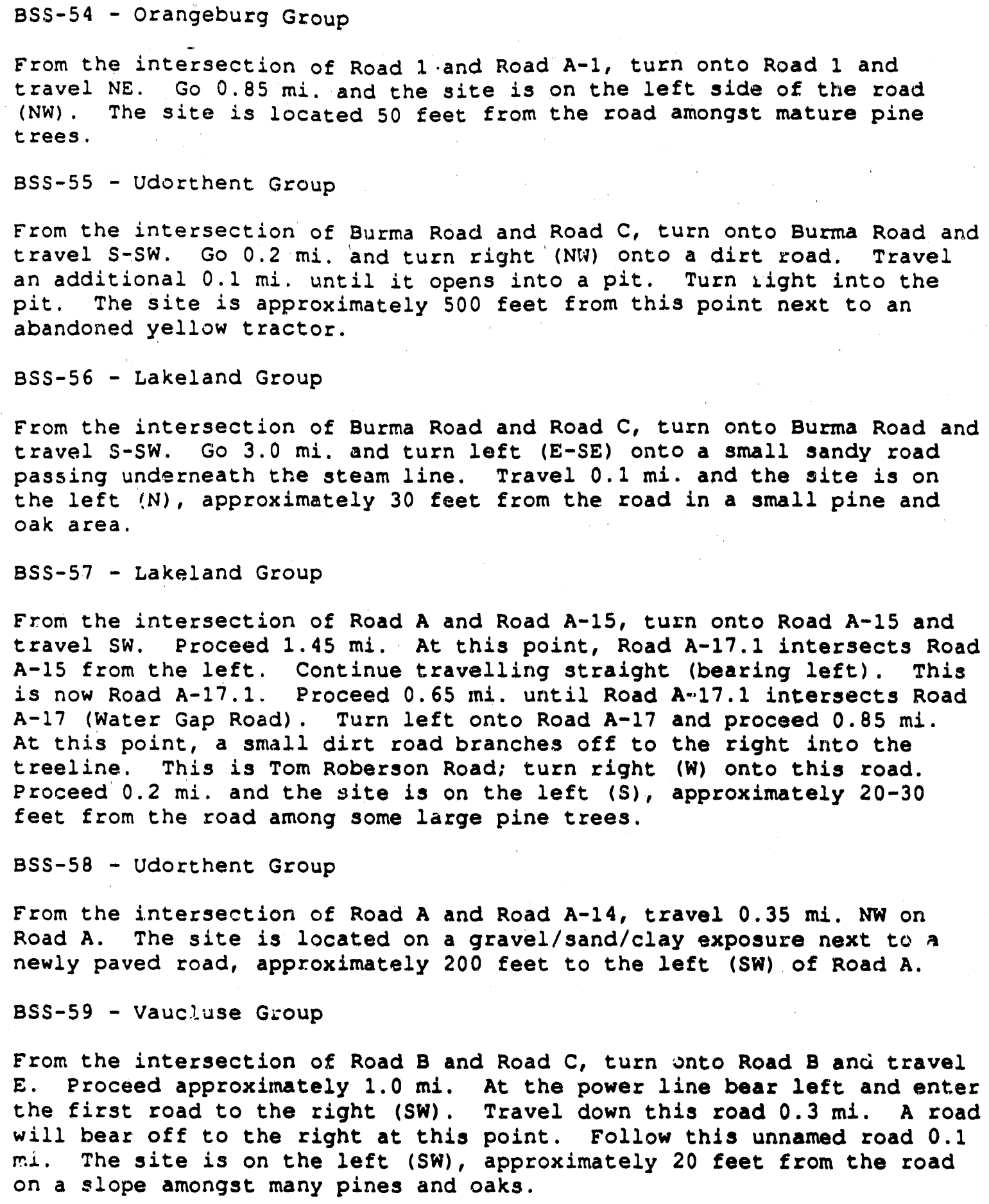


GEOCHEMICAL AND PHYSICAL PROPERTIES OF SOILS AND SHALLOW SEDIMENTS AT THE SAVANNAH RIVER SITE

\section{Appendix B.2}

ASTM Classification of Background Soil Samples 


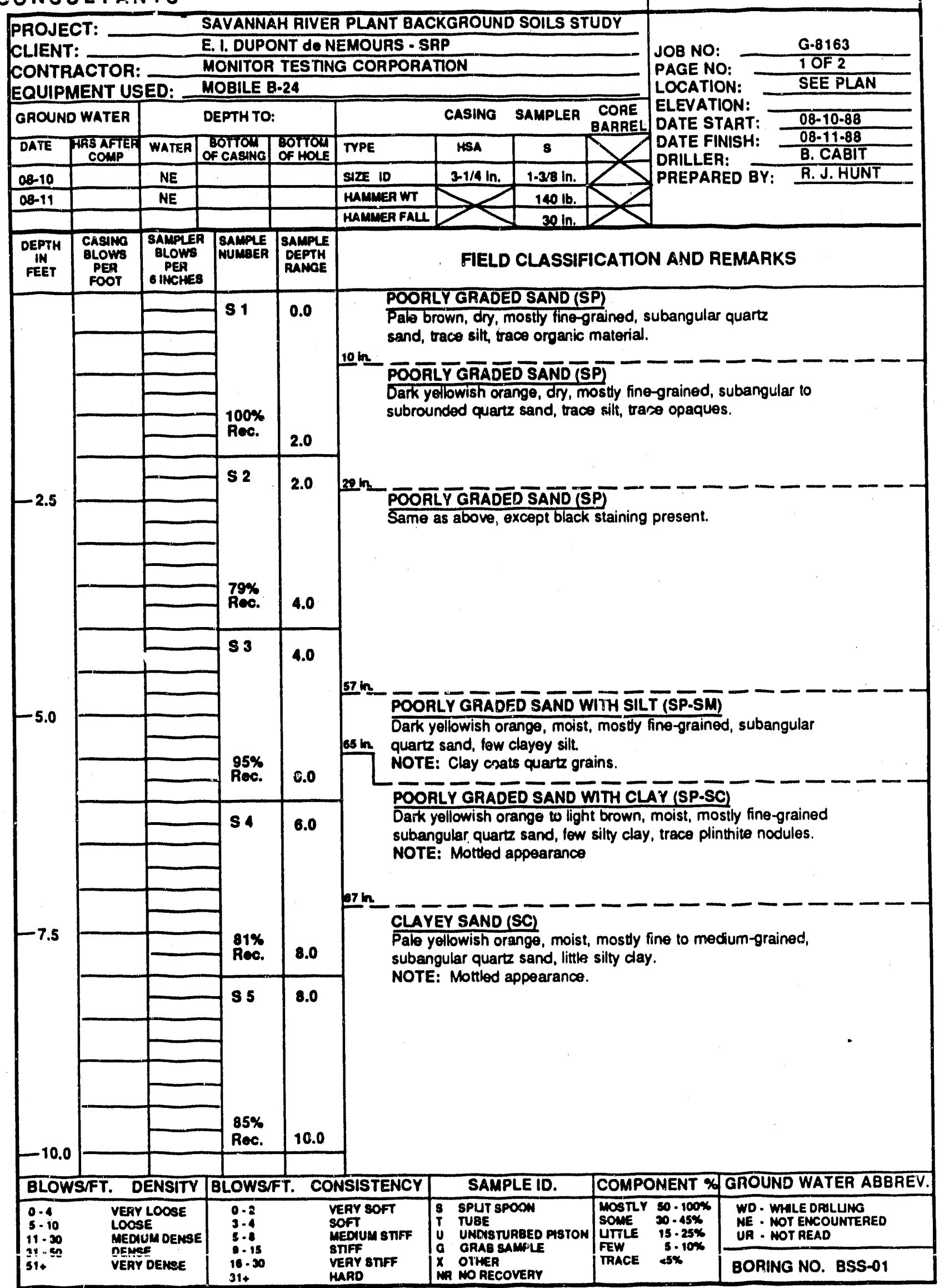


CONSULTANTS

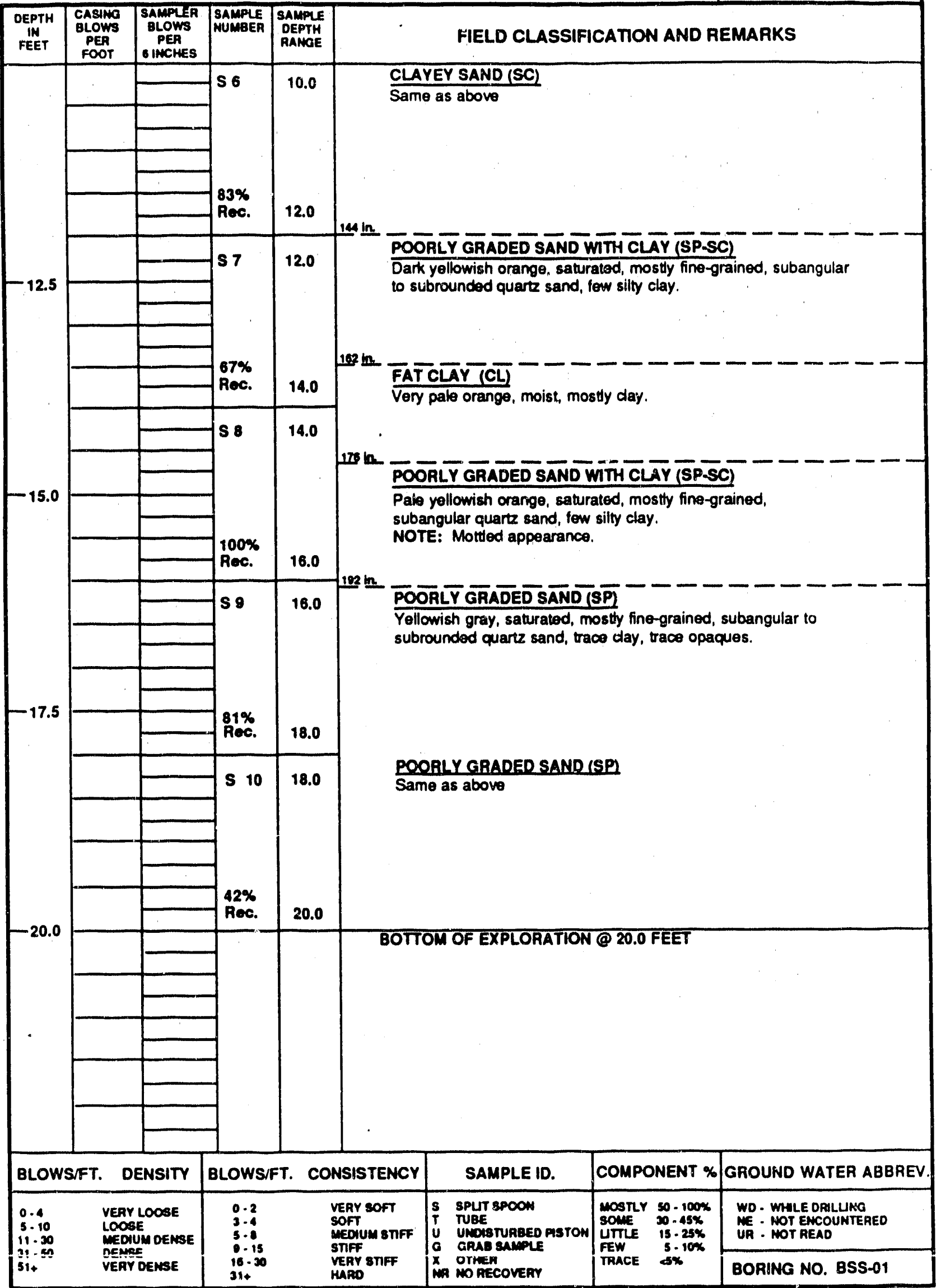




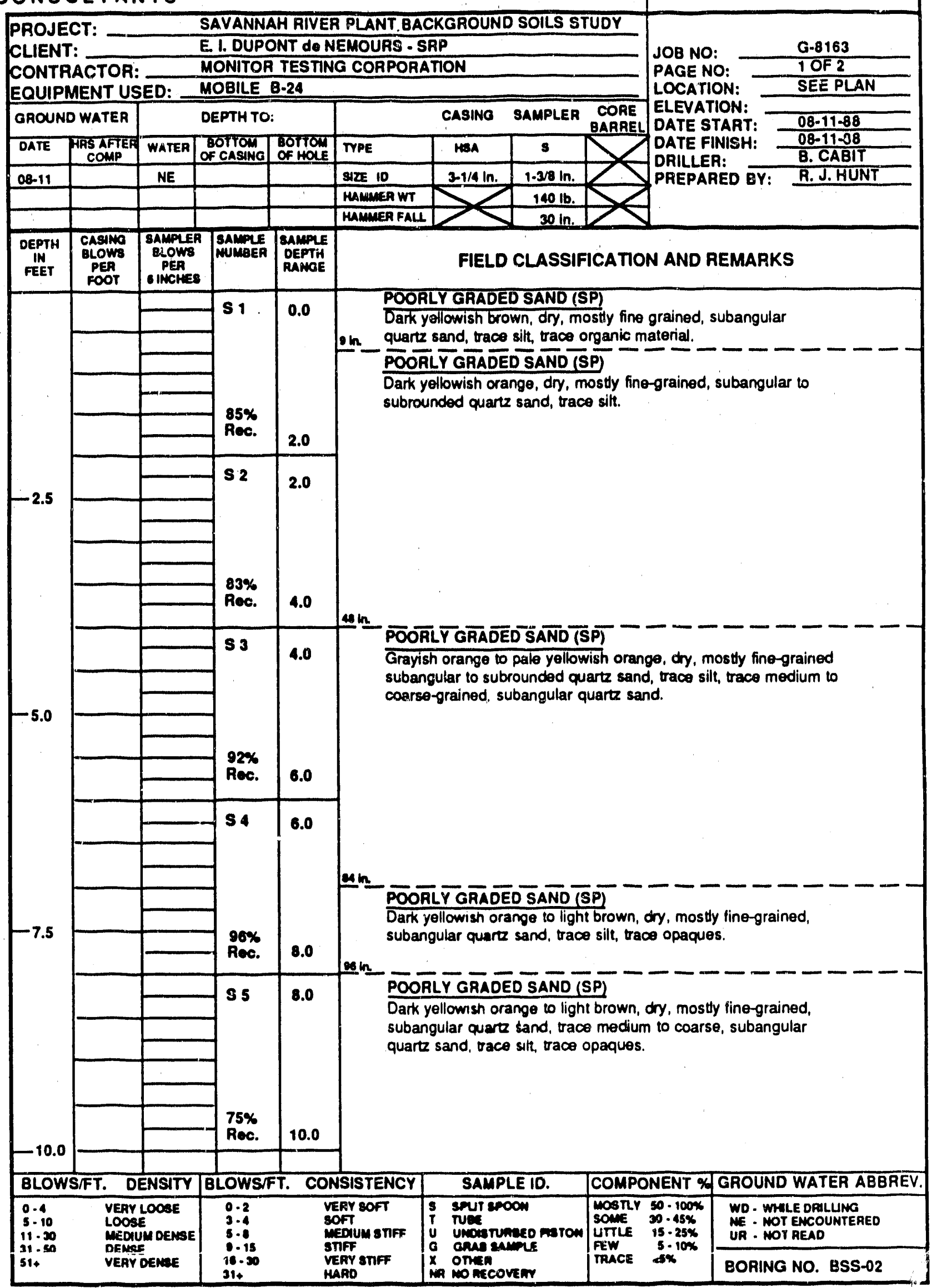




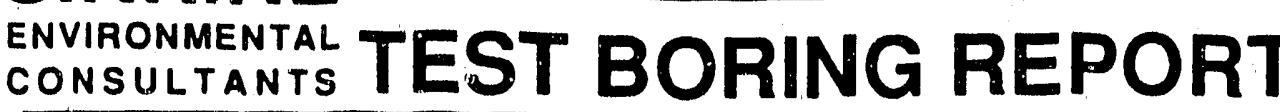

BOFING NO. BSS-02

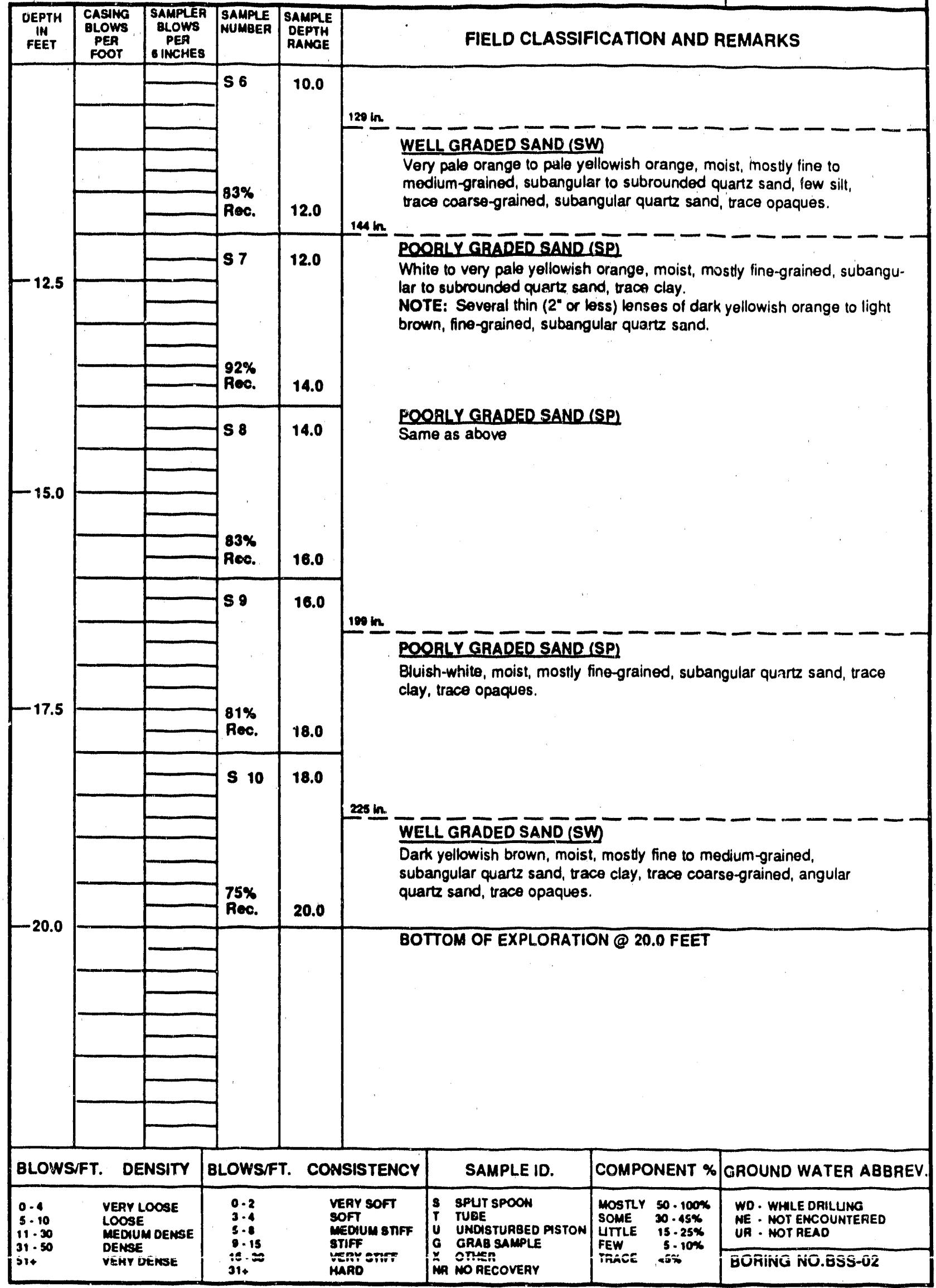


SIRRINE

ENVIRONMENTAL

CONSULTANTS

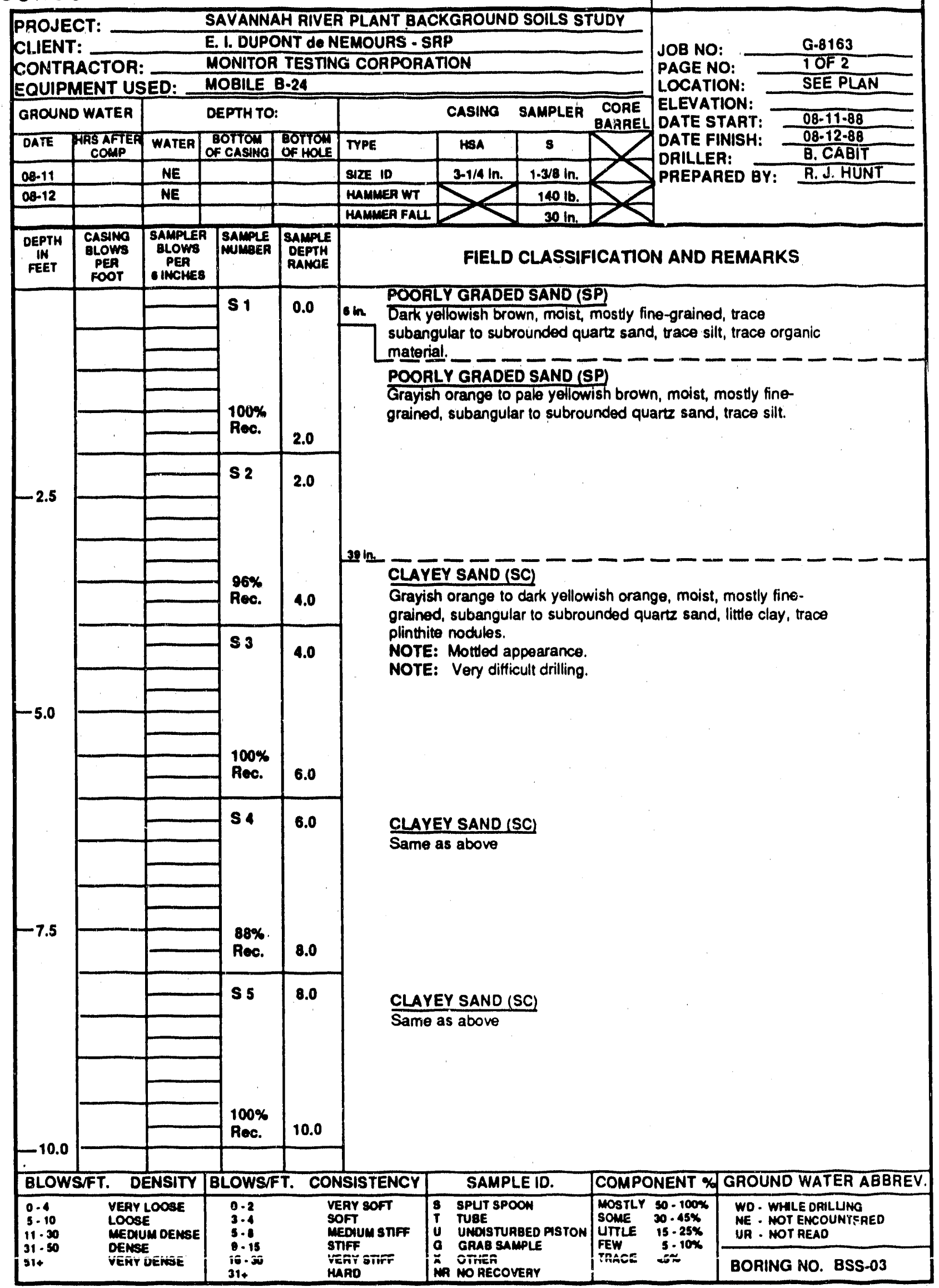




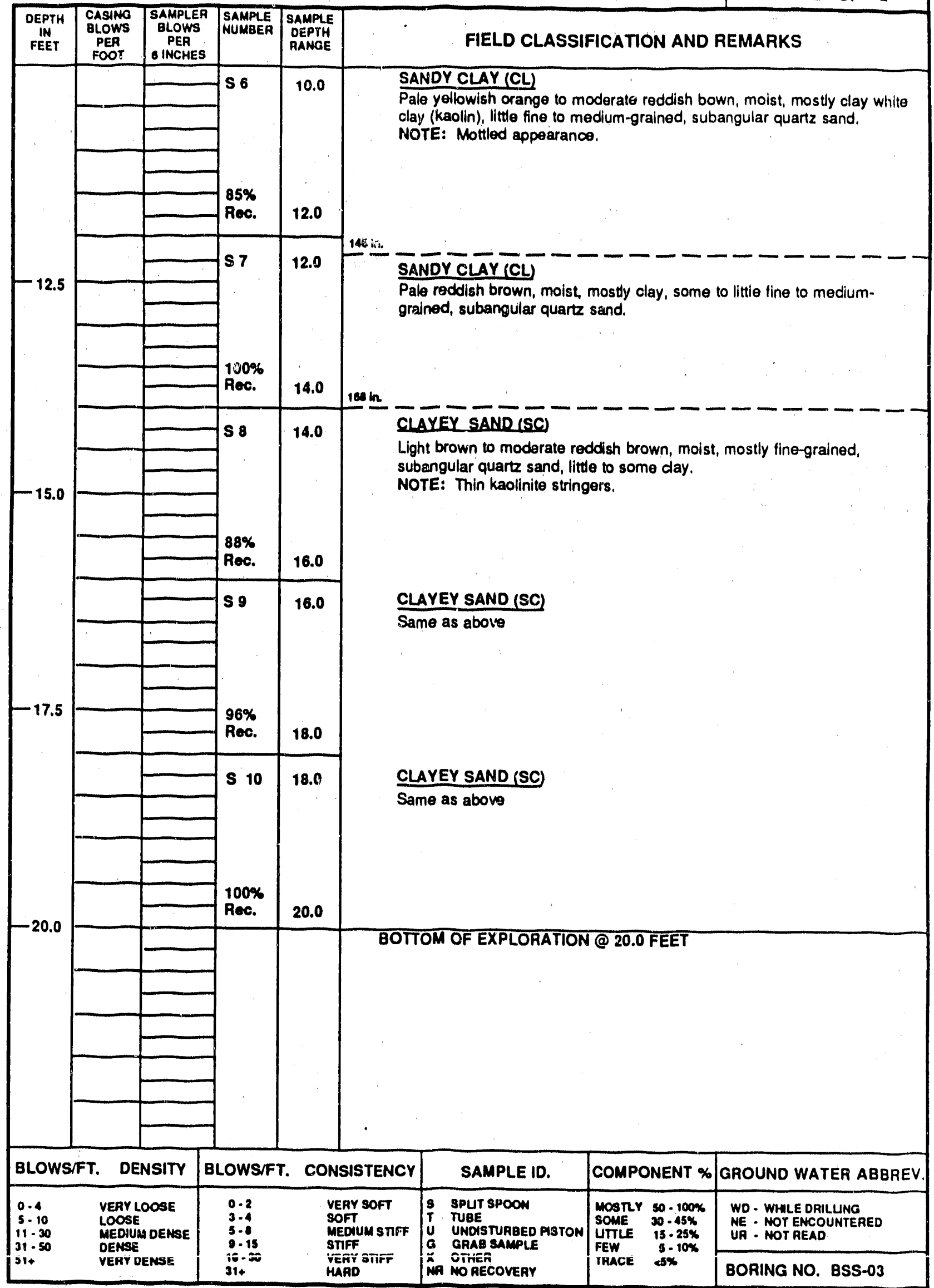


SIRRINE

ENVIRONMENTAL

CONSULTANTS

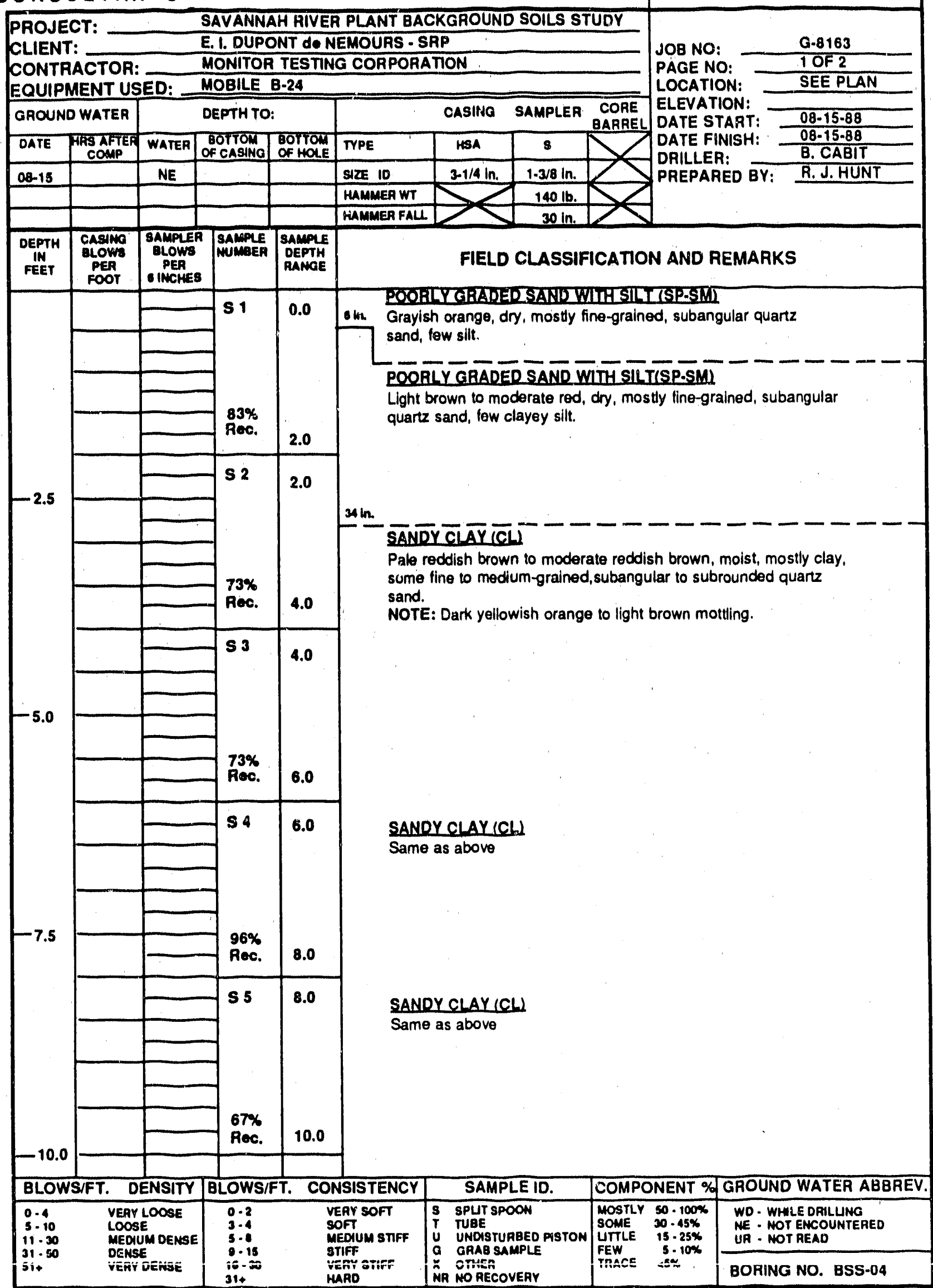



14. PAGE 2 OF 2

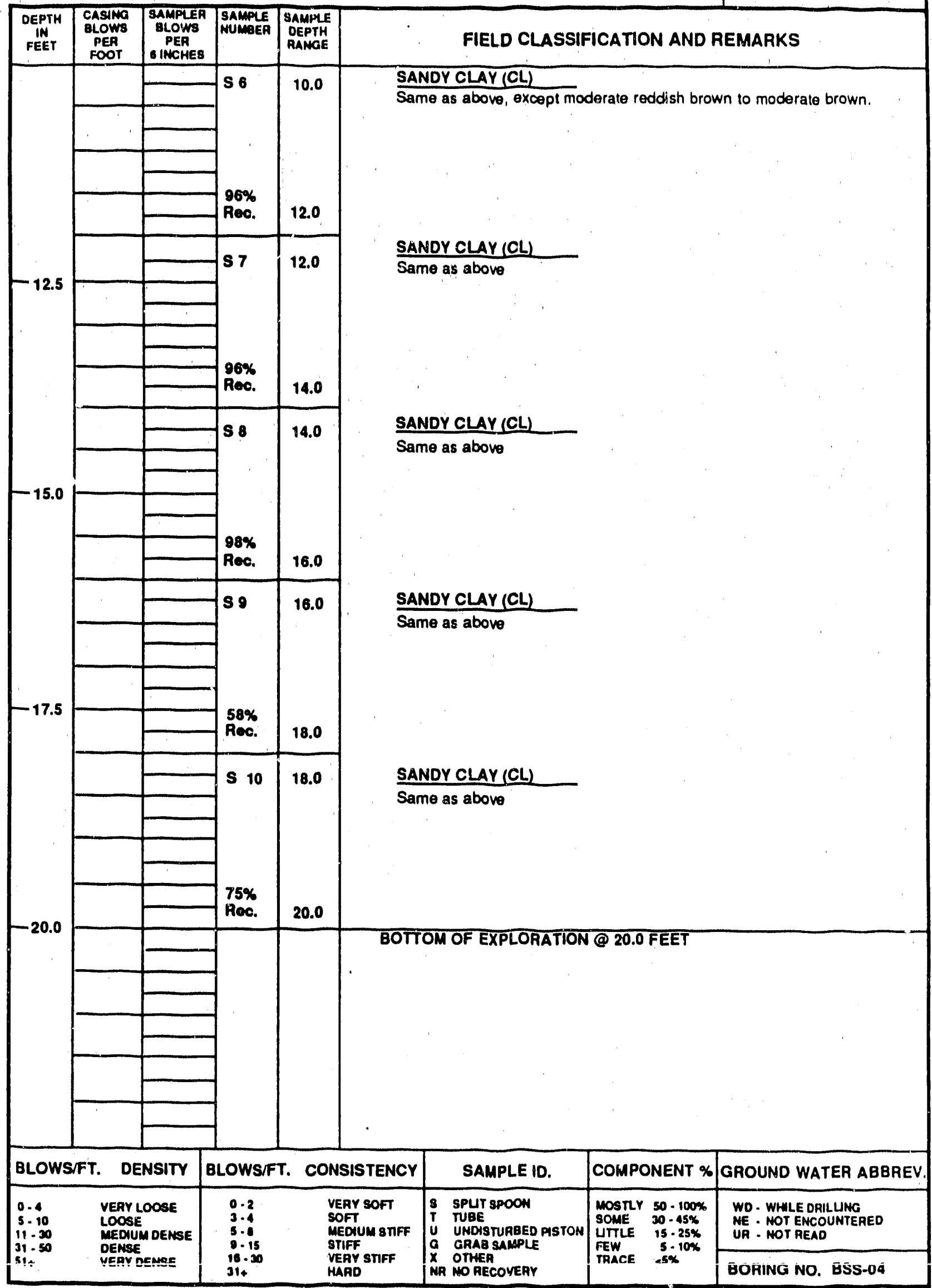


SIRRINE

ENVIRONMENTAL

CONSULTANTS

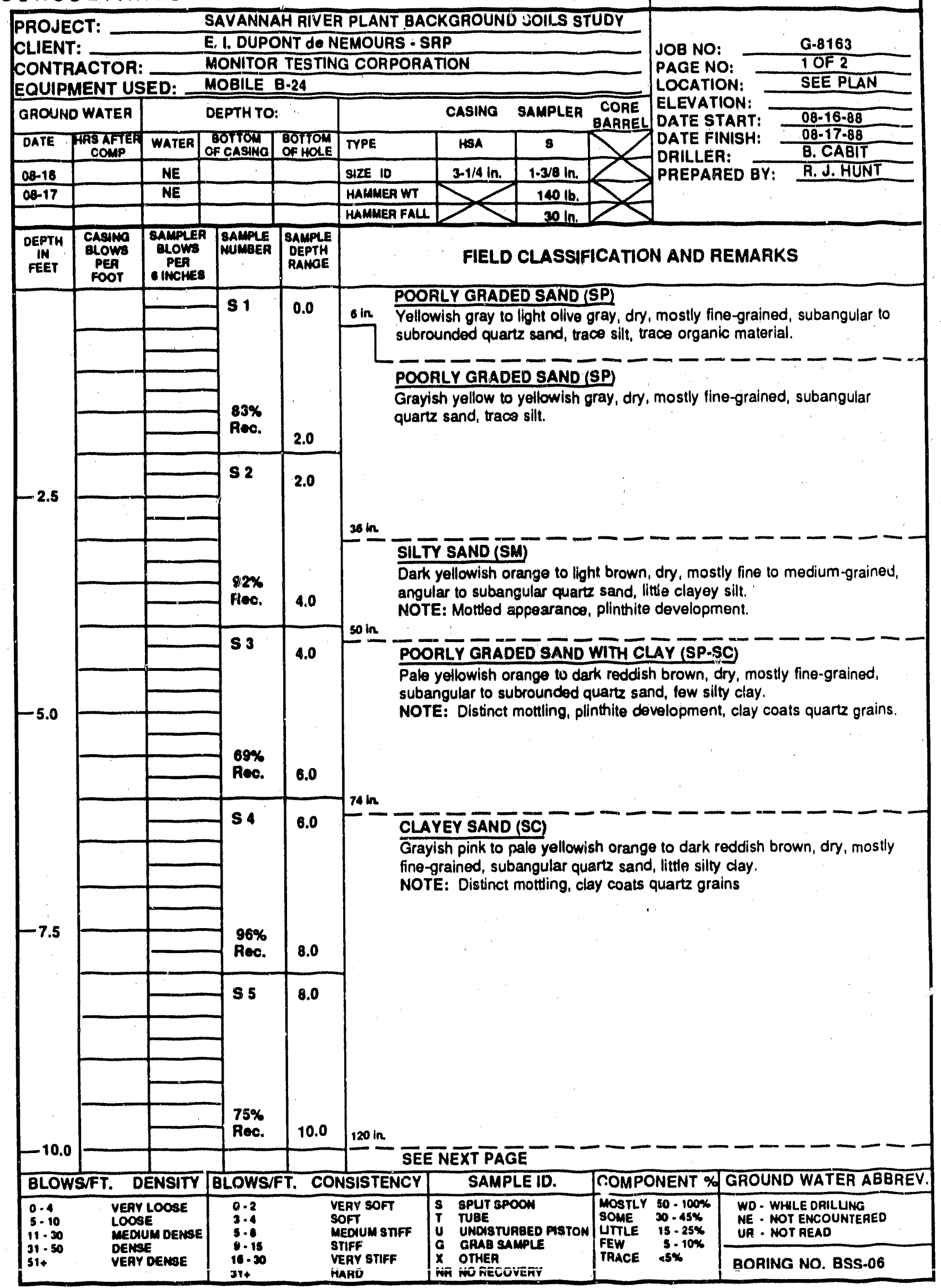




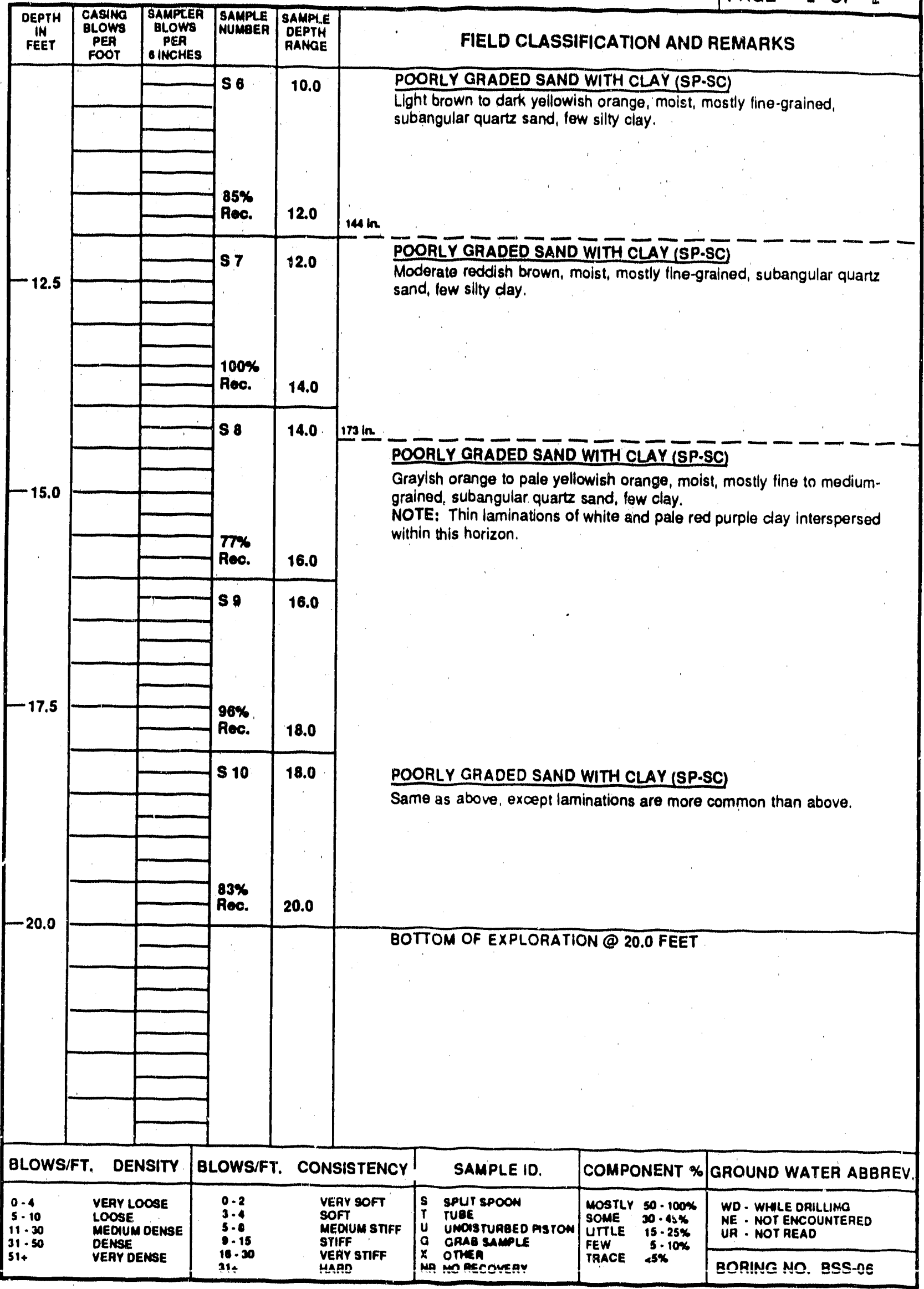


SIRRINE ENVIRONMENTAL CONSULTANTS

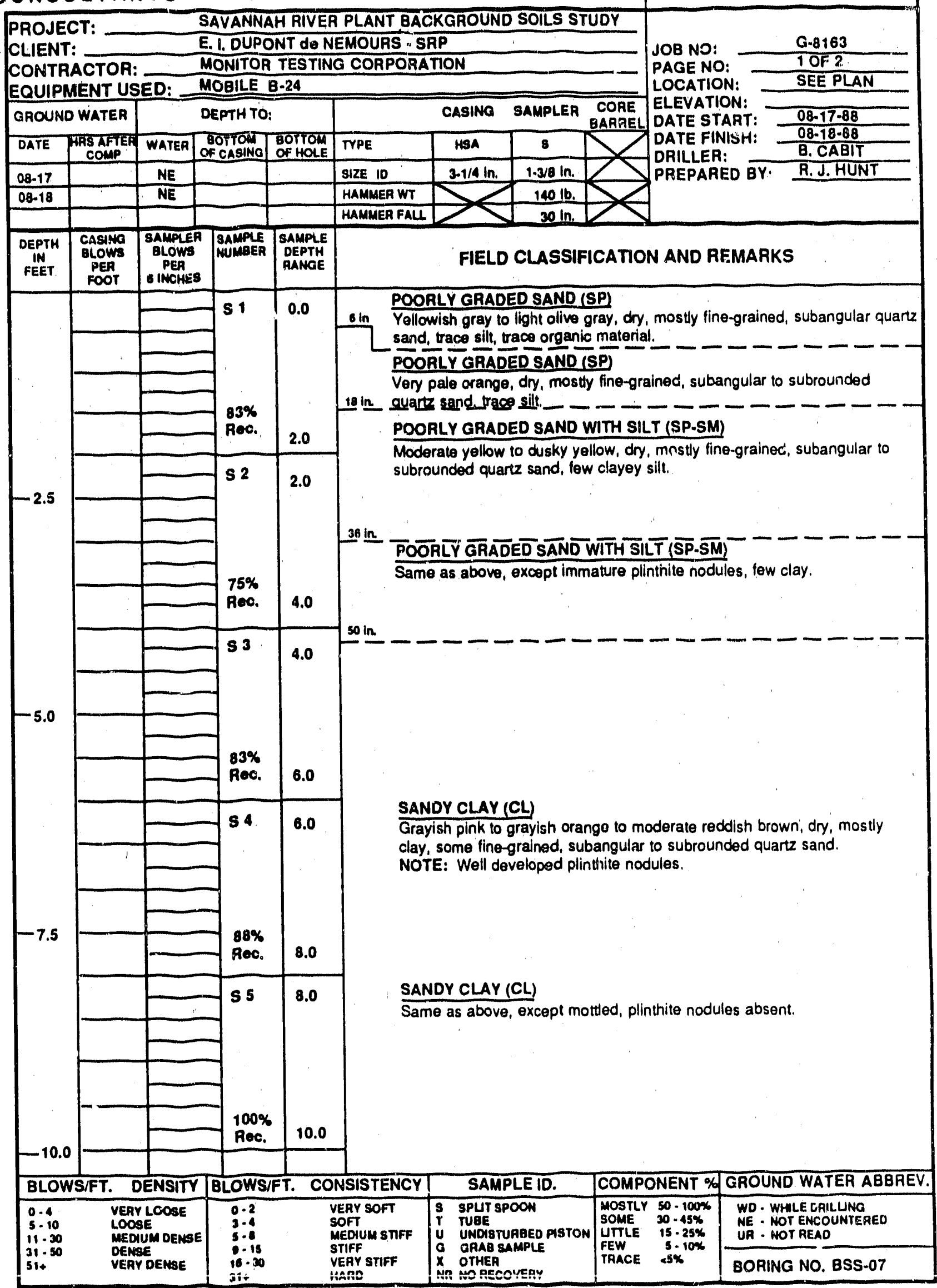


encomerem CONSUUTANTS TEST BORING REPORT

BORING NO. BSS.07

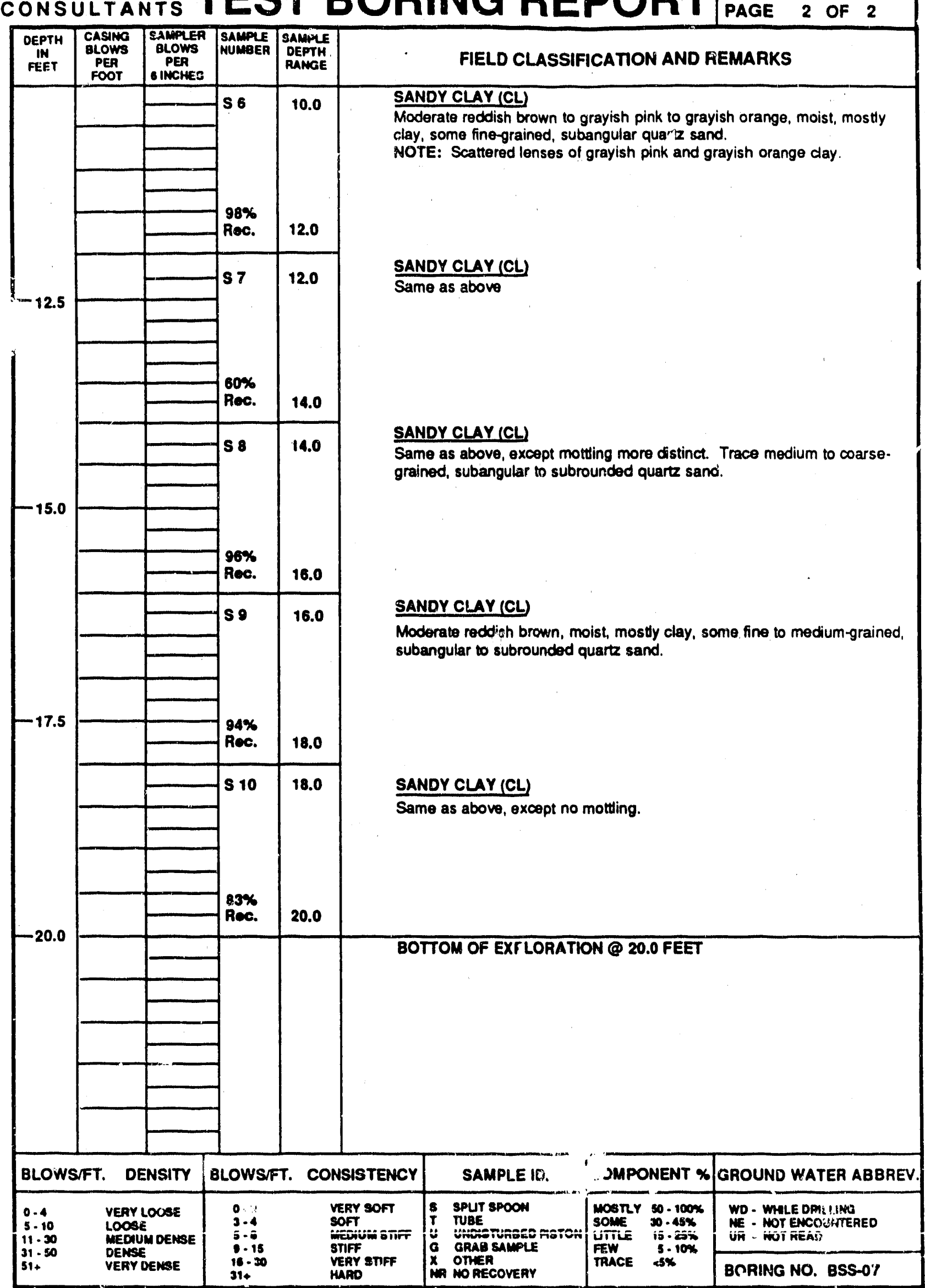




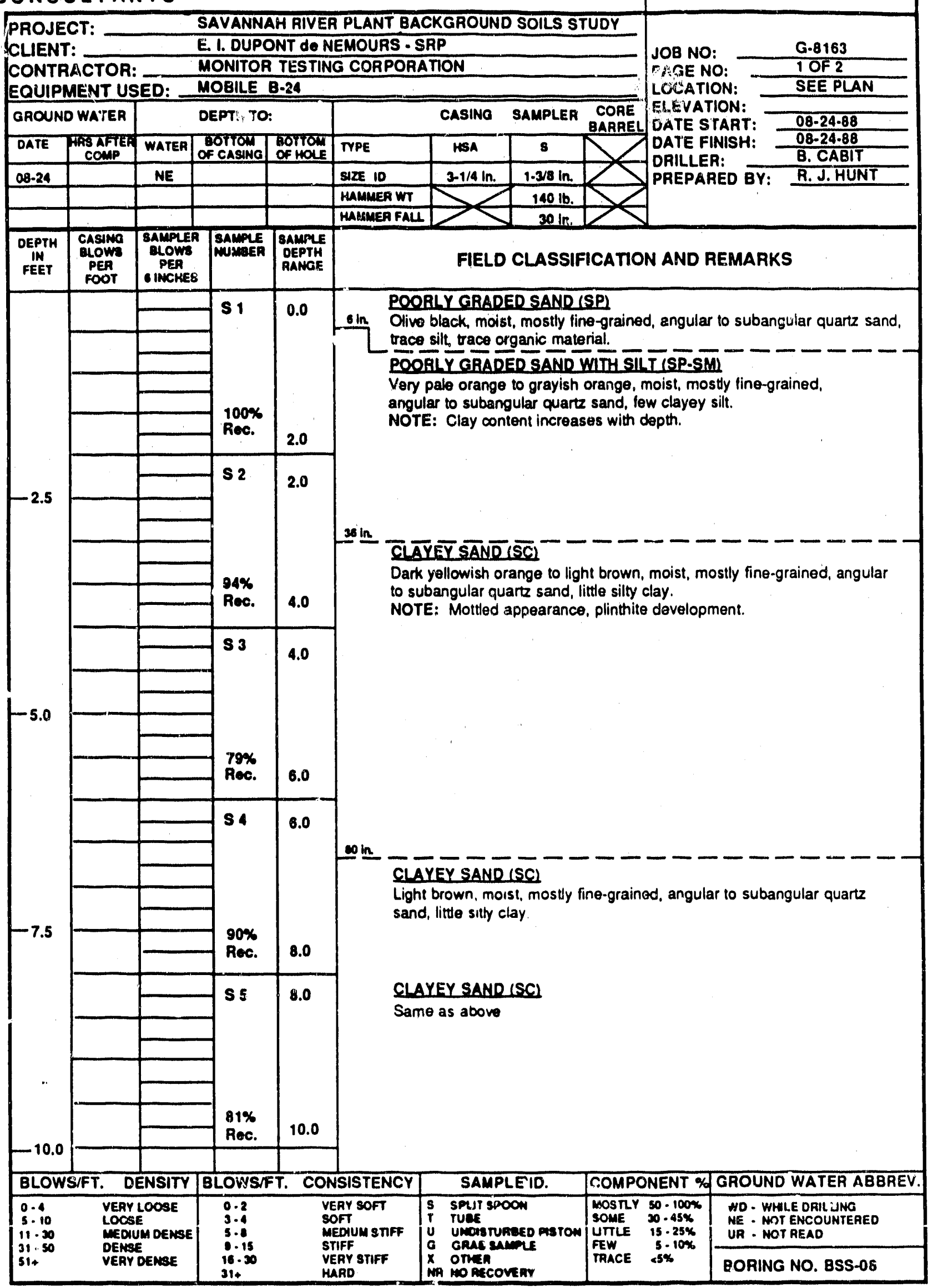




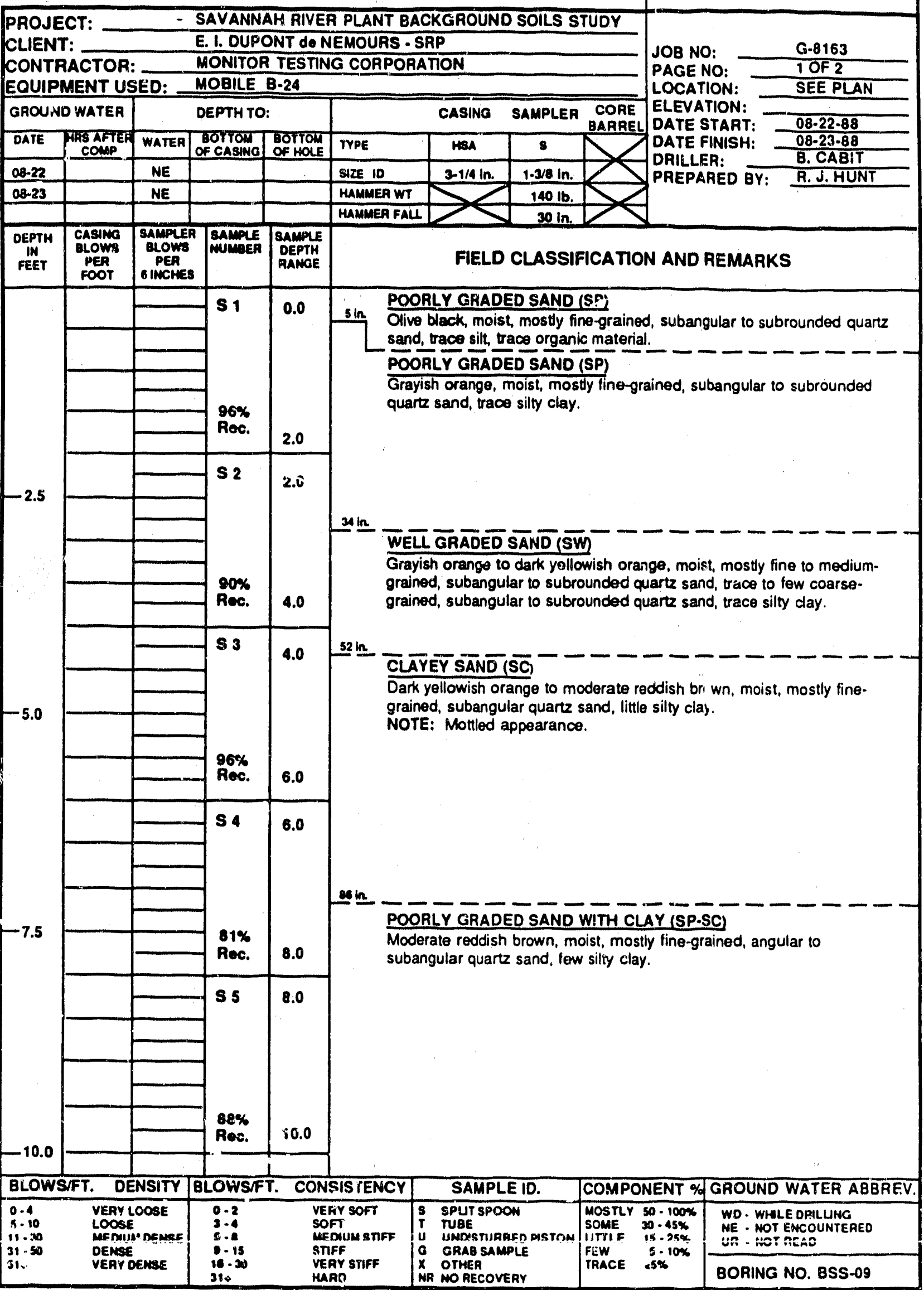




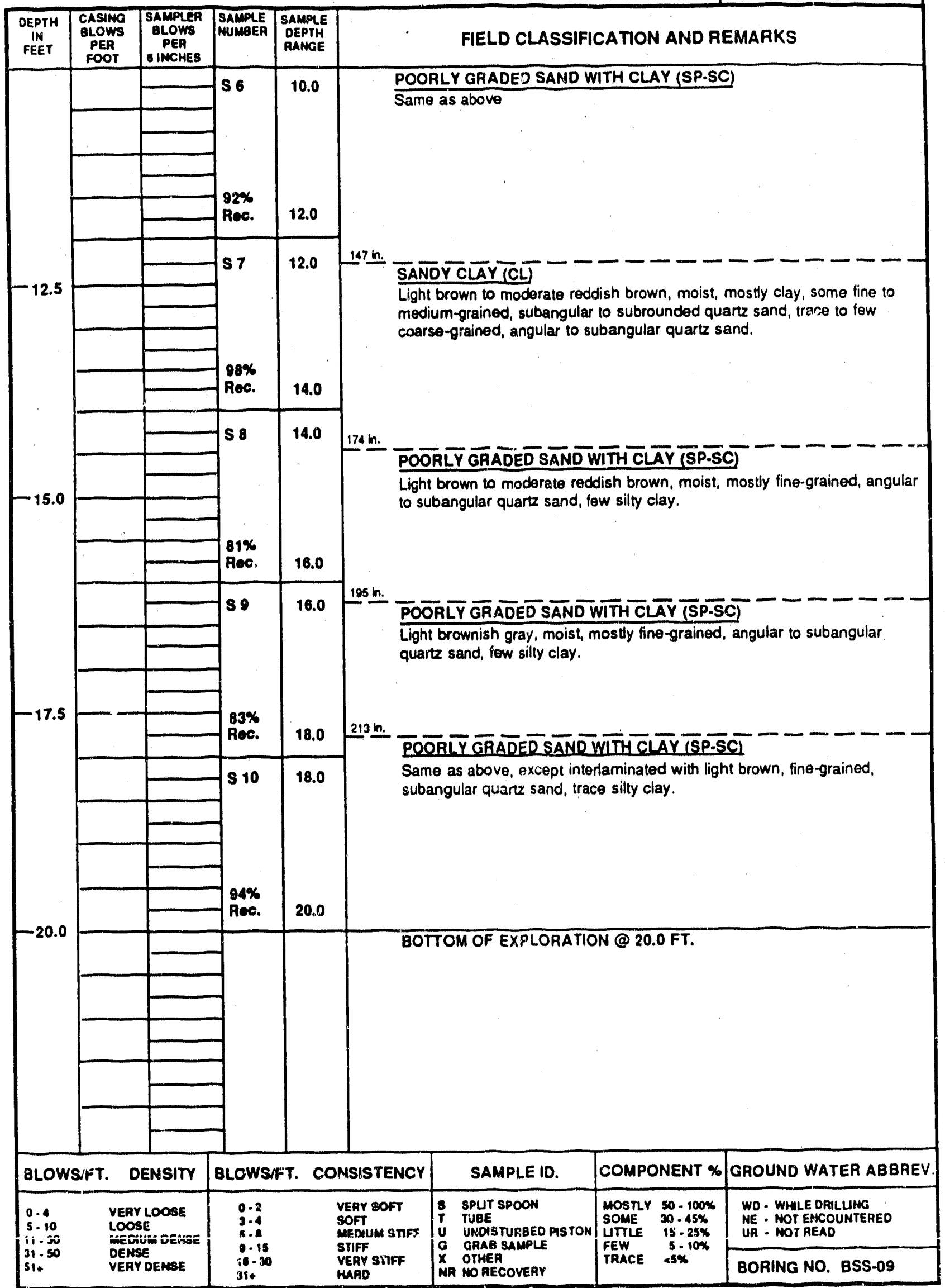


SIRRINE

ENVIRONMENTAL
CONSULTANTS

TEST BORING REPORT

BORING NO. BSS-10

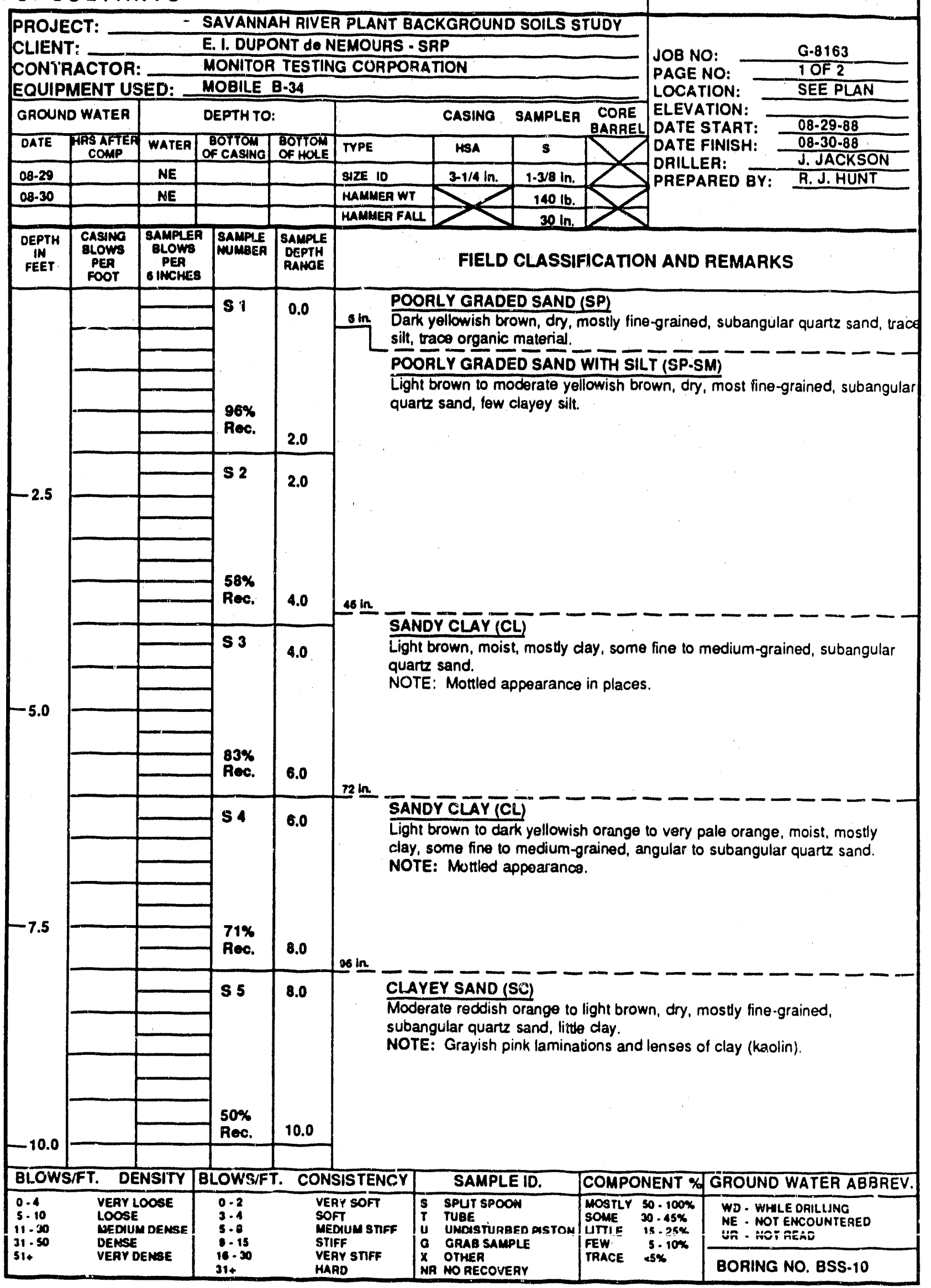




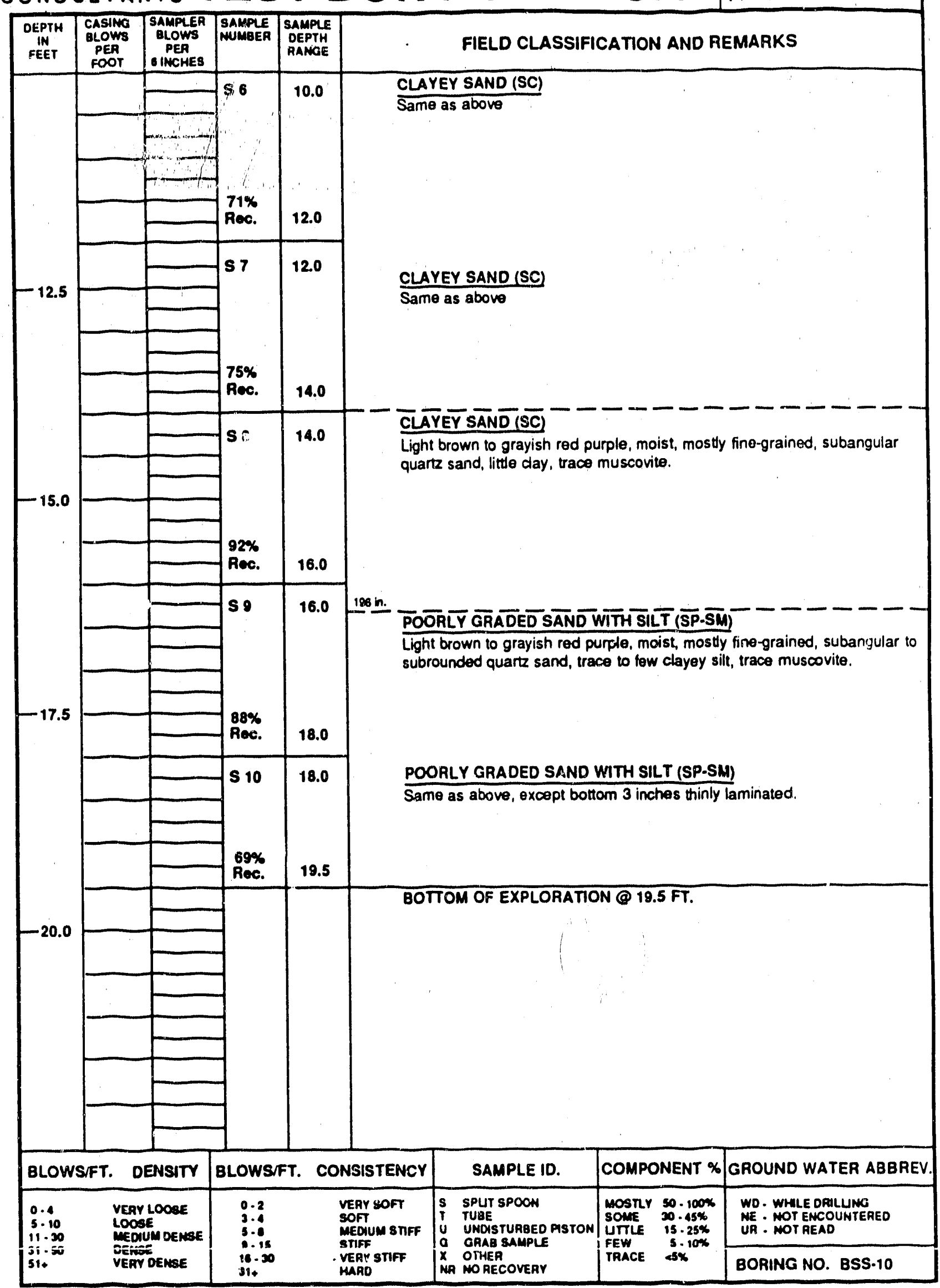


CONSULTANTS

TEST BORING REPORT

PROJECT:

CONTRACTOR:

SAVANNAH RIVER PLANT BACKGROUND SOILS STUDY

E. I. DUPONT dO NEMOURS - SRP MONITOR TESTING CORPORATION

EQUIPMENT USED: MOBILE B.28

\begin{tabular}{|c|c|c|c|c|c|c|c|c|}
\hline \multicolumn{2}{|c|}{ GROUND WATER } & \multicolumn{3}{|c|}{ DEPTH TO: } & & \multirow{2}{*}{ CASING } & \multirow{2}{*}{\multicolumn{2}{|c|}{$\begin{array}{c}\text { SAMPLER } \\
\text { CORE } \\
\mathrm{s}\end{array}$}} \\
\hline DATE & $\begin{array}{l}\text { MAS AFTEF } \\
\text { COMP }\end{array}$ & WATER & $\begin{array}{l}\text { BOTTOM } \\
\text { of CASING }\end{array}$ & $\begin{array}{l}\text { BOTTOM } \\
\text { Of HOLE }\end{array}$ & TYPE & & & \\
\hline \multirow[t]{3}{*}{$09-02$} & & NE & & & SIZE 10 & $3-1 / 4$ in. & $1-3 / 8 \mathrm{ln}$. & \\
\hline & & & & & HAMMEA WT & & $140 \mathrm{lb}$ & \\
\hline & & & & & HAMMER FALL & & $30 \ln$. & \\
\hline
\end{tabular}

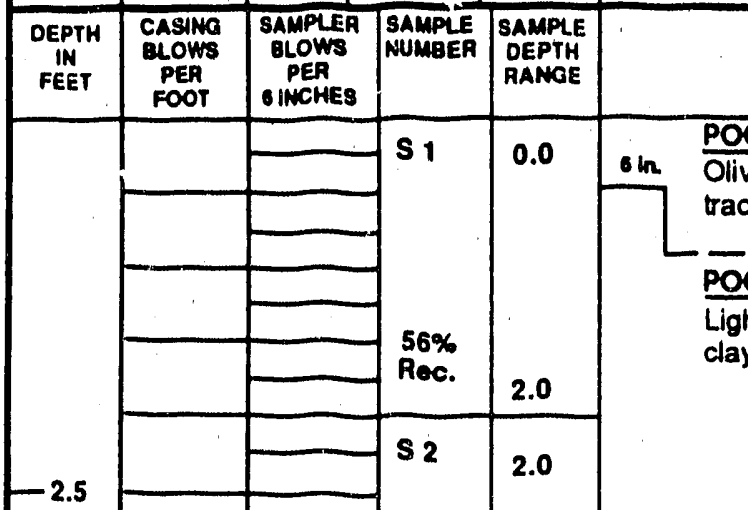

$-2.5$

FIELD CLASSIFICATION AND REMARKS

POALY GRADED SAND (SP)

Olive black, moist, mostly fine-grained, subangular quartz sand trace silt, trace organic material.

POORLY GRADED SAND WITH SILTISP.SM

Light brown, moist, mosty fine-grained, subangular quartz sand, few clayey silt.
BORING NO. BSS-11

JOB NO:

G.8163

PAGE NO: $\quad 1$ OF 1 LOCATION: SEE PLAN ELEVATION:

DRILLER: $\quad$ E. BARRON

PREPARED BY: R.J.HUNT
DATE FINISH: $\quad 09.02 .88$

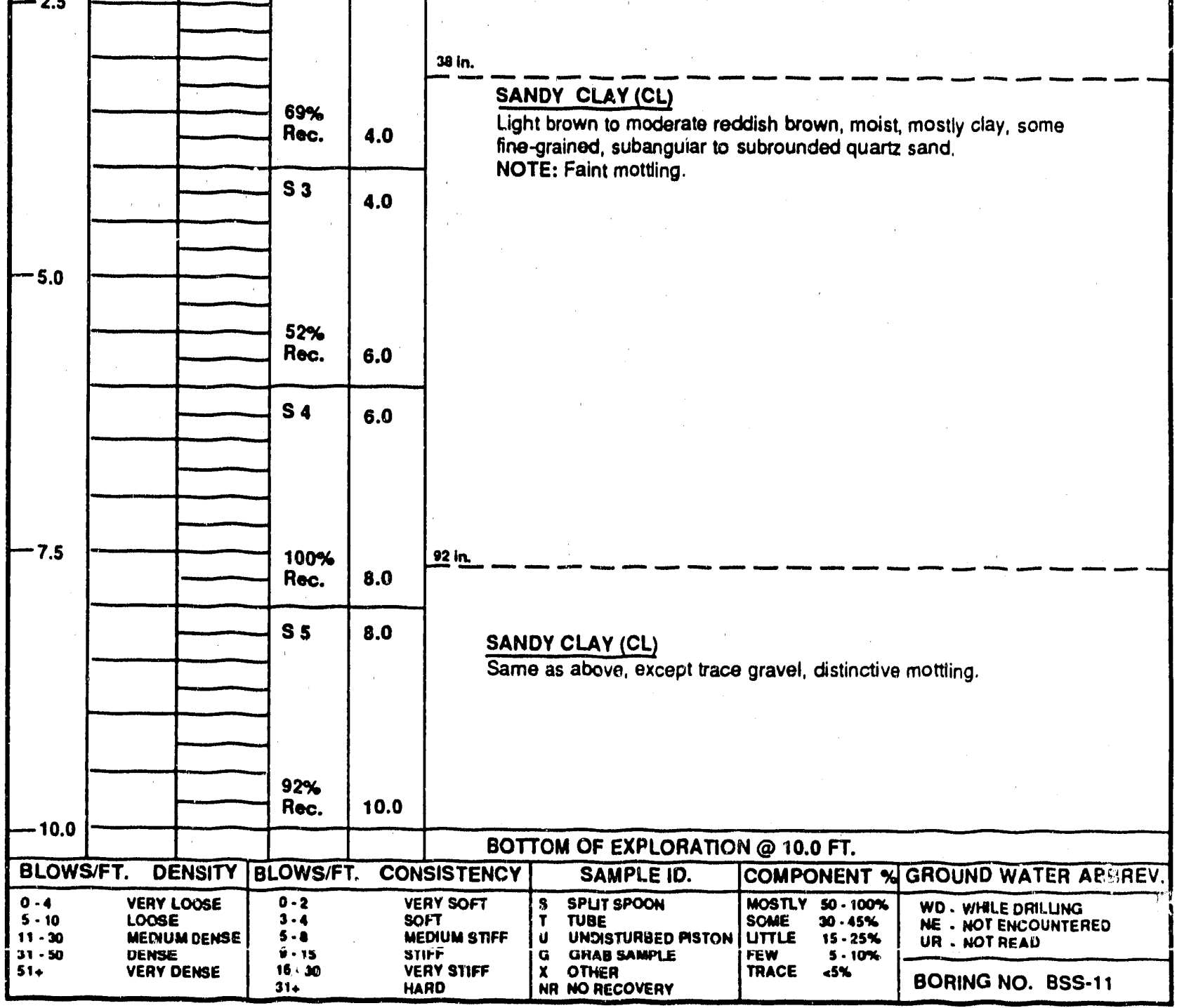




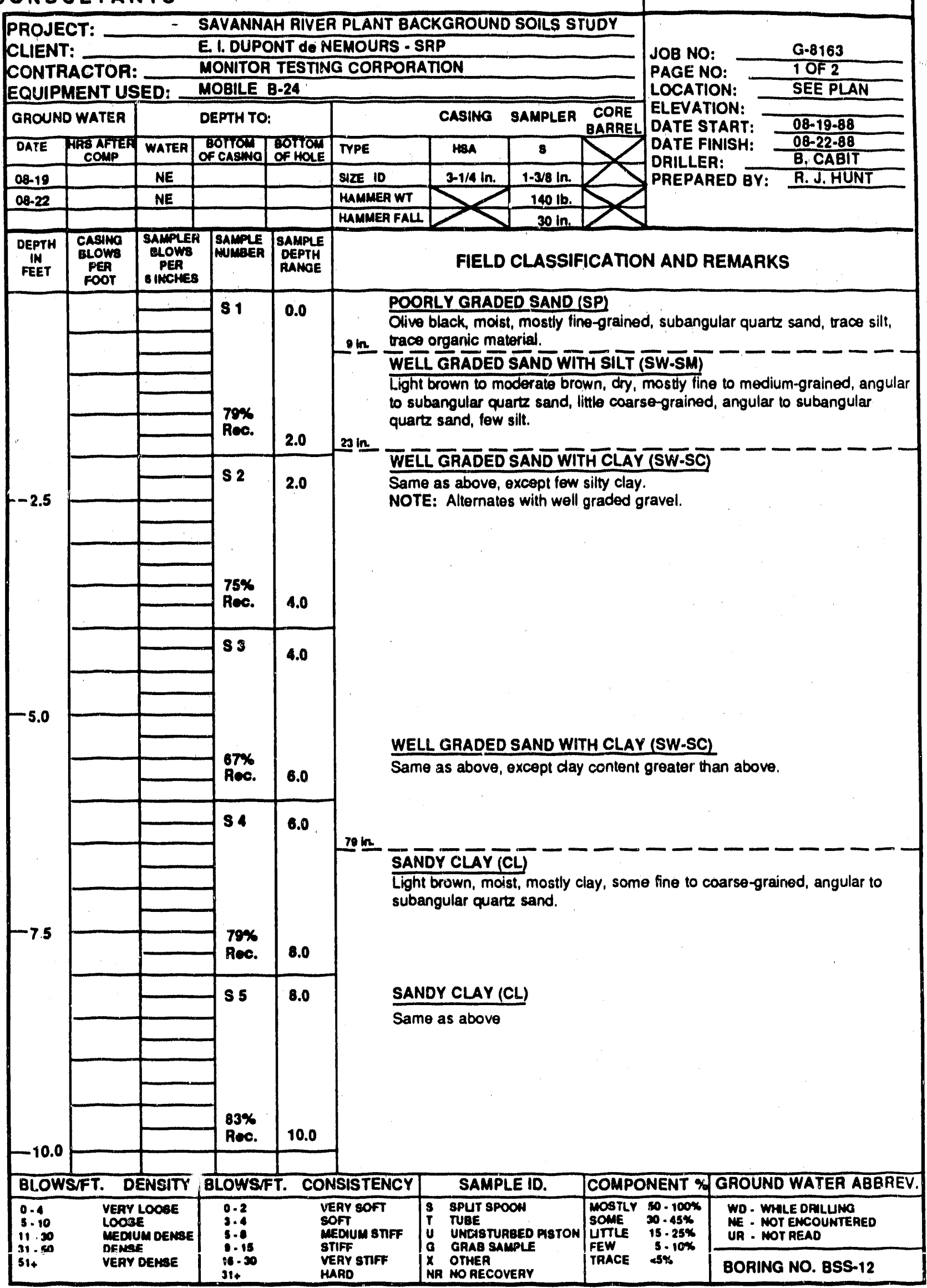




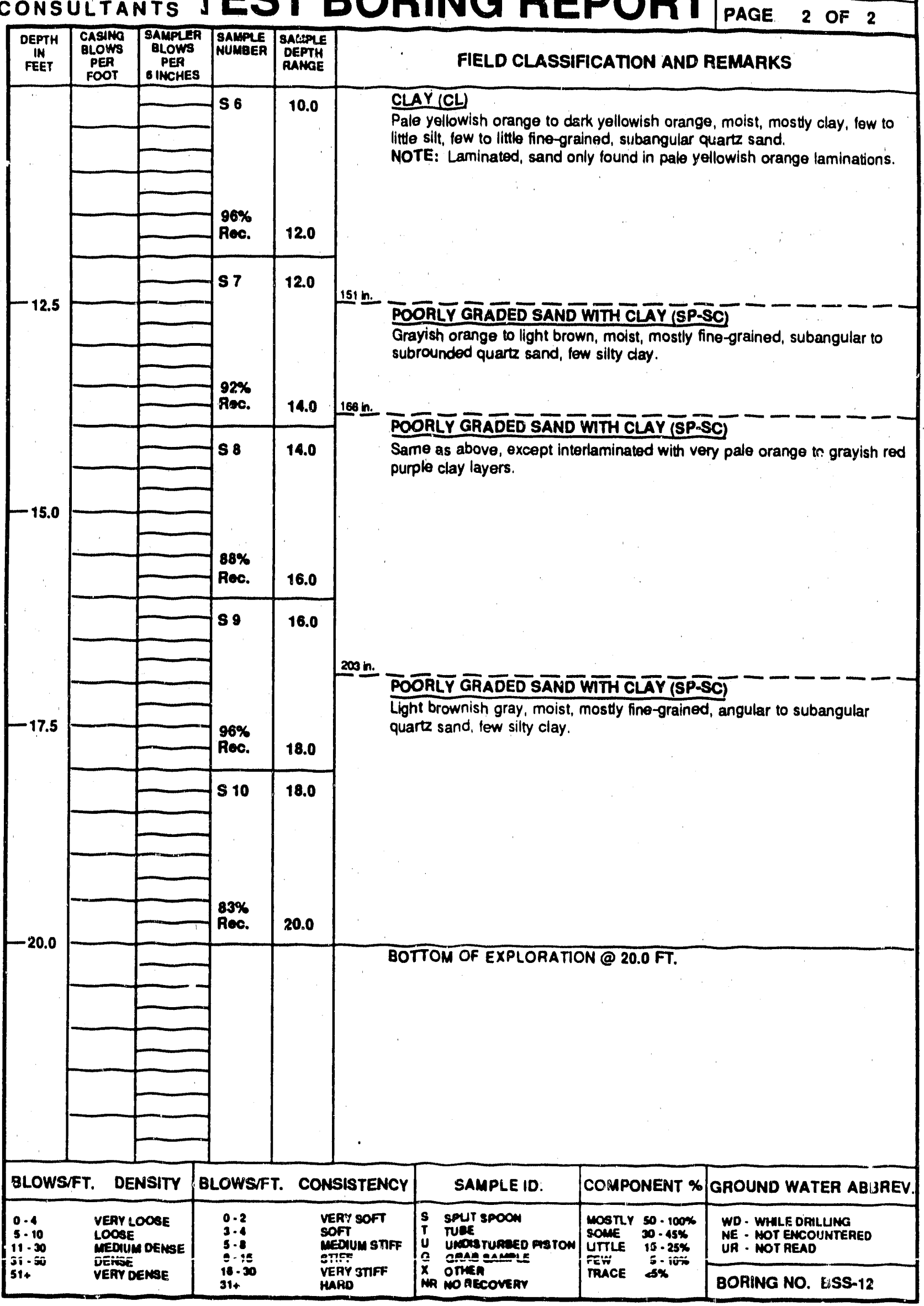


TEST BORING REPORT

BORING NO. BSS-13

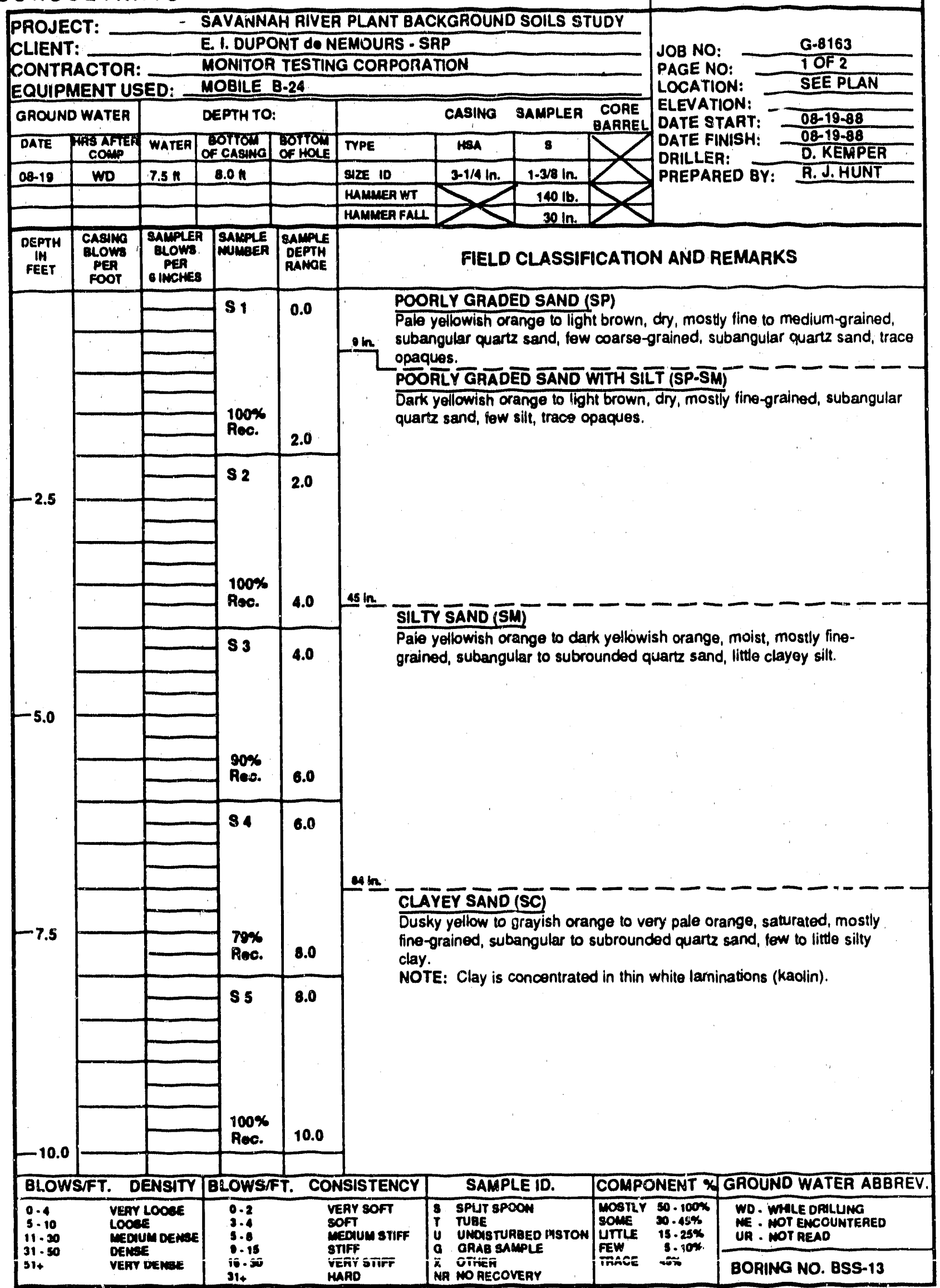



CONSULTANTS

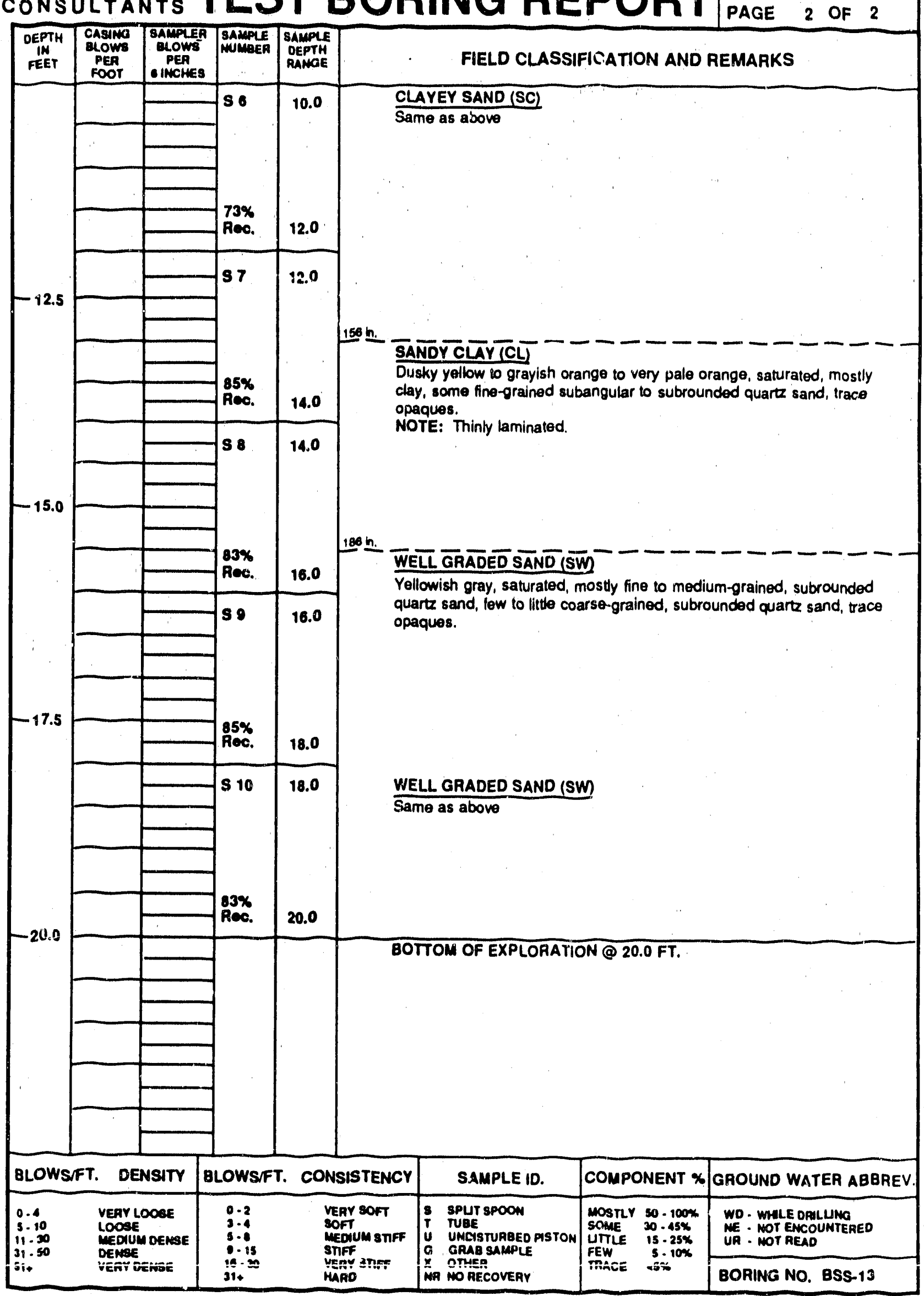


SIRRINE

\section{ENVIRONMENTAL}

CONSULTANTS

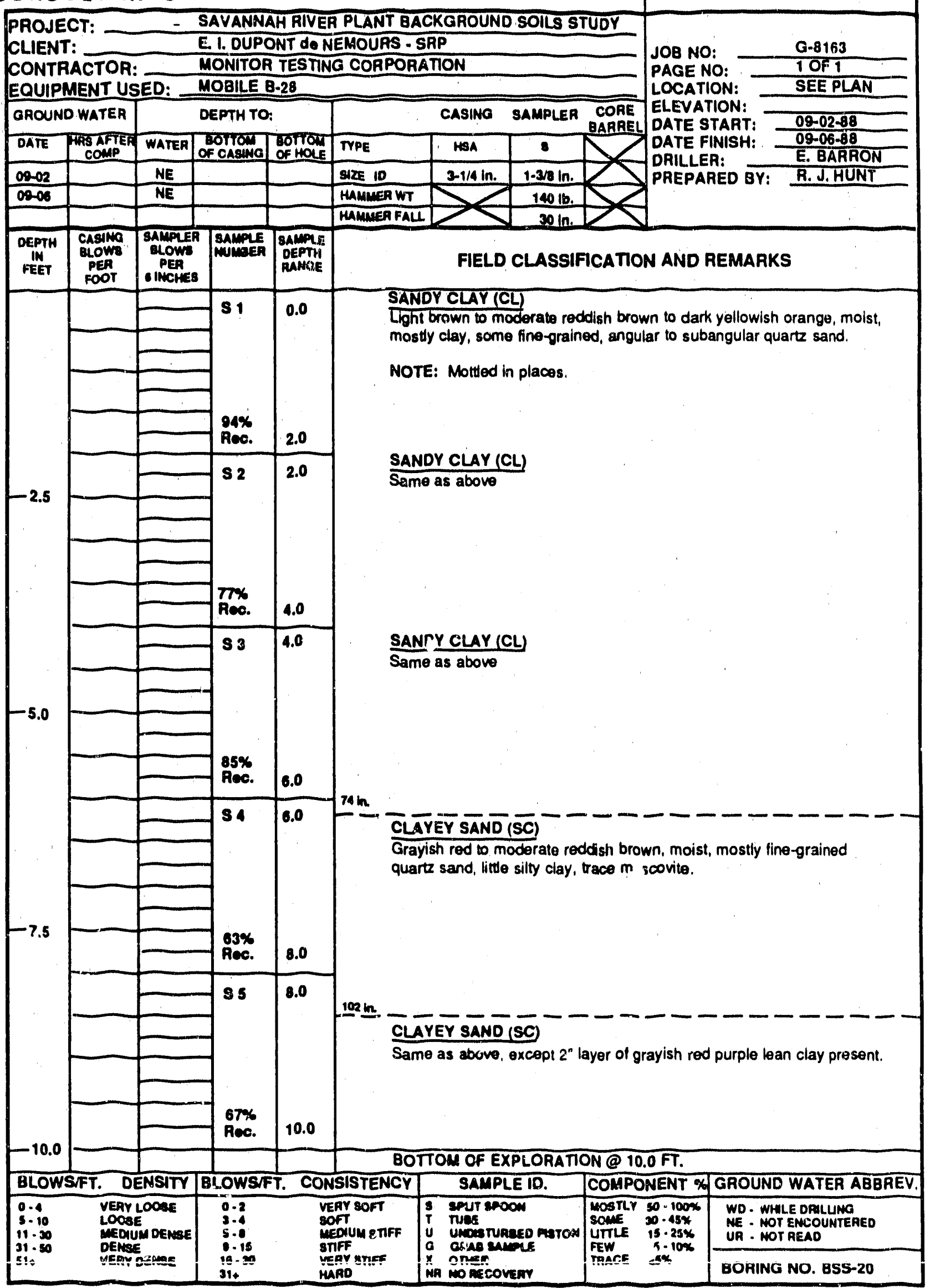




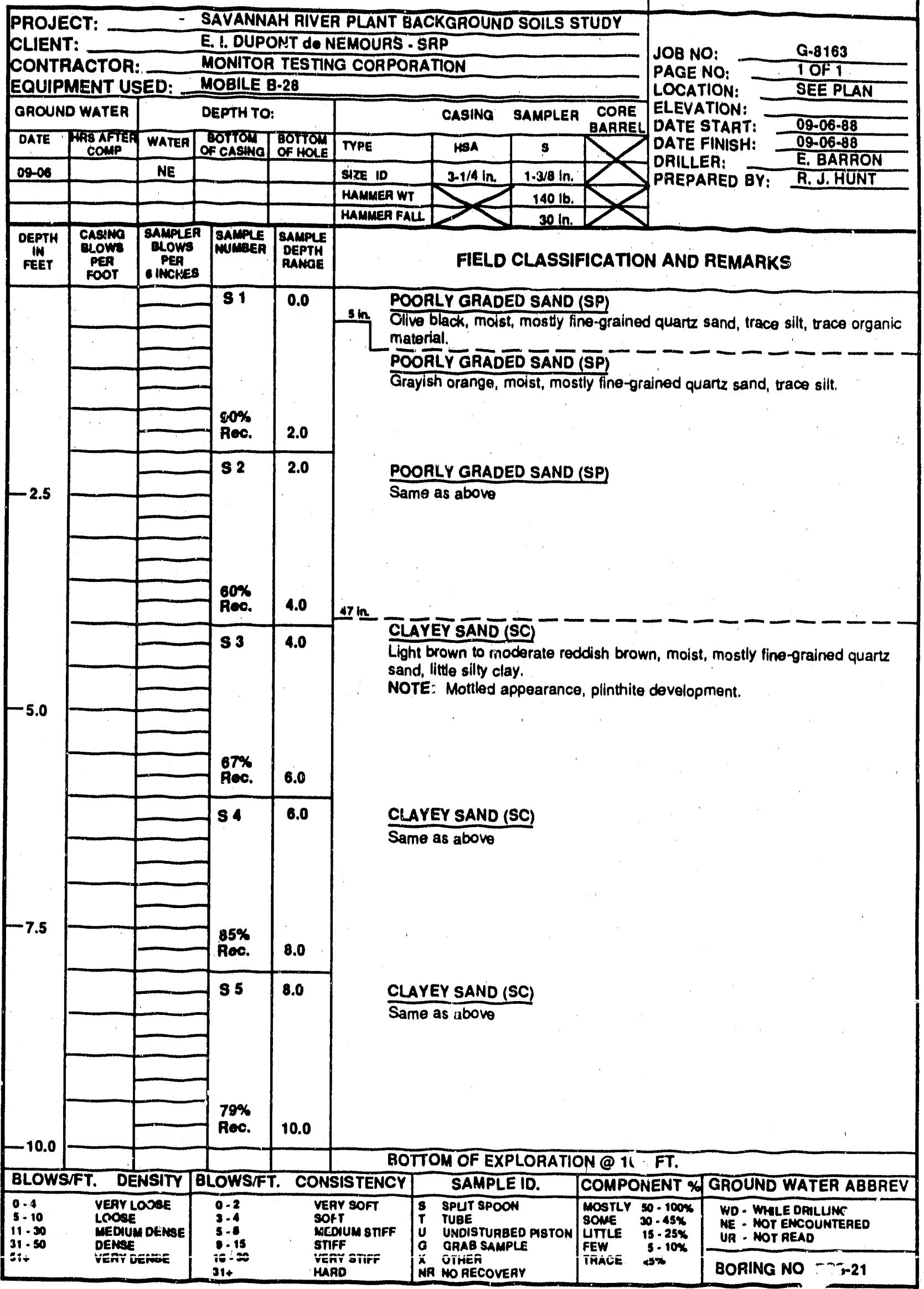


ENVIRONMENTAL

CONSULTANTS

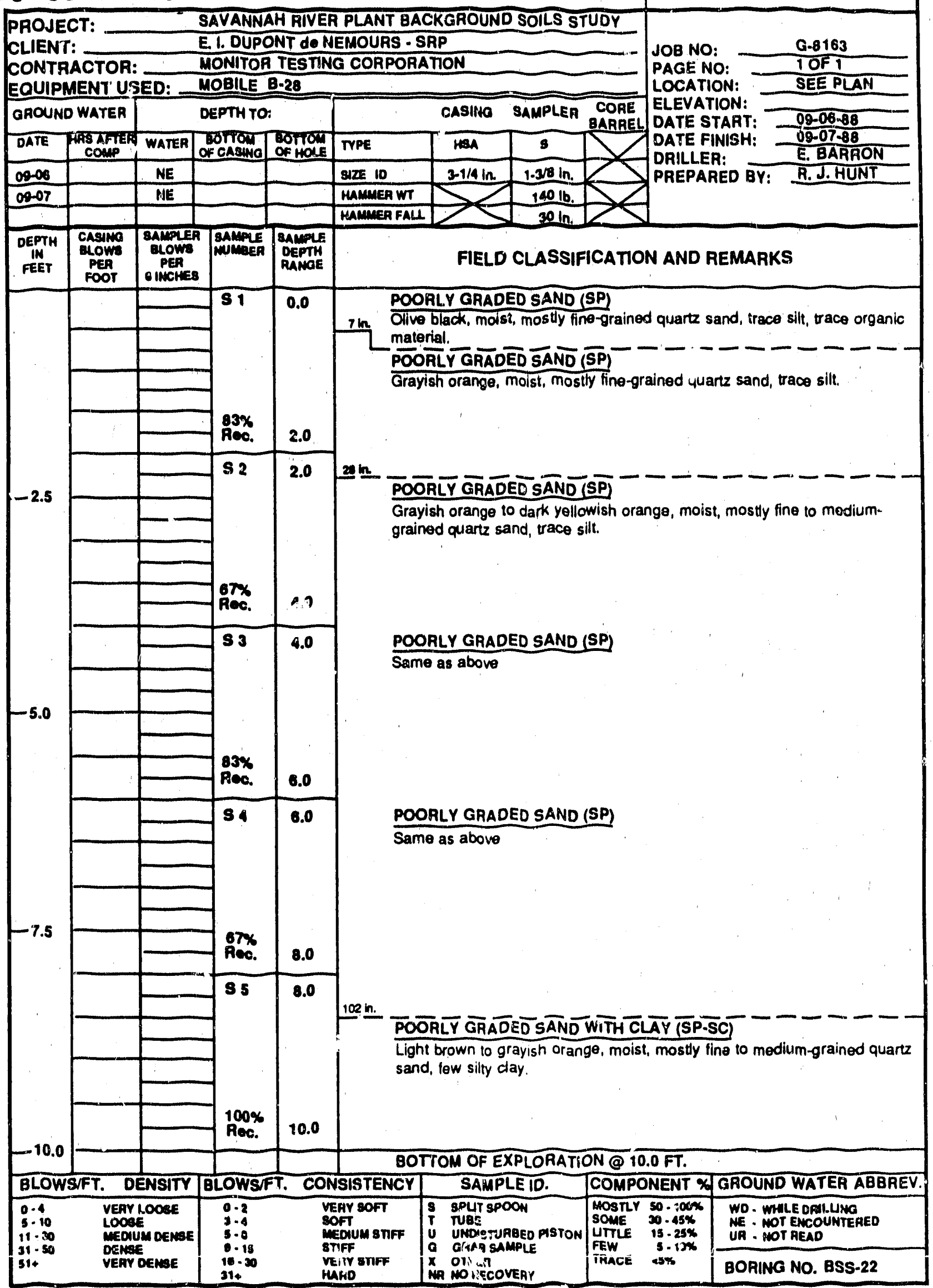


CONSULTANTS

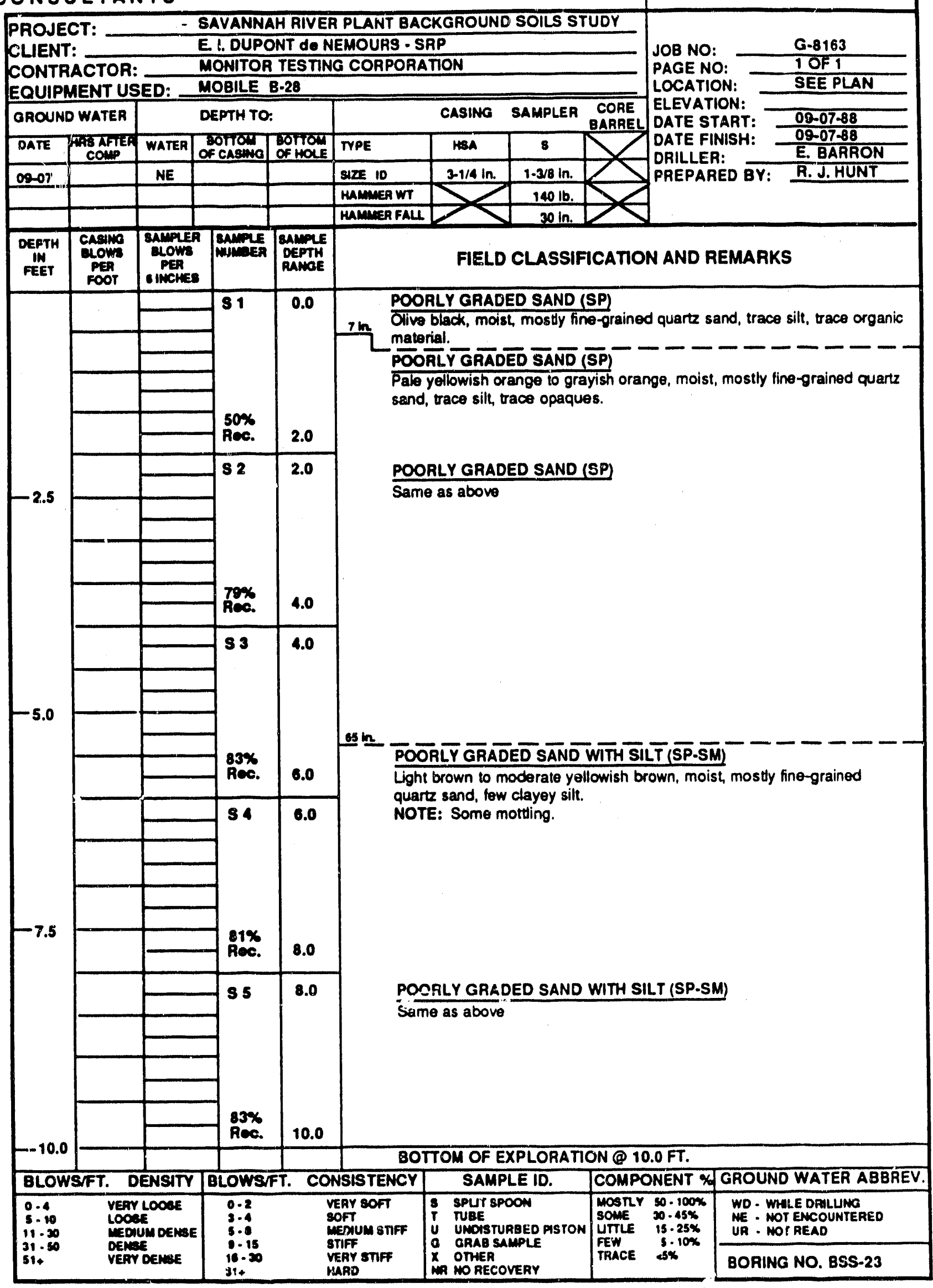




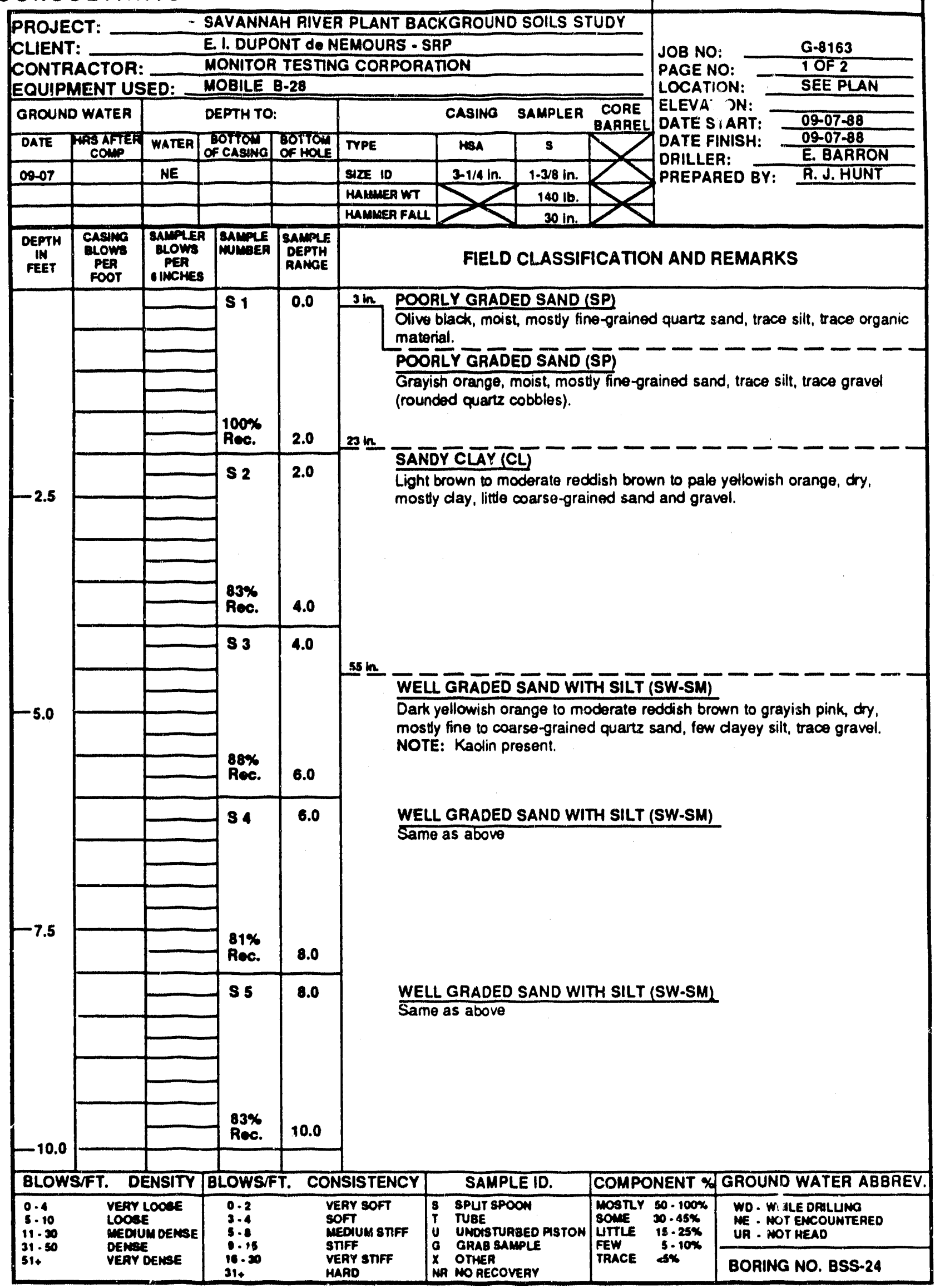




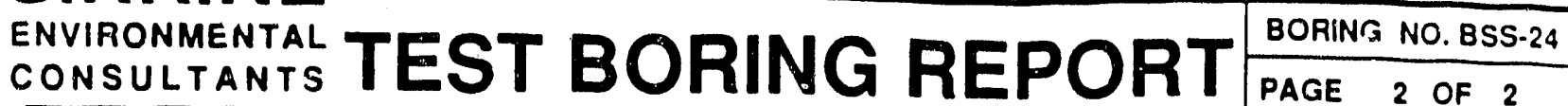

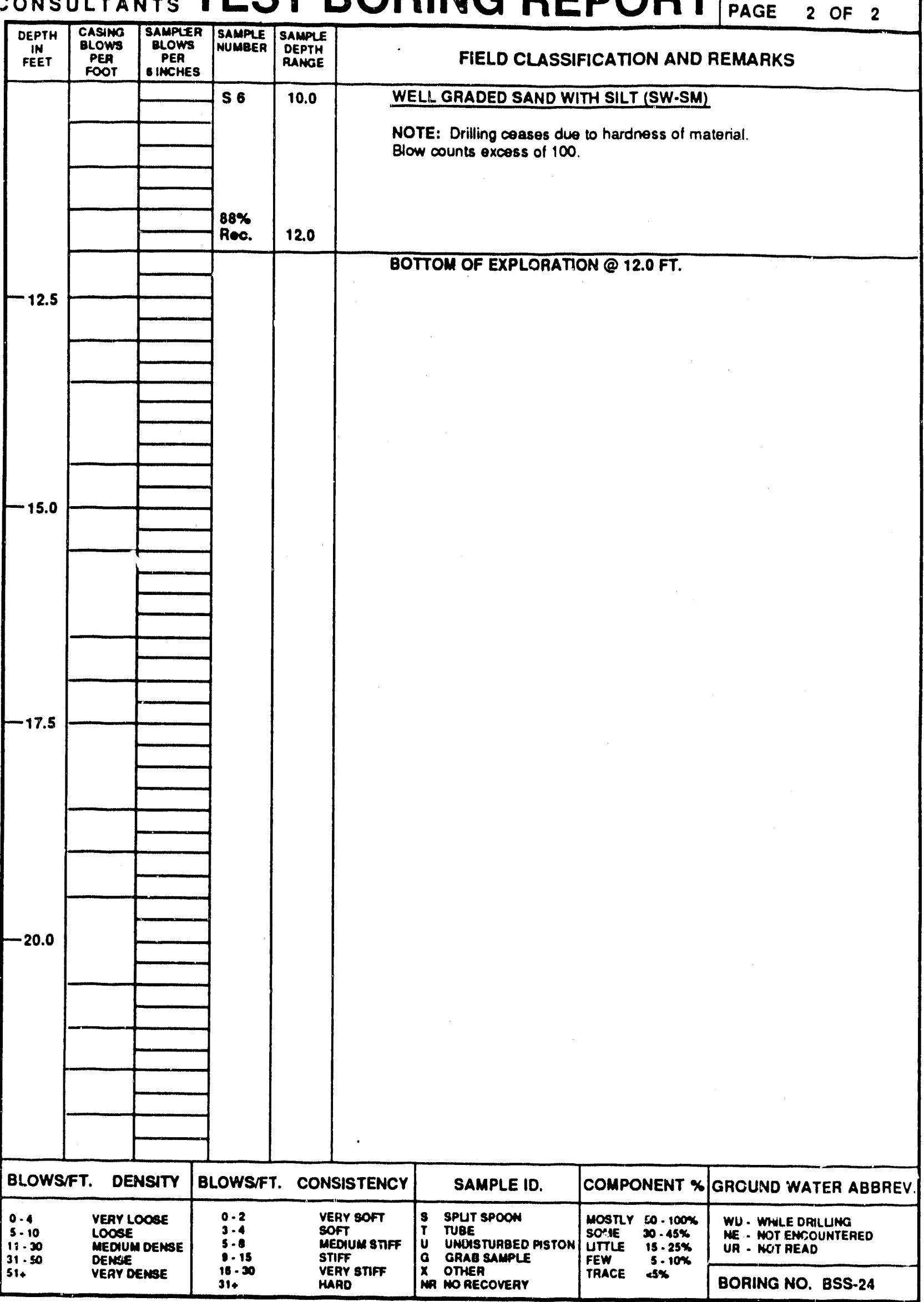




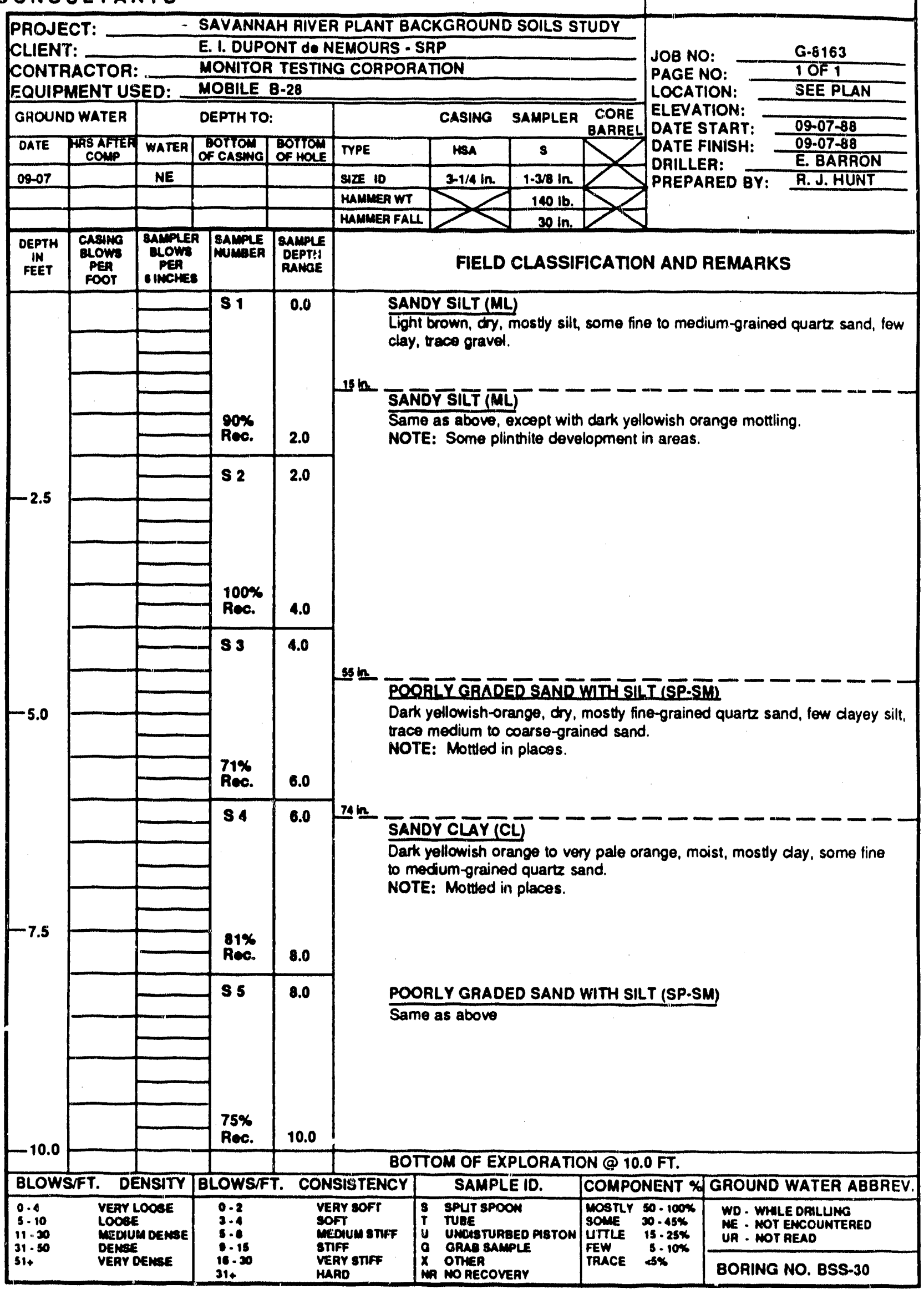


PROJECT:

SAVANNAH RIVER PLANT.BACKGROUND SOILS STUDY

CLIENT:

E. I. DUPONT dO NEMOURS - SRP

CONTRACTOR: MONITOR TESTING CORPOAATION

EQUIPMENT USED:

MOBILE B-28

GROUND WATER

DEPTH TO:

\begin{tabular}{|c|c|c|c|c|c|c|c|c|}
\hline \multicolumn{2}{|c|}{ GROUND WATER } & \multicolumn{3}{|c|}{ DEPTHTO: } & \multicolumn{2}{|r|}{ CASING } & \multirow{2}{*}{\multicolumn{2}{|c|}{ SAMPLEA $\begin{array}{c}\text { CORE } \\
\text { BARREL }\end{array}$}} \\
\hline DATE & $\begin{array}{c}\text { MAS AFTEF } \\
\text { comp }\end{array}$ & WATEA & $\begin{array}{c}\text { Bortom } \\
\text { of casing }\end{array}$ & $\begin{array}{l}\text { BOTrom } \\
\text { Of HOLE }\end{array}$ & TYPE & HSA & & \\
\hline $00-08$ & & NE & & & Suze 10 & $3-1 / 4 \mathrm{in}$ & $1.3 / 3 \mathrm{ln}$ & \\
\hline & & & & & MAMMEA WT & & $140 \mathrm{lb}$ & \\
\hline & & & & & HAMMES FALL. & & $30 \mathrm{in}$. & \\
\hline DEPTH & $\begin{array}{l}\text { CASNMB } \\
\text { BLOWS }\end{array}$ & SAMPU: & 8Acive & SAMPLE & & & & \\
\hline
\end{tabular}

JOB NO:

PAGE NO:

G.8163

LOCATION:

SEE PLAN

DEVATION:

DATE START: $09-08-88$

DATE FINISH: $09.08-88$

DRILLER:

E. BARAON

PREPARED BY: R.J.HUNT

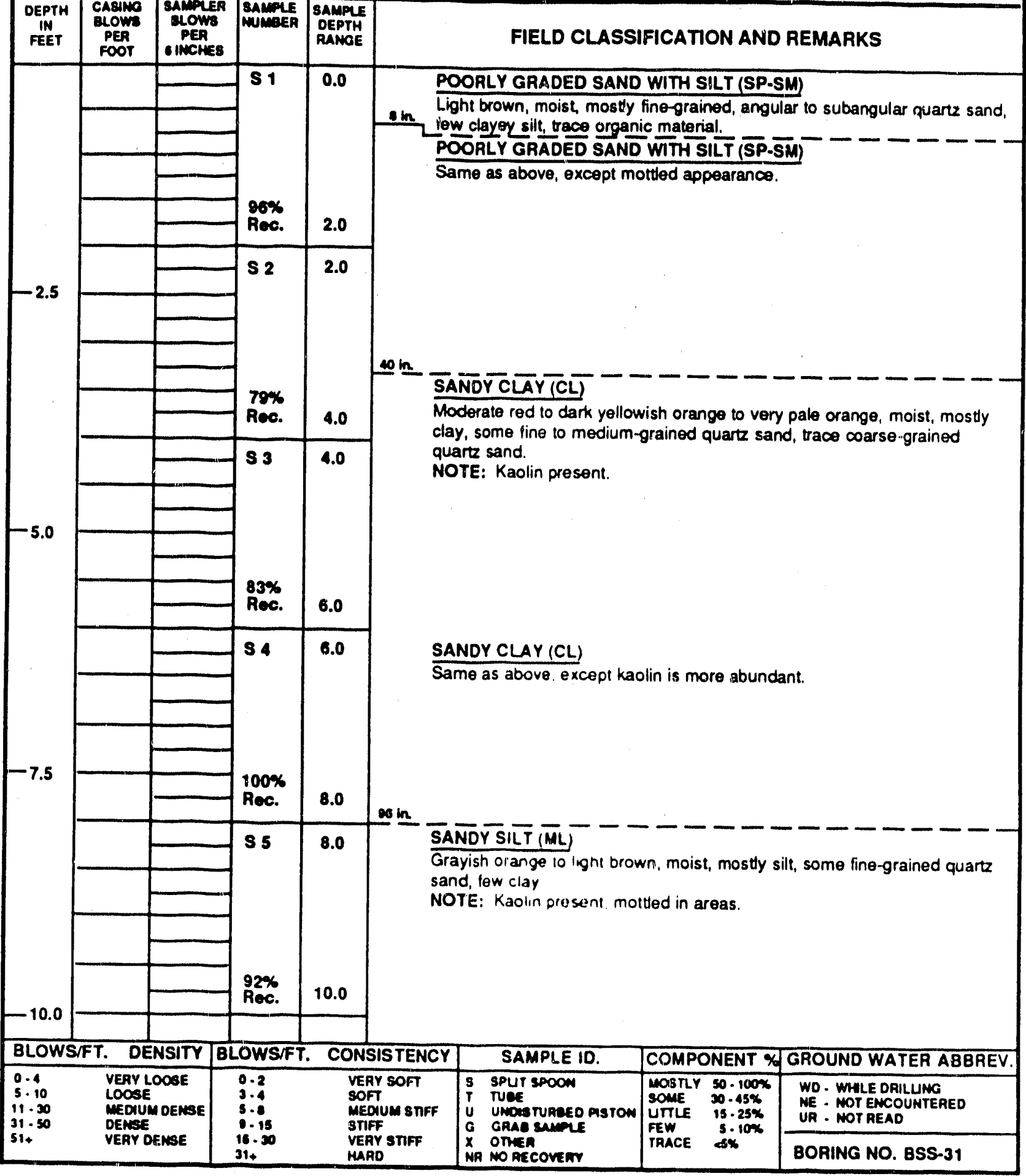


SIRRINE

ENVIRONMENTAL

CONSULTANTS

TEST BORING REPORT

BORING NO. BSS-31

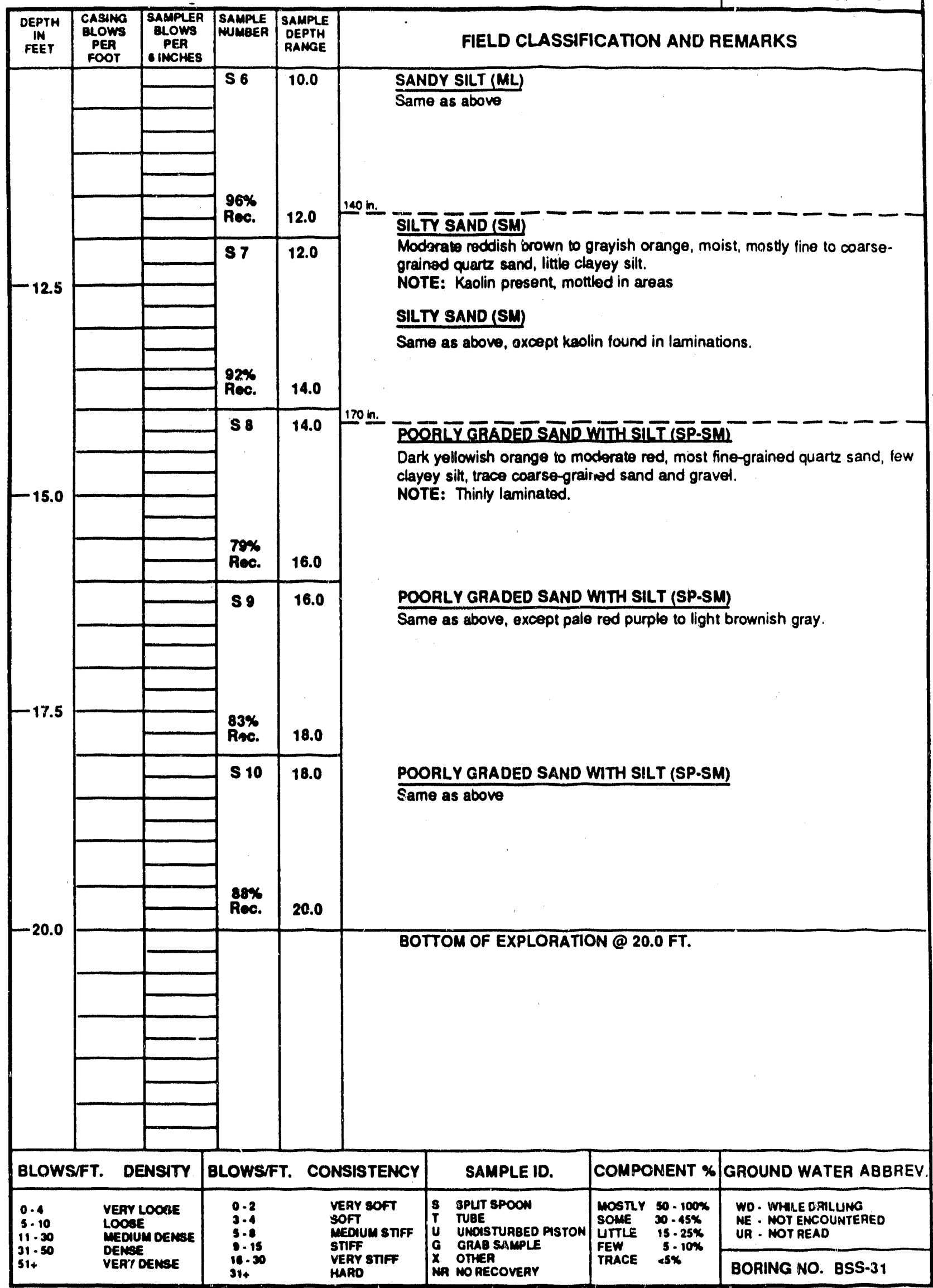


SIRRINE

ENVIRONMENTAL

CONSULTANTS

\section{TEST BORING REPORT}

BORING NO.

BSS-32

PROJECT:

CLIENT:

SAVANNAH RIVER PLANT BACKGROUND SOILS STUDY

CONTRACTOR:

EQUIPMENT USED: MOBILE 8-28

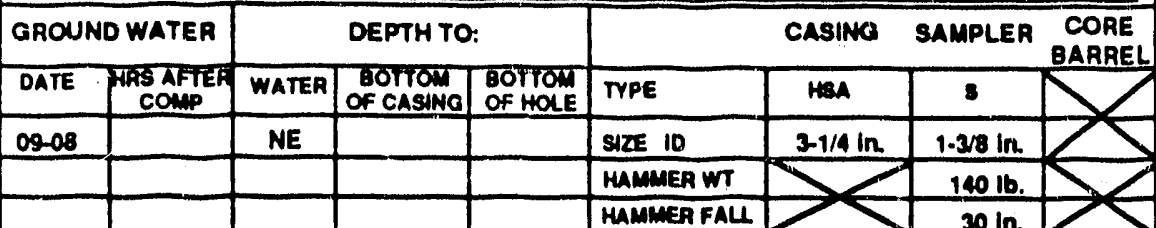

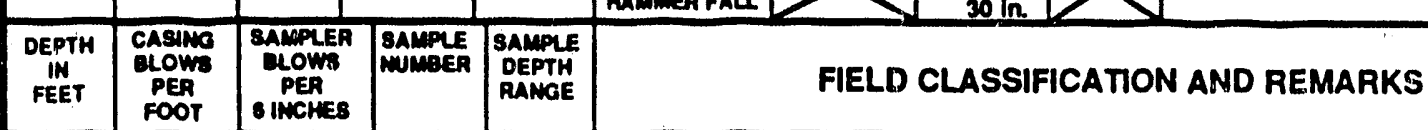

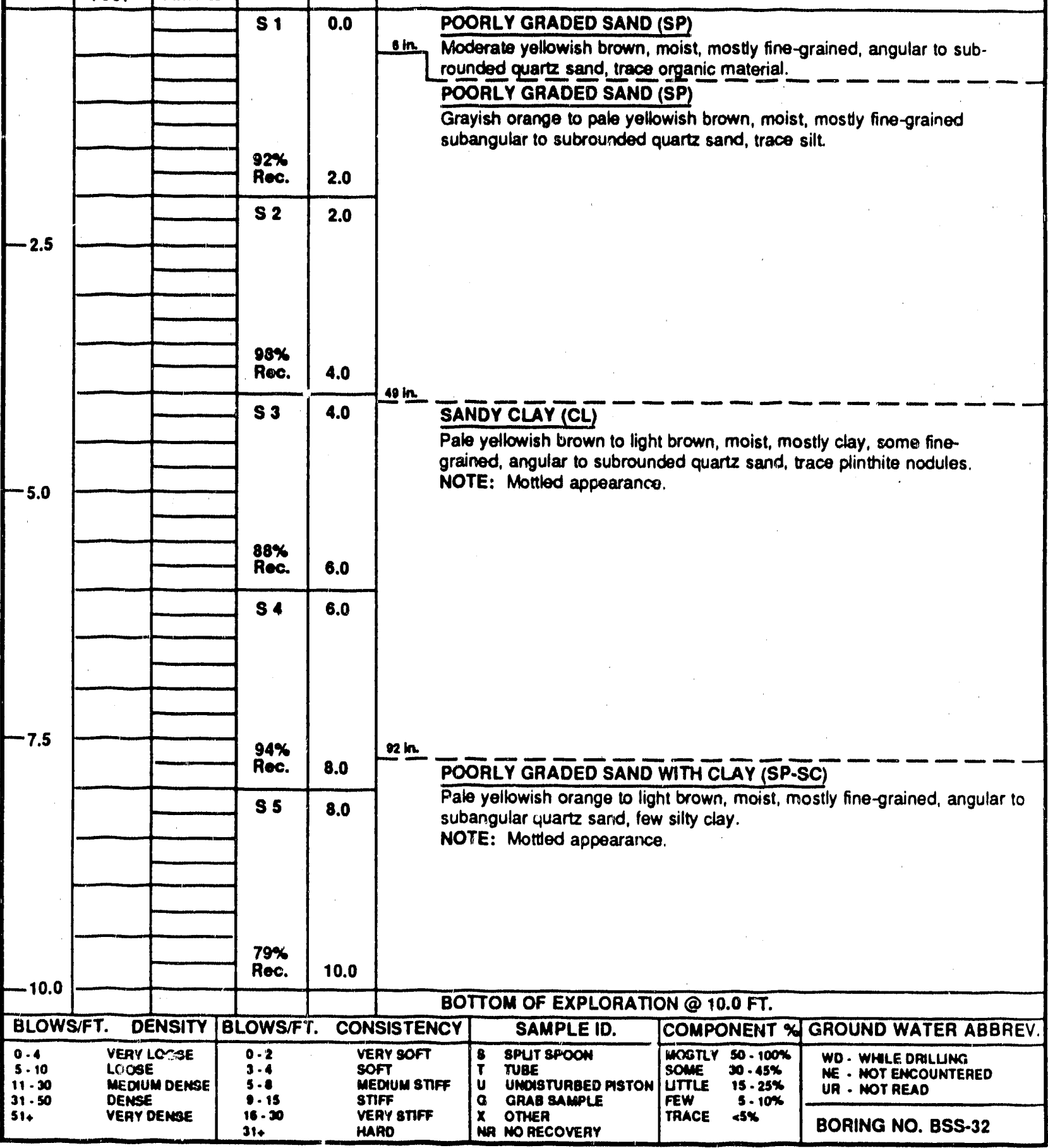




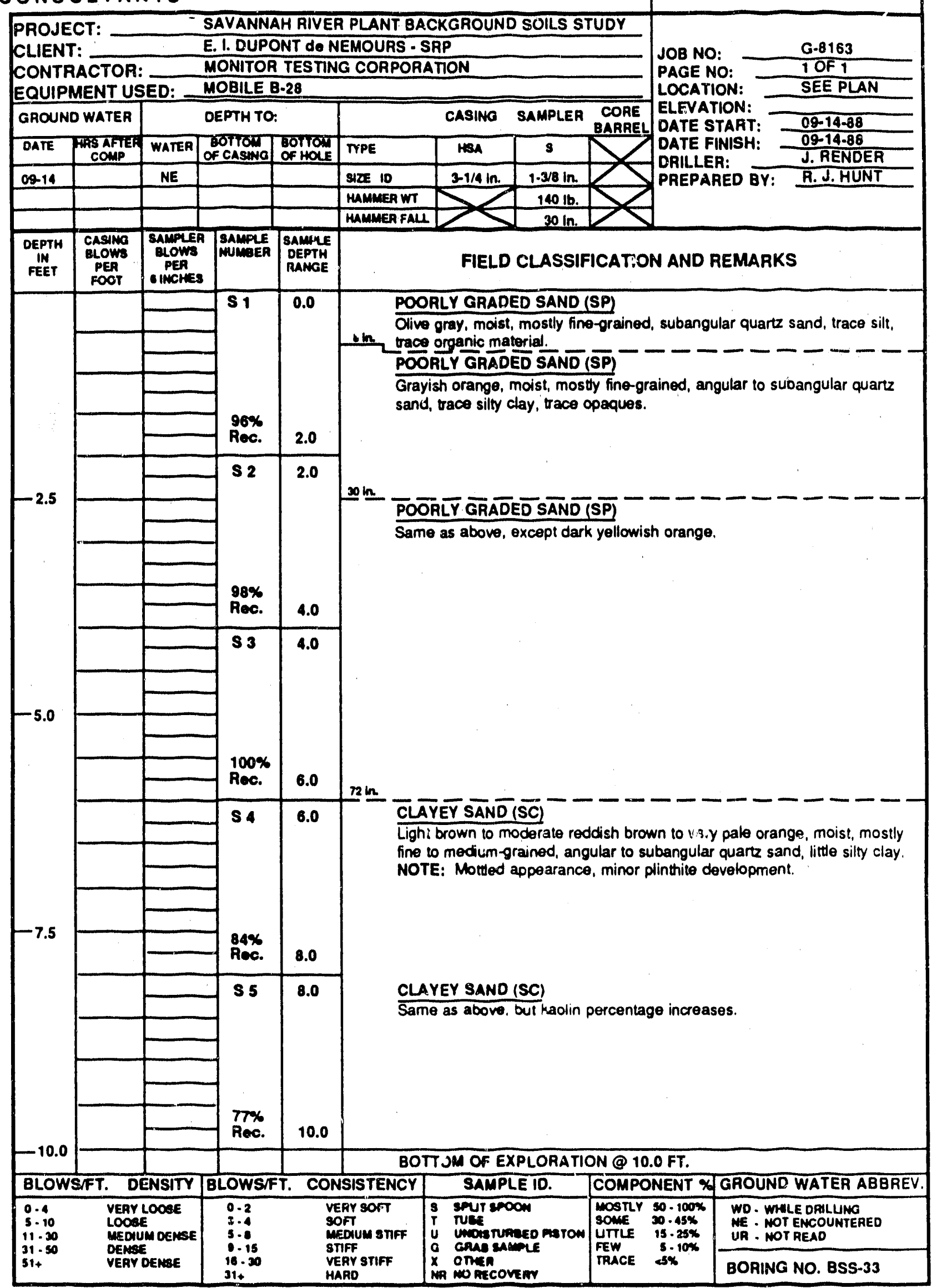


ENVIRONMENTAL

CONSULTANTS

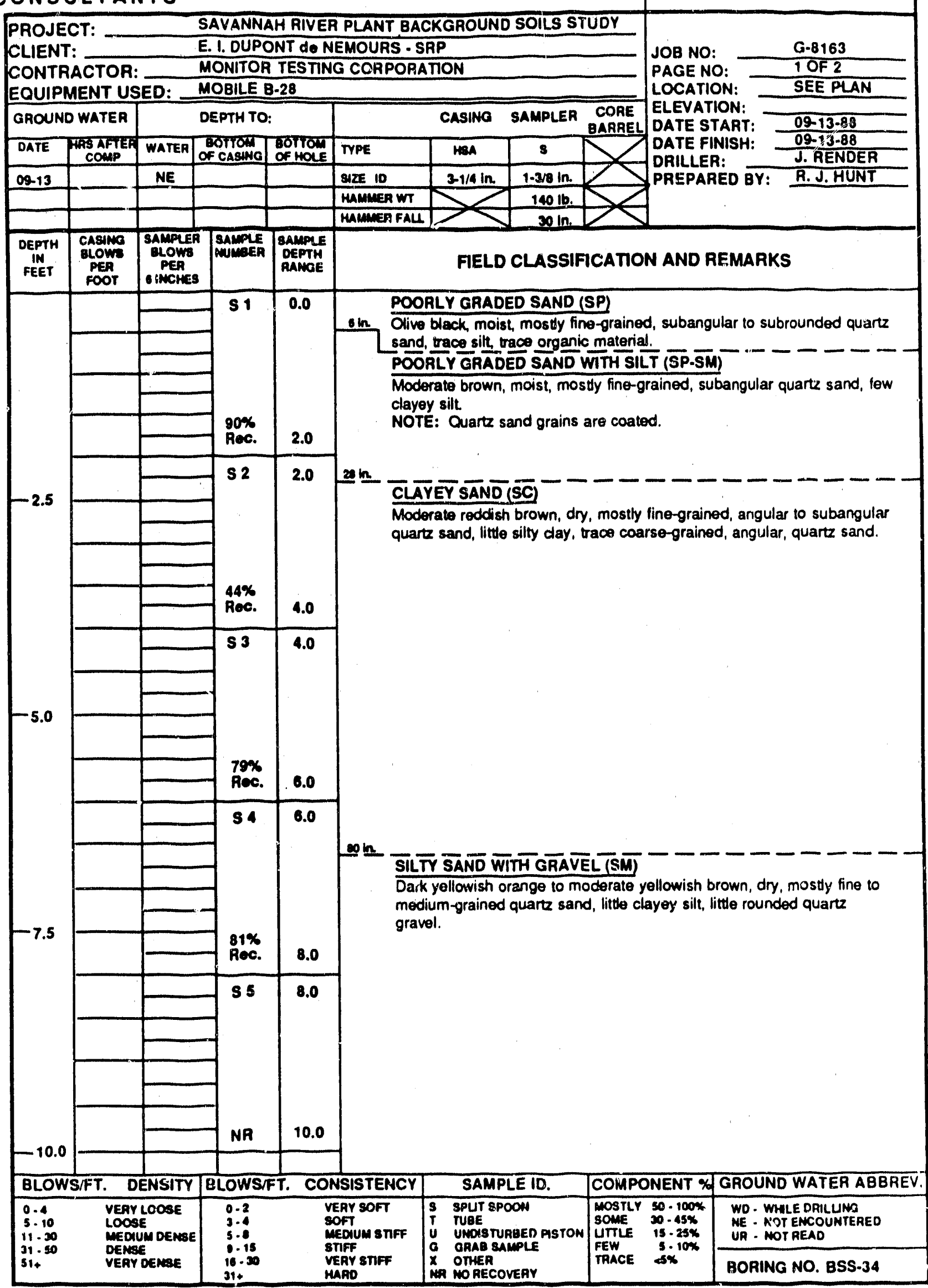




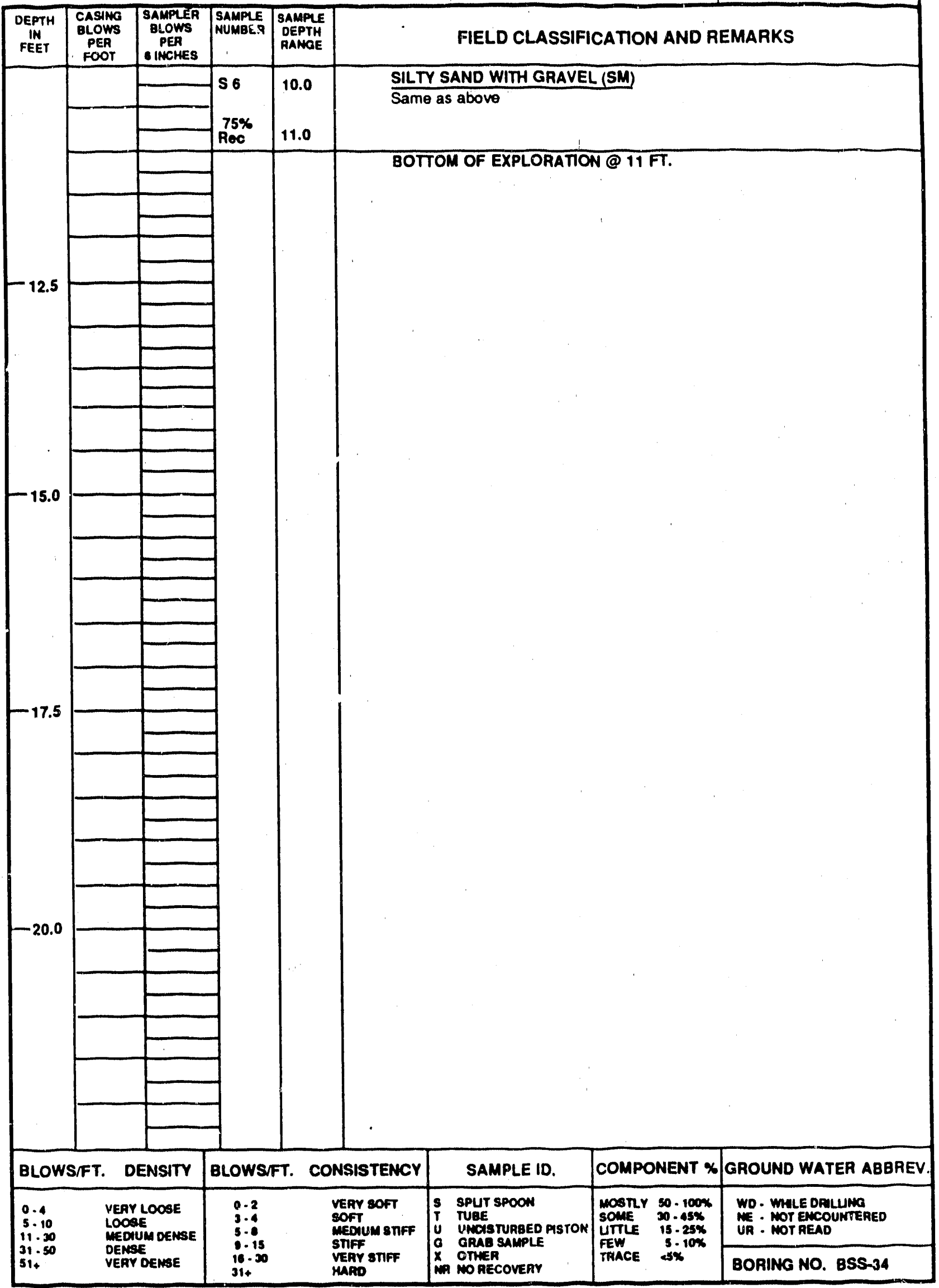


PROJECT:

CLIENT:

CONTRACTOR: EQUIPMENT USED:

\begin{tabular}{|l|l|l|}
\hline GROUND WATER & \\
\hline DATE & $\begin{array}{c}\text { MASTAFEA } \\
\text { COMP }\end{array}$ & WATER \\
\hline
\end{tabular}

\begin{tabular}{|c|c|c|c|c|c|c|c|c|}
\hline DATE & $\begin{array}{c}\text { WHSAFEA } \\
\text { COUP } \\
\end{array}$ & WATER & $\begin{array}{l}\text { borion } \\
\text { of CAsina }\end{array}$ & $\begin{array}{l}\text { EOTTON } \\
\text { OF HOLE }\end{array}$ & TYPE & MSA & 8 & \\
\hline 0909 & & NE & & & SIZE 10 & $3-1 / 4$ in. & $1.3 / 8$ in. & \\
\hline & & & & & HAMMES WT & & 14016. & \\
\hline & & & & & HAMAER FALL & & $30 \mathrm{ln}$ & \\
\hline
\end{tabular}

\begin{tabular}{|c|c|c|c|c|c|}
\hline $\begin{array}{l}\text { DEPTH } \\
\text { IN } \\
\text { FEET }\end{array}$ & $\begin{array}{c}\text { CASUNO } \\
\text { aLOW' } \\
\text { PEA } \\
\text { FoOT }\end{array}$ & $\begin{array}{l}\text { SAMPLER } \\
\text { DLOW' } \\
\text { PEA } \\
\text { OINCHES }\end{array}$ & $\begin{array}{l}\text { SAMPLE } \\
\text { MUMPEA }\end{array}$ & $\begin{array}{l}\text { SAMPLE } \\
\text { DEPTH } \\
\text { RAMGE }\end{array}$ & \\
\hline & & & 91 & 0.0 & \\
\hline
\end{tabular}

2.5

\begin{tabular}{|l|}
\hline \\
\hline
\end{tabular}

$-5.0$

$-7.5$

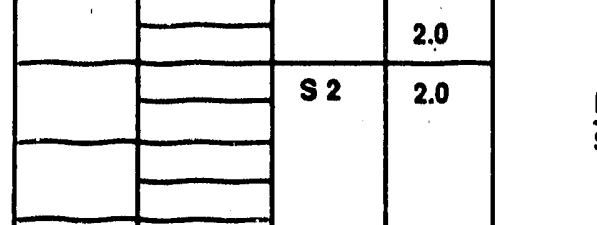

0
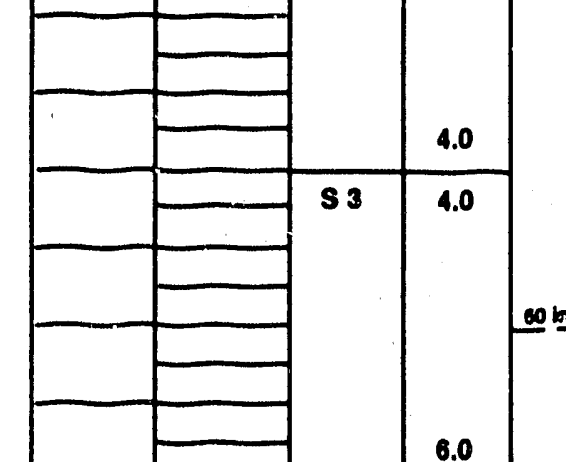

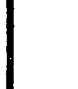

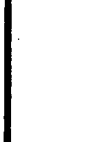

$-7.5$

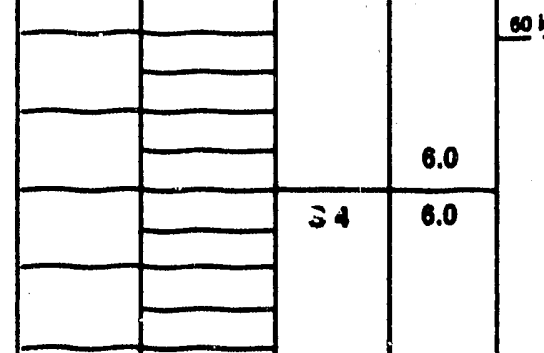

POORLY GRADED SAND (SP)

Olive gray, moist, mostly fine-grained, angular to subangular quartz sand, trace organics.

POORLY GRADED SAND (SP)

Light brown, moist, mostly fine-grained, subangular to subrounded quartz sand, trace opaques.

POORLY GRADED SAND (SP)

Same as above

$60 \mathrm{~h}$

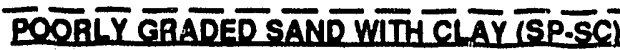

Light brown to moderate reddish brown, moist, mostly fine-grained, subangular to subrounded quartz sand, few silty clay.

NOTE: Motted appearance in places.
BORING NO. BSS-35

JOB NO:

G-8163

PAGE NO: $\quad$ TOF 1

LOCATION: SEE PLAN

ELEVATION:

OATE START: 0909.88

DATE FINISH: $\quad 09-09-88$

DRILLER:

E. BARRON

PREPARED BY: R.J.HUNT 


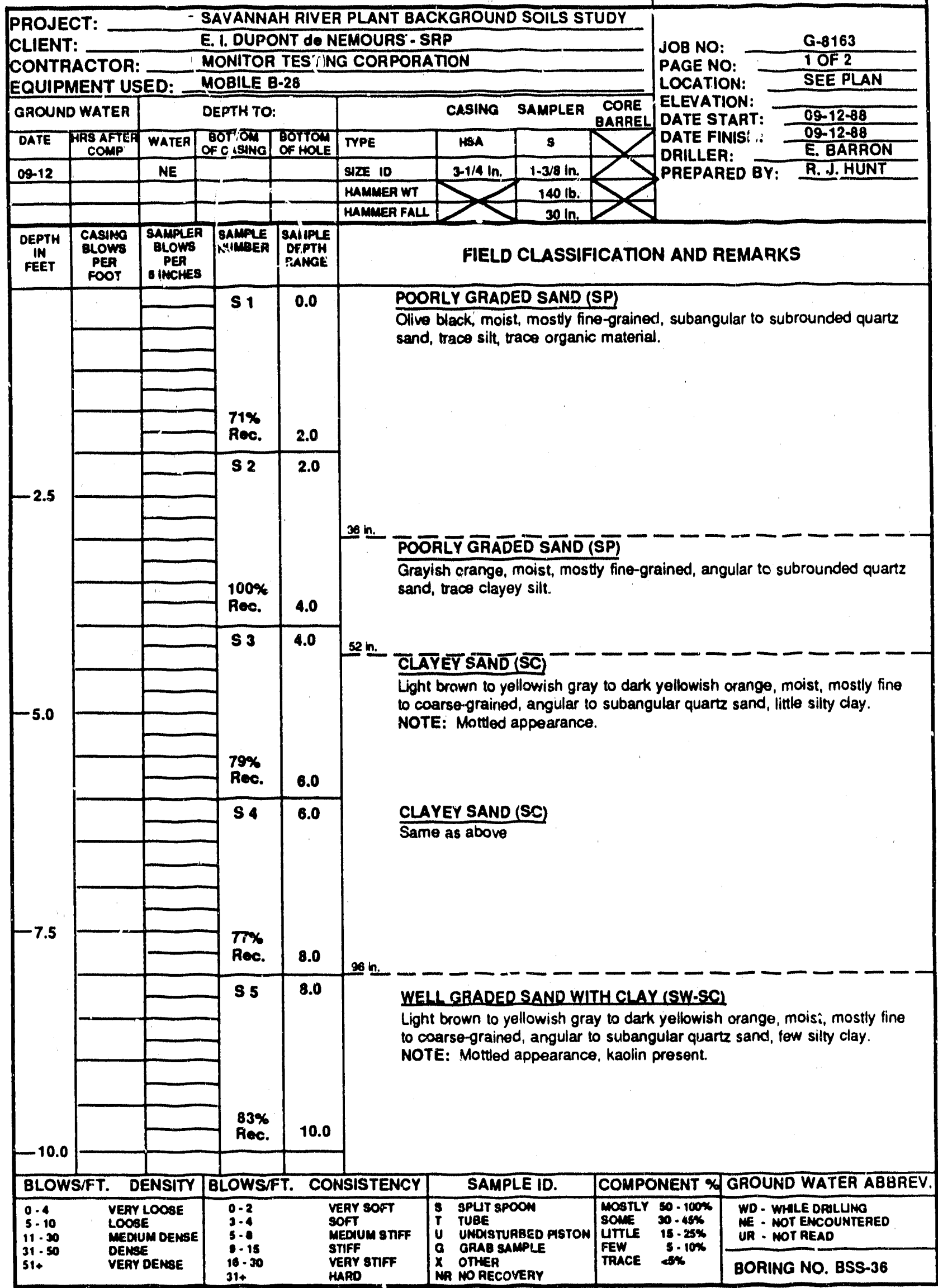




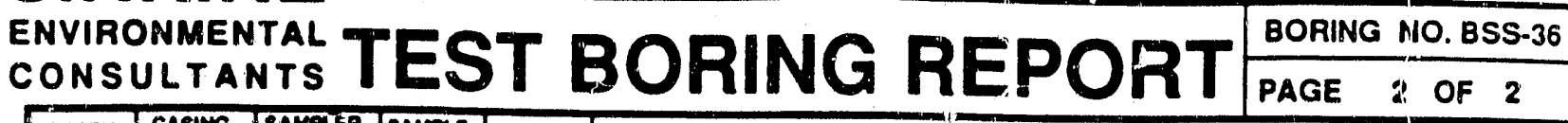

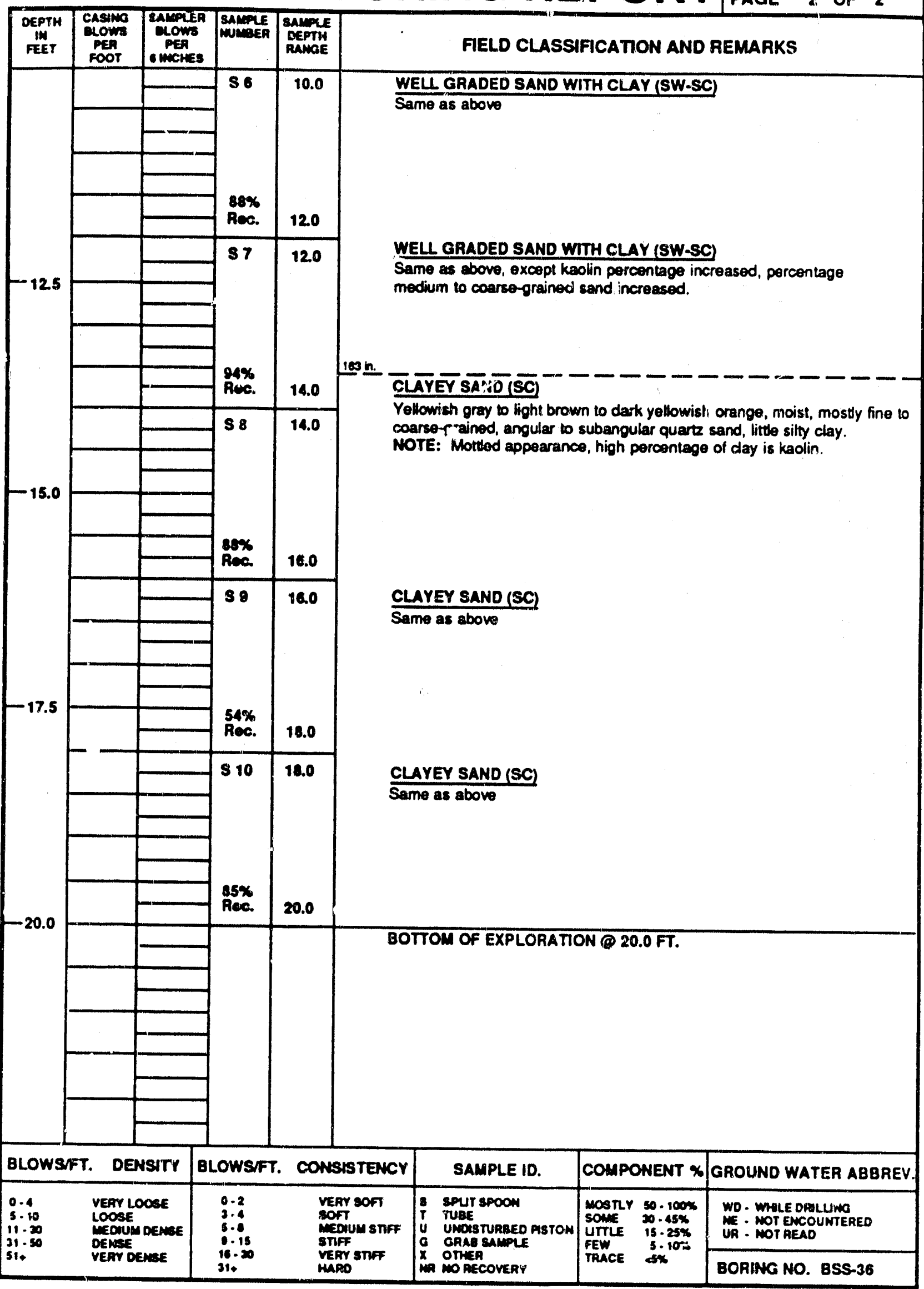




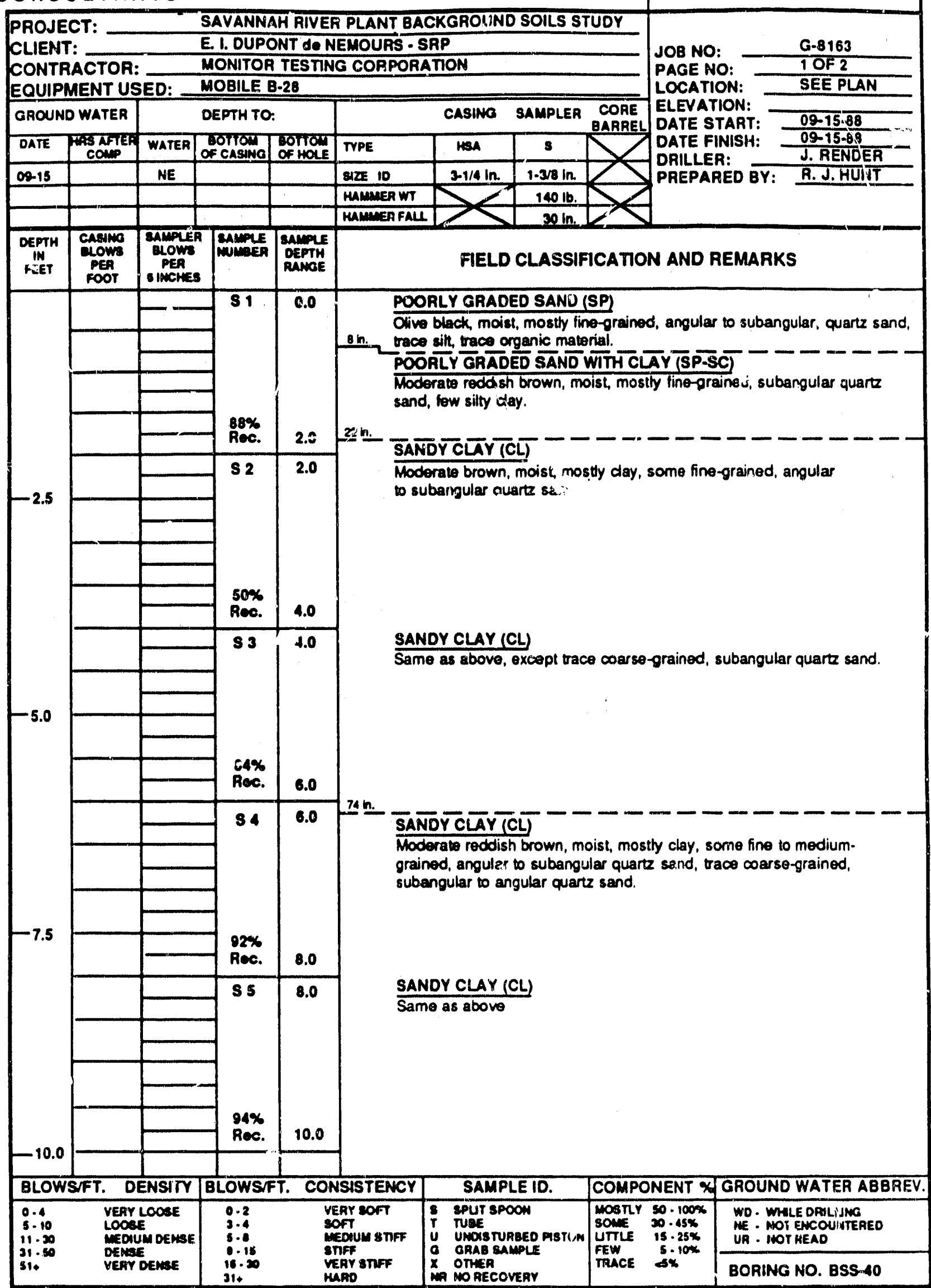


ENVIPONMENTAL

CONSULTANTS

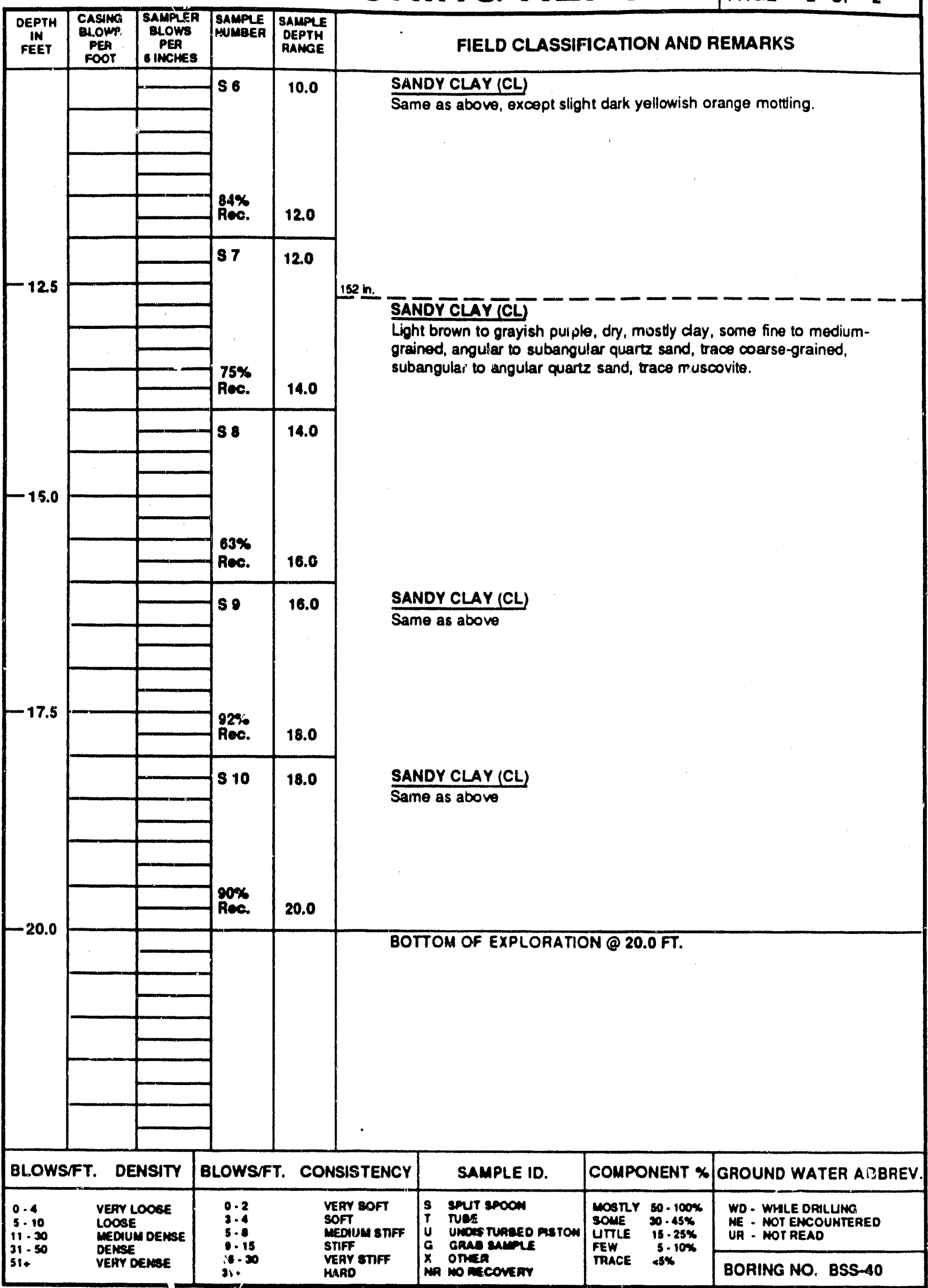


TEST BORING REPORT

BORING NO. BSS-41

PROJECT:

- SAVANNAH RIVER PLANT BACKGROUND SOILS STUDY

CLIENT: E. I. DUPONT dO NEMOURS - SRP CONTRACTOR: MONITOR TESTING CORPORATION EQUIPMENT USED: GROUND WATER DATE ThS AFTEK WATER MOBILE B-28 casing samplea core DEPTHTO:

\begin{tabular}{|c|c|c|c|c|c|c|c|}
\hline \\
\hline DATE & $\begin{array}{c}\text { WhS Afrite: } \\
\text { comp }\end{array}$ & WATER & $\begin{array}{l}\text { Eoriom } \\
\text { of casina }\end{array}$ & $\begin{array}{l}\text { Boriom } \\
\text { of Houe }\end{array}$ & TPPE & MSA & 8 \\
\hline $09-14$ & & NE & & & SIZE 10 & $3-1 / 4 \mathrm{ln}$ & 1-3/8 in. \\
\hline & & & & & HAMMEA WT & & $140 \mathrm{lb}$. \\
\hline & & & & & MAMMER FALL & & $30 \mathrm{ln}$. \\
\hline
\end{tabular}

BARAE

FIELD CLASSIFICATION AND REMARKS

\begin{tabular}{|c|c|}
\hline $\begin{array}{c}\text { DEPTH } \\
\text { IN } \\
\text { FEET }\end{array}$ & $\begin{array}{l}\text { PLOW } \\
\text { PER } \\
\text { FOOT }\end{array}$ \\
\hline
\end{tabular}

$-2.5$

\begin{tabular}{l|l|l}
\hline & \\
\hline &
\end{tabular}

81

\subsection{PDORLY GRADED SAND (SP)}

Olive gray, moist, mostly fine-grained, angular to subangular quartz sand, trace silt, trace organic material. POORTY GRADED SAND (SP)

Grayish orange, moist, mostly fine-grained, angular to subangular quartz sand, trace clayey silt.

POORLY GRADED SAND (SP)

Same as above

\begin{tabular}{l|l} 
Roc. & 2.0 \\
\hline
\end{tabular}

$\mathbf{S 2}$

2.0

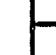

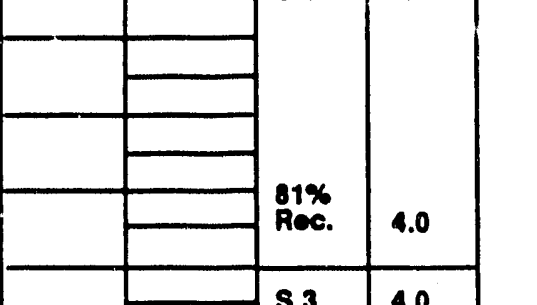

s 3

4.0

JOB NO:

G.8163

PAGE NU: $10 F 2$

LOCATION: SEE PLAN

ELEVATION:

DATE START: $09-14-88$

DATE FINISH: 09-14-88

DRILLER: J.RENDER

PREPARED BY: R.J.HUNT

$-5.0$

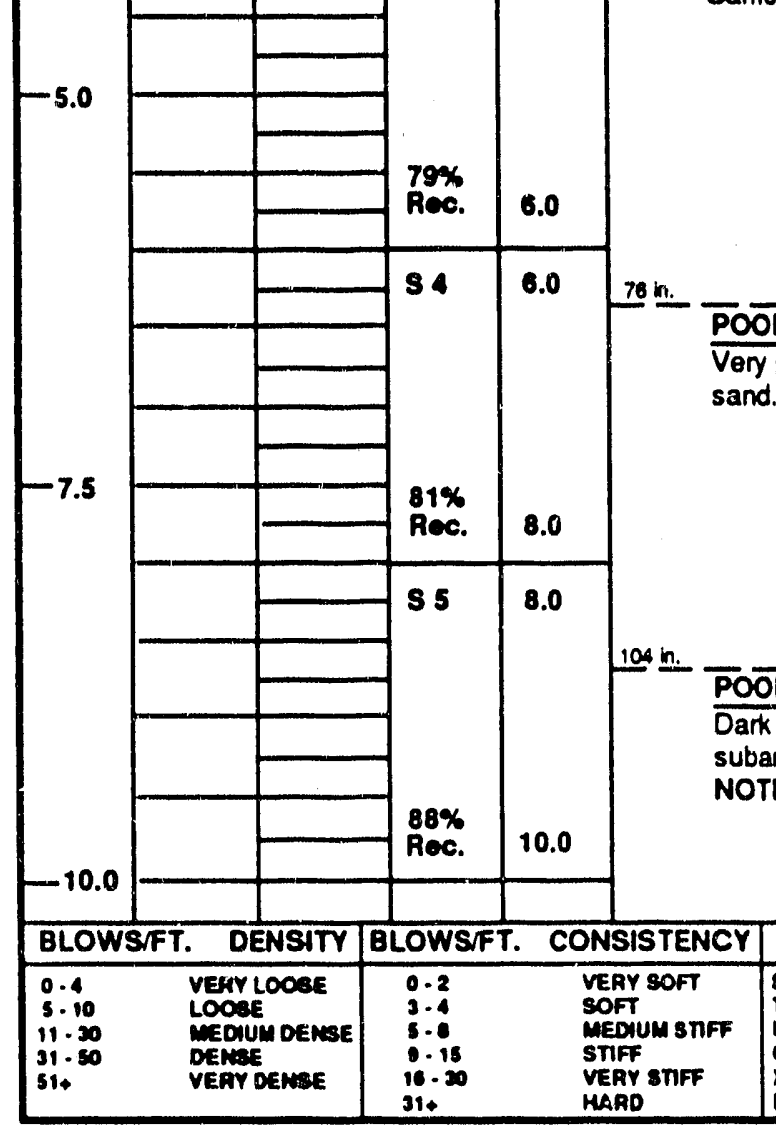

Samo as abovo

yellowish orange to light brown, moist, mostly fine-grained, angular to subangular quartz sand, fow silty clay.

NOTE: Molded appearanco. 


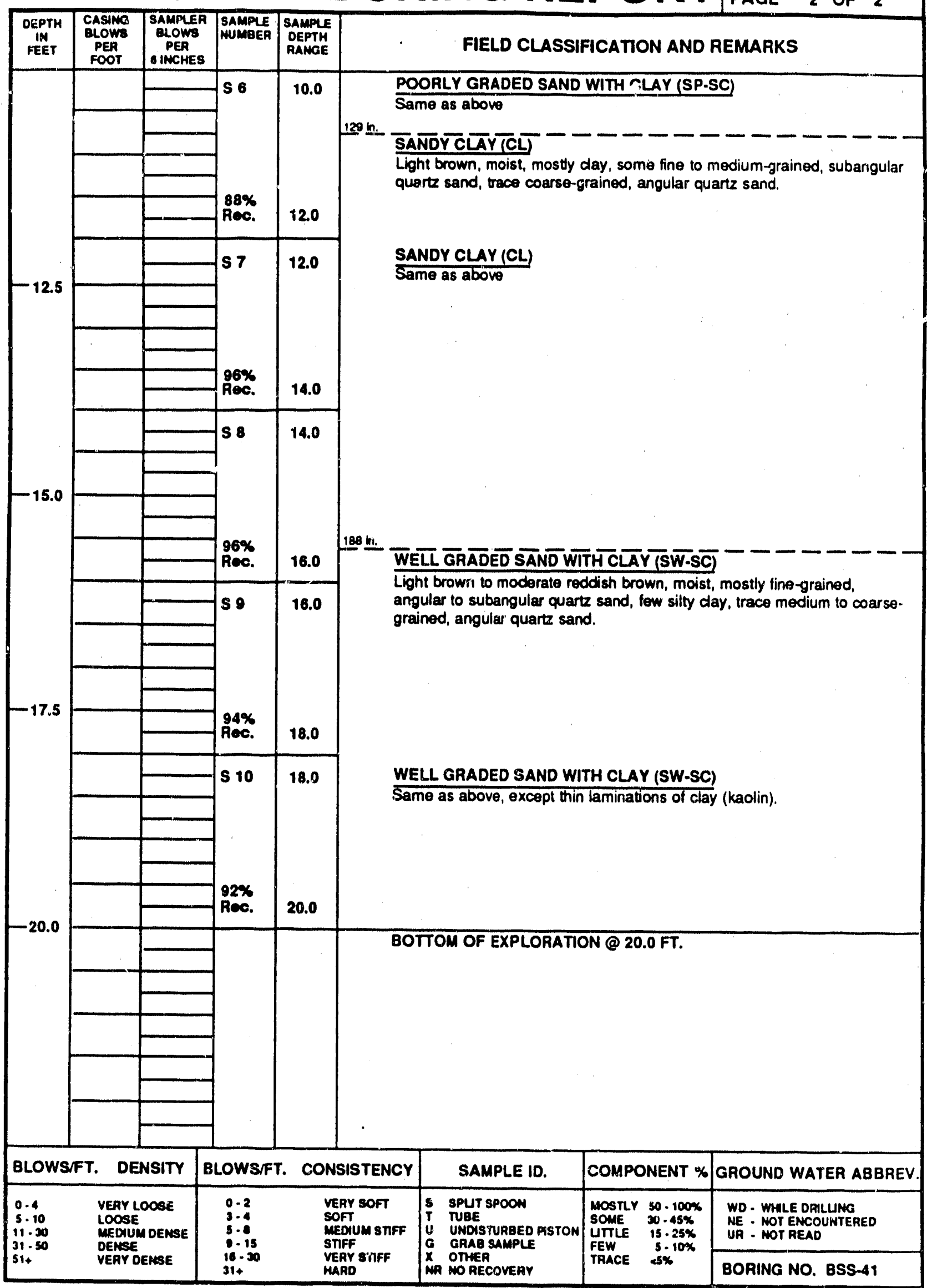


CONSULTANTS

TEST BORING REPORT

BORING NO. BSS-42

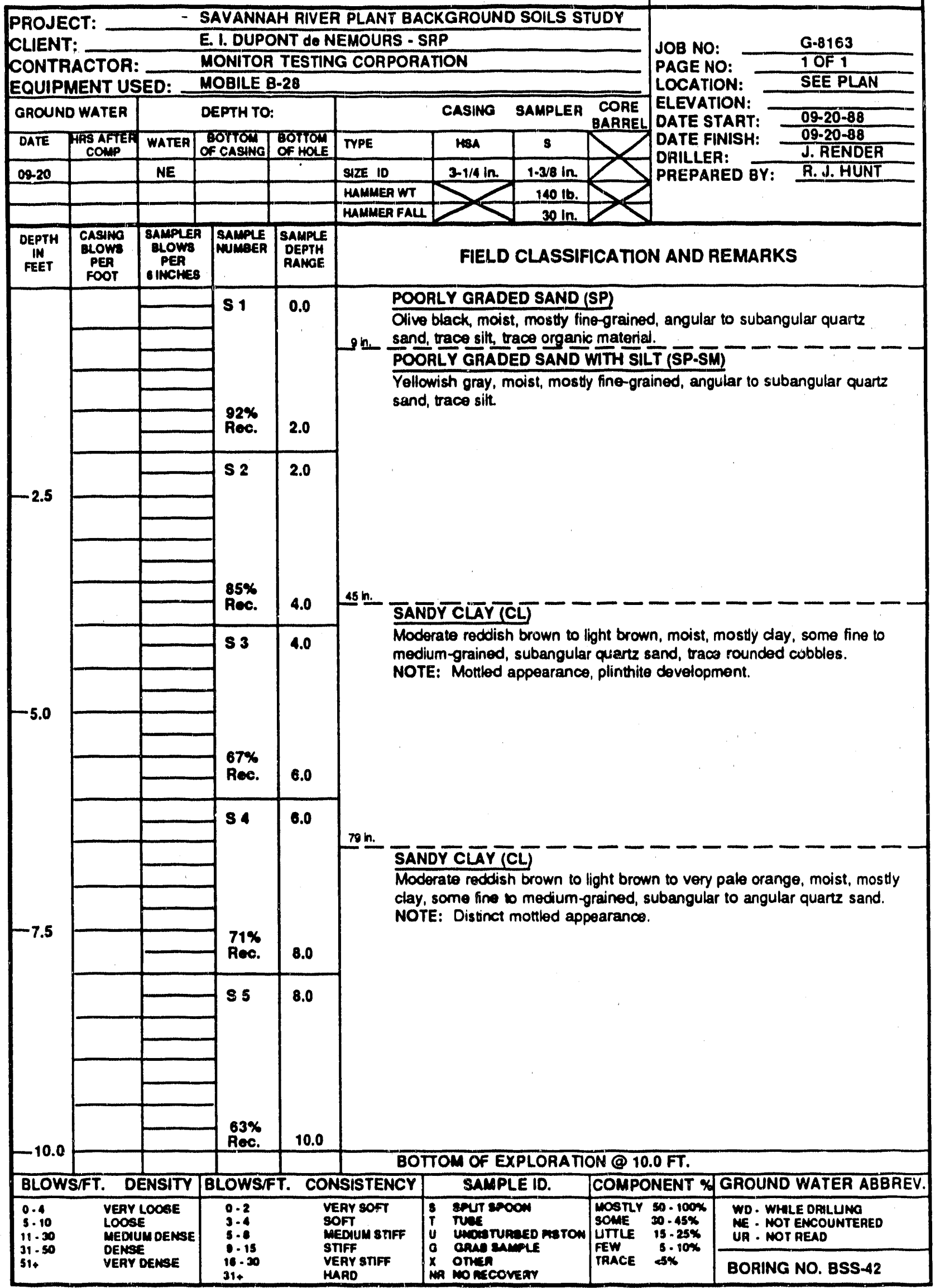


TEST BORING REPORT

PROJECT:

CLIENT: CONTRACTOR: EQUIPMENT USED: GROUND WATER

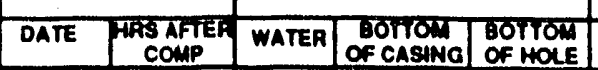

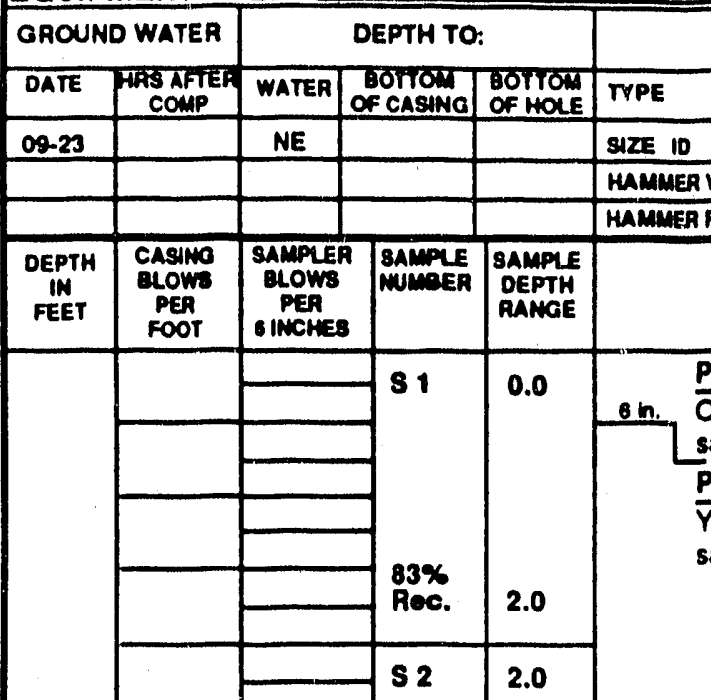

$-2.5$

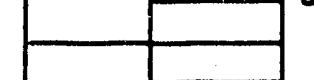

E. I. DUPONT dO NEMOURS - SRP MONITOA TESTING CORPORATION MOBILE B.28

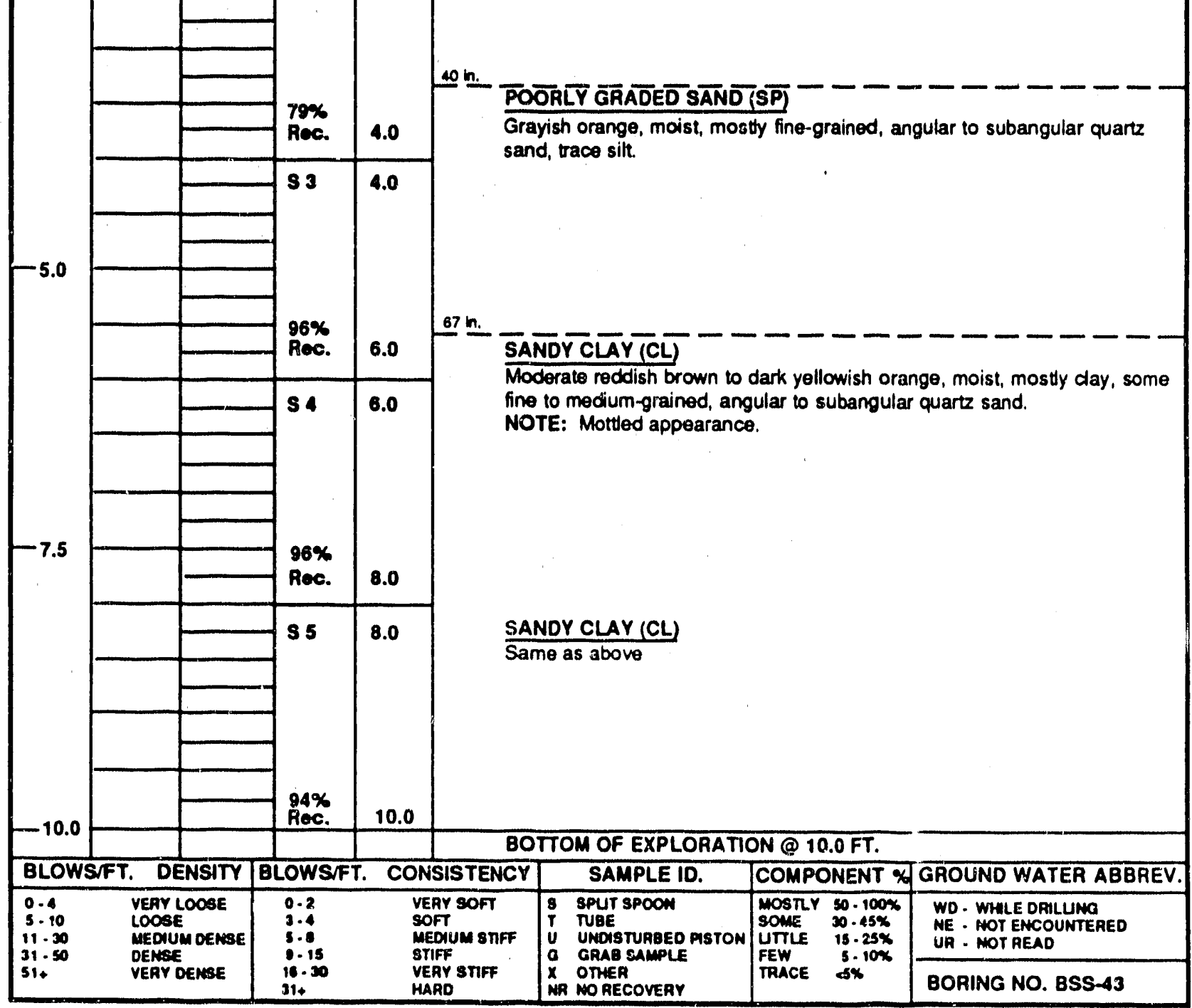

BORING NO. BSS-43

JOB NO: G-8163

PAGE NO: 1 OF 1

ELEVATION:

DATE START: $09-23.88$

PREPARED BY: R.J.HUNT LOCATION: SEE PIAN

DATE FINISH: $09-23-88$

DRILLER:
POORLY GRADED SAND (SP)

Olivo black, moist, mostly fine-grained, angular to subangular quartz sand, trace silt, trace organic material.

POORLY GRADED SAND (SP)

Yellowish gray, moist, mostly fine-grained, angular to subangular quartz sand, trace silt.

\section{FIELD CLASSIFICATION AND REMARKS}




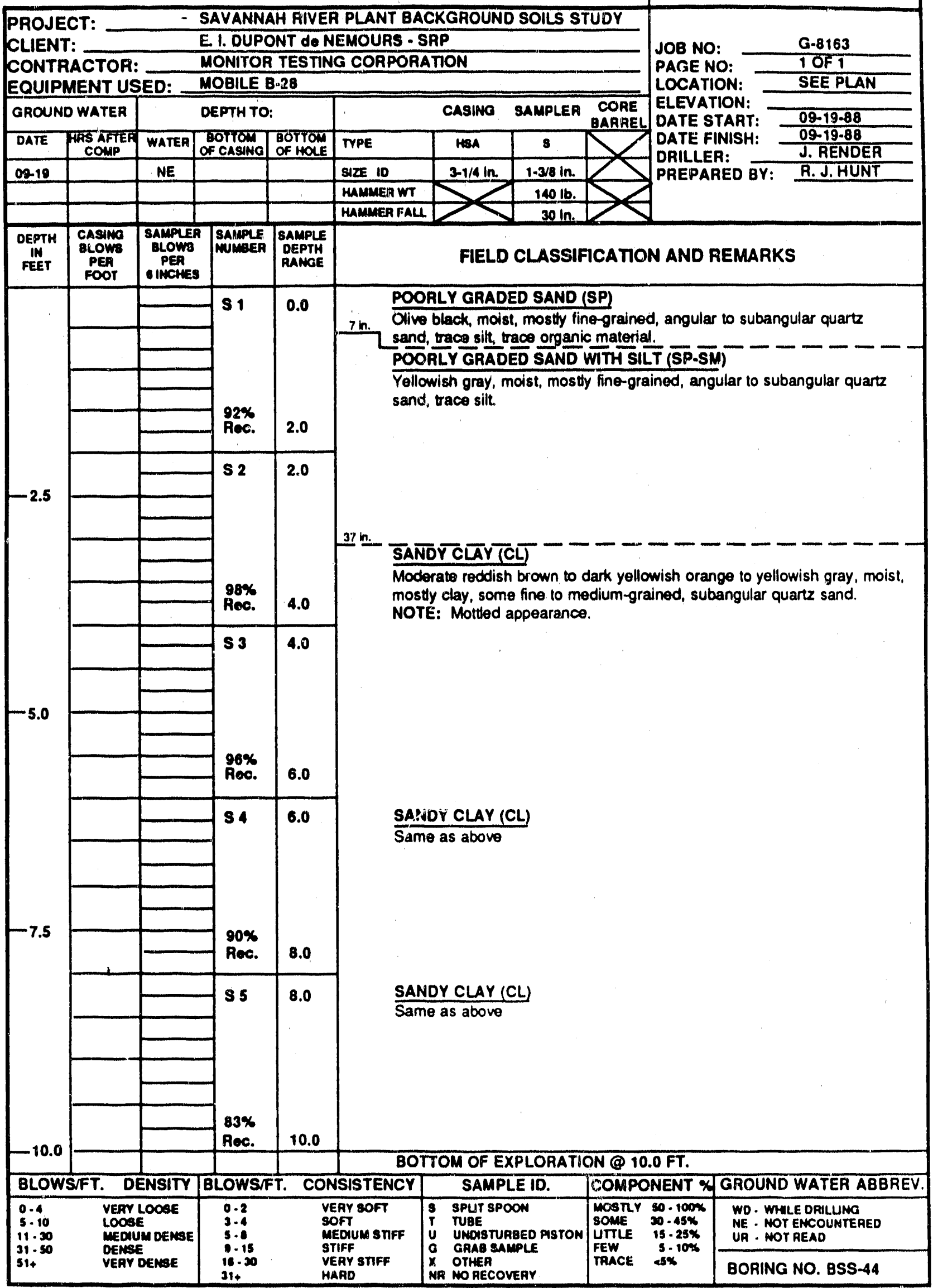




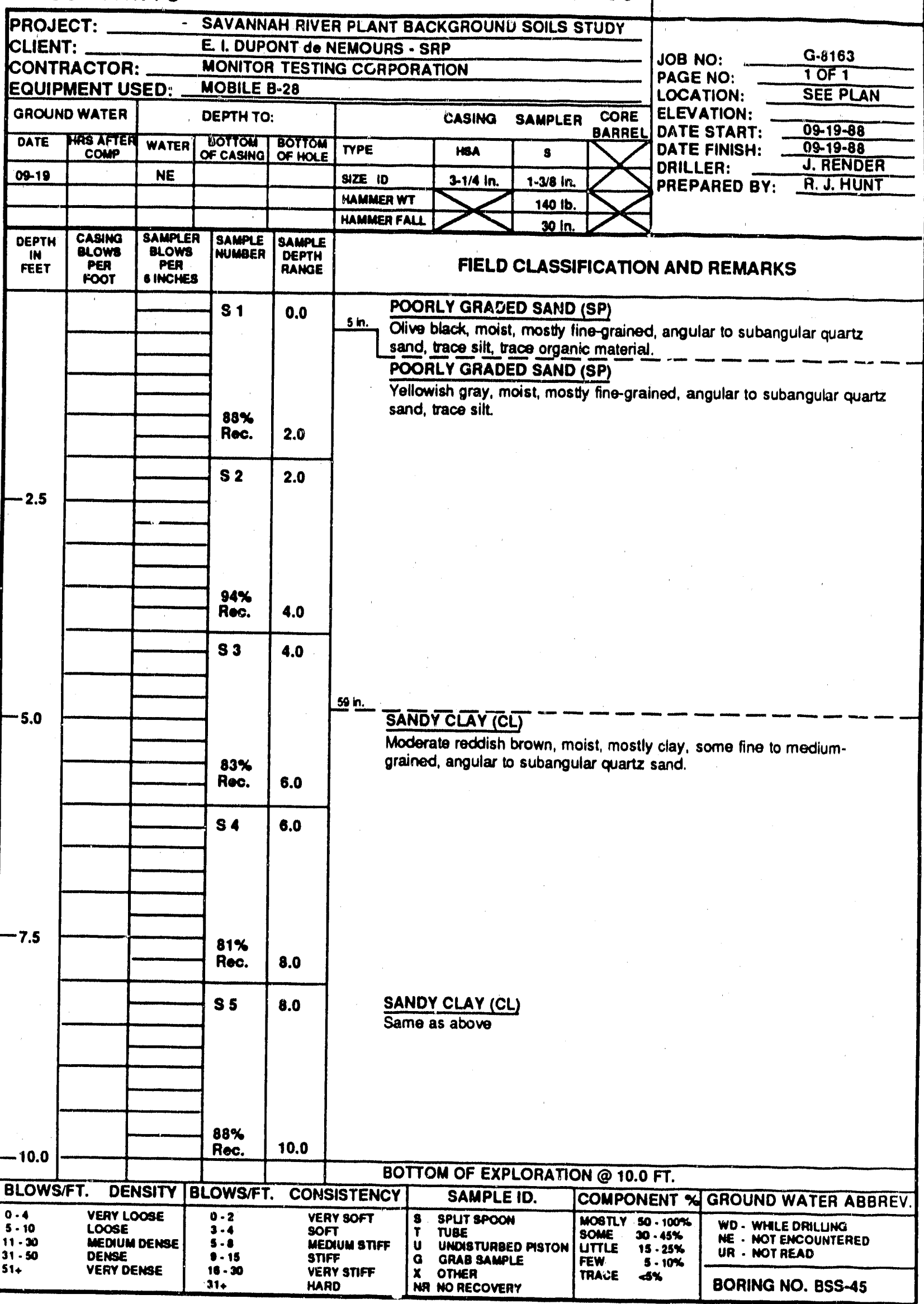


TEST BORING REPORT

SAVANNAH RIVER PLANT BACKGROUND SOILS STUDY

E. I. DUPONT de NEMOURS - SRP

CLIENT: MONITOR TESTING CORPORATION

CONTRACTOR: MOBILE B.28 EQUIPMENT USED: GROUNO WATER

\begin{tabular}{|c|c|c|c|c|c|c|c|c|}
\hline \multicolumn{2}{|c|}{ GROUND WATER } & \multicolumn{3}{|c|}{ DEPTH TO: } & \multicolumn{2}{|r|}{ CASING } & \multirow{2}{*}{\multicolumn{2}{|c|}{$\begin{array}{c}\text { SAMPLER } \begin{array}{c}\text { CORE } \\
\text { BARAEL }\end{array} \\
\mathrm{s}\end{array}$}} \\
\hline DGTE & $\begin{array}{l}\text { TAS AFFE } \\
\text { COMP }\end{array}$ & WATER & $\begin{array}{l}\text { Bofton } \\
\text { Of CASINO }\end{array}$ & $\begin{array}{l}\text { EOTTOW } \\
\text { Of HOLE }\end{array}$ & TYPE & HSA & & \\
\hline $09-75$ & & NE & & & SIZE 10 & $3-1 / 4 \mathrm{in}$. & 1-3/8 in. & \\
\hline $09-18$ & & NE & & & HAMAAEA WT & & $140 \mathrm{lb}$. & \\
\hline & & & & & HAMMEA FALL & & $30 \mathrm{ln}$ & \\
\hline
\end{tabular}

\begin{tabular}{c|c|c|c|c}
\hline DEPTH & $\begin{array}{c}\text { CASIMG } \\
\text { BLOWS } \\
\text { IN } \\
\text { PEET }\end{array}$ & $\begin{array}{c}\text { SAMPLER } \\
\text { BLOWS } \\
\text { PEER } \\
\text { PEOT }\end{array}$ & $\begin{array}{c}\text { SAMPLE } \\
\text { MUMBER }\end{array}$ & $\begin{array}{c}\text { SAMPLE } \\
\text { OEPTH } \\
\text { RAMGE }\end{array}$ \\
\hline
\end{tabular}

\begin{tabular}{l} 
PER \\
FOOT PER \\
\hline INCHES
\end{tabular}

$-2.5$

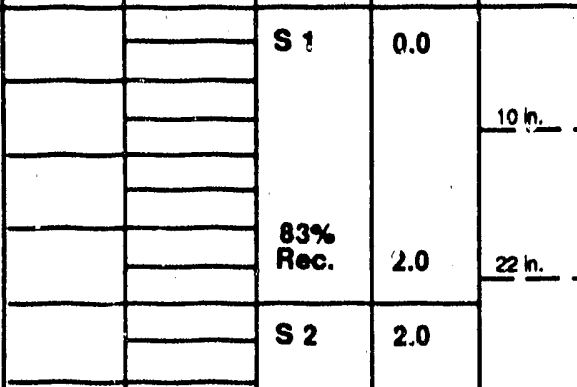

$-5.0$

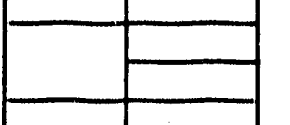

$-7.5$

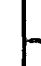
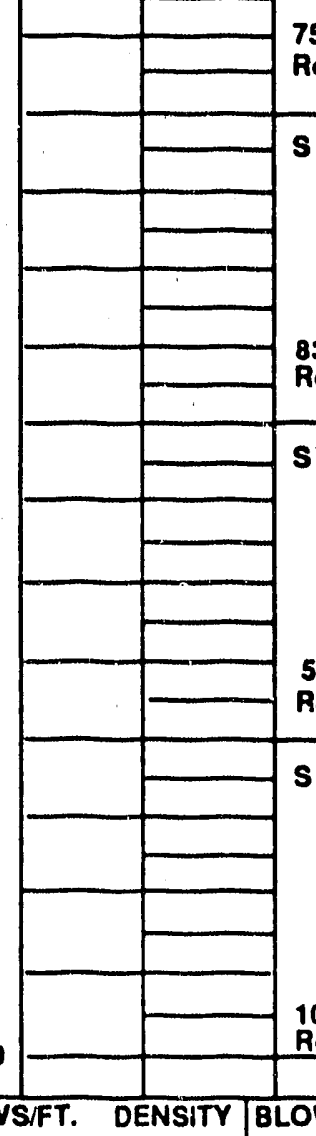

$75 \%$

Rec. 4.0

$\mathbf{S} 3$

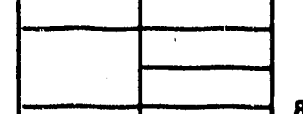

$83 \%$

Rec. 6.0

$\mathbf{s} 4$

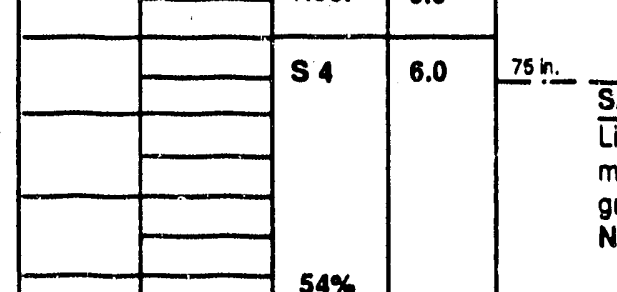

SANDYTCLAY (CL)

Light brown to dark yellowish orange, dry, mostly clay, some fine to medium-grained, angular to subangular quartz sand, trace coarsegrained, angular to subangular quartz sand. NOTE: Mottled appearance. POOALY GRADED SAND (SP)

Olive black, moist, mostly fine-grained, subangular to angular quartz sand, trace silt, trace organic material.

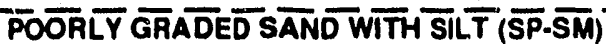
Light brown, moist, mostly fine-grained, subangular to angular quarzz sand, few clayey silt.

SANDY CLAY $\overline{(C L)}$

Light brown to moderate reddish brown, moist, mostly clay, some finegrained, subangular quartz sand.
BORING NO. BSS-46

ELEVATION: $09-15-88$

DATE FINISH: $09-16-88$

DAILLER: J.RENDER

PREPA.RED BY: R.J.HUNT
JOB NO: $\quad$ G-8163

PAGE NO: 1 OF 2

LOCATION: SEE PLAN

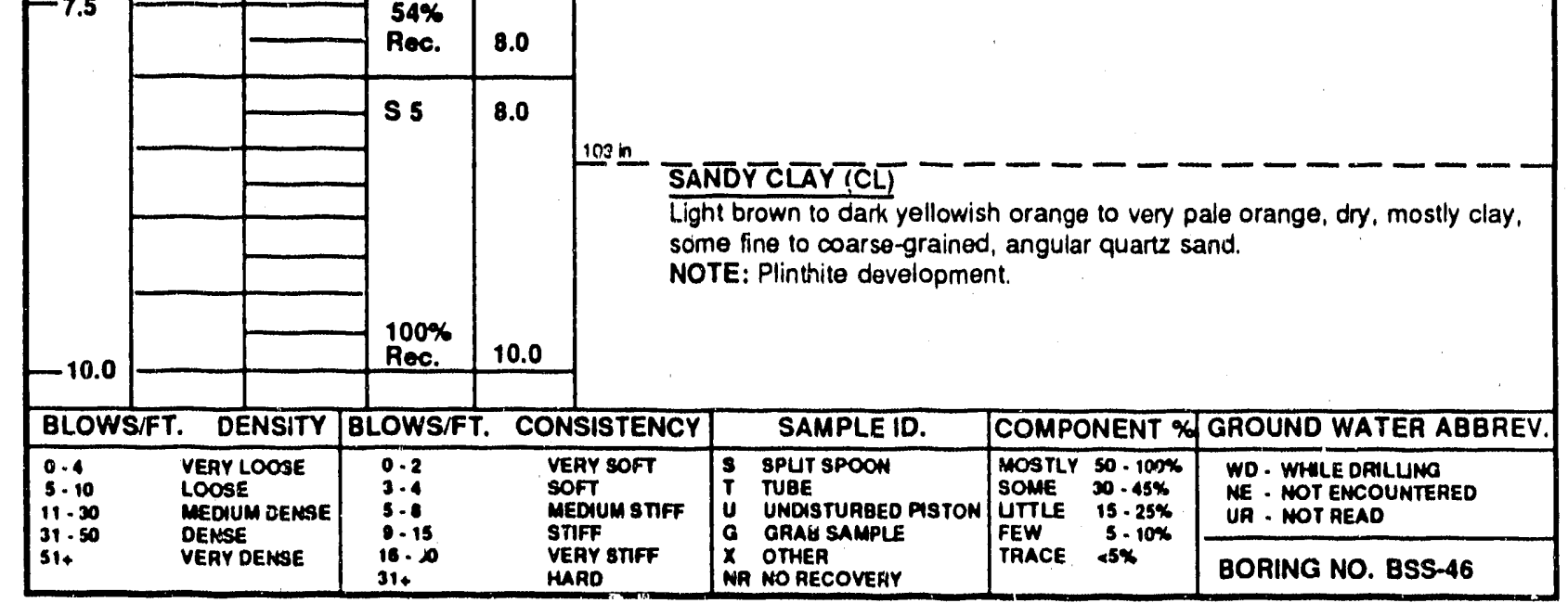


ENVIRONMENTAL CONSULTANTS

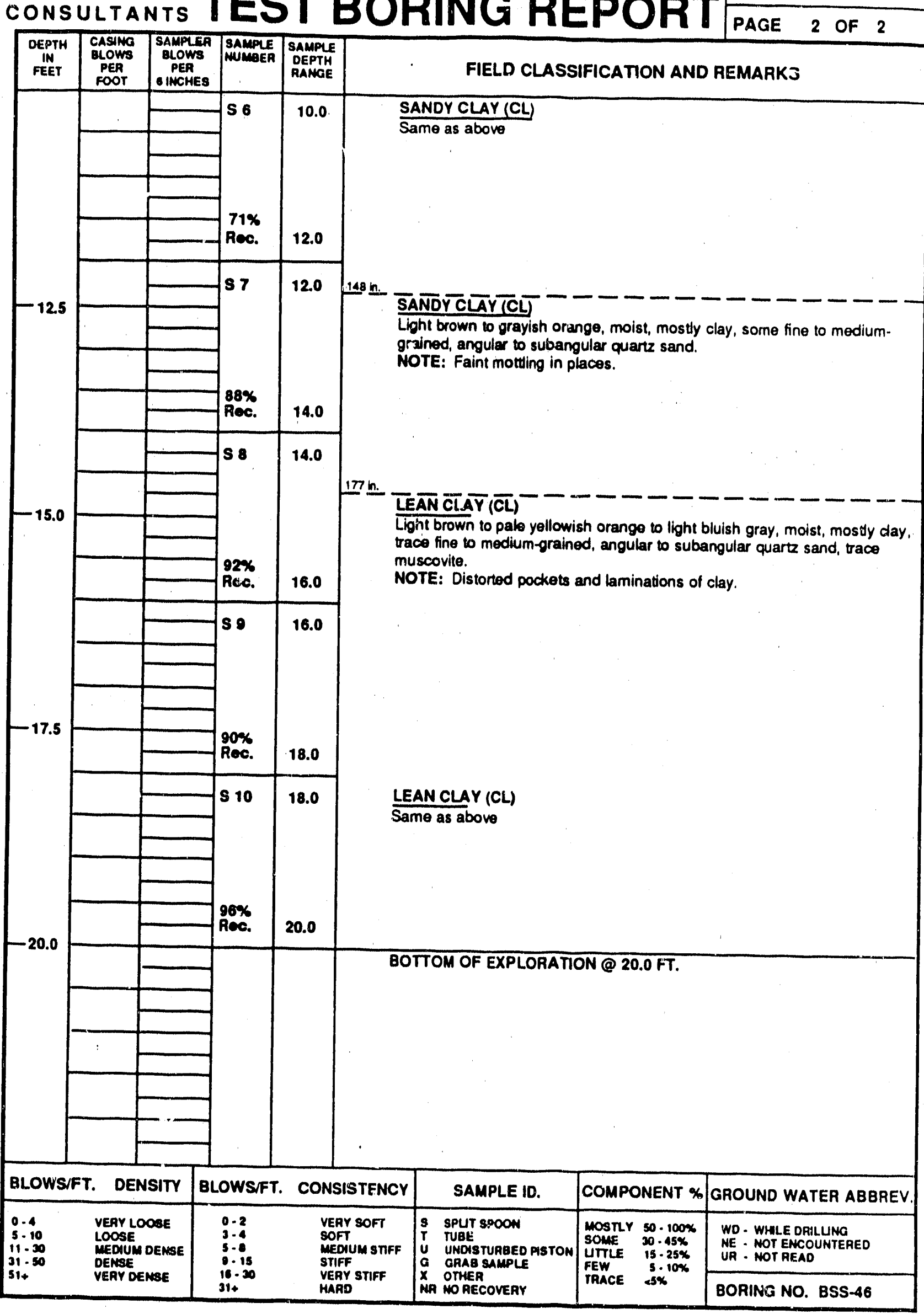




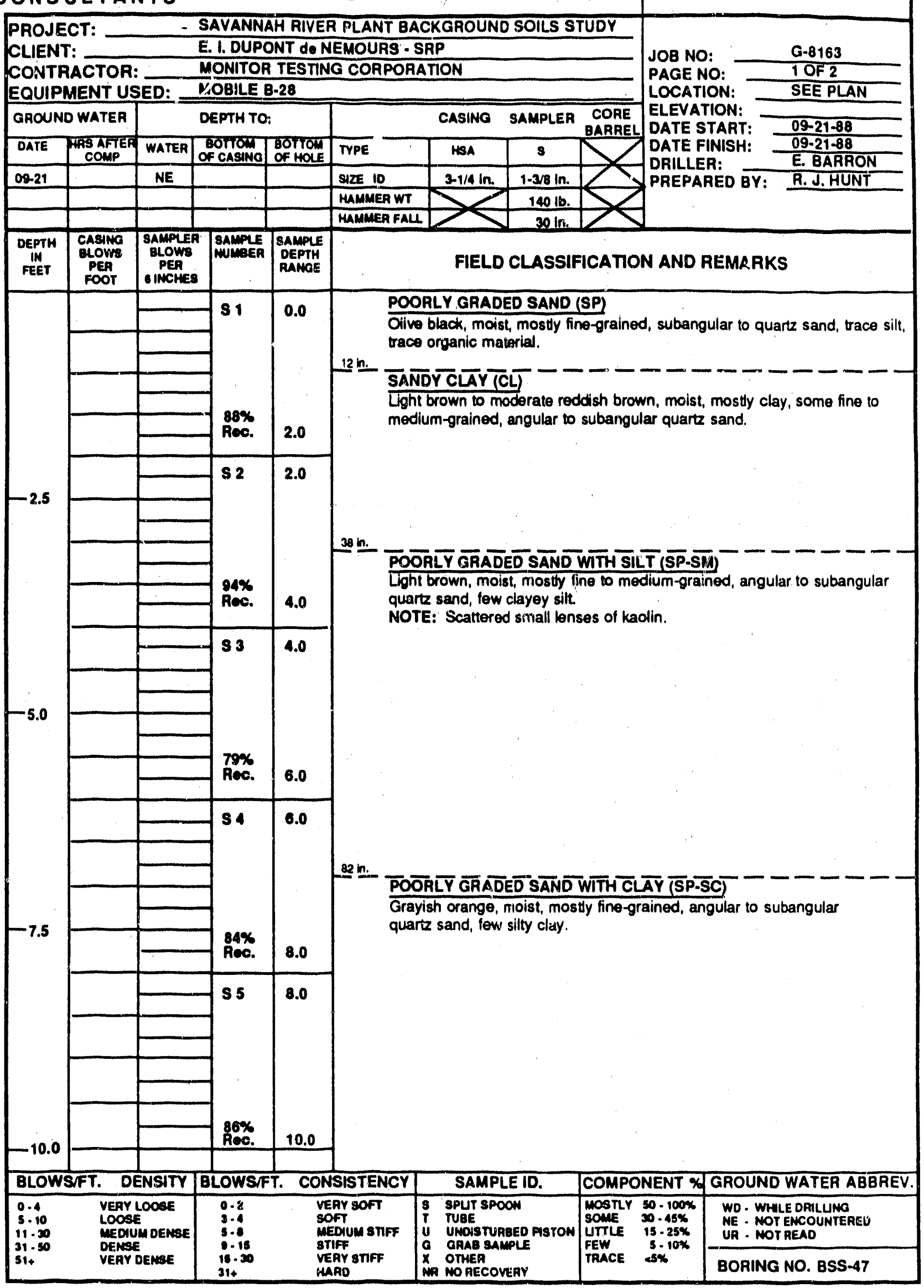




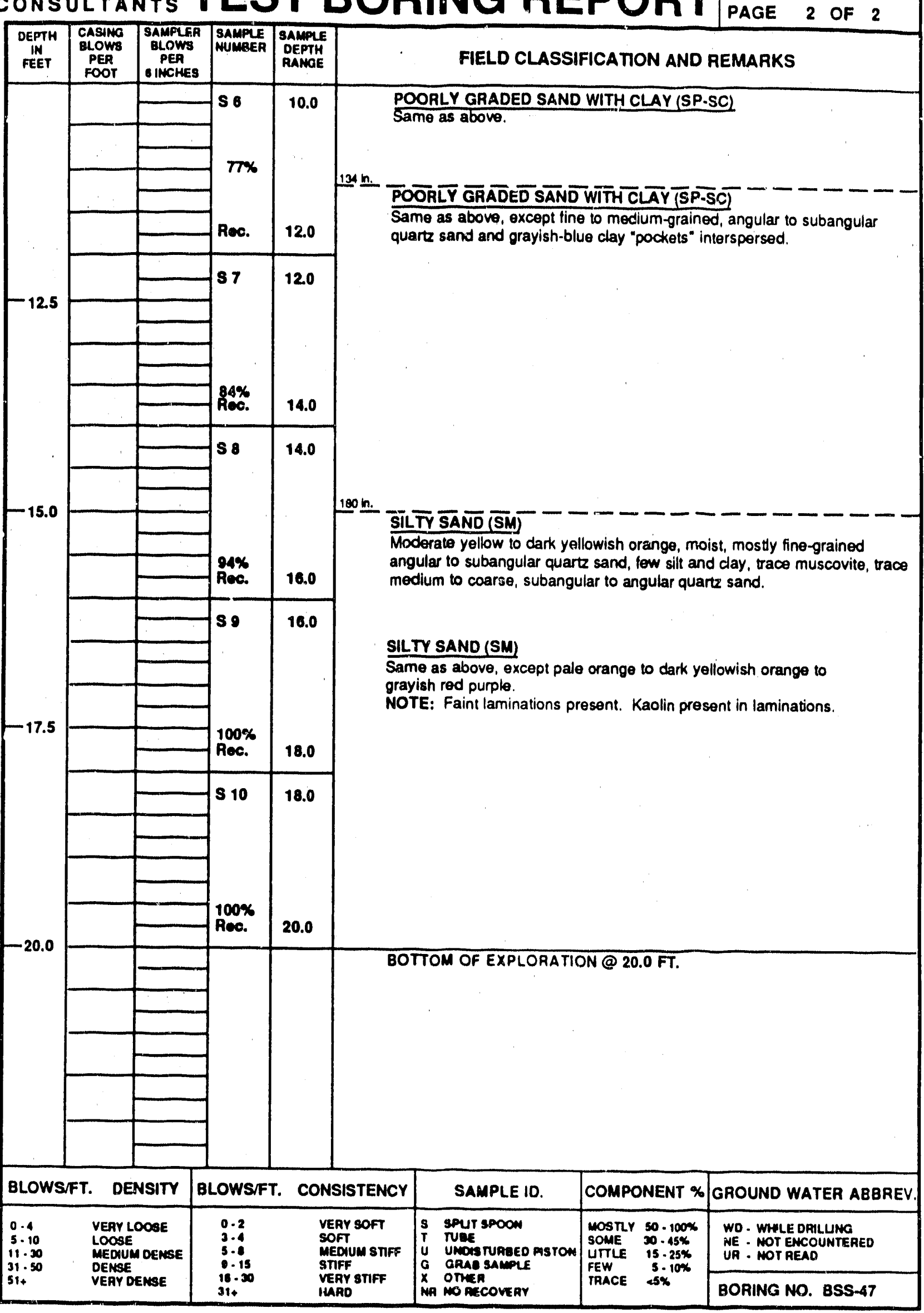


TEST BORING REPORT

PROJECT:

CLIENT:

CONTRACTOR:

E. I. DUPONT dO NEMOURS - SRP
MONITOR TESTING CORPOAATIOM

EQUIPMENT USED: GHOUND WATER

DATE TAS AFTEH WATER

MOBILE B.28

\begin{tabular}{|c|c|c|c|c|c|c|c|c|}
\hline & & \multicolumn{2}{|r|}{ 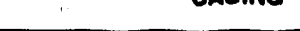 } & \\
\hline DATE & $\begin{array}{l}\text { HAS AFTEH } \\
\text { COMP }\end{array}$ & WATER & $\begin{array}{l}80 \text { Tiom } \\
\text { Of CASING }\end{array}$ & $\begin{array}{l}\text { BofTom } \\
\text { OF HOLE }\end{array}$ & TYPE & HSA & 8 & \\
\hline $\cos 21$ & & NE & & & SIZE ID & $3-1 / 4$ ln. & $1-3 / 8 \mathrm{ln}$ & \\
\hline & & & & & MAMMER WT & & $140 \mathrm{lb}$ & \\
\hline & & & & & HAMMEA FALL & & $30 \mathrm{ln}$. & \\
\hline DEPTH & $\begin{array}{l}\text { CASING } \\
\text { OLOWB }\end{array}$ & $\begin{array}{l}\text { SAMPLE } \\
\text { BLWWB }\end{array}$ & $\begin{array}{l}\text { A SAMPLE } \\
\text { MUMBER }\end{array}$ & SAMPLE & & & & \\
\hline
\end{tabular}

FIELD CLASSIFICATION AND REMARKS

POORLY GRADED SAND (SP)

Olive black, moist, mosty fine-grained, angular to subangular quartz sand, trace silt, trace organic material.

POORLY GRADED SAND (SP)

Light brown to dark yellowish orange, moist, mostly fine-grained, angular to subangular quartz sand, trace clayey silt, trace opaques.

POORLY GRADED SAND (SP)

Same as above

$-2.5$

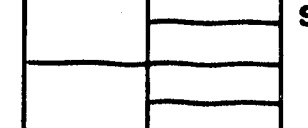

$83 \%$

Rec. 2.0

\begin{tabular}{l|l}
$\mathbf{S} 2$ & 2.0
\end{tabular}

$-5.0$

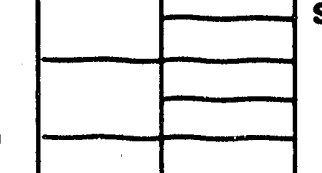

$75 \%$

Rec.

83

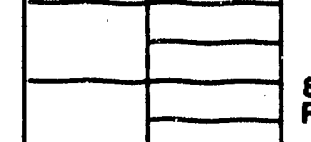

$88 \%$

Roc.

$\mathbf{S} 4$

6.0

$-7.5$

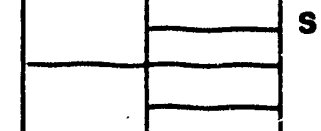

$-10.0$

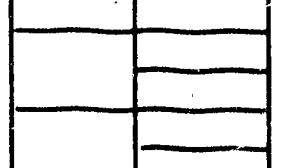

6.0

POORLY GAADED CAR 'D (SP)

Same as above, except light brown.

POORLY GRADED SAND (SP)

Same as above
BOHING NO. BSS-48

JOBNO: G-8163

LOCATION:

SEE PLAN

DATE START:

PREPARED BY: R.J.HUNT
PAGE NO: 1 OF 1

$09-21-88$

DATE FINISH:

DRILLER: E. BARRON
POORLY GRADED SAND $S \overline{S P}$

Same as above, except some thin horizons of very pale orange sand.

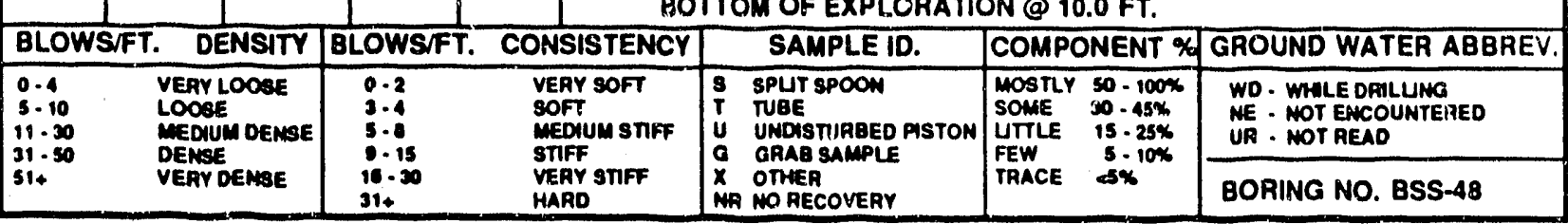


TEST BORING REPORT

PROJECT:

CLIENT:

CONTRACTOR:

SAVANTAAH RIVER PLANT BACKGROUND SOILS STUDY

E. L. OUPONT dO NEMOURS : SRP

EQUIPMENT USED: MONITOR TESTING CORPORATION

\begin{tabular}{|c|c|c|c|c|c|c|c|c|}
\hline \multicolumn{2}{|c|}{ GROUND WATER } & \multicolumn{3}{|c|}{ DEPTH TO: } & \multirow[b]{2}{*}{ TYPE } & \multirow{2}{*}{$\begin{array}{c}\text { CAsing } \\
\text { misa }\end{array}$} & \multirow{2}{*}{$\begin{array}{l}\text { SAMPLER } \\
8\end{array}$} & \multirow{2}{*}{$\begin{array}{r}\text { CORE } \\
\text { BARREI }\end{array}$} \\
\hline DATE & $\begin{array}{c}\text { FiSAFTEF } \\
\text { ftomp }\end{array}$ & WATER & $\begin{array}{l}\text { Eortom } \\
\text { of casing }\end{array}$ & $\begin{array}{l}\text { EOTIOW } \\
\text { OF MOLE }\end{array}$ & & & & \\
\hline $05-21$ & & NE & & & Siat 10 & $3-1 / 4 \mathrm{ln}$. & $1-3 / 8 \ln$. & \\
\hline & & & & & Mamines wr & & $140 \mathrm{lb}$. & \\
\hline & & & & & MAMMER FAL & & 39 In. & \\
\hline DEPT & CAsino & sAmpe & salmove & sumpe & & & & \\
\hline
\end{tabular}

\begin{tabular}{|c|c|c|c|c|}
\hline $\begin{array}{l}\text { DEPTH } \\
\text { INEET }\end{array}$ & $\begin{array}{l}\text { CASING } \\
\text { CLOW' } \\
\text { PEA } \\
\text { Foot }\end{array}$ & 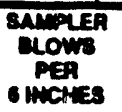 & $\begin{array}{l}\text { gampue } \\
\text { numenes }\end{array}$ & 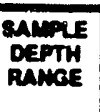 \\
\hline & & & 81 & 0.0 \\
\hline
\end{tabular}

FIELD CLASSIFICATION AND REMARKS

POORLY GRADED SAND (SP)

Olive black, moist, mosty fine-grained, angular to subangular quartz

2. sand, trace sit, trace organic material.

POORLY GRADED SAND (SP)

Grayish orange, moist, mostly fine-grained, angular to subangular quarte sand, trace opeques.

$-2.5$

$-5.0$

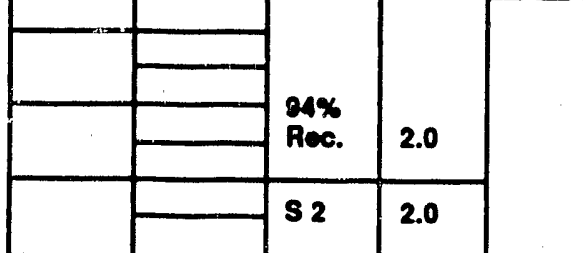

$-7.5$
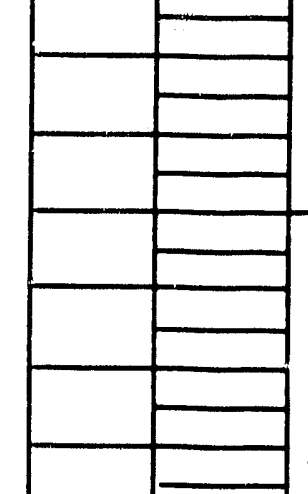

(20)

Rec.

4.0

$41 \mathrm{n}$.

\section{SANDYCTAYT(C)}

Light brown to moderale reddish brown, moist, mostly clay, some fine to medium-grained, angular to subangular quartz sand. NOTE: Motted appearance, plinthite development.

\section{SANDY CLAY (CL)}

Same as above

$77 x$

Rec.

54

6.0

Sameas above

1

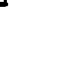

ELEVATION:

DATE START: $-09-21-83$

DATE FINISH: $\frac{09-21-88}{}$

DRILLER:

PREPARED BY: R.J.HUNT

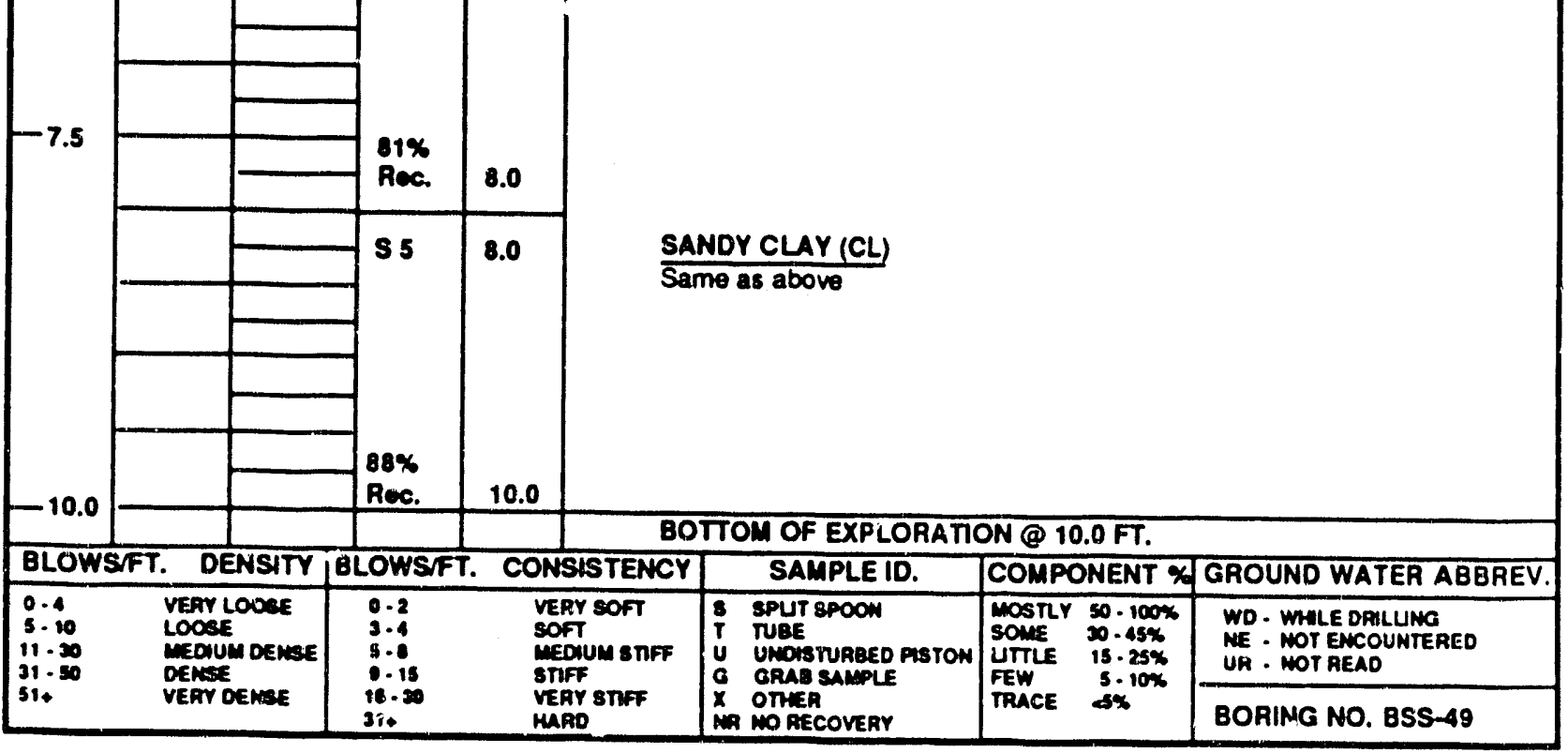


CONSULTANTS

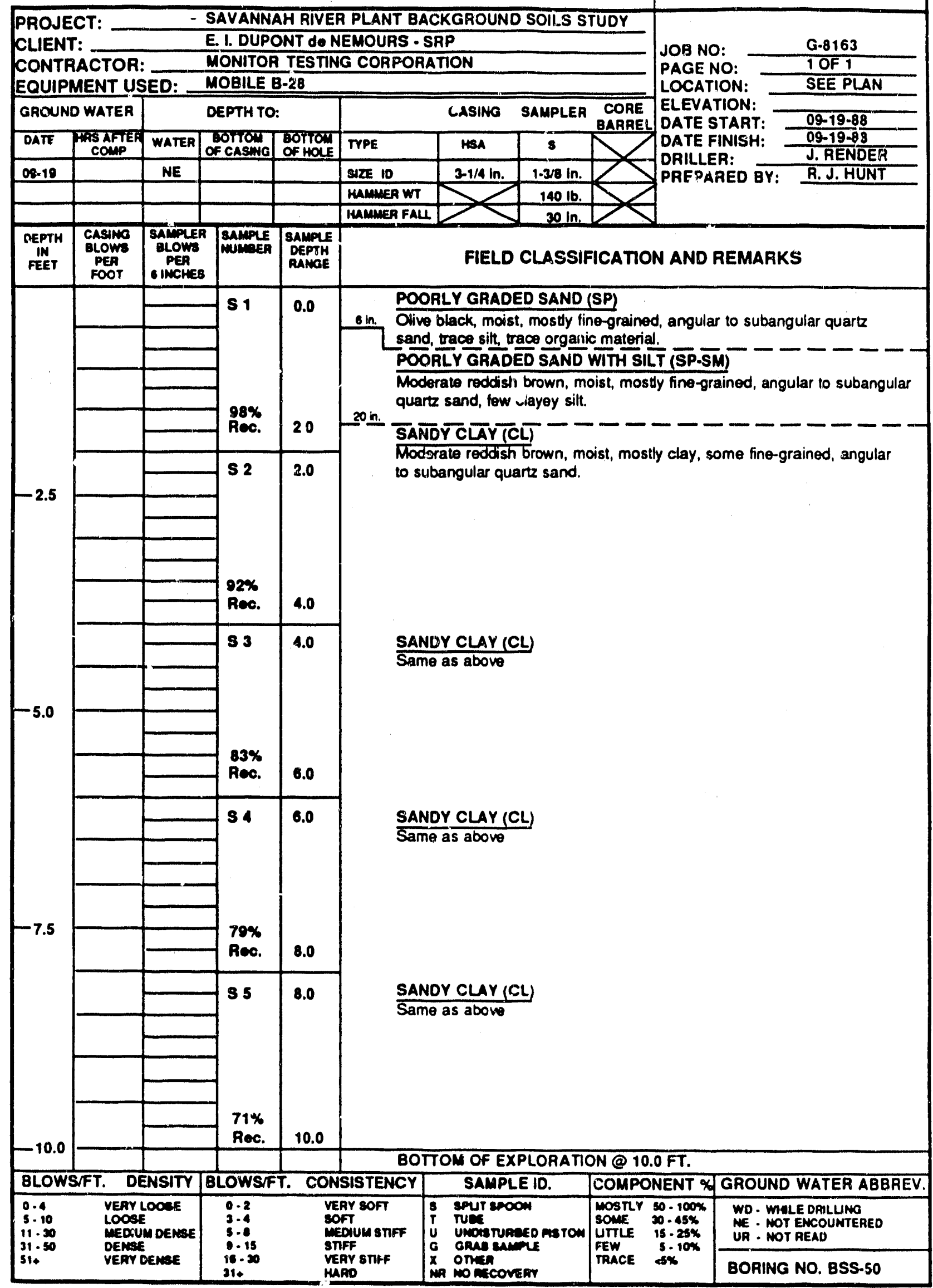


PROJECT:

TEST BORING REPORT

BORING NO. BSS-51

CLIENT:

SAVANNAH RIVER PLANT BACKGROUND SOILS STUDY

CONTRACTOR:

E. I. DUPONT DO NEMOURS - SRP

EOUIPMENT USED: MONITOR TESTING CORPORATION

JOB NO:

G.8163

PAGE NO:

1 OF 2

\begin{tabular}{|c|c|c|c|c|c|c|c|c|}
\hline \multicolumn{8}{|c|}{ EOUIPMENT USED: MOBILE B-28 } & \multirow{4}{*}{$\begin{array}{l}\text { LOCATION: } \\
\text { ELEVATION: } \\
\text { DATE START: } \\
\text { DATE FINISH: } \frac{\text { SEE PLAN }}{\text { O9-19.88 }} \\
\text { DRILLER: } \\
\text { PREPARED BY. }\end{array}$} \\
\hline \multicolumn{2}{|c|}{ GROUND WATER } & \multicolumn{3}{|c|}{ DEPTH TO: } & & CAsino & SAMPLER & \\
\hline DATE & $\begin{array}{c}\text { HAS AFFIEF } \\
\text { comp }\end{array}$ & WATER & $\begin{array}{l}\text { bortov } \\
\text { of casinga }\end{array}$ & $\begin{array}{l}\text { botid } \\
\text { Of Houe }\end{array}$ & TVPE & MSA & $\mathbf{s}$ & \\
\hline $00-10$ & & NE & & & Size 10 & $3-1 / 4 \ln$. & $1-3 / 8 \mathrm{ln}$. & \\
\hline & & & & & HALMEER WT & & $i \leq 0 \mid b$. & \\
\hline & & & & & HAMMER FALL & & & \\
\hline $\begin{array}{l}\text { DEPTH } \\
\text { IN } \\
\text { FEET }\end{array}$ & $\begin{array}{l}\text { CASING } \\
\text { BLOWW } \\
\text { PER }\end{array}$ & $\begin{array}{l}\text { SAMPLE } \\
\text { BLOW } \\
\text { PER }\end{array}$ & 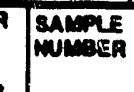 & $\begin{array}{l}\text { SAMPLE } \\
\text { DEPTH } \\
\text { RAMGE }\end{array}$ & & & 7. & \\
\hline
\end{tabular}

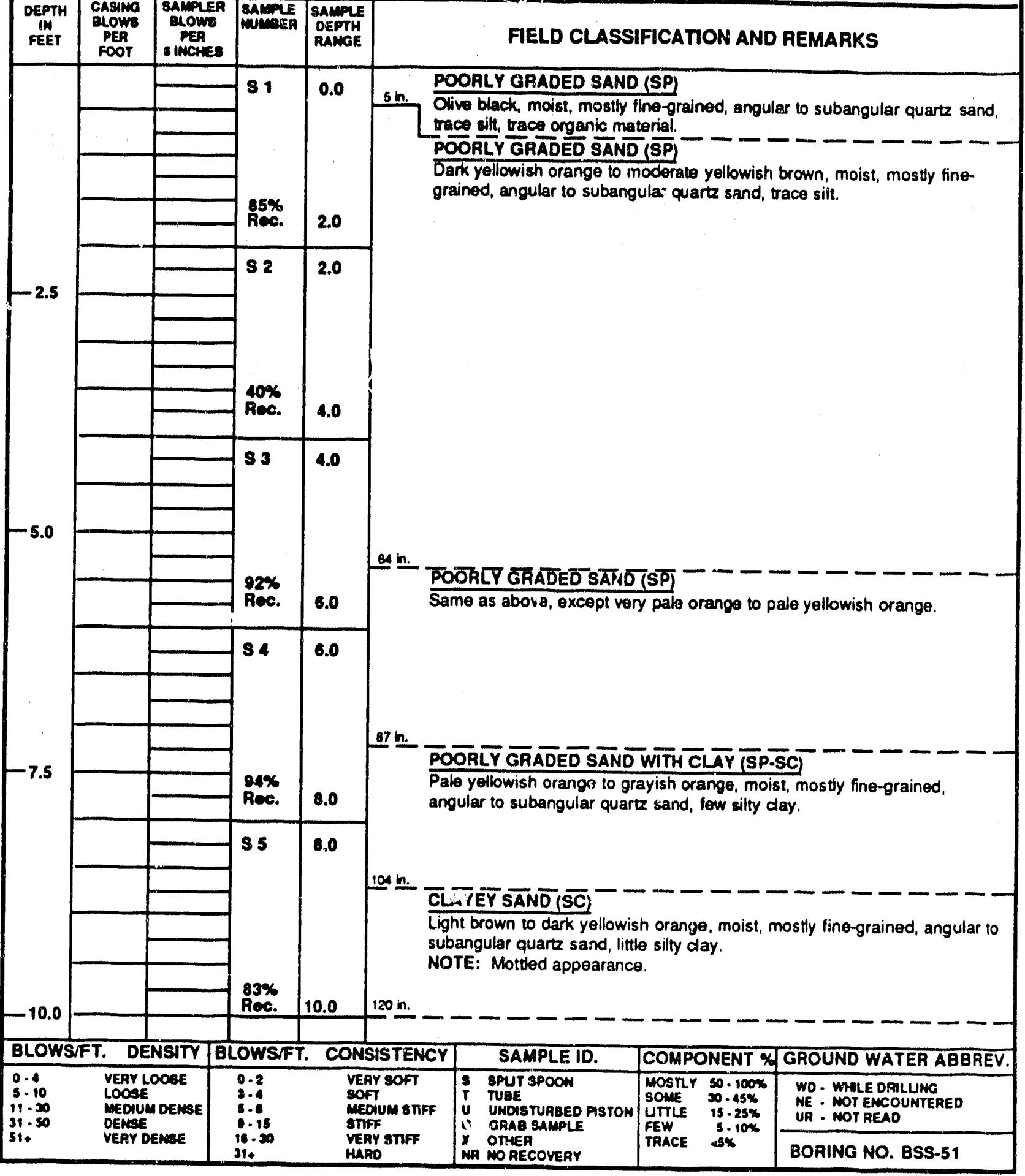


SIRRINE

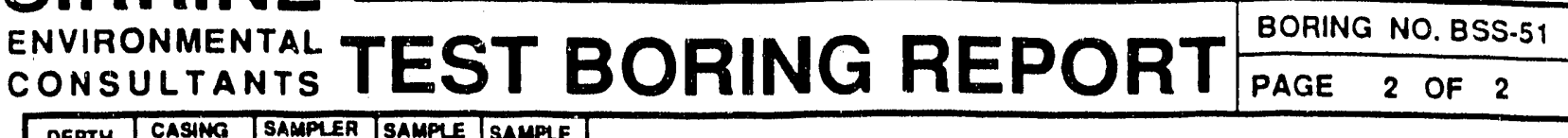

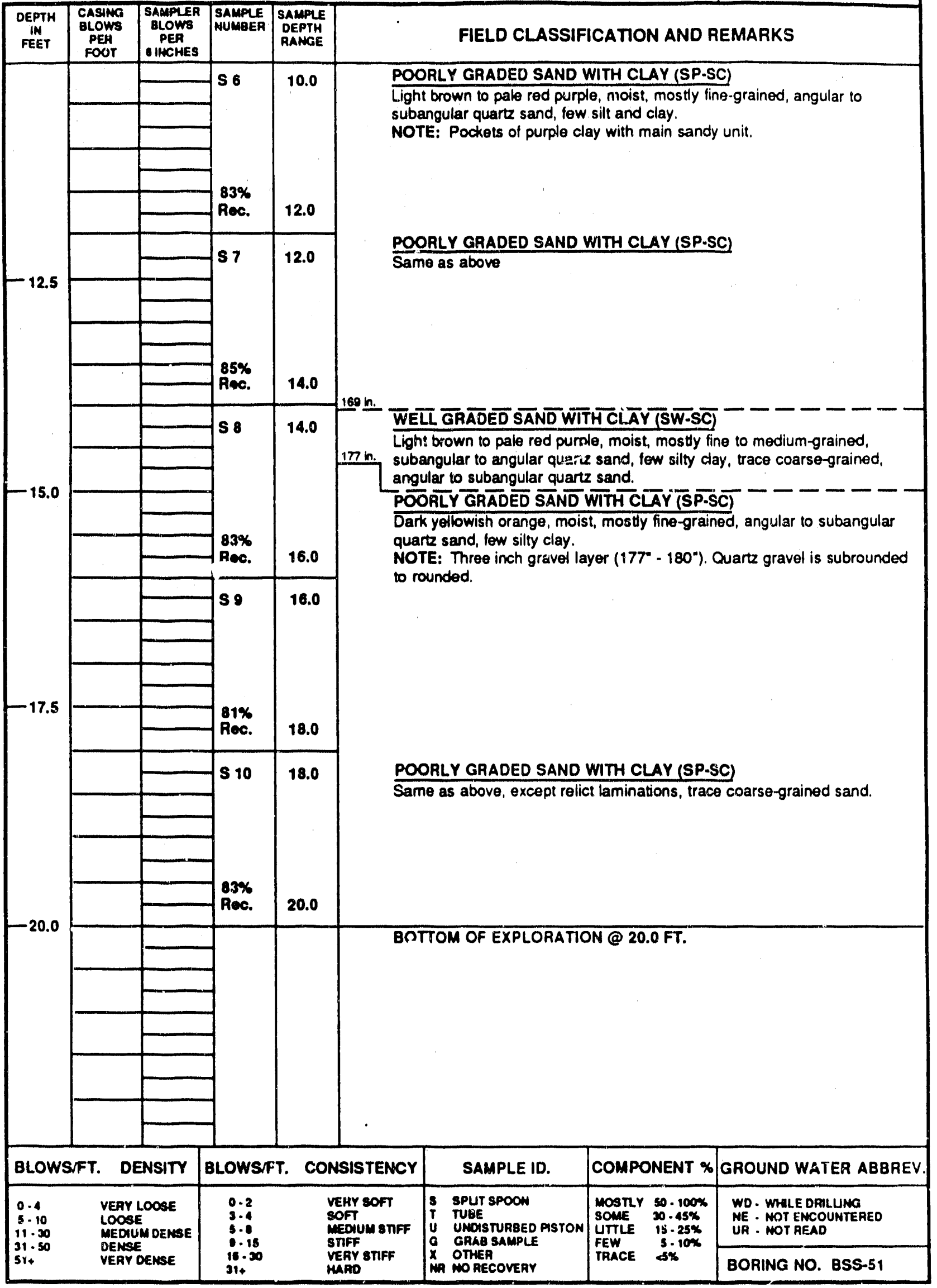


SIRRINE

ENVIRONMENTAL

CONSULTANTS

TEST BORING REPORT

BORING NO. BSS-52

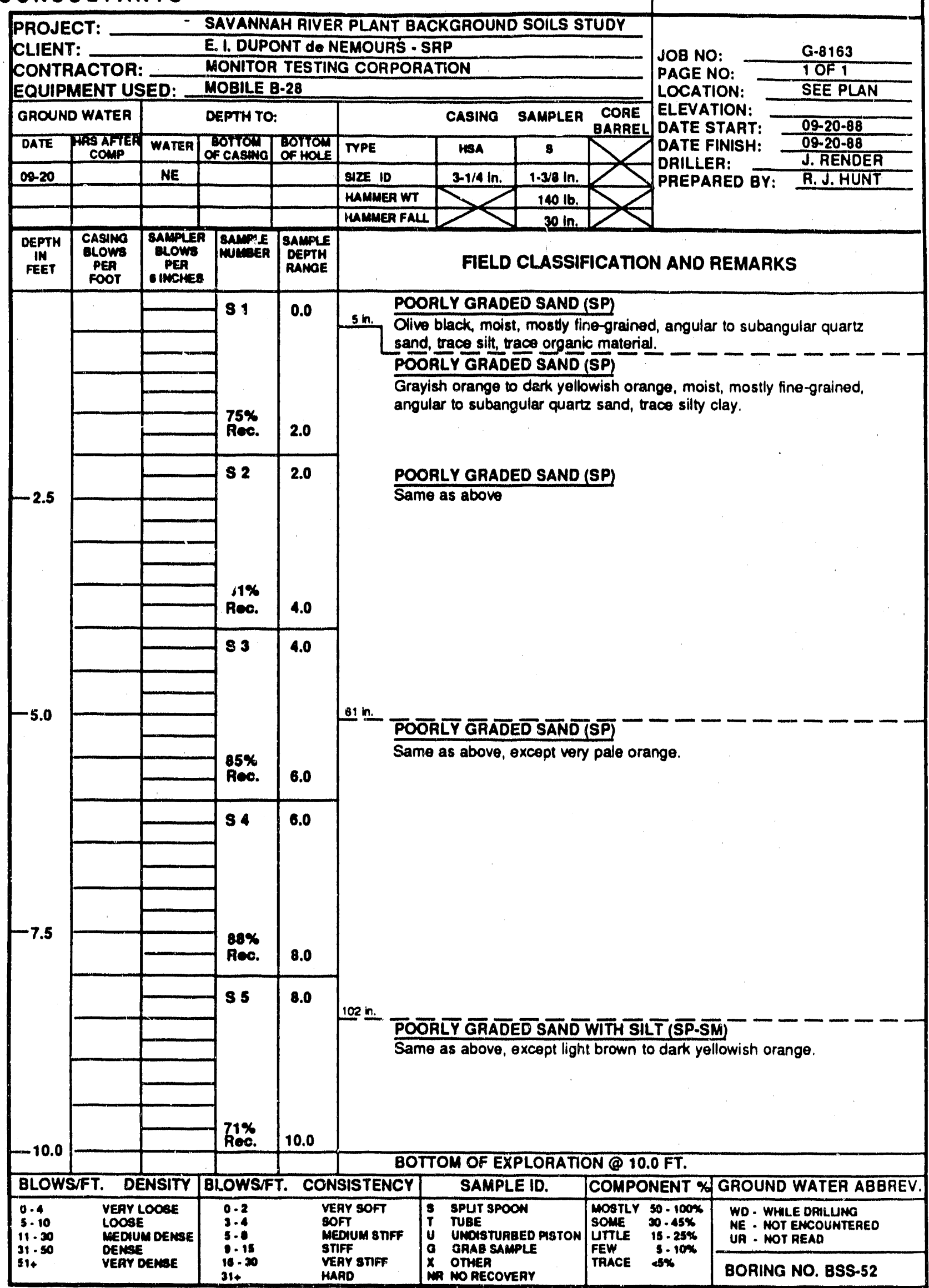




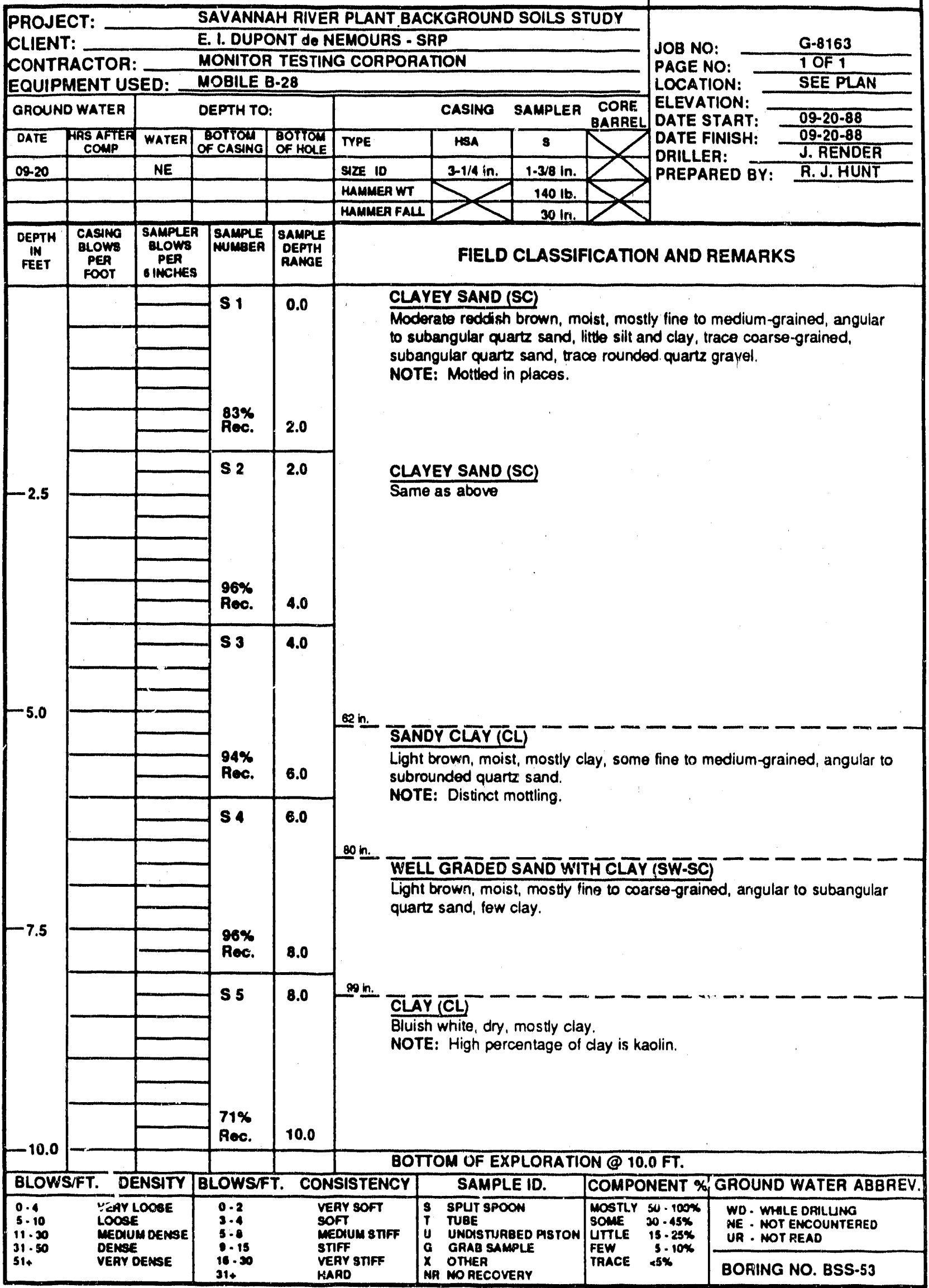


CONSULTANTS

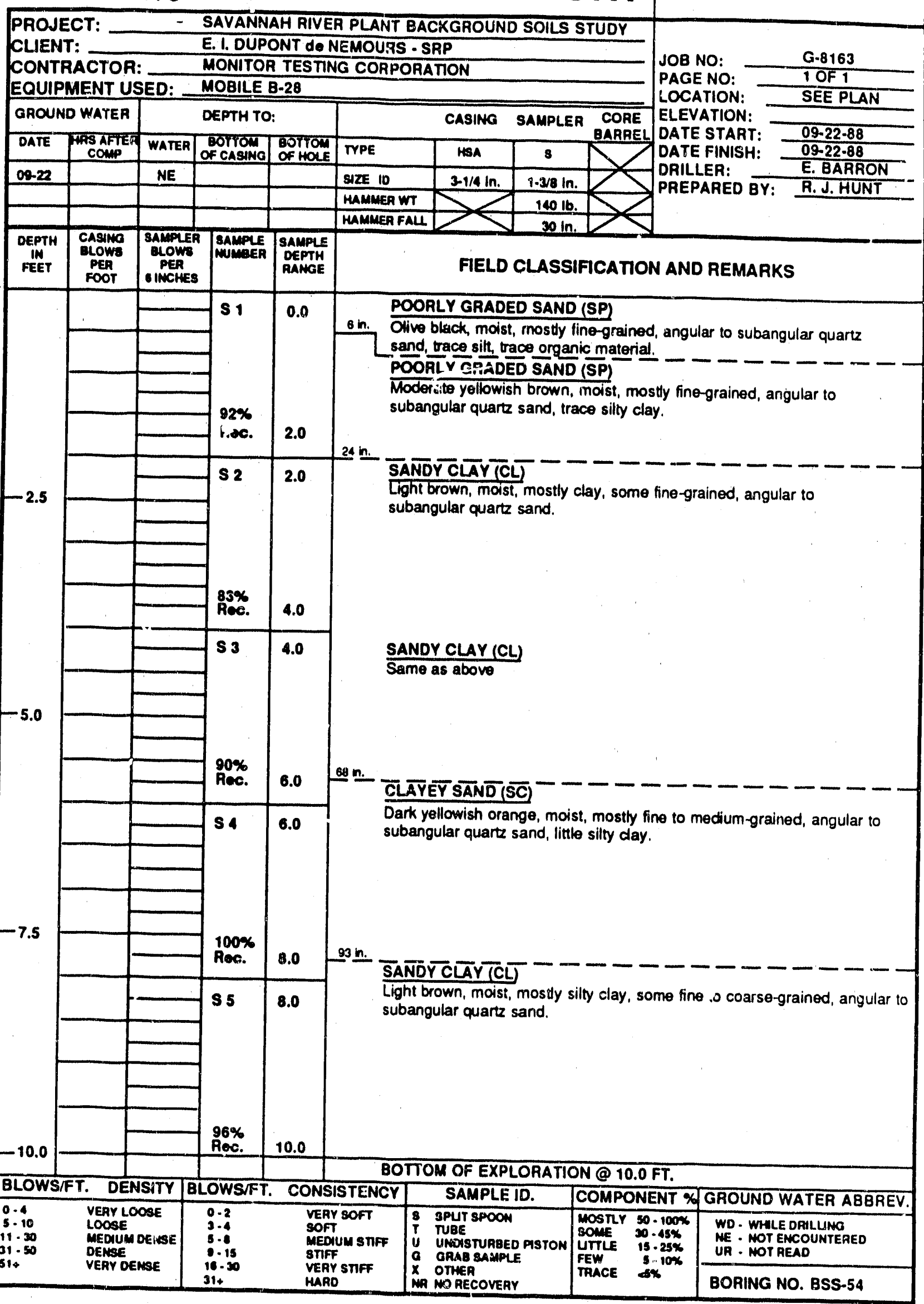




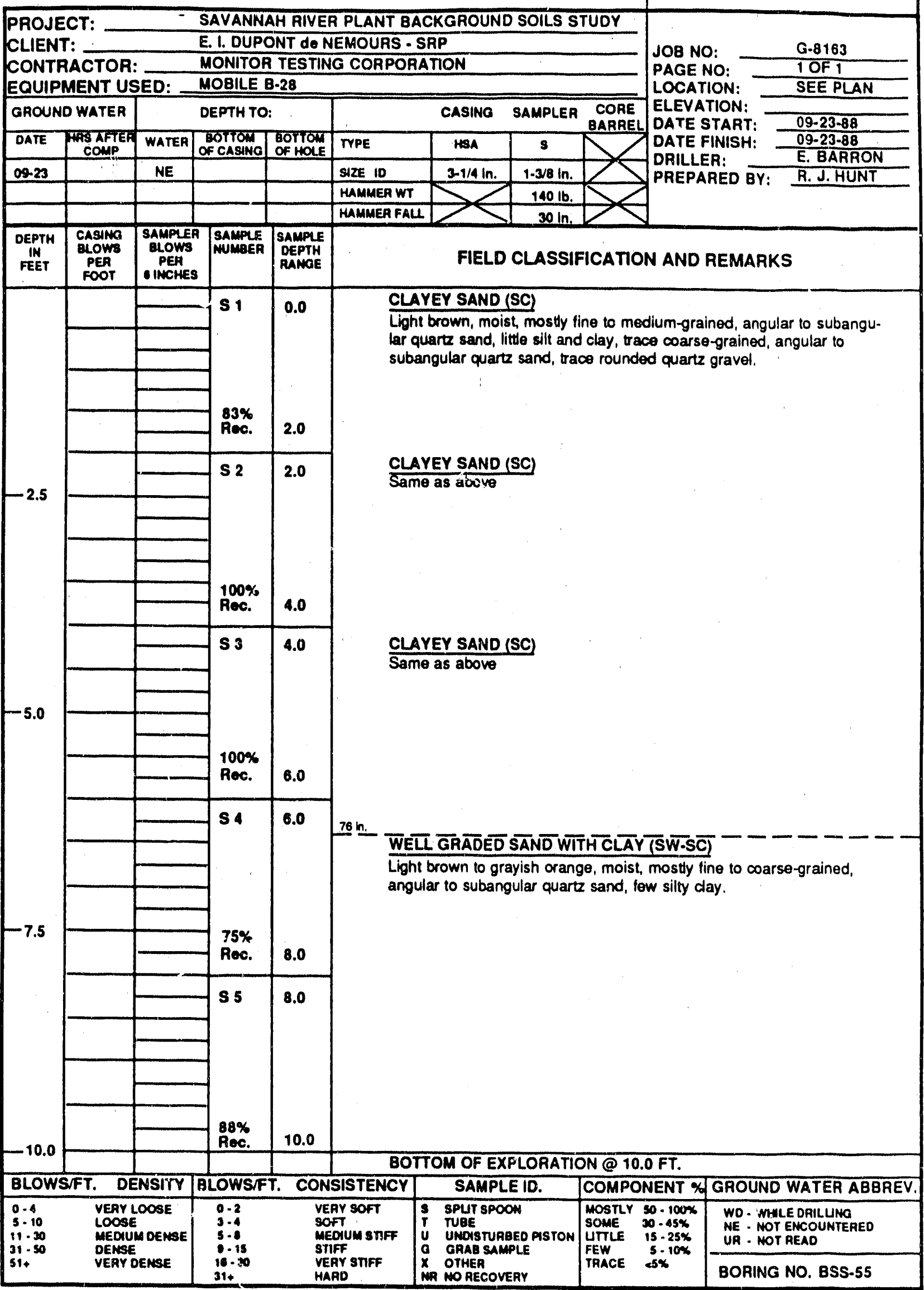


TEST BORING REPORT BORING NO. BSS-56

PROJECT:

SAVANNAH RIVER PLANT BACKGROUND SOILS STUDY

CLIENT:

E. I. DUPONT dO NEMOURS - SAP

CONTRACTOR: MONITOR TESTING CORPORATION EQUIPMENT USED:

\begin{tabular}{|c|c|c|c|c|c|c|c|c|}
\hline \multicolumn{2}{|c|}{ GAOUND WATER } & \multicolumn{3}{|c|}{ DEPTH TO: } & \multicolumn{2}{|r|}{ CASING } & \multicolumn{2}{|c|}{ 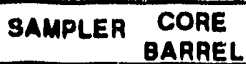 } \\
\hline DATE & $\begin{array}{c}\text { HAS AFTEF } \\
\text { COMP }\end{array}$ & WATER & $\begin{array}{l}\text { BOTTOM } \\
\text { Of CASING }\end{array}$ & $\begin{array}{l}\text { BOTTOM } \\
\text { OF HOLE }\end{array}$ & TYPE & NSA & 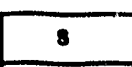 & \\
\hline $09-20$ & & NE & & & SIयE 10 & $3-1 / 4 \mathrm{ln}$. & $1.3 / 8 \mathrm{ln}$. & \\
\hline & & & & & MAMMEA WT & & $140 \mathrm{lb}$ & \\
\hline & & & & & MAMMEA FAUL & & $30 \mathrm{ln}$. & \\
\hline
\end{tabular}
MOBILE B-28

JOB NO

PAGE NO:

G.8163

LOCATION:

OF 1

ELEVATION:

09-20-88

DRILLEA:

PREPARED BY: R.J.HUNT

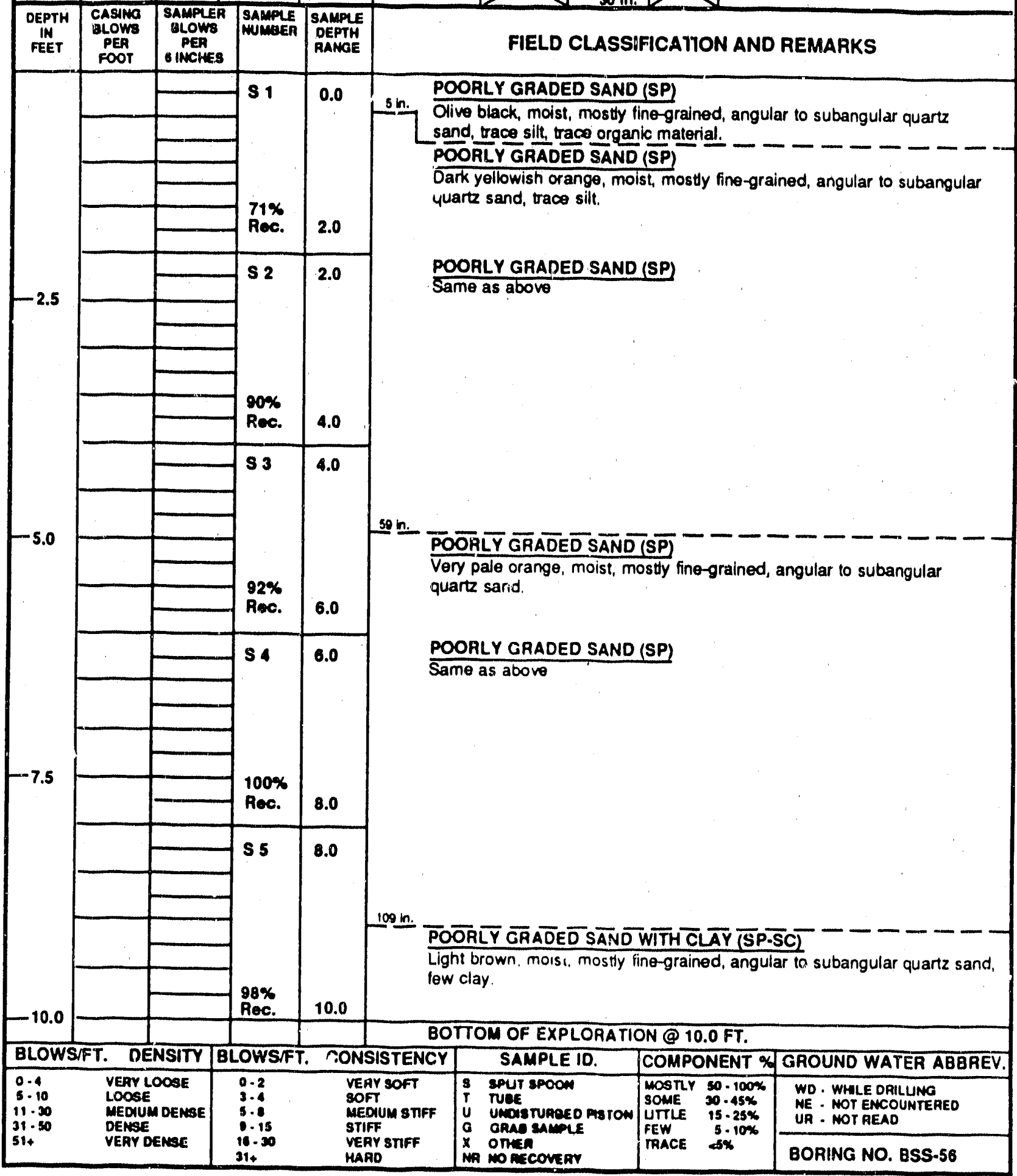


CONSULTANTS

TEST BORING REPORT

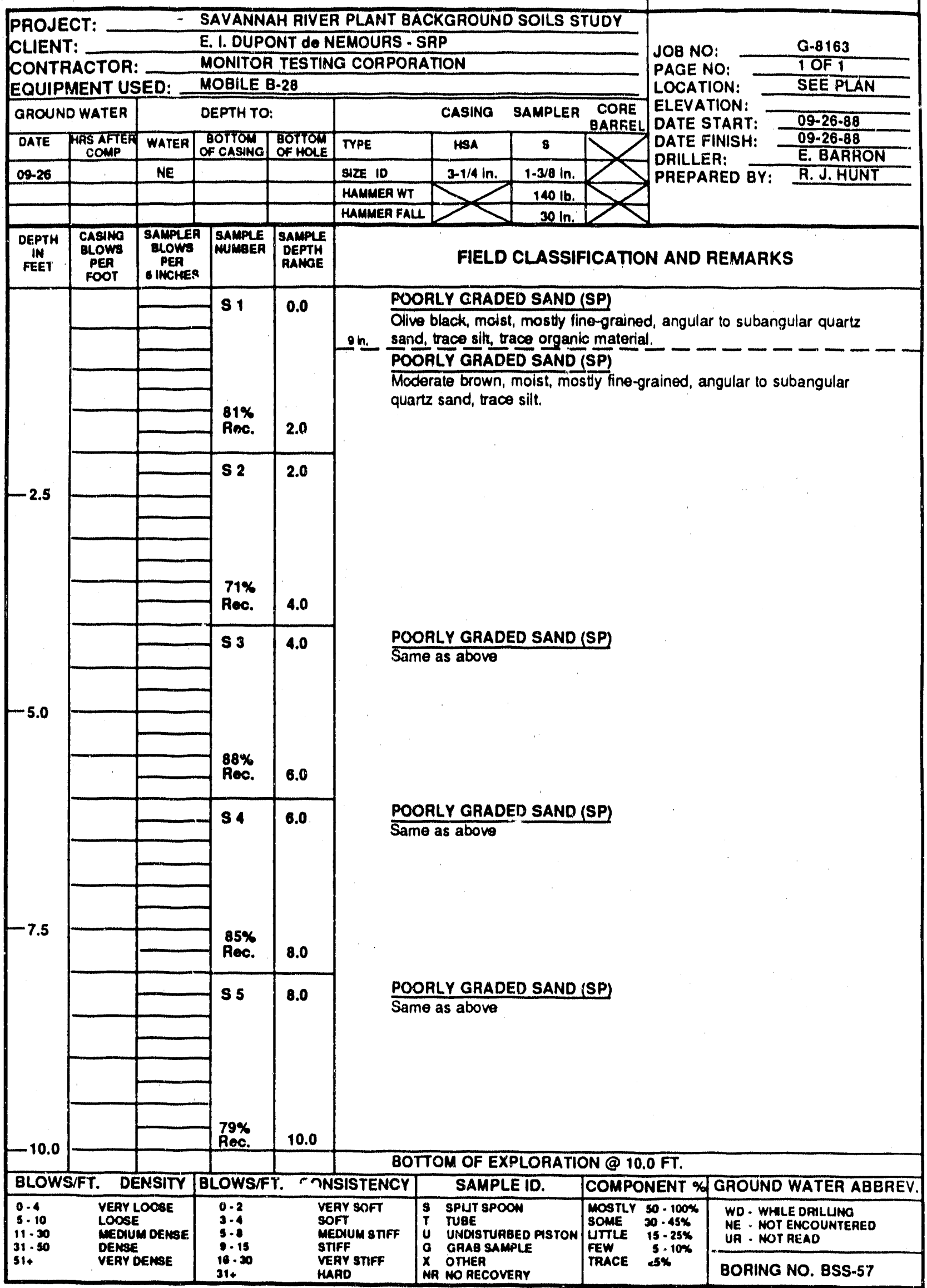


SIRRINE

\section{ENVIRONMENTAL}

CONSULTANTS

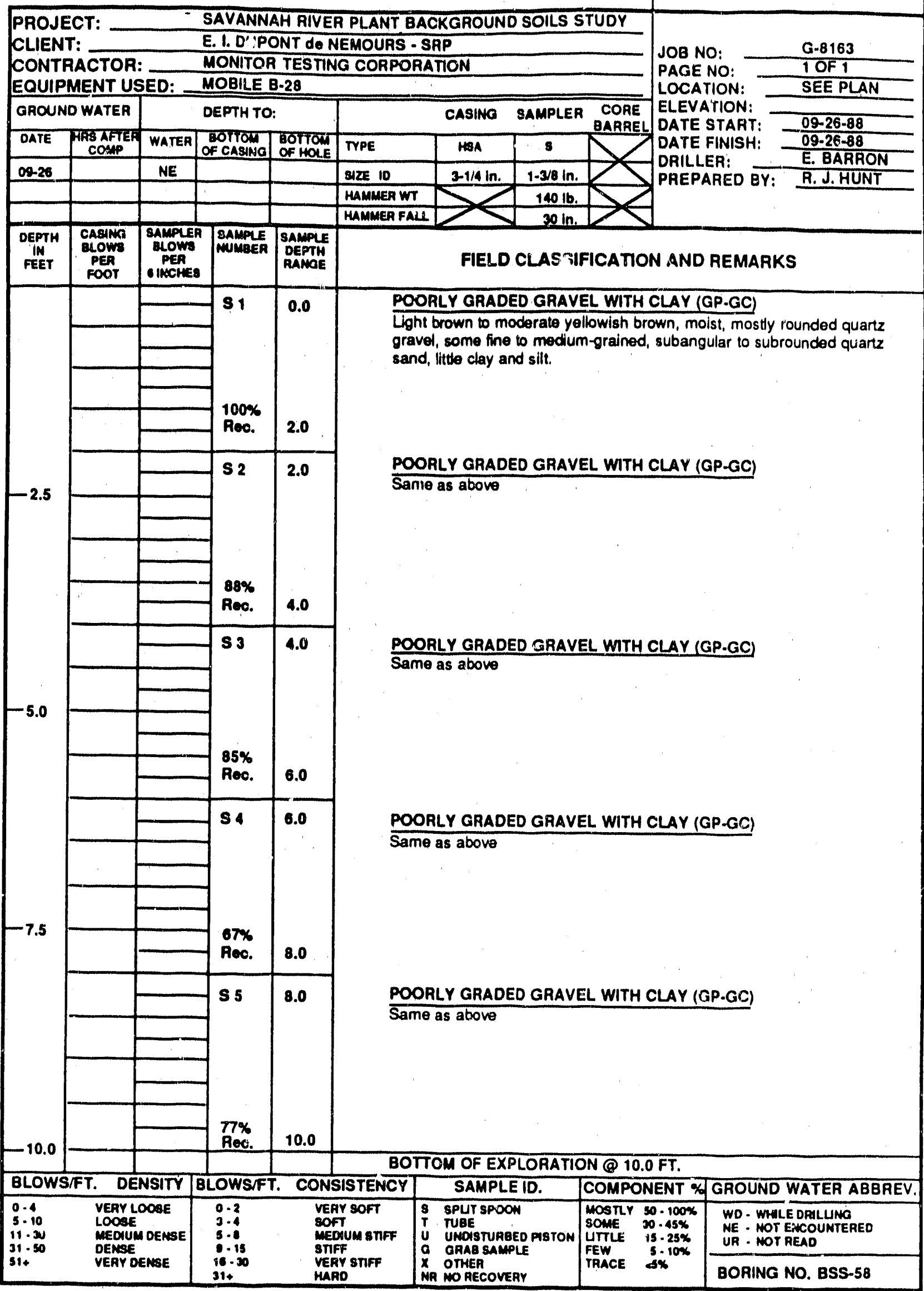




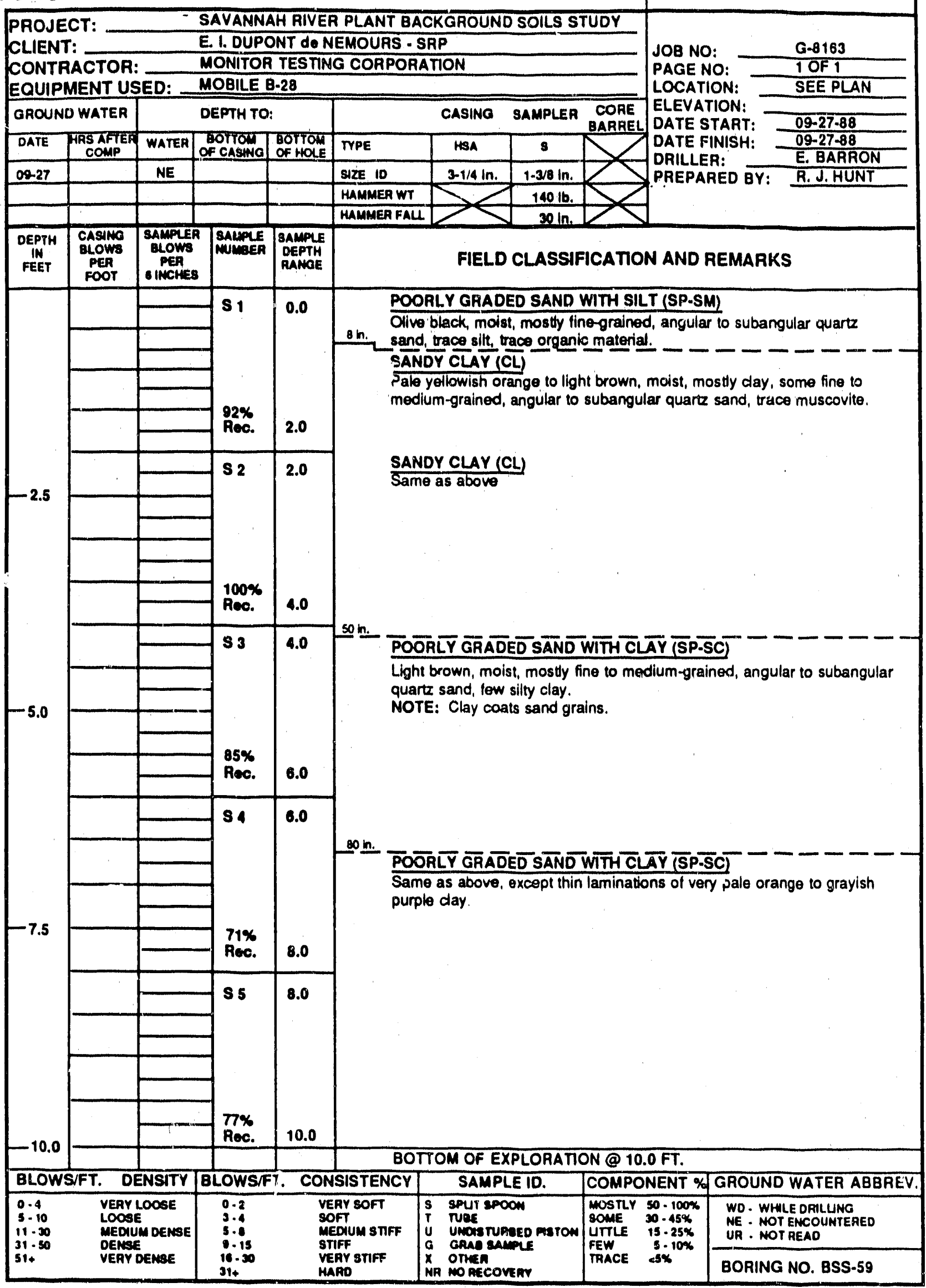


GEOCHEMICAL AND PHYSICAL PROPERTIES OF SOILS AND

SHALLOW SEDIMENTS AT THE SAVANNAH RIVER SITE

\author{
Appendix B.3
}

Modified Wentworth Classification of Background Soil Samples 
FIELD GEOLOGIC LOG

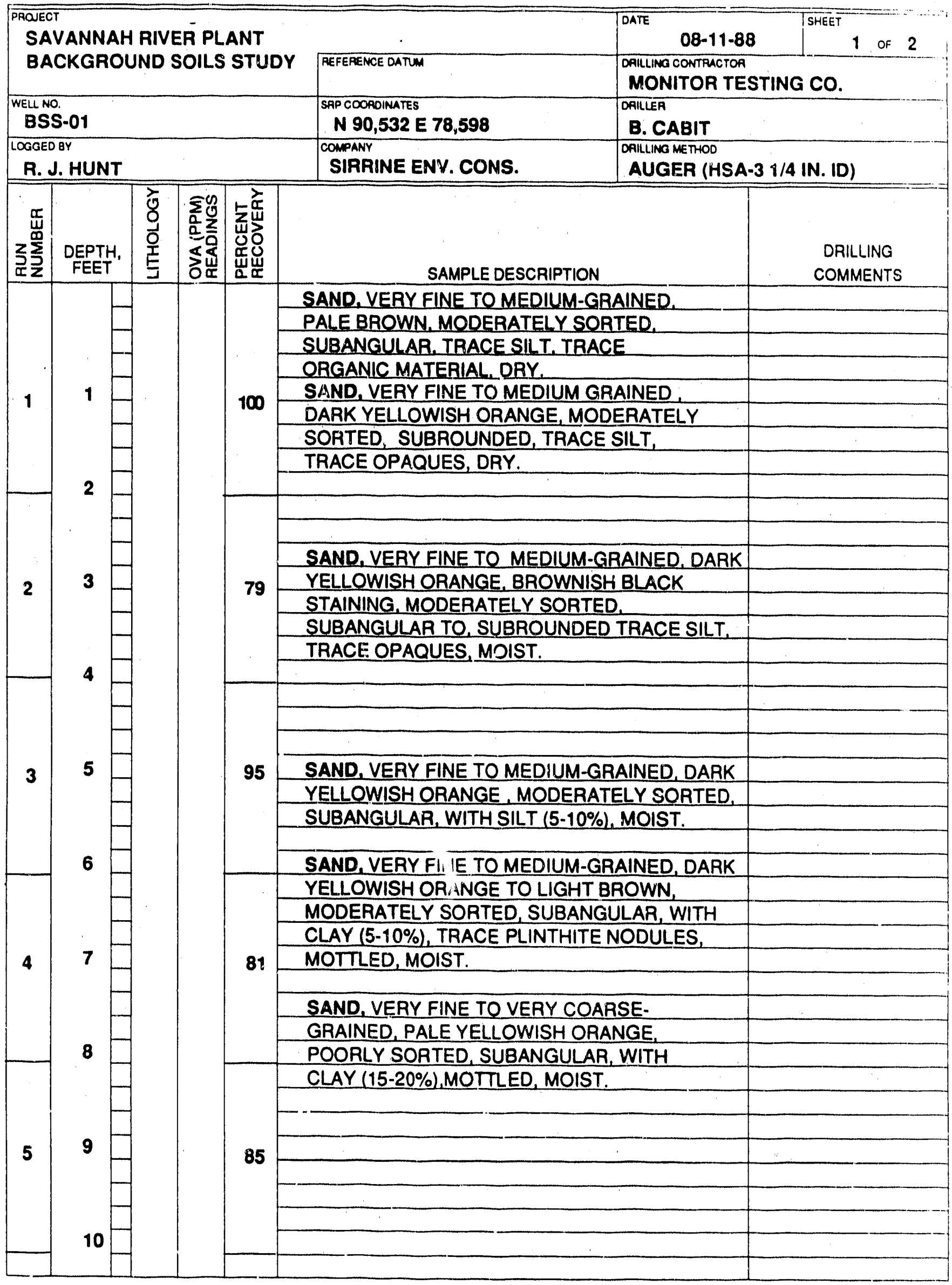




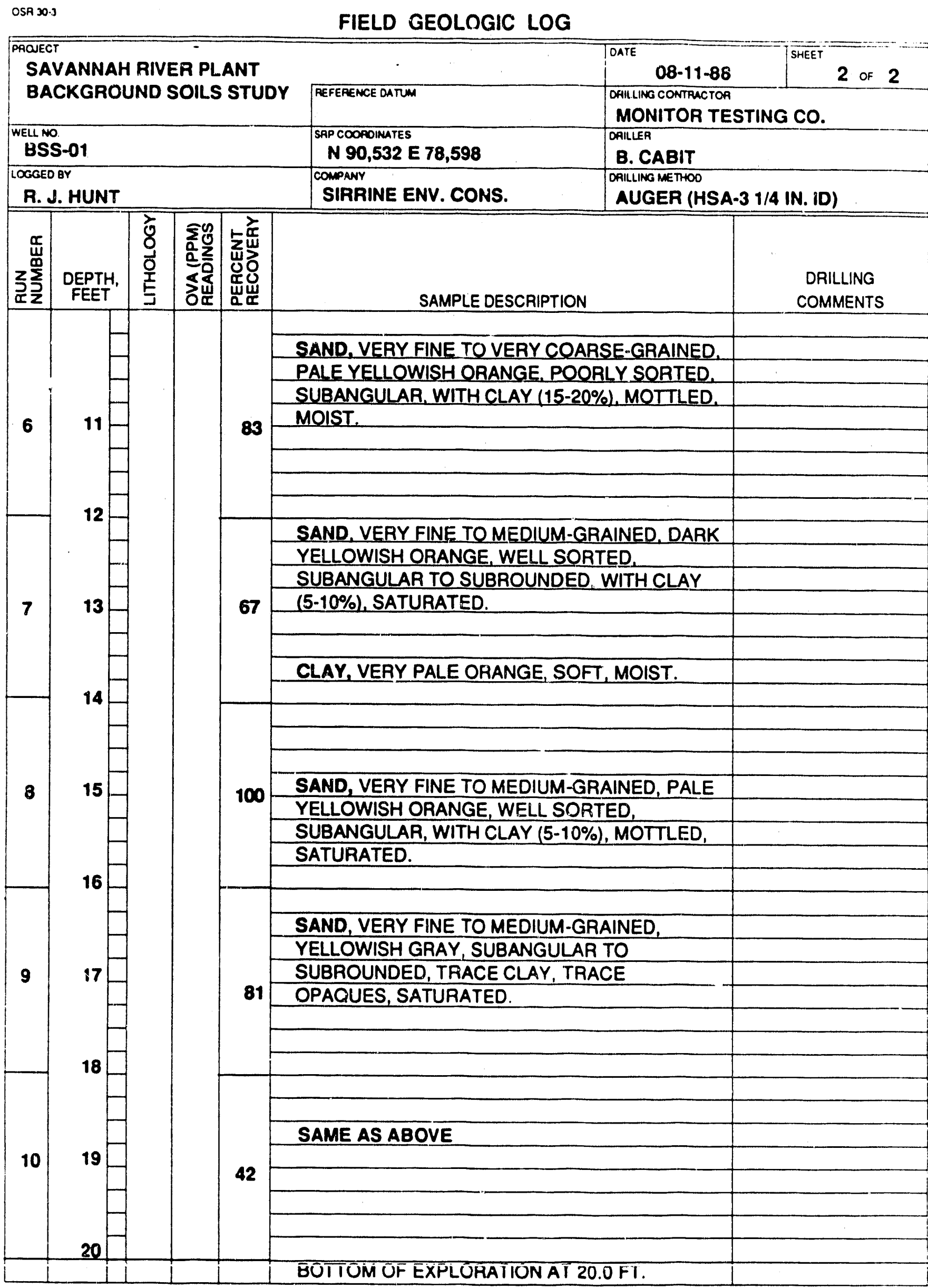


FIELD GEOLOGIC LOG

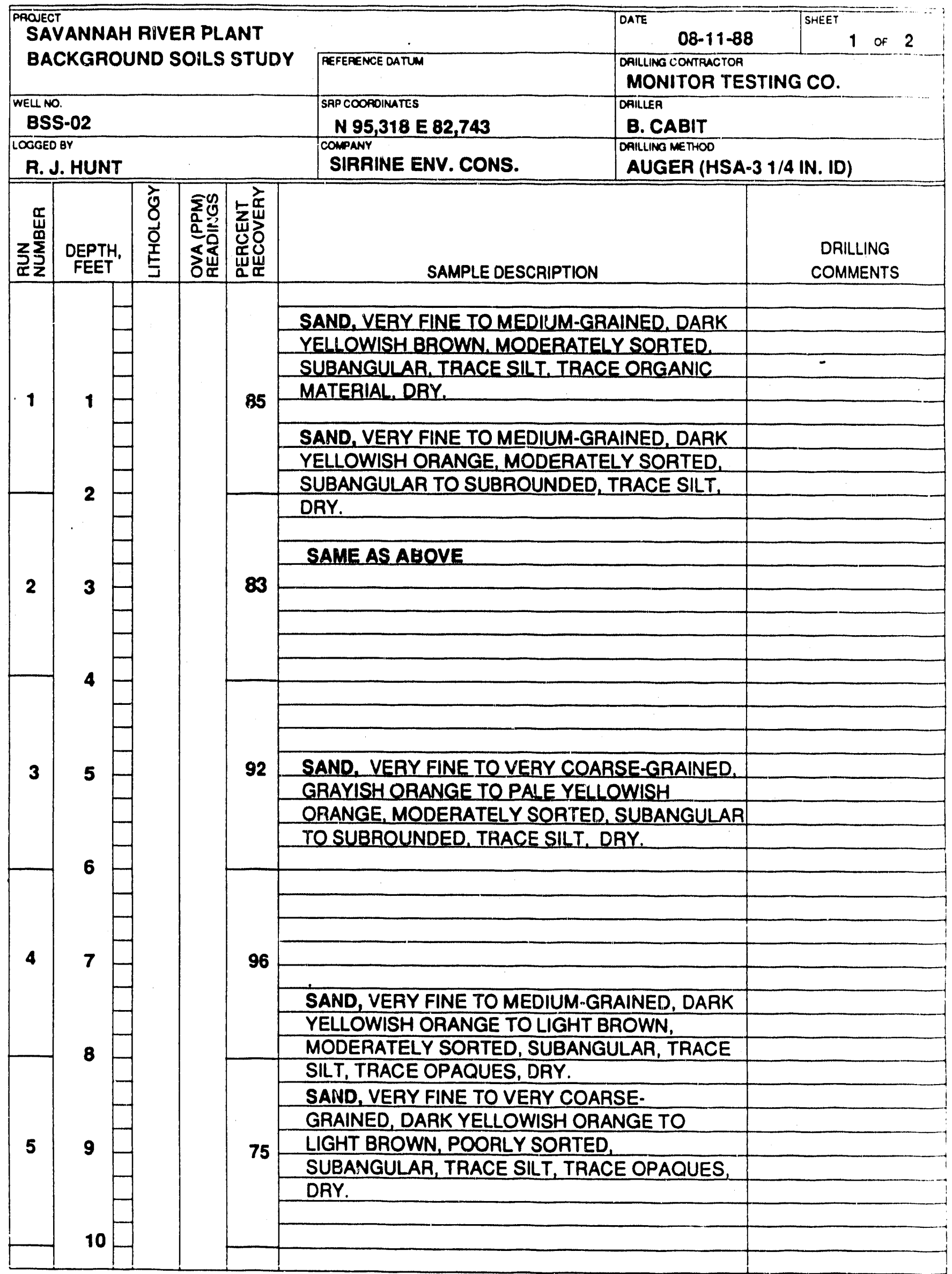




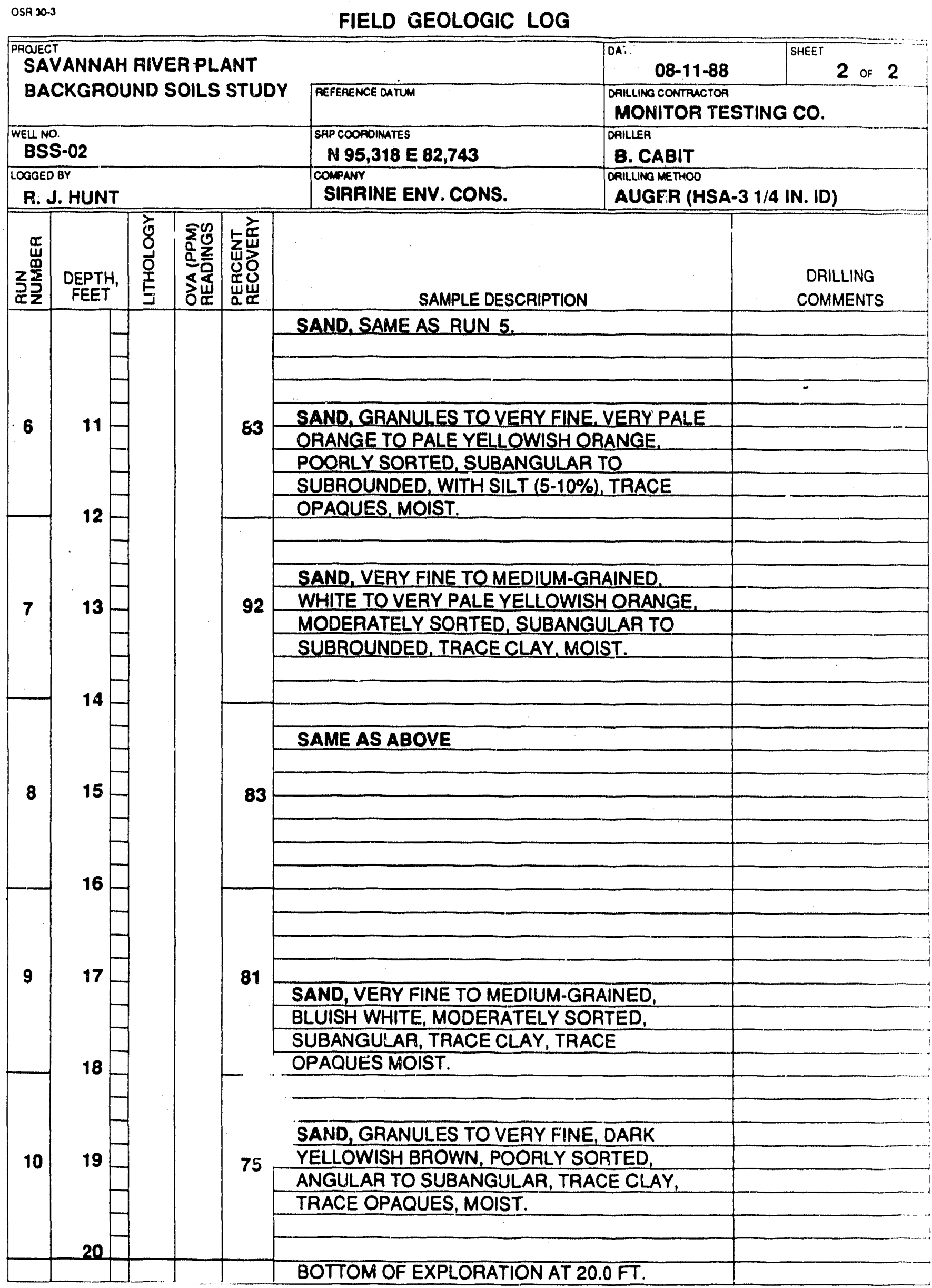


FIELD GEOLOGIC LOG

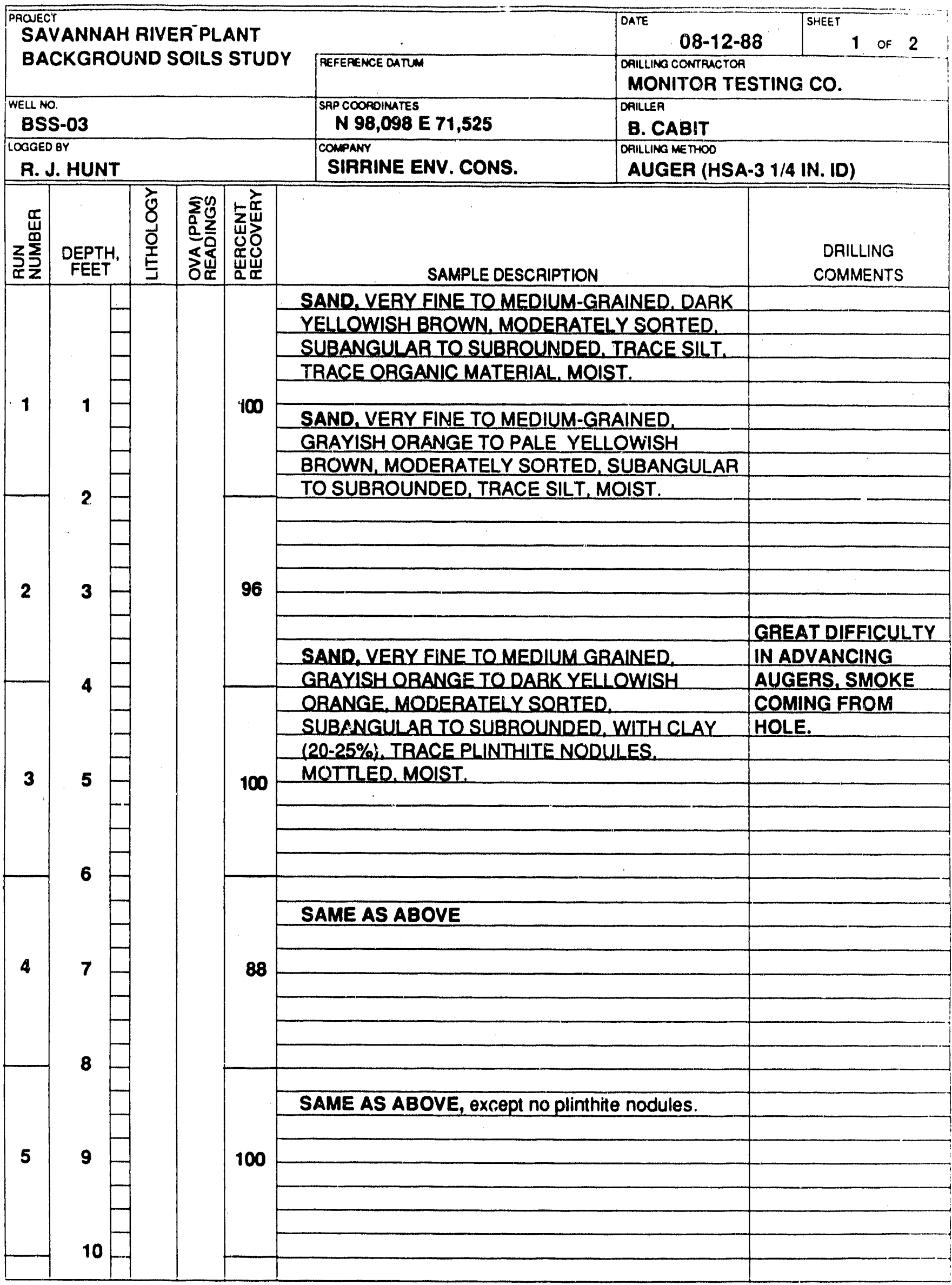




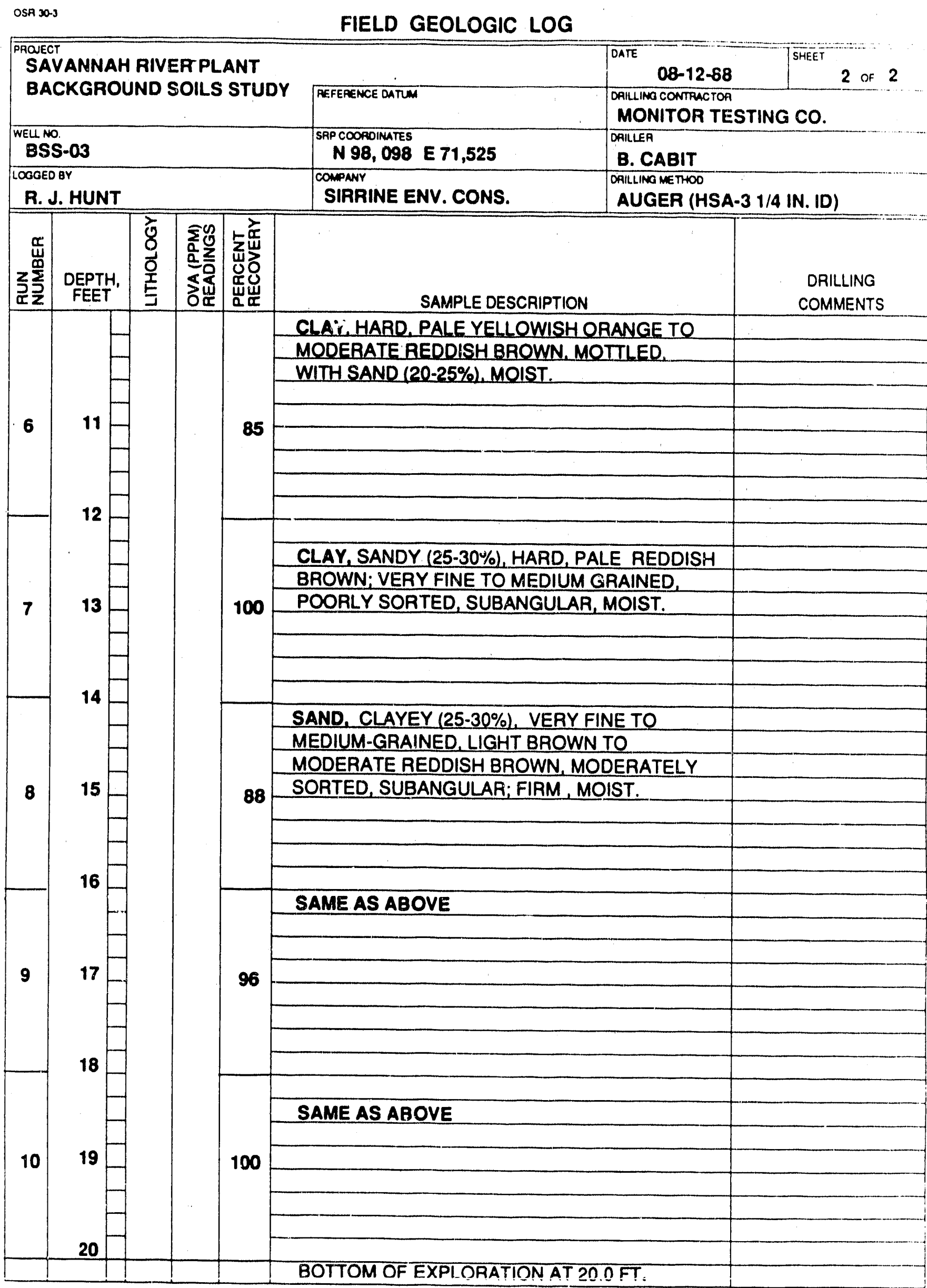


FIELD GEOLOGIC LOG

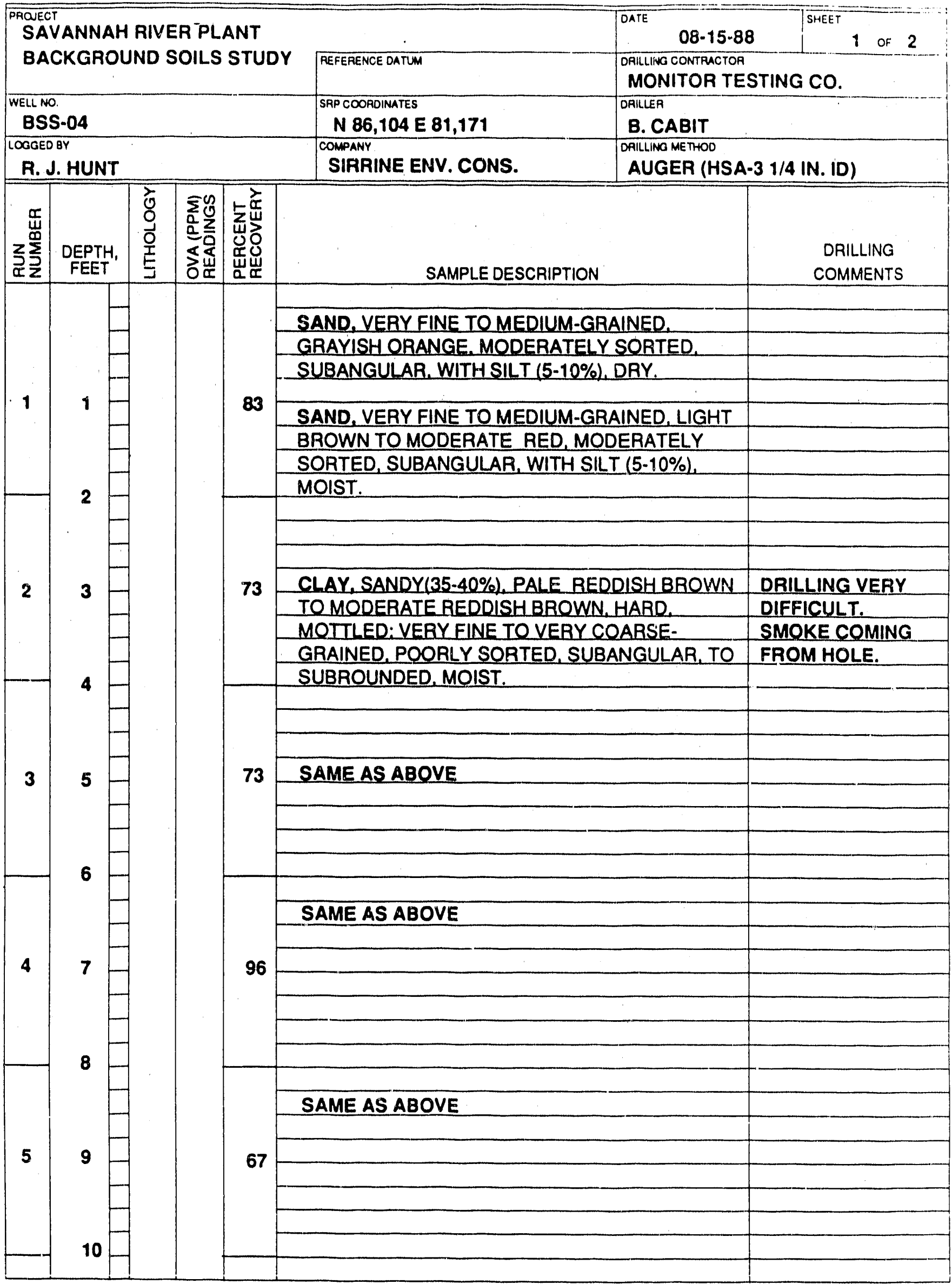


FIELD GEOLOGIC LOG

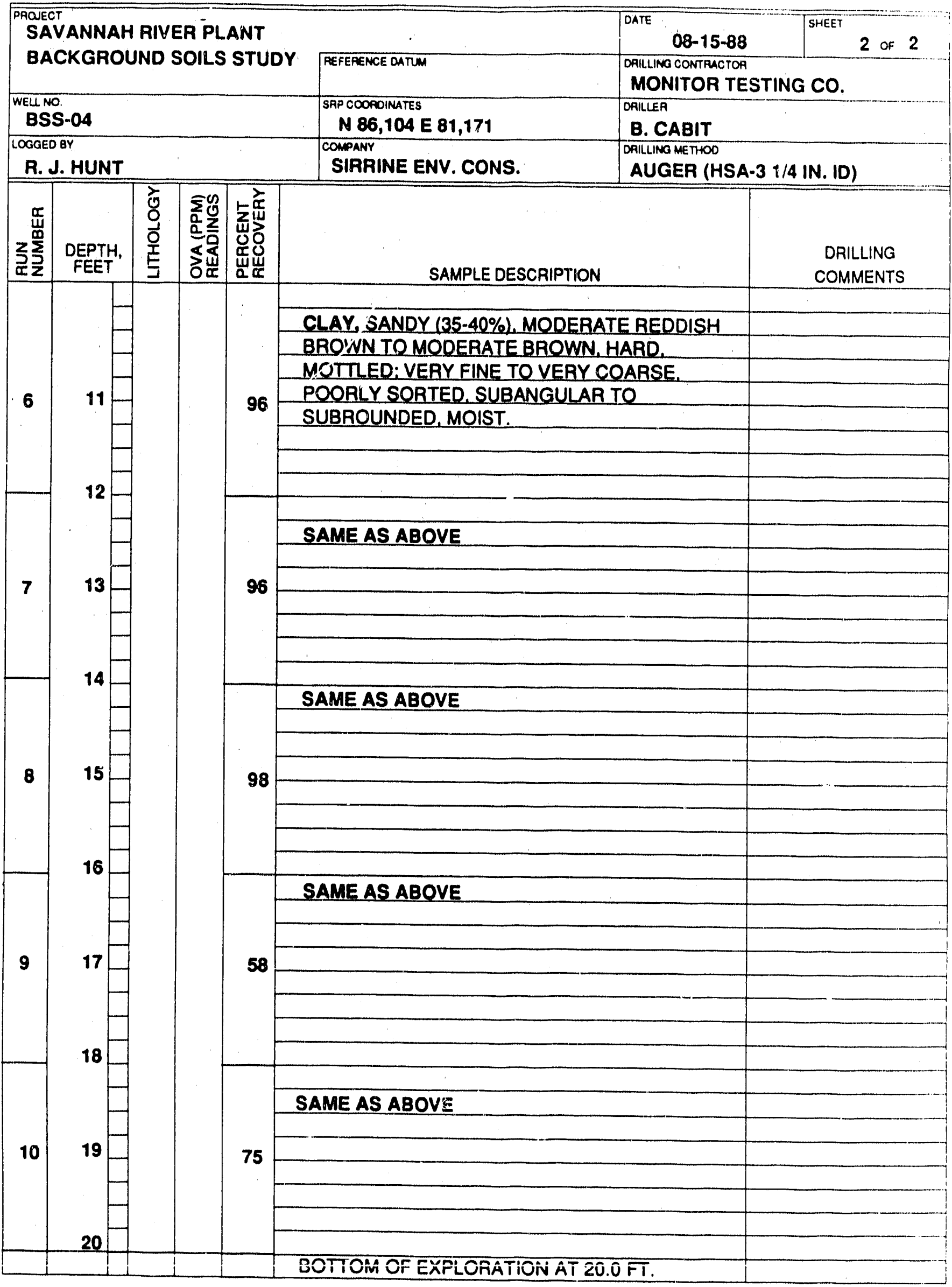


FIELD GEOLOGIC LOG

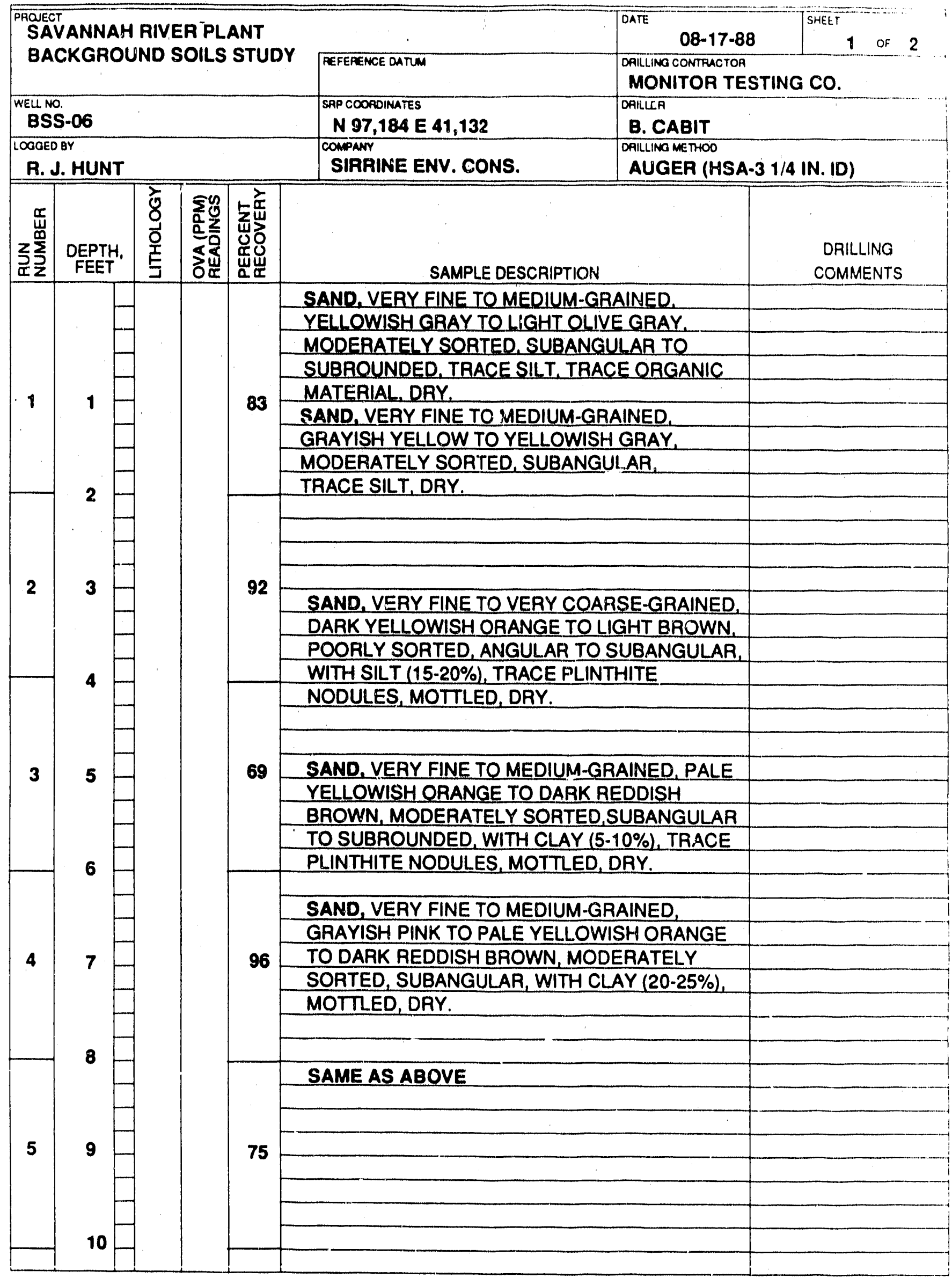


FIELD GEOLOGIC LOG

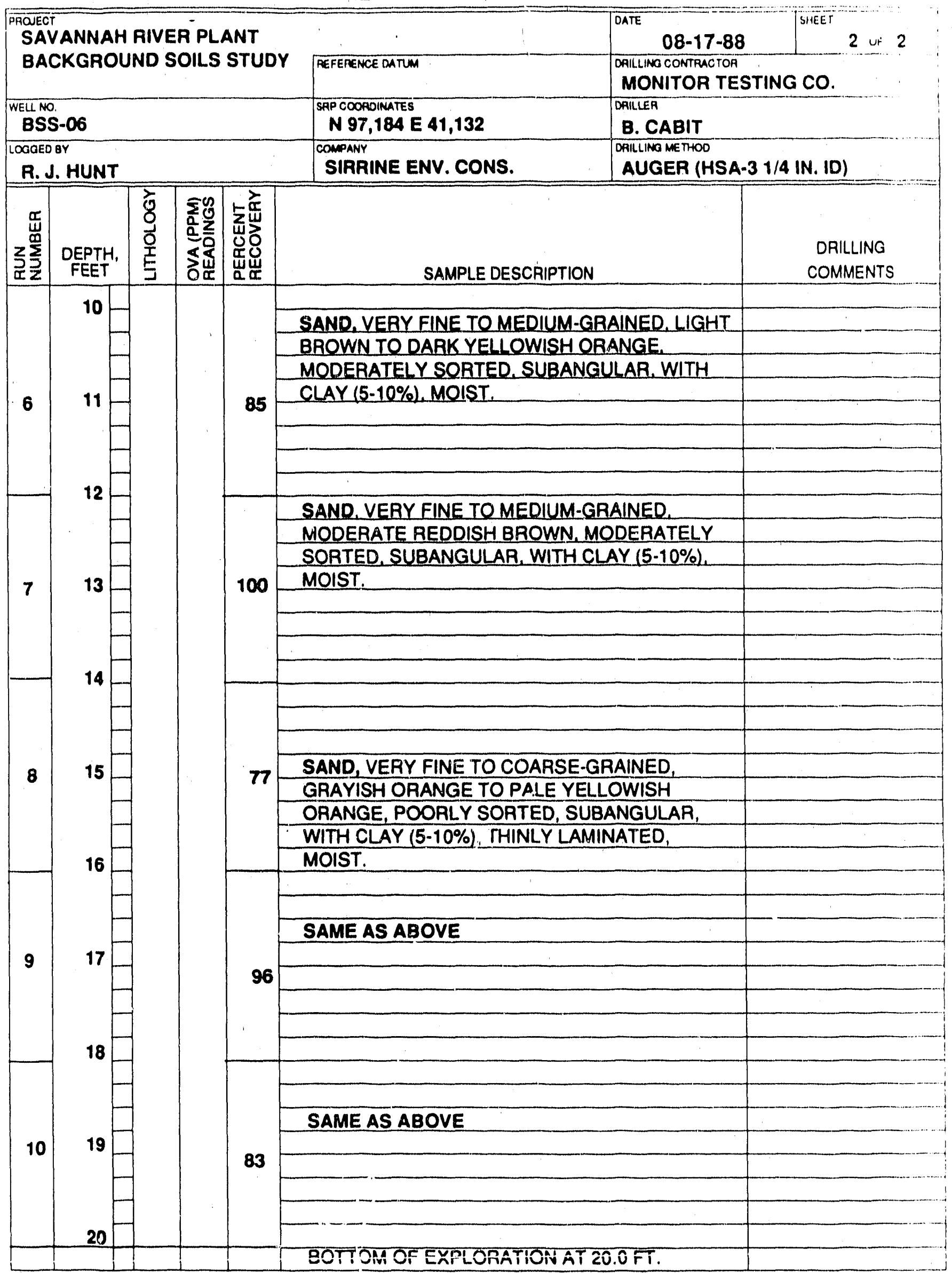


FIELD GEOLOGIC LOG

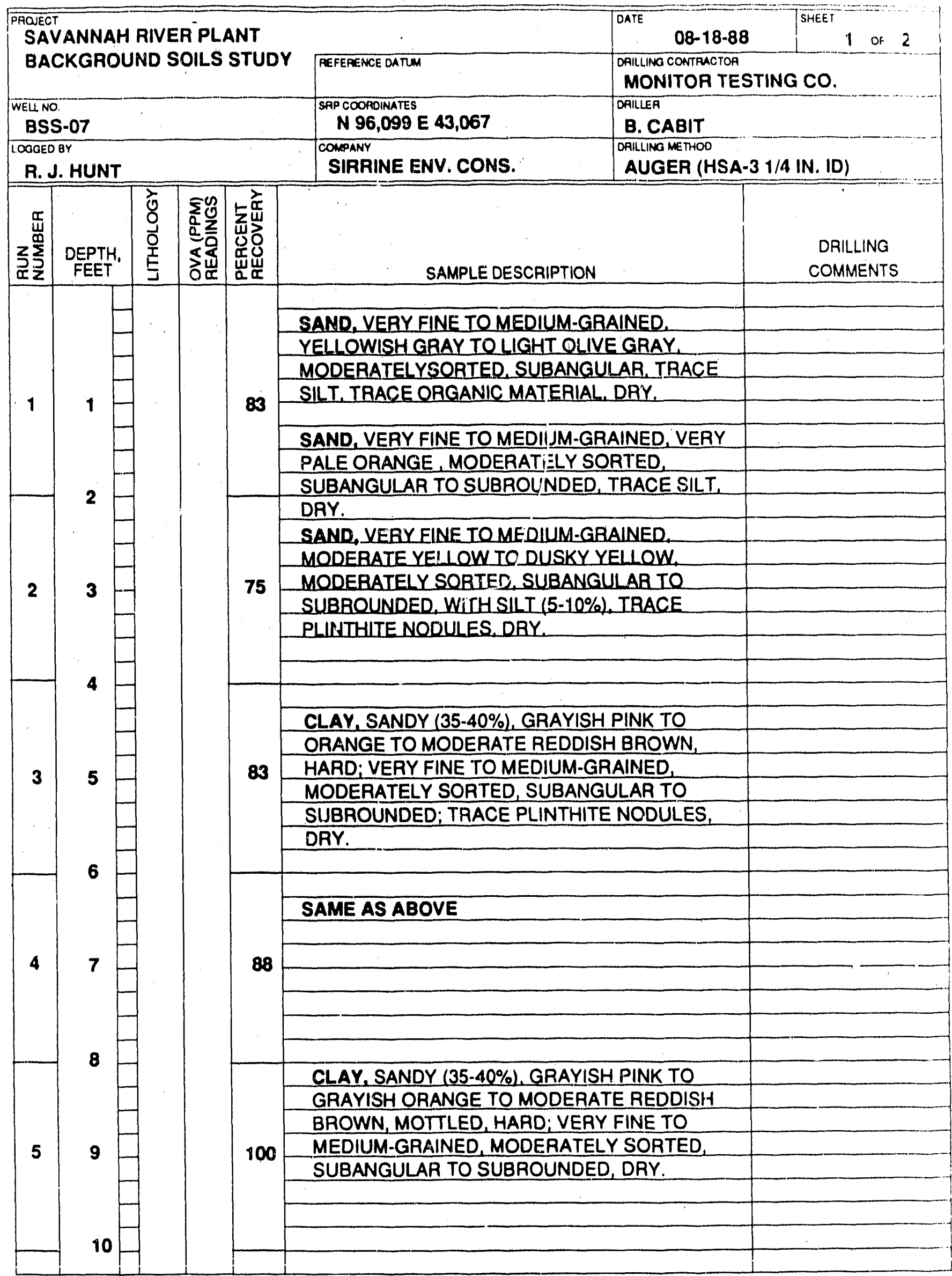


FIELD GEOLOGIC LOG

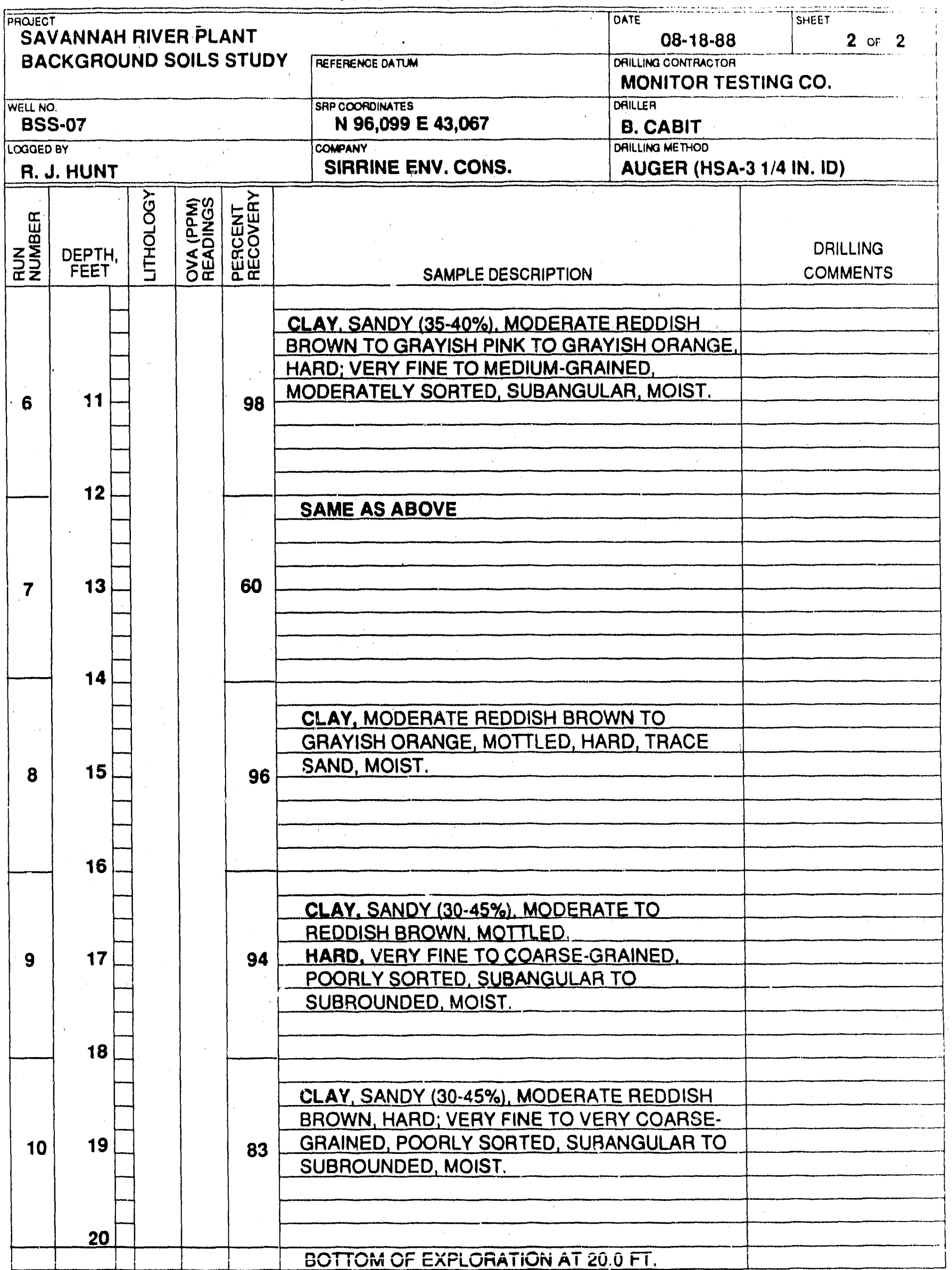


FIELD GEOLOGIC LOG

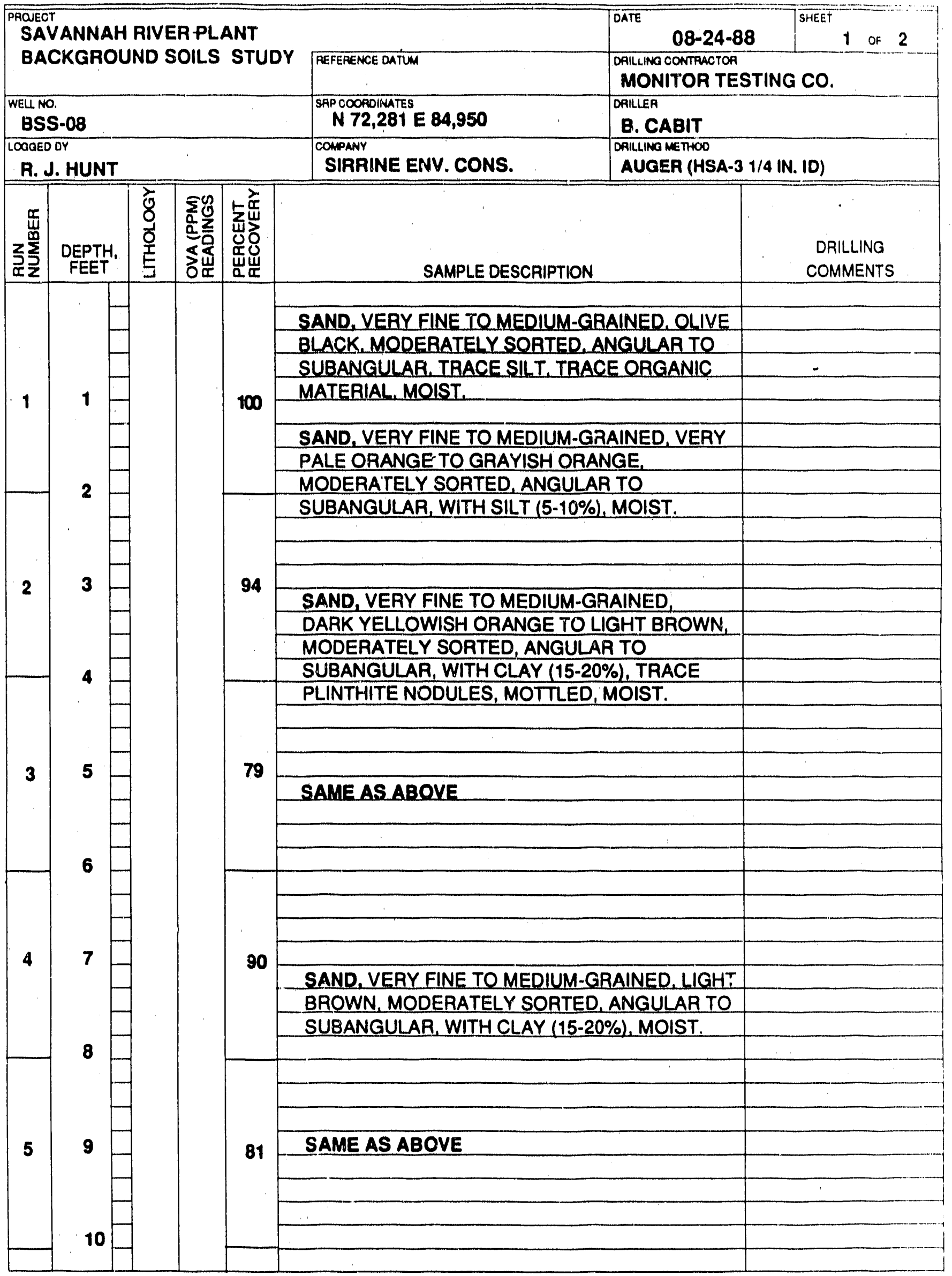


FIELD GEOLOGIC LOG

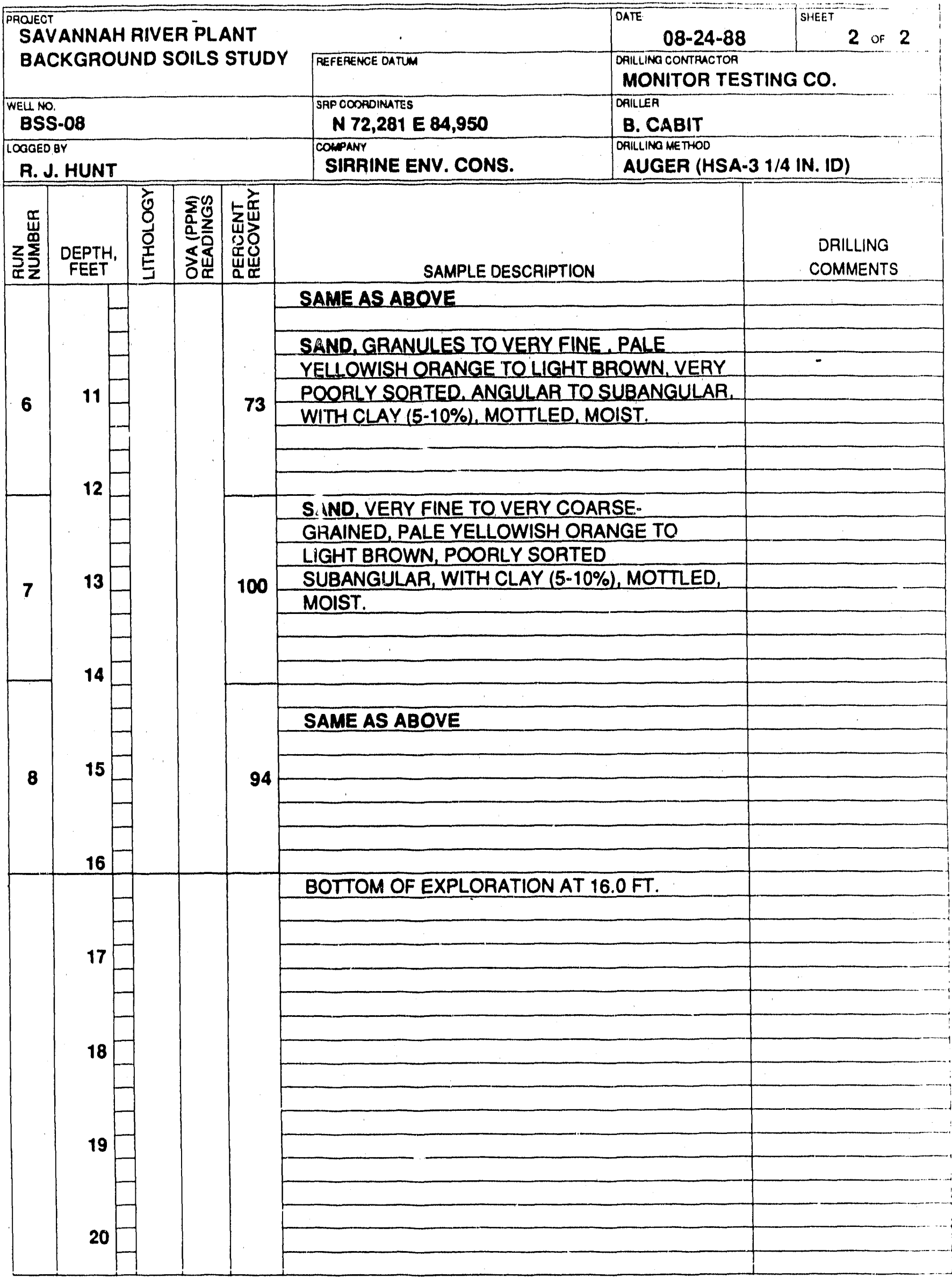


FIELD GEOLOGIC LOG

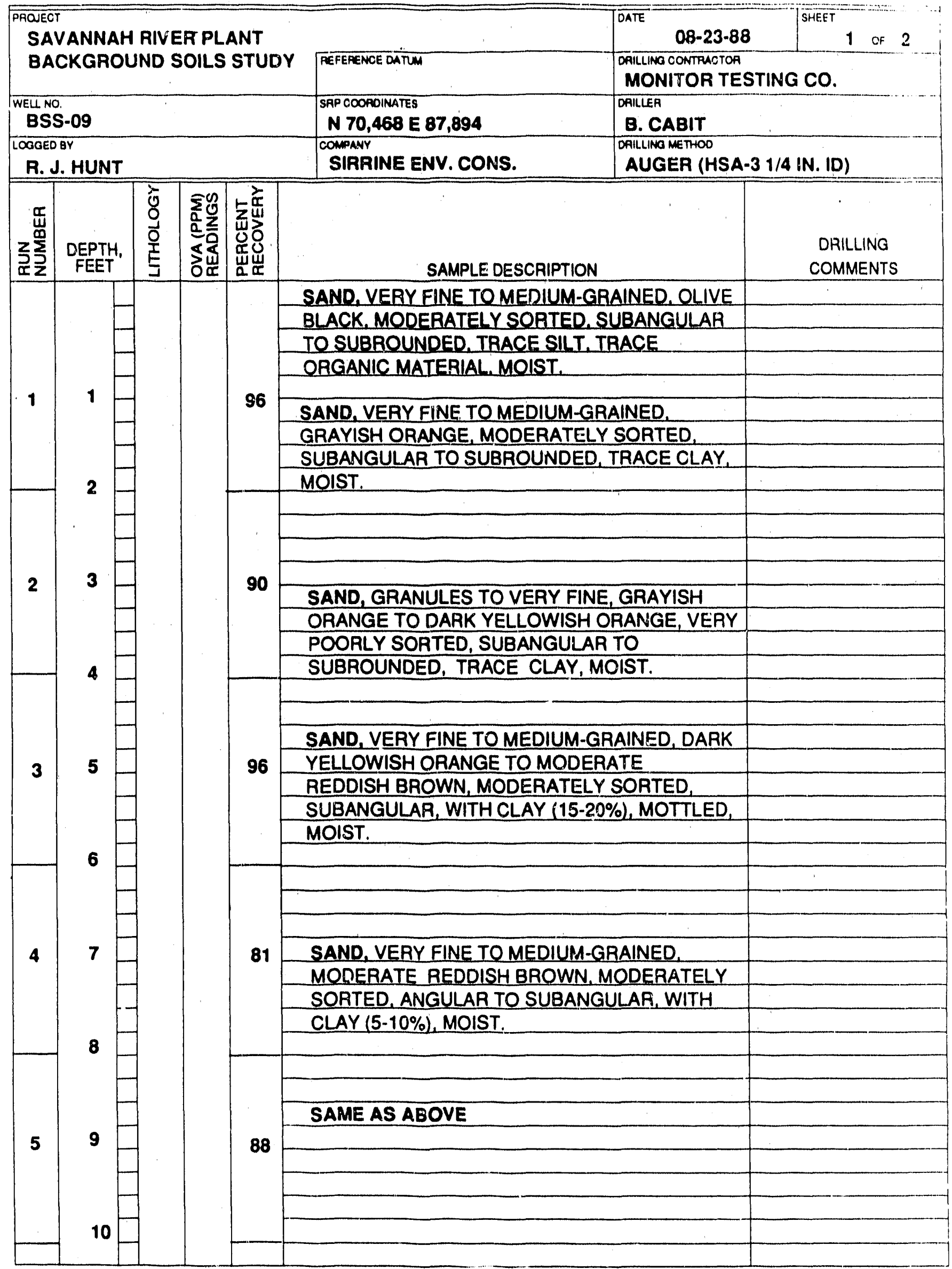




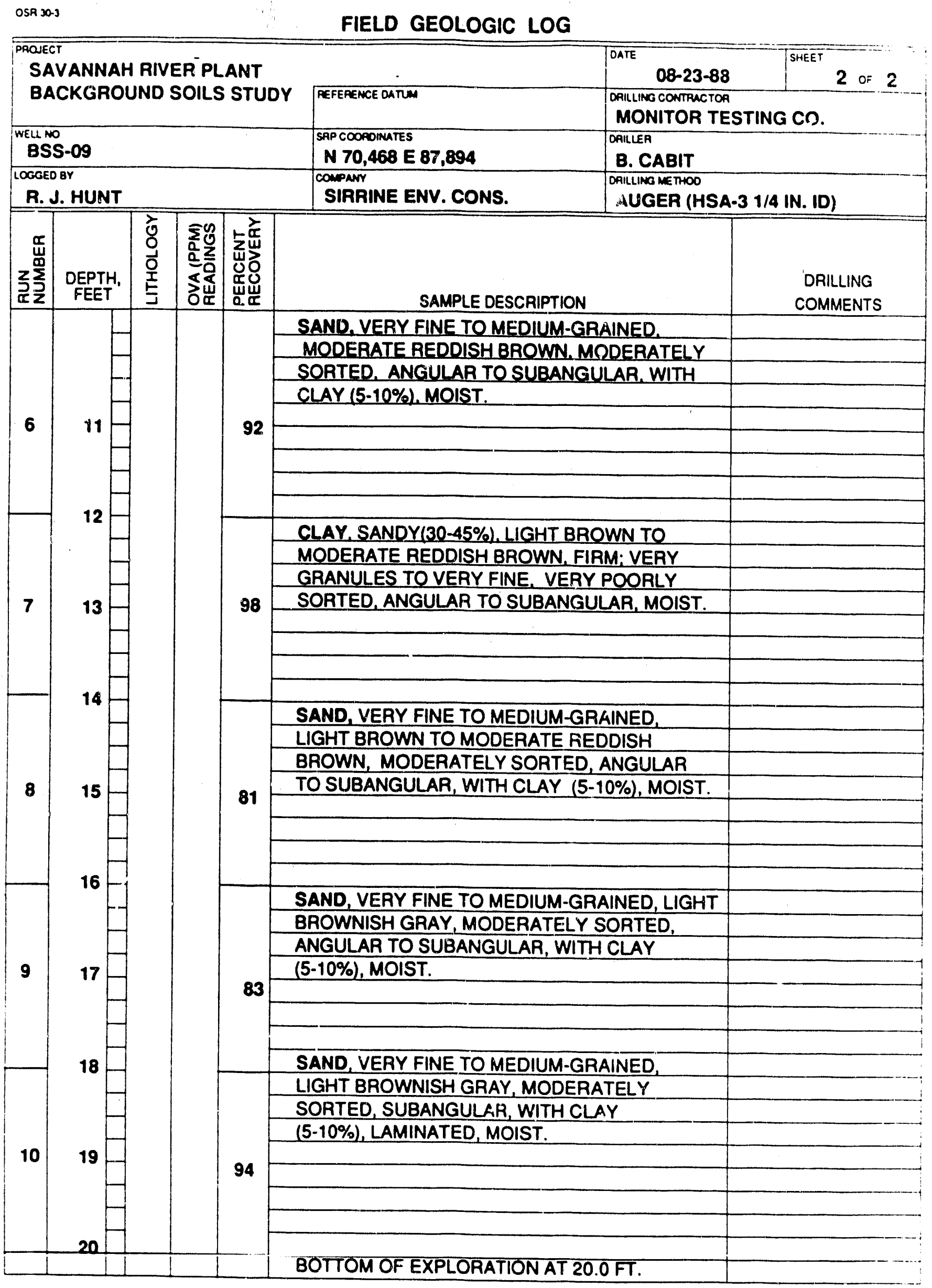


FIELD GEOLOGIC LOG

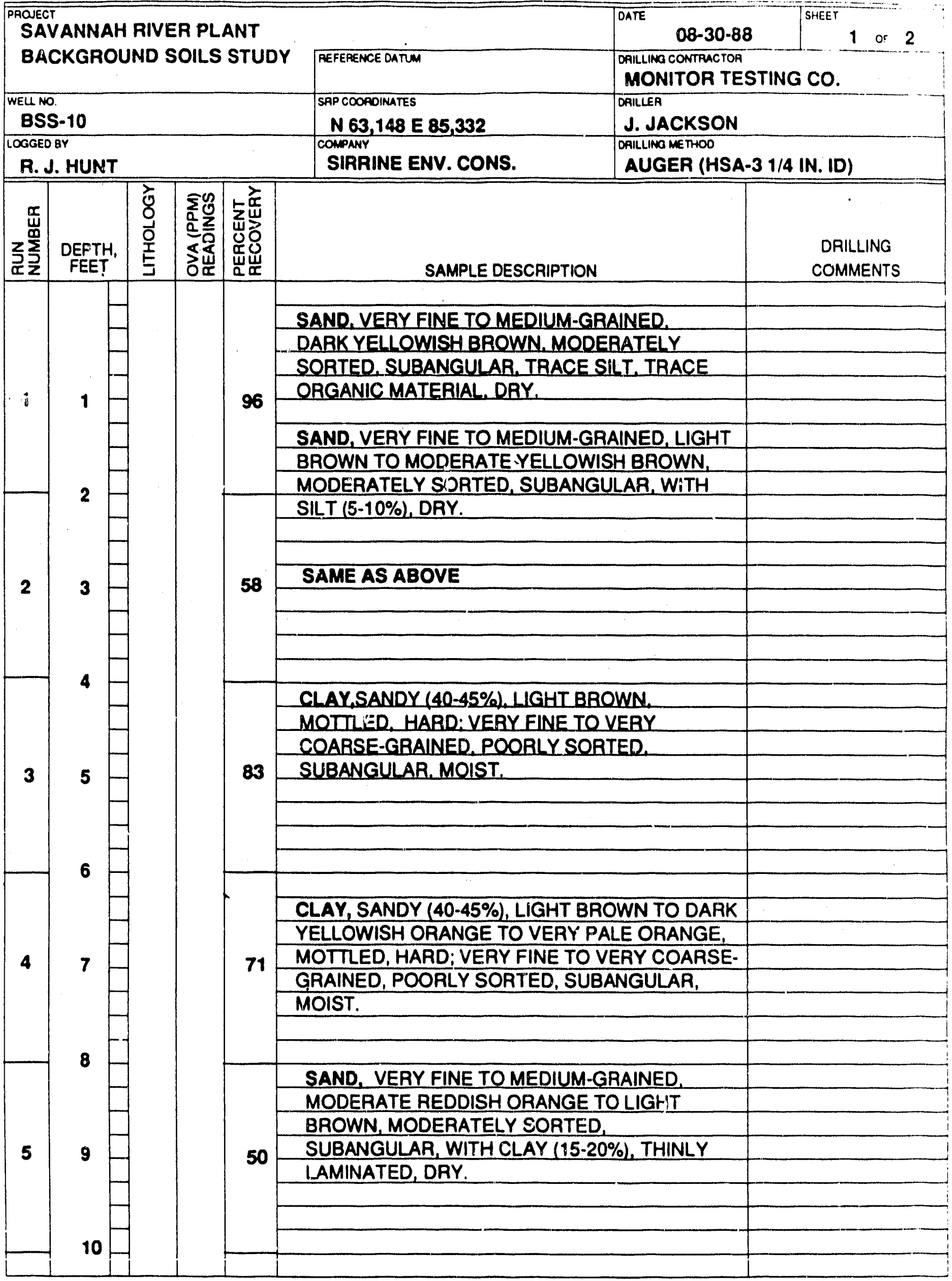




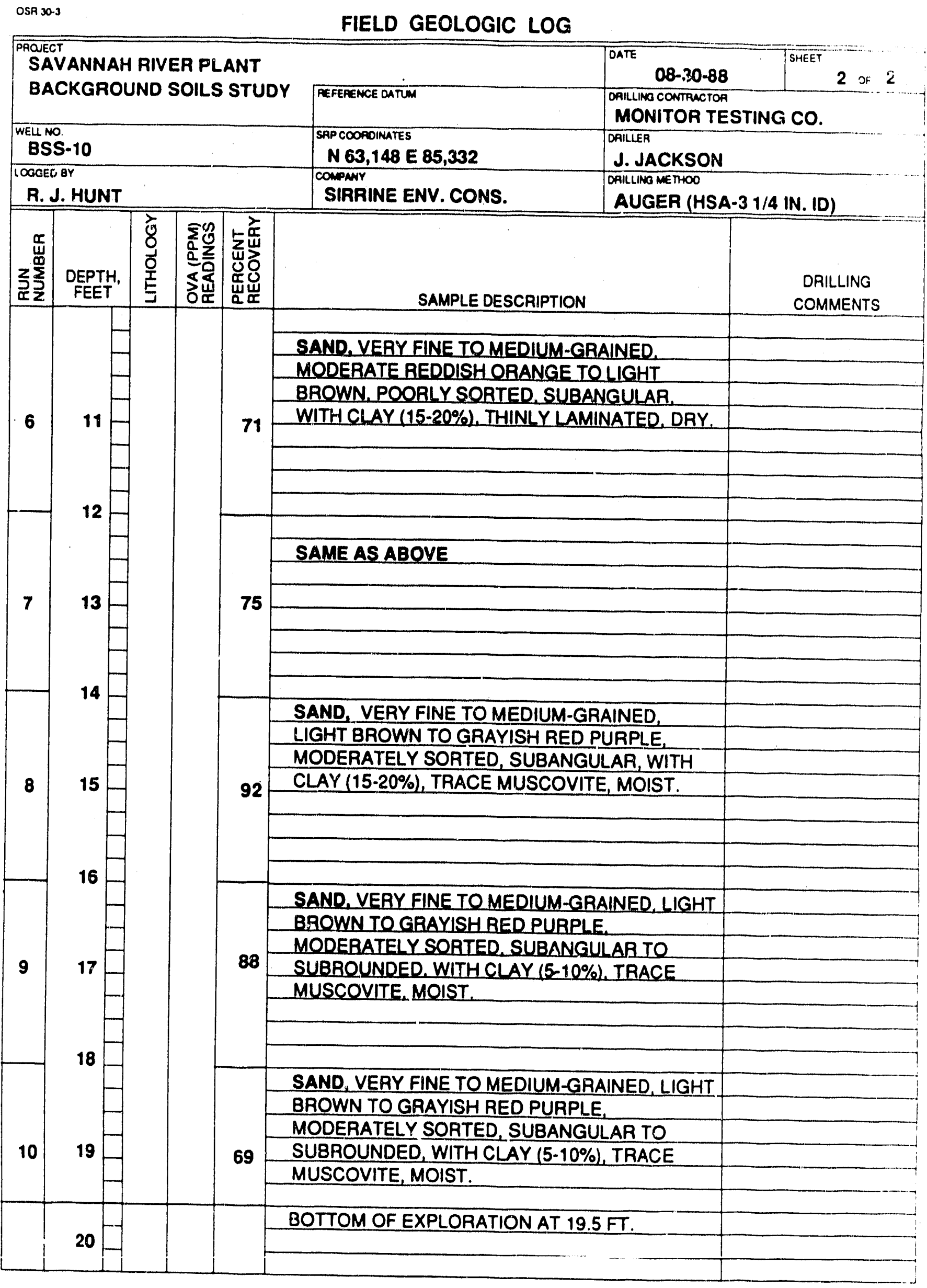


FIELD GEOLOGIC LOG

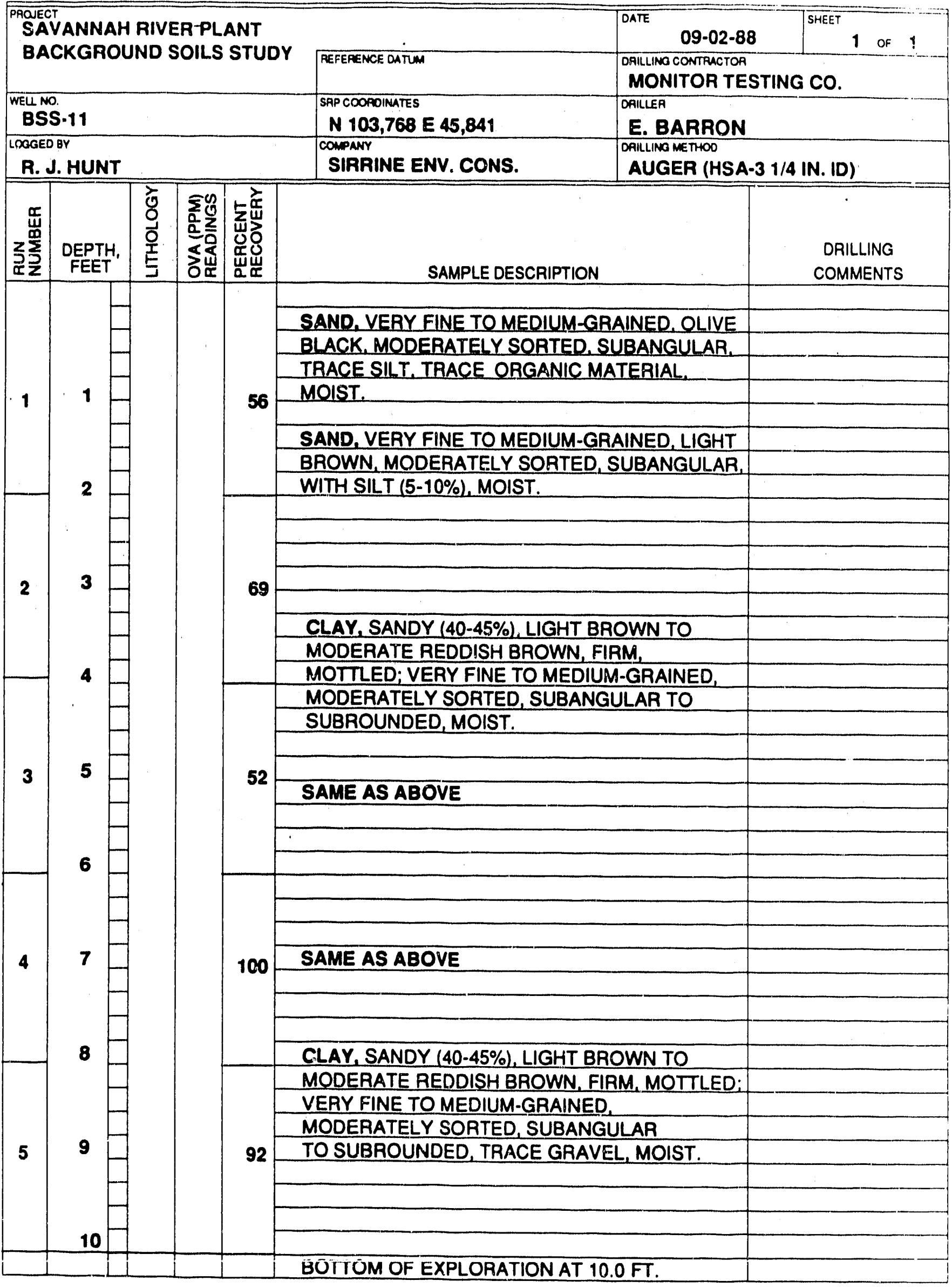


FIELD GEOLOGIC LOG

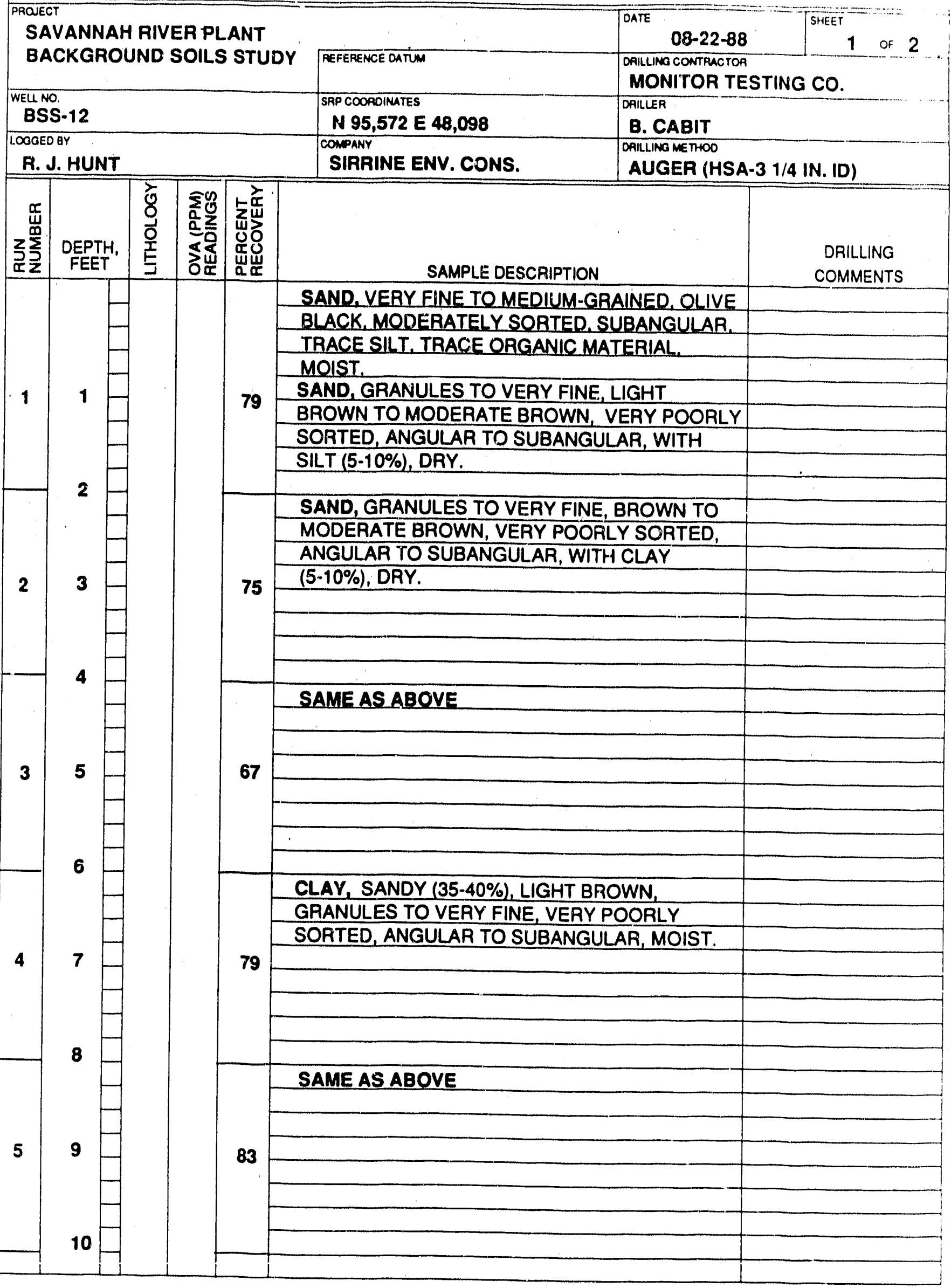




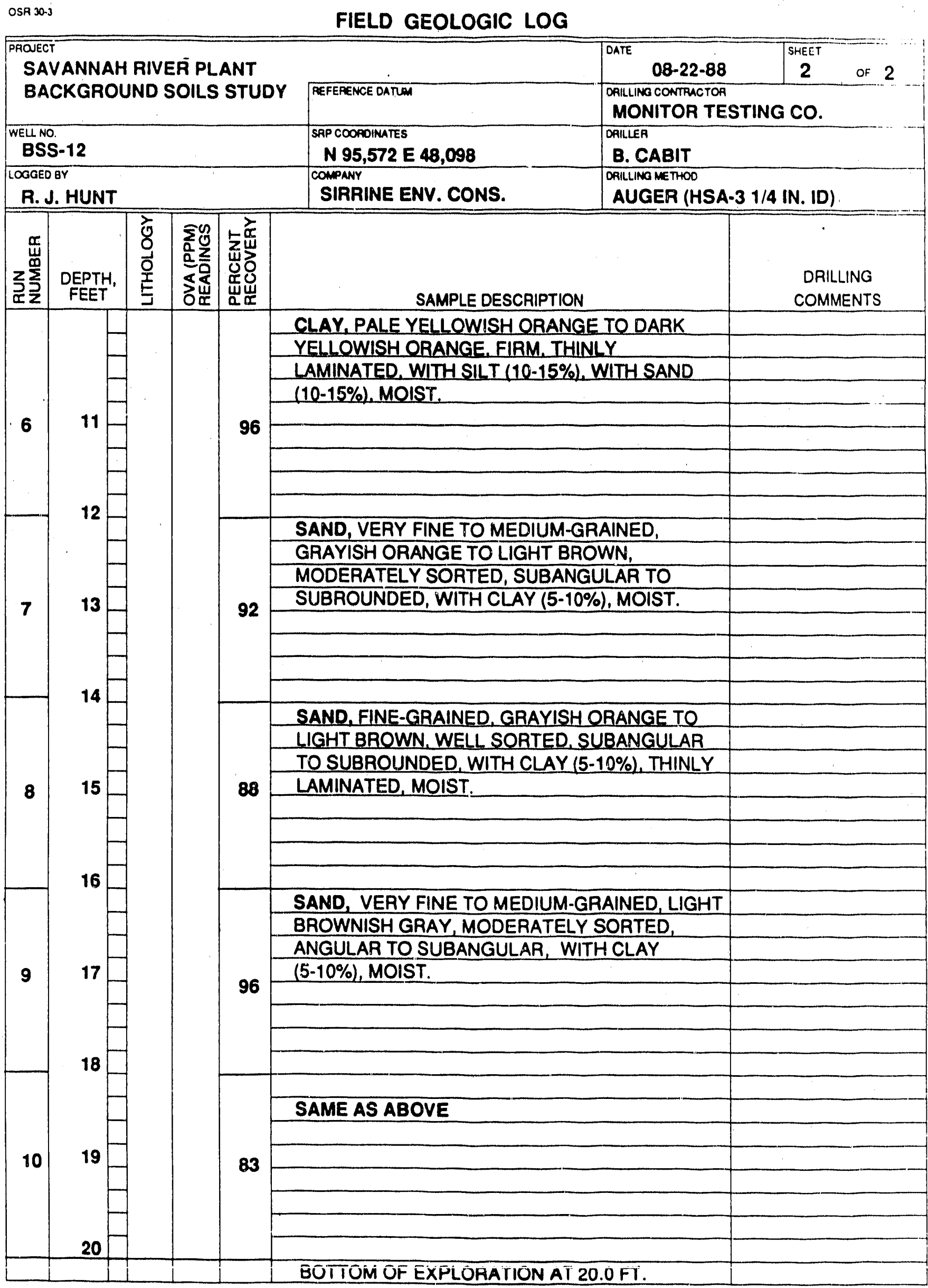


FIELD GEOLOGIC LOG

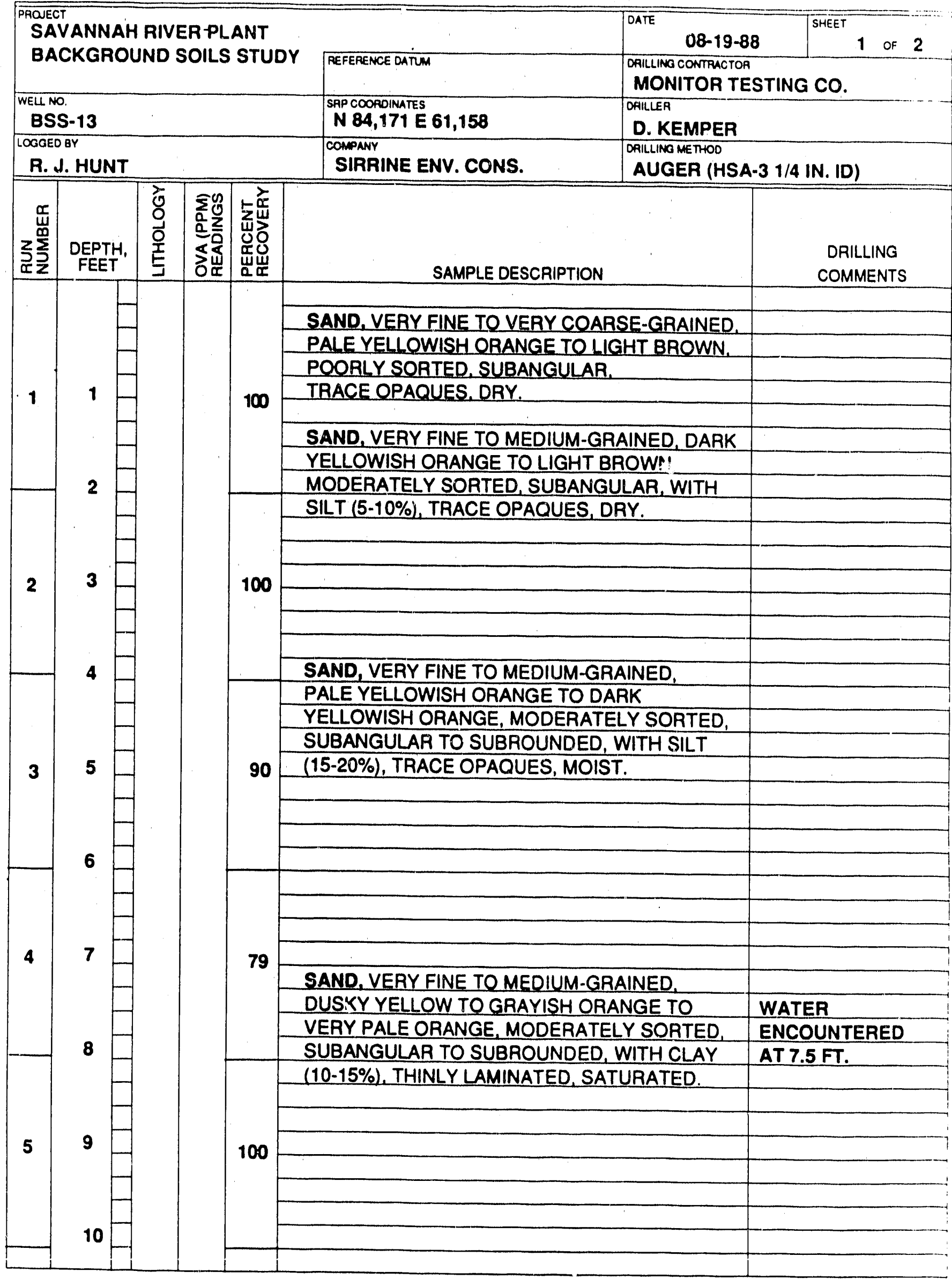


FIELD GEOLOGIC LOG

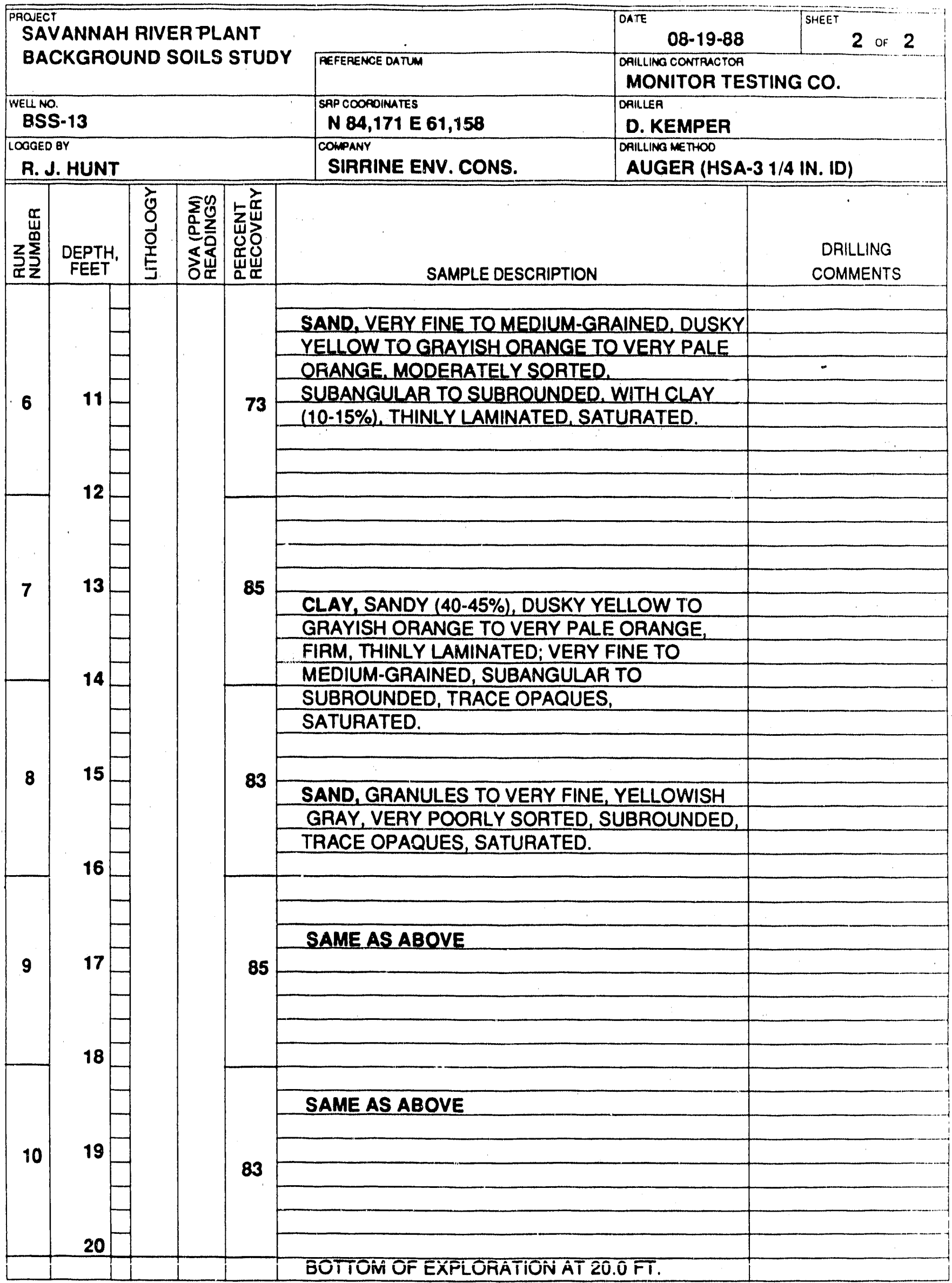




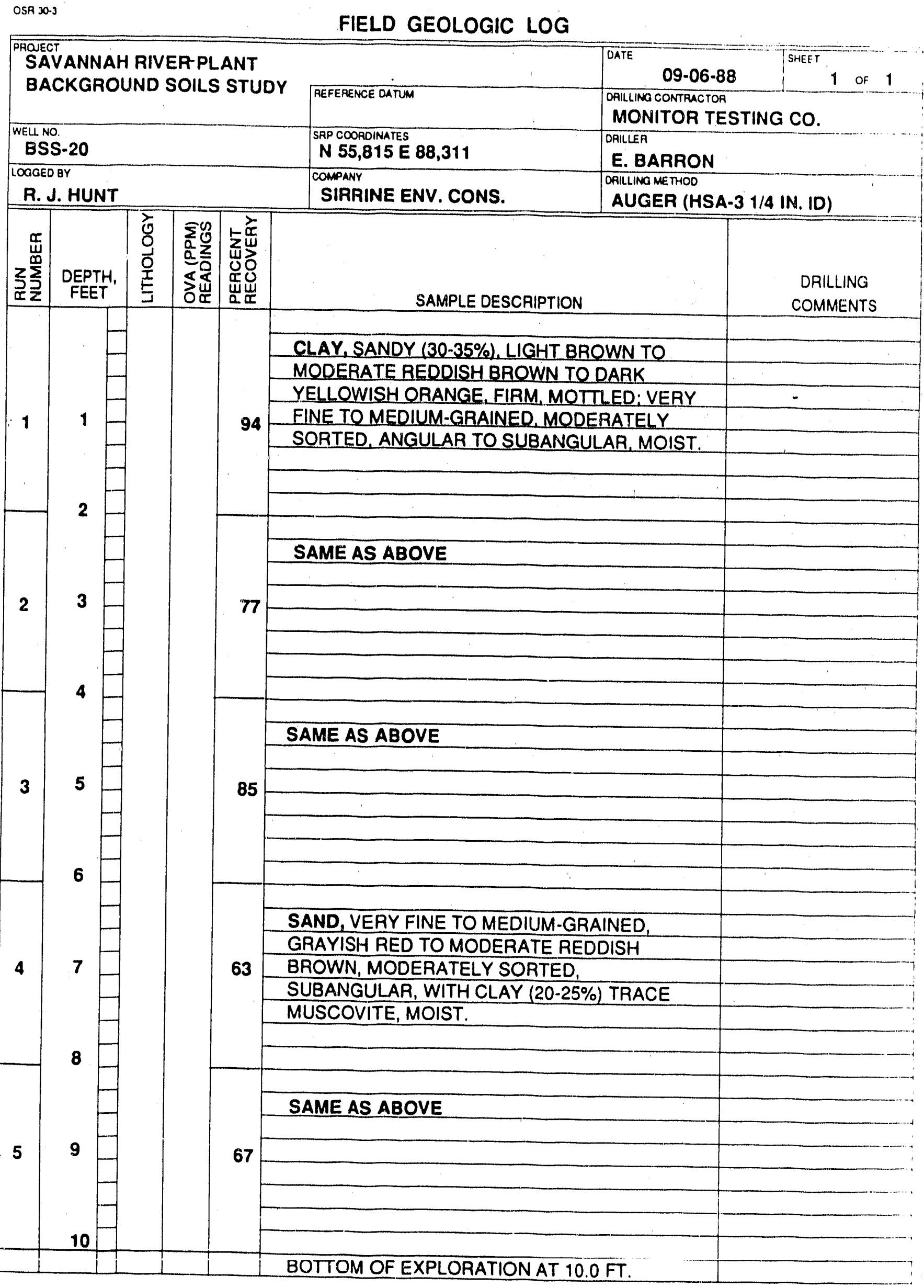


FIELD GEOLOGIC LOG

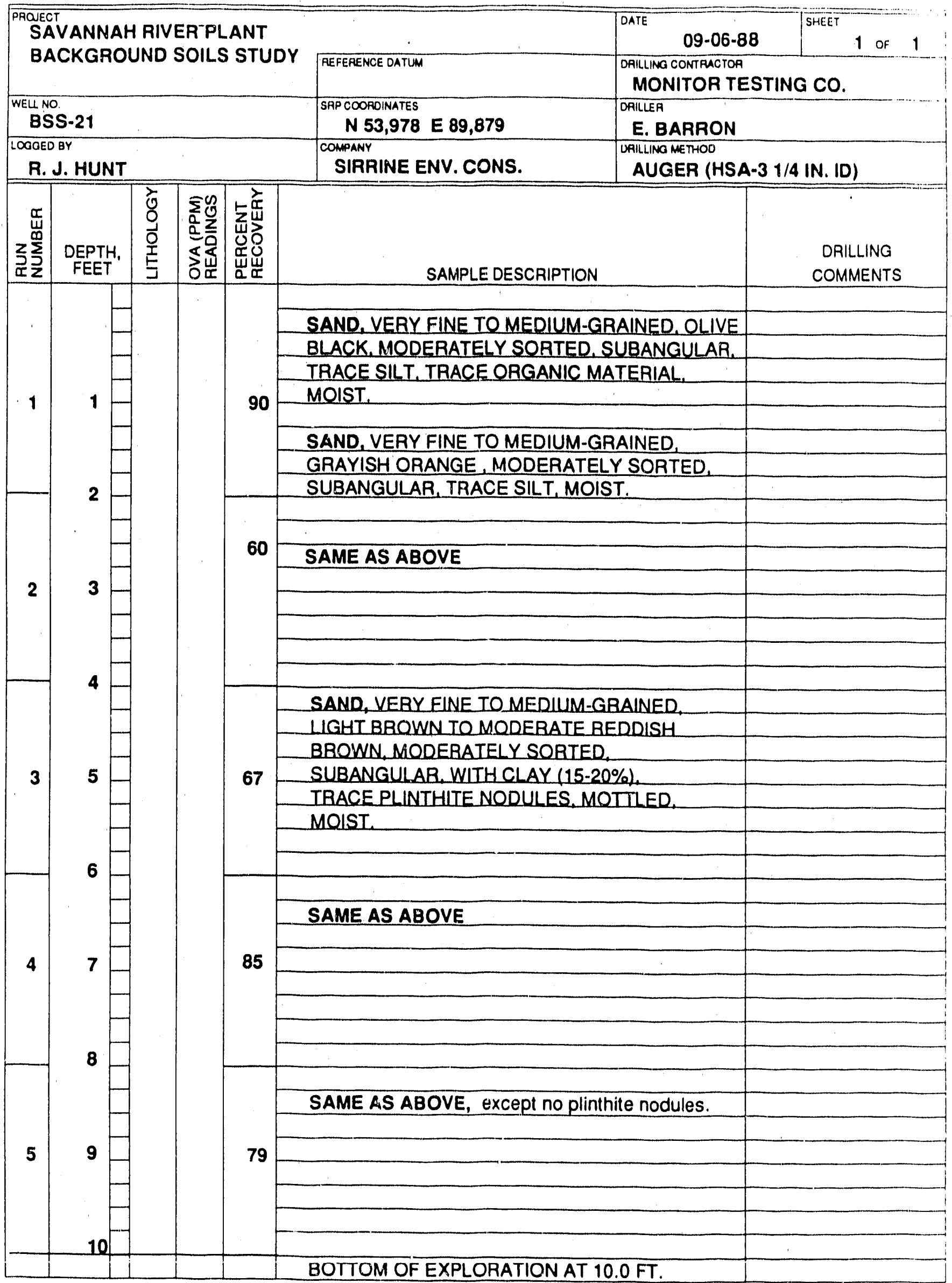


FIELD GEOLOGIC LOG

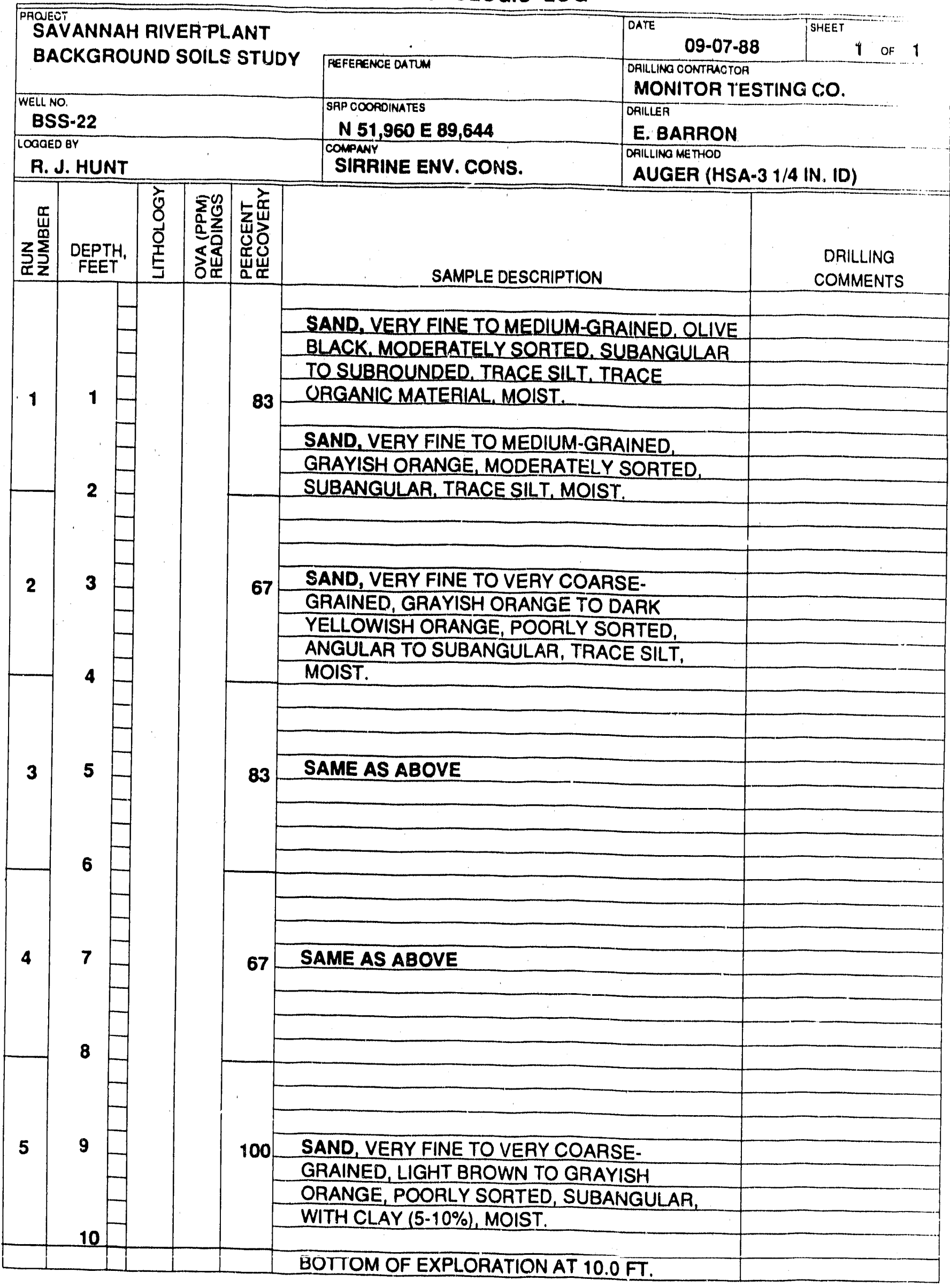


FIELD GEOLOGIC LOG

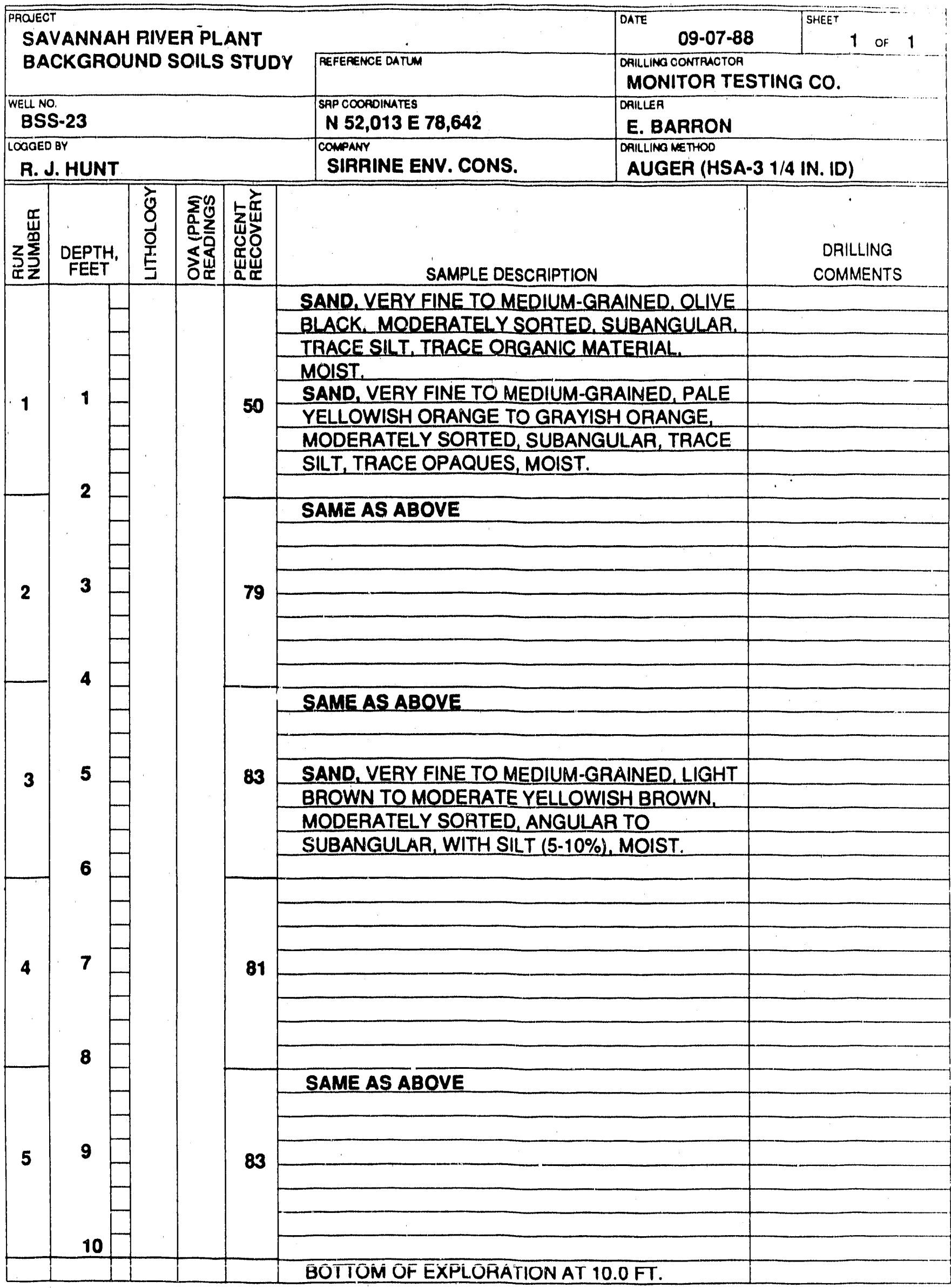


OSR 30.3

FIELD GEOLOGIC LOG

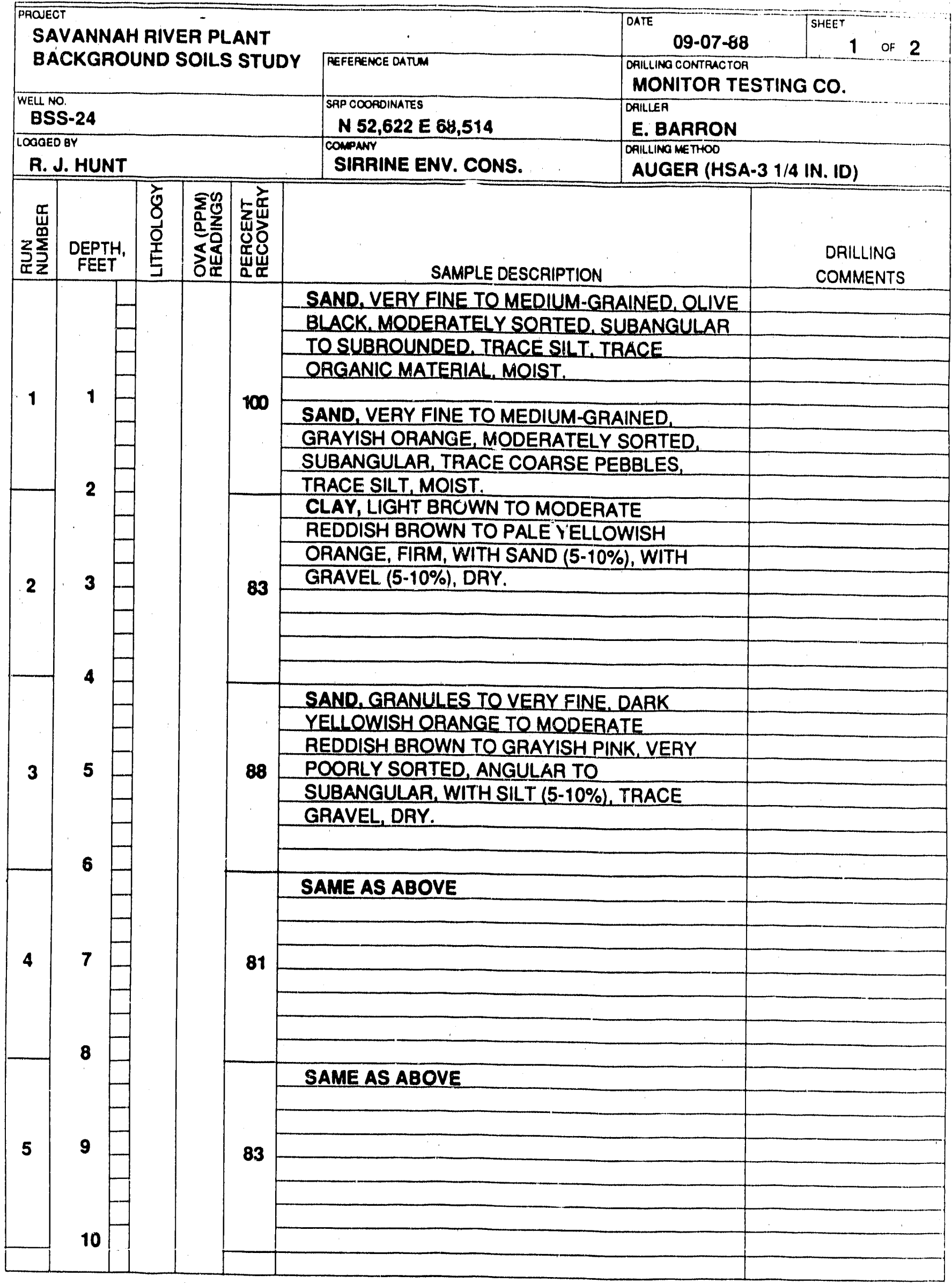


FIELD GEOLOGIC LOG

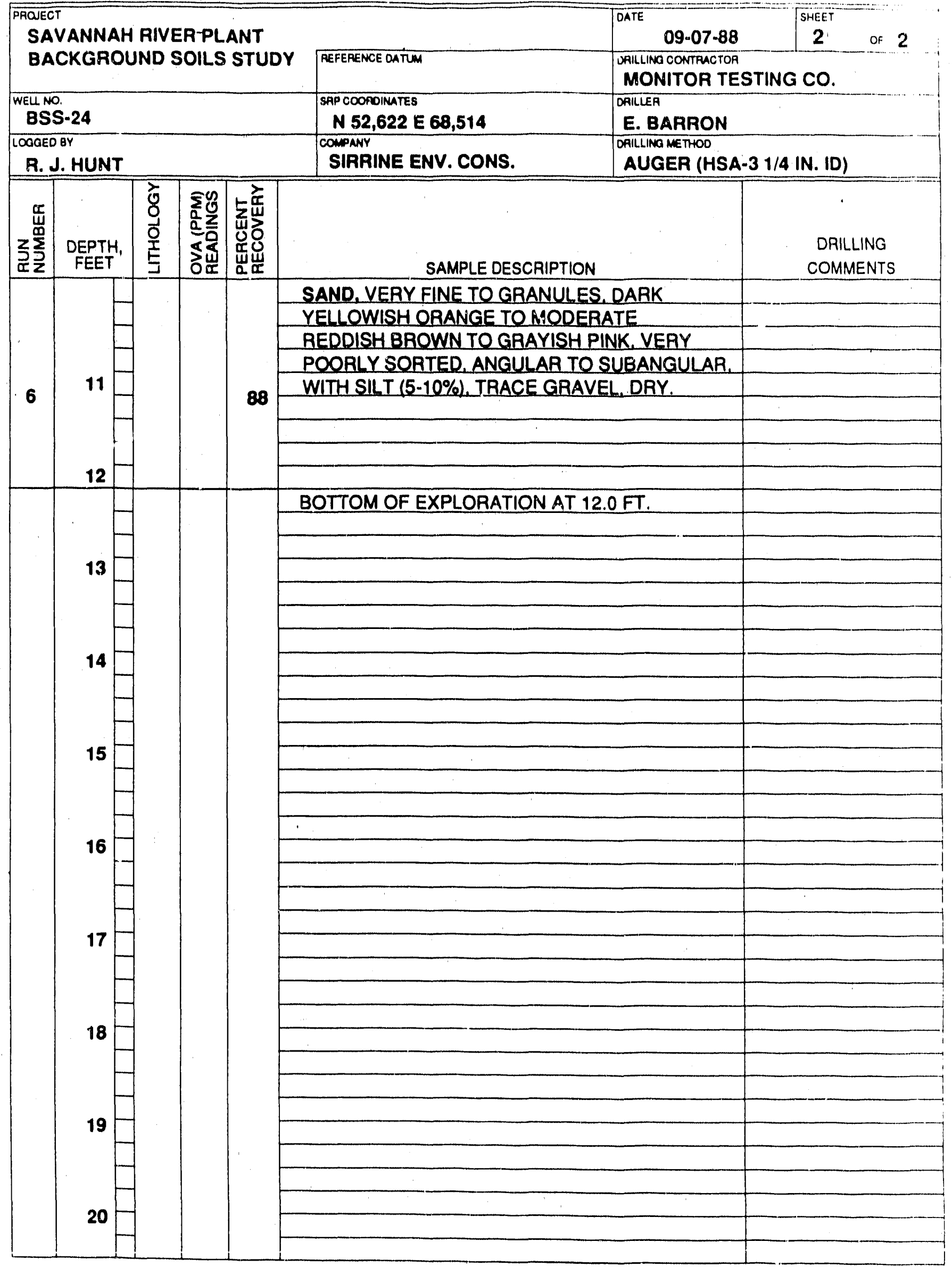


FIELD GEOLOGIC LOG

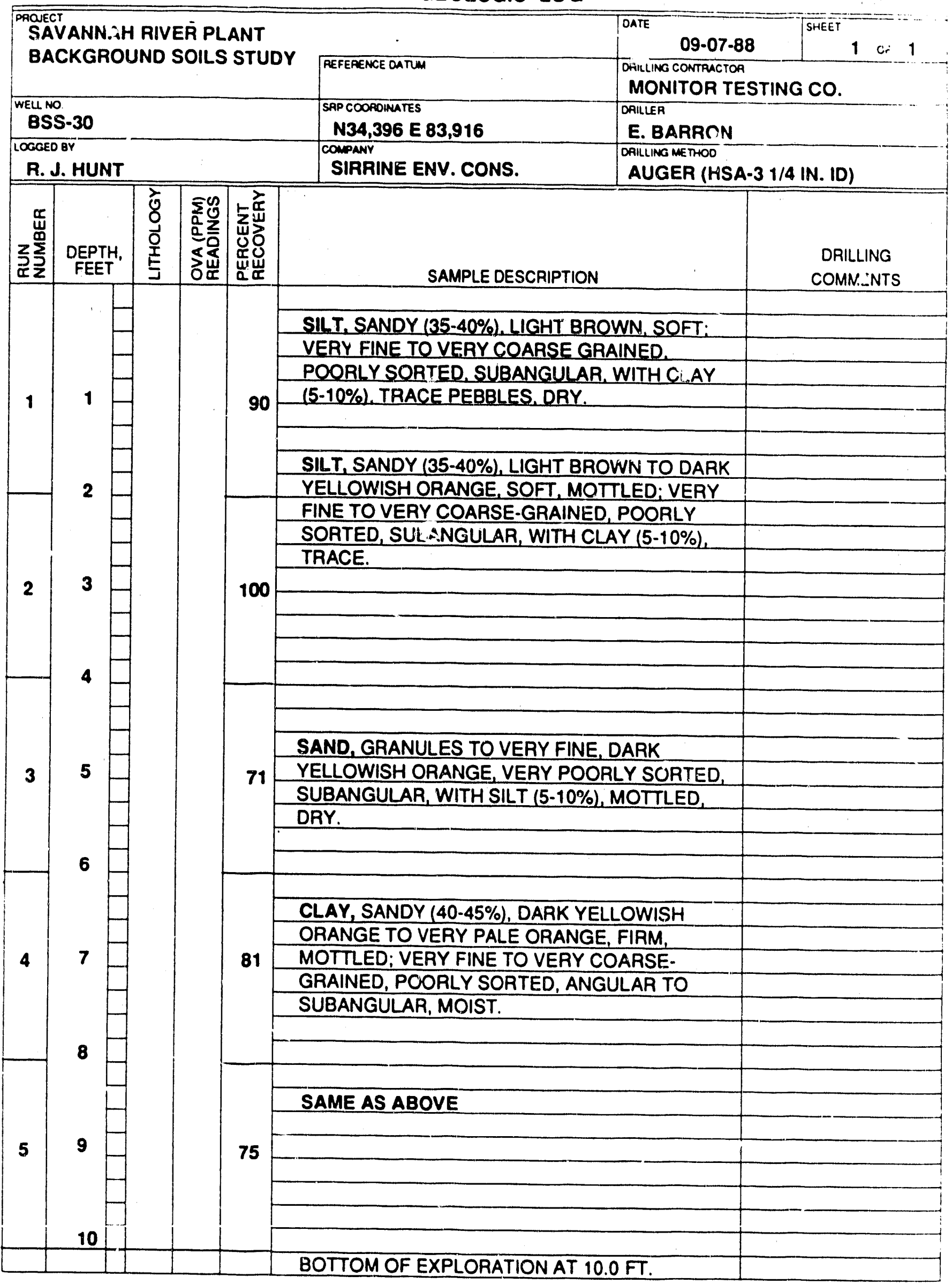


FIELD GEOLOGIC LOG

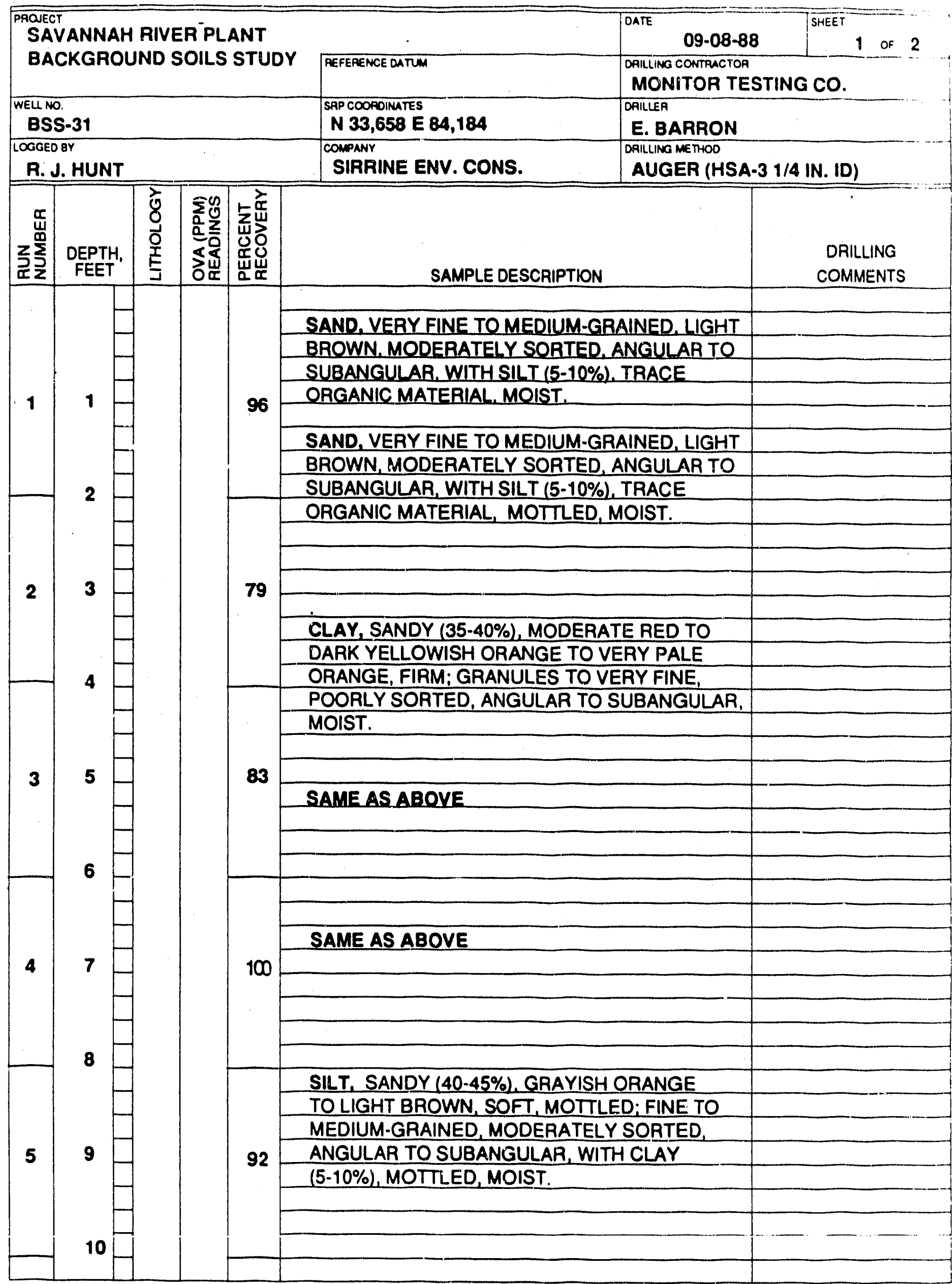




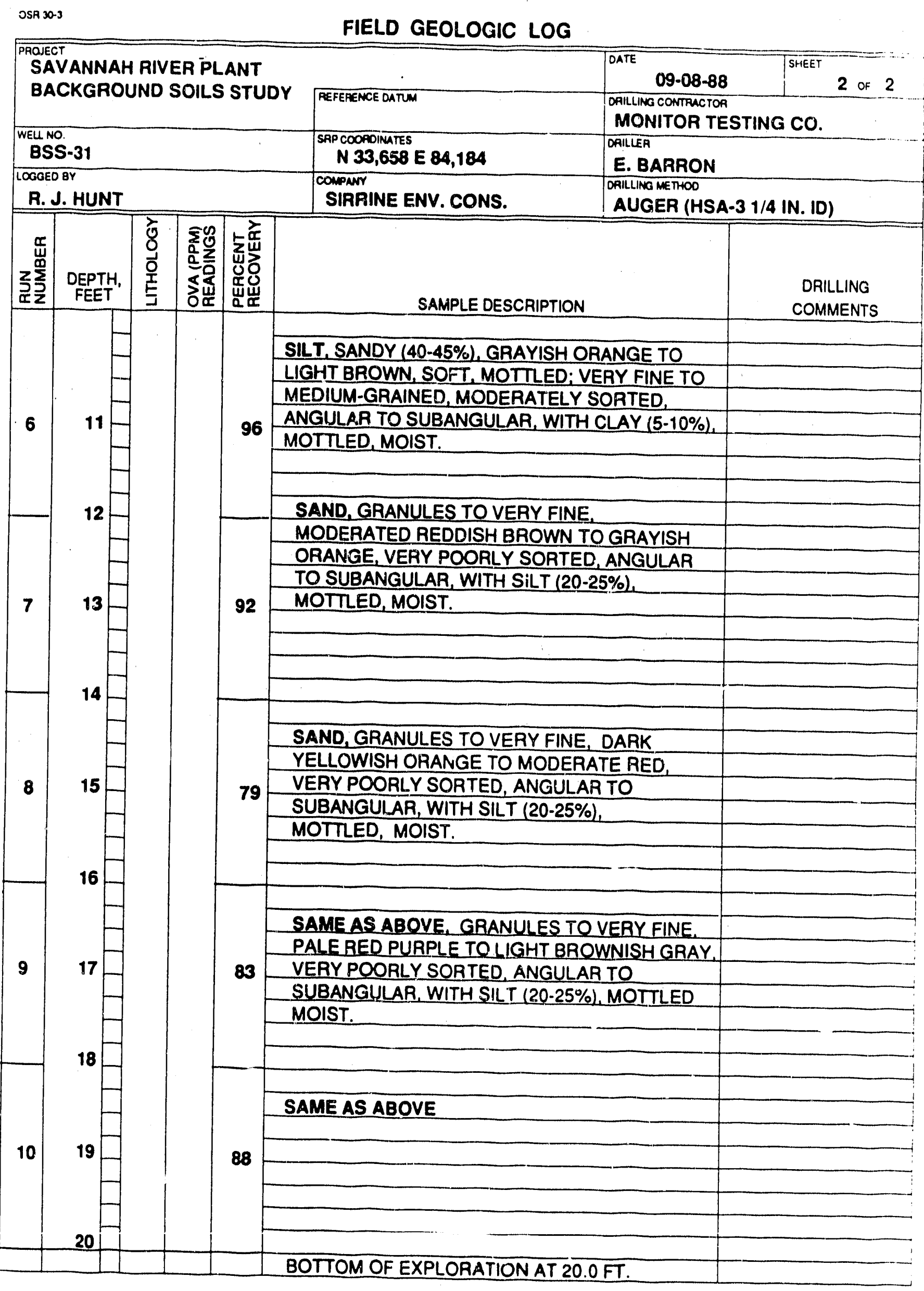


FIELD GEOLOGIC LOG

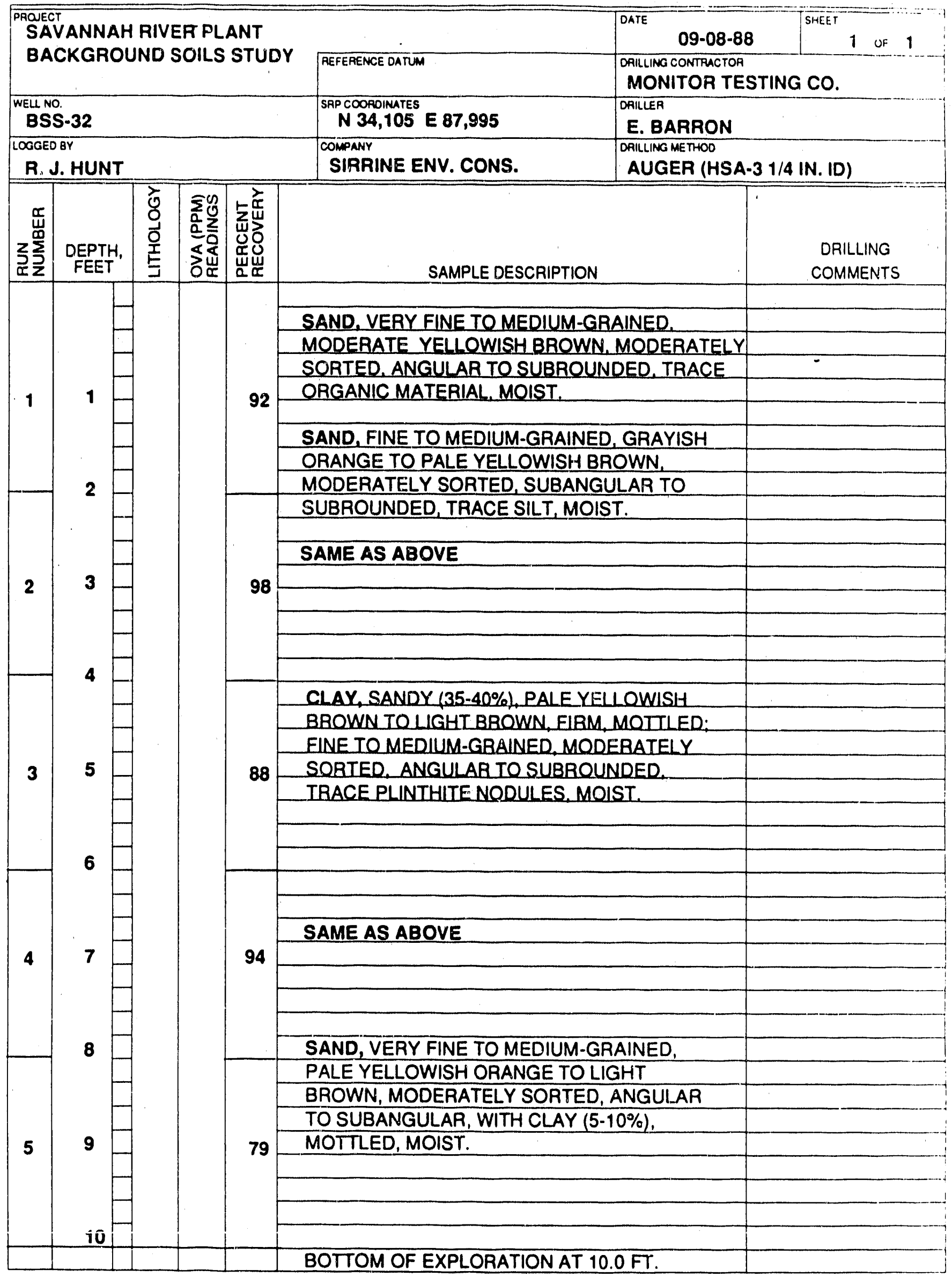




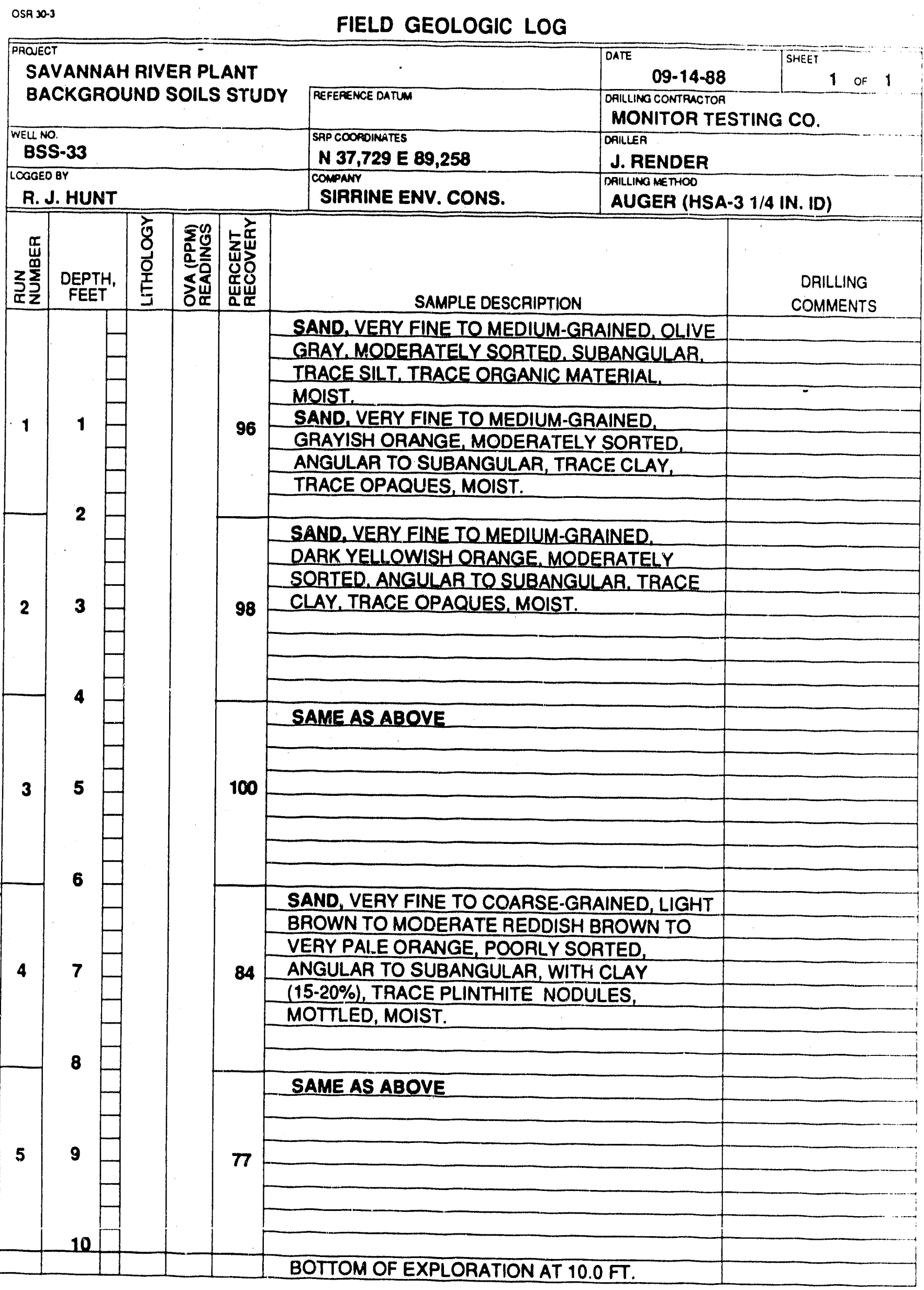


FIELD GEOLOGIC LOG

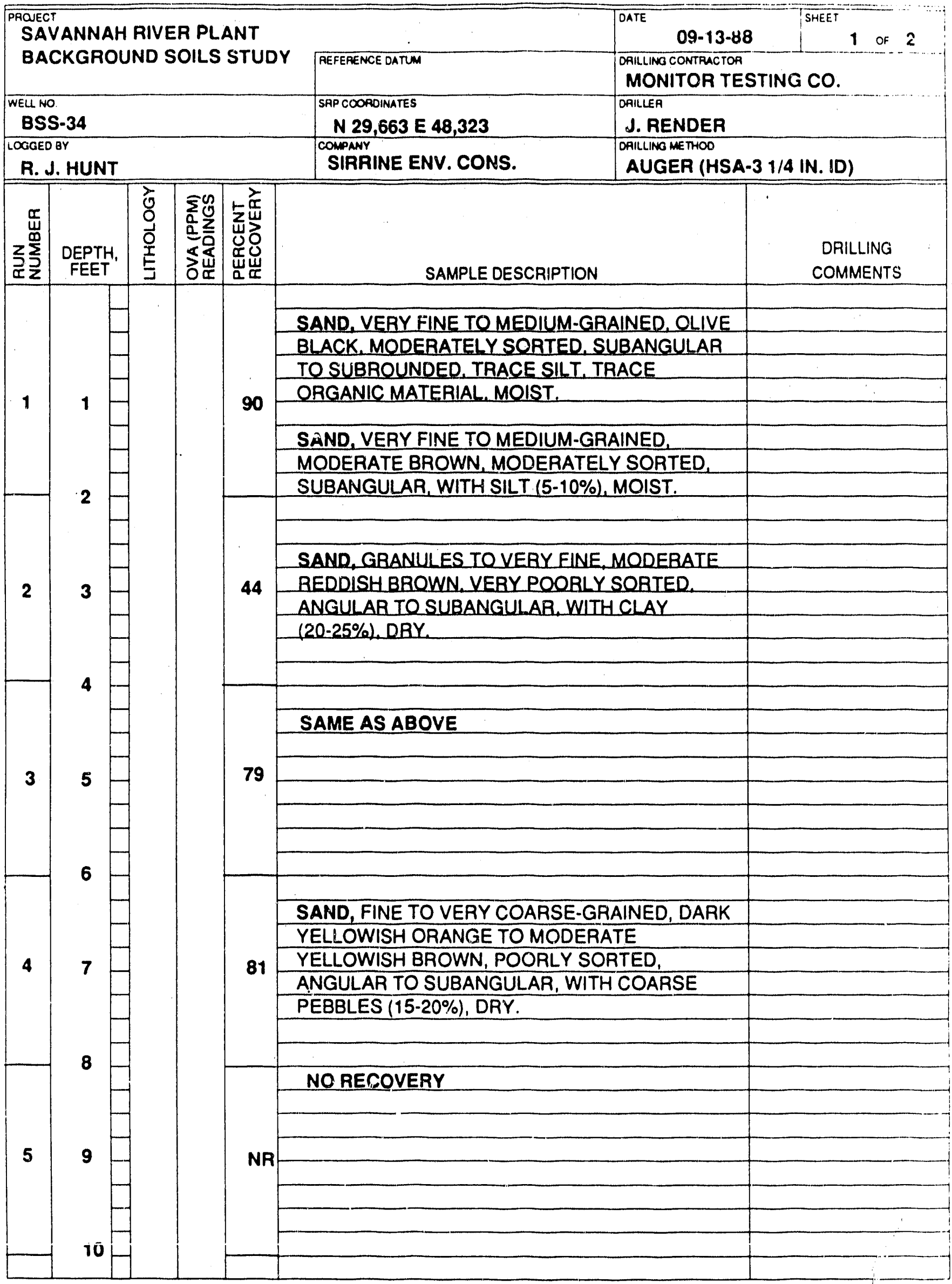


FIELD GEOLOGIC LOG

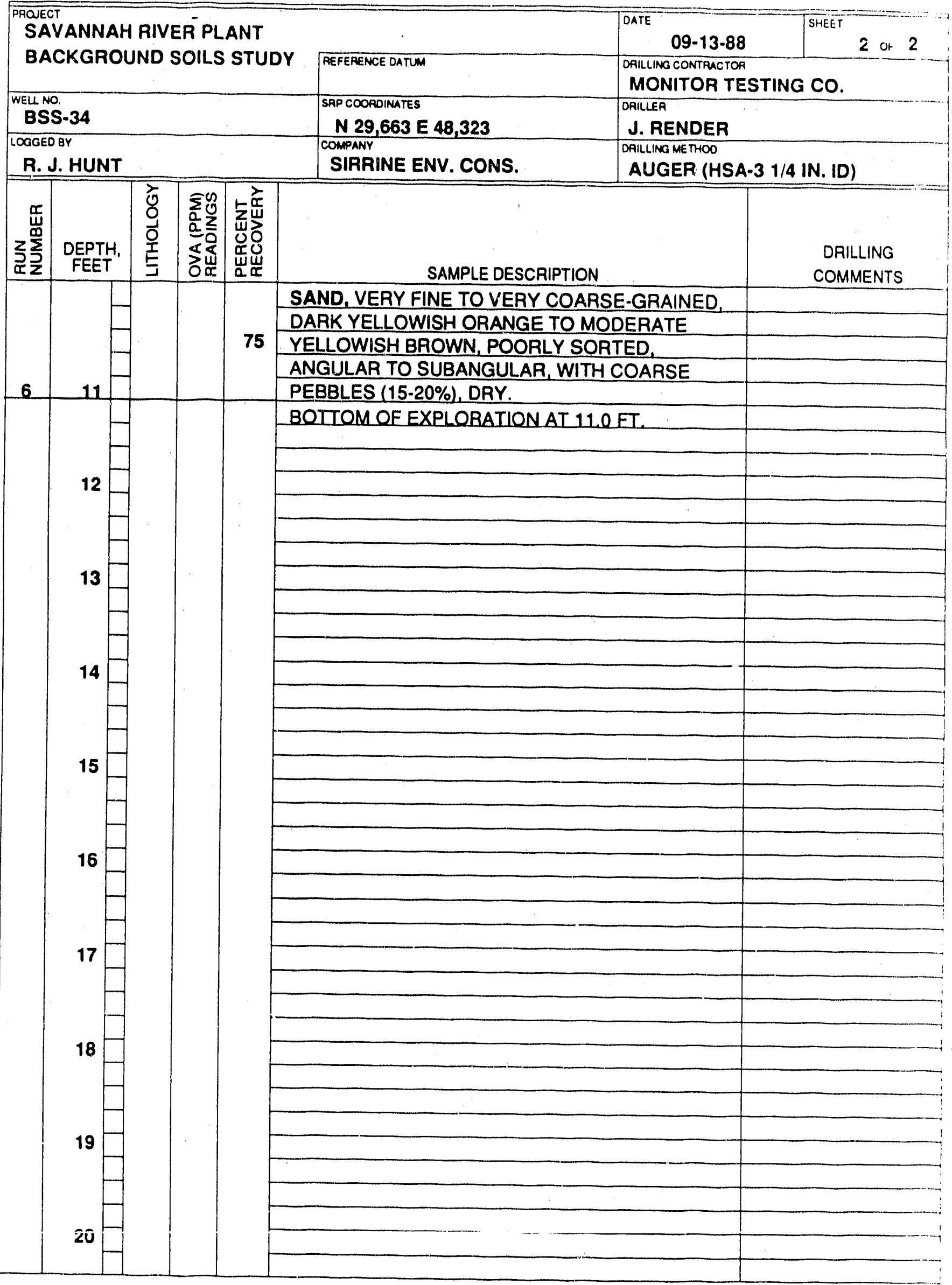


FIELD GEOLOGIC LOG

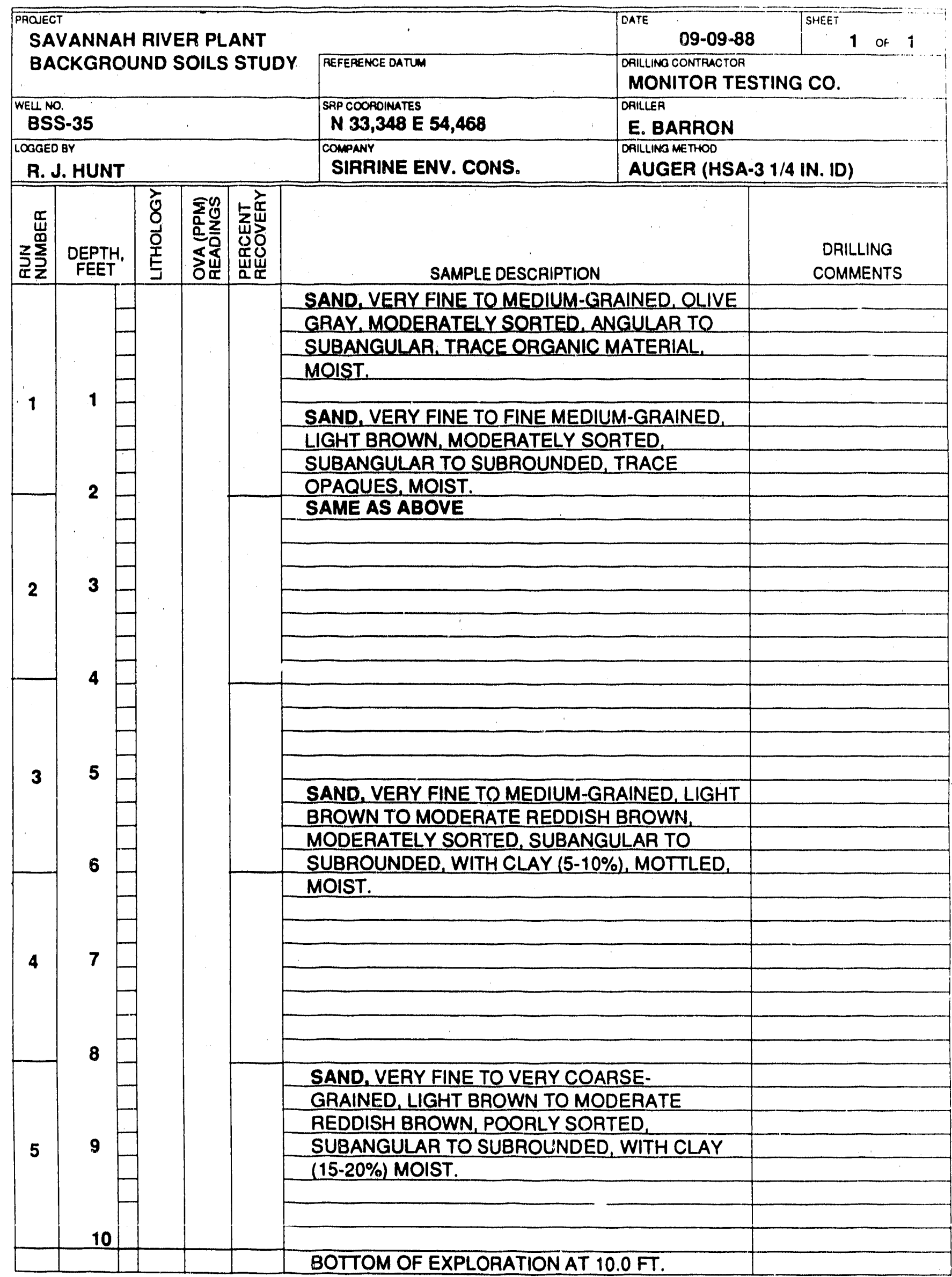


FIELD GEOLOGIC LOG

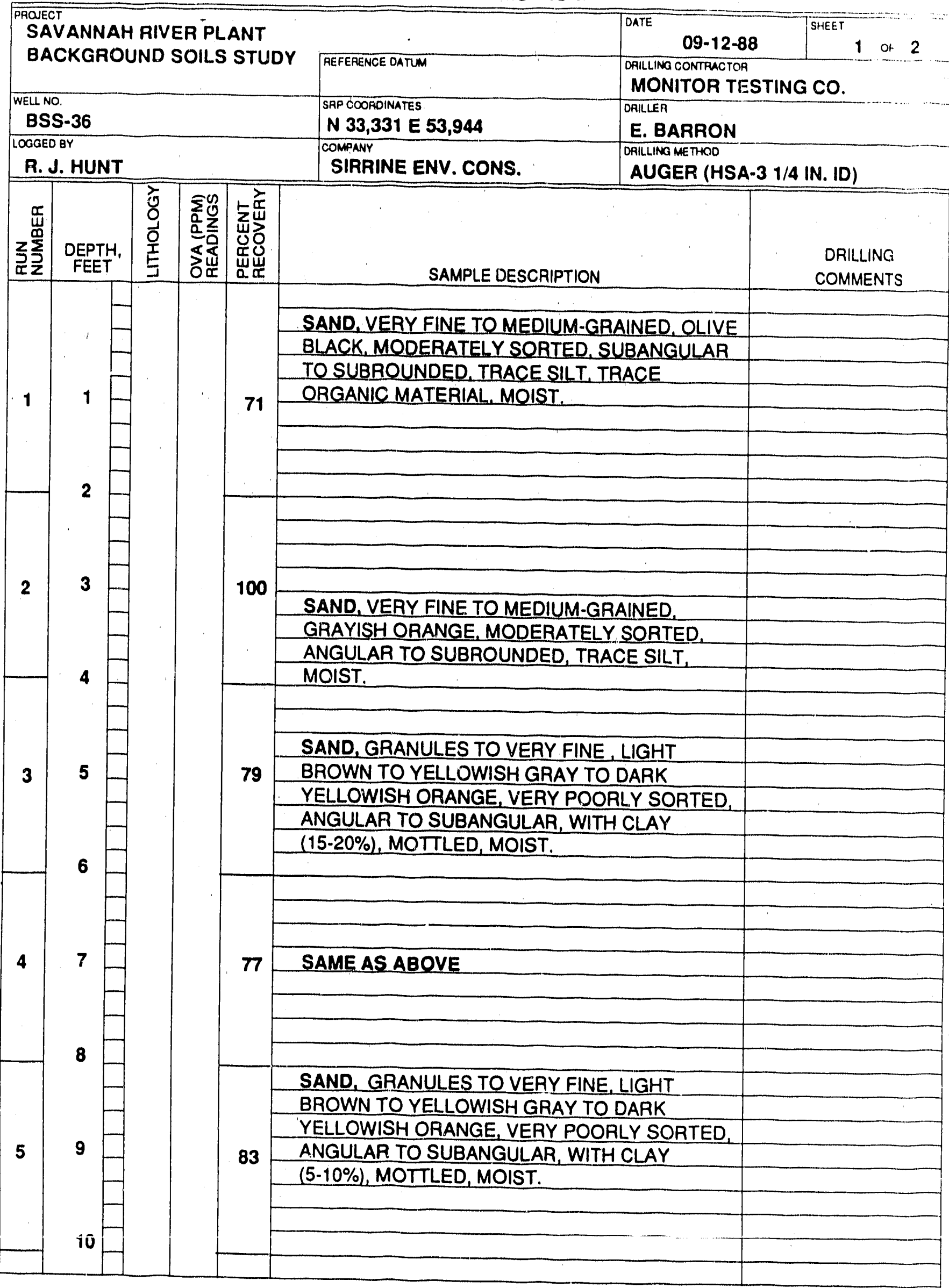


FIELD GEOLOGIC LOG

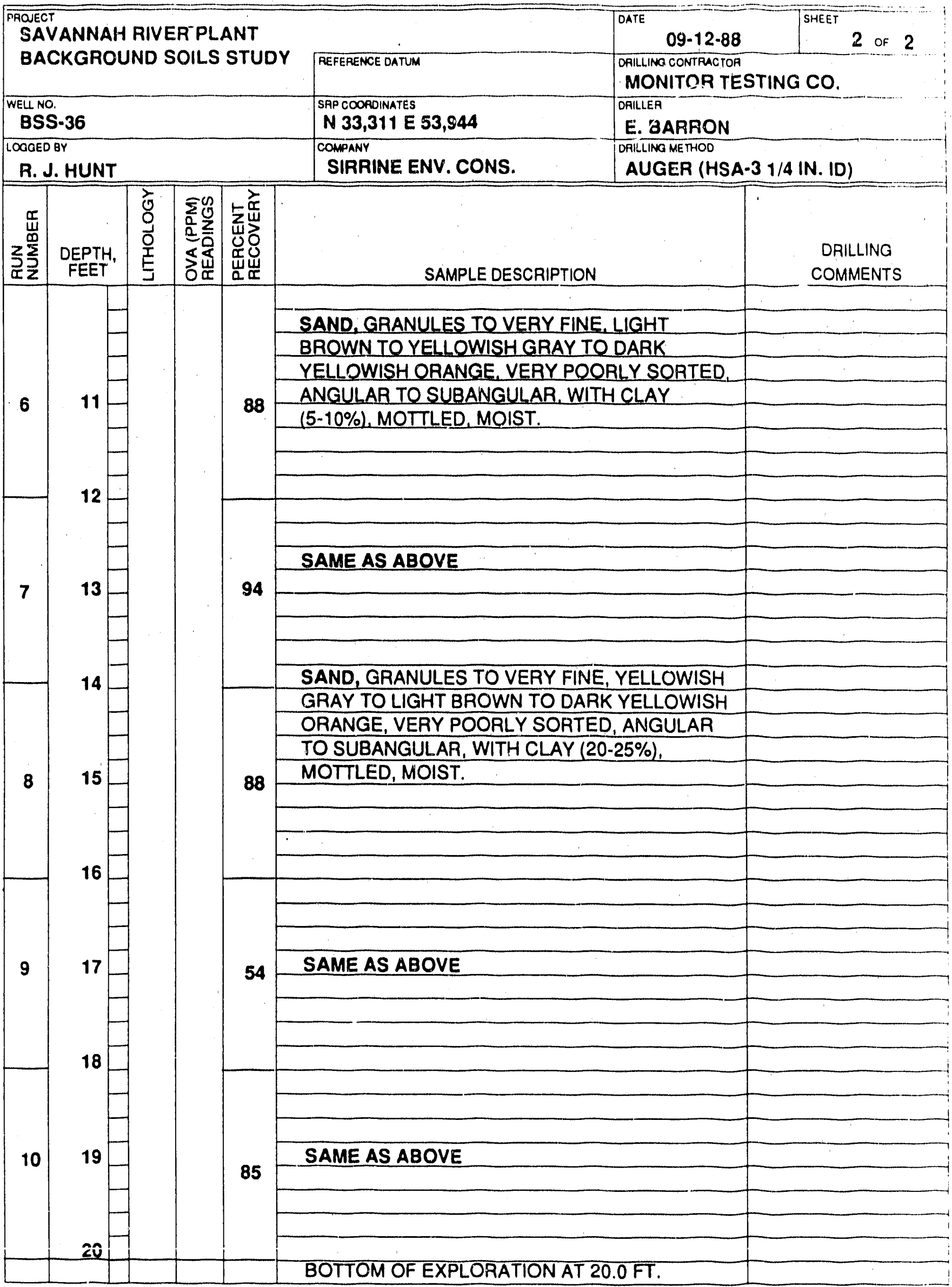


FIELD GEOLOGIC LOG

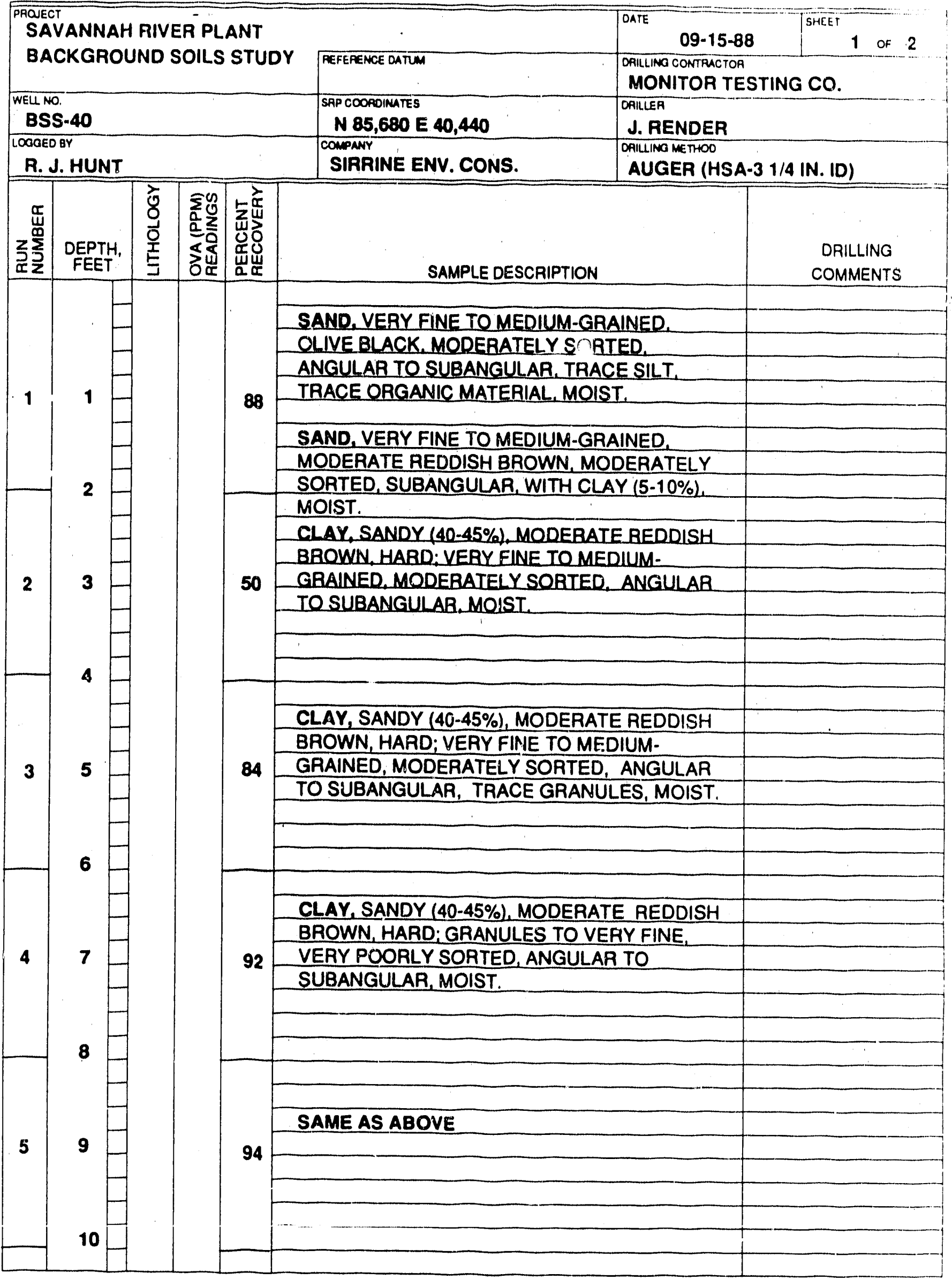


FIELD GEOLOGIC LOG

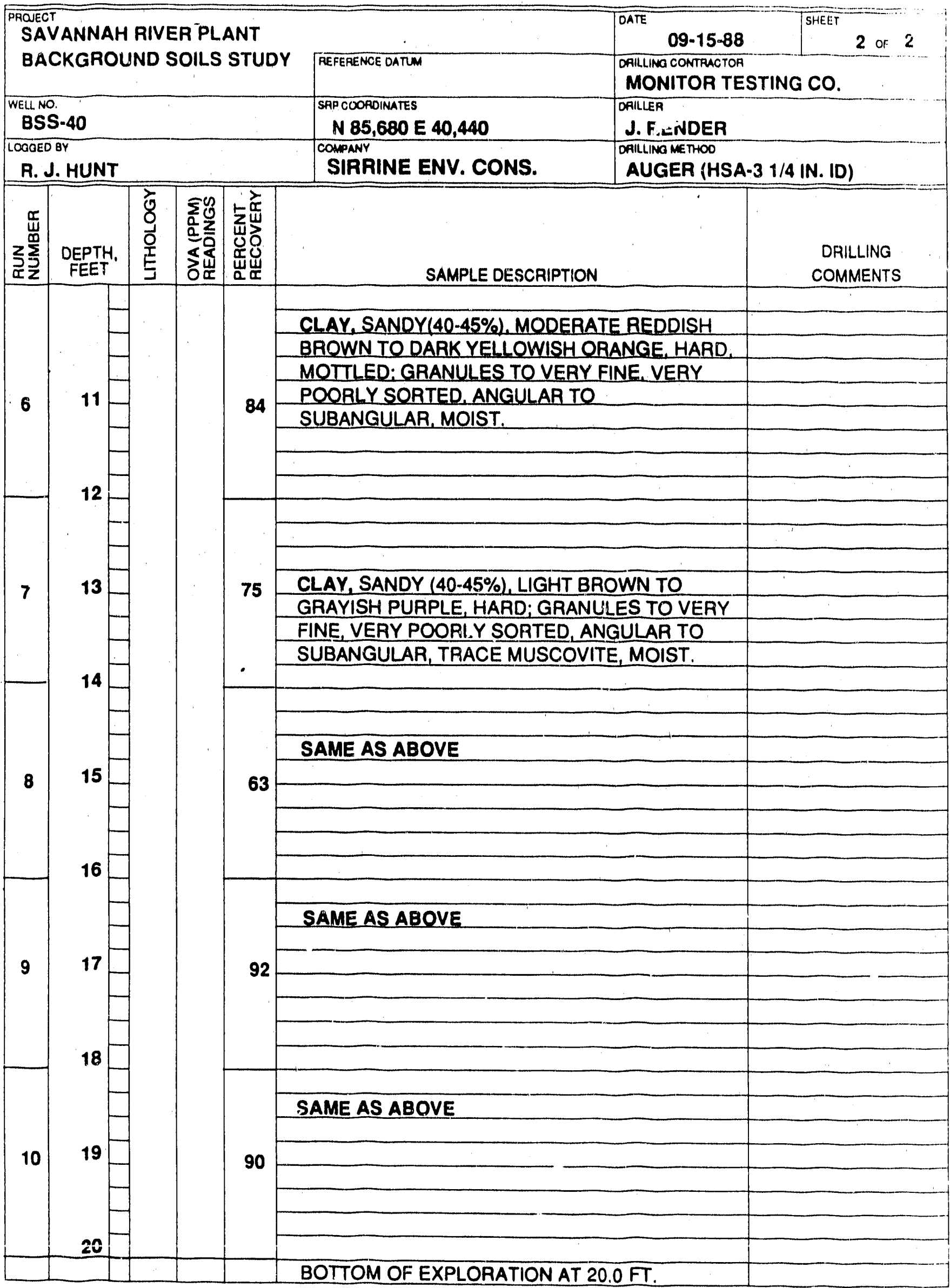




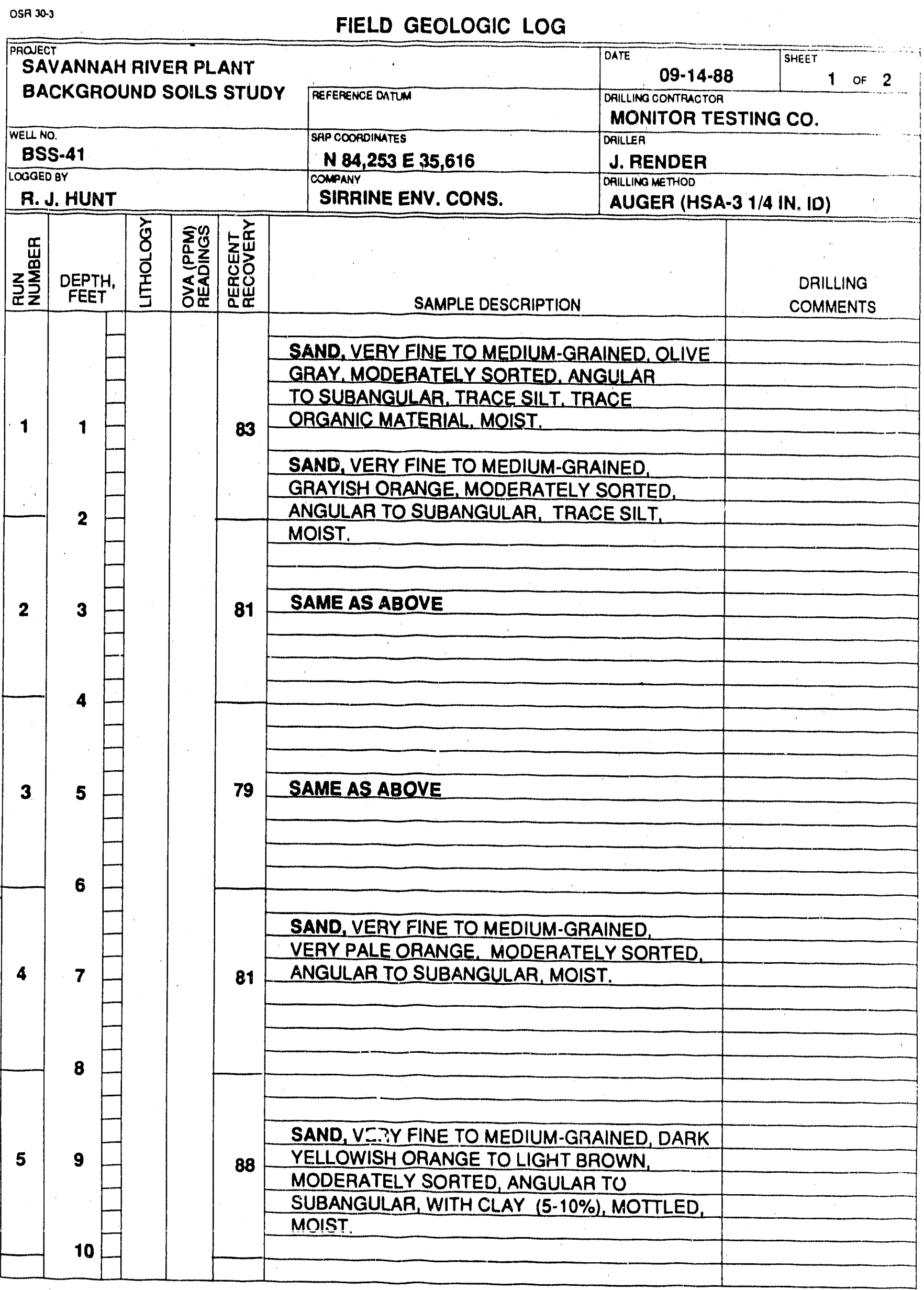


FIELDD GEOLOGIC LOG

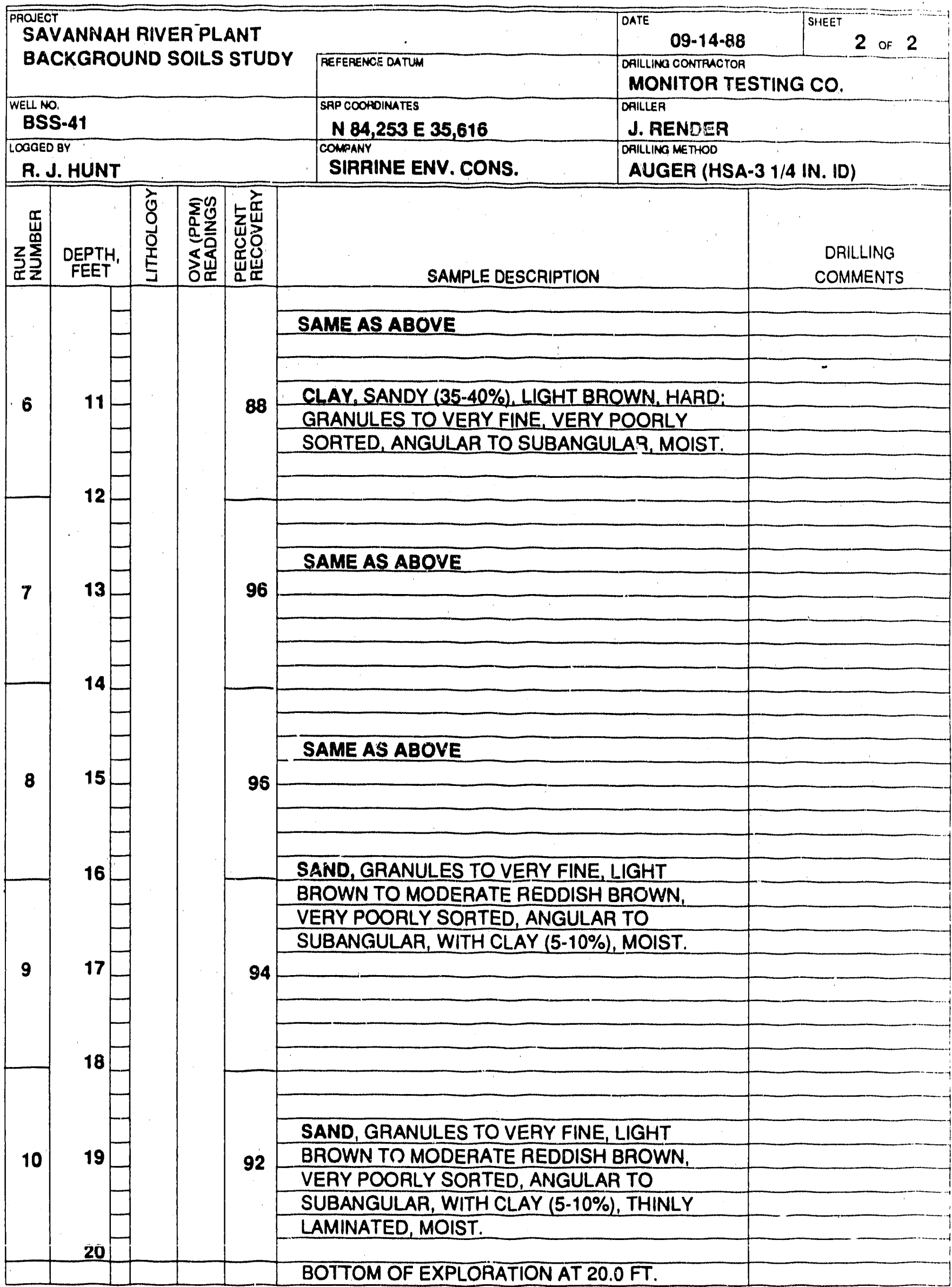


FIELD GEOLOGIC LOG

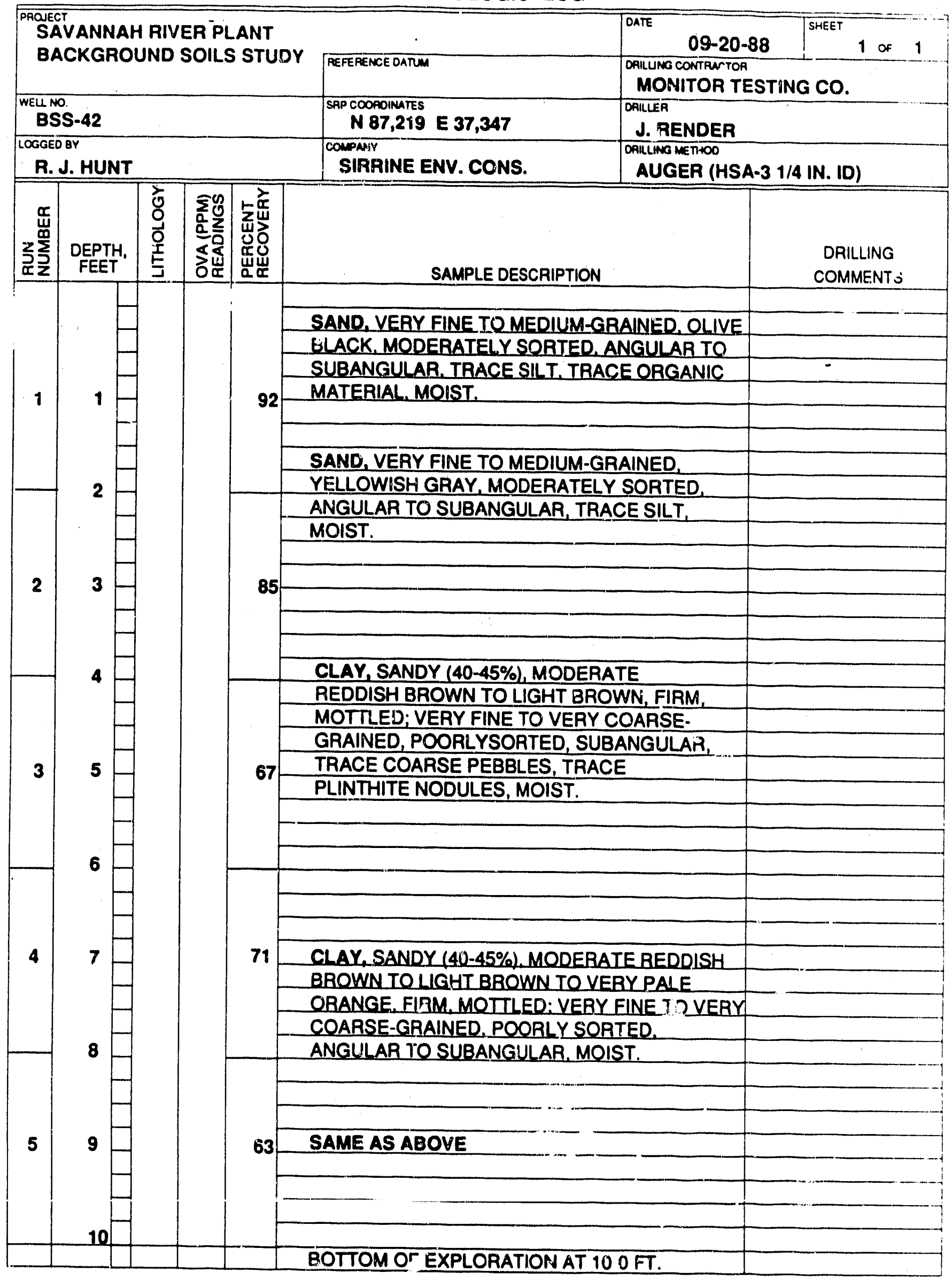




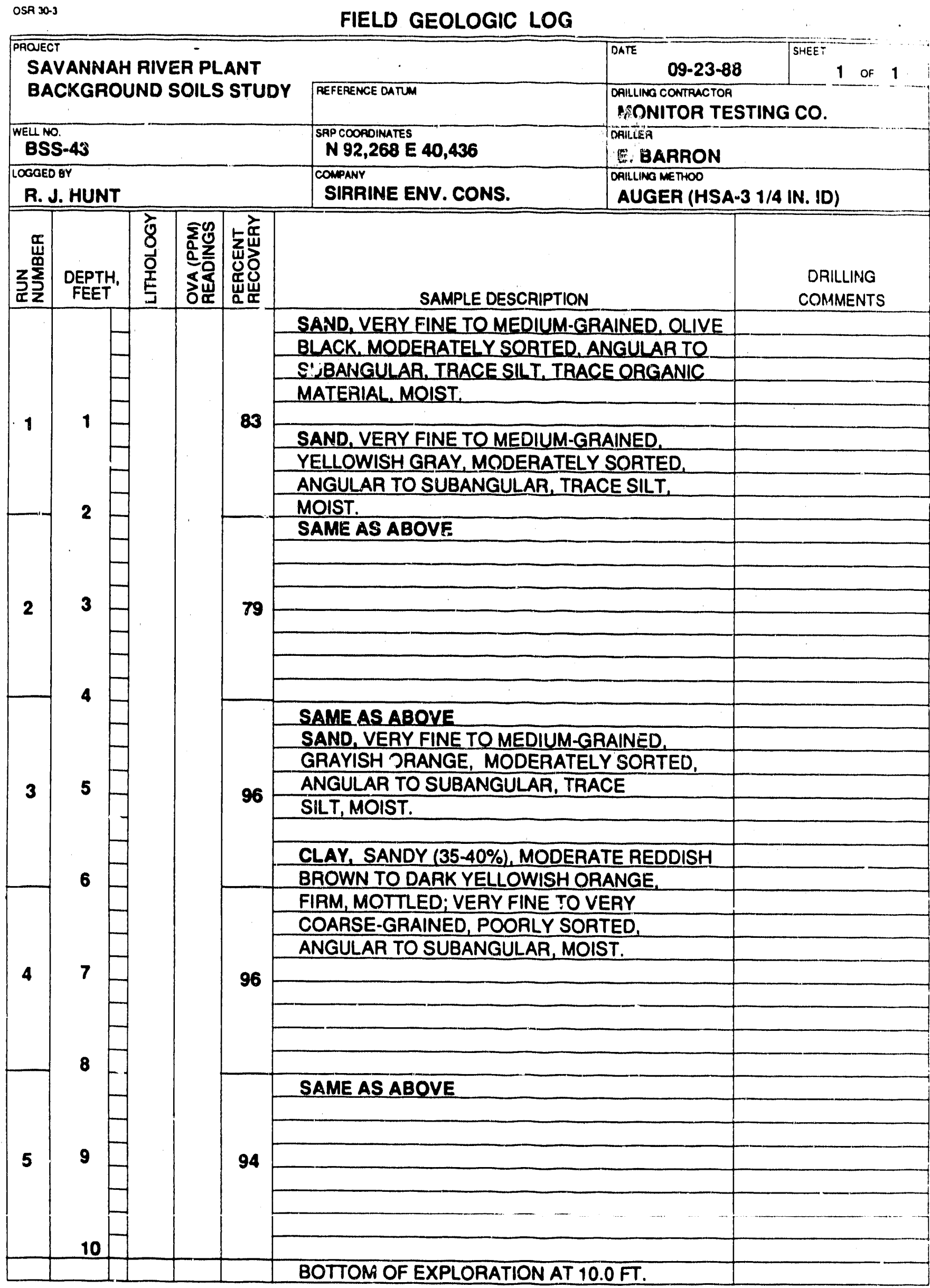




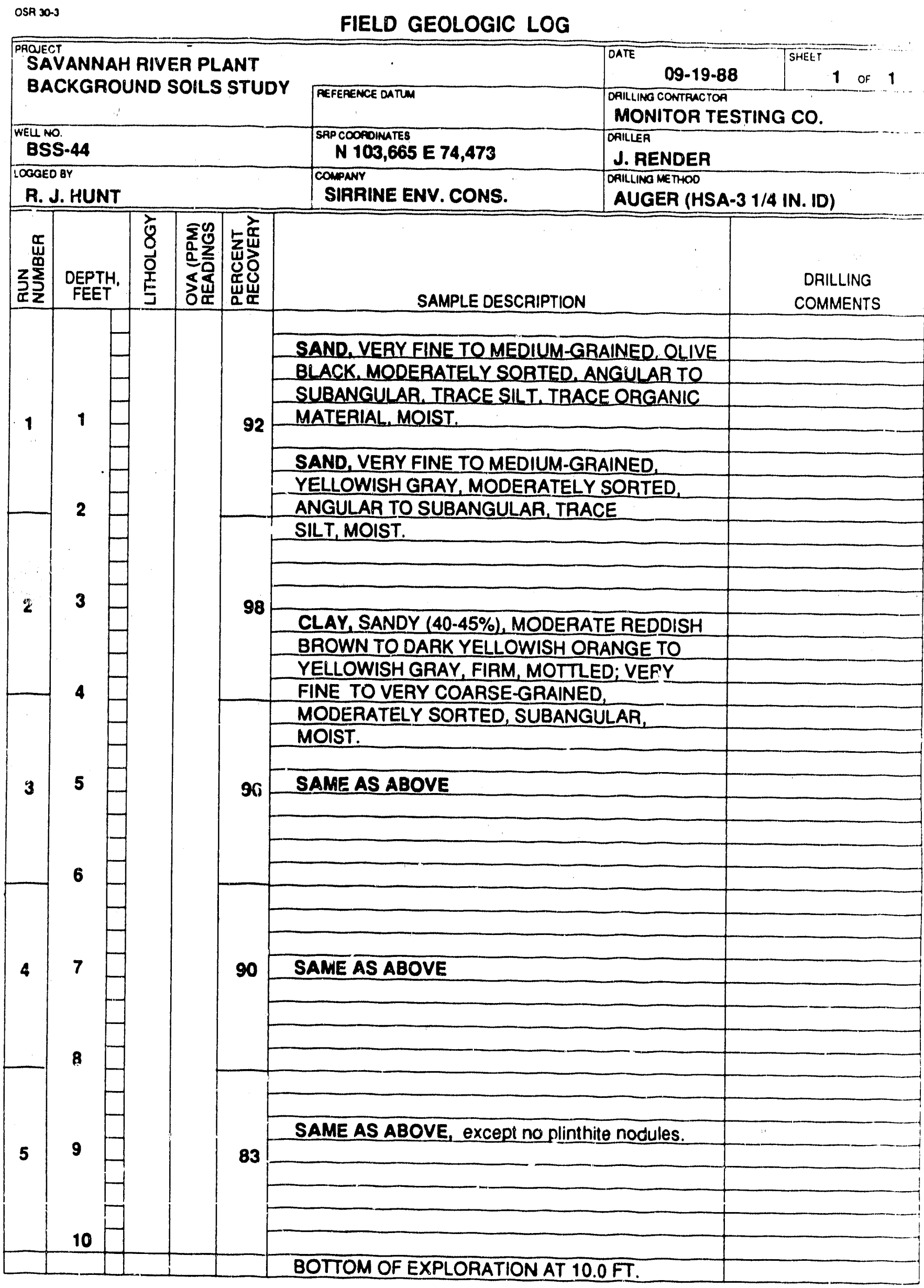


FIELD GEOLOGIC LOG

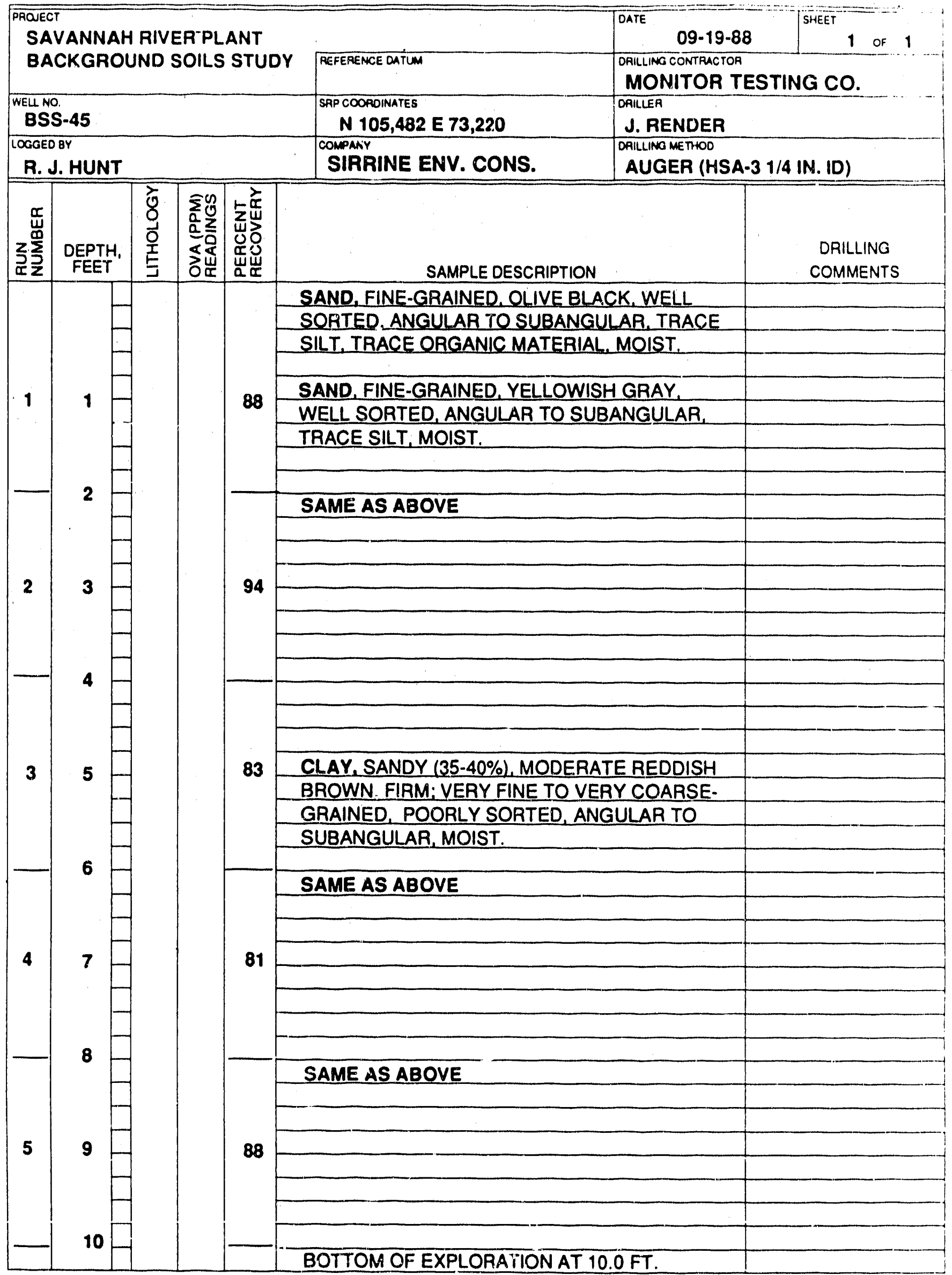


FIELD GEOLOGIC LOG

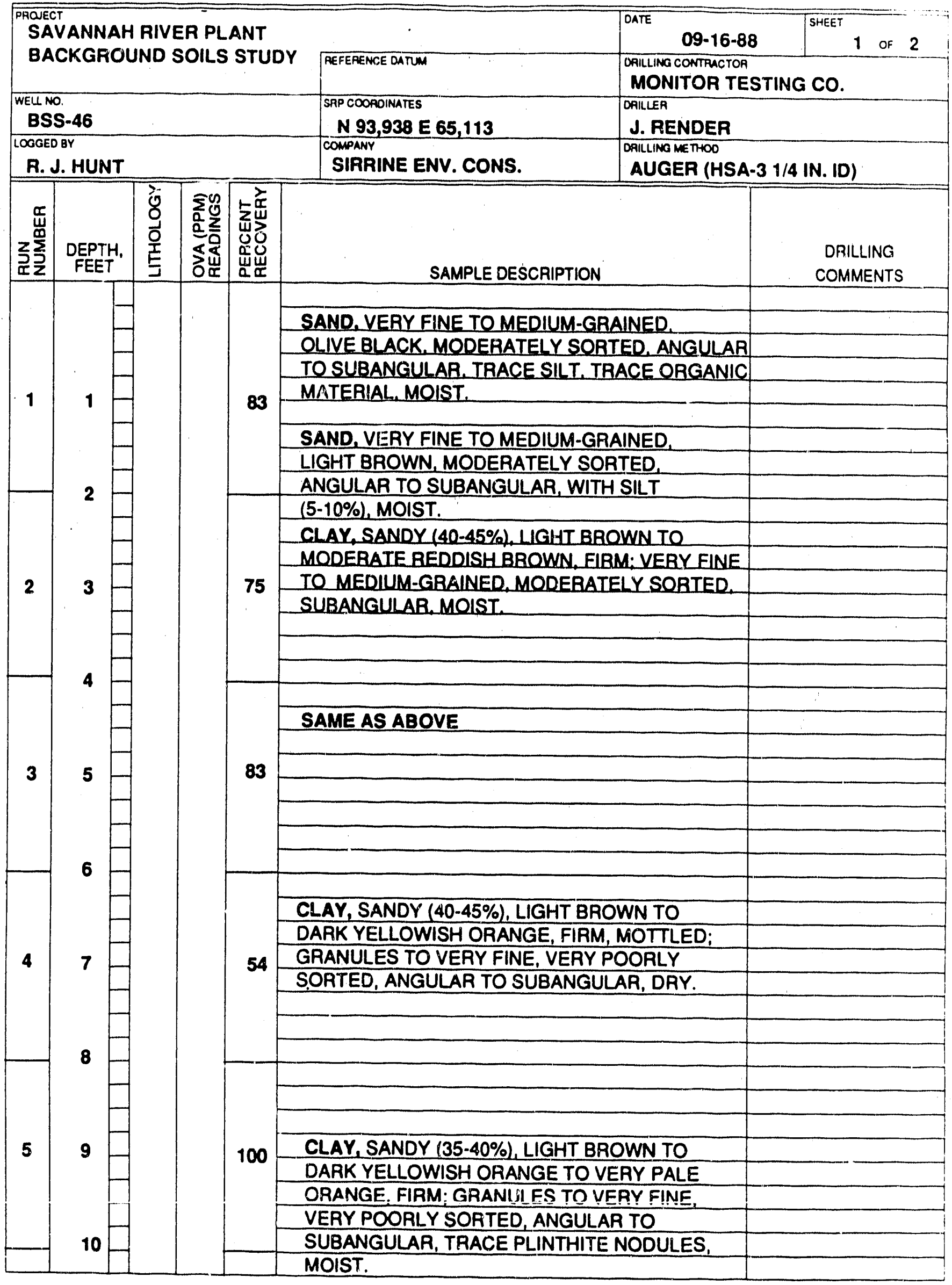




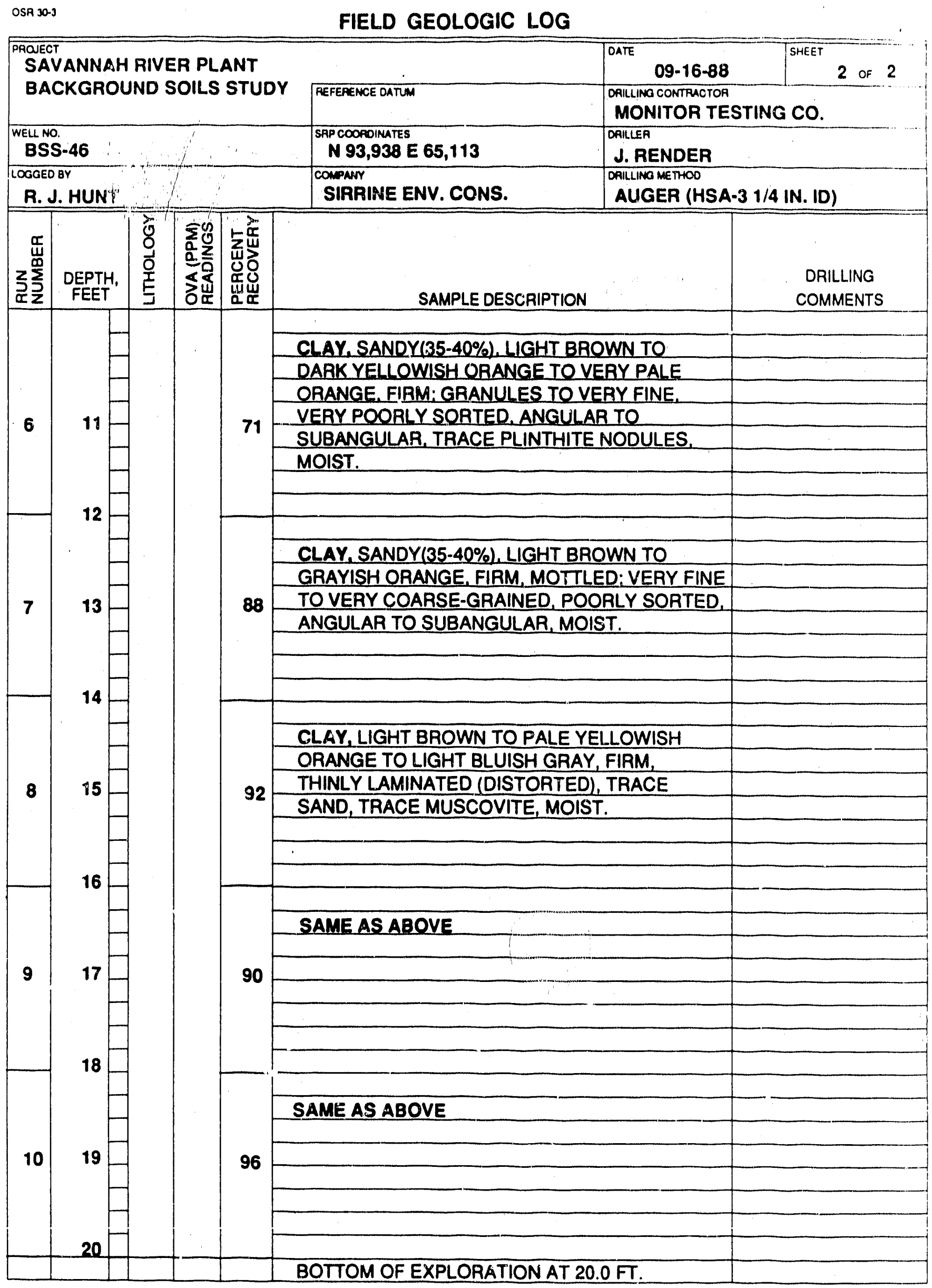




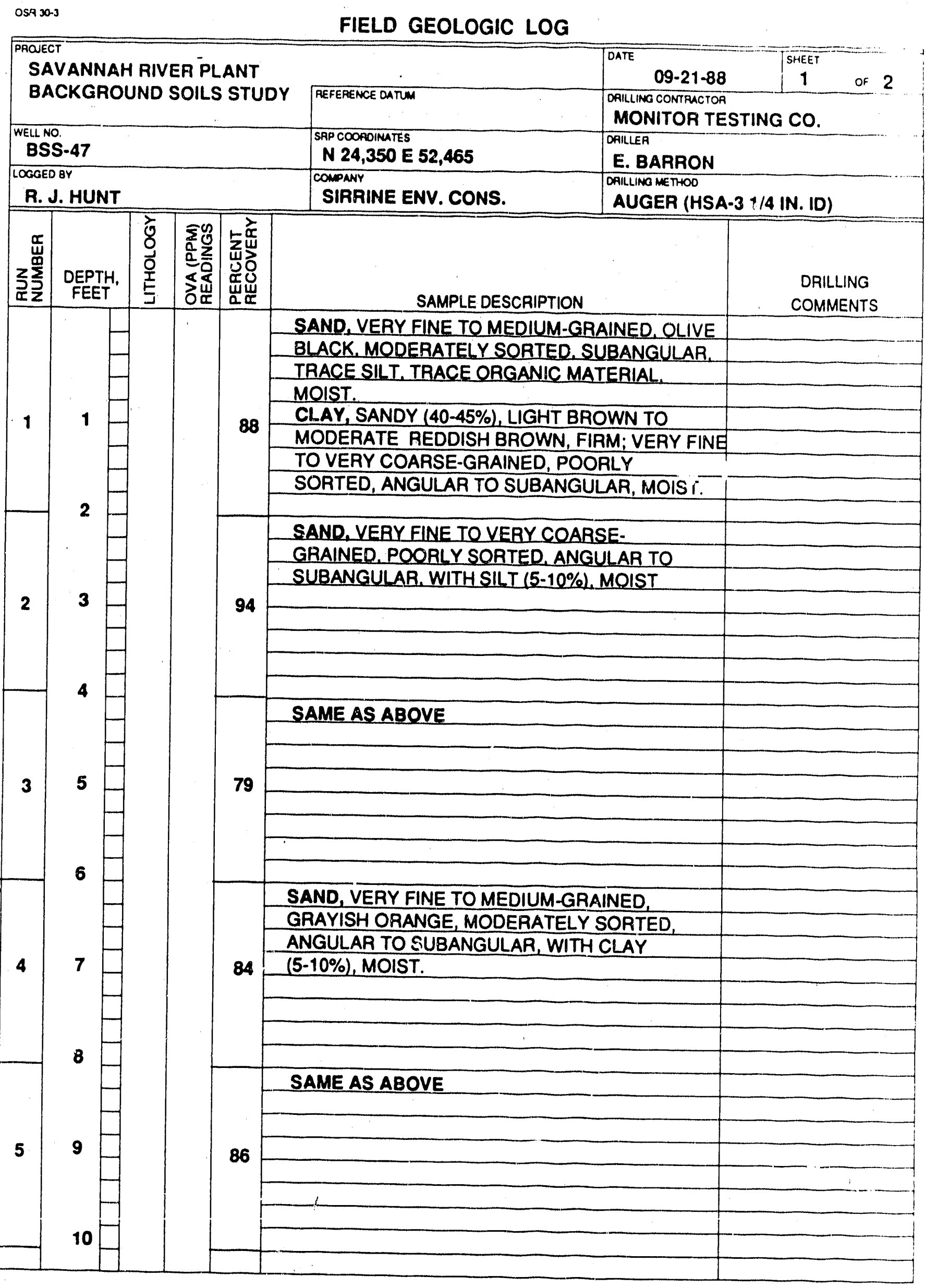




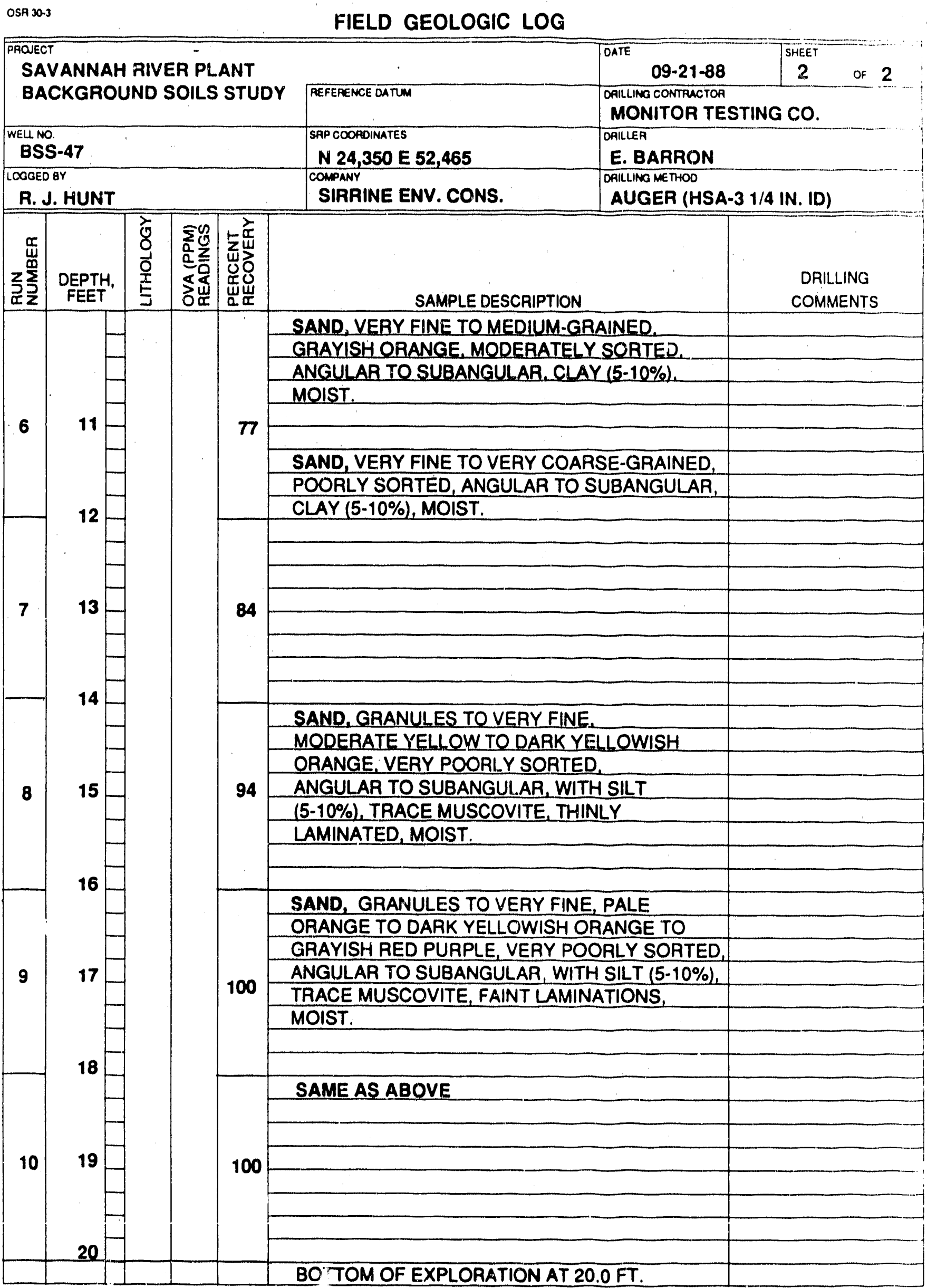




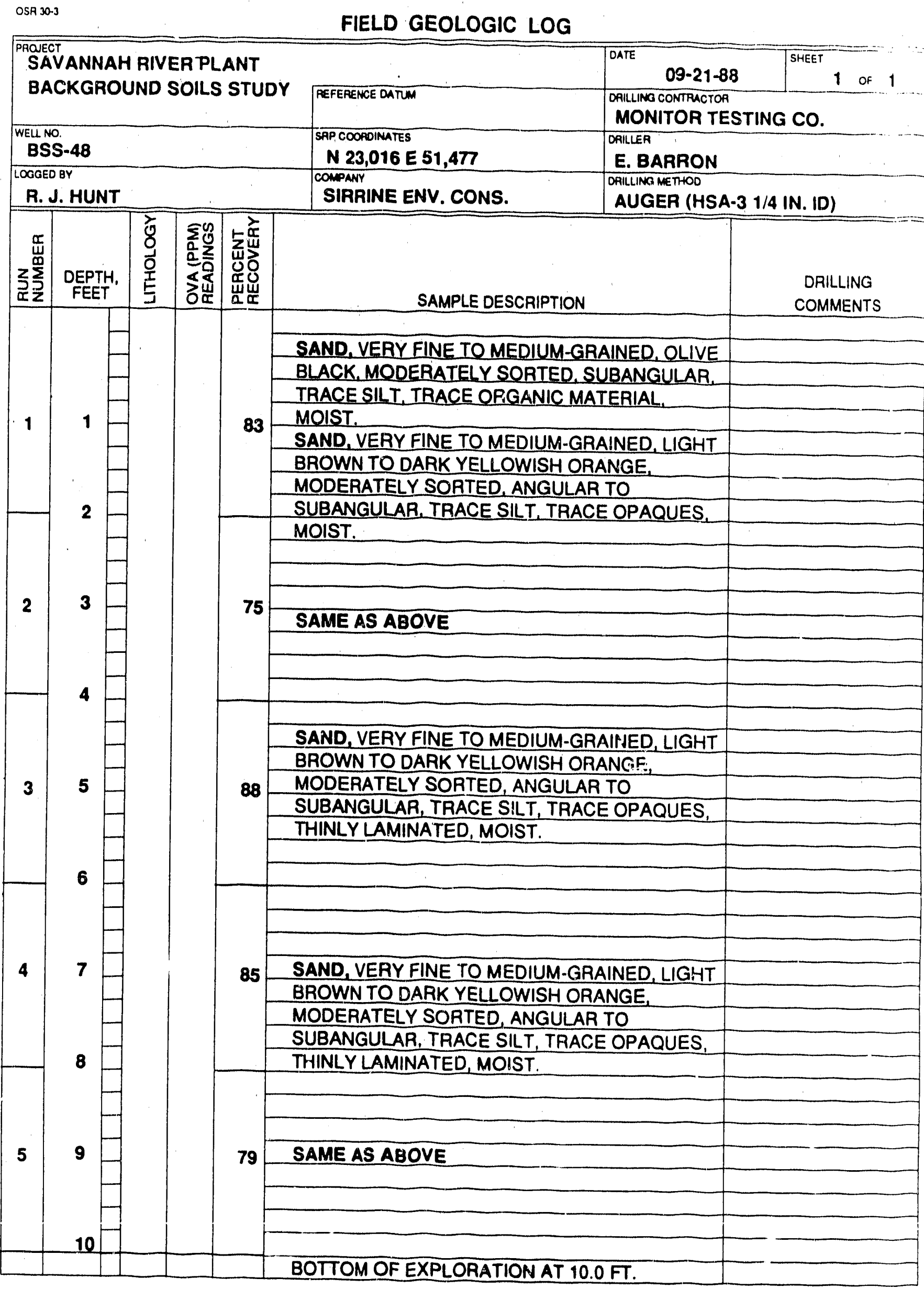


FIELD GEOLOGIC LOG

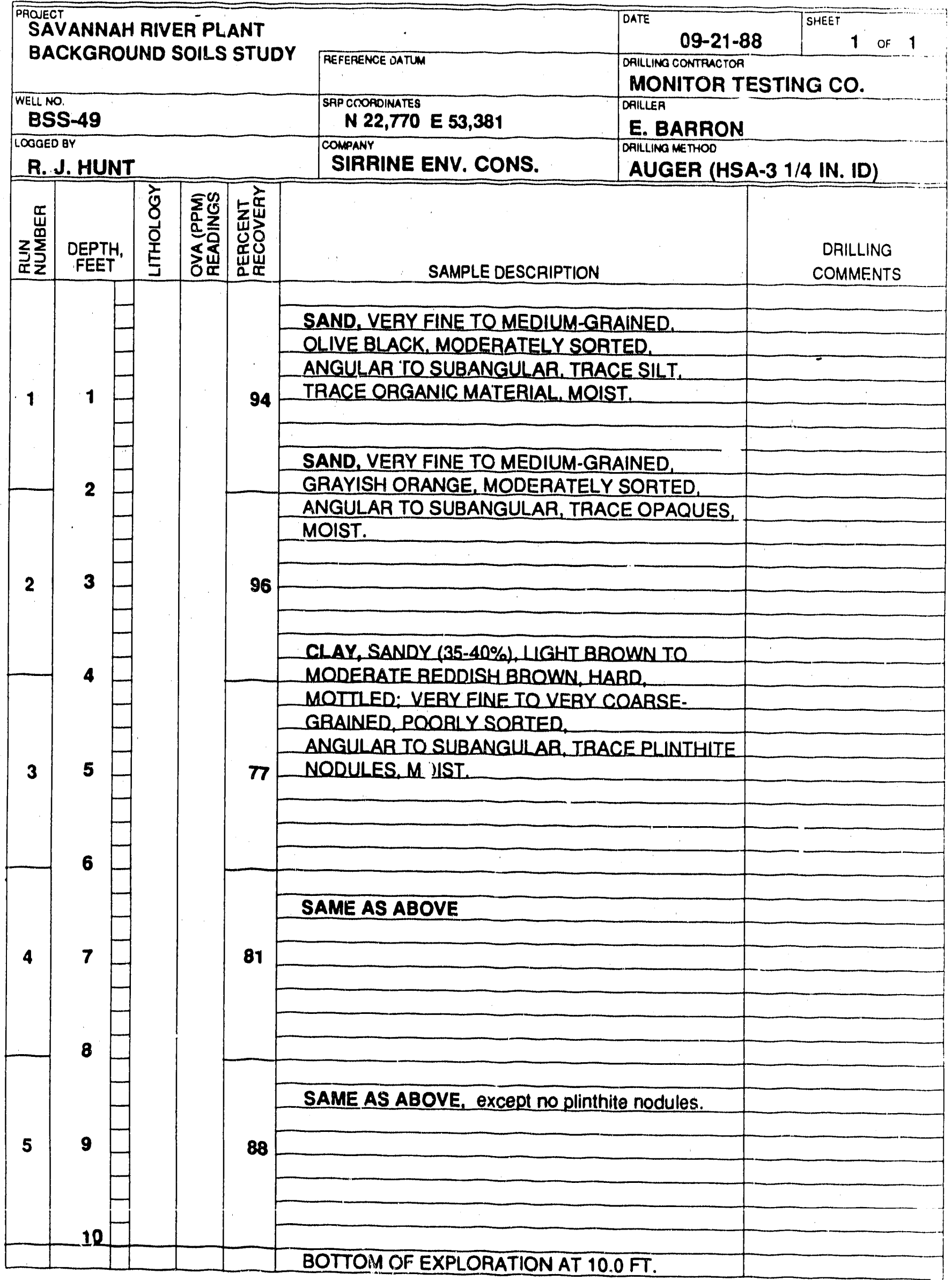


FIELD GEOLOGIC LOG

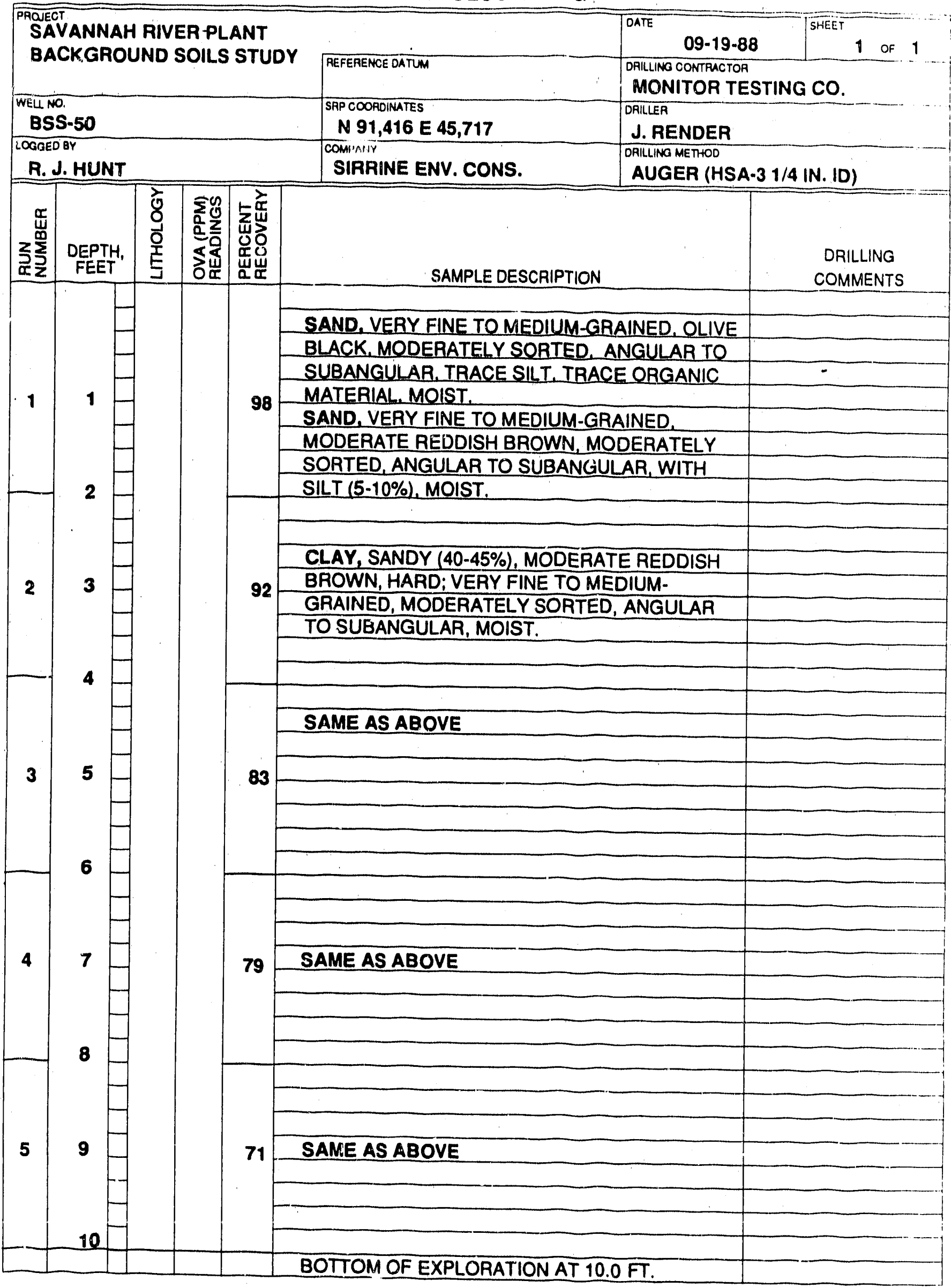


osa 30-3

FIELD GEOLOGIC LOG

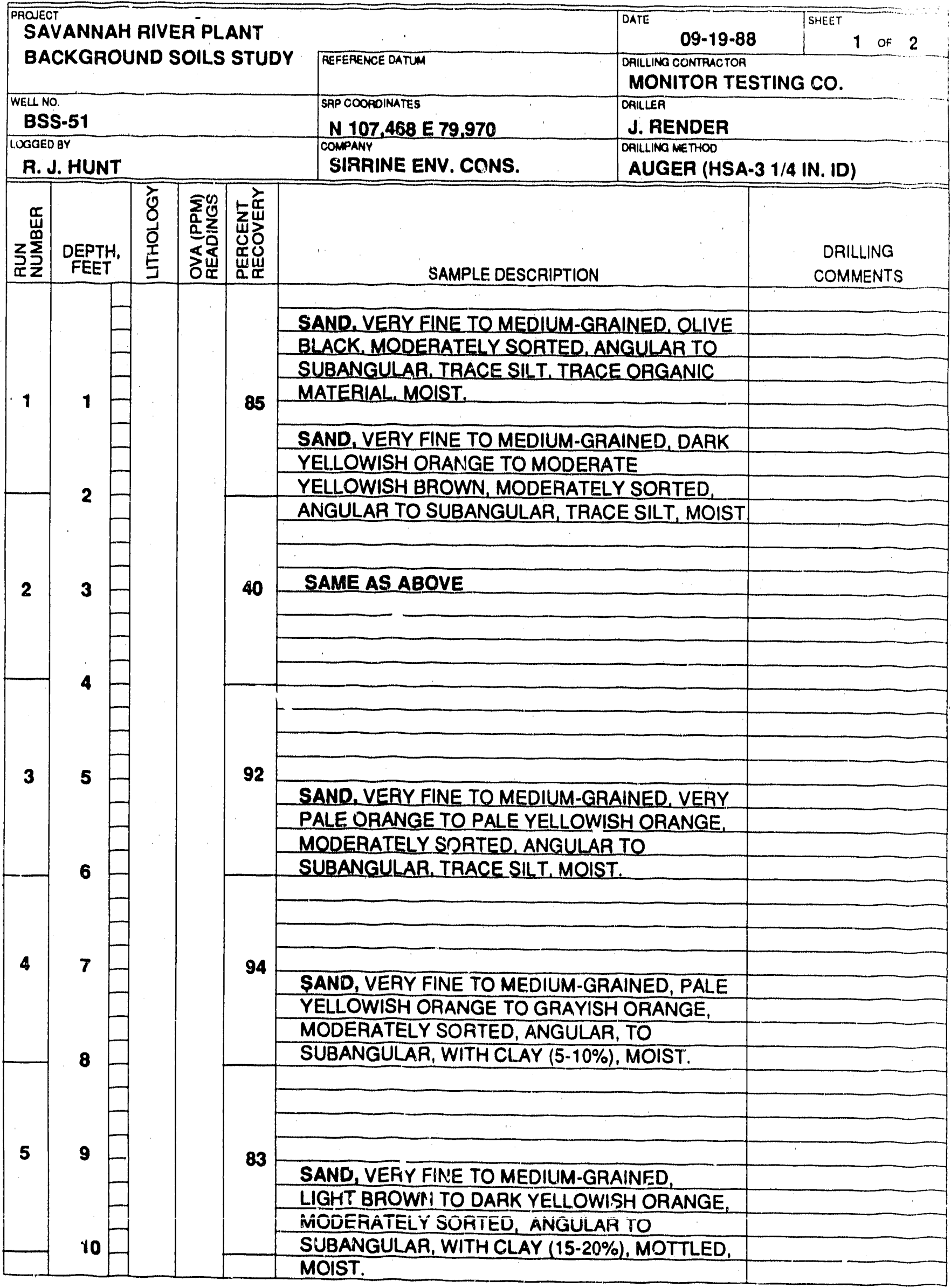


FIELD GEOLOGIC LOG

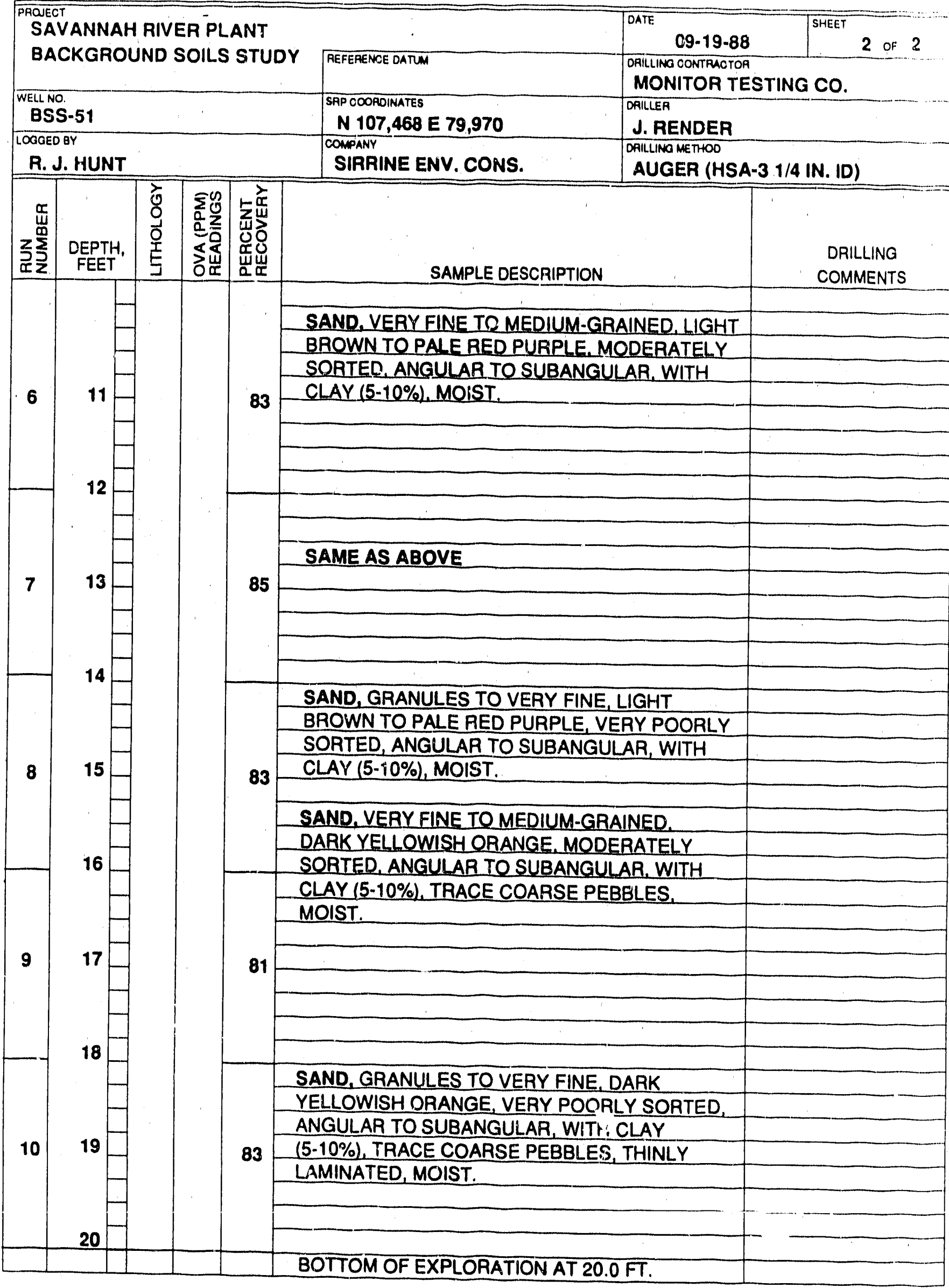


FIELD GEOLOGIC LOG

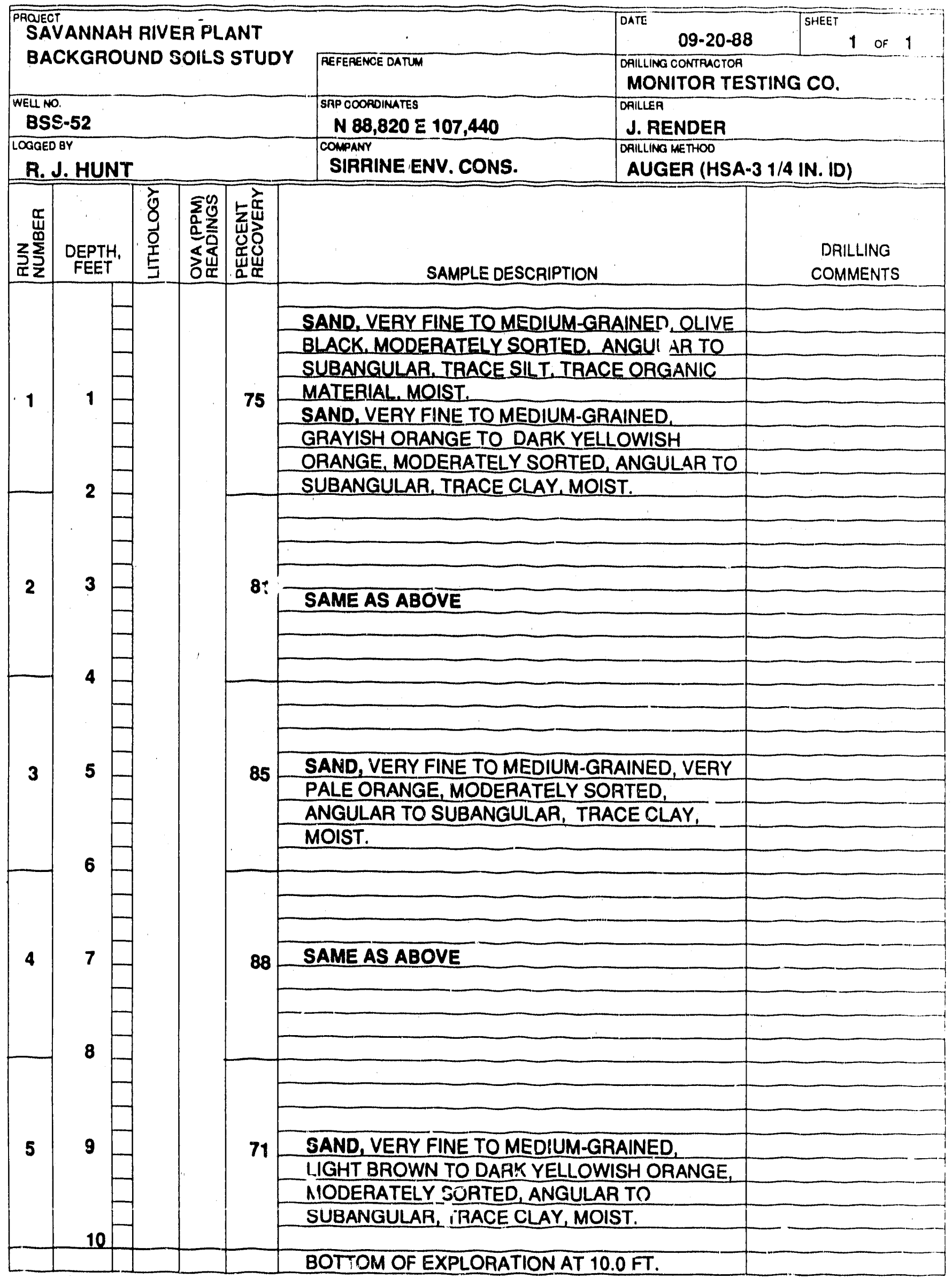


FIELD GEOLOGIC LOG

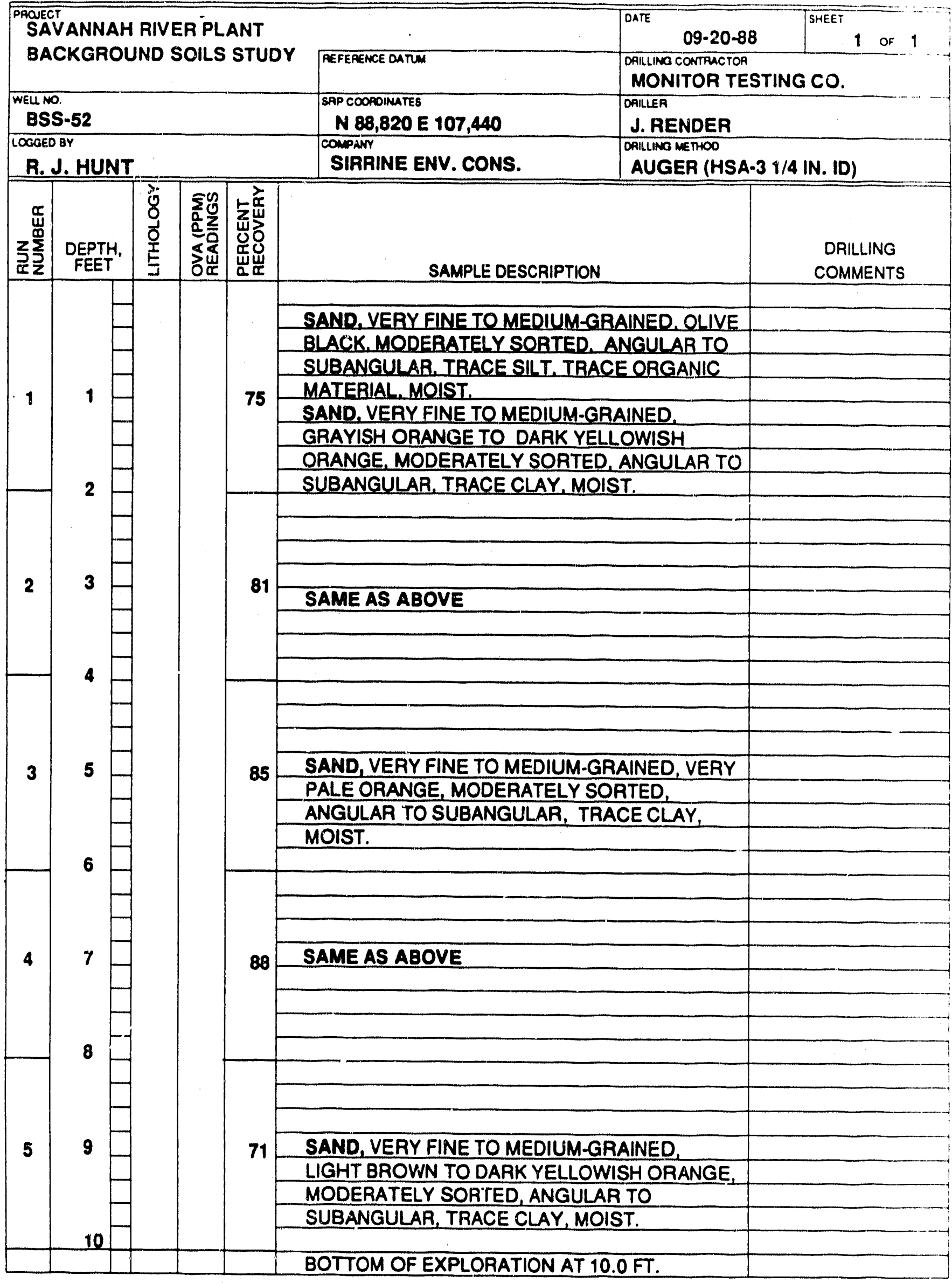


FIELD GEOLOGIC LOG

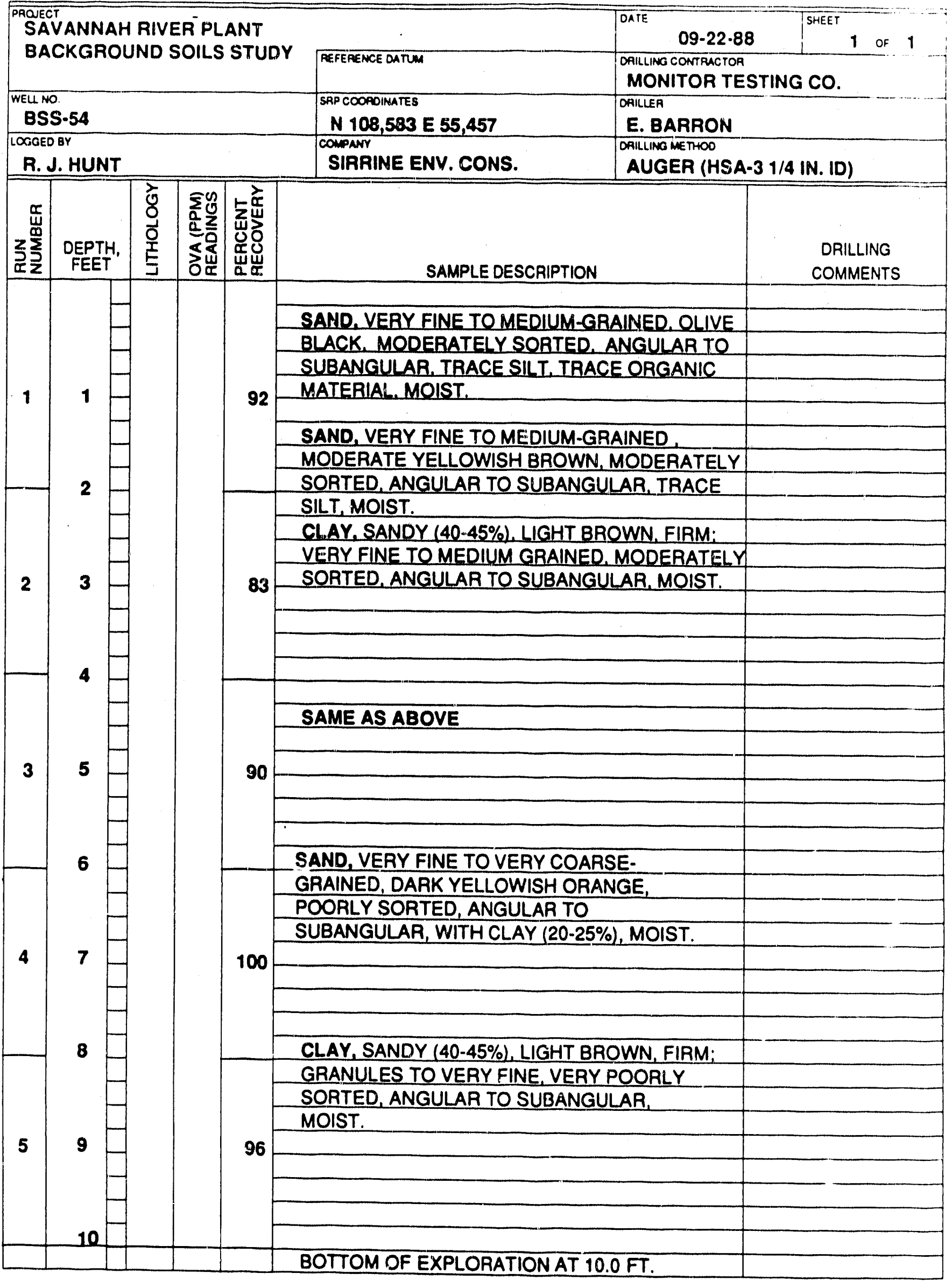


FIELD GEOLOGIC LOG

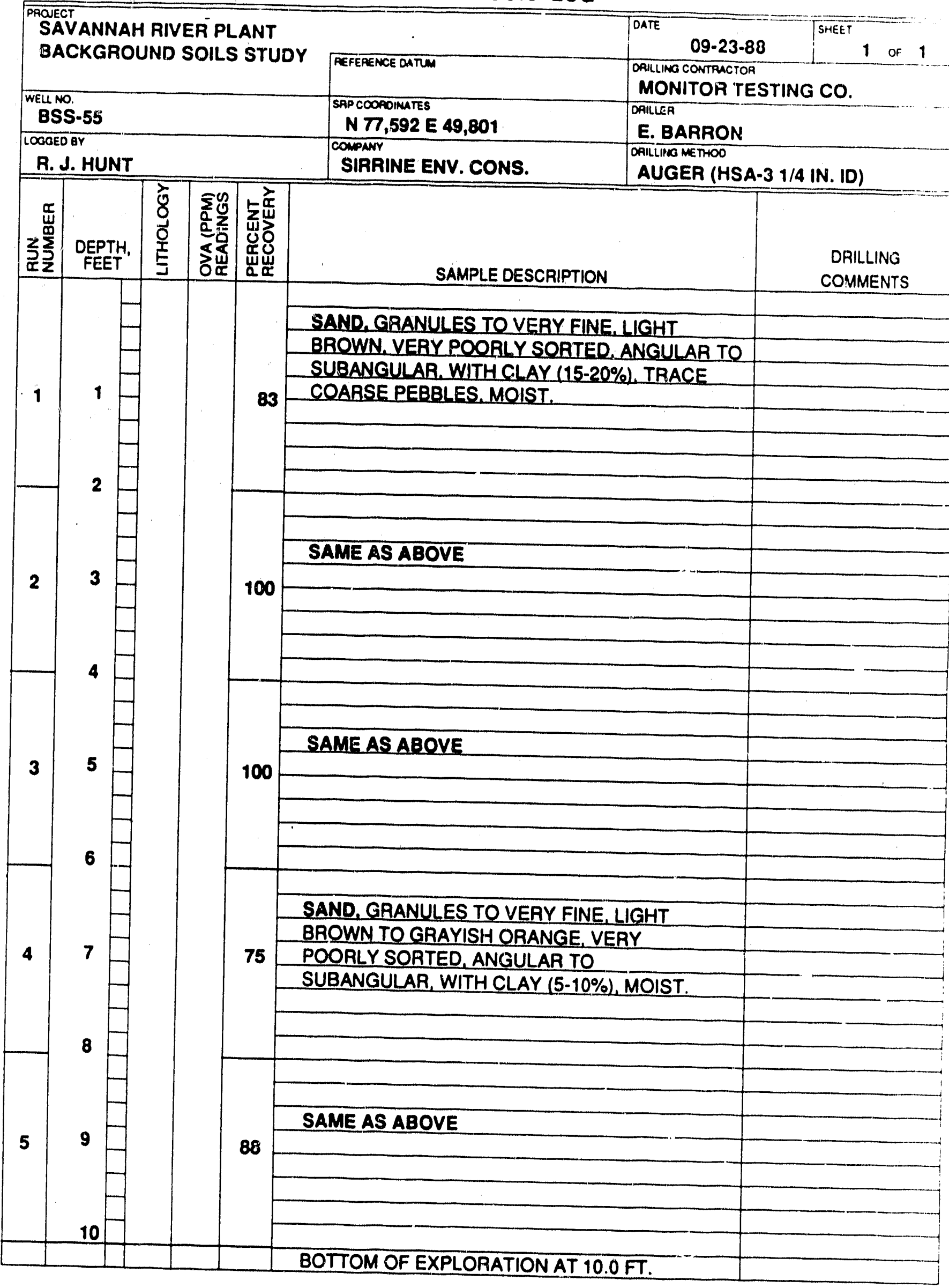


FIELD GEOLOGIC LOG

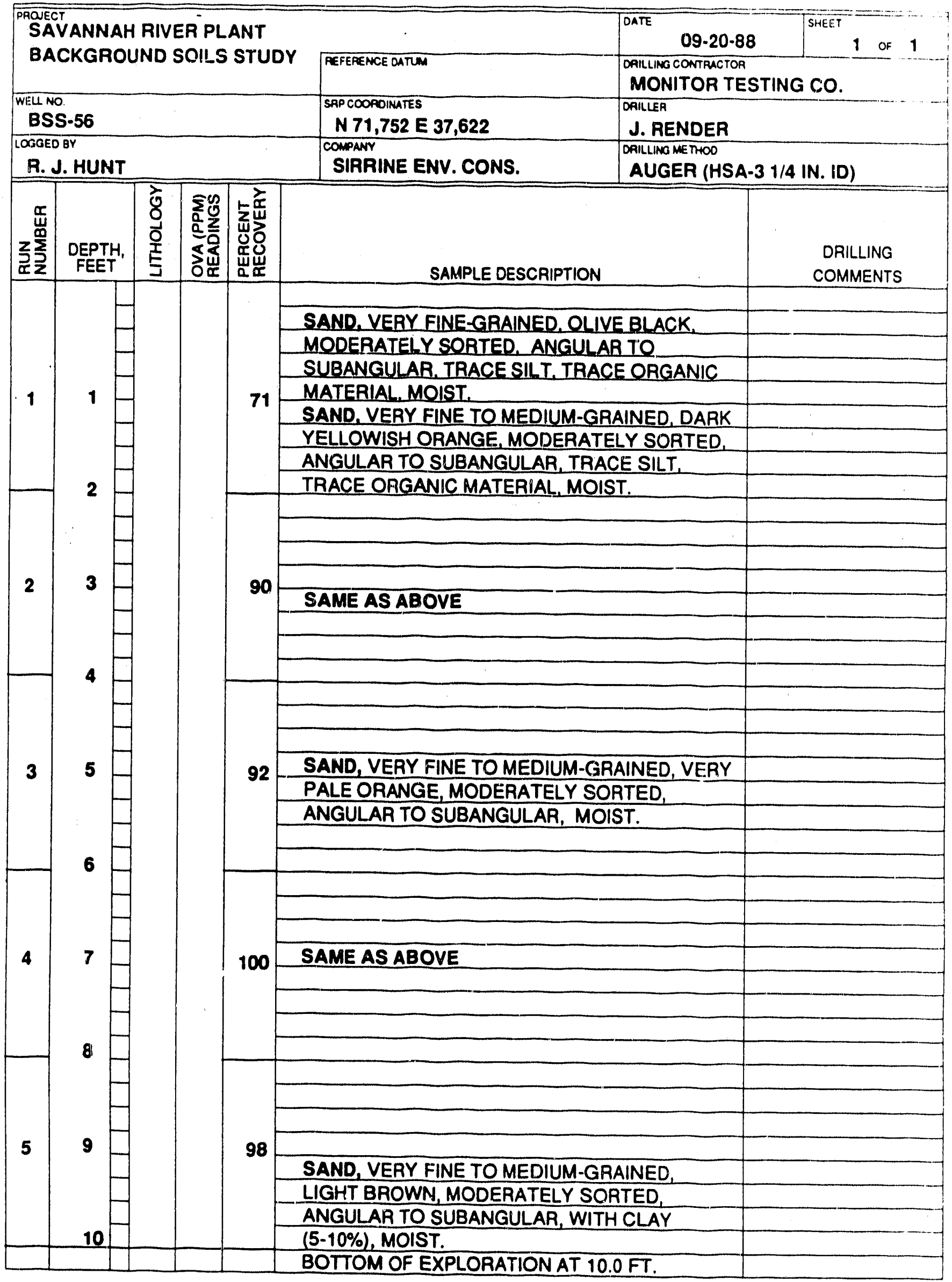


FIELD GEOLOGIC LOG

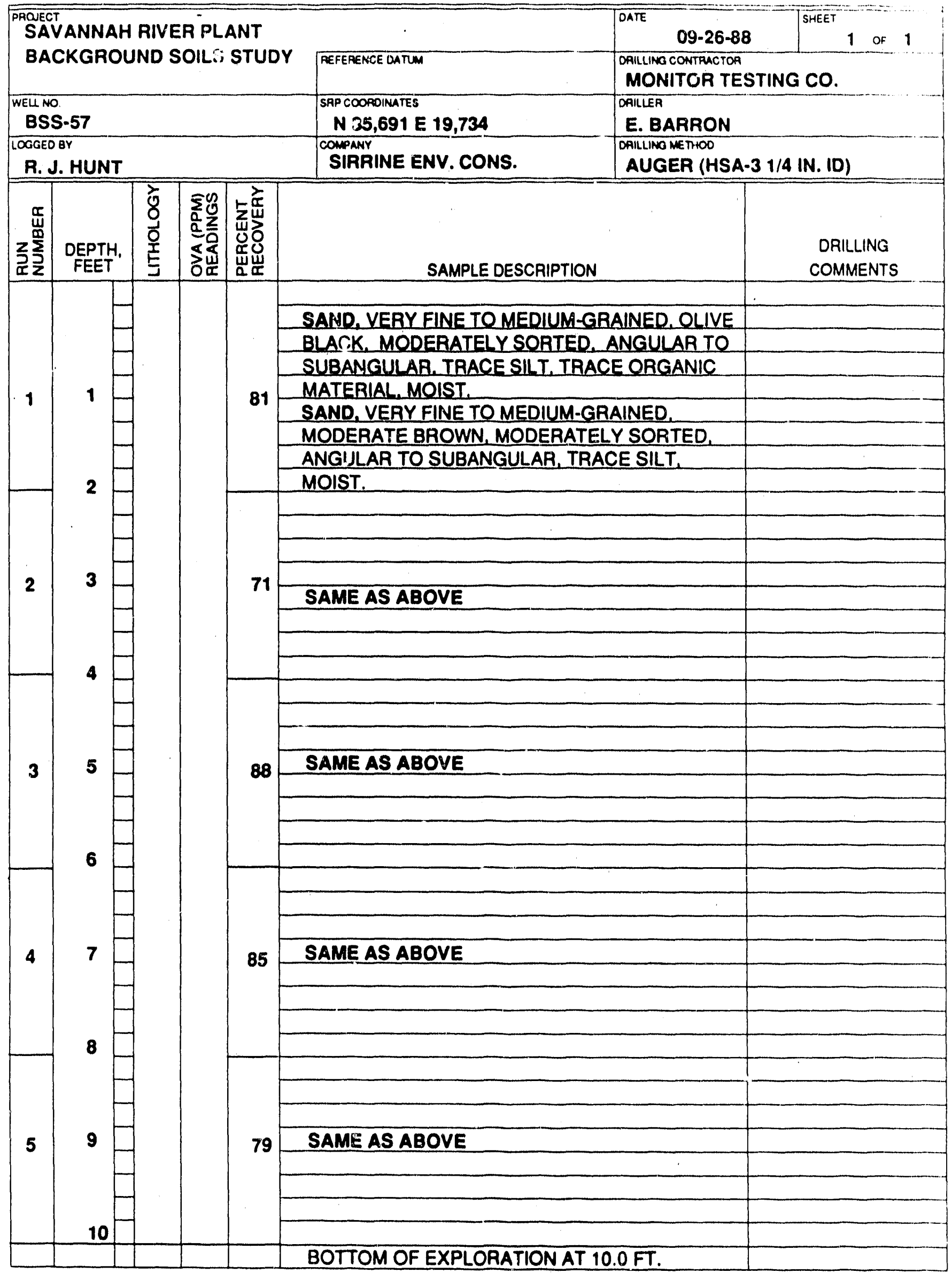




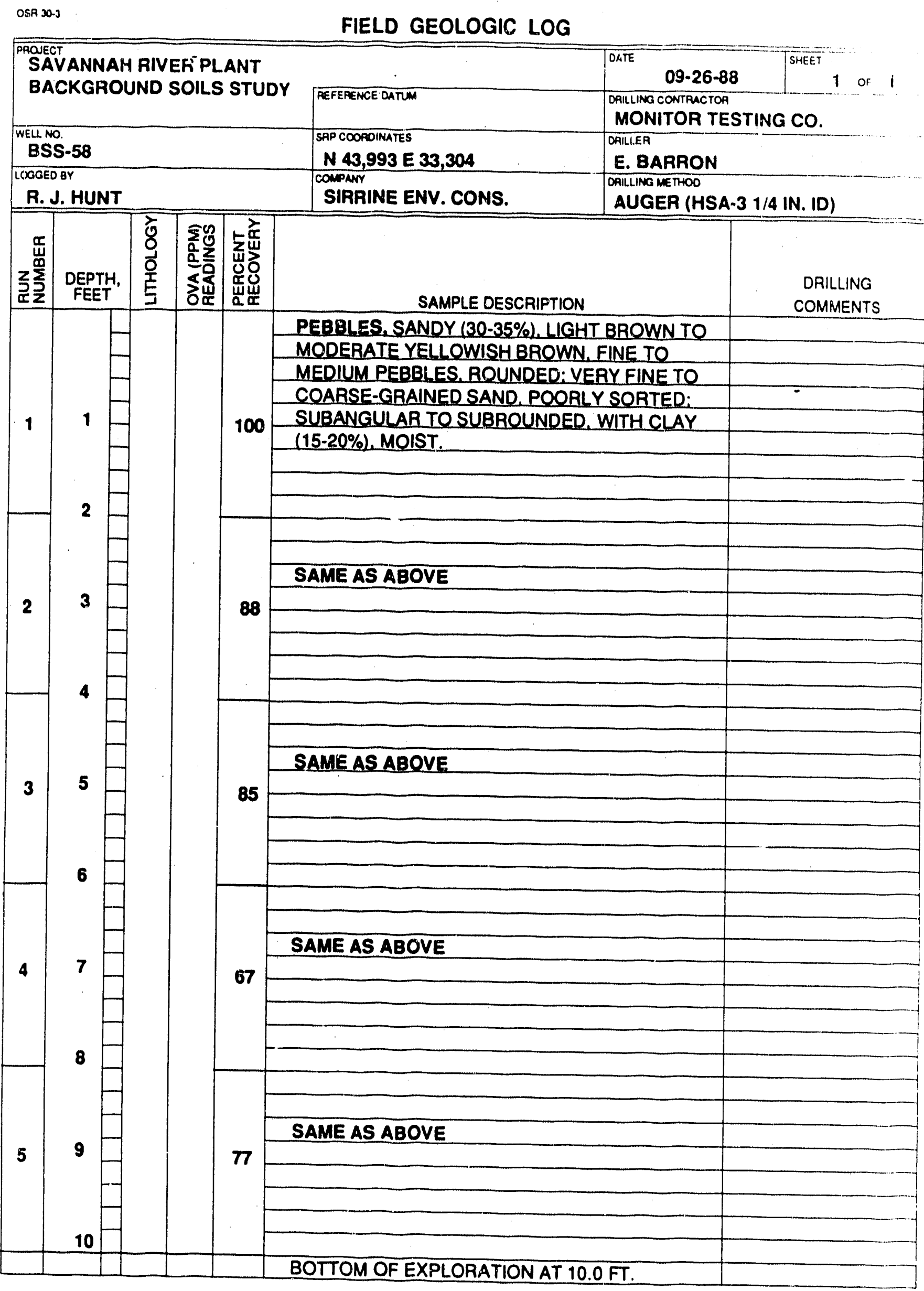




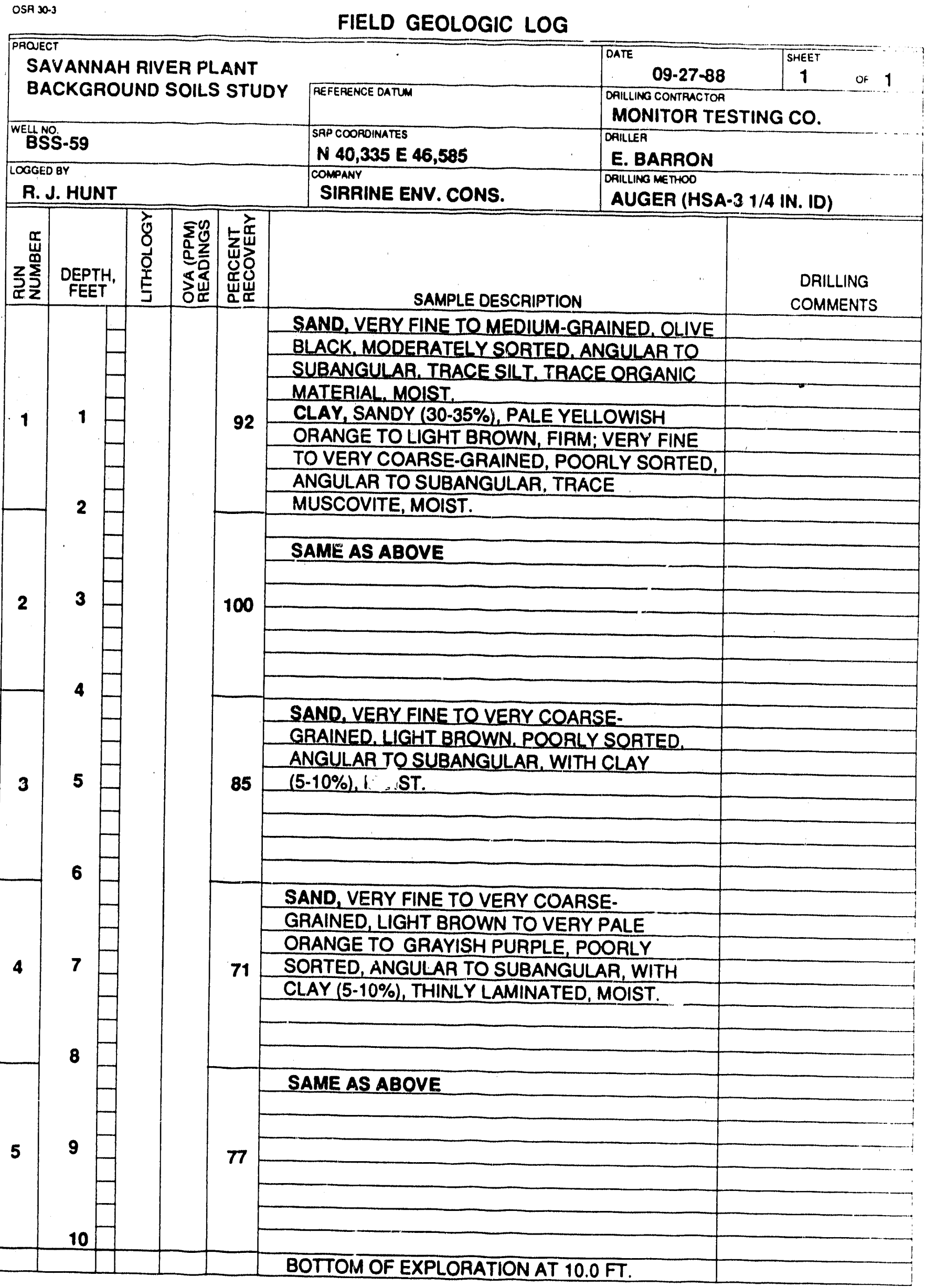


GEOCHEMICAL AND PHYSICAL PROPERTIES OF SOILS AND SHALLOW SEDIMENTS AT THE SAVANNAH RIVER SITE

Appendix C.1

Metal Data Summary 


\begin{tabular}{|c|c|c|c|c|}
\hline \multicolumn{5}{|l|}{ Aluminum } \\
\hline Unit: micx & & & \multicolumn{2}{|c|}{. } \\
\hline \multicolumn{3}{|c|}{ First Layer Interval (inches) } & Modifier & Rasult \\
\hline BSS-011 & 0 & -7 & & 715 \\
\hline BSS-061 & 0 & -6 & & 1476 \\
\hline BSS-091 & 0 & -5 & & 4150 \\
\hline BSS -231 & 0 & -7 & & 2509 \\
\hline BSS -331 & 0 & -8 & & 2075 \\
\hline BSS-351 & 0 & -7 & & 21200 \\
\hline BSS -431 & 0 & -6 & & 2485.7 \\
\hline$B S S-451$ & 0 & -5 & & 2358.4 \\
\hline \multicolumn{5}{|c|}{ :econd Iayer } \\
\hline BSS-012 & 10 & -65 & & 842 \\
\hline BSS-062 & 6 & -50 & & 17450 \\
\hline$B S S-092 A$ & 5 & -52 & & 4520 \\
\hline$B S S-232$ & 7 & -61 & & 19510 \\
\hline$B S S-332$ & 8 & -72 & & 7271.4 \\
\hline BSS -3.52 & 7 & -60 & & 14020 \\
\hline BSS -432 & 6 & -67 & & 2426.8 \\
\hline$B S S-452$ & 5 & -59 & & 3366.2 \\
\hline \multicolumn{5}{|l|}{ Third Layer } \\
\hline$B S S-013$ & 65 & -87 & & 2550 \\
\hline BSS-063 & 50 & -74 & & 13390 \\
\hline BSS-093A & 52 & -72 & & 18400 \\
\hline BSS-233 & 61 & -70 & & 7534 \\
\hline BSS -333 & 72 & -84 & & 16370 \\
\hline BSS -35.3 & 60 & -72 & & 20010 \\
\hline BSS -433 & 67 & -77 & & 12527 \\
\hline BSS -453 & 59 & -72 & & 27512 \\
\hline \multicolumn{5}{|l|}{ Fourth Layer } \\
\hline$B S S-014$ & 111 & -124 & & 1969 \\
\hline BSS-064 & 96 & -120 & & 9016 \\
\hline$B S S-094 A$ & 96 & -120 & & 7140 \\
\hline BSS-234 & 96 & -120 & & 6614 \\
\hline BSS -334 & 96 & -120 & & 14593 \\
\hline BSS -354 & 96 & -120 & & 5213 \\
\hline BSS-434 & 96 & -120 & & 10083 \\
\hline BSS-454 & 96 & -120 & & 10540 \\
\hline
\end{tabular}


Arsenic

Unit: microgram/gram

First Layer

BSS-011

BSS-061

BSS-091

BSS -231

BSS -331

BSS -351

BSS-431

BSS -451

Second Layer

BSS-012

BSS-062

BSS-092A

BSS -232

BSS -332

BSS -352

BSS -432

BSS -452

Third Layer

BSS-013

BSS-063

BSS-093A

BSS-233

BSS -333

BSS -353

BSS -433

BSS -453

Eourth Layer

BSS-014

BSS-064

BSS-094A

BSS -234

BSS-334

BSS- 354

ESS- 434

BSS- 454

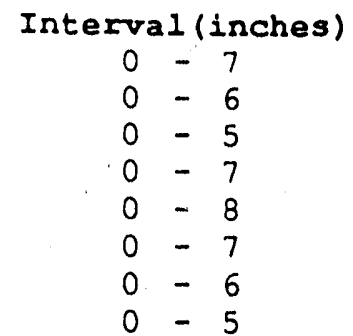

Modifier
LT
LT

LT
LT
LT
LT

Result

0.5

0.5

0.73

1
2

1.07

2
2

$10-65$

$6-50$

LT

0.5

$5-52$

$7-61$

$8-72$

$7-60$

$6-67$

$5-59$

1.28

0.79

LT

LT

1

1.33

LT

LT

2

$65-87$

$50-74$

LT

0.5

$52-72$

$61-70$

$72-84$

$60-72$

$67-77$

$39-72$

LT

LT

2.71

5.29

1.11

7.05

2

2.8

$111-124$

LT

0.5

$96-120$

1.89

6.18

$96-120$

$96-120$

$96-120$

$96-120$

$96-120$
1. 61

LT
5.33

5.33
3.6

2.2 


\begin{tabular}{|c|c|c|c|c|}
\hline & & & Modiller & Result \\
\hline & 0 & -7 & & 2.8 \\
\hline BSS-061 & 0 & -6 & & 5.35 \\
\hline BSS-091 & 0 & -5 & & 25.4 \\
\hline BSS -231 & 0 & -7 & & 7.683 \\
\hline BSS -331 & 0 & -8 & & 11.6 \\
\hline BSS -351 & 0 & -7 & & 21.99 \\
\hline BSS -431 & 0 & -6 & & 10.4 \\
\hline BSS -451 & 0 & -5 & & 7.6 \\
\hline Second Layex & & & & \\
\hline BSS-012 & 10 & -65 & & 3 \\
\hline BSS -062 & 6 & -50 & & 30.7 \\
\hline BSS-092A & 5 & -52 & & 14 \\
\hline BSS-232 & 7 & -61 & LT & 8.9 \\
\hline BSS -332 & 8 & -72 & & 31.6 \\
\hline BSS- -352 & 7 & -60 & & 9.119 \\
\hline BSS -432 & 6 & -67 & 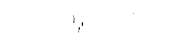 & 12.6 \\
\hline$B S S-452$ & 5 & -59 & & 11.6 \\
\hline Third Layer & & & & \\
\hline BSS-013 & 65 & -87 & & 6.4 \\
\hline BSS-063 & 50 & -74 & & 10.6 \\
\hline BSS-09.3A & 52 & -72 & & 7.6 \\
\hline$B S S-233$ & 61 & -70 & & 6.683 \\
\hline$B S S-333$ & 72 & -84 & & 18 \\
\hline$B S S-353$ & 60 & -72 & & 13.55 \\
\hline BSS -433 & 67 & -77 & & 25.9 \\
\hline BSS -453 & 59 & -72 & & 25.2 \\
\hline Fourth Laye & & & & \\
\hline BSS -014 & 111 & -124 & & 4.6 \\
\hline BSS-064 & 96 & -120 & & 6.06 \\
\hline BSS-094A & 96 & -120 & & 2.4 \\
\hline BSS-234 & 96 & -120 & & 4.435 \\
\hline BSS -334 & 96 & -120 & & 13.6 \\
\hline BSS -354 & 96 & -120 & & 11.93 \\
\hline BSS -434 & 96 & -120 & & 10.5 \\
\hline BSS -454 & 96 & -120 & & 9.2 \\
\hline
\end{tabular}


Cadmium

Unit: microgram/gram

First Layer

BSS-0.11

BSS-061

BSS-091

BSS-231

BSS-331

BSS-351

BSS -431

BSS-451

Second Layer

BSS-0 012

BSS-0 062

BSS-092A

BSS -232

BSS -332

BSS -352

BSS -432

BSS -452

Third Layer

BSS-013

BSS-063

BSS-093A

BSS -233

BSS -333

BSS -353

BSS -433

BSS -453

Fourth Layer

BSS-014

BSS-064

BSS-094A

BSS-234

BSS -334

BSS -354

BSS -434

BSS -454

\section{Interval (inches)}

$0-7$

$0-6$

$0-5$

0 .. 7

$0-8$

$0-7$

$0-6$

$0-5$

$10-65$

$6-50$

$5-52$

$7-61$

$8-72$

$7-60$

$6-67$

$5-59$

$65-87$

$50-74$

$52-72$

$61-70$

$72-84$

$60-72$

$67-77$

$59-72$

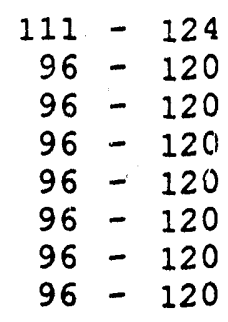

Modifier

LT

LT

LT

LT

LT

LT

LT

LT

LT

LT

LT

LI'

LT

LT

LT

LT

LT

LT

LT

LT

LT

LT

LT

LT

LT

LT
Result

0.5

0.5

1

0.6

0.9282

0.6

0.6

0.5

0.5

1

0.6

0.8619

0.6

0.6

0.5

0.5

0.6

0.9945

0.6

0.5304

0.6

0.6

0.5

0.5

0.2652

0.6

0.3978

0.6
0.6 
Chromium

Unit: microgram/gram

Eirst Layer

BSS-011

BSS-061

BSS-091

BSS-231

BSS-331

BSS-351

BSS -431

BSS-451

Second Layex

BSS-012

BSS-062

BSS-092A

BSS-232

BSS-? 32

BSS-352

BSS- 432

BSS -452

Third Iayer

BSS-013

BSS -063

BSS-093A

BSS-233

BSS -333

BSS-353

BSS -433

BSS- 453

Fourth Layex

BSS-014

BSS-064

BSS-094A

BSS-234

BSS -334

BSS- 354

BSS -434

BSS- 454

Interval (inches)
$0-7$
$0-6$
$0-5$
$0-7$
$0-8$
$0-7$
$0-6$
$0-5$

Modifier

Result

1.7

2.18

12.2

4. 388

2.8

27.97

3.3

8.2

$10-65$

$6-50$

$5-52$

$7-61$

$8-72$

$7-60$

$6-67$

$5-5$ ?

1.8

11.9

8.4

4.3

6.4

24.54

3.1

4. 6

$65-87$

3.1

$50-74$

$52-72$

$61-70$

$72-84$

$60-72$

$67-77$

$59-72$

14.8

23.4

8.09

16.2

31.54

21.4

32.8

$$
\begin{array}{r}
111-124 \\
96-120 \\
96-120 \\
96-120 \\
96-120 \\
96-120 \\
96-120 \\
96-120
\end{array}
$$

3.4

13.1

17.6

7.953

15

6.307

35.2

15 


\begin{tabular}{|c|c|c|c|c|}
\hline \multicolumn{5}{|l|}{ Copper } \\
\hline Unit: micro & & & \multirow[b]{2}{*}{ Modifier } & \multirow{3}{*}{ Result } \\
\hline First Layer & Interva & al (inches) & & \\
\hline BSS-011 & 0 & -7 & & \\
\hline BSS-061 & 0 & -6 & & 0.36 \\
\hline BSS-091 & 0 & -5 & & 2.26 \\
\hline BSS -231 & 0 & -7 & & 1.985 \\
\hline BSS -331 & 0 & -8 & & 2 \\
\hline BSS-351 & 0 & -7 & & 7.009 \\
\hline BSS-431 & 0 & -6 & & 1.3 \\
\hline BSS-451 & 0 & -5 & & 2.4 \\
\hline \multicolumn{5}{|l|}{ Second Layer } \\
\hline BSS-012 & 10 & -65 & & 0.57 \\
\hline BSS-062 & $f$ & -50 & & 2.71 \\
\hline$B S S-092 A$ & 5 & -52 & & 2.02 \\
\hline BSS -232 & 7 & -61 & LT & 0.6 \\
\hline BSS -332 & 8 & .- 72 & & 2.6 \\
\hline BSS -352 & 7 & -60 & & 6.147 \\
\hline BSS -432 & 6 & -67 & & 1 \\
\hline BSS -452 & 5 & -59 & & 1.8 \\
\hline \multicolumn{5}{|l|}{ Third Iayer } \\
\hline BSS-013 & 65 & -87 & & $0 . .5$ \\
\hline BSS-063 & 50 & -74 & & 2.78 \\
\hline BSS-093A & 52 & -72 & & 6.4 \\
\hline BSS-233 & 61 & -70 & & 2.872 \\
\hline BSS-333 & 72 & -84 & & 6 \\
\hline BSS -353 & 60 & -72 & & 7.615 \\
\hline BSS -433 & 67 & -77 & & 5 \\
\hline$B S S-453$ & 59 & -72 & & 8.2 \\
\hline \multicolumn{5}{|l|}{ Fourth Layer } \\
\hline BSS-014 & 111 & -124 & & 1 \\
\hline BSS-064 & 96 & -120 & & 1.42 \\
\hline BSS-094A & 96 & -120 & & 2.6 \\
\hline BSS-234 & 96 & -120 & & 2.834 \\
\hline BSS-334 & 96 & -120 & & 4.8 \\
\hline BSS-354 & 96 & -120 & & 2.475 \\
\hline BSS -434 & 96 & -120 & & 5.4 \\
\hline BSS- 454 & 96 & -120 & & 4.6 \\
\hline
\end{tabular}




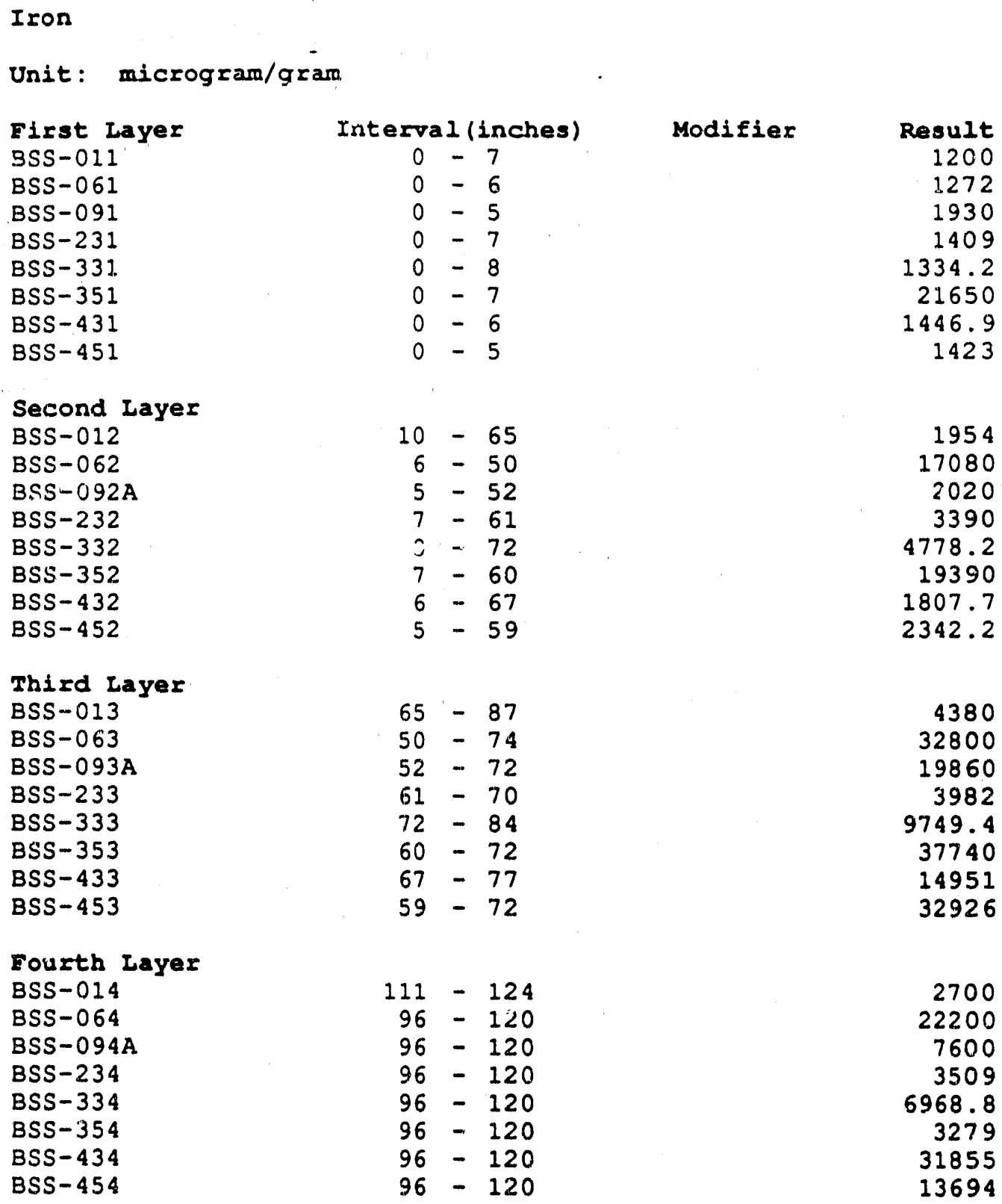


Lead

Unit: microgram/gram

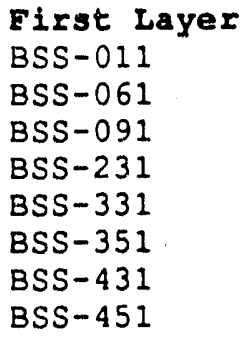

Third Layer BSS-013

BSS-063

BSS-093A

BSS -233

BSS- 333

BSS- 353

BSS -433

BSS -453

Fourth Layer

BSS-014
BSS-064
$B S S-094 A$
$B S S-234$
$B S S-334$
$B S S-354$
$B S S-434$
$B S S-454$

Interval (inches) $\quad$ Modifier
$0-7$
$0-6$
$0-5$
$0-7$
$0-8$
$0-7$
$0-6$
$0-5$

$10-65$

$6-50$

$5-52$

$7-61$

$8-72$

$7-60$

$6-67$

$5-59$

LT

$65-87$

$50-74$

$52-72$

$61-70$

$72-84$

$60-72$

$67-77$

$59-72$

0.72

3.71

6.54

2.09

5.1

3.8

4.4

7.2

$111-124$
$96-120$
$96-120$
$96-120$
$96-120$
$96-120$
$96-120$
$96-120$

0.79

5.6

7.84

2.16

5. 56

6

4.3 
Lithium

Unit: microgram/gram

Eirst Layer
BSS-011
BSS-061
BSS-091
BSS-231
BSS-331
BSS-351
BSS-431
$B S S-451$

Interval (inches)
$0-7$
$0-6$
$0-5$
$0-7$
$0-8$
$0-7$
$0-6$
$0-5$

$\begin{array}{cr}\text { Modifier } & \text { Result } \\ \text { LT } & 10 \\ \text { LT } & 10 \\ \text { LT } & 10 \\ \text { LT } & 10 \\ \text { LT } & 10 \\ \text { LT } & 10 \\ \text { LT } & 10 \\ \text { LT } & 10\end{array}$

Second Layer

BSS-012

BSS-062

BSS-092A

BSS -232

BSS -332

BSS -352

BSS -432

BSS -452

$10-65$
$5-50$
$5-52$
$7-61$
$8-72$
$7-60$
$6-67$
$5-59$

$\begin{array}{ll}\text { LT } & 10 \\ \text { LT } & 10 \\ \text { LT } & 10 \\ \text { LT } & 10 \\ \text { LT } & 10 \\ \text { LT } & 10 \\ \text { LT } & 10 \\ \text { LT } & 10\end{array}$

Third Layer

BSS-013

BSS -063

BSS-093A

BSS -233

BSS -333

BSS -353

BSS -433

BSS -453

$$
\begin{aligned}
& 65-87 \\
& 50-74 \\
& 52-72 \\
& 61-70 \\
& 72-84 \\
& 60-72 \\
& 67-77 \\
& 59-72
\end{aligned}
$$

Fourth Layer

BSS-014

BSS-065

BSS-094A

3SS -234

BSS -334

BSS -354

BSS -434

BSS -454
$111-124$
$96-120$
$96-120$
$96-120$
$96-120$
$96-120$
$96-120$
$96-120$
LT 10

IT 10

LT 10

LT 10

LT 10

LT 10

LT 10

IT 10

$\begin{array}{ll}\text { LT } & 10 \\ \text { LT } & 10 \\ \text { LT } & 10 \\ \text { LT } & 10 \\ \text { LT } & 10 \\ \text { LT } & 10 \\ \text { LT } & 10 \\ \text { LT } & 10\end{array}$


Magnesium

Unit: microgram/gram

Eirst Layex

BSS-011

BSS-061

BSS-091

BSS-231

BSS-331

BSS-351

BSS- 431

BSS- 451

\section{Second Iayer}

BSS-012

BSS-062

BSS-092A

BSS -232

BSS- 332

BSS -352

BSS -432

BSS -452

Third Layer

BSS-013

BSS-063

BSS-093A

BSS -233

BSS- 333

BSS -353

BSS -433

BSS-453

Fourth Layer
BSS-014
BSS-064
BSS-094A
BSS-234
BSS- 334
BSS-354
BSS- 434
BSS -454

Interval (inches)
$0-7$
$0-6$
$0-5$
$0-7$
$0-8$
$0-7$
$0-6$
$0-5$

Modifier

Result

20.09

42.4

117.4

69.17

86.18

107

34.65

54.78

$$
\begin{array}{r}
10-65 \\
6-50 \\
5-52 \\
7-61 \\
8-72 \\
7-60 \\
6-67 \\
5-59
\end{array}
$$

25.94

188.5

123.8

167.1 100

232.9

91.71.

79.54

45.55

$65-87$

$50-74$

$52-72$

$61-70$

$72-84$

$60-72$

$67-77$

$59-72$

112.7

$1: 1$

73.32

135.4

296.7

121.1

185.9

$111-124$
$96-120$
$96-120$
$96-120$
$96-120$
$96-120$
$96-120$
$96-120$

163.5

62.11

43.23

48.07

75.53

108.4

137.2

76.64 


\section{Manganese}

Unit: microgram/gram

Eirst Layer
BSS-011
BSS-061
BSS-091
BSS-231
BSS-331
BSS-351
BSS-431
BSS -451

Interral (inches)
$0-7$
$0-6$
$0-5$
$0-7$
$0-8$
$0-7$
$0-6$
$0-5$

Modifier Resuit
12.72
22.79
138.5
18.46
30.56
70.93
32.25
15.47

Second Layer

BSS-012

BSS-062

BSS-092A

BSS -232

BSS-332

BSS -352

BSS -432

BSS -452

$$
\begin{array}{r}
10-65 \\
6-50 \\
5-52 \\
7-61 \\
8-72 \\
7-60 \\
6-67 \\
5-59
\end{array}
$$

3.409

13.83

27.05

11.41

9.268

30.56

12.64

4.29

Third Layer

BSS-013

$65-87$

$50-74$

$52-72$

$61-70$

BSS-093A

BSS -233

BSS -333

BSS-353

BSS -433

BSS -453

$72-84$

$60-72$

$67-77$

$59-72$

LT

3.983

2.834

15.28

1.762

1.6

2.451

3.294

IT 1.6

Eourth Iayer

BSS-014

$111-124$

2.758

$96-120$

3.485

BSS-094A

BSS -234

BSS-334

$96-120$

12.12

$96-120$

BSS-354

$96-120$

2.298

BSS -434

$96-120$

LT

1.6

2.528

3.37

$96-120$
$96-120$

2.375 


\section{Mercury}

Unit: microgram/gram

Eirst Layer

BSS-011

BSS-061

BSS-091

BSS -231

BSS-331

BSS -351

BSS-431

BSS -451

Second Layer

BSS-0 012

BSS-062

BSS-092A

BSS -232

BSS -332

BSS- 352

BSS- 432

BSS -452

Third Iayer

BSS-013

BSS-063

BSS-093A

BSS-233

BSS- 333

BSS -353

BSS -433

BSS -453

Eourth Layer

BSS-014

BSS-064

BSS-094A

BSS-234

BSS -334

BSS-354

BSS -434

BSS -454

Interval (inches)
$0-7$
$0-6$
$0-5$
$0-7$
$0-8$
$0-7$
$0-6$
$0-5$

Modifier
LT
LT
LT
LT
LT
LT
LT

Result

0.1

0.1

0.19

0.1

0.1

0.1

0.1

0.1

$10-65$

$6-50$

$5-52$

$7-61$

$8-72$

$7-60$

$6-67$

$5-59$

LT

0.1

0.1

0.14

LT

LT

LT

0.1

0.1

0.1

0.15

LT

0.1

$65-87$

$50-74$

$52-72$

$61-70$

$72-84$

$60-72$

$67-77$

$59-72$

LT

LT

0.1

LT

0.1

0.25

0.1

0.1

0.1

0.1

0.1

$$
\begin{array}{r}
111-124 \\
96-120 \\
96-120 \\
96-120 \\
96-120 \\
96-120 \\
96-120 \\
96-120
\end{array}
$$

$\mathrm{LT}$

0.1

0.13

0.15

LT

0.1

LT

0.1

0.19

LT

0.1

0.1 


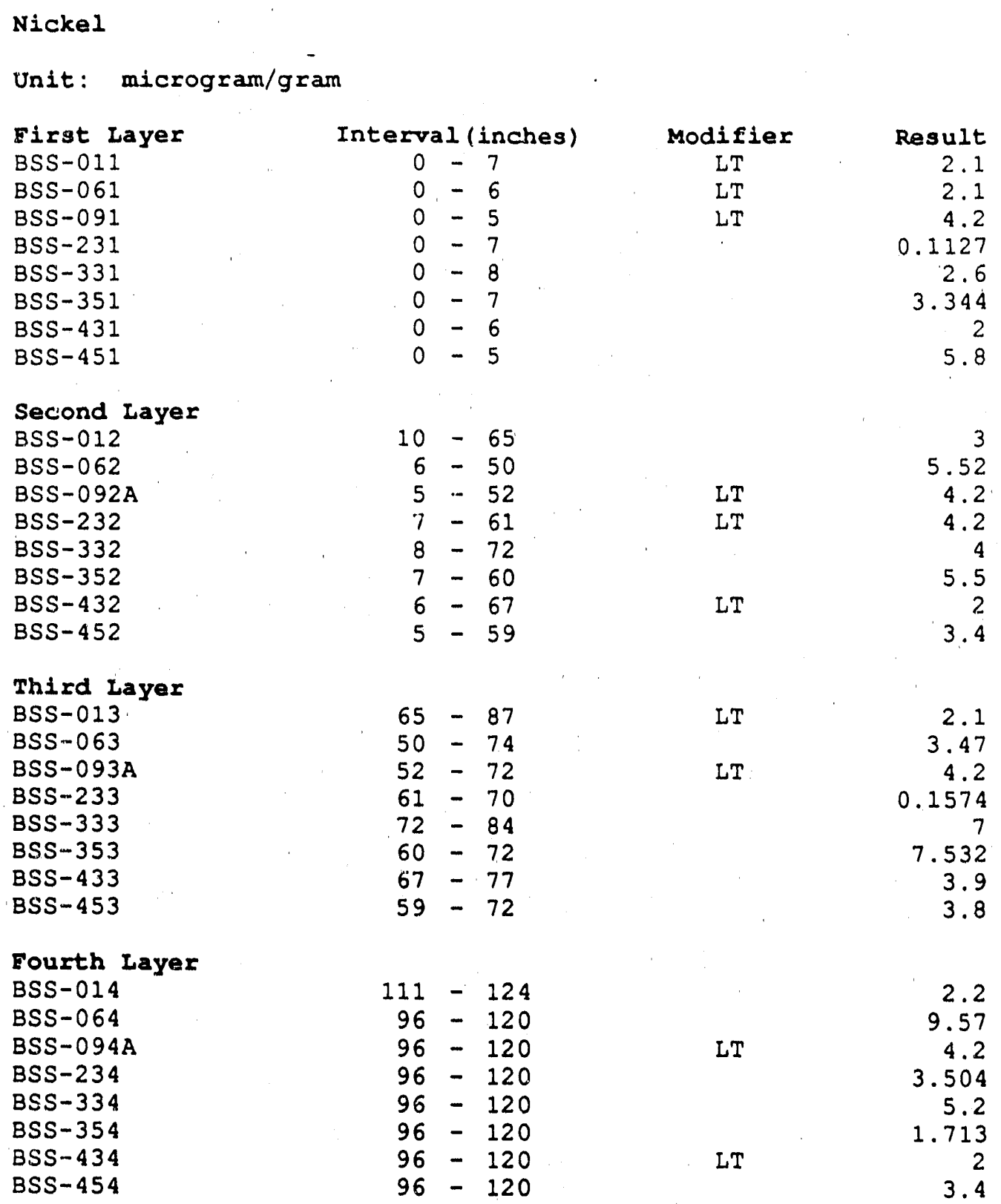




\begin{tabular}{|c|c|c|c|c|}
\hline \multicolumn{5}{|l|}{ Potassium } \\
\hline \multicolumn{5}{|c|}{ Unit: microgram/gram } \\
\hline First Layer & \multicolumn{2}{|c|}{ Interval (inches) } & \multirow{2}{*}{$\begin{array}{c}\text { Modifier } \\
\text { LT }\end{array}$} & \multirow{2}{*}{$\begin{array}{r}\text { Result } \\
40\end{array}$} \\
\hline BSS -011 & 0 & -7 & & \\
\hline BSS-061 & 0 & -6 & LT & 40 \\
\hline BSS-091 & 0 & -5 & LT & 360 \\
\hline BSS -231 & 0 & -7 & LT & 326 \\
\hline BSS-331 & 0 & -8 & LT & 180 \\
\hline$B S S-351$ & 0 & -7 & & 139.4 \\
\hline$B S S-431$ & 0 & -6 & LT & 180 \\
\hline BSS -451 & 0 & -5 & $\mathrm{LT}$ & 180 \\
\hline \multicolumn{5}{|l|}{ Second Layex } \\
\hline BSS-012 & 10 & -65 & $\mathrm{LT}$ & 40 \\
\hline BSS-062 & 6 & -50 & $\mathrm{LT}$ & 40 \\
\hline BSS-092A & 5 & -52 & LT & 360 \\
\hline BSS -232 & 7 & -61 & LT & 800 \\
\hline BSS -332 & 8 & -72 & LT & 180 \\
\hline BSS -352 & 7 & -60 & LT & 326 \\
\hline BSS -432 & 6 & -67 & LT & 180 \\
\hline BSS -452 & 5 & -59 & & 188 \\
\hline \multicolumn{5}{|l|}{ Third Layer } \\
\hline BSS-013 & 65 & & LT & 40 \\
\hline BSS-063 & 50 & $-7,8$ & LT & 40 \\
\hline BSS-093A & 52 & -72 & LT & 360 \\
\hline BSS -233 & 61 & -70 & & 82.96 \\
\hline BSS -333 & 72 & -84 & LT & 180 \\
\hline BSS -353 & 60 & -72 & & 89.6 \\
\hline$B S S-433$ & 67 & -77 & LT & 180 \\
\hline$B S S-453$ & 59 & -72 & & 224 \\
\hline \multicolumn{5}{|l|}{ Fouxth Layer } \\
\hline BSS -014 & 111 & -124 & LT & 40 \\
\hline BSS-064 & 96 & -120 & LT & 40 \\
\hline$B S S-094 A$ & 96 & -120 & LT & 360 \\
\hline BSS-234 & 96 & -120 & & 59.73 \\
\hline BSS -334 & 96 & -120 & LT & 180 \\
\hline BSS -354 & 96 & -120 & & 159.3 \\
\hline$B S S-434$ & 96 & -120 & LT & 180 \\
\hline BSS -454 & 96 & -120 & LT & 180 \\
\hline
\end{tabular}




\section{Selenium}

Unit: microgram/gram

Eirst Layer
BSS -011
BSS-061
BSS-091
$B S S-231$
BSS -331
BSS -351
$B S S-431$
$B S S-451$

BSS -451

Second Layer

BSS -012

BSS-062

BSS-092A

BSS -232

BSS -332

BSS -352

BSS -432

BSS -452

Third Layer

BSS -013

BSS -063

BSS-093A

BSS -233

BSS -333

BSS -353

BSS -433

BSS -453

rourth Layer

BSS-014
BSS-064
BSS-094A
BSS-234
BSS-234
BSS-354
BSS-434
BSS- 454

Interval (inches)
$0-7$
$0-6$
$0-5$
$0-7$
$0-8$
$0-7$
$0-6$
$0-5$

$10-65$

$6-50$

$5-52$

$7-61$

$8-72$

$7-60$

$6-67$

$5-59$

$$
\begin{aligned}
& 65-87 \\
& 50-74 \\
& 52-72 \\
& 61-70 \\
& 72-84 \\
& 60-72 \\
& 67-77 \\
& 59-72
\end{aligned}
$$

$$
\begin{array}{r}
111-124 \\
96-120 \\
96-120 \\
96-120 \\
96-120 \\
96-120 \\
96-120 \\
96-120
\end{array}
$$

$\begin{array}{cr}\text { Modifier } & \text { Result } \\ \text { LT } & 0.2 \\ \text { LT } & 0.2 \\ \text { LT } & 0.4 \\ & 0.44 \\ \text { LT } & 0.4 \\ \text { LT } & 0.4 \\ \text { LT } & 0.4 \\ \text { LT } & 0.4\end{array}$

$\mathrm{J} \mathrm{T}$

0.2

0.35

0.4

L'T

LT

LT

LT

LT

LT

LT

0.2

0.64

0.45

0.4

0.4

0.8

0.4

1.15

LT

0.2

0.58

0.4

0.4

0.4

0.4

0.42

IT $\quad 0.4$ 


\section{Silver}

Unit: microgram/gram

\section{Eirst Layer}

BSS-011

BSS-061

BSS-091

BSS-231

BSS-331

BSS-351

BSS-431

BSS- 451

Second Layer

BSS-0 12

BSS-002

BSS-092A

BSS -232

BSS -332

BSS-352

BSS -432

BSS -452

Third Layer

BSS-013

BSS -063

BSS-093A

BSS- -33

BSS-333

BSS -353

BSS -433

BSS -453

Fourth Layer

BSS-014

BSS-064

BSS-094A

BSS-234

BSS -334

BSS-354

BSS -434

BSS -454

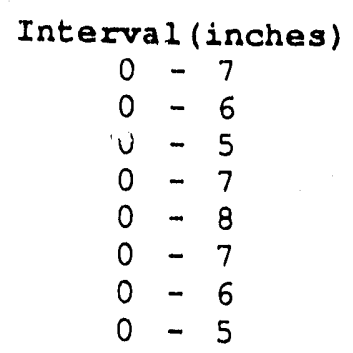

Modifier
LT
LT
LT

LT
LT
LT
LT

Result

0.3

0.3

0.6

0.2395

1

0.6

1
LT

LT

LT

LT

$\mathrm{LT}$

LT

LT

LT

0.3

0.3

0.6

0.6

1

0.6

1
1

0.3

0.3

0.6

0.6

1

0.6

1

$$
\begin{array}{r}
11.1-124 \\
96-120 \\
96-120 \\
96-120 \\
96-120 \\
96-120 \\
96-120 \\
96-120
\end{array}
$$

0.3

0.3

0.6

0.6

1

0.6 1 


\begin{tabular}{|c|c|c|c|c|}
\hline \multicolumn{5}{|l|}{ Sodium } \\
\hline \multicolumn{5}{|c|}{ Unit: microgram/gram } \\
\hline First Layer & \multicolumn{2}{|c|}{ Interval (inches) } & \multirow[t]{9}{*}{ Modifier } & Result \\
\hline BSS-011 & 0 & -7 & & 23.7 \\
\hline BSS-061 & 0 & -6 & & 30.4 \\
\hline BSS-091. & 0 & -5 & & 39.8 \\
\hline BSS -231 & 0 & -7 & & 38.05 \\
\hline BSS-331 & 0 & -8 & & 78 \\
\hline BSS -351 & 0 & -7 & & 45.54 \\
\hline$B S S-431$ & 0 & -6 & & 26.7 \\
\hline$B S S-451$ & 0 & -5 & & 31.8 \\
\hline \multicolumn{5}{|l|}{ Second Layer } \\
\hline BSS-012 & 10 & -65 & & 22.2 \\
\hline BSS-062 & 6 & -50 & & 26.5 \\
\hline BSS-092A & 5 & -52 & & 56.4 \\
\hline BSS-232 & 7 & -61 & & 44.36 \\
\hline BSS -332 & 8 & -72 & & 109.4 \\
\hline BSS -352 & 7 & -60 & & 43.2 \\
\hline BSS -432 & 6 & -67 & & 26.1 \\
\hline BSS -452 & 5 & -59 & & 60 \\
\hline \multicolumn{5}{|l|}{ Third Layez } \\
\hline BSS-013 & 65 & -87 & & 20.1 \\
\hline BSS-063 & 50 & -74 & & 47.4 \\
\hline BSS-093A & 52 & -72 & & 45.6 \\
\hline BSS-233 & 61 & -70 & & 37.12 \\
\hline BSS -333 & 72 & -84 & & 42.4 \\
\hline BSS -353 & 60 & -72 & & 38.05 \\
\hline$B S S-433$ & 67 & -77 & & 43.5 \\
\hline BSS -453 & 59 & -72 & & 58 \\
\hline \multicolumn{5}{|l|}{ Fourth Layer } \\
\hline BSS -014 & 111 & -124 & & 24.1 \\
\hline BSS-064 & 96 & -120 & & 24 \\
\hline BSS-094A & 96 & -120 & & 40.6 \\
\hline$B S S-234$ & 96 & -120 & & 35.71 \\
\hline BSS -334 & 96 & -120 & & 42.4 \\
\hline BSS-35, 4 & 96 & -120 & & 45.38 \\
\hline BSS -434 & 96 & -120 & & 52 \\
\hline BSS -454 & 96 & -120 & & 57.6 \\
\hline
\end{tabular}


zinc

Unit: microgram/gram

Eirst Layer

BSS-011

BSS-061

B'SS-091

BSS-231

BSS -331

BSS-351

BSS -431

BSS -451

\section{Serond Layer}

BSS -012

BSS -062

BSS-092A

BSS -232

BSS -332

BSS -352

BSS -432

BSS -452

Third Layer

BSS-013

BSS-063

BSS-093A

BSS -233

BSS -333

BSS -353

BSS -433

BSS -453

Fourth Layer

BSS-014

BSS-064

BSS-094A

BSS -234

BSS -334

BSS - 354

BSS -434

BSS-454

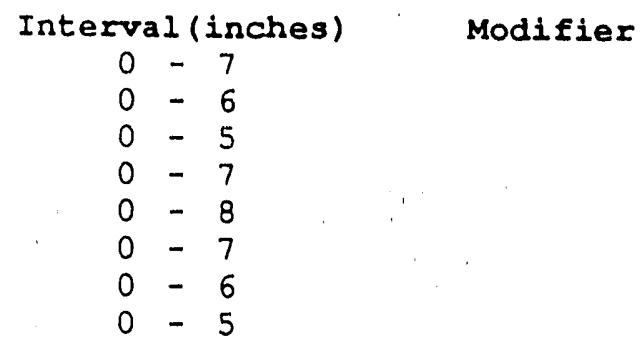

$$
\begin{array}{r}
10-65 \\
6-50 \\
5-52 \\
7-61 \\
8-72 \\
7-60 \\
6-67 \\
5-59
\end{array}
$$

6.2

14.5

12.6

11.

10.96

4.7

3

10.8

9.27

10.5

6.829

8.2

13.12

13.6

5.8

$67-77$

$59-72$

$$
\begin{array}{r}
111-124 \\
96-120 \\
96-120 \\
96-120 \\
96-120 \\
96-120 \\
96-120 \\
96-120
\end{array}
$$

12.7

13.5

7.65

8.594

10.8

9.864

24.6

3. 4 


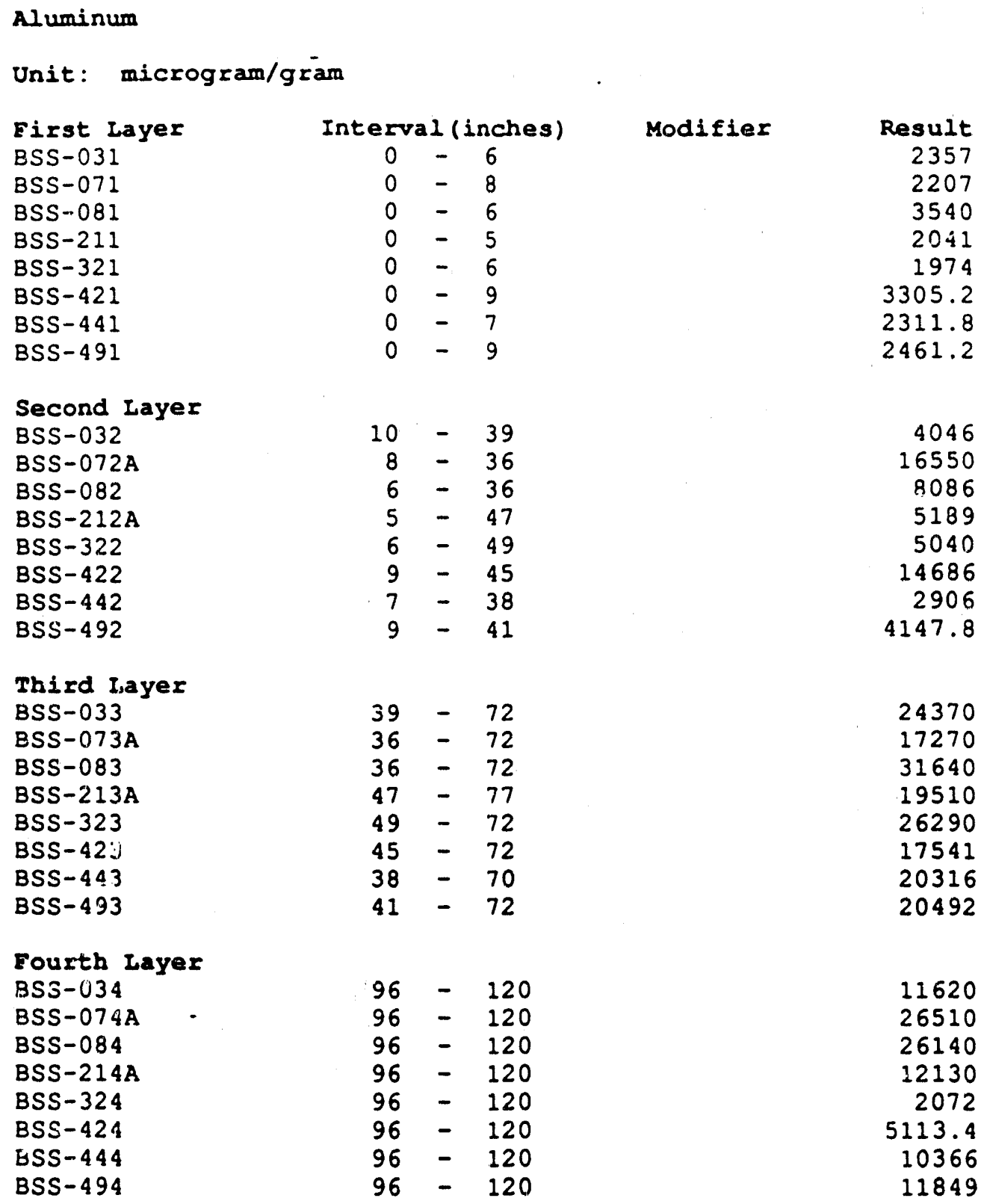


Arsenic

Unit: microgram/gram

Eirst Layer

BSS-031

BSS-071

BSS-081

BSS-211

BSS-321

BSS- 421

BSS-441

BSS -491

Second Layer

BSS-0 32

BSS-072A

BSS -082

BSS-212A

BSS -322

BSS- 422

BSS -442

BSS-492

Third Layer

BSS-033

BSS-073A

BSS-083

BSS-213A

BSS -323

BSS -423

BSS -443

BSS -493

Fourth Layer

BSS-034

BSS-074A

BSS-084

BSS-214A

BSS-324.

BSS- 424

BSS- 444

BSS -494

Interval (inches)
$0-6$
$0-8$
$0-6$
$0-5$
$0-6$
$0-9$
$0-7$
$0-9$

Modifier
LT
LT
LT
LT
LT
LT
LT
LT

Result

0.5

0.5

2

1

1

2

2

$10-39$

$\mathrm{LT}$

0.5

1.46

LT

LT

LT

LT

LT

LT

2

36
$-\quad 47$

-49
$-\quad 45$

-45
$-\quad 38$

- 38

- 41

$39-72$

$36-72$

$36-72$

$47-77$

$49-72$

$45-72$

$38-70$

$41-72$

2.34

4.62

11.6

6.23

7.26

2

2.4

2.4

$96-120$
$96-120$
$96-120$
$96-120$
$96-120$
$96-120$
$96-120$
$96-120$

2.19

15.2

6.49

3.73

1.84

3.2
LT

LT 


\begin{tabular}{|c|c|c|c|c|}
\hline \multicolumn{5}{|l|}{ Barium } \\
\hline \multicolumn{5}{|c|}{ Unit: microgram/gram } \\
\hline First Layer & Interva & al (inches) & Modifier & Result \\
\hline$B S S-031$ & 0 & -6 & & 4.57 \\
\hline$B S S-071$ & 0 & 8 & & 11.9 \\
\hline BSS-081. & 0 & -6 & & 28.7 \\
\hline BSS-211 & 0 & -5 & & 9.557 \\
\hline$B S S-3 \div 1$ & 0 & -6 & & 10.18 \\
\hline BSS -421 & 0 & -9 & & 17.4 \\
\hline BSS- 441 & 0 & -7 & & 7.6 \\
\hline$B S S-491$ & 0 & -9 & & 15.5 \\
\hline \multicolumn{5}{|l|}{ Second Layer } \\
\hline BSS -032 & 10 & 39 & & 9.72 \\
\hline $\mathrm{BSS}-072 \mathrm{~A}$ & 8 & 36 & & 42.6 \\
\hline BSS-082 & 6 & -36 & & 16.5 \\
\hline BSS-212A & 5 & $-\quad 47$ & & 17.49 \\
\hline BSS -322 & 6 & 49 & & 24.02 \\
\hline BSS -422 & 9 & -45 & & 50.4 \\
\hline BSS -442 & 7 & -38 & & 8.4 \\
\hline BSS -492 & 9 & -41 & & 20.6 \\
\hline \multicolumn{5}{|l|}{ Third Layer } \\
\hline BSJ -033 & 39 & 72 & & 19.2 \\
\hline BSS-073A & 36 & 72 & & 15.3 \\
\hline$B S S-083$ & 36 & -72 & & 11.4 \\
\hline$B S S-213 A$ & 47 & 77 & & 14.36 \\
\hline BSS -323 & 49 & 72 & & 34.73 \\
\hline BSS -423 & 45 & -72 & & 23 \\
\hline BSS -443 & 38 & -70 & & 20 \\
\hline$B S S-493$ & 41 & -72 & & 19.1 \\
\hline \multicolumn{5}{|l|}{ Fourth Layer } \\
\hline BSS -034 & 96 & $-\quad 120$ & & 6.24 \\
\hline$B S S-074 A$ & 96 & -120 & & 12.2 \\
\hline BSS-084 & 96 & -120 & & 9.44 \\
\hline$B S S-214 A$ & 96 & -120 & & 4.622 \\
\hline$B S S-324$ & 96 & -120 & & 14.87 \\
\hline BSS -424 & 96 & -120 & & 3 \\
\hline BSS-444 & 96 & -120 & & 6.4 \\
\hline BSS-494 & 96 & 120 & & 7.4 \\
\hline
\end{tabular}


Cadmium

Unit: microgram/gram

\section{First Layer}

BSS-031

BSS-071

BSS-081

BSS-211

BSS-321

BSS-421

BSS-441

BSS-491

Second Layer

BSS -0.32

BSS-072A

BSS-082

BSS-212A

BSS -322

BSS -422

BS: $1-442$

BS! -492

Third Layer

BSS-03.3

BSS-073A

BSS-083

BSS-213A

BSS -323

BSS -423

BSS -443

BSS -4.93

Fourth Layer

BSS-034

BSS-074A

BSS-084

BSS-214A

BSS- 324

BSS-424

BSS- 444

BSS -494

$\begin{array}{cc}\text { Interval (inches) } & \text { Modifier } \\ 0-6 & \text { LT } \\ 0-8 & \text { LT } \\ 0-6 & \text { LT } \\ 0-5 & \\ 0-6 & \\ 0-9 & \text { LT } \\ 0-7 & \text { LT } \\ 0-9 & \text { LT }\end{array}$

$10-39$

- 36

- 36

$-\quad 47$
$-\quad 49$

- 49

- 45

- 38

- 41

$39-72$
$36-72$
$36-72$
$47-77$
$49-72$
$45-72$
$38-70$
$41-72$

LT

LT

LT

LT

LT

LT

LT

LT

LT

LT

LT

LT

LT

LT

Result

0.5

0.5

0.8619

0.7293

0.6

0.6

0.6

0.5

0.5

0.5967

1

0.6

0.6

$96-120$
$96-120$
$96-120$
$96-120$
$96-120$
$96-120$
$96-120$
$96-120$

LT

LT

LT

0.5

0.5

1

0.5301

0.6

0.6

0.6

LT

LT
0.5

0.5

0.5304

0.8619

0.6

0.6

0.6 


\begin{tabular}{|c|c|c|c|c|}
\hline \multicolumn{5}{|l|}{ Chromiun } \\
\hline \multicolumn{5}{|c|}{ Unit: microgram/gram } \\
\hline First Layer & Interva & al (inches) & Modifier & Result \\
\hline$B S S-031$ & 0 & -6 & & 2.28 \\
\hline BSS-071 & 0 & -8 & & 2.34 \\
\hline BSS-081 & 0 & -6 & & 30.6 \\
\hline BSS-211 & 0 & -5 & & 4.936 \\
\hline$B S S-321$ & 0 & -6 & & 3.154 \\
\hline BSS -421 & 0 & -9 & & 3.8 \\
\hline BSS -441 & 0 & -7 & & 2.4 \\
\hline BSS -491 & 0 & -9 & & 3.8 \\
\hline \multicolumn{5}{|l|}{ Second Layer } \\
\hline BSS -032 & 10 & -39 & & 3.5 \\
\hline $\mathrm{BSS}-072 \mathrm{~A}$ & 8 & -36 & & 10.2 \\
\hline BSS -082 & 6 & -36 & & 39.4 \\
\hline$B S S-212 A$ & 5 & 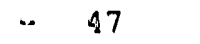 & & 6.719 \\
\hline BSS-322 & 6 & $-\quad 49$ & & 6.3 \\
\hline BSS -422 & 9 & -45 & & 13.8 \\
\hline BSS -442 & 7 & $-\quad 38$ & & 10.6 \\
\hline BSS -492 & 9 & -41 & & 3.7 \\
\hline \multicolumn{5}{|l|}{ Third Layer } \\
\hline BSS-033 & 39 & -72 & & 30.9 \\
\hline BSS-073A & 36 & -72 & & 30.4 \\
\hline BSS-083 & 36 & .. 72 & & 53.4 \\
\hline$B S S-213 A$ & 47 & -77 & & 31.98 \\
\hline BSS-323 & 49 & $-\quad ?$ & & 31.67 \\
\hline BSS -423 & 45 & 72 & & 25 \\
\hline BSS -443 & 38 & 70 & & 39.2 \\
\hline BSS -493 & 41 & -72 & & 21.9 \\
\hline \multicolumn{5}{|l|}{ Fourth Layer } \\
\hline BSS-034 & 96 & & & 30.4 \\
\hline$B S S-074 A$ & 96 & $-\quad 120$ & & 33.7 \\
\hline BSS-084 & 96 & $-\quad 120$ & & 105.1 \\
\hline$B S S-214 A$ & 96 & -120 & & 18.1 \\
\hline BSS-324 & 96 & -120 & & 2.331 \\
\hline BSS-424 & 96 & $-\quad 120$ & & 24.2 \\
\hline BSS- 444 & 96 & -120 & & 28.4 \\
\hline BSS-494 & 96 & -120 & & 13.4 \\
\hline
\end{tabular}




\begin{tabular}{|c|c|c|c|c|}
\hline RSS -031 & & $\begin{array}{l}1 \text { (inches) } \\
-6\end{array}$ & Modifier & Result \\
\hline BSS-071 & 0 & $\begin{array}{l}-6 \\
-\quad 8\end{array}$ & & 0.71 \\
\hline BSS-081 & 0 & $\begin{array}{l}-\quad 8 \\
-\quad 6\end{array}$ & & 1.42 \\
\hline$B S S-211$ & 0 & $\begin{array}{l}-6 \\
-\quad 5\end{array}$ & & $\begin{array}{r}2.3 \\
2.133\end{array}$ \\
\hline BSS-321 & 0 & -6 & & 1.262 \\
\hline BSS -421 & 0 & -9 & & 1.8 \\
\hline BSS -441 & 0 & -7 & & 1 \\
\hline BSS- 491 . & 0 & -9 & & 2.3 \\
\hline \multicolumn{5}{|l|}{ Second Layer } \\
\hline BSS-032 & 10 & $-\quad 39$ & & 0.85 \\
\hline$B S S-072 A$ & 8 & $-\quad 36$ & & 3.99 \\
\hline BSS-082 & 6 & -35 & & 1.16 \\
\hline$B S S-212 A$ & 5 & -47 & & 3.493 \\
\hline BSS-322 & 6 & -49 & & 1.4 \\
\hline BSS -422 & 9 & -45 & & 5 \\
\hline BSS -442 & 7 & $-\quad 38$ & & 1.4 \\
\hline BSS -492 & 9 & -41 & & 1.5 \\
\hline \multicolumn{5}{|l|}{ Third Layer } \\
\hline BSS -033 & 39 & -72 & & 4.2 \\
\hline BSS-073A & 36 & 72 & & 0.78 \\
\hline BSS-083 & 36 & $-\quad 72$ & & 4.32 \\
\hline$B S S-213 A$ & 47 & 77 & & 3.64 \\
\hline BSS-323 & 49 & $-\quad 72$ & & 7.766 \\
\hline BSS -423 & 45 & -72 & & 8 \\
\hline BSS -443 & 38 & 70 & & 13 \\
\hline BSS -493 & 41 & 72 & & 7.1 \\
\hline \multicolumn{5}{|l|}{ Fourth Layer } \\
\hline BSS-034 & 36 & $-\quad 120$ & & 0.64 \\
\hline BSS-074A & 96 & $-\quad 120$ & & 4.77 \\
\hline BSS-084 & 96 & 120 & & 11.1 \\
\hline BSS-214A & 96 & 120 & & 8.784 \\
\hline BSS -324 & 96 & 120 & & 1.974 \\
\hline$B S S-424$ & 96 & $-\quad 120$ & & 4.4 \\
\hline BSS -444 & 96 & -120 & & 6 \\
\hline BSS-494 & 96 & 120 & & 4.3 \\
\hline
\end{tabular}




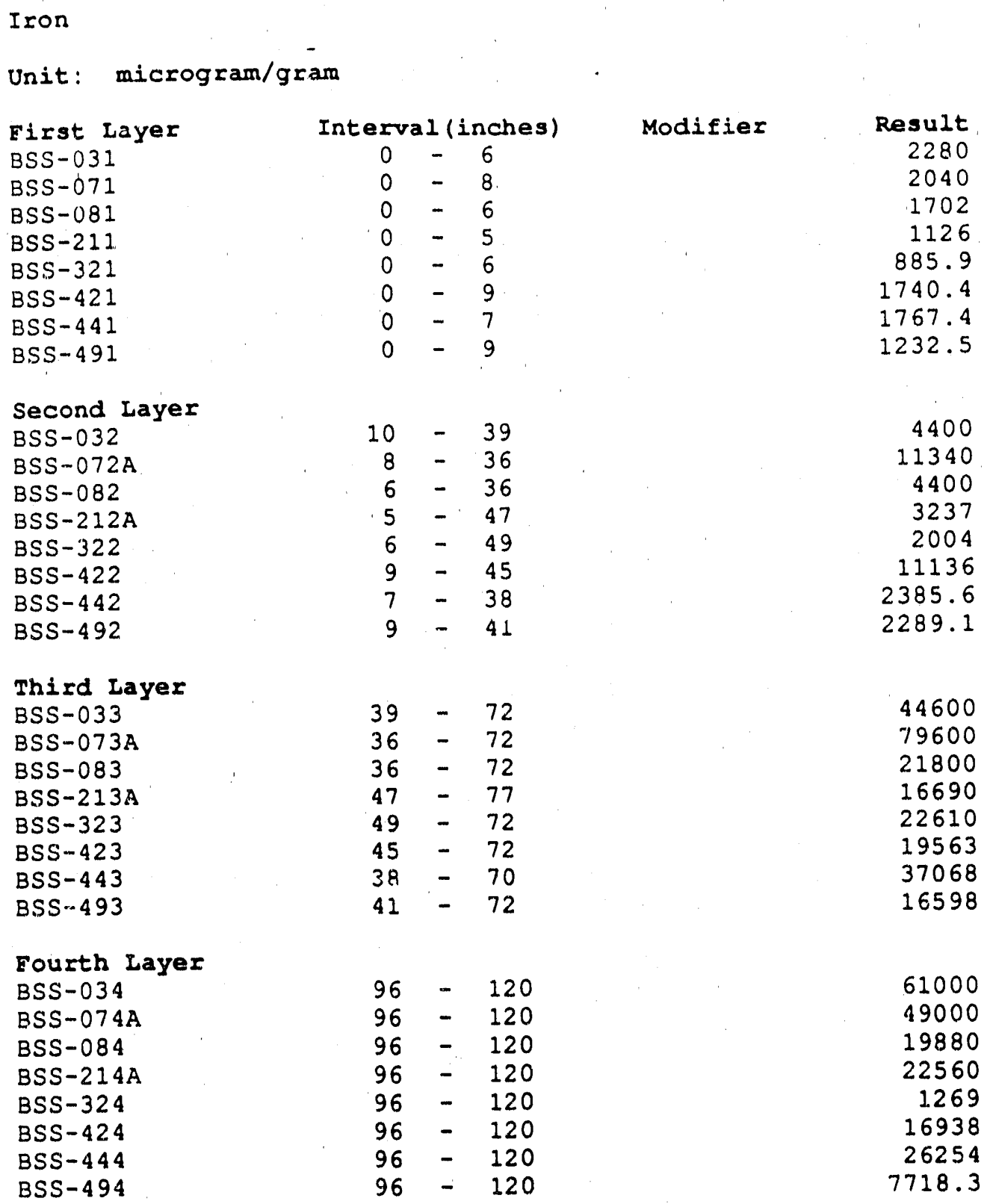




\section{Lead}

Unit: microgram/gram

First Layer

BSS-031

BSS-071

BSS-081

BSS-211

BSS-321

BSS-421

BSS-441

BSS-491

\section{Second Layer}

BSS-032

BSS $-072 \mathrm{~A}$

BSS-082

BSS-2.12A

ESS-3i2

BSS -422

BSS -442

BSS -492

Third Layer

BSS -033
BSS $-073 A$
$B S S-083$
$B S S-213 A$
$B S S-323$
$B S S-423$
$B S S-443$
$B S S-493$

Fourth Layer

BSS- 034

BSS-074A

BSS-084

BSS-214A

BSS-324

BSS- 424

BSS-444

BSS-494

Interval (inches) Modifier
$0-6$
$0-8$
$0-6$
$0-5$
$0-6$
$0-9$
$0-7$
$0-9$

Result

2.05

4.22

3.72

4.01

4.23

5.8

4.9

3.5

$10-39$
$8-36$
$6-36$
$5-47$
$6-49$
$9-45$
$7-38$
$9-41$

2.32

3.05

2.02

3.07

3.82

8.6

2.4

3.2

$39-72$
$36-72$
$36-72$
$47-77$
$49-72$
$45-72$
$38-70$
$41-72$

5.75

7.12

6.75

3.82

6.8

8.6

4.7

$96-120$

6.08

10.9

4.15

6.67

16.4

5.1

5.7

2. 3 


\begin{tabular}{|c|c|c|c|c|}
\hline & Interv & 1 (inches) & Modifier & Result \\
\hline BSS-071 & 0 & $\begin{array}{l}-6 \\
-\quad 8\end{array}$ & $\begin{array}{l}\mathrm{LT} \\
\mathrm{LT}\end{array}$ & $\begin{array}{l}10 \\
10\end{array}$ \\
\hline BSS-081 & 0 & -6 & LT & $\begin{array}{l}10 \\
10\end{array}$ \\
\hline$B S S-211$ & 0 & 5 & LT & 10 \\
\hline BSS -321 & 0 & -6 & LT & 10 \\
\hline BSS -421 & 0 & -9 & LT & 10 \\
\hline BSS -441 & 0 & -7 & LT & 10 \\
\hline BSS -491 & 0 & -9 & LT & 10 \\
\hline Second Layer & & & & \\
\hline BSS-032 & 10 & $-\quad 39$ & LT & 10 \\
\hline BSS-072A & 8 & -36 & LT & 10 \\
\hline BSS-082 & 6 & -36 & LT & 10 \\
\hline$B S S-212 A$ & 5 & $-\quad 4 \%$ & LT & 10 \\
\hline BSS-322 & 6 & -49 & LT & 10 \\
\hline BSS -422 & 9 & -45 & LT & 10 \\
\hline BSS -442 & 7 & -38 & LT & 10 \\
\hline BSS-492 & 9 & -41 & LT & 10 \\
\hline Third Layer & & & & \\
\hline BSS -033 & 39 & -72 & LT & 10 \\
\hline$B S S-073 A$ & 36 & -72 & LT & 10 \\
\hline BSS-083 & 36 & -72 & LT & 10 \\
\hline BSS $-213 A$ & 47 & -77 & LT & 10 \\
\hline BSS-323 & 49 & -72 & & 12.59 \\
\hline BSS -423 & 45 & -72 & LT & 10 \\
\hline BSS -443 & 38 & -70 & LT & 10 \\
\hline$B S S-49^{\prime} 3$ & 41 & -72 & LT & 10 \\
\hline Fourth Layed & & & & \\
\hline BSS-034 & 96 & 120 & LT & 10 \\
\hline$B S S-074 A$ & 96 & $-\quad 120$ & LT & 10 \\
\hline BSS-084 & 96 & -120 & LT & 10 \\
\hline$B S S-214 A$ & 96 & $-\quad 120$ & LT & 10 \\
\hline BSS-324 & 96 & $-\quad 120$ & LT & 10 \\
\hline BSS -424 & 96 & 120 & LT & 10 \\
\hline BSS -444 & 96 & -120 & $L T$ & 10 \\
\hline$B S S-494$ & 96 & 120 & LT & 10 \\
\hline
\end{tabular}




\begin{tabular}{|c|c|c|c|c|}
\hline \multicolumn{5}{|l|}{ Magnesium } \\
\hline \multicolumn{5}{|c|}{ Unit: microgram/gram } \\
\hline First Layer & \multicolumn{2}{|c|}{ Interval (inches) } & \multirow[t]{9}{*}{ Modifier } & \multirow{2}{*}{$\begin{array}{r}\text { Result } \\
58.86\end{array}$} \\
\hline BSS-031 & 0 & -6 & & \\
\hline BSS-071 & 0 & -8 & & 57.86 \\
\hline BSS-081 & 0 & -6 & & 96.32 \\
\hline BSS-211 & 0 & - & & 72.55 \\
\hline BSS-321 & 0 & - & & 66.95 \\
\hline BSS -421 & 0 & -9 & & 58.65 \\
\hline BSS -441 & 0 & -7 & & 40.53 \\
\hline $8 S S-491$ & 0 & -9 & & 57.27 \\
\hline \multicolumn{5}{|l|}{ Second Layer } \\
\hline BSS-032 & 10 & 39 & & 86.15 \\
\hline$B S S-072 A$ & 8 & 36 & & 436.1 \\
\hline BSS-082 & 6 & -36 & & 131 \\
\hline$B S S-212 A$ & 5 & $-\quad 47$ & & 145.4 \\
\hline BSS-322 & 6 & 49 & & 197.7 \\
\hline BSS -422 & 9 & 45 & & 296.7 \\
\hline USS -442 & 7 & $-\quad 38$ & & 91.37 \\
\hline BSS -492 & 9 & -41 & & 93.58 \\
\hline \multicolumn{5}{|l|}{ Third Layer } \\
\hline BSS -033 & 39 & -72 & & 207.2 \\
\hline$B S S-073 A$ & 36 & -72 & & 348.7 \\
\hline BSS-083 & 36 & -72 & & 219.3 \\
\hline BSS-213A & 47 & 77 & & 244.8 \\
\hline BSS -323 & 49. & -72 & & 514 \\
\hline BSS -423 & 45 & -72 & & 178.6 \\
\hline BSS-443 & 38 & -70 & & 158 \\
\hline BSS -493 & 41 & -72 & & 166.5 \\
\hline \multicolumn{5}{|l|}{ Fourth Layer } \\
\hline BSS-034 & 96 & -120 & & 94.2 \\
\hline BSS-074A & 96 & -120 & & 194.1 \\
\hline BSS-084 & 96 & -120 & & 157 \\
\hline BSS-214A & 96 & -120 & & 71.93 \\
\hline BSS-324 & 96 & $-\quad 120$ & & 171.5 \\
\hline BSS -424 & 96 & $-\quad 120$ & & 49.25 \\
\hline BSS -444 & 96 & -120 & & \\
\hline BSS -4.94 & 96 & $-\quad 120$ & & 81.48 \\
\hline
\end{tabular}




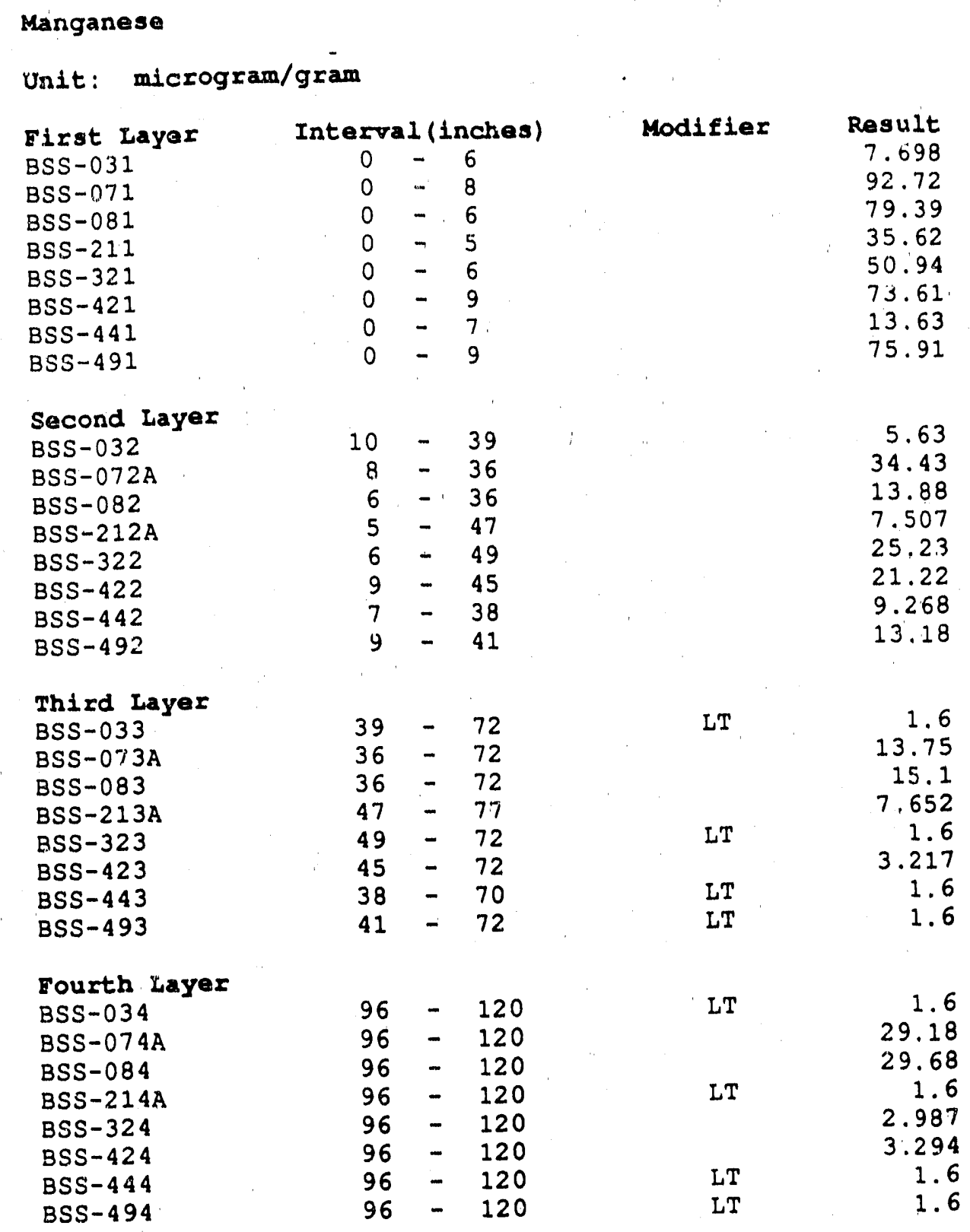




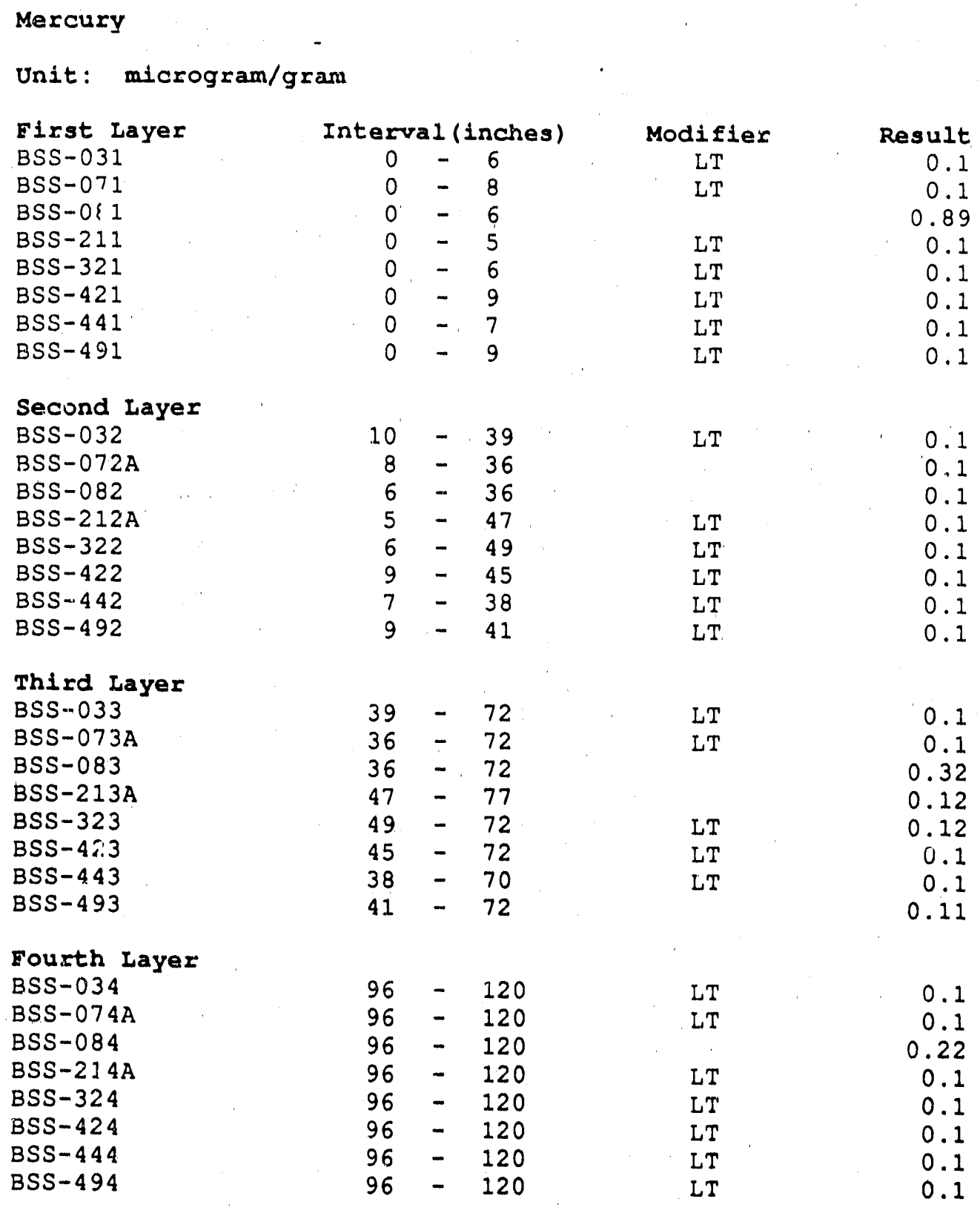


Nickel

Unit: microgram/gram

Eirst Layer

BSS-031

BSS-071

BSS -081

BSS-211

BSS -321

BSS -421

BSS -441

BSS -491

Second Layer

BSS -032

BSS-072A

BSS -082

BSS-212A

BSS -322

BSS -422

BSS -442

BSS -492

Third Layer

BSS -033

BSS-073A

BSS -083

BSS-213A

BSS -323

BSS -423

BSS -443

BSS -493

Fourth Layer

BSS-034

BSS-074A

BSS-084

BSS-214A

BSS -324

BSS -424

BSS -444

BSS -494

Interval (inches)
$0-6$
$0-8$
$0-6$
$0-5$
$0-6$
$0-9$
$0-7$
$0-9$

Modifier

LT

LT.

Result

2.1

2.1

8.1

3.797

LT

4.2

LT

2

2

$$
\begin{array}{r}
10-39 \\
8=36 \\
6-36 \\
5-47 \\
6=49 \\
9-45 \\
7=38 \\
9-41
\end{array}
$$

LT

2.99

6.24

5.86

2.677

LT

4.2

5.2

4.2

2

$39-72$

$36-72$

$36-72$

$47-77$

$49-72$

$45-72$

$38-70$

$41-72$

5.32

8.35

7.42

LT

4.2

6.483

6.8

4.6

3.7

$96-120$

$96-120$

$96-120$

- 120

- 120

- 120

- 120

- 120
4.4

7.54

1.7 .9

3.739

1.713

LT

LT

2
2
2 


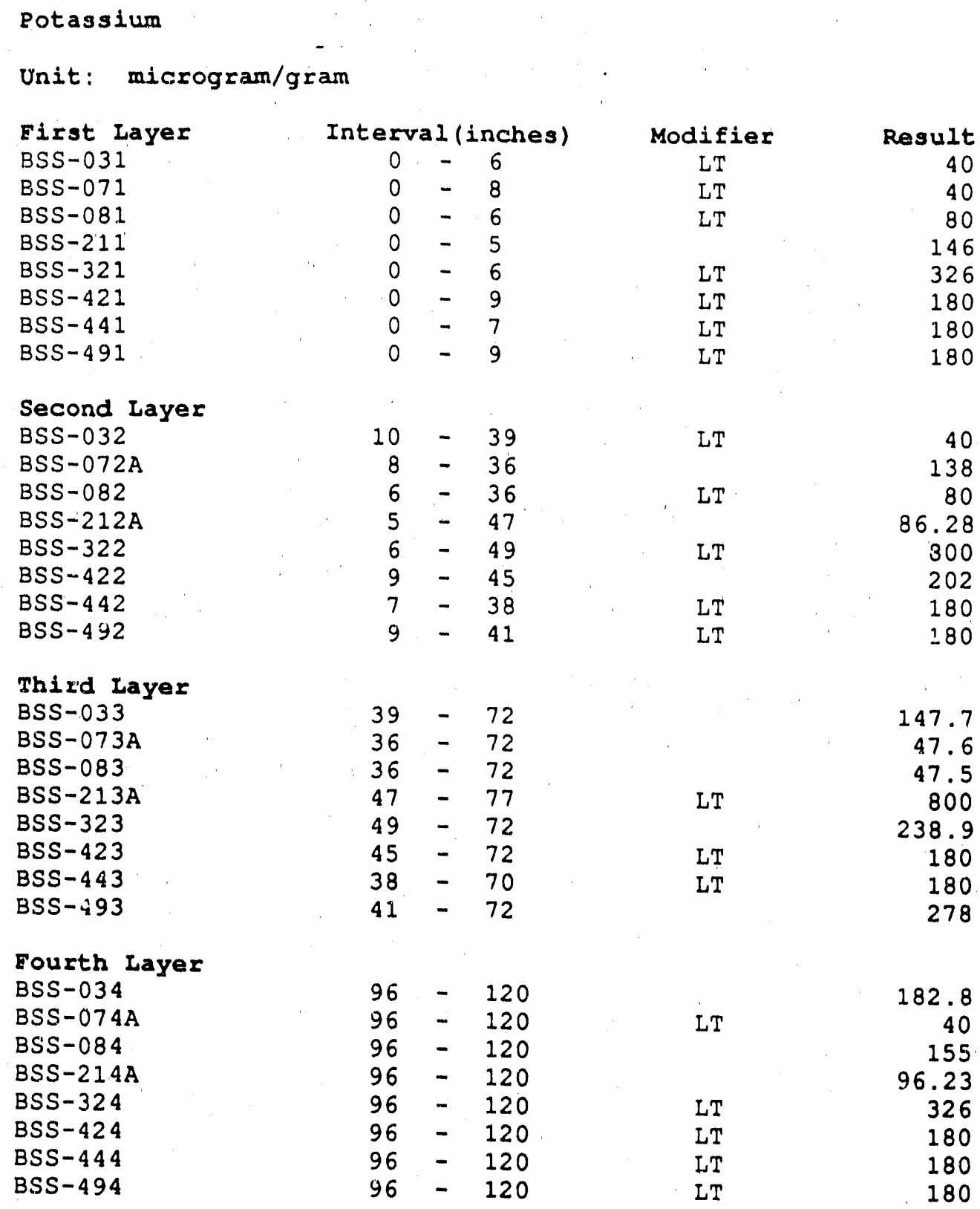


Selenium

Unit: microgram/gram

First Layer

BSS-031

BSS-071

BSS-081

BSS-211

BSS-321

BSS -421

BSS -441

BSS-491

Second Iayer

BSS-032

BSS-072A

BSS -082

BSS-212A

BSS -322

BSS -422

BSS -442

BSS- 492

Third Layer

BSS -033

BSS-073A

BSS-083

BSS-213A

BSS -323

BSS -423

BSS -443

BSS -493

Fourth Iaper

BSS-034

BSS-074A

BSS-084

BSS-214A

BSS-324

BSS-424

BSS-444

BSS-494

\section{Interval (inches)}

$0-6$
$0-8$
$0-6$
$0-5$
$0-6$
$0-9$
$0-7$
$0-9$

Modifier

$10-39$
$8-36$

- 36

-47
$-\quad 46$

- 49

- 45

- 38

- 41

$$
\begin{aligned}
& 39-72 \\
& 36-72 \\
& 36-72 \\
& 47-77 \\
& 49-72 \\
& 45-72 \\
& 38-70 \\
& 41-72
\end{aligned}
$$

$96-120$
$96-120$
$96-120$
$96-120$
$96-120$
$96-120$
$96-120$
$96-120$

LT

LT

LT

LT

LT

LT,

LT

LT

LT

Result

0.2

0.2

0.4

0.4

0.4

0.4

0.4

0.4

0.2

0.38

0.4

0.4

1

0.4

0.4

$\begin{array}{ll}\mathrm{LT} & 0.4 \\ \mathrm{LT} & 0.4\end{array}$

$\begin{array}{lr} & 0.51 \\ \text { LT } & 0.79 \\ \text { LT } & 0.4 \\ & 1 \\ \text { LT } & 1.66 \\ & 0.4 \\ \text { LT } & 0.93\end{array}$

$\begin{array}{rr} & 0.25 \\ \text { LT } & 0.55 \\ & 0.4 \\ & 0.5 \\ \text { LT } & 0.59 \\ \text { LT } & 0.4 \\ \text { LT } & 0.4 \\ & 0.4\end{array}$




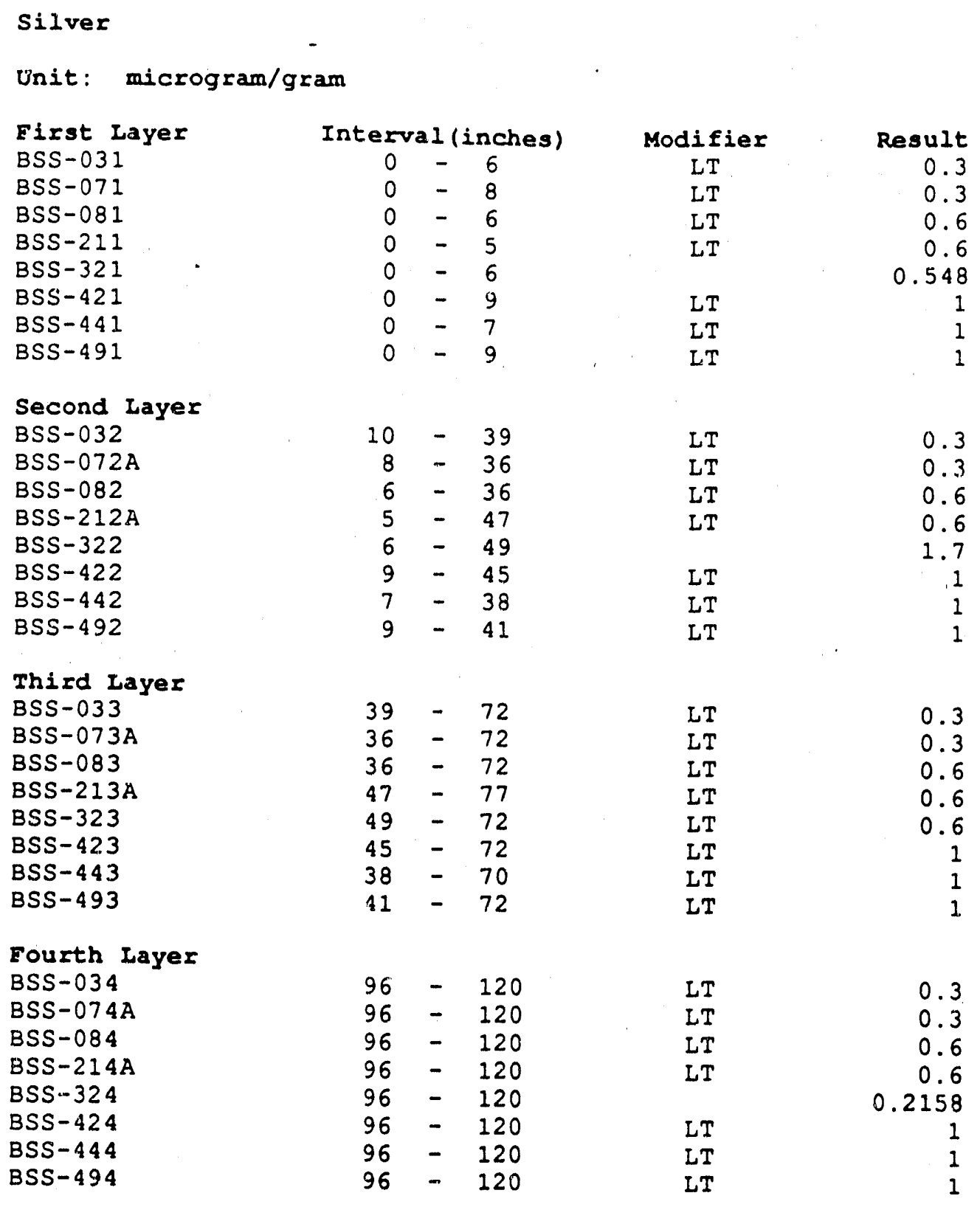


Sodium

Unit: microgram/gram

First Layer

BSS-0 31

BSS-071

BSS-081

BSS-211

BSS-321

BSS- 421

BSS -441

BSS -491

Second Layer

BSS-0 032

BSS-072A

BSS-082

BSS-212A

BSS -322

BSS -422

BSS -442

BSS -492

Third Layer

BSS-0 33

BSS-073A

BSS -083

BSS-213A

BSS -323

BSS -423

BSS -443

BSS -493

Eourth Layer

BSS-034

BSS-074A

BSS-084

BSS-214A

BSS- 324

BSS-424

BSS -444

BSS -494

Interval (inches) $\quad$ Modifier
$0-6$
$0-8$
$0-6$
$0-5$
$0-6$
$0-9$
$0=7$
$0-9$

$10-39$

- 36

- 36

$-\quad 47$
$-\quad 49$

$-\quad 49$
$-\quad 45$

- 45

- 38

- 41

$39-72$

$36-72$

$36-72$

$47-77$

$49-72$

$45-72$

$38-70$

$41-72$

19.3

37.6

28.6

47.88

72.15

58.2

64.6

21.6

36.8

28.6

48.6

63.34

58.95

64.6

52.6

45.6

$96-120$
$96-120$
$96-120$
$96-120$
$96-120$
$96-120$
$96-120$
$96-120$

26.8

41.5

50

41.64

42.88

39.8

39

25.1 
$z$ inc

Unit: microgram/gram

Eirst Layer

BSS-031

BSS-071

BSS-081

BSS-211

BSS- 321

BSS -421

BSS- 441

BSS -491

Second Layer

BSS -032

BSS-072A

BSS -082

BSS-212A

BSS -322

BSS -422

BSS -442

BSS -492

Third Layer

BSS-033

BSS-073A

BSS-083

BSS -213 A

BSS -323

BSS -423

BSS -443

BSS -493

Fourth Layer

BSS-034

BSS-074A

BSS-084

BSS-214A

BSS-324

BSS -424

BSS -444

BSS-494

Interval (inches) $\quad$ Modifier
$0-6$
$0-8$
$0-6$
$0-5$
$0-6$
$0-9$
$0-7$
$0-9$

$10-39$

$8-36$

$6-36$

$5-47$

$6-49$

$9-45$

$7-38$

$9-41$

$$
\begin{aligned}
& 39-72 \\
& 36-72 \\
& 36-72 \\
& 47-77 \\
& 49-72 \\
& 45-72 \\
& 38-70 \\
& 41-72
\end{aligned}
$$

4.69

16.8

7.18

10.76

6.04

9.8

2.2

4.1

10.6

19.6

14.7

15.82

15.11

12

5.4

4.2

$96-120$
$96-120$
$96-120$
$96-120$
$96-120$
$96-120$
$96-120$
$96-120$

9.75

13.7

14.4

12.67

8.178

3.4

4

8.3 
Aluminum

Unit: microgram/gram

Eirst Layer

BSS-021

BSS-221

BSS- 411

BSS-481

BSS-511

BSS-521

BSS-561

BSS-571

Second Layer

BSS-022

BSS $-222 \mathrm{~A}$

BSS -412

BSS-48:

BSS -512

BSS -522

BSS -562

BSS -572

Third Layer

BSS-023

BSS-223A

BSS -413

BSS -483

BSS -513

BSS-523

BSS -563

BSS -573

Interval (inch
$0-8$
$0-7$
$0-5$
$0-4$
$0-5$
$0-5$
$0-5$
$0-9$

Modifier

ches)

7

5

$-4$

$-5$

$-9$

$-\quad 84$
$-\quad 80$

- 80

- 80

-80
$-\quad 80$

- 80

- 80

- 80

$-80$

$110-124$
$96-120$
$96-120$
$96-120$
$96-120$
$96-120$
$96-120$
$96-120$

Result

1347

2502

2143

1399.6

4367.6

1708.2

1964.8

4169.6

3612

2010

2090

1886

3726.4

1966

1221.4

8959.5

4092

1669

4146

3407.2

21504

4697

1087.2

5219.7 
Arsenic

Unit: microgram/gram

Eirst Layer

BSS-021

BSS-221

BSS-411

BSS-481

BSS-511

BSS- 521

BSS-561

BSS-571

Second Layer

BSS-022

BSS-222A

BSS -412

BSS -482

BSS -512

BSS -522

BSS -562

BSS -572

Third Layer

BSS-023

BSS-223A

BSS-413

BSS -483

BSS-513

BSS -523

BSS -563

BSS -573

\begin{tabular}{|c|c|c|}
\hline 0 & - & 8 \\
\hline 0 & - & 7 \\
\hline 0 & - & 5 \\
\hline 0 & - & 4 \\
\hline 0 & - & 5 \\
\hline 0 & - & 5 \\
\hline 0 & - & 5 \\
\hline 0 & - & 9 \\
\hline
\end{tabular}

Modifier
LT
LT
LT
LT
LT
LT
LT

Result

0.5

1.02

1

2

2

2

2

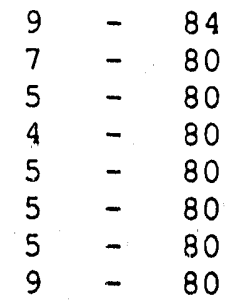

LT

LT

LT

LT

LT

LT

0.5

0.85

1

2

2

2

2

$110-124$
$96-120$
$96-120$
$96-120$
$96-120$
$96-120$
$96-120$
$96-120$

LT

LT

LT

0.5

0.6

4. 81

2

2.2

LT

LT 


\section{Barium}

Unit: microgram/gram

First Layer
BSS -021
BSS -221
BSS -411
BSS -481
BSS -511
BSS -521
BSS -561
BSS -571

Interval (inches)
$0-8$
$0-7$
$0-5$
$0-4$
$0-5$
$0-5$
$0-5$
$0-9$

Result 7.61 3.993

13.55

3

5.2

8.2

58.2

Second Layer

$$
\text { BSS-022 }
$$

BSS-222A

BSS-412

BSS -482

BSS -5.12

BSS-522

BSS -562

BSS-572

$9-84$
$7-80$
$5-80$
$4-80$
$5-80$
$5-80$
$5-80$
$9-80$

12.3

5.011

9.764

5.4

12.6

5.8

3.6

32.3

Third Layer

BSS-023

BSS-223A

BSS -413

BSS -483

BSS -513

BSS-523

BSS-563

BSS -573

$110-124$
$96-120$
$96-120$
$96-120$
$96-120$
$96-120$
$96-120$
$96-120$

7.79

4.071

18.61

36.6

8.4

2.6

65.2 
Cadmium

Unit: microgram/gram

Eirst Layer

BSS-021

BSS-221

BSS-411

BSS-481

BSS-511

BSS-521

BSS-561

BSS-571

\section{Second Layer}

BSS-022

BSS-222A

BSS -412

BSS -432

BSS- 512

BSS -522

BSS -562

BSS -572

Third Iayer

BSS-023

BSS-223A

BSS -413

BSS -483

BSS -513

BSS -523

BSS-563

BSS -573

Interva (inches)
$0-8$
$0-7$
$0-5$
$0-4$
$0-5$
$0-5$
$0-5$
$0-5$

Modifier
LT
LT
LT
LT
LT
LT
LT

Result

0.52

1

0.6

0.6

0.6

0.6

0.6

0.5

1

0.6

0.6

0.6

0.6

0.6

$110-124$
$96-120$
$96-120$
$96-120$
$96-120$
$96-120$
$96-120$
$96-120$

LT

LT

LT

LT

LT

LT

LT

LT
0.5

1
1

0.6

0.6

0.6

0.6

0.6 


\section{Chromium}

Unit: microgram/gram

Eirst Layer

BSS-021

BSS-221

BSS - 411

BSS -481

BSS-511

BSS -521

BSS-561

BSS -571

\section{Second Iayer}

BSS-022

BSS-222A

$B S S-412$

BSS -482

BSS -512

BSS -522

BSS -562

BSS -572

Third Layer

BSS-023

BSS-223A

BSS -413

BSS-483

BSS-513

BSS-523

BSS-563

BSS -573

$\begin{array}{ccc}\text { Interval (inches) } & \text { Modifier } \\ 0 & -8 \\ 0 & -7 \\ 0 & -5 \\ 0 & -4 \\ 0 & -5 \\ 0 & - & 5 \\ 0 & - & 5 \\ 0 & - & 9\end{array}$

Result

1.31

4. 33

3.01

5
4

4. 2

5.8

7.1

3.33

3.862

3.364

6.2

4.8

. 4

5.8 .

6.8

4.85

8.425

18.29

35.2

6.6

11.4

6.4 


\begin{tabular}{|c|c|c|c|c|}
\hline BSS-021 & 0 & -8 & & 1 \\
\hline BSS-221 & 0 & -7 & & 2.116 \\
\hline BSS-411 & 0 & -5 & & 1.422 \\
\hline$B S S-481$ & 0 & -4 & & 1.6 \\
\hline BSS - 511 & 0 & -5 & & 2.2 \\
\hline BSS -521 & 0 & - & & 1.8 \\
\hline BSS-561 & 0 & -5 & & 2 \\
\hline BSS-571 & 0 & -9 & & 2.7 \\
\hline Second Layex & & & & \\
\hline BSS-022 & 9 & 84 & & 2.14 \\
\hline$B S S-222 A$ & 7 & 80 & & 2.108 \\
\hline BSS-412 & 5 & 80 & & 1.282 \\
\hline BSS -482 & 4 & 80 & & 2 \\
\hline BSS-512 & 5 & 80 & & 2.2 \\
\hline BSS -522 & 5 & 80 & & 2.2 \\
\hline BSS -562 & 5 & 80 & & 1.6 \\
\hline BSS -572 & 9 & 80 & & 5.5 \\
\hline Third Layer & & & & \\
\hline BSS - 023 & 110 & 124 & & 2.71 \\
\hline$B S S-223 A$ & 9.5 & 120 & & 1.849 \\
\hline BSS - 413 & 96 & 120 & & 1.282 \\
\hline BSS -483 & 96 & 120 & & 2.6 \\
\hline BSS -513 & 96 & 120 & & 9.6 \\
\hline BSS -523 & 96 & 120 & & 2.4 \\
\hline BSS -563 & 96 & 120 & & 4.2 \\
\hline BSS-573 & 96 & $-\quad 120$ & & 4.4 \\
\hline
\end{tabular}




\begin{tabular}{|c|c|c|c|c|}
\hline & & & Modifler & Result \\
\hline BSS-021 & 0 & 8 & & 1456 \\
\hline BSS-221 & 0 & -7 & & 1534 \\
\hline$B S S-411$ & 0 & -5 & & 1474 \\
\hline BSS -481 & 0 & 4 & & 1634.6 \\
\hline BSS -511 & 0 & - & & 2653.6 \\
\hline BSS -521 & 0 & - & & 1416.6 \\
\hline$B S S-561$ & 0 & - & & 2.183 .4 \\
\hline BSS-571 & $c$ & - & & 2580.6 \\
\hline \multicolumn{5}{|l|}{ Second Layer } \\
\hline BSS-022 & 9 & 84 & & 5780 \\
\hline$B S S-222 A$ & 7 & 80 & & 1426 \\
\hline BSS -412 & 5 & 80 & & $\begin{array}{r}1758 \\
\end{array}$ \\
\hline$B S S-482$ & 4 & 80 & & 2243.8 \\
\hline BSS -512 & 5 & 80 & & 2632.2 \\
\hline$B S S-522$ & 5 & 80 & & 1477 \\
\hline BSS -562 & 5 & 80 & & 1929.4 \\
\hline BSS -572 & 9 & 80 & & 6909.3 \\
\hline \multicolumn{5}{|l|}{ Third Layer } \\
\hline BSS-023 & 110 & 124 & & 6900 \\
\hline$B S S-223 A$ & 96 & 120 & & 1313 \\
\hline BSS-413 & 96 & 120 & & 1758 \\
\hline BSS -483 & 96 & 120 & & 4192.2 \\
\hline BSS-513 & 96 & 120 & & 26308 \\
\hline BSS -523 & 96 & 120 & & 3645.6 \\
\hline BSS-563 & 95 & 120 & & 3899 \\
\hline BSS-573 & 96 & 120 & & 5112.8 \\
\hline
\end{tabular}


Iead

Unit: microgram/gram

inst Layer

BSS-021

BSS-221

BSS -411

BSS -481

BSS-511

BSS-521

BSS-561

BSS-571

Second Layer

BSS-022

BSS-222A

BSS -412

BSS -482

BSS -512

BSS -522

BSS-562

BSS-572

Third Layer

BSS-023

BSS-223A

BSS -413

BSS -483

BSS -513

BS.S -523

BSS -563

BSS -573

Interval (Inches) $\quad$ Modifier
$0-8$
$0-7$
$0-5$
$0-4$
$0-5$
$0-5$
$0-5$
$0-9$

LT

84

-80
$-\quad 80$

- 80

$-80$

- 80

-80
$-\quad 80$

- 80

LT

1.76

7.32

2.26

$110-124$
$96-120$
$96-120$
$96-120$
$96-120$
$96-120$
$96-120$
$96-120$

1.05

4.68

2.4

LT

LT
1

1. 9

1. 2

1. 4

6.3

5.2

2.4

1.6

3. 1

4.7 2

1 
Lakeland Soll Series

Lithium

Unit: microgram/gräm

First Layer

BSS-021

BSS -221

BSS -411

BSS -481

BSS-511

BSS -521

BSS -561

BSS-571

Second Layer

BSS -022

BSS-222A

BSS -412

BSS -482

BSS -512

BSS -522

BSS -562

BSS -572

Third Layer

BSS-023

BSS-223A

BSS -413

BSS -483

BSS -513

BSS -523

BSS -563

BSS -573

Interval (inches)
$0-8$
$0-7$
$0-5$
$0-4$
$0-5$
$0-5$
$0-5$
$0-9$

Modifier

LT

LT

L'T

LT

LT

LT

LT

IT

Result

10

10

10

10

10

10

10

10

$9-84$
$7-80$
$5-80$
$4-80$
$5-80$
$5-80$
$5-80$
$9-80$

LT

$\mathrm{LT}$

LT

LT

LT

LT

LT

LT

10

10

10

10

1.0

10

10

10

$110-124$
$96-120$
$96-120$
$96-120$
$96-120$
$96-120$
$96-120$
$96-120$

LT 10

LT 10

IT 10

LT 10

IT 10

LT 1.0

LT 10
LT 10 
Lakeland soil Series

\begin{tabular}{|c|c|c|c|c|}
\hline \multicolumn{5}{|l|}{ Magnesium } \\
\hline \multicolumn{5}{|c|}{ Unit: microgram/gram } \\
\hline First Layer & \multicolumn{2}{|c|}{ Interval (inches) } & \multirow[t]{9}{*}{ Modifier } & \multirow{9}{*}{$\begin{array}{r}\text { Result } \\
32.65 \\
58.49 \\
99.26 \\
60.04 \\
106.2 \\
35.27 \\
79.75 \\
140.1\end{array}$} \\
\hline$B S S-021$ & 0 & -8 & & \\
\hline$B S S-221$ & 0 & -7 & & \\
\hline BSS -411 & 0 & -5 & & \\
\hline$B S S-481$ & 0 & -4 & & \\
\hline$B S S-511$ & 0 & -5 & & \\
\hline$B S S-521$ & 0 & 5 & & \\
\hline BSS -561 & 0 & -5 & & \\
\hline BSS-571. & 0 & -9 & & \\
\hline \multicolumn{5}{|l|}{ Second Layer } \\
\hline BSS -022 & 9 & 84 & & 145.1 \\
\hline$B S S-222 A$ & 7 & 80 & & 63.06 \\
\hline BSS -412 & 5 & 80 & & 108.2 \\
\hline$B S S-482$ & 4 & 80 & & 47.72 \\
\hline BSS -512 & 5 & 8.0 & & 95.45 \\
\hline$B S S-522$ & 5 & 80 & & 45.37 \\
\hline BSS -562 & 5 & 80 & & 102.8 \\
\hline BSS $-57 \hat{z}$ & 9 & -80 & & 140.6 \\
\hline \multicolumn{5}{|l|}{ Third Layer } \\
\hline BSS-023 & 110 & 124 & & 63.43 \\
\hline$B S S-223 A$ & 96 & 120 & & 50.1 \\
\hline BSS -413 & 96 & 120 & & 107.4 \\
\hline BSS- 483 & 96 & 120 & & 25.25 \\
\hline BSS-513 & 96 & -120 & & 254.6 \\
\hline BSS -523 & 96 & $-\quad 120$ & & 79.68 \\
\hline BSS- 563 & 96 & -120 & & 59.21 \\
\hline BSS -573 & 96 & 120 & & 137.5 \\
\hline
\end{tabular}


Manganese

Unit: microgram/gram

Eirst Layer

BSS-021

BSS-221

BSS -411

BSS -481

BSS-5.11

BSS- 521

BSS-561

$B S S-571$

Seccad Layer

BSS-022

BSS-222A

BSS -412

BSS- 482

BSS -512

BSS- 522

BSS- 562

BSS -572

Third Layer

BSS-023

BSS-223A

BSS-413

BSS -483

BSS -513

BSS -523

BSS -563

BSS-573
Interval (inches)

$0-8$

-7
$-\quad 5$

$-5$

- 4

- 5

- 5

$-5$

Mesult
69.21
3.738
127.9
5.132
104.6
2.987
9.881
498.2

$9-84$

$7-80$

$5-80$

$4-80$

$5-80$

$5-80$

$5-80$

$9-80$

45.42

4.724

17.39

6.358

12.79

14.25

5.898

141.2

$110-124$
$96-120$
$96-120$
$96-120$
$96-120$
$96-120$
$96-120$
$96-120$

11.6

4.299

5.445

4. 673

5.515

3.907

5.898

169.3 


\begin{tabular}{|c|c|c|c|c|}
\hline \multicolumn{5}{|l|}{ Mercury } \\
\hline \multicolumn{5}{|c|}{ Unit: microgram/gram } \\
\hline First Layer & Interv & al (inches) & Modifier & Result \\
\hline$B S S-021$ & 0 & -8 & LT & 0.1 \\
\hline BSS-221 & 0 & 7 & LT & 0.1 \\
\hline$B S S-411$ & 0 & -5 & LT & 0.1 \\
\hline BSS -481 & 0 & -4 & LT & 0.1 \\
\hline BSS-511 & 0 & -5 & LT & 0.1 \\
\hline BSS-521 & 0 & 5 & LT & 0.1 \\
\hline BSS-561 & 0 & 5 & LT & 0.1 \\
\hline$B S S-571$ & 0 & - & LT & 0.1 \\
\hline \multicolumn{5}{|l|}{ Second Layer } \\
\hline BSS-022 & 9 & 84 & LT & 0.1 \\
\hline$B S S-222 A$ & 7 & 80 & & 0.1 \\
\hline BSS-412 & 5 & 80 & LT & 0.1 \\
\hline BSS-482 & 4 & 80 & LT & 0.1 \\
\hline BSS -512 & 5 & 80 & LT & 0.1 \\
\hline BSS -522 & 5 & 80 & LT & 0.1 \\
\hline BSS -562 & 5 & 80 & LT & 0.1 \\
\hline BSS-572 & 9 & 80 & LT & 0.1 \\
\hline \multicolumn{5}{|l|}{ Third Layer } \\
\hline BSS-023 & 110 & 124 & LT & 0.1 \\
\hline$B S S-223 A$ & 96 & 120 & & 0.14 \\
\hline BSS-413 & 96 & 120 & LT & 0.1 \\
\hline BSS -483 & 96 & 120 & LT & 0.1 \\
\hline BSS-513 & 96 & 120 & $\mathrm{LT}$ & 0.1 \\
\hline BSS -523 & 96 & 120 & LT & 0.1 \\
\hline BSS-563 & 96 & 120 & LT & 0.1 \\
\hline BSS-573 & 96 & 120 & LT & 0.1 \\
\hline
\end{tabular}




\begin{tabular}{|c|c|c|c|c|}
\hline & Hot & 1 (inches) & Modifier & Result \\
\hline BSS-021 & 0 & -8 & $\mathrm{LT}$ & 2.1 \\
\hline BSS-221 & 0 & -7 & LT & 5.2 \\
\hline BSS-411 & 0 & -5 & & 1.081 \\
\hline$B S S-481$ & 0 & -4 & & 2.8 \\
\hline BSS-511 & 0 & -5 & & 3 \\
\hline BSS -521 & 0 & 5 & & 2.6 \\
\hline BSS -561 & 0 & - & & 2.2 \\
\hline$B S S-571$ & 0 & - & & 3.1 \\
\hline Second Layex & & & & \\
\hline BSS-022 & 9 & 84 & & 3.33 \\
\hline$B S S-222 A$ & 7 & 80 & LT & 5.2 \\
\hline BSS-412 & 5 & 80 & & 4.406 \\
\hline BSS -482 & 4 & 80 & & 2.4 \\
\hline BSS -512 & 5 & 80 & & 2.6 \\
\hline BSS -522 & 5 & 80 & & 2 \\
\hline BSS -562 & 5 & 80 & LT & 2 \\
\hline BSS -572 & 9 & 80 & & 4.2 \\
\hline Third Layer & & & & \\
\hline BSS-023 & 110 & 124 & LT & $\begin{array}{r}2.1 \\
6.762\end{array}$ \\
\hline$B S S-223 A$ & 96 & 120 & & 6.762 \\
\hline BSS-413 & 96 & 120 & & 4.406 \\
\hline$B S S-483$ & 96 & 120 & LT & 2 \\
\hline BSS -513 & 96 & 120 & & 4.6 \\
\hline BSS -523 & 96 & $-\quad 120$ & LT & 2 \\
\hline BSS-563 & 96 & $-\quad 120$ & LT & 2 \\
\hline BSS -573 & 96 & 120 & & 3.3 \\
\hline
\end{tabular}




\begin{tabular}{|c|c|c|c|c|}
\hline BSS-021 & 0 & -8 & LT & 40 \\
\hline BSS-221 & 0 & -7 & LT & 800 \\
\hline BSS -411 & 0 & -5 & & 145.2 \\
\hline$B S S-481$ & 0 & - & & 196 \\
\hline BSS-511 & 0 & -5 & LT & 180 \\
\hline BSS -521 & 0 & -5 & & 254 \\
\hline BSS -561 & 0 & -5 & & 220 \\
\hline$B S S-571$ & 0 & -9 & LT & 180 \\
\hline \multicolumn{5}{|l|}{ Second Layer } \\
\hline BSS-022 & 9 & 84 & & 28.6 \\
\hline$B S S-222 A$ & 7 & 80 & LT & 800 \\
\hline BSS -412 & 5 & 80 & & 170.5 \\
\hline $3 S S-482$ & 4 & 80 & & 274 \\
\hline BSS -512 & 5 & 80 & LT & 180 \\
\hline BSS -522 & 5 & 80 & LT & 180 \\
\hline BSS -562 & 5 & 80 & LT & 180 \\
\hline BSS -572 & 9 & -80 & LT & 180 \\
\hline \multicolumn{5}{|l|}{ Third Layer } \\
\hline BSS-023 & 110 & 124 & & 73.5 \\
\hline$B S S-223 A$ & 96 & 120 & LT & 800 \\
\hline BSS-413 & 96 & 120 & & 170.5 \\
\hline BSS -483 & 96 & 120 & & 184 \\
\hline BSS -513 & 96 & 120 & LT & 180 \\
\hline BSS-523 & 96 & 120 & & 190 \\
\hline BSS-563 & 96 & 120 & LT & 180 \\
\hline BSS-573 & 96 & -120 & $\mathrm{LT}$ & 180 \\
\hline
\end{tabular}




\section{Selenium}

Unit: microgram/gräm

First Layer BSS-021

BSS-221

BSS- 411

BSS -481

BSS-511

BSS- 521

BSS-561

BSS-571

\section{Second Layer}

BSS-022

BSS-222A

BSS - 412

BSS- 482

BSS -512

BSS-522

BSS -562

BSS -572

Third Layer

BSS-023

BSS-223A

BSS- 413

BSS -483

BSS -513

BSS- 523

BSS-563

BSS -573

\section{Interval (inches)}

$0-8$
$0-7$
$0-5$
$0-4$
$0-5$
$0-5$
$0-5$
$0-9$

Modifier

-80
$-\quad 80$

$110-124$
$96-120$
$96-120$
$96-120$
$96-120$
$96-120$
$96-120$
$96-120$

LT

$\mathrm{LT}$

LT

LT

LT

LT

L'T

LT

LT

LT

LT

LT

LT

LT

LT

LT

Result

0.2

0.4

0.4

0.4

0.4

0.4

0.4

0.4

0.2

0.4

1

4

0.4

0.4

0.4

LT

$$
\text { LT }
$$

LT

LT

0.2

0.4

0.4

0.4

0.75

0.4

0.4

0.4 


\begin{tabular}{|c|c|c|c|c|}
\hline \multicolumn{5}{|l|}{ Silver } \\
\hline \multicolumn{5}{|c|}{ Unit: microgram/gram } \\
\hline First Layer & Interv & al (inches) & Modifier & Result \\
\hline BSS-021 & 0 & -8 & $\mathrm{LT}$ & 0.3 \\
\hline$B S S-221$ & 0 & -7 & LT & 1.4 \\
\hline BSS-411 & 0 & - & & 0.6071 \\
\hline$B S S-481$ & 0 & - & LT & 1 \\
\hline BSS-511 & 0 & -5 & LT & 1 \\
\hline BSS-521 & 0 & -5 & LT & 1 \\
\hline BSS -561 & 0 & - & LT & 1 \\
\hline BSS-571 & 0 & -9 & LT & $i$ \\
\hline \multicolumn{5}{|l|}{ Second Layer } \\
\hline BSS -022 & 9 & 84 & LT & 0.3 \\
\hline$B S S-222 A$ & 7 & 80 & LT & 1.4 \\
\hline BSS -412 & 5 & 80 & $\mathrm{LT}$ & 0.6 \\
\hline$B S S-482$ & 4 & 80 & $\mathrm{LT}$ & 1 \\
\hline BSS -512 & 5 & 80 & $\mathrm{LT}$ & 1 \\
\hline$B S S-522$ & 5 & 80 & $\mathrm{LT}$ & 1 \\
\hline BSS -562 & 5 & 80 & $\mathrm{LT}$ & 1. \\
\hline BSS -572 & 9 & 80 & $\mathrm{LT}$ & 1 \\
\hline \multicolumn{5}{|l|}{ Third Layer } \\
\hline BSS -023 & 110 & 124 & LT & 0.3 \\
\hline$B S S-223 A$ & 96 & 120 & LT & 1.4 \\
\hline BSS -413 & 96 & 120 & $\mathrm{LT}$ & 0.6 \\
\hline BSS -483 & 96 & 120 & LT & 1 \\
\hline BSS -513 & 96 & 120 & LT & 1 \\
\hline BSS -523 & 96 & -120 & LT & 1 \\
\hline BSS -563 & 96 & -120 & LT & 1 \\
\hline BSS -573 & 96 & 120 & $\mathrm{LT}$ & 1 \\
\hline
\end{tabular}




\begin{tabular}{|c|c|c|c|c|}
\hline \multicolumn{5}{|l|}{ Sodium } \\
\hline \multicolumn{5}{|c|}{ Unit: microgram/gram } \\
\hline First Layer & Inter & 1 (inches) & Modifier & Result \\
\hline $\mathrm{BSS}-021$ & 0 & -8 & & 26.2 \\
\hline BSS -221 & 0 & -7 & & 42.49 \\
\hline BSS -411 & 0 & -5 & & 60.44 \\
\hline BSS- 481 & 0 & -4 & & 24.6 \\
\hline BSS-511 & 0 & -5 & & 36.2 \\
\hline BSS -521 & 0 & -5 & & 28.4 \\
\hline BSS -561 & 0 & - & & 34 \\
\hline BSS -571 & 0 & - & & 354.6 \\
\hline \multicolumn{5}{|l|}{ Second Layer } \\
\hline BSS -022 & 9 & 84 & & 24.6 \\
\hline$B S S-222 A$ & 7 & 80 & & 36.34 \\
\hline BSS -412 & 5 & 80 & & 41.55 \\
\hline BSS -482 & 4 & 80 & & 99.4 \\
\hline BSS -512 & 5 & 80 & & 50.6 \\
\hline BSS -522 & 5 & 80 & & 19.6 \\
\hline BSS -562 & 5 & 80 & & 14.8 \\
\hline BSS -572 & 9 & 80 & & 28.2 \\
\hline \multicolumn{5}{|l|}{ Third Layer } \\
\hline BSS -023 & 110 & 124 & & 27.7 \\
\hline$B S S-223 A$ & 96 & 120 & & 33.14 \\
\hline BSS -413 & 96 & 120 & & 41.55 \\
\hline BSS -4.83 & 96 & 120 & & 123.2 \\
\hline BSS -513 & 96 & 120 & & 73.6 \\
\hline BSS -523 & 96 & 120 & & 28.8 \\
\hline BSS -563 & 96 & 120 & $\mathrm{LT}$ & 7 \\
\hline BSS -573 & 96 & 120 & & 35.1 \\
\hline
\end{tabular}




\begin{tabular}{|c|c|c|c|c|}
\hline \multicolumn{5}{|l|}{ zinc } \\
\hline \multicolumn{5}{|c|}{ Unit: microgram/gram } \\
\hline First Layer & Interv & al (inches) & Modifier & Result \\
\hline BSS -021 & 0 & -8 & & 11.8 \\
\hline BSS-221 & 0 & - & & 8.531 \\
\hline BSS-411 & 0 & - & & 6.852 \\
\hline BSS -481 & 0 & - & & 5.4 \\
\hline BSS-511 & 0 & - & & 7.6 \\
\hline BSS -521 & 0 & - & & 4.4 \\
\hline$B S S-561$ & 0 & - & & 3.6 \\
\hline BSS-571 & 0 & -9 & & 8.9 \\
\hline \multicolumn{5}{|l|}{ Second Layer } \\
\hline BSS-022 & 9 & 84 & & 17.2 \\
\hline $\mathrm{BSS}-222 \mathrm{~A}$ & 7 & 80 & & 10.11 \\
\hline BSS -412 & 5 & 80 & & 6.981 \\
\hline$B S S-482$ & 4 & 80 & & 13.2 \\
\hline BSS-512 & 5 & 80 & & 7.2 \\
\hline BSS -522 & 5 & 80 & & 4.8 \\
\hline BSS -562 & 5 & 80 & & 5.6 \\
\hline BSS-572 & 9 & 80 & & 16.1 \\
\hline \multicolumn{5}{|l|}{ Third Layer } \\
\hline BSS-023 & 1.10 & 124 & & 10.2 \\
\hline BSS-223A & 96 & 120 & & 7.72 \\
\hline BSS-413 & 96 & 120 & & 6.981 \\
\hline BSS -483 & 96 & 120 & & 4.2 \\
\hline BSS -513 & 96 & 120 & & 11.8 \\
\hline BSS-523 & 96 & 120 & & 7 \\
\hline BSS - 563 & 96 & 120 & & 11 \\
\hline BSS-573 & 96 & 120 & & 12,1 \\
\hline
\end{tabular}


Aluninum

Unit: microgram/gram

Eirst Layer

BSS-041

BSS-101

BSS-111

BSS-341

BSS-401

BSS-461

BSS - 50.1

BSS-54I

Second Layer

BSS-042A

BSS-102A

BSS-112A

BSS -342

BSS -402

BSS -462

BSS -502

BSS-542

Third Layer

BSS-043A

BSS-103A

BSS -343

BSS -403

BSS -463

BSS- 503

BSS -543

\section{Eourth Layer}

\section{BSS - 044A}

BSS-104A

BSS-113A

BSS-114A

BSS -344

BSS -404

BSS -464

BSS -504

BSS-544

Fifth Layer

BSS - 045A

BSS-105A

BSS-115A

BSS-345

BSS- 405

BSS-465

BSS-505

BSS- 545

Interval (inches)
$0 \quad-6$
$0-6$
$0-6$
$0-6$
$0-7$
$0-10$
$0-6$
$0-6$

Modifier

Result

4849

4175

9080

1871.2

3607

8034.6

4313.2

4418.3

$$
6-25
$$

29930

12460

50100

17030

22470

25894

21426

13022

$25-34$
$16-34$
$28-48$
$20-48$
$22-50$
$20-34$
$24-40$

26300

31000

18667

24690

24804

22268

21989

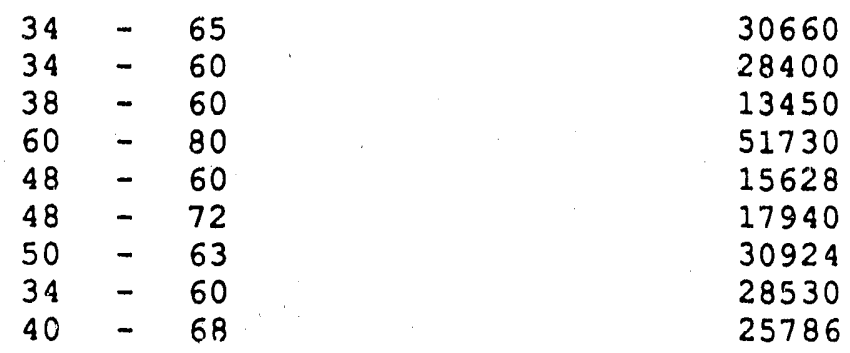

$89-103$
$90-120$
$96-120$
$108-132$
$96-120$
$96-120$
$96-120$
$96-120$

53530

9520

26850

7651.4

16340

18775.2

17117

19520 
Arsenic

Unit: microgram/gram

Eirst Layer

BSS-041

BSS-101

BSS-111

BSS -341

BSS- 401

BSS -461

BSS -501

BSS -541

Second Layer

BSS-042A

BSS-102A

BSS-112A

BSS-342

BSS-402

BSS- 462

BSS-502

BSS- 542

Third Layer

BSS-043A

BSS-103A

BSS-343

BSS-403

BSS -463

BSS-503

BSS- 543

Fourth Layer

BSS-044A

BSS-104A

BSS-113A

BSS - $114 \mathrm{~A}$

BSS-344

BSS-404

BSS-464

BSS-504

BSS-544

Fifth Layer

BSS-045A

BSS-105A

BSS-115A

BSS- 345

BSS -405

BSS -465

BSS -505

BSS- 545

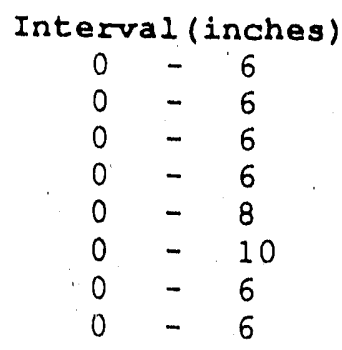

$\underset{\text { LT }}{\operatorname{Modifier}}$
LT
LT
LT
LT

Result

0.5

1.87

1.93

2

1. 32

2

2
2

$6-25$
$6-16$
$6-38$
$6-28$
$8-20$
$10-22$
$6-20$
$6-24$

3. 42

2.19

4.87

LT

2

7.93

LT

IT

3.2

$25-34$
$16-34$
$28-48$
$20-48$
$22-50$
$20-34$
$24-40$

4. 1

LT

LT

LT

LT

3
2
5
2
2
2

5.78

2. 96

3.28

6.84

LT

80
$-\quad 60$

- 72

- 63

- 60

- 68

$\mathrm{LT}$

LT

LT

10.8

2

2

7.01

2.17

8. 42

- 120

- 132

- 120

- 120

- 120

$\begin{array}{r}-120 \\ \hline\end{array}$

$\begin{array}{lr}\text { LT } & 2 \\ \text { LT } & 4 \\ \text { LT } & 2 \\ & 2.8 \\ \text { LT } & 2\end{array}$




\section{Barium}

Unit: microgram/gram

First Layer
BSS-041
$B S S-101$
$B S S-111$
$B S S-341$
$B S S-401$
$B S S-461$
$B S S-501$
$B S S-541$

Interval (inches)
$0-6$
$0-6$
$0-6$
$0-6$
$0-8$
$0-10$
$0-6$
$0-6$

Modifier

Result

12.85

9.2

17.68

28.4

12.87

32

25.8

17.1

Second Layer

BSS-042A

BSS-102A

BSS $-112 A$

BSS -342

BSS -402

BSS -462

BSS -502

BSS -542

$6-25$
$6-16$
$6-38$
$6-28$
$8-20$
$10-22$
$6-20$
$6-24$

61.3

18.4

48.84

56.6

65.13

77.4

71.6

47.1

Third Layer

BSS-043A

$25-34$

53.4

BSS-103A

$16-34$

15.4

BSS -343

BSS -403

$28-48$

$20-48$

BSS -463

BSS -503

BSS -543

- 50

- 34

$24-40$

36.99

44.4

52.8

36.6

Eourth Layer

BSS-044A

BSS-104A

BSS-113A

BSS-114A

BSS -344

BSS-404

BSS -464

BSS-504

BSS-544

$34-65$

36.9

10.5

7.308

$38-60$

22.74

14.2

21.25

28.4

31.6

24.2

Fifth Layer

BSS - 045A

BSS-105A

BSS-115A

BSS -345

BSS-405

BSS -465

BSS -505

BSS -545

$89-103$
$90-120$
$96-120$
$108-13 ?$
$96-120$
$96-120$
$96-120$
$96-120$

28.6

34.2

1.37

13.32

12.8

18.2

8.2 


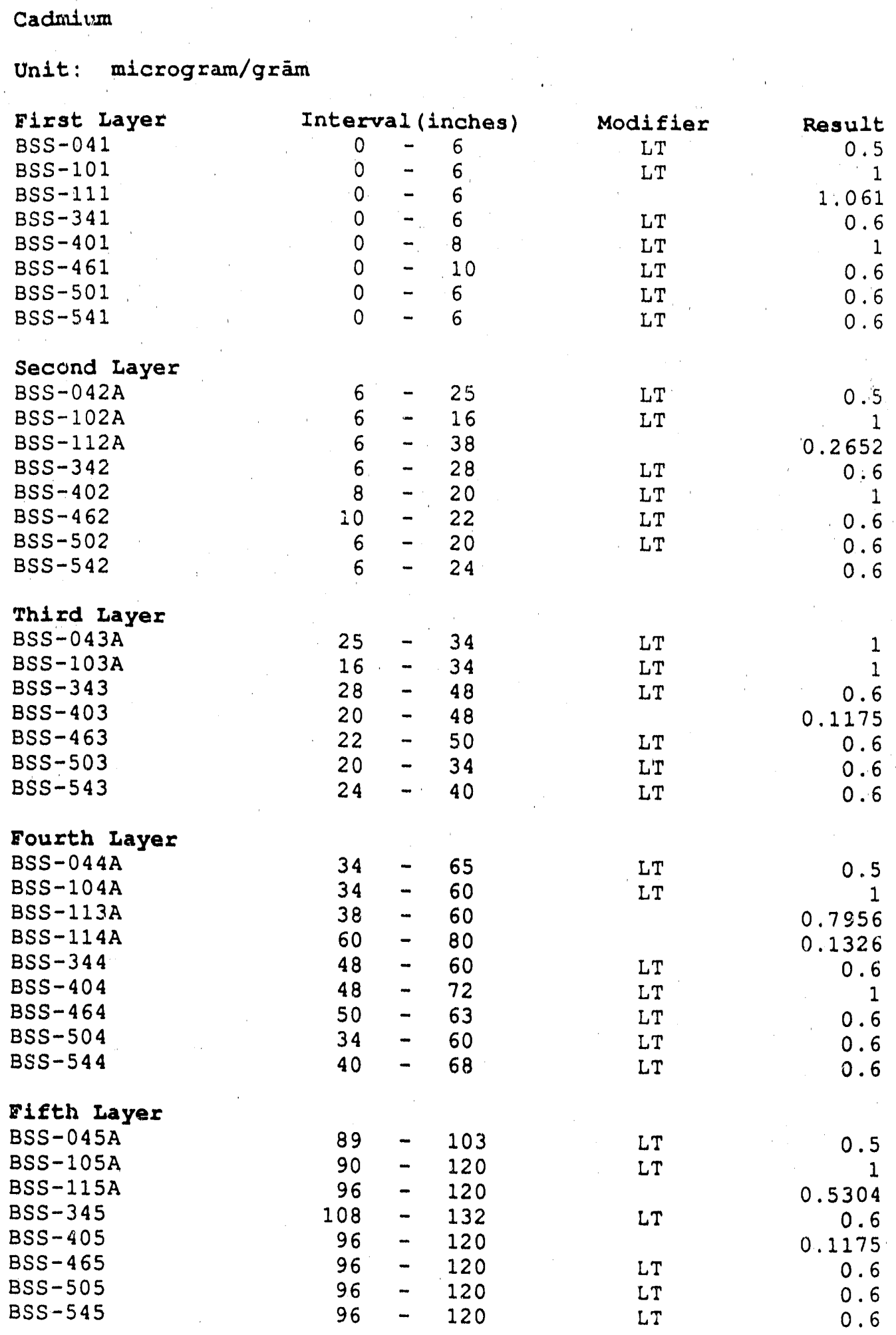




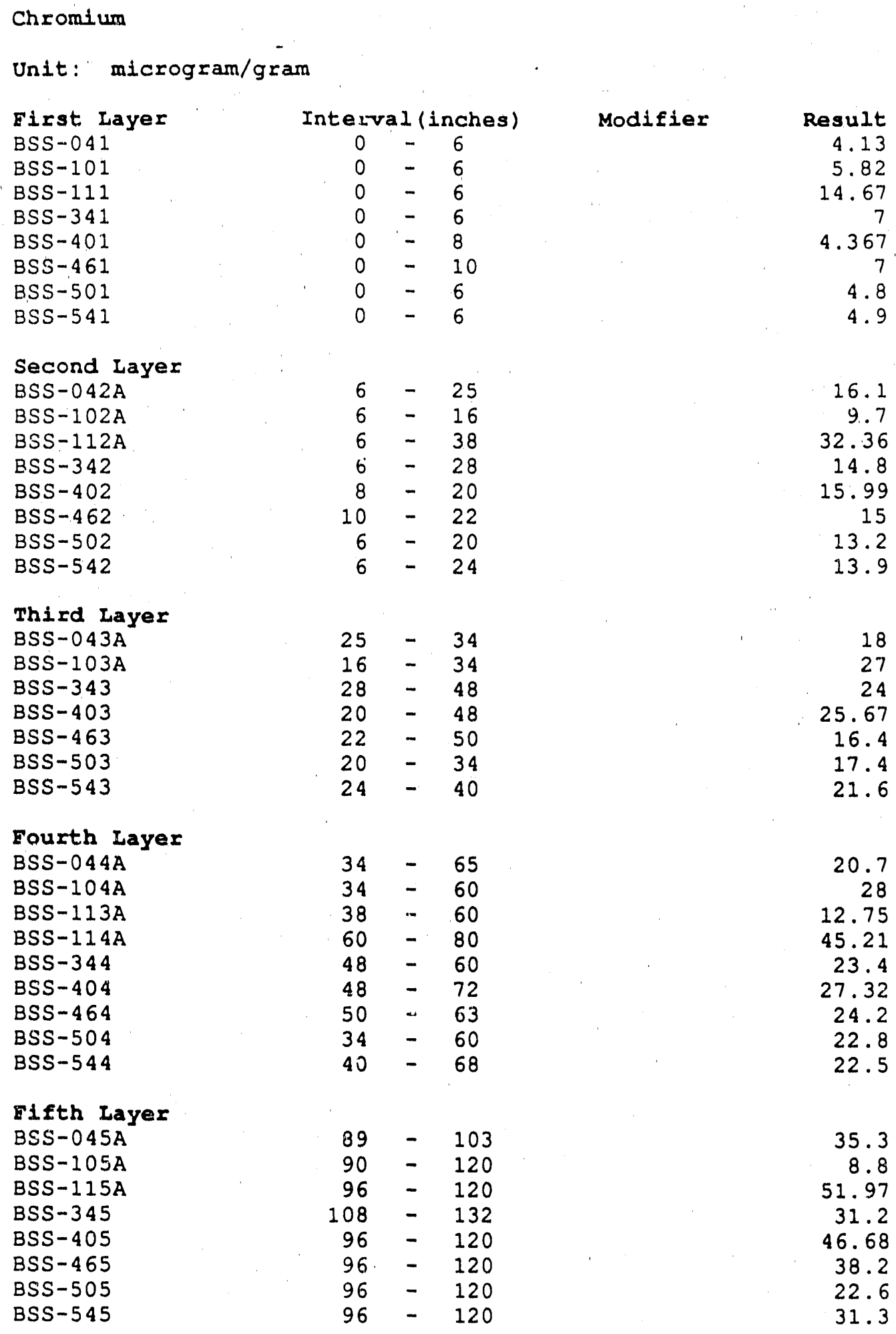




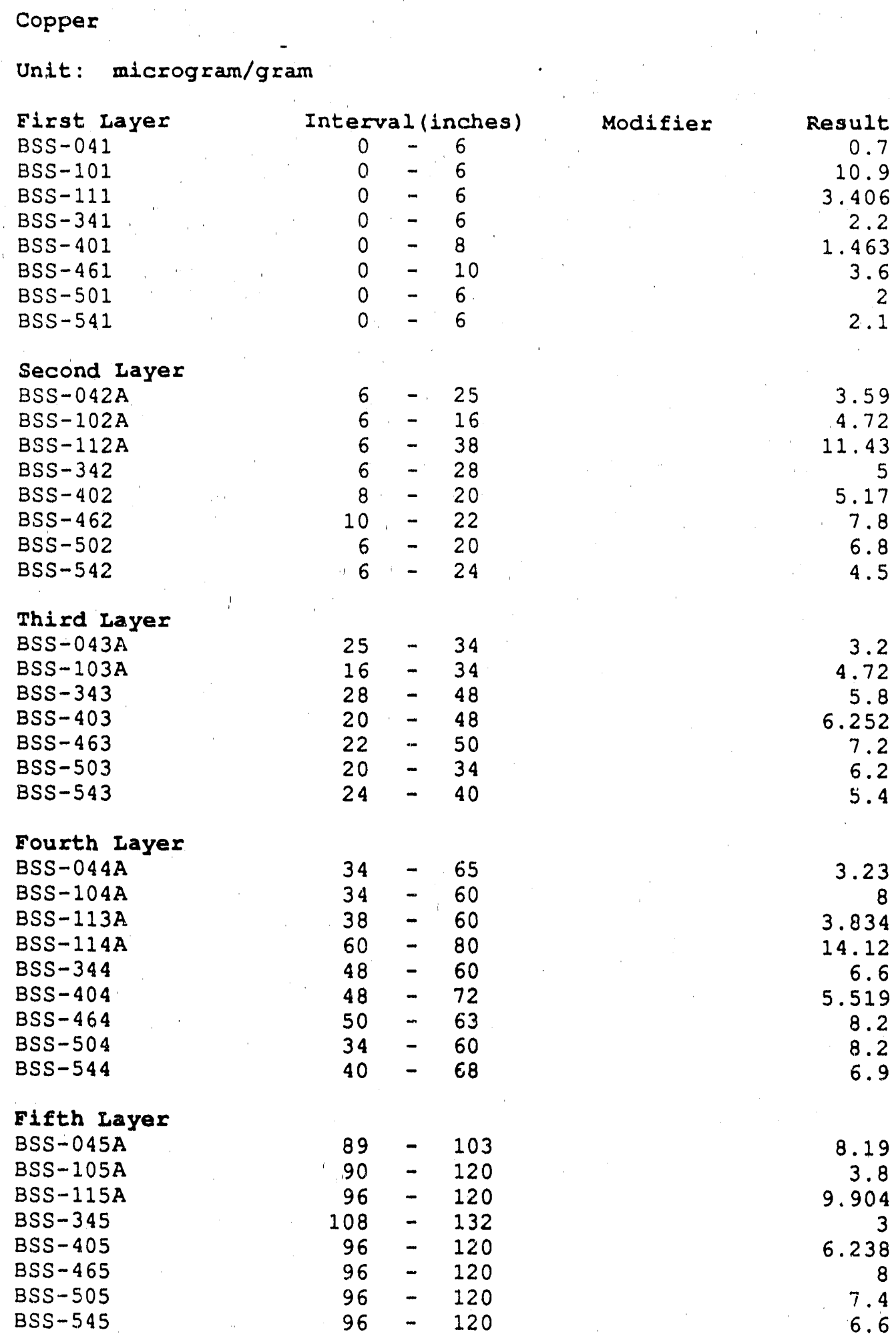


Iron

Unit: microgram/gräm

First Layer

BSS-041

BSS-101

BSS-111

BSS-341

BSS- 401

BSS-461

BSS-50I

BSS-541

\section{Second Layer}

BSS-042A

BSS-102A

BSS-112A

BSS- 342

BSS-402

BSS -462

BSS -502

BSS- 542

Third Layer

BSS-043A

BSS-103A

BSS -343

BSS- 403

BSS -463

BSS-503

BSS- 543

Eourth Layer

BSS-044A

BSS-104A

BSS-113A

BSS-114A

BSS-344

BSS -404

BSS -464

BSS-504

BSS-544

\section{Fifth Iayer}

BSS-045A

BSS-105A

BSS-115A

BSS -345

BSS -405

BSS -465

BSS-505

BSS -545

Interval (inches)
$0-6$
$0-6$
$0-6$
$0-6$
$0-8$
$0-10$
$0-6$
$0-6$

Modifier

Result

4540

3230

8526

3576.4

4986

3208.8

2621.4

2067.1

$$
\begin{array}{r}
6-25 \\
6-16 \\
6-38 \\
6-28 \\
8-20 \\
10-22 \\
6-20 \\
6-24
\end{array}
$$

24600

8660

27300

11990

11660

9764

11959

7003.1

$$
\begin{aligned}
& 25-34 \\
& 16-34 \\
& 28-48 \\
& 20-48 \\
& 22-50 \\
& 20-34 \\
& 24-40
\end{aligned}
$$

12660

28600

18579

14990

11395.8

16749

15525

$34-65$
$34-60$
$38-60$
$60-60$
$48-60$
$48-72$
$50-63$
$34-60$
$40-68$

34400

35800

7502

27510

19131

20090

16699.4

22028

16981

62600

15300

30170

15642

25010

39468

20972

27941 
Lead

Unit: microgram/gram

Eirst Layer
BSS-041
BSS-101
BSS-111
BSS-341
BSS-401
$B S S-461$
$B S S-501$
$B S S-541$

Second Layer

BSS-042A

BSS - 102A

BSS-112A

BSS-342

BSS -402

BSS -462

BSS-502

BSS -542

Third Layer

BSS-043A

BSS-103A

BSS -343

BSS -403

BSS -463

BSS-503

BS3-543

Eourth Iayer

BSS-044A

BSS-104A

BSS-113A

BSS-114A

BSS-344

BSS-404

BSS -464

BSS-504

BSS - 544

\section{Fifth Layer}

BSS-045A

BSS-105A

BSS-115A

BSS- 315

BSS-405

BSS -465

BSS-505

BSS- 545

Interval (inches)
$0-6$
$0-6$
$0-6$
$0-6$
$0-8$
$0-10$
$0-6$
$0-6$

Modifier

Result

3.96

5.15

9.04

6.4

6.72

5.6

4. $\frac{3}{6}$

11.4

6.52

15.4

7.9

14.5

7.3

5. 4

9.2

11.6

9.53

7.8

7.66

4.1

6.1

13.1

6.6

4.89

12.7

6.3

9.76

11.9

6.9

$40-68$

$89-103$

14

4.45

14.6

12.3

9.5

2. 8

5.6 


\begin{tabular}{|c|c|c|c|c|}
\hline \multicolumn{5}{|l|}{ Bum } \\
\hline i: microgram/gram & & . & & \multirow[b]{2}{*}{ Result } \\
\hline .rst Layer & \multicolumn{2}{|c|}{ Interval (inches) } & Modifier & \\
\hline $3 S S-041$ & 0 & -6 & $L T$ & 10 \\
\hline BSS-10\}. & 0 & -6 & LT & 10 \\
\hline BSS-111 & 0 & -6 & LT & 10 \\
\hline BSS -341 & 0 & -6 & LT & 10 \\
\hline BSS. 401 & 0 & -8 & LT & 10 \\
\hline BSS- 461 & 0 & -10 & $\mathrm{LT}$ & 10 \\
\hline BSS- 501 & 0 & -6 & LT & 10 \\
\hline BSS-541 & 0 & -6 & LT & 10 \\
\hline \multicolumn{5}{|l|}{$\begin{array}{l}\text { Second Layer } \\
\text { BSS-042A }\end{array}$} \\
\hline BSS-042A & 6 & $-\quad 25$ & & 10.82 \\
\hline BSS-102A & 6 & $-\quad 16$ & LT & 10 \\
\hline BSS-112A & 6 & -38 & & 19.87 \\
\hline BSS - 342 & 6 & $-\quad 28$ & LT & 10 \\
\hline BSS -402 & 8 & -20 & $\mathrm{LT}$ & 10 \\
\hline BSS-462 & 10 & $-\quad 22$ & LT & 10 \\
\hline BSS-502 & 6 & -20 & LT & 10 \\
\hline BSS- 542 & 6 & -24 & LT & 10 \\
\hline \multicolumn{5}{|l|}{$\begin{array}{l}\text { Third Layer } \\
\text { BSS-043A }\end{array}$} \\
\hline BSS $-043 A$ & 25 & $-\quad 34$ & LT & 10 \\
\hline BSS-103A & 16 & $-\quad 34$ & LT & 10 \\
\hline $\begin{array}{l}\text { BSS }-343 \\
B S S-403\end{array}$ & 28 & $-\quad 48$ & LT & 10 \\
\hline BSS -403 & 20 & -48 & LT & 10 \\
\hline BSS -463 & 22 & -50 & LT & 10 \\
\hline BSS -503 & 20 & $-\quad 34$ & LT & 10 \\
\hline BSS-543 & 24 & -40 & LT & 10 \\
\hline \multicolumn{5}{|l|}{$\begin{array}{l}\text { Eourth Layer } \\
\text { BSS }-044 \mathrm{~A}\end{array}$} \\
\hline BSS-044A & 34 & & & 14.85 \\
\hline BSS-104A & 34 & -60 & LT & 10 \\
\hline BSS-113A & 38 & -60 & LT & 10 \\
\hline BSS-114A & 60 & -80 & & 17.07 \\
\hline BSS-344 & 48 & -60 & LT & 10 \\
\hline BSS-404 & 48 & -72 & LT & 10 \\
\hline BSS -464 & 50 & -63 & $\mathrm{LT}$ & 10 \\
\hline BSS-504 & 34 & -60 & $\mathrm{LT}$ & 10 \\
\hline BSS-5么4 & 40 & -68 & $\mathrm{LT}$ & 10 \\
\hline \multicolumn{5}{|l|}{$\begin{array}{l}\text { Fifth Layer } \\
\text { BSS }-045 \mathrm{~A}\end{array}$} \\
\hline BSS-045A & 89 & -103 & $\mathrm{LT}$ & 10 \\
\hline BSS-105A & 90 & -120 & $\mathrm{LT}$ & 10 \\
\hline$B S S-115 A$ & 96 & -120 & $\mathrm{LT}$ & 10 \\
\hline BSS-345 & 108 & $-\quad 132$ & LT & 10 \\
\hline BSS- 405 & 96 & $-\quad 120$ & $\mathrm{LT}$ & 10 \\
\hline BSS -465 & 96 & -120 & LT & 10 \\
\hline $\begin{array}{l}\text { BSS }-505 \\
\text { BSS }-545\end{array}$ & 96 & $-\quad 120$ & LT & 10 \\
\hline & 96 & $-\quad 120$ & LT & 10 \\
\hline
\end{tabular}




\section{Magnesium}

Unit: microgram/gram

First Layer

BSS-041

BSS- 101

BSS-111

BSS-341

BSS-401

BSS-461

BSS-501

BSS-541

\section{Second Layex}

BSS-042A

BSS-102A

BSS-112A

BSS-342.

BSS -402

BSS -462

BSS -502

BSS-542

Third Layer

BSS-043A

BSS-103A

BSS -343

BSS -403

BSS -463

BSS -503

BSS -543

Eourth Layer

BSS-044A

BSS-104A

BSS-113A

BSS-114A

BSS-344

BSS-404

BSS-464

BSS-504

BSS-544

Eifth Layex

BSS-045A

BSS-105A

BSS-115A

BSS -345

BSS -405

BSS -465

BSS-505

BSS-545

$\begin{array}{ccr}\text { Interval (inches) } & \text { Modifier } & \text { Result } \\ 0 & -6 & 124.4 \\ 0 & -6 & 74.98 \\ 0 & -6 & 171.5 \\ 0 & -6 & 81.89 \\ 0 & -8 & 110.5 \\ 0 & -10 & 97.18 \\ 0 & -6 & 48.42 \\ 0 & -6 & 61.28\end{array}$

591.2

115.5

759.4

134.7

456.2

375.3

181.2

163.4

$25-34$
$16-34$
$28-48$
$20-48$
$22-50$
$20-34$
$24-40$

398

135.6

214.5

402.8

205.3

123

111.6

519.1

212.8

120.5

445.5

162.6

236.8

459.7

136.8

122.9

241.7

55.47

208.5

52.22

281.4

133.5

87.29

94.62 


\section{Manganese}

Unit : microgram/gram

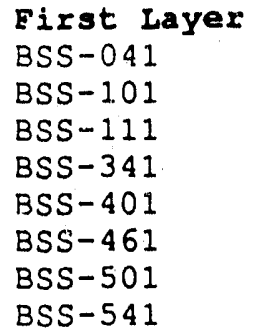

Interval (inches)
$0-6$
$0-6$
$0-6$
$0-6$
$0-8$
$0-10$
$0-6$
$0-6$

Modifier

$$
\begin{array}{r}
6-25 \\
6-16 \\
6-38 \\
6-28 \\
8-20 \\
10-22 \\
6-20 \\
6-24
\end{array}
$$

50.67

13.71

35.47

181.2

78.86

155.7

241.2

125.9

$25-34$
$16-34$
$28-48$
$20-48$
$22-50$
$20-34$
$24-40$

LT

24.78

1.6

23.06

48.92

34.47

72.46

10.88

$$
\begin{aligned}
& 34-65 \\
& 34-60 \\
& 38-60 \\
& 60-60 \\
& 48-60 \\
& 48-72 \\
& 50-63 \\
& 34-60 \\
& 40-68
\end{aligned}
$$

24.93

6.051

7.583

31.79

17.31

32.93

31.56

41.9

7.503

$$
\begin{array}{r}
89-103 \\
90-120 \\
96-120 \\
108-132 \\
96-120 \\
96-120 \\
96-120 \\
96-120
\end{array}
$$

38.72

$$
3.6
$$

34.09

LT

1.6

32.67

LT 35.47

3.6 


\begin{tabular}{|c|c|c|c|c|}
\hline \multicolumn{5}{|l|}{ Mercury } \\
\hline \multicolumn{5}{|c|}{ Unit: microgram/gram } \\
\hline First Layer & \multicolumn{2}{|c|}{ Interval (inches) } & Modifier & \multirow{2}{*}{ Result } \\
\hline BSS-041 & 0 & -6 & $\mathrm{LT}$ & \\
\hline BSS-101 & 0 & -6 & LT & 0.1 \\
\hline BSS-111 & 0 & 6 & $\mathrm{LT}$ & 0.1 \\
\hline BSS -341 & 0 & -6 & LT & 0.1 \\
\hline BSS -401 & 0 & 8 & LT & 0.1 \\
\hline$B S S-461$ & 0 & -10 & $\mathrm{LT}$ & 0.1 \\
\hline BSS-501 & 0 & -6 & $L T$ & 0.1 \\
\hline$B S S-541$ & 0 & -6 & LT & 0.1 \\
\hline \multicolumn{5}{|l|}{ Second Layer } \\
\hline$B S S-042 A$ & 6 & 25 & LT & 0.1 \\
\hline$B S S-102 A$ & 6 & -16 & LT & 0.1 \\
\hline$B S S-112 A$ & 6 & $-\quad 38$ & LT & 0.1 \\
\hline$B S S-342$ & 6 & $-\quad 28$ & $\mathrm{LT}$ & 0.1 \\
\hline BSS -402 & 8 & -20 & LT & 0.1 \\
\hline BSS -462 & 10 & $-\quad 22$ & LT & 0.1 \\
\hline BSS -502 & 6 & 20 & $\mathrm{I}, \mathrm{T}$ & 0.1 \\
\hline BSS -542 & 6 & -24 & $\mathrm{LT}$ & 0.1 \\
\hline \multicolumn{5}{|l|}{ Third Layer } \\
\hline$B S S-043 A$ & 25 & 34 & LT & 0.1 \\
\hline BSS-103A & 16 & -34 & & 0.11 \\
\hline BSS-343 & 28 & -48 & & 0.11 \\
\hline BSS -403 & 20 & $-\quad 48$ & LT & 0.1 \\
\hline BSS -463 & 22 & -50 & LT & 0.1 \\
\hline BSS -503 & 20 . & -34 & LT & 0.1 \\
\hline BSS-543 & 24 & -40 & LT & 0.1 \\
\hline \multicolumn{5}{|l|}{ Fourth Layer } \\
\hline BSS-044A & 34 & -65 & LT & 0.1 \\
\hline BSS-104A & 34 & -60 & & 0.19 \\
\hline BSS-113A & 38 & -60 & LT & 0.1 \\
\hline$B S S-114 A$ & 60 & -80 & LT & 0.01 \\
\hline BSS -344 & 48 & -60 & LT & 0.1 \\
\hline BSS $=404$ & 48 & $-\quad 72$ & LT & 0.1 \\
\hline BSS- 464 & 50 & -63 & $\mathrm{LT}$ & 0.1 \\
\hline BSS-504 & 34 & 60 & LT & 0.1 \\
\hline BSS - 544 & 40 & 68 & & 0.17 \\
\hline \multicolumn{5}{|l|}{ Fifth Layer } \\
\hline BSS-045A & 89 & -103 & LT & 0.1 \\
\hline BSS-105A & 90 & $-\quad 120$ & & 0.17 \\
\hline BSS-115A & 96 & -120 & LT & 0.1 \\
\hline BSS-345 & 108 & $-\quad 132$ & LT & 0.1 \\
\hline BSS-405 & 96 & $-\quad 120$ & LT & 0.1 \\
\hline BSS - 465 & 96 & $-\quad 120$ & LT & 0.1 \\
\hline BSS-505 & 96 & $-\quad 120$ & LT & 0.1 \\
\hline BSS -545 & 96 & $-\quad 120$ & LT & 0.1 \\
\hline
\end{tabular}




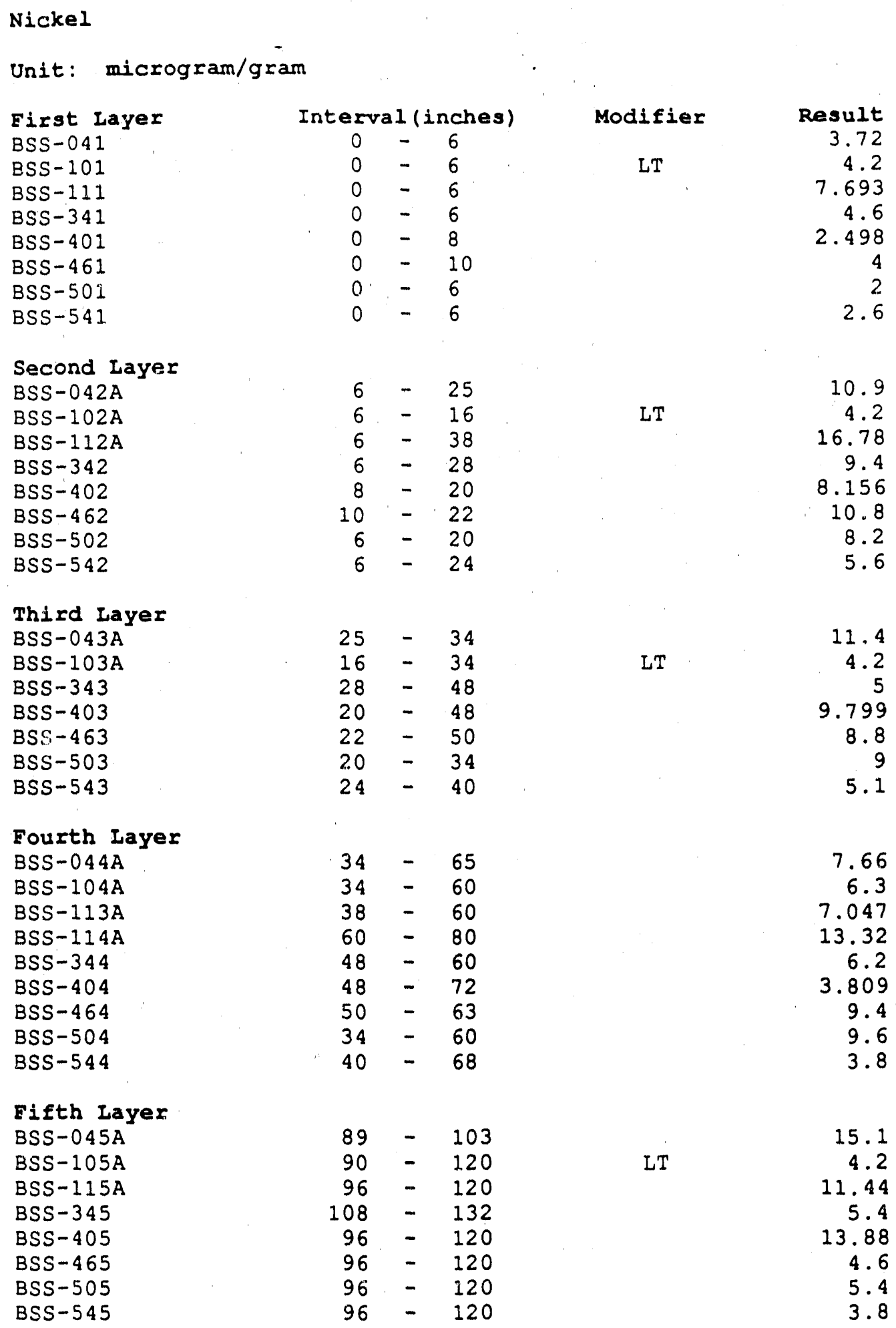




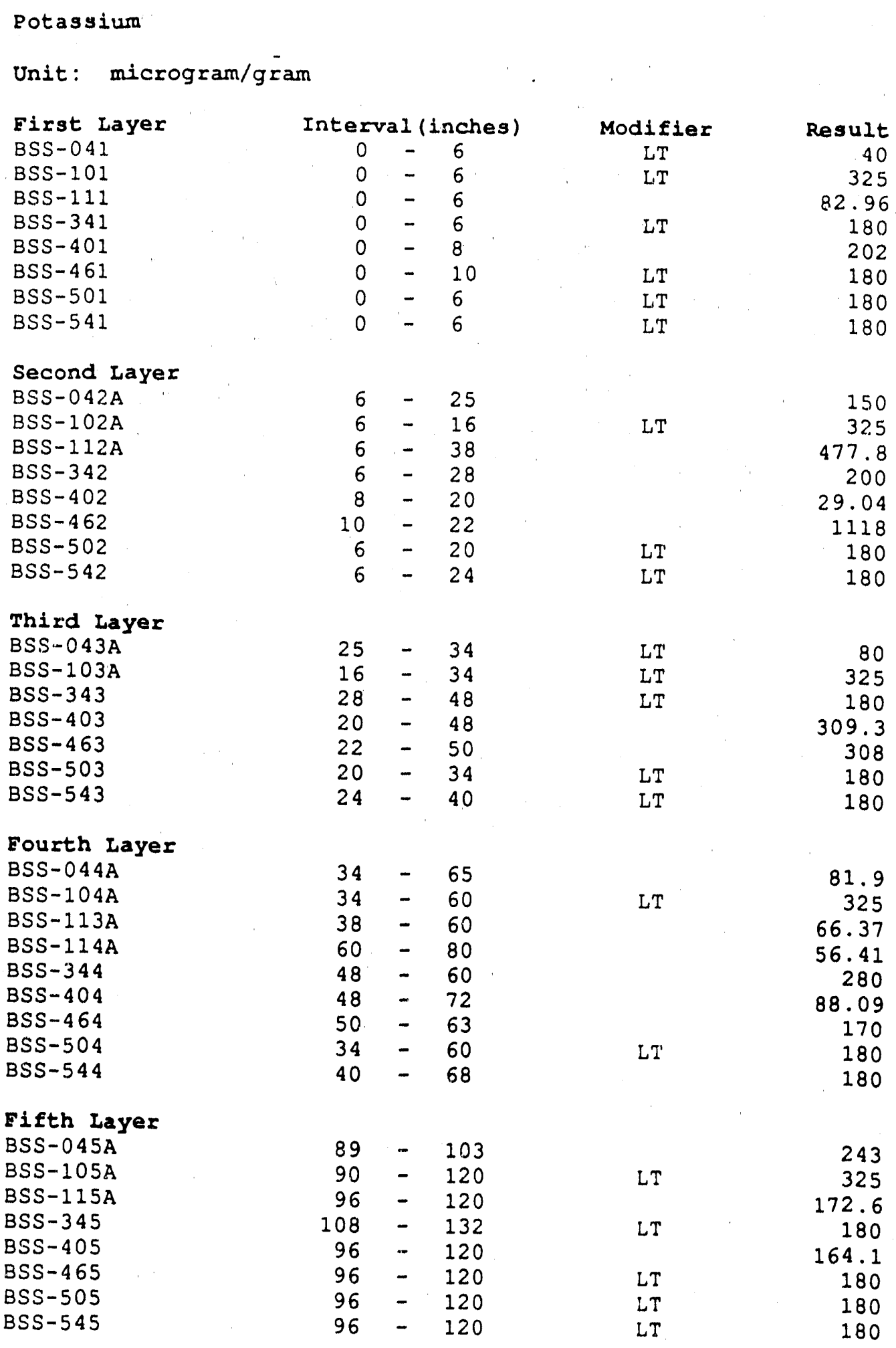




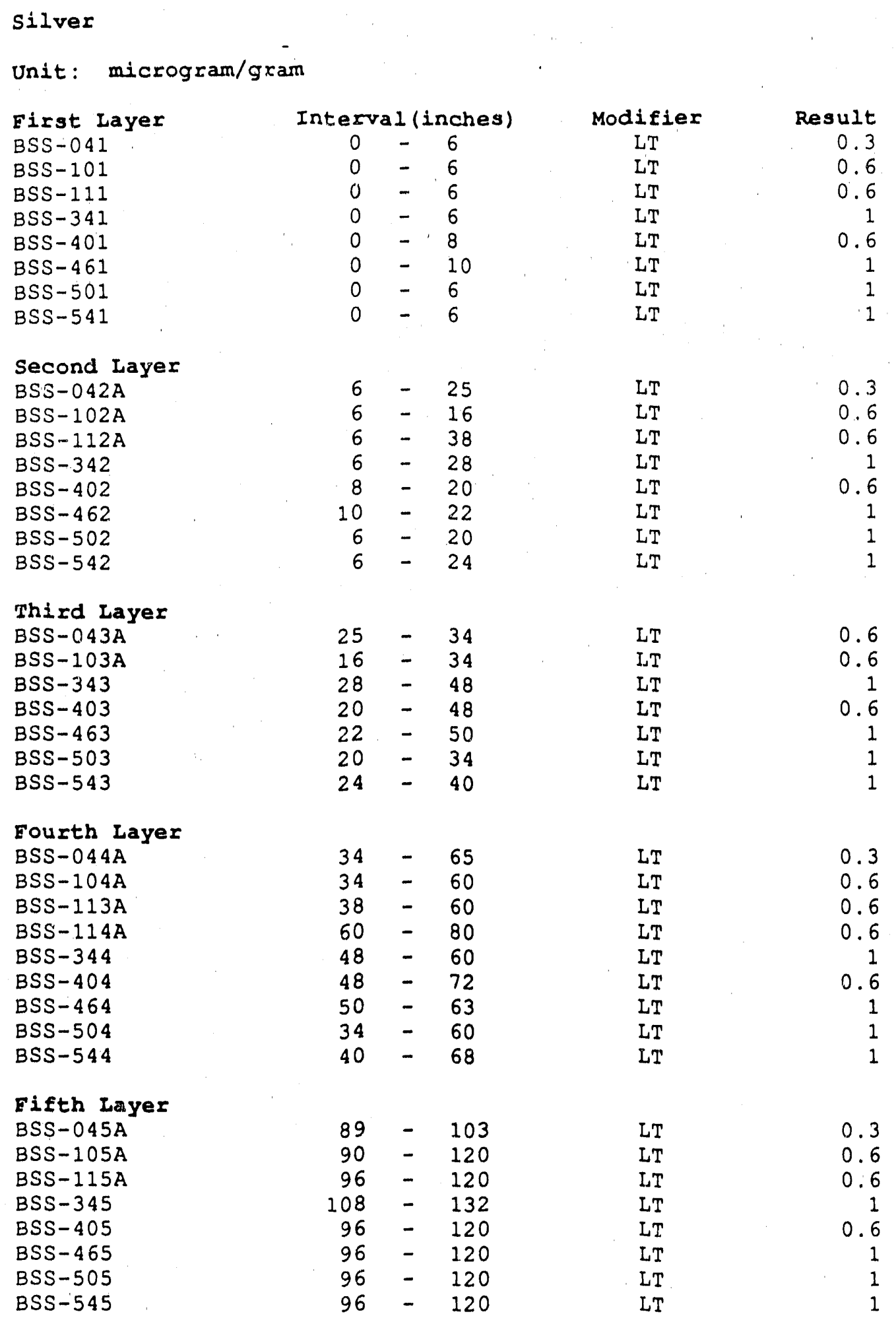




\section{Selenium}

Unit: microgram/gràm

Eirst Layer

BSS-041

BSS-101

BSS-111

BSS -341

BSS-401

BSS -461

BSS-501

BSS -541

\section{Second Layer}

BSS-042A

BSS-102A

BSS-112A

BSS -342

BSS -402

BSS -462

BSS -502

BSS -542

\section{Third Layer}

BSS-043A

BSS - 103A

BSS -343

BSS -403

BSS -463

BSS- 503

BSS -543

\section{Fourth Layer}

BSS-044A

BSS-104A

BSS-113A

BSS-114A

BSS-344

BSS -404

BSS -464

BSS -504

BSS- 544

Fifth Layer

BSS-045A

BSS-105A

BSS-115A

BSS -345

BSS -405

BSS -465

BSS -505

BSS -545

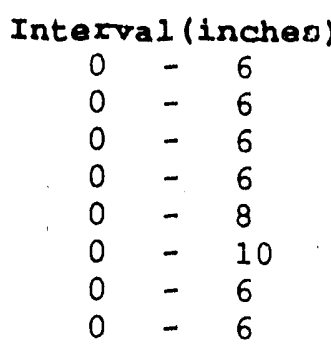

Modifier
LT
LT
LT
LT
LT
LT
LT
LT

Result

0.2

0.4

0.4

0.4

0.4

0.4

0.4

0.4

$$
\begin{array}{r}
6-25 \\
6-16 \\
6-38 \\
6-28 \\
8-20 \\
10-22 \\
6-20 \\
6-24
\end{array}
$$

LT

0.37

0.4

0.92

0.55

LT

0.4

0.4

0.4

LT

LT

0.4

$$
\begin{aligned}
& 25-34 \\
& 16-34 \\
& 28-48 \\
& 20-48 \\
& 22-50 \\
& 20-34 \\
& 24-40
\end{aligned}
$$

LT

LT

LT

LT

0.31

0.42

0.43

0.4

0.4

0.4

0.4

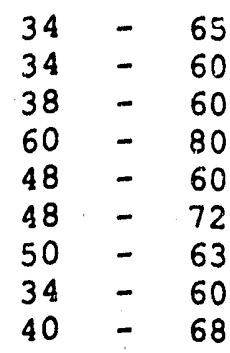

0.99

LT

0.49

LT

1

0.49

LT

0.4

0.47

LT

LT

0.4

$89-103$
$90-120$
$96-120$
$108-132$
$96-120$
$96-120$
$96-120$
$96-120$

LT

0.84

LT

0.4

0.4

0.52

LT

0.4

0.72

LT

0.55 
Sodium

Unit: microgram/gram

Eirst Layer

BSS-0 41

BSS- 101

BSS-111

BSS-341

BSS-401

BSS-461

BSS-501

BSS -541

Second Layer

BSS-042A

BSS-102A

BSS-112A

BSS -342

BSS- 402

BSS -462

BSS -502

BSS -542

Thixd Layer

BSS-043A

BSS-103A

BSS -343

BSS -403

BSS -463

BSS-503

BSS -543

Fourth Layer

BSS-044A

BSS - 104A

BSS-113A

BSS-114A

BSS -344

BSS -404

BSS-464

BSS -504

BSS-544

Fifth Layer

BSS-045A

BSS-105A

BSS-115A

BSS -345

BSS -405

BSS-465

BSS -505

BSS-545

Interval (inches)
$0-6$
$0-6$
$0-6$
$0-6$
$0-8$
$0-10$
$0-6$
$0-6$

Sodifier

Result

18.3

288

65.34

42

52.03

65.2

44.4

31.4

33.9

210

78.75

123.6

52.82

93

79.4

42.6

20
$-\quad 24$

41

760

86.8

72.97

84.4

66.8

49.1

- 34

\begin{tabular}{l}
$-\quad 40$ \\
\hline
\end{tabular}

25.9

321

70.64

88.11

90.2

351

82.2

60.4

44.3

68

39.5

236

73.76

47.6

55.68

75.2

78

44.3 
zinc

Unit: microgram/gram

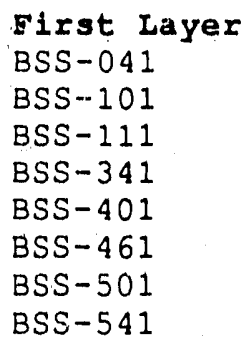

\section{Second Layer}

BSS-042A

BSS-102A

BSS-112A

BSS -342

BSS -402

BSS- 462

BSS-502

BSS-542

Third Layer

BSS-043A

BSS - $103 A$

BSS -343

BSS -403

BSS -463

BSS -503

BSS-543

Fourth Layer

BSS-044A

BSS-104A

BSS-113A

BSS-114A

BSS- 344

BSS-404

BSS- 464

BSS- 504

BSS -544

\section{Fifth Layer}

BSS-045A

BSS-105A

BSS-115A

BSS-345

BSS -405

BSS -465

BSS- 505

BSS -545

Interval (inches)
$0-6$
$0-6$
$0-6$
$0-6$
$0-8$
$0-10$
$0-6$
$0-6$

Modifier

Result

11.84

24.6

8.729

10.8

6.306

7.8

6.6

5.2

21.3

16.8

28.46

22

16.75

- 28

- 20

- 22

- 20

- 24

13.4

12.3

18.4

14.2

- 34

-48
$-\quad 48$

- 48

-58
$-\quad 50$

-. 34

- 40

15.32

15.8

12.2

13.4

15.6

18

23.94

33.42

11.63

14

12.8

9.8

28.6

11.2

22.37

7.4

14.16

7

11. 2

9.8 
Aluminum

Unit: microgram/gram

\begin{tabular}{|c|c|c|c|c|}
\hline rirst Layer & Interv & 1 (inches) & Modifier & Result \\
\hline$B S S-131$ & 0 & -12 & & 43320 \\
\hline BSS-132 & 12 & 24 & & 5934 \\
\hline BSS-201A & 0 & $-\quad 24$ & & 21260 \\
\hline BSS-301 & 0 & $-\quad 24$ & & 16290 \\
\hline BSS-311 & 0 & -24 & & 7032 \\
\hline BSS-361 & 0 & $-\quad 24$ & & 14167 \\
\hline BSS - 531 & 0 & $-\quad 24$ & & 6073 \\
\hline BSS -551 & 0 & $-\quad 24$ & & 4551.6 \\
\hline BSS-581 & 0 & -24 & & 3391.3 \\
\hline Second Layer & & & & \\
\hline$B S S-133$ & 24 & 36 & $\therefore$ & 25540 \\
\hline$B S S-202 A$ & 24 & -48 & . & 19080 \\
\hline $\mathrm{BSS}-302$ & 24 & 48 & 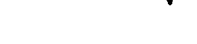 & 13670 \\
\hline$B S S-312$ & 24 & -48 & & 7384 \\
\hline BSS- 362 & 24 & 48 & & 18279 \\
\hline BSS -532 & 24 & 48 & & 5730 \\
\hline$B S S-552$ & 24 & -48 & & 3015.7 \\
\hline BSS -582 & 24. & -48 & & 3756.4 \\
\hline Third Layer & & & & \\
\hline$B S S-203 A$ & 48 & -72 & & 4809 \\
\hline BSS -303 & 48 & -72 & & 16080 \\
\hline BSS -313 & 48 & -72 & & 11070 \\
\hline BSS -363 & 48 & -72 & & 11869 \\
\hline BSS-533 & 48 & $-\quad 72$ & & 8795.4 \\
\hline BSS-553 & 48 & $-\quad 72$ & & 2899.3 \\
\hline BSS-583 & 48 & -72 & & 3843.5 \\
\hline
\end{tabular}




\begin{tabular}{|c|c|c|c|c|}
\hline \multicolumn{5}{|l|}{ Arsenic } \\
\hline \multicolumn{5}{|c|}{ Unit: microgran/gram } \\
\hline First Layer & \multicolumn{2}{|c|}{ Interval (inches) } & \multirow[t]{2}{*}{ Modifier } & \multirow{2}{*}{$\begin{array}{r}\text { Result } \\
4.47\end{array}$} \\
\hline BSS -131 & 0 & -12 & & \\
\hline BSS -132 & 12 & $-\quad 24$ & & 5.44 \\
\hline$B S S-201 A$ & 0 & -24 & & 4.45 \\
\hline BSS-301 & 0 & -24 & & 2.64 \\
\hline BSS-311 & 0 & -24 & & 1.95 \\
\hline BSS -361 & 0 & -24 & LT & 2 \\
\hline BSS -531 & 0 & -24 & $\mathrm{LT}$ & 2 \\
\hline BSS -551 & 0 & -24 & $\mathrm{LT}$ & 2 \\
\hline$B S S-581$ & 0 & -24 & & 3.6 \\
\hline \multicolumn{5}{|l|}{ Second Layer } \\
\hline BSS-133 & 24 & -36 & & 3.79 \\
\hline$B S S-202 A$ & 24 & -48 & & 4.61 \\
\hline BSS - 302 & 24 & -48 & & 3.64 \\
\hline$B S S-312$ & 24 & -48 & & 5.93 \\
\hline BSS -362 & 24 & -48 & LT & 2 \\
\hline BSS -532 & 24 & $-\quad 48$ & LT & 2 \\
\hline BSS -552 & 24 & -48 & LT & 2 \\
\hline BSS -582 & 24 & -48 & LT & 2 \\
\hline \multicolumn{5}{|l|}{ Third Layer } \\
\hline$B S S-203 A$ & 48 & & & 4.41 \\
\hline BSS -303 & 48 & -72 & & 3.75 \\
\hline BSS -313 & 48 & -72 & & 5.99 \\
\hline BSS -363 & 48 & -72 & LT & 2 \\
\hline BSS-533 & 48 & -72 & $\mathrm{LT}$ & 2 \\
\hline BSS -553 & 48 & $-\quad 72$ & $\mathrm{~L}, \mathrm{~T}$ & 2 \\
\hline BSS -583 & 48 & $-\quad 72$ & $\mathrm{LT}$ & 2 \\
\hline
\end{tabular}




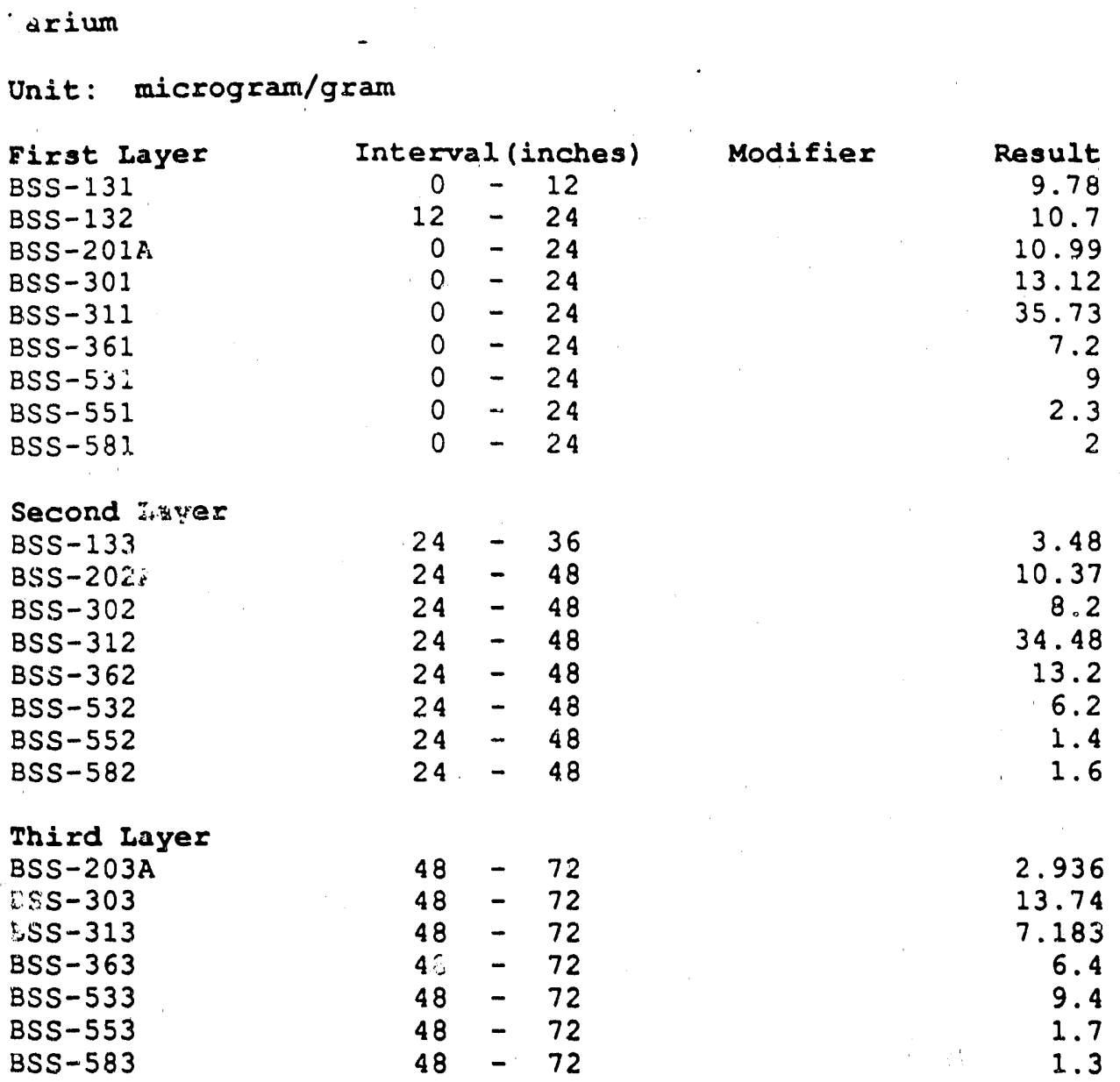




\section{Cadmium}

Unit: microgram/gram

Eirst Layer
BSS-131
BSS -132
BSS-201A
BSS -301
BSS-311
BSS -361
BSS -531
BSS -551
BSS -581

$\begin{array}{cc}\text { Interval (inches) } & \text { Modifier } \\ 0-12 & \text { LT } \\ 12-24 & \text { LT } \\ 0-24 & \\ 0-24 & \\ 0-24 & \\ 0-24 & \text { LT } \\ 0-24 & \text { LT } \\ 0-24 & \text { LT } \\ 0-24 & \text { LT }\end{array}$

Result

0.5

0.5304

0.3978

1.193

0.6

0.6

0.6

Second Layer

BSS-133

BSS-202A

BSS -302

BSS -312

BSS-362

BSS -532

BSS -552

BSS-582

Third Layer

BSS-203A

BSS -303

BSS-313

BSS -363

BSS -533

BSS -553

BSS -583

$24-36$
$24-48$
$24-48$
$24-48$
$24-48$
$24-48$
$24-48$
$24-48$

$48-72$
$48-72$
$48-72$
$48-72$
$48-72$
$48-72$
$48-72$

LT

LT

LT

LT

LT

LT

$\begin{array}{lr} & 0.5967 \\ & 0.663 \\ \text { LT } & 0.5304 \\ \text { LT } & 0.6 \\ \text { LT } & 0.6 \\ \text { LT } & 0.6 \\ & 0.6\end{array}$




\begin{tabular}{|c|c|c|c|c|}
\hline BSS-131 & & 1 (inches) & Modifier & Result \\
\hline & 0 & $-\quad 12$ & & 27.3 \\
\hline BSS-132 & 12 & $-\quad 24$ & & 28 \\
\hline BSS-201A & 0 & -24 & & 27.15 \\
\hline BSS-301 & 0 & $-\quad 24$ & & 26.05 \\
\hline BSS-311 & 0 & -24 & & 10.42 \\
\hline BSS-361 & 0 & -24 & & 26.6 \\
\hline$B S S-531$ & 0 & -24 & & 15.4 \\
\hline BSS -551 & 0 & -24 & & 21.4 \\
\hline BSS- 581 & 0 & $-\quad 24$ & & 10.7 \\
\hline Second Laye & & & & \\
\hline BSS -133 & 24 & -36 & & 24.4 \\
\hline BSS-202A & 24 & -48 & & 23.72 \\
\hline BSS -302 & 24 & $-\quad 48$ & & 15.8 \\
\hline BSS -312 & 24 & -48 & & 31.95 \\
\hline BSS -362 & 24 & -48 & & 21.2 \\
\hline BSS -532 & 24 & -48 & & 19.8 \\
\hline BSS -552 & 24 & -48 & & 7.3 \\
\hline BSS -582 & 24 & -48 & & 9 \\
\hline Third Layer & & & & \\
\hline BSS- $203 \mathrm{~A}$ & 48 & -72 & & 5.485 \\
\hline BSS-303 & 48 & -72 & & 16.73 \\
\hline BSS-313 & 48 & 72. & & 34.21 \\
\hline BSS -363 & 48 & -72 & & 22.6 \\
\hline BSS -533 & 48 & -72 & & 19.4 \\
\hline BSS -553 & 48 & -72 & & 10.8 \\
\hline BSS -583 & 48 & 72 & & 14.6 \\
\hline
\end{tabular}




$\begin{array}{lrrr}\text { Copper } & & & \\ \text { Unit: microgram/gram } & & \\ \text { First Layer } & \text { Interval(inches) } & \text { Modifier } & \text { Result } \\ \text { BSS-131 } & 0-12 & 1.57 \\ \text { BSS-132 } & 12-24 & 1.99 \\ \text { BSS-201A } & 0-24 & 8.016 \\ \text { BSS-301 } & 0-24 & 5.696 \\ \text { BSS-311 } & 0-24 & 5.568 \\ \text { BSS-361 } & 0-24 & 5 \\ \text { BSS-531 } & 0-24 & 3.8 \\ \text { BSS-551 } & 0-24 & 3.5 \\ \text { BSS-581 } & 0-24 & 1.9 \\ \text { Second Layer } & & & \\ \text { BSS-133 } & 24-36 & 1.85 \\ \text { BSS-202A } & 24-48 & 8.642 \\ \text { BSS-302 } & 24-48 & 2.3 \\ \text { BSS-312 } & 24-48 & 10.69 \\ \text { BSS-362 } & 24-48 & 6.4 \\ \text { BSS-532 } & 24-48 & 4 \\ \text { BSS-552 } & 245 & 1.3 \\ \text { BSS-582 } & 24-48 & 2 \\ \text { Third Layer } & 24-48 & \\ \text { BSS-203A } & & & \\ \text { BSS-303 } & 48-72 & 5.172 \\ \text { BSS-313 } & 48-72 & 4.348 \\ \text { BSS-363 } & 48-72 & 5.4 \\ \text { BSS-533 } & 48-72 & 4 \\ \text { BSS-553 } & 48-72 & 2.1 \\ \text { BSS-583 } & 48-72 & \\ & 48-72 & \end{array}$




\section{Iron}

Unit: microgram/gräm

First Layer
BSS-131
BSS-132
BSS-201A
BSS-301
BSS -311
BSS-361
BSS-531
BSS -551
BSS -581

Interval (inches)
$0-12$
$12-24$
$0-24$
$0-24$
$0-24$
$0-24$
$0-24$
$0-24$
$0-24$

Modifier
35400
34200
25730
18000
3637
21464
12566
8575.6
9303.2

\section{Second Layer}

BSS-133

BSS-202A

BSS- 302

$24-36$

30600

$24-48$

$24-48$

25040

11700

5909

BSS -312

BSS- 362

BSS -532

$24-48$

17251

$24-48$

13498

$24-48$

4432.3

BSS-582

$24-48$

8182

Third Layer

BSS-203A

$48-72$
$48-72$
$48-72$
$48-72$
$48-72$
$48-72$
$48-72$

7731

11590

22430

24140

15487

4799.2

BSS-553

BSS-583

$48-72$
$48-72$

10532 


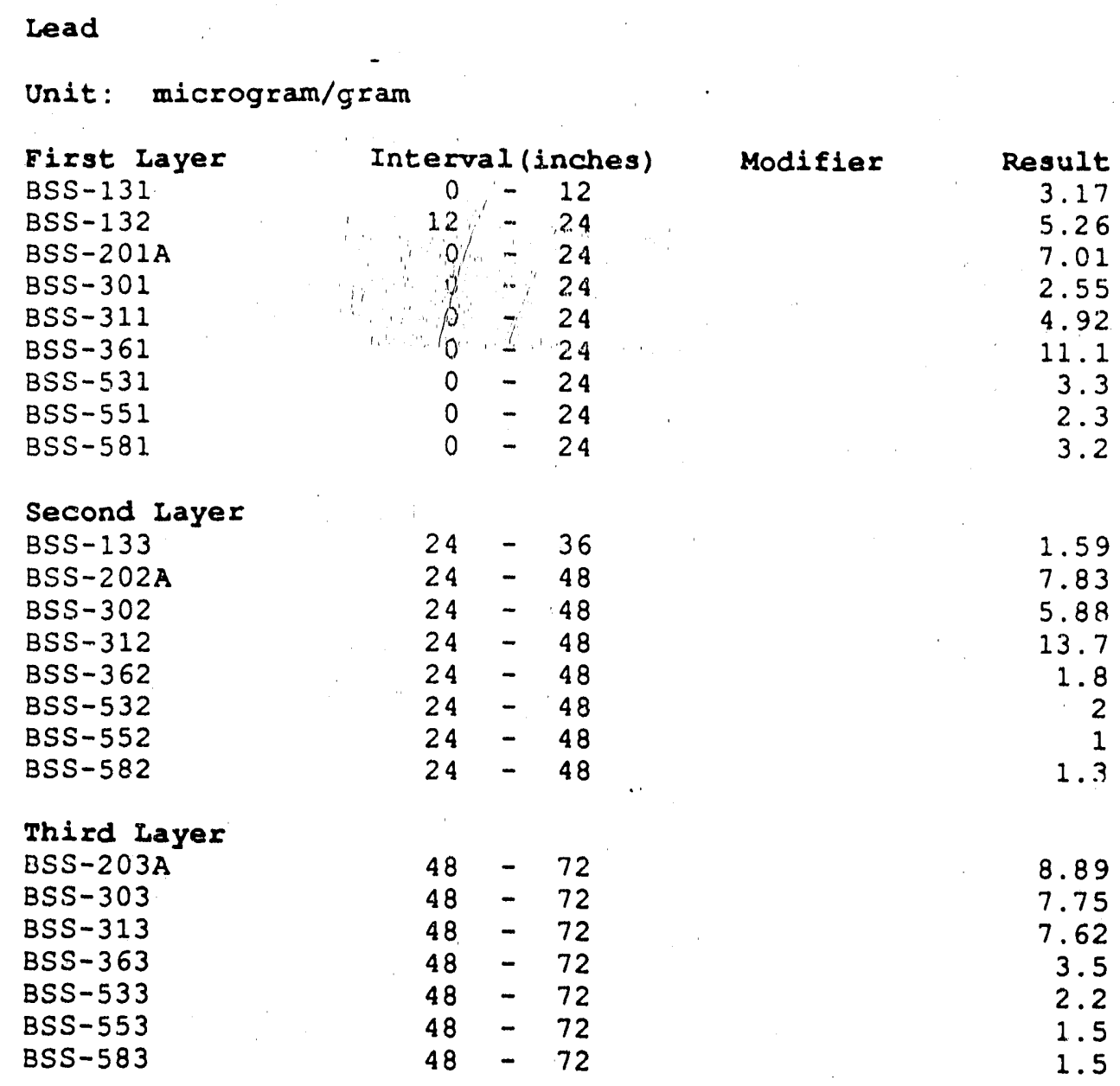




\section{Lithium}

Unit: microgram/gräm

First Layer
BSS-131
BSS-132
BSS-201A
BSS-301
BSS-311
BSS-361
BSS-531
BSS-551
BSS-581

Interval (inches)
$0-12$
$12-24$
$0-24$
$0-24$
$0-24$
$0-24$
$0-24$
$0-24$
$0-24$

$\begin{array}{cr}\text { Modifier } & \text { Result } \\ \text { LT } & 10 \\ \text { LT } & 10 \\ \text { LT } & 10 \\ \text { LT } & 10 \\ \text { LT } & 10 \\ \text { LT } & 10 \\ \text { LT } & 10 \\ \text { LT } & 10 \\ \text { LT } & 10\end{array}$

Second Layer

BSS -133

BSS-202A

BSS -302

BSS- 312

BSS -362

BSS -532

BSS-552

BSS -582

$24-36$
$24-48$
$24-48$
$24-48$
$24-48$
$24-48$
$24-48$
$24-48$

LT

LT 10

IT 10

LT 10

LT 10

$\mathrm{LT} \quad 10$

Third Iayer

BSS-203A

BSS -303

BSS -313

BSS -363

BSS -533

BSS -553

BSS -583

$48-72$
$48-72$
$48-72$
$48-72$
$48-72$
$48-72$
$48-72$

LT

10

10

10

10

10

10

10 


\begin{tabular}{|c|c|c|c|c|}
\hline \multicolumn{5}{|l|}{ Magnesium } \\
\hline \multicolumn{5}{|c|}{ Unit: microgram/gram } \\
\hline First Layer & \multicolumn{2}{|c|}{ Interval (inches) } & \multirow[t]{10}{*}{ Modifier } & Result \\
\hline BSS -131 & 0 & -12 & & \\
\hline BSS -132 & 12 & -24 & & 104.4 \\
\hline BSS-201A & 0 & -24 & & 165.9 \\
\hline BSS -301 & 0 & -24 & & 209.4 \\
\hline BSS -311 & 0 & -24 & & 111.5 \\
\hline BSS -361 & 0 & -24 & & 156.4 \\
\hline$B S S-531$ & 0 & -24 & & 94.07 \\
\hline BSS -551 & 0 & -24 & & 30.85 \\
\hline$B S S-581$ & 0 & -24 & & 87.7 \\
\hline \multicolumn{5}{|l|}{ Second Layer } \\
\hline BSS -133 & 24 & -36 & & 51.22 \\
\hline BSS-202A & 24 & -48 & & 159.1 \\
\hline BSS-302 & 24 & -48 & & 166.6 \\
\hline BSS -312 & 24 & -48 & & 110.1 \\
\hline BSS -362 & 24 & -48 & & 44.13 \\
\hline BSS -532 & 24 & -48 & & 36.24 \\
\hline BSS-552 & 24 & -48 & & 34.86 \\
\hline BSS -582 & 24 & -48 & & 31.12 \\
\hline \multicolumn{5}{|l|}{ Third Layex } \\
\hline$B S S-203 A$ & 48 & -72 & & 43.58 \\
\hline BSS -303 & 48 & -72 & & 255.4 \\
\hline BSS -313 & 48. & -72 & & 98.77 \\
\hline BSS -363 & 48 & -72 & & 57.82 \\
\hline BSS -533 & 48 & -72 & & 73.87 \\
\hline BSS -553 & 48 & -72 & & 31.4 \\
\hline BSS -583 & 48 & -72 & & 24.35 \\
\hline
\end{tabular}




\section{Manganese}

Unit: microgram/gram

Eirst Layer

BSS-131

BSS -132

BSS-201A

BSS-301

BSS -311

BSS-361

BSS-531

BSS -551

BSS- 581

Second Layer

BSS-133

BSS-202A

BSS-302

BSS-312

BSS -362

BSS -532

BSS -552

BSS- 582

Third Layer

BSS-203A

BSS -303

BSS -313

BSS -363

BSS -533

BSS -553

BSS-583

$\begin{array}{cc}\text { Interval (inches) } & \text { Modifier } \\ 0-12 & \\ 12-24 & \text { LT } \\ 0-24 & \text { LT } \\ 0-24 & \\ 0-24 & \\ 0-24 & \\ 0-24 & \\ 0-24 & \\ 0-24 & \end{array}$

$24-36$

$24-48$

$24-48$

$24-48$

$24-48$

$24-48$

$24-48$

$24-48$

$48-72$
$48-72$
$48-72$
$48-72$
$48-72$
$48-72$
$48-72$
LT

Result

5.63

1.6

1.6

2. 987

2. 987

4.979

7.583

3.294

11.87

LT

1.6

2.068

10.28

1.762

1.6

2.068

3.524

12.18

2.083

4.673

1.6

1.6

1.6

2.834

27.96 
Mercury

Unit: microgram/gram

\begin{tabular}{|c|c|c|c|c|}
\hline \multirow{2}{*}{ First Layer } & \multicolumn{2}{|c|}{ Interval (inches) } & \multirow{2}{*}{$\begin{array}{c}\text { Modifier } \\
\text { LT }\end{array}$} & Result \\
\hline & 0 & -12 & & 0.1 \\
\hline BSS -132 & 12 & 24 & $\mathrm{LT}$ & 0.1 \\
\hline$B S S-201 A$ & 0 & 24 & $\mathrm{LT}$ & 0.1 \\
\hline BSS-301 & 0 & -24 & $\mathrm{LT}$ & 0.1 \\
\hline BSS -311 & 0 & 24 & LT & 0.1 \\
\hline BSS -361 & 0 & -24 & $\mathrm{LT}$ & 0.1 \\
\hline BSS -531 & 0 & 24 & LT & 0.1 \\
\hline BSS -551 & 0 & -24 & LT & 0.1 \\
\hline$B S S-581$ & 0 & -24 & LT & 0.1 \\
\hline \multicolumn{5}{|l|}{ Second Layex } \\
\hline BSS -133 & 24 & 36 & & 0.1 \\
\hline$B S S-202 A$ & 24 & -48 & $\mathrm{~L} \mathrm{~T}$ & 0.1 \\
\hline BSS -302 & 24 & 48 & LT & 0.1 \\
\hline BSS -312 & 24 & 48 & $\mathrm{LT}$ & 0.1 \\
\hline$B S S-362$ & 24 & 48 & $\mathrm{LT}$ & 0.1 \\
\hline BSS -532 & 24 & -48 & LT & 0.1 \\
\hline BSS -552 & 24 & 48 & & 0.15 \\
\hline$B S S-582$ & 24 & -48 & $\mathrm{LT}$ & 0.1 \\
\hline \multicolumn{5}{|l|}{ Third Layer } \\
\hline$B S S-203 A$ & 48 & 72 & $\mathrm{LT}$ & 0.1 \\
\hline BSS-303 & 48 & -72 & $\mathrm{LT}$ & 0.1 \\
\hline BSS-313 & 48 & 72 & $\mathrm{LT}$ & 0.1 \\
\hline BSS -363 & 48 & 72 & $\mathrm{LT}$ & 0.1 \\
\hline$B S S-533$ & 48 & -72 & LT & 0.1 \\
\hline BSS -553 & 48 & -72 & $\mathrm{LT}$ & 0.1 \\
\hline BSS -583 & 48 & $-\quad 72$ & $\mathrm{LT}$ & 0.1 \\
\hline
\end{tabular}


Nickel

Unit: microgram/gram

First Layer
BSS -131
BSS -132
BSS -201 A
BSS -301
BSS -311
BSS -361
BSS -531
BSS -551
BSS -581

Interval (inches)
$0-12$
$12-24$
$0-24$
$0-24$
$0-24$
$0-24$
$0-24$
$0-24$
$0-24$

Modifier
LT
LT
LT

Result

3.66

3.14

5.121

3.374

1.983

Second Layer

BSS -133

BSS $-202 A$

BSS -302

BSS -312

BSS -362

BSS -532

BSS -552

BSS -582

$$
\begin{aligned}
& 24-36 \\
& 24-48 \\
& 24-48 \\
& 24-48 \\
& 24-48 \\
& 24-48 \\
& 24-48 \\
& 24-48
\end{aligned}
$$

Third Layer

BSS-203A

BSS -303

BSS -313

BSS -363

BSS -533

BSS -553

BSS -583

$48-72$
$48-72$
$48-72$
$48-72$
$48-72$
$48-72$
$48-72$

LT

LT

LT

LT

2.29

8.531

4.2

3.857

5.2

2

2

2.875

4.956

3.89

5.6

LT $\quad 0.2$

LT

0.2

2.8 
Potassium

Unit: microgram/gram

First Layer

BSS-131.

BSS -132

BSS-201A

BSS-301

BSS-311

BSS -361

BSS -531

BSS -551

BSS-581

$\begin{array}{cc}\text { Interval (inches) } & \text { Modifier } \\ 0-12 & \\ 12-24 & \\ 0-24 & \\ 0-24 & \\ 0-24 & \\ 0-24 & \text { LT } \\ 0-24 & \\ 0-24 & \text { LT } \\ 0-24 & \text { LT }\end{array}$

Result

65.8

70.9

258.8

112.8

43.14

180

206

180

Second Layer

BSS- 133

BSS-202A

BSS -302

BSS -312

BSS -362

BSS -532

BSS -552

BSS- 582

$24-36$
$24-48$
$24-48$
$24-48$
$24-48$
$24-48$
$24-48$
$24-48$

LT

180

Third Layer

BSS-203A

BSS -303

ESS -313

BSS -363

BSS -533

BSS -553

BSS -583

$48-72$
$48-72$
$48-72$
$48-72$
$48-72$
$48-72$
$48-72$


Selenium

Unit: microgram/gram

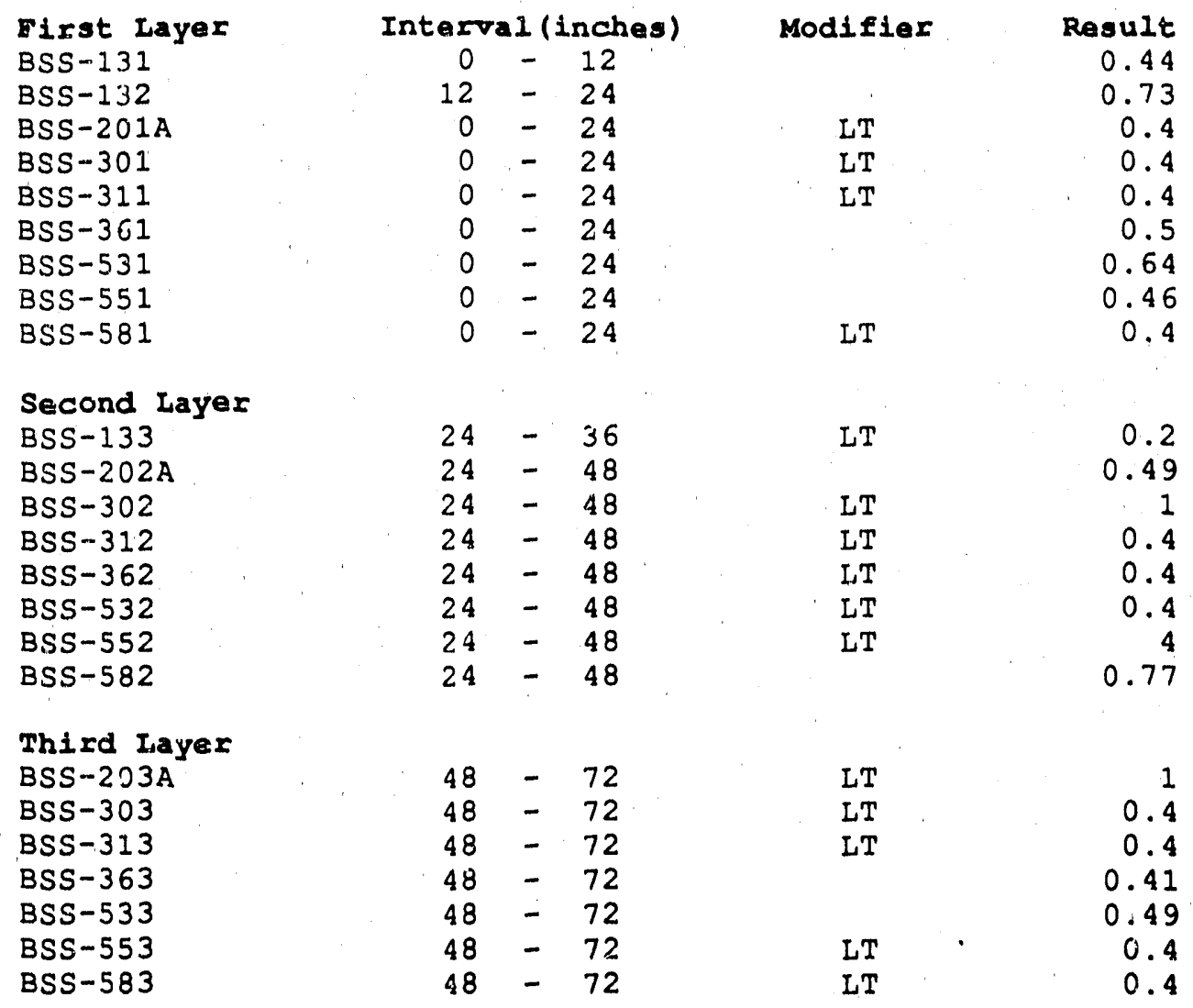


Silver

Unit : microgram/gram

Eirat Layer

BSS-131

BSS -132

BSS-201A

BSS-301

BSS-311

BSS-361

BSS-531

BSS-551

BSS -581

Second Layer

BSS-133

BSS-202A

BSS- 302

BSS -312

BSS- 362

BSS -532

BSS -552

BSS -582

Third Layer

BSS-203A

BSS -303

BSS -313

BSS -363

BSS -533

BSS -553

BSS -583

$\begin{array}{cc}\text { Interval(inches) } & \text { Modifier } \\ 0-12 & \text { LT } \\ 12-24 & \text { LT } \\ 0-24 & \text { LT } \\ 0-24 & \text { LT } \\ 0-24 & \text { LT } \\ 0-24 & \text { LT } \\ 0-24 & \text { LT } \\ 0-24 & \text { LT } \\ 0-24 & \text { LT }\end{array}$

$24-36$

$24-48$

$24-48$

$24-48$

$24-48$

$24-48$

$24-48$

$24-48$

LT

LT

LT

LT

LT

LT

LT

$48-72$
$48-72$
$48-72$
$48-72$
$48-72$
$48-72$
$48-72$

LT

LT

LT

L'

$\mathrm{L} T$

Result

0.3

0.3

0.6

0.6

0.6

1

1

0.3
0.6
0.6
0.0111
1
1
1
1

0.6
0.6
0.2369
1
1
1.8
1 


\begin{tabular}{|c|c|c|c|c|}
\hline \multicolumn{5}{|l|}{ Sodium } \\
\hline Unit: micro & ram & & & \\
\hline First Layer & Interv & 1 (inches) & Modifier & Result \\
\hline$B S S-131$ & 0 & -12 & & 18.5 \\
\hline BSS-132 & 12 & -24 & & 26.6 \\
\hline BSS-201A & 0 & -24 & & 47.25 \\
\hline BSS-301 & 0 & -24 & & 41.33 \\
\hline BSS-311 & 0 & -24 & & 76.1 \\
\hline BSS-361 & 0 & -24 & & 51.8 \\
\hline BSS-531 & 0 & -24 & & 13.8 \\
\hline BSS-551 & 0 & -24 & & 24.4 \\
\hline BSS-581 & 0 & -24 & & 20.5 \\
\hline Second Layer & & & & \\
\hline BSS-133 & 24 & -36 & & 14.8 \\
\hline$B S S-202 A$ & 24 & -48 & & 54.11 \\
\hline BSS-302 & 24 & -48 & & 37.95 \\
\hline BSS -312 & 24 & -48 & & 45.69 \\
\hline BSS -362 & 24 & 48 & & 223.2 \\
\hline BSS -532 & 24 & 48 & & 65.8 \\
\hline BSS -552 & 24 & 48 & & 20.5 \\
\hline BSS -582 & 24 & -48 & & 16.4 \\
\hline Third Layer & & & & \\
\hline$B S S-203 A^{-}$ & 48 & 72 & & 51.93 \\
\hline BSS-303 & 48 & -72 & & 42.26 \\
\hline BSS -313 & 48 & 72 & & 46.32 \\
\hline BSS -363 & 48 & -72 & & 381.6 \\
\hline$B S S-533$ & 48 & -72 & & 31.6 \\
\hline BSS-553 & 48 & -72 & & 47.5 \\
\hline BSS -583 & 48 & 72 & & 28.1 \\
\hline
\end{tabular}




\begin{tabular}{|c|c|c|c|c|}
\hline \multicolumn{5}{|l|}{ zinc } \\
\hline \multicolumn{5}{|c|}{ Unit: microgram/gram } \\
\hline Eirst Layer & Interv & I (inches) & Modifier & Result \\
\hline BSS -131 & 0 & -12 & & 23.5 \\
\hline BSS - 132 & 12 & -24 & & 19.3 \\
\hline BSS-201A & 0 & -24 & & 10.51 \\
\hline BSS-301 & 0 & -24 & & 15.12 \\
\hline BSS -311 & 0 & -24 & & 9.597 \\
\hline BSS -361 & 0 & -24 & & 5.2 \\
\hline BSS -531 & 0 & -24 & & 3.8 \\
\hline BSS -551 & 0 & -24 & & 4.2 \\
\hline BSS -581 & 0 & -24 & & 1.8 \\
\hline \multicolumn{5}{|l|}{ Second Layer } \\
\hline BSS -133 & 24 & -36 & & 21.95 \\
\hline BSS-202A & 24 & -48 & & 12.05 \\
\hline BSS -302 & 24 & $-\quad 48$ & & 9.7 \\
\hline BSS -312 & 24 & $-\quad 48$ & & 30.14 \\
\hline BSS -362 & 24 & -48 & & 11.4 \\
\hline BSS -532 & 24 & -48 & & 5.4 \\
\hline BSS -552 & 24 & -48 & & 4.9 \\
\hline$B S S-582$ & 24 & -48 & & 4.7 \\
\hline \multicolumn{5}{|l|}{ Third Layer } \\
\hline BSS-203A & 48 & -72 & & 10.46 \\
\hline BSS-303 & 48 & -72 & & 10.99 \\
\hline BSS -313 & 48 & -72 & & 7.021 \\
\hline BSS -363 & 48 & -72 & & 16.4 \\
\hline BSS -533 & 48 & -72 & & 7.2 \\
\hline BSS-553 & 48 & -72 & & 4.1 \\
\hline BSS -583 & 48 & 72 & & 5 \\
\hline
\end{tabular}


Aluminum

Unit: microgram/gram

First Layer BSS- 121

BSS-241

BSS -471

BSS -591

Second Layer

BSS- 122

BSS -242

BSS -472

BSS -592

Third Layer

BSS -123

BSS -243

BSS -473

BSS -593

Fourth Layer

BSS-124

BSS -244

BSS -474

BSS - 594

Interval (inches)
$0-23$
$0-22$
$0-12$
$0-23$

$$
23-65
$$

$22-59$

$12-38$

$23-50$

$$
\begin{aligned}
& 65-82 \\
& 59-74 \\
& 38-72 \\
& 50-72
\end{aligned}
$$

$95-119$

$96-120$

$96-120$

$96-120$
Modifier

Result

21510

2826

6435

9194.5

9345

13286

8353.8

8152.5

7850

5089

4850.6

4096.2

11330

2592

3559.6

5195.4 


\section{Arsenic}

Unit: microgram/gram

First Layer

BSS-121

BSS-241

BSS -471

BSS-591

Second Layer

BSS- 122

BSS -242

BSS -472

BSS -592

Third Layer

BSS- 123

BSS -243

BSS -473

BSS -593

Fourth Layer

BSS- 124

BSS-244

BSS -474

BSS-594

$\begin{array}{cc}\text { Interval (inches) } & \text { Modifier } \\ 0-23 & \\ 0-22 & \text { LT } \\ 0-12 & \\ 0-23 & \text { LT }\end{array}$

$23-65$

$22-59$

$12-38$

$23-50$

$65-82$
$59-74$
$38-72$
$50-72$

$95-119$

$96-120$

$96-120$

$96-120$
LT

Result

5.04

1
2

3.34

4.88

2

3.11

2.35

LT

LT

2

0.96

1.01

LT 


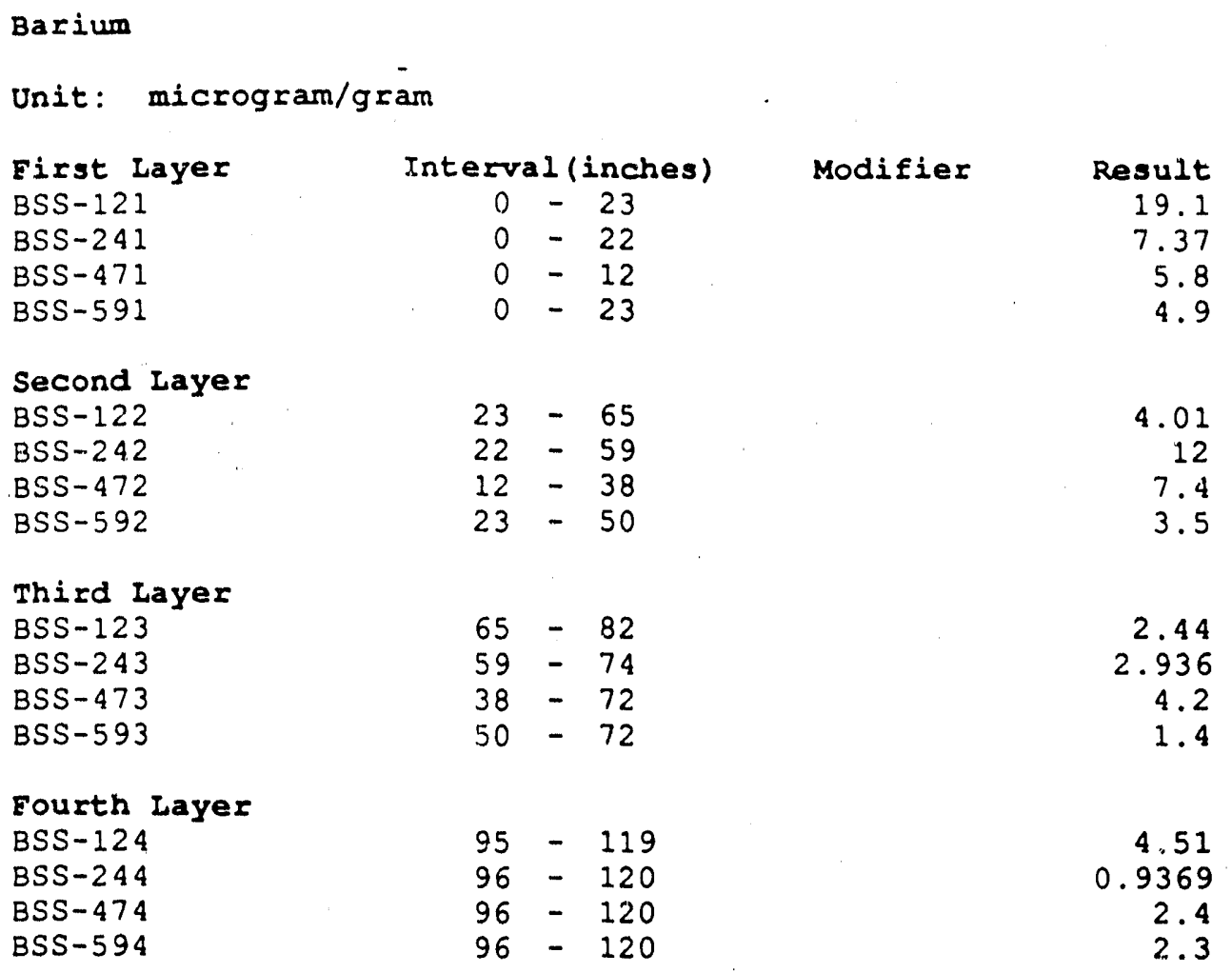




\section{Cadmium}

Unit: microgram/gram

First Layer

BSS-121

BSS-241

BSS -471

BSS -591

Second Layer

BSS- 122

BSS-242

BSS -472

BSS -592

Third Layex

BSS -123

BSS -243

BSS -473

BSS -593

Fourth Layer

BSS- 124

BSS-244

BSS -474

BSS -594

Interval (inches)
$0-23$
$0-22$
$0-12$
$0-23$

$23-65$

$22-59$

$12-38$

$23-50$

$65-82$
$59-74$
$38-72$
$50-72$

$95-119$

$96-120$

$96-120$

$96-120$
Modifier

LT

LT

LT

LT.

LT

LT

LT

LT

LT

LT

LT

LT
Result

0.5

0.3978

0.6

0.6

0.5

0.6

0.6

0.5

0.9945

0.6

0.6

0.5

0.1989

0.6

0.6 


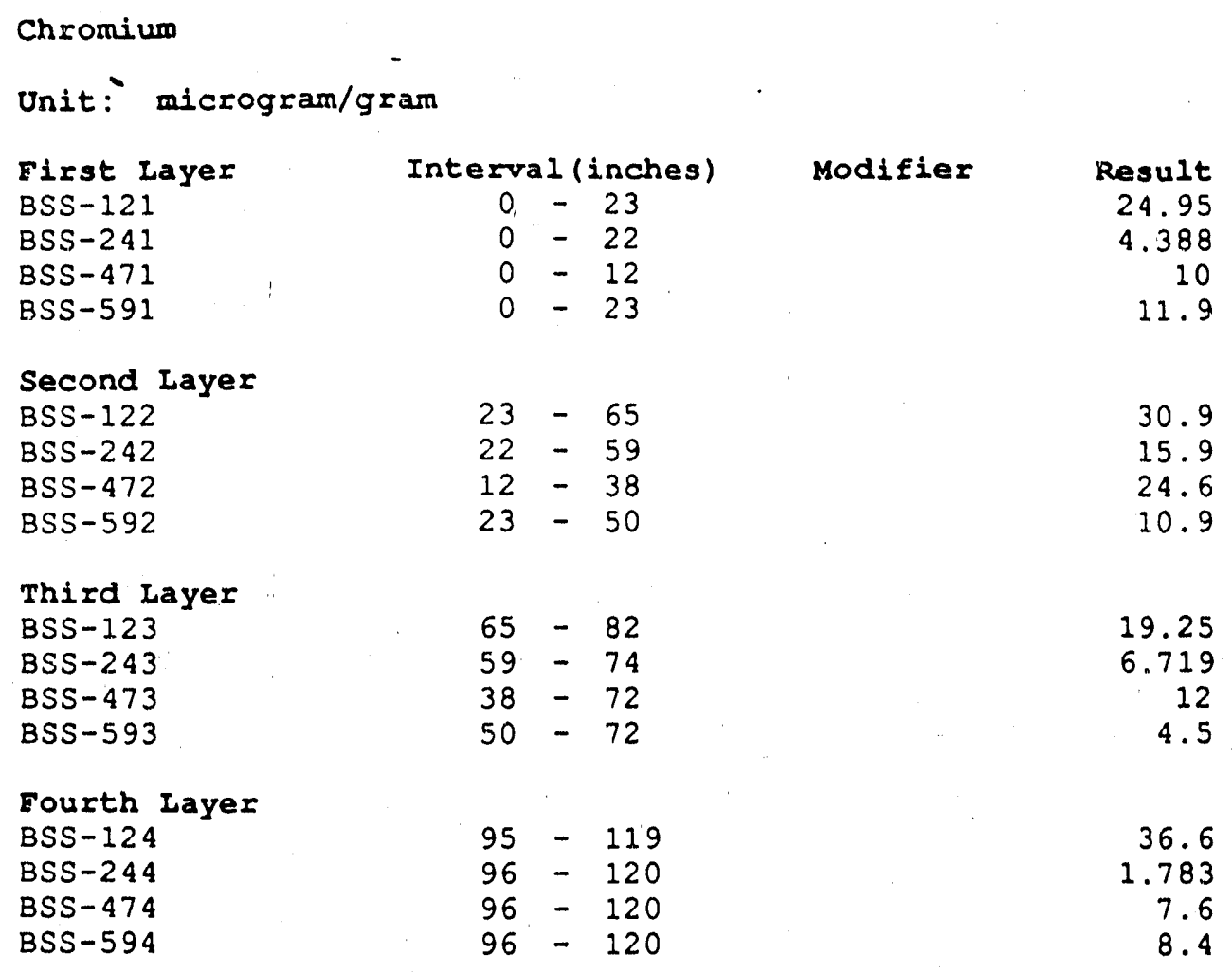




\begin{tabular}{|c|c|c|c|c|}
\hline \multicolumn{5}{|l|}{ Copper } \\
\hline \multicolumn{3}{|c|}{ Unit: wicrogram/gram } & \multirow[b]{2}{*}{ Modifier } & \multirow[b]{2}{*}{$\begin{array}{r}\text { Result } \\
2.35 \\
1.013 \\
2.6 \\
4.8\end{array}$} \\
\hline $\begin{array}{l}\text { First Layer } \\
\text { BSS }-121 \\
\text { BSS }-241 \\
\text { BSS }-471 \\
\text { BSS }-591\end{array}$ & $\begin{array}{r}\text { Interv } \\
0 \\
0 \\
0 \\
0\end{array}$ & $\begin{array}{l}1 \text { (inches) } \\
=\quad 23 \\
=\quad 22 \\
=12 \\
-\quad 23\end{array}$ & & \\
\hline \multicolumn{5}{|l|}{ Second Layer } \\
\hline BSS -122 & 23 & -65 & & 2.14 \\
\hline $\mathrm{ESS}-242$ & 22 & -59 & LT & \\
\hline BSS -472 & 12 & -38 & & 5.2 \\
\hline BSS-592 & 23 & -50 & & 4.5 \\
\hline \multicolumn{5}{|l|}{ Third Layer } \\
\hline BSS -12.3 & 65 & -82 & & 2.85 \\
\hline BSS -243 & 59 & -74 & & 2.195 \\
\hline BSS -473 & 38 & -72 & & 3.8 \\
\hline BSS -593 & 50 & -72 & & 2.1 \\
\hline \multicolumn{5}{|l|}{ Fourth Layer } \\
\hline BSS-124 & 95 & -119 & & 2.92 \\
\hline BSS -244 & 96 & -120 & & 1.335 \\
\hline BSS -474 & 96 & -120 & & 2.6 \\
\hline BSS-594 & 96 & -120 & & 4.3 \\
\hline
\end{tabular}


Iron

Unit: microgram/gram

First Layer

BSS-121

BSS-241

BSS -471

BSS-591

Second Iayer

BSS- 122

BSS -242

BSS -472

$3 S S-592$

Third Layer

BSS- 123

BSS -243

BSS -473

BSS -593

Fourth Layer

BSS- 124

BSS-244

BSS -474

BSS -594

Interval (inches)
$0-23$
$0-22$
$0-12$
$0-23$

Modifier

$23-65$

$22-59$

$12-38$

$23-50$

$65-82$

$59-74$

$58-72$

$50-72$

$95-119$

$96-120$

$96-120$

$96-120$
Result

33000

2024

6341.8

11637

3100

13648

15371

11083

33400

4032

6893.6

5172.3

65600

1790

4531

8133.8 


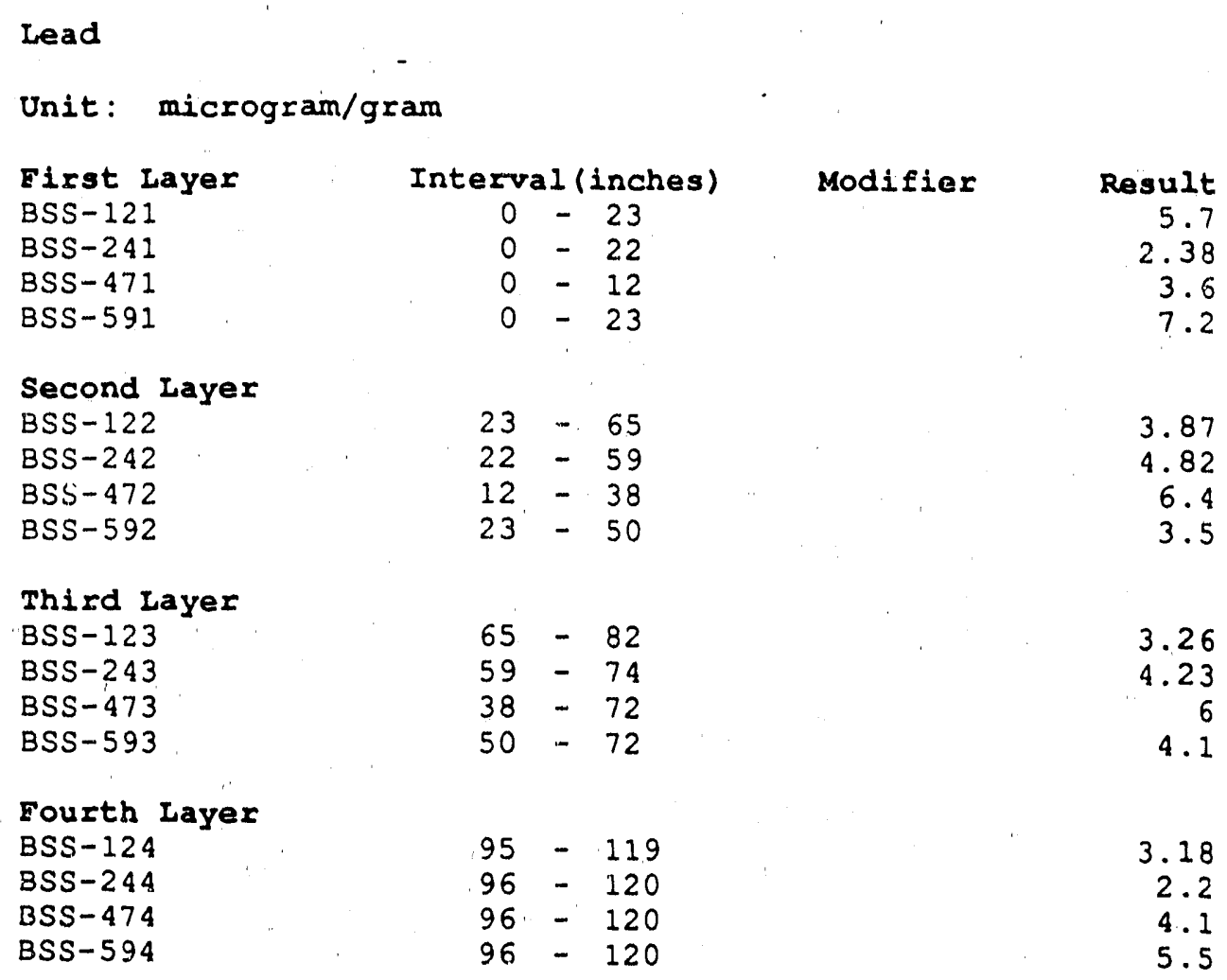


Lithium

Unit: microgram/gram

Eirst Layer

BSS-121

BSS-241

BSS -471

BSS-591

Second Layer

BSS- 122

BSS-242

BSS -472

BSS -592

Third Layer

BSS- 123

BSS -243

BSS -473

BSS -593

Eourth Layer

BSS-124

BSS-244

BSS -474

BSS-59A

Interval (inches)
$0-23$
$0-22$
$0-12$
$0-23$

$23-65$

$22-59$

$12-38$

$23-50$

$65-82$
$59-74$
$38-72$
$50-72$

$95-119$

$96-120$

$96-120$

$96-120$

Modifier
LT
LT
LT
LT

Result

10

10

10

10

10

10

10

10

10

10

10

10

10

10

10

10 


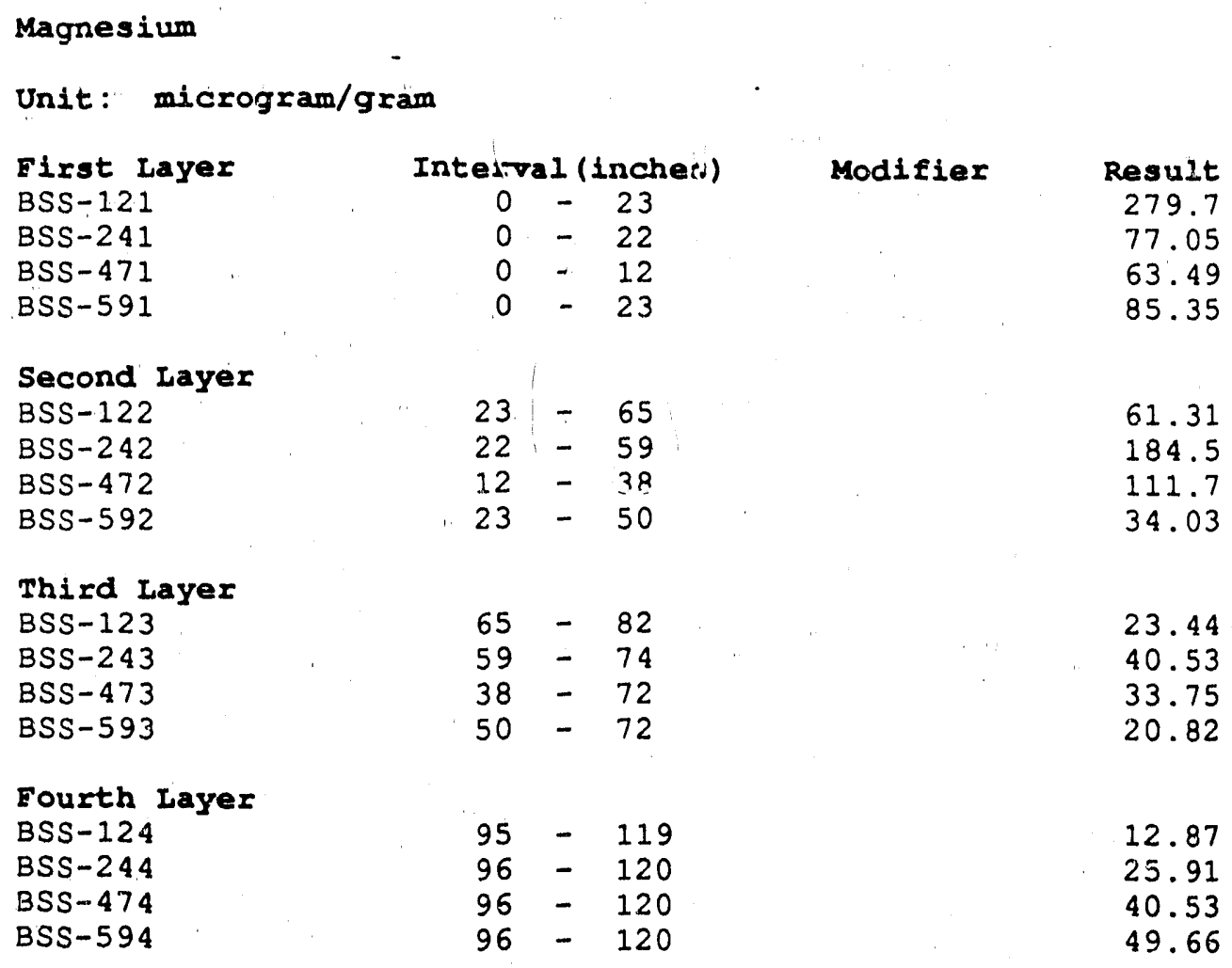




\section{Manganese}

Unt.t: microgram/gram

\begin{tabular}{|c|c|c|c|c|}
\hline $\begin{array}{l}\text { First Layer } \\
\text { BSS-121 } \\
\text { BSS-241 } \\
\text { BSS-471 } \\
\text { BSS-591 }\end{array}$ & $\begin{array}{r}\text { Interv } \\
0 \\
0 \\
0 \\
0\end{array}$ & $\begin{array}{l}1 \text { (inches) } \\
-\quad 23 \\
-\quad 22 \\
-\quad 12 \\
-\quad 23\end{array}$ & rodizier & $\begin{array}{r}\text { Result } \\
18 \\
7.583 \\
4.519 \\
2.145\end{array}$ \\
\hline $\begin{array}{l}\text { Second Layer } \\
\text { BSS-122 } \\
\text { BSS-242 } \\
\text { BSS- } 472 \\
\text { BSS-592 }\end{array}$ & $\begin{array}{l}23 \\
22 \\
12 \\
23\end{array}$ & $\begin{array}{l}-\quad 65 \\
-\quad 59 \\
-\quad 38 \\
-\quad 50\end{array}$ & L'T & $\begin{array}{r}3.118 \\
1.6 \\
4.213 \\
3.37\end{array}$ \\
\hline $\begin{array}{l}\text { Third Layer } \\
\text { BSS-123 } \\
\text { BSS-243 } \\
\text { BSS-473 } \\
B S S-593\end{array}$ & $\begin{array}{l}65 \\
59 \\
38 \\
50\end{array}$ & $\begin{array}{l}-\quad 32 \\
-\quad 74 \\
-\quad 72 \\
-\quad 72\end{array}$ & $\begin{array}{l}\mathrm{LT} \\
\mathrm{LT}\end{array}$ & $\begin{array}{r}1.6 \\
1.6 \\
8.043 \\
3.447\end{array}$ \\
\hline $\begin{array}{l}\text { Eourth Layer } \\
\text { BSS-124 } \\
\text { BSS-244 } \\
\text { BSS }-474 \\
\text { BSS }-594\end{array}$ & $\begin{array}{l}95 \\
96 \\
96 \\
96\end{array}$ & $\begin{array}{l}119 \\
-\quad 120 \\
-\quad 120 \\
-\quad 120\end{array}$ & LT & $\begin{array}{r}4.215 \\
1.6 \\
2.528 \\
8.579\end{array}$ \\
\hline
\end{tabular}




\begin{tabular}{|c|c|c|c|c|}
\hline \multicolumn{5}{|l|}{ Mercury } \\
\hline \multicolumn{5}{|c|}{ Unit: microgram/gram } \\
\hline First Layer & Interva & 1 (inches) & Modifier & Result \\
\hline BSS-121 & 0 & -23 & LT & 0.1 \\
\hline BSS -241 & 0 & -22 & LT & 0.01 \\
\hline BSS -471 & 0 & -12 & LT & 0.1 \\
\hline BSS-591 & 0 & -23 & $\mathrm{LT}$ & 0.1 \\
\hline \multicolumn{5}{|l|}{ Second Layer } \\
\hline BSS -122 & 23 & -65 & LT & 0.1 \\
\hline$B S S-242$ & 22 & -59 & LT & 0.1 \\
\hline BSS -472 & 12 & -38 & LT & 0.1 \\
\hline BSS- 592 & 23 & -50 & LT & 0.1 \\
\hline \multicolumn{5}{|l|}{ Third Layer } \\
\hline BSS-123 & 65 & -82 & LT & 0.1 \\
\hline BSS - 243 & 59 & -74 & $\mathrm{LT}$ & 0.1 \\
\hline$B S S=473$ & 38 & -72 & LT & 0.1 \\
\hline$B S S-593$ & 50 & -72 & LT & 0.1 \\
\hline \multicolumn{5}{|l|}{ Fourth Layer } \\
\hline BSS-124 & 95 & -119 & LT & 0.1 \\
\hline BSS-244 & 96 & -120 & LT & $r 1$ \\
\hline BSS -474 & 96 & -120 & LT & 0.1 \\
\hline BSS-594 & 96 & -120 & LT & 0.1 \\
\hline
\end{tabular}


Nickel

Unit: microgram/gram

First Layer

BSS-121

BSS-241

BSS-471

BSS-591

Second Layer

BSS -122

BSS -242

ESS-472

BSS -592

Third Iayer

ISS- 123

BSS -243

BSS -473

BSS -593

Fourth Jayer

BSS-124

BSS-244

BSS -474

BSS-594

Interval (inches)
$0-23$
$0-22$
$0-12$
$0-23$

$23-65$

$22-59$

$12-38$

$23-50$

$65-82$
$59-74$
$38-72$
$50-72$

$95-119$

$96-120$

$96-120$

$96-120$
Modifier

LT

LT

LT

3.4

4. 2

3.4

2. 2

3.1. 5

LT

LT

4.2

3

6.84

1.916

2.6

LT 


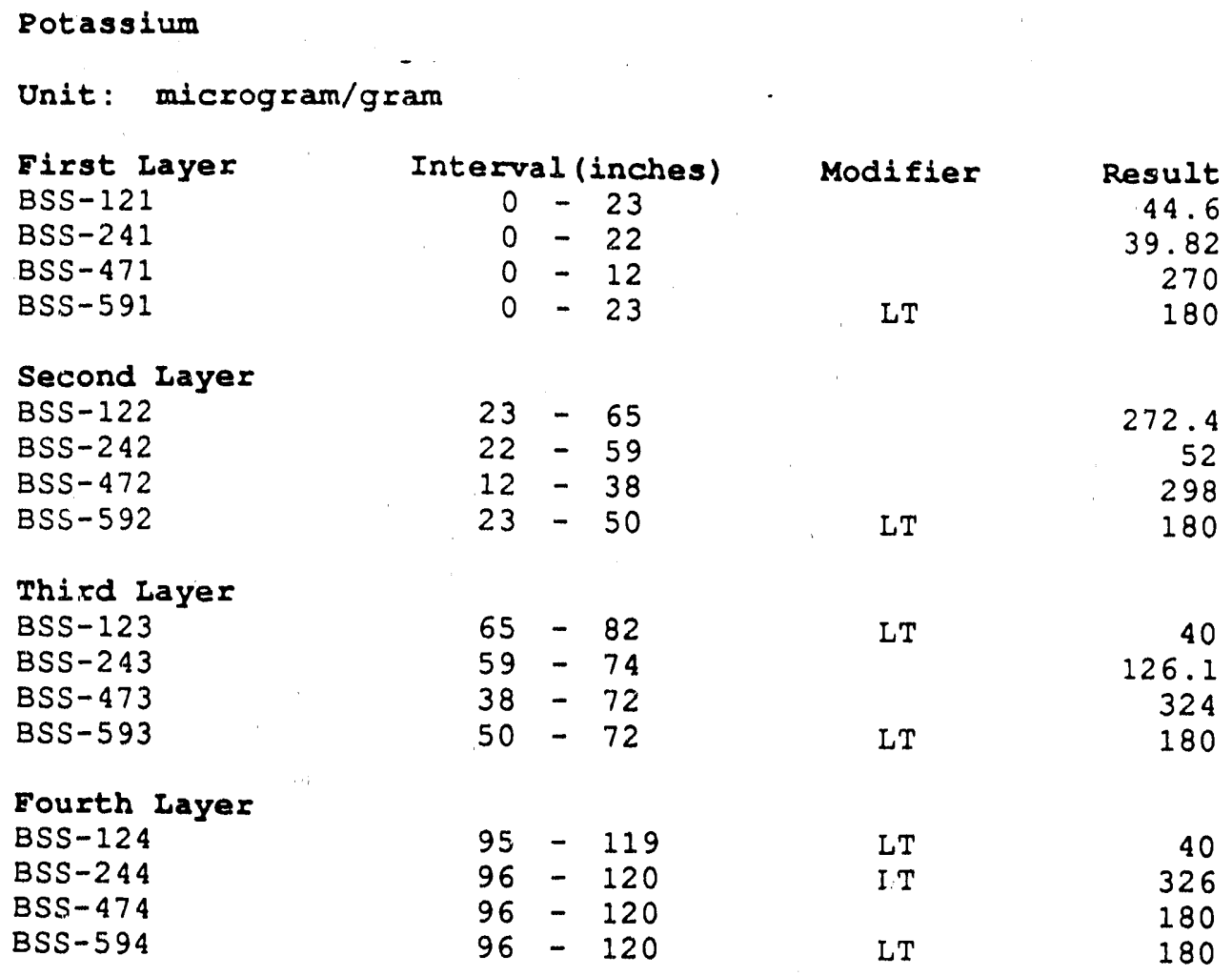


Selenium

Unit : microgram/gram

\begin{tabular}{|c|c|c|c|c|}
\hline First Layer & Interv & 1 (inches) & Modtfier & Result \\
\hline BSS-121 & 0 & -23 & & 0.23 \\
\hline BSS-241 & 0 & -22 & LT & 0.4 \\
\hline BSS -471 & 0 & $-\quad 12$ & $\mathrm{LT}$ & 0.4 \\
\hline BSS-591 & C & -23 & & 0.57 \\
\hline \multicolumn{5}{|l|}{ Second Layer } \\
\hline BSS-122 & 23 & -65 & $\mathrm{LT}$ & 0.2 \\
\hline$B S S-242$ & 22 & $-\quad 59$ & LT & 1 \\
\hline$B S S-472$ & 12 & -38 & $\mathrm{LT}$ & 0.4 \\
\hline BSS -592 & 23 & -50 & & 0.41 \\
\hline \multicolumn{5}{|l|}{ Third Layer } \\
\hline BSS - 123 & 65 & -82 & $\mathrm{LT}$ & 0.2 \\
\hline$B S S-243$ & 59 & -74 & & 0.49 \\
\hline$B S S-473$ & 38 & -72 & $\mathrm{LT}$ & 4 \\
\hline BSS-593 & 50 & $-\quad 72$ & & 0.51 \\
\hline \multicolumn{5}{|l|}{ Eourth Layer } \\
\hline BSS-124 & 95 & $-\quad 119$ & LT & 0.2 \\
\hline BSS-244 & 96 & $-\quad 120$ & & 0.45 \\
\hline BSS -474 & 96 & 120 & LT & 0.4 \\
\hline BSS-594 & 96 & $-\quad 120$ & LT & 0.4 \\
\hline
\end{tabular}


Silver

Unit: microgram/gram

Eirst Layer

BSS- 121

BSS-241

BSS-471

BSS -591

Second Layer

BSS -122

BSS -242

BSS -472

BSS -592

Third Layer

BSS- 123

BSS -243

BSS -473

BSS -593

Fourth Layer

BSS-124

BSS -244

BSS -474

BSS- 594

$\begin{array}{cc}\text { Interval (inches) } & \text { Modifier } \\ 0-23 & \text { LT } \\ 0-22 & \text { LT } \\ 0-12 & \text { LT } \\ 0-23 & \text { LT }\end{array}$

$$
\begin{aligned}
& 23-65 \\
& 22-59 \\
& 12-38 \\
& 23-50
\end{aligned}
$$

LT

LT

LT

LT

$$
\begin{aligned}
& 65-82 \\
& 59-74 \\
& 38-72 \\
& 50-72
\end{aligned}
$$

LT

$$
\text { LT }
$$

LT

$$
\begin{aligned}
& 95-119 \\
& 96-120 \\
& 96-120 \\
& 96-120
\end{aligned}
$$

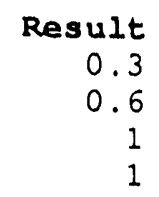

0.3
0.6
1
1

0.3

0.6854

1
1

0.3

0.6

1
1 


\section{Sodium}

Unit: microgram/gram

First Layex

BSS- 121

BSS-241

BSS -471

BSS-591

Second Layer

BSS -122

BSS -242

BSS -472

BSS -592

Third Layer

BSS-123

BSS -243

BSS -473

BSS -593

Fourth Layer

BSS-124

BSS-244

BSS -474

BSS -594

Interval (inches) Modifier
$0-23$
$0-22$
$0-12$
$0-23$

$23-65$

$22-59$

$12-38$

$23-50$

$65-82$

$59-74$

$38-72$

$50-72$

$95-119$

$96-120$

$96-120$

$96-120$
Result

26.6

27.29

46.4

28.7

32.2

28.8

38

78.5

14.5

29.63

29.6

59.9

18.6

30.1

42.8

70.3 


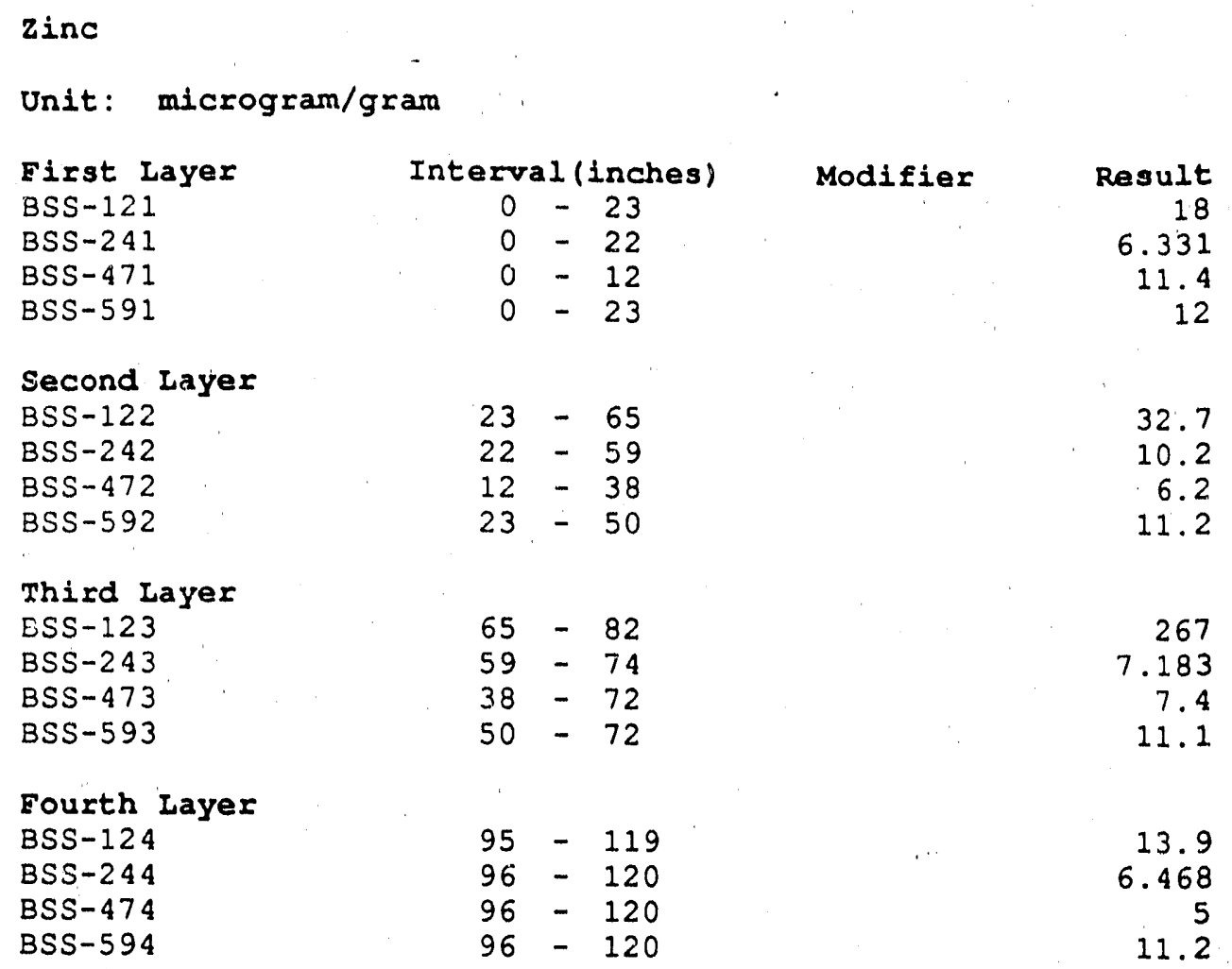


GEOCHEMICAL AND PHYSICAL PROPERTIES OF SOILS AND SHALLOW SEDIMENTS AT THE SAVANNAH RIVER SITE

\section{Appendix C.2}

Other Inorganic Data Summary 


\begin{tabular}{|c|c|c|c|c|}
\hline BSS-011 & Interva & 11 (1nches) & Modifier & Result \\
\hline & 0 & -7 & LT & 0.25 \\
\hline & 0 & -6 & LT & 0.25 \\
\hline & 0 & -5 & $\mathrm{LT}$ & 0.25 \\
\hline & $c$ & -7 & $\mathrm{LT}$ & 0.25 \\
\hline $\begin{array}{l}\text { BSS }-331 \\
\text { BSS }-351\end{array}$ & 0 & -8 & $\mathrm{LT}$ & 0.25 \\
\hline BSS-351 & 0 & -7 & LT & 0.25 \\
\hline BSS-431 & 0 & -6 & LT & 0.25 \\
\hline$B S S-451$ & 0 & -5 & LT & 0.25 \\
\hline \multicolumn{5}{|l|}{ Second Layer } \\
\hline BSS-012 & 10 & -65 & LT & 0.25 \\
\hline BSS-062 & 6 & -50 & LT & 0.25 \\
\hline$B S S-092 A$ & 5 & -52 & LT & 0.25 \\
\hline BSS-232 & 7 & -61 & LT & 0.25 \\
\hline BSS -332 & 8 & -72 & LT & 0.25 \\
\hline BSS-352 & 7 & -60 & LT & 0.25 \\
\hline BSS -432 & 6 & -67 & LT & 0.25 \\
\hline$B S S-452$ & 5 & -59 & LT & 0.25 \\
\hline \multicolumn{5}{|l|}{ Third Layer } \\
\hline BSS-013 & 65 & -87 & LT & 0.25 \\
\hline BSS-063 & 50 & -74 & LT & 0.25 \\
\hline BSS-093A & 52 & -72 & LT & 0.25 \\
\hline BSS-233 & 61 & -70 & $\mathrm{LT}$ & 0.25 \\
\hline BSS-333 & 72 & -84 & LT & 0.25 \\
\hline BSS -353 & 60 & -72 & LT & 0.25 \\
\hline BSS -433 & 67 & -77 & $\mathrm{LT}$ & 0.25 \\
\hline BSS- 453 & 59 & -72 & LT & 0.25 \\
\hline \multicolumn{5}{|l|}{ Fourth Layer } \\
\hline BSS-014 & 111 & -124 & LT & 0.25 \\
\hline BSS-064 & 96 & -120 & LT & 0.25 \\
\hline$B S S-094 A$ & 96 & -120 & LT & 0.25 \\
\hline$B S S-234$ & 96 & -120 & LT & 0.25 \\
\hline BSS-334 & 96 & -120 & LT & 0.25 \\
\hline BSS-354 & 96 & -120 & LT & 0.25 \\
\hline BSS -434 & 96 & -120 & L'T & 0.25 \\
\hline BSS -454 & 96 & -120 & J.T & 0.25 \\
\hline
\end{tabular}




\section{Chloride}

Untt: mlcrogram/gram

First Layer
BSS-011
BSS-061
BSS-091
BSS-231
BSS-331
BSS-351
BSS-431
BSS-451

Interval (inches)
$0-7$
$0-6$
$0-5$
$0-7$
$0-8$
$0-7$
$0-6$
$0-5$

Modifier Result
2.1
7.7
3.8
1.7
7.9
2.5
2.4
3

Second Layer BSS-012 BSS-062 BSS-092A

BSS-232

BSS-332

BSS-352

BSS -432

BSS -452

$$
\begin{array}{r}
10-65 \\
6-50 \\
5-52 \\
7-61 \\
8-72 \\
7-60 \\
6-67 \\
5-59
\end{array}
$$

1.25
1.9
2.8
LT $\quad 1.25$
LT $\quad 1.25$
1.6
3.2

Third Layer BSS-013

BSS-063

BSS-093A

BSS-233

BSS -333

BSS -353

BSS -433

BSS -453

$$
\begin{aligned}
& 65-87 \\
& 50-74 \\
& 52-72 \\
& 61-70 \\
& 72-84 \\
& 60-72 \\
& 67-77 \\
& 59-72
\end{aligned}
$$

\section{Fourth Iayer}

BSS-014

BSS-064

BSS-094A

BSS-234

BSS-334

BSS-354

BSS -434

BSS-454

$$
\begin{array}{r}
111-124 \\
96-120 \\
96-120 \\
96-120 \\
96-120 \\
96-120 \\
96-120 \\
96-120
\end{array}
$$

1. 3

2.6

8.6

LT 1.25

1.3

1.4

2. 6

4.5

1. 4

2.9

6.1

1.5

2.4

11.2

1.8

4.6 


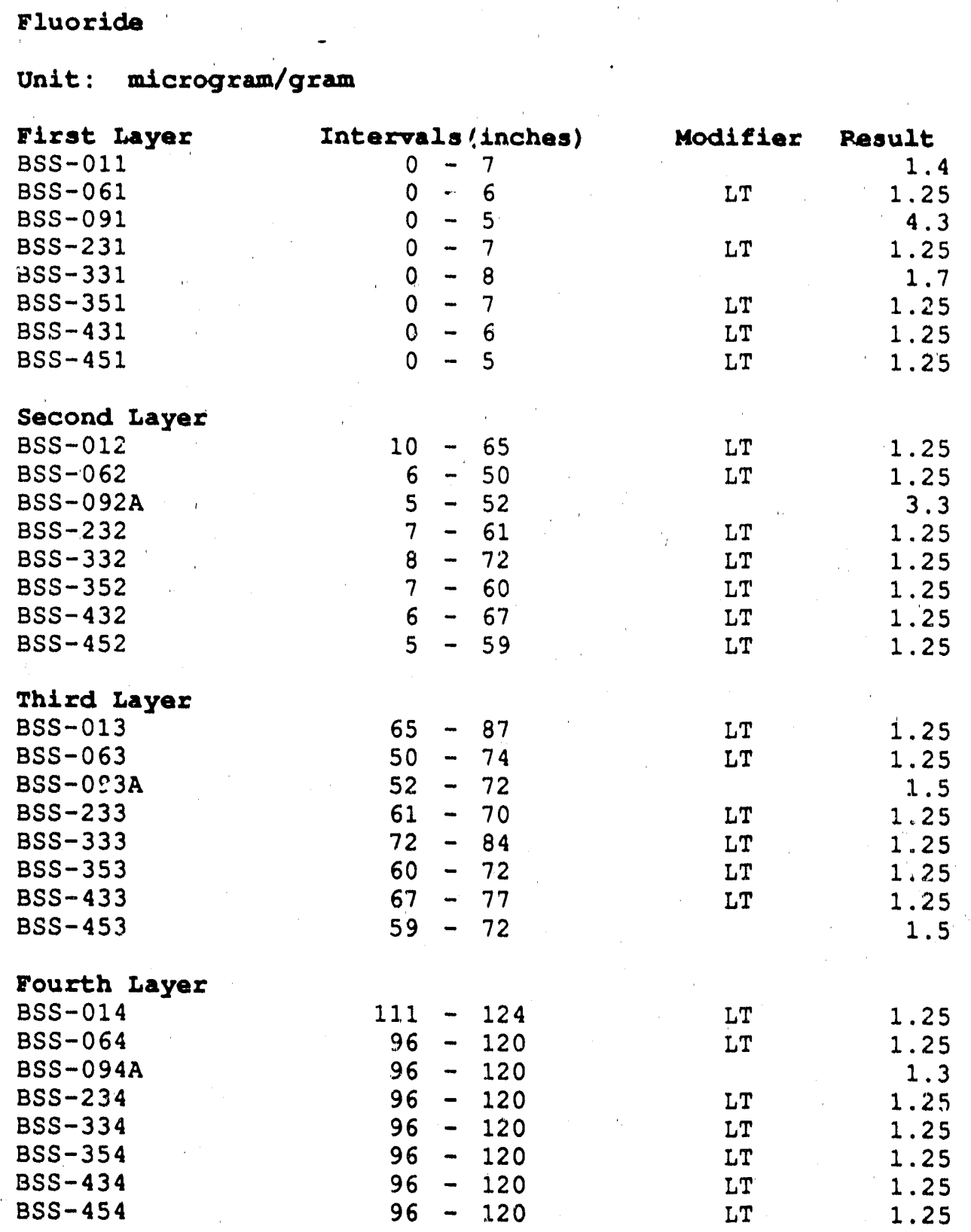




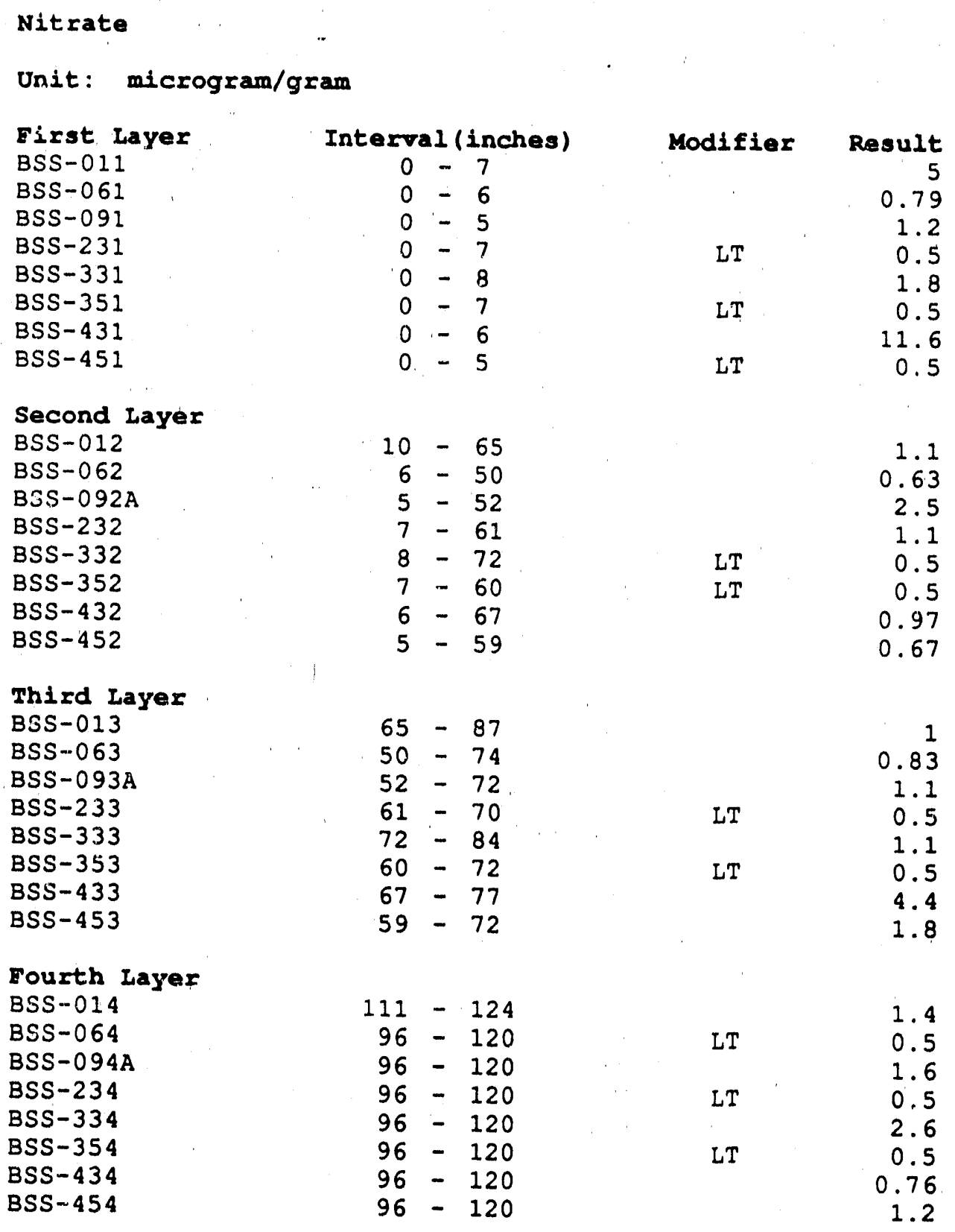




\section{Nitrite}

Unit: microgram/gram

First Layer

BSS-011

BSS-061

BSS-091

BSS-231

BSS -331

BSS -351

BSS-431

BSS -451

\section{Second Layer}

BSS-012

BSS -062

BSS-092A

BSS -232

BSS -332

BSS -352

BSS -432

BSS -452

Third Layer

BSS-013

BSS-063

BSS-093A

BSS- 233

BSS -333

BSS -353

BSS -433

BSS -453

Fourth Layer

BSS-014

BSS-064

BSS-094A

BSS-234

BSS-334

BSS- 354

BSS -434

BSS-454

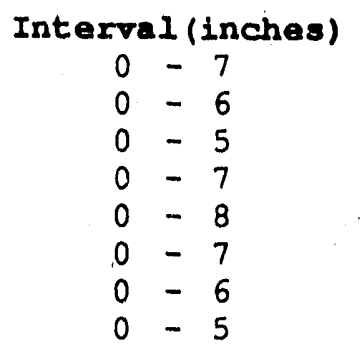

$\begin{array}{cr}\text { Modifier } & \text { Result } \\ \text { LT } & 1.25 \\ \text { LT } & 1.25 \\ \text { LT } & 1.25 \\ \text { LT } & 1.25 \\ \text { LT } & 1.25 \\ \text { LT } & 1.25 \\ \text { LT } & 1.25 \\ \text { LT } & 1.25\end{array}$

$10-65$

$6-50$

$5-52$

$7-61$

$8-72$

$7-60$

$6-67$

$5-59$

$\begin{array}{ll}\mathrm{LT} & 1.25 \\ \mathrm{LT} & 1.25 \\ \mathrm{LT} & 1.25 \\ \mathrm{LT} & 1.25 \\ \mathrm{LT} & 1.25 \\ \mathrm{LT} & 1.25 \\ \mathrm{LT} & 1.25 \\ \mathrm{LT} & 1.25\end{array}$

$65-87$

$50-74$

$52-72$

$61-70$

$72-84$

$60-72$

$67-77$

$59-72$

$111=124$
$96=120$
$96=120$
$96=120$
$96=120$
$96=120$
$96=120$
$96=120$
1.25

1.25

1.2.5

1.25

1.25

1.25

1.25

1.25

LT

1.25

1.25

1.25

1.25

1. 25

1.25

1.25

1.25 


\section{Phosphate}

Unit: microgram/gram

Eirst Iayer
BSS -011
BSS -061
BSS -091
BSS -231
BSS -331
BSS -351
$B S S-431$
$B S S-451$

Interval (inches)
$0-7$
$0-6$
$0-5$
$0-7$
$0-8$
$0-7$
$0-6$
$0-5$

$\begin{array}{cr}\text { Modifier } & \text { Result } \\ \text { LT } & 5 \\ \text { LT } & 5 \\ \text { LT } & 5 \\ \text { LT } & 5 \\ \text { LT } & 5 \\ \text { LT } & 5 \\ \text { LT } & 5 \\ \text { LT } & 5\end{array}$

Second Layer

BSS-012

BSS-062

BSS-092A

BSS-232

BSS-332

BSS -352

BSS -432

BSS- 452

$$
\begin{array}{r}
10-65 \\
6-50 \\
5-52 \\
7-61 \\
8-72 \\
7-60 \\
6-67 \\
5-59
\end{array}
$$

$\begin{array}{ll}\text { LT } & 5 \\ \text { LT } & 5 \\ \text { LT } & 5 \\ \text { LT } & 5 \\ \text { LT } & 5 \\ \text { LT } & 5 \\ \text { LT } & 5 \\ \text { LT } & 5\end{array}$

Third Layer

BSS-013

BSS-063

BSS-093A

BSS-233

BSS-333

BSS- 353

BSS -433

BSS- 453

Fourth Iayex

BSS-014
BSS-064
BSS-094A
BSS-234
BSS-334
BSS-354
BSS- 434
BSS- 454

$65-87$

$50-74$

$52-72$

$61-70$

$72-84$

$60-72$

$67-77$

$59-72$

$$
\begin{array}{r}
111-124 \\
96-120 \\
96-120 \\
96-120 \\
96-120 \\
96-120 \\
96-120 \\
96-120
\end{array}
$$

$\begin{array}{lr}\text { LT } & 5 \\ \text { LT } & 5 \\ \text { LT } & 5 \\ \text { LT } & 5 \\ \text { LT } & 5 \\ \text { LT } & 5 \\ L T & 12.2 \\ \text { LT } & 5\end{array}$

$\begin{array}{ll}\text { LT } & 5 \\ \text { LT } & 5 \\ \text { LT } & 5 \\ \text { LT } & 5 \\ \text { LT } & 5 \\ \text { LT } & 5 \\ \text { LT } & 5 \\ \text { LT } & 5\end{array}$




\section{Sulfate}

Unit: microgram/gram

First Layer

BSS-011

BSS-061

BSS-091

BSS-231

BSS-331

BSS- 351

BSS -431

BSS-451

\section{Second Layer}

BSS-0 012

BSS-062

BSS-092A

BSS-232

BSS- 332

BSS-352

BSS -432

BSS -452

\section{Third Layer}

BSS-013

BSS-063

BSS - 093A

BSS-233

BSS-333

BSS -353

BSS-433

BSS-453

\section{Eourth Layer}

BSS-014

BSS-064

BSS-094A

BSS-234

BSS- 334

BSS-354

BSS- 434

BSS-454

Interval (inches)
$0-7$
$0-6$
$0-5$
$0-7$
$0-8$
$0-7$
$0-6$
$0-5$

$$
\begin{array}{r}
10-65 \\
6-50 \\
5-52 \\
7-61 \\
8-72 \\
7-60 \\
6-67 \\
5-59
\end{array}
$$

Rodifiex
11.7
6.5
15
10
18.2
7.8
6.6
12.1

$$
\begin{aligned}
& 65-87 \\
& 50-74 \\
& 52-72 \\
& 51-70 \\
& 72-84 \\
& 60-72 \\
& 67-77 \\
& 59-72
\end{aligned}
$$

$$
\begin{array}{r}
7.7 \\
8.8 \\
16.9 \\
9 \\
13.5 \\
8.8 \\
8.7 \\
16.8
\end{array}
$$

$$
\begin{array}{r}
111-124 \\
96-120 \\
96-120 \\
96-120 \\
96-120 \\
96-120 \\
96-120 \\
96-120
\end{array}
$$

LT

9.6

5.3

5

9.4

11.5

14.3

7.1

96

$\begin{array}{lr}\text { LT } & 5 \\ \text { LT } & 5 \\ \text { IT } & 5 \\ & 5.2 \\ & 5.7 \\ \text { LT } & 14.3 \\ \text { LT } & 5 \\ & 5\end{array}$




\section{Cyanide}

Unit: microgram/gram

First Layer

BSS-031

BSS-071

BSS-081

BS - 211

BSS -321

BSS -421

BSS -441

BSS -491

\section{Second Layer}

BSS -032

BSS-072A

BSS -082

BSS-212A

BSS -322

BSS -422

BSS -442

BSS -492

Third Layar

BSS-033

BSS-073A

BSS -083

BSS-213A

BSS -323

BSS -423

BSS -443

BSS -493

Fourth Layex

BSS-034

BSS-074A

BSS-084

BSS-214A

BSS- 324

BSS-42.4

BSS -444

BSS-494

Interval (inches)
$0=6$
$0-8$
$0=6$
$0=5$
$0=9$
$0=9$
$0=$
$0=9$
$0=9$

Modifier

LT

LT

LT

LT

LT

LT

LT

LT

Rasuit

0.25

0.25

0.25

0.25

0.25

0.25

0.25

0.25

$10-39$
$8-36$
$6-36$
$5=47$
$6=49$
$9-45$
$7-38$
$9-41$

LT

LT

LT

LT

LT

LT

LI

LT

0.25

0.25

0.25

0.25

0.25

0.25

0.25

0.25

$39-72$
$36-72$
$36-72$
$47-77$
$49-72$
$45-72$
$38=70$
$41-72$

LT

LT

L'T

LT

LT

LT

LT

LT

0.25

0.25

0.25

0.25

0.25

0.25

0.25

0.25

$96-120$
$96-120$
$96-120$
$96-120$
$96-120$
$96-120$
$96-120$
$96-120$

$\begin{array}{ll}\text { LT } & 0.25 \\ \text { LT } & 0.25 \\ \text { LT } & 0.25 \\ \text { LT } & 0.25 \\ \text { LT } & 0.25 \\ \text { LT } & 0.25 \\ \text { LT } & 0.25 \\ \text { LT } & 0.25\end{array}$

0.25

0.25

0.25

0.25

0.25 
Chloride

Uait: microgram/gram

Eirst Layer

BSS-031

BSS-071

BSS-081

BSS-211

BSS-321

BSS-421

BSS-441

BSS-49I

\section{Second Layer}

BSS-032

BSS-072A

BSS-082

BSS-212A

BS, -322

BS? -422

BSS -442

BSS -492

Third Layex

\section{BSS-033}

BSS-073A

BSS-083

BSS-213A

BSS -323

BSS -423

BSS -443

BSS -493

\section{Fourth Layer}

BSS -034

BSS-074A

BSS-084

BSS $-214 \mathrm{~A}$

BSS-324

BSS -424

BSS- 444

BSS -494

Interval (inches)
$0-6$
$0-8$
$0-6$
$0-5$
$0-6$
$0-9$
$0-7$
$0-9$

Modifier

Result

2.1

5.4

3.7

2.1

4.6

3

2.1

$10-39$
$8-36$
$6-36$
$5-47$
$6-49$
$9-45$
$7-38$
$9-41$

LT

1.25

1.9

2.4

LT

1.25

LT

1.25

3.2

1.8

LT

1.25

$39-72$
$36-72$
$36-72$
$47-77$
$49-72$
$45-72$
$38-70$
$41-72$

1.4

9.1

2. 1

2

10.7

5.1

3. 9

2.8

$96-120$
$96-120$
$96-120$
$96-120$
$96-120$
$96-120$
$96-120$
$96-120$

2. 3

5.9

2

2. 3

1. 8

5. 6

6.1

2. 9 


\begin{tabular}{|c|c|c|c|c|}
\hline \multicolumn{5}{|c|}{ Unit: microgram/gram } \\
\hline First Layer & Interv & 1 (inches) & Modifler & Result \\
\hline BSS-031 & 0 & -6 & LT & 1.25 \\
\hline BSS-071 & 0 & -8 & LT & 9.25 \\
\hline$B S S-081$ & 0 & -6 & $\mathrm{LT}$ & 1.25 \\
\hline BSS-21] & 0 & -5 & LT & 1.25 \\
\hline BSS -321 & 0 & -6 & $\mathrm{LT}$ & 1.25 \\
\hline BSS -421 & 0 & -9 & & 2.3 \\
\hline$B S S-441$ & 0 & -7 & & 1.9 \\
\hline$B S S-491$ & 0 & -9 & $\mathrm{LT}$ & 1.25 \\
\hline \multicolumn{5}{|l|}{ Second Layer } \\
\hline BSS -032 & 10 & 39 & LT & 1.25 \\
\hline BSS-072A & 8 & -36 & LT & 1.25 \\
\hline BSS -082 & 6 & $-\quad 36$ & $\mathrm{LT}$ & 1.25 \\
\hline BSS $-212 A$ & 5 & -47 & $\mathrm{LT}$ & 1.25 \\
\hline BSS-322 & 6 & -49 & $\mathrm{LT}$ & 1.25 \\
\hline$B S S-422$ & 9 & -45 & LT & 1.25 \\
\hline BSS -442 & 7 & $-\quad 38$ & LT & 1.25 \\
\hline BSS -492 & 9 & -41 & LT & 1.25 \\
\hline \multicolumn{5}{|l|}{ Third Layer } \\
\hline BSS-033 & 39 & -72 & LT & 1.25 \\
\hline BSS-073A & 36 & -72 & LT & 1.25 \\
\hline BSS -083 & 36 & -72 & $\mathrm{LT}$ & 1.25 \\
\hline$B S S-213 A$ & 47 & -75 & $\mathrm{LT}$ & 1.25 \\
\hline BSS -323 & 49 & $-7 \%$ & LT & 1.25 \\
\hline BSS -423 & 45 & -72 & LT & 1.25 \\
\hline$B S S-443$ & 38 & -70 & & 1.4 \\
\hline$B S S-493$ & 41 & -72 & $\mathrm{LT}$ & 1.25 \\
\hline \multicolumn{5}{|l|}{ Fourth Layer } \\
\hline BSS -034 & 96 & $-\quad 120$ & LT & 1.25 \\
\hline$B S S-074 A$ & 96 & -120 & LT & 1.25 \\
\hline BSS-084 & 96 & -120 & LT & 1.25 \\
\hline$B S S-214 A$ & 96 & -120 & LT & 1.25 \\
\hline BSS-324 & 96 & $-\quad 120$ & $\mathrm{LT}$ & 1.25 \\
\hline BSS -424 & 96 & -120 & LT & 1.25 \\
\hline BSS -444 & 96 & -120 & LT & 1.25 \\
\hline BSS -494 & 96 & -120 & LT & 1.25 \\
\hline
\end{tabular}




\begin{tabular}{|c|c|c|c|c|}
\hline & (2) & & Modifier & Result \\
\hline BSS-071 & 0 & -8 & & 1.2 \\
\hline BSS-081 & 0 & -6 & & 0.89 \\
\hline BSS-211 & 0 & -5 & LT & 0.5 \\
\hline BSS-321 & 0 & -6 & LT & 0.5 \\
\hline$B S S-421$ & 0 & -9 & & 2.2 \\
\hline BSS-441 & 0 & -7 & LT & 0.5 \\
\hline BSS-491 & 0 & -9 & & , \\
\hline \multicolumn{5}{|l|}{ Second Layer } \\
\hline BSS-032 & 10 & 39 & & 1.2 \\
\hline $\mathrm{BSS}-072 \mathrm{~A}$ & 8 & 36 & & 0.99 \\
\hline BSS-082 & 6 & -36 & & 1.1 \\
\hline$B S S-212 A$ & 5 & $-\quad 47$ & LT & 0.5 \\
\hline BSS-322 & 6 & $-\quad 49$ & & 0.67 \\
\hline BSS -422 & 9 & -45 & & \\
\hline BSS -442 & 7 & $-\quad 38$ & & 0.9 \\
\hline BSS-492 & 9 & -41 & & 1.6 \\
\hline \multicolumn{5}{|l|}{ Third Layer } \\
\hline BSS-033 & 39 & 72 & & 1.2 \\
\hline BSS-073A & 36 & -72 & & 0.78 \\
\hline BSS-083 & 36 & -72 & & 0.73 \\
\hline BSS-213A & 47 & $-\quad 77$ & & 1.4 \\
\hline BSS-323 & 49 & -72 & & 2.3 \\
\hline BSS-423 & 45 & $-\quad 72$ & & 1.4 \\
\hline BSS- 443 & 38 & -70 & & 2.1 \\
\hline BSS -493 & 41 & $-\quad 72$ & LT & 0.5 \\
\hline \multicolumn{5}{|l|}{ Fourth Layer } \\
\hline BSS-034 & 96 & 120 & & 0.94 \\
\hline BSS-074A & 96 & $-\quad 120$ & LT & 0.5 \\
\hline BSS-084 & 96 & -120 & & 0.78 \\
\hline$B S S-214 A$ & 96 & -120 & LT & 0.5 \\
\hline BSS-324 & 96 & $-\quad 120$ & LT & 0.5 \\
\hline BSS -424 & 96 & $-\quad 120$ & & 1.2 \\
\hline$B S S-444$ & 96 & -120 & & 1.4 \\
\hline$B S S-494$ & 96 & 120 & & 2.3 \\
\hline
\end{tabular}


Nitrite

Unit: microgram/gram

Eirst Layer
BSS-031
BSS-071
BSS-081
BSS -211
BSS -321
BSS -421
BSS -441
BSS -491

Interval (inches)
$0-6$
$0-8$
$0-6$
$0-5$
$0-6$
$0-9$
$0-7$
$0-9$

$\begin{array}{cr}\operatorname{Modifier} & \text { Result } \\ \text { LT } & 1.25 \\ \text { LT } & 1.25 \\ \text { LT } & 1.25 \\ \text { LT } & 1.25 \\ \text { LT } & 1.25 \\ \text { LT } & 1.25 \\ \text { LT } & 1.25 \\ \text { LT } & 1.25\end{array}$

Second Iayer BSS-032

BSS-072A

BSS-082

BSS-2.12A

BSS -322

BSS -422

BSS- 442

BSS -492

$10-39$
$8-36$
$6-36$
$5-47$
$6-49$
$9-45$
$7-38$
$9-41$

LT

LT

LT

LT

LT

LT

LT

LT

1.25

1.25

1.25

1.25

1.25

1.25

1.25

1.25

Third Layer

BSS-033

BSS-073A

BSS -083

BSS-213A

BSS -323

BSS -423

BSS -443

BSS -493

Fourth Layex

BSS-034
BSS-074A
BSS-084
BSS-214A
BSS-324
BSS-424
BSS-444
BSS-494

$39-72$
$36-72$
$36-72$
$47-77$
$49-72$
$45-72$
$38-70$
$41-72$

$96-120$

$96-120$

$96-120$

$96-120$

$96-120$

$96-120$

$96-120$

$96-120$

$\begin{array}{ll}\text { LT } & 1.25 \\ \text { LT } & 1.25 \\ \text { LT } & 1.25 \\ \text { LT } & 1.25 \\ \text { LT } & 1.25 \\ \text { LT } & 1.25 \\ \text { LT } & 1.25 \\ \text { LT } & 1.25\end{array}$

$\begin{array}{ll}\text { LT } & 1.25 \\ \text { LT } & 1.25 \\ \text { LT } & 1.25 \\ \text { LT } & 1.25 \\ \text { LT } & 1.25 \\ \text { LT } & 1.25 \\ \text { LT } & 1.25 \\ \text { LT } & 1.25\end{array}$




\section{Phosphate}

Unit: microgram/gram

virst Layex

BSS-031

BSS-071

BSS-081

BSS-211

BSS-321

BSS -421

BSS- 441

BSS-491

\section{Second Layer}

BSS -032

BSS-072A

BSS-082

BSS-212A

BSS -322

BSS -422

BSS -442

BSS-492

Third Layer

BSS-033

BSS-073A

BSS -083

BSS-213A

BSS -323

BSS -423

BSS- 443

BSS -493

Fourth Iayer

BSS-034

BSS-074A

BSS-084

BSS-214A

BSS-324

BSS -424

BSS-444

BSS-494

Interval (inches)
$0-6$
$0-8$
$0-6$
$0-5$
$0-6$
$0-9$
$0-7$
$0-9$

Modifier

Result

$\mathrm{LT}$
$\mathrm{LT}$
$\mathrm{LT}$
$\mathrm{LT}$
$\mathrm{LT}$
$\mathrm{LT}$
$\mathrm{LT}$

5
5

5

5

5

5

5
5

$10-39$
$8-36$
$6-36$
$5-47$
$6-49$
$9-45$
$7-38$
$9-41$

$\begin{array}{lr}\text { LT } & 5 \\ \text { LT } & 5 \\ \text { LT } & 5 \\ \text { LT } & 5 \\ \text { LT } & 5 \\ \text { LT } & 5 \\ \text { LT } & 5 \\ & 9.2\end{array}$

$39-72$
$36-72$
$36-72$
$47-77$
$49-72$
$45-72$
$38-70$
$41-72$

LT

LT

LT

LT

LI

LT

LT

7.5

$96-120$
$96-120$
$96-120$
$96-120$
$96-120$
$96-120$
$96-120$
$96-120$

$\begin{array}{lr}\text { LT } & 5 \\ \text { LT } & 5 \\ \text { LT } & 5 \\ \text { LT } & 5 \\ \text { LT } & 5 \\ \text { LT } & 5 \\ \text { LT } & 5 \\ & 12\end{array}$




\section{Sulfate}

Unit: microgram/gram

\section{First Layer}

BSS-031

BSS-071

BSS-081

BSS -211

BSS- 321

BSS-421

BSS-441

BSS -491

\section{Second Layer}

BSS-032

BSS-072A

BSS-082

BSS-212A

BSS -322

BSS -422

BSS -442

BSS -492

Third Layer

BSS -033

BSS-073A

BSS-083

BSS-213A

BSS -323

BSS -423

BSS -443

BSS -493

Eourth Layer

BSS-034
$B S S-074 A$
$B S S-084$
$B S S-214 A$
$B S S-324$
$B S S-424$
$B S S-444$
$B S S-494$

Interval (inches)
$0-6$
$0-8$
$0-6$
$0-5$
$0-6$
$0-9$
$0-7$
$0-9$

Modifier

Result

6.4

8.7

15.4

10.1

10.5

19.5

10.2

8.1

$10-39$
$8-36$
$6-36$
$5-47$
$6-49$
$9-45$
$7-38$
$9-41$

8.4

19.2

14.3

11.3

7.3

14.8

10.7

10.9

$39-72$
$36-72$
$36-72$
$47-77$
$49-72$
$45-72$
$38-70$
$41-72$

5.6

LT

7.2

5

10.5

LT

5
6.5

6.5
4.4

$96-120$
$96-120$
$96-120$
$96-120$
$96-120$
$96-120$
$96-120$
$96-120$

LT

$\mathrm{LT}$

LT

LT

LT

LT

LT

5
5
5
5
10.5
5
5
5


Cyantde

Unit: microgram/gram

Iirst Inyer

BSS-021

BSS-221

BSS-411

BSS- 481

BSS -511

BSS-521

BSS-561

BSS -571

Second Layer

BSS-022

BSS-222A

BSS-412

BSS-482

BSS- 512

.3SS-522

BSS -562

BSS- 572

Third Iayer

BSS-023

BSS-223A

BSS -413

BSS -483

BSS -513

BSS -523

BSS-563

BSS -573
Interval (inches)

$0-8$
$0-7$
$0-5$
$0-4$
$0-5$
$0-5$
$0-5$
$0-9$

Modifier

LT

LT

LT

LT

LT

LT

LT

LT

$\mathrm{LT}$

LT

LT

LT

LT

LT

LT

LT

LT

$110-124$

$96-120$

$96-120$

$96-120$

$96-120$

$96-120$

$96-120$

$96-120$
Result

0.25

0.25

0.25

0.25

0.25

0.25

0.25

0.25

0.2 .5

0.25

0.25

0.25

0.25

0.25

0.25

0.25

0.25

0.25

0.5

0.25

0.25

0.25

0.25

0.25 


\section{Chloride}

Unit : 'microgram/gram

E1rst Layer
BSS -021
BSS -221
BSS -411
BSS -481
BSS -511
BSS -521
BSS -561
$B S S-571$

Second Layer

BSS-022

BSS-222A

BSS-412

BSS -482

BSS-512

BSS-522

BSS -562

BSS-572

Third Layer

BSS-023

BSS-223A

BSS- 413

BSS -483

BSS-513

BSS -523

BSS -563

BSS -573

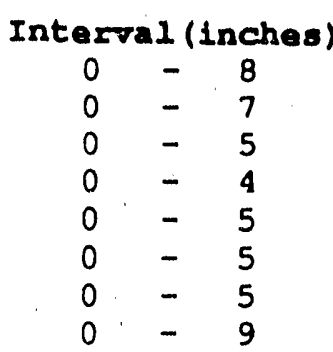

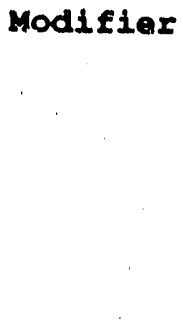
Result
5.2
2.3
5.1
6.6
2.1
4.8
118.4

$9-84$
$7-80$
$5-80$
$4-80$
$5-80$
$5-80$
$5-80$
$9-80$

LT

5.2

1.25

3.7

2. 5

2.2

3.6

5.6

LT

1.25

$110-124$
$96-120$
$96-120$
$96-120$
$96-120$
$96-120$
$96-120$
$96-120$

1.3

1.25

1.25

8.7

1.8

50.9

3.5

1.4 


\section{Eluoride}

Unit: microgram/gram

rirst Layer
BSS-021
BSS-221
BSS-411
BSS-481
BSS-511
BSS-521
BSS-561
BSS-571

\section{Second Layer}

BSS-022

BSS-222A

BSS -412

BSS -482

BSS -512

BSS- 522

BSS- 562

BSS-572

Third Iayer

BSS-023

BSS-223A

BSS -413

BSS -483

BSS -513

BSS -523

BSS -563

BSS -573

Interval (inches)
$0-8$
$0-7$
$0-5$
$0-4$
$0-5$
$0-5$
$0-5$
$0-9$

$\begin{array}{cr}\text { Modifiex } & \text { Result } \\ \text { LT } & 1.25 \\ \text { LT } & 1.25 \\ \text { LT } & 1.25 \\ \text { LT } & 1.25 \\ \text { LT } & 1.25 \\ \text { LT } & 1.25 \\ & 1.4 \\ \text { LT } & 1.25\end{array}$

$9-84$

$7-80$

$5-80$

-80
$-\quad 80$

-80
$-\quad 80$

- 80

$-80$

$-80$

LT

LT

1.25

1.25

1.6

1. 25

1.25

1.25

1.25

1.25

$110-124$
$96-120$
$96-120$
$96-120$
$96-120$
$96-120$
$96-120$
$96-120$

LT

1.25

1.25

1.25

1.25

1. 25

1.25

1.25

1.25 


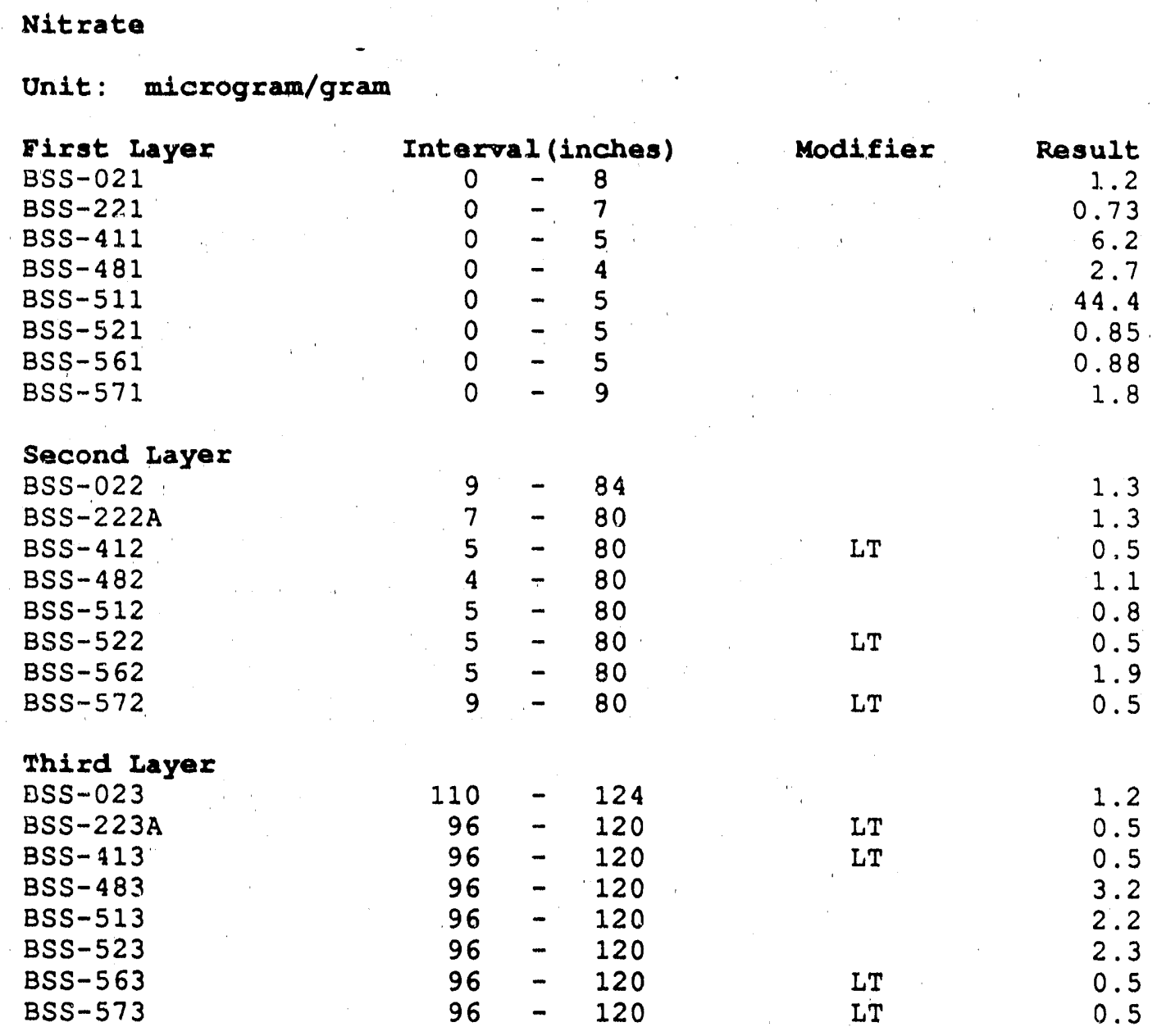


Nitrite

Unit: microgram/gram

\begin{tabular}{|c|c|c|c|c|}
\hline First Layer & Inter & I (inches) & Modifier & Result \\
\hline$B S S-021$ & 0 & -8 & LT & 1.25 \\
\hline BSS -221 & 0 & - & LT & 1.25 \\
\hline BSS -411 & 0 & -5 & LT & 1.25 \\
\hline BSS -481 & 0 & - & LT & 1.25 \\
\hline BSS-511 & 0 & 5 & LT & 1.25 \\
\hline BSS-521 & 0 & 5 & LT & 1.25 \\
\hline BSS-56I & 0 & 5 & LT & 1.25 \\
\hline BSS-571 & 0 & -9 & LT & 1.25 \\
\hline Second Layer & & & & \\
\hline BSS-022 & 9 & 84 & LT & 1.25 \\
\hline BSS-222A & 7 & 80 & LT & 1.25 \\
\hline BSS- 412 & 5 & 80 & LT & 1.25 \\
\hline BSS -482 & 4 & 80 & LT & 1.25 \\
\hline BSS-512 & 5 & 80 & $L T$ & 1.25 \\
\hline BSS -522 & 5 & 80 & LT & 1.25 \\
\hline BSS -562 & 5 & 80 & LT & 1.25 \\
\hline BSS-572 & 9 & 80 & LT & 1.25 \\
\hline Third Layer & & & & \\
\hline BSS -023 & 110 & 124 & LT & 1.25 \\
\hline$B S S-223 A$ & 96 & 120 & LT & 1.25 \\
\hline BSS-413 & 96 & 120 & LT & 1.25 \\
\hline BSS -483 & 96 & 120 & LT & 1.25 \\
\hline BSS -513 & 96 & 120 & LT & 1.25 \\
\hline BSS -523 & 96 & 120 & LT & 1.25 \\
\hline BSS -553 & 96 & $-\quad 120$ & LT & 1.25 \\
\hline BSS -573 & 96 & $-\quad 120$ & LT & 1.25 \\
\hline
\end{tabular}




\begin{tabular}{|c|c|c|c|c|}
\hline & 0 & -8 & $\begin{array}{c}\text { Modifier } \\
\text { LT }\end{array}$ & 5 \\
\hline BSS-221 & 0 & -7 & LT & 5 \\
\hline BSS-411 & 0 & -5 & LT & 5 \\
\hline BSS-481 & 0 & -4 & LT & 5 \\
\hline BSS-511 & 0 & -5 & LT & 5 \\
\hline BSS-521 & 0 & -5 & LT & 5 \\
\hline BSS-561 & 0 & -5 & LT & 5 \\
\hline BSS-571 & 0 & -9 & LT & 5 \\
\hline \multicolumn{5}{|l|}{ Second Layer } \\
\hline BSS-022 & 9 & 84 & LT & 5 \\
\hline$B S S-222 A$ & 7 & -80 & & 9.7 \\
\hline$B S S-412$ & 5 & -80 & LT & 5 \\
\hline BSS -482 & 4 & -80 & LT & 5 \\
\hline BSS-512 & 5 & 80 & LT & 5 \\
\hline BSS-522 & 5 & 80 & LT & 5 \\
\hline BSS-562 & 5 & 80 & LT & 5 \\
\hline BSS-572 & 9 & -80 & LT & 5 \\
\hline \multicolumn{5}{|l|}{ Third Layer } \\
\hline BSS-023 & 110 & $-\quad 124$ & LT & 5 \\
\hline$B S S-223 A$ & 96 & 120 & LT & 5 \\
\hline BSS-413 & 96 & 120 & $\mathrm{LT}$ & 5 \\
\hline BSS-483 & 96 & $-\quad 120$ & & 1.3 .7 \\
\hline BSS -513 & 96 & $-\quad 120$ & LT & 5 \\
\hline BSS-523 & 96 & $-\quad 120$ & LT & 5 \\
\hline BSS-563 & 96 & $-\quad 120$ & LT & 5 \\
\hline BSS-573 & 96 & $-\quad 120$ & LT & 5 \\
\hline
\end{tabular}




\section{Sulfate}

Unit: microgram/gram

Eirst Layer
BSS -021
BSS -221
BSS-411
BSS-481
BSS-511
BSS-521
BSS-561
$B S S-571$

\begin{tabular}{|c|c|c|}
\hline \multicolumn{3}{|c|}{ Interval (inches) } \\
\hline 0 & - & 8 \\
\hline 0 & - & 7 \\
\hline 0 & - & 5 \\
\hline 0 & - & 4 \\
\hline 0 & - & 5 \\
\hline 0 & - & 5 \\
\hline 0 & - & 5 \\
\hline 0 & - & 9 \\
\hline
\end{tabular}

Modifiex

$$
\begin{aligned}
& 9-84 \\
& 7-80 \\
& 5-80 \\
& 4-80 \\
& 5-80 \\
& 5-80 \\
& 5-80 \\
& 9-80
\end{aligned}
$$$$
\text { BSS-022 }
$$

BSS-222A

BSS -412

BSS -482

BSS -512

BSS -522

BSS -562

BSS -572

\section{Third Layer}

BSS-023

BSS-223A

BSS -413

BSS -483

BSS -513

BSS -523

BSS -563

BSS- 573

$110-124$
$96-120$
$96-120$
$96-120$
$96-120$
$96-120$
$96-120$
$96-120$

Result

18.1

11.2

13.5

7.6

7.1

8.1

1.1. 2

19.9

10.8

9.7

8.7

8.2

11.3

7. 4

8.2

8.3

5.5

12.

12.7

17.8

11

LT

11. 7

9.1 


\section{Cyaride}

Unit: microgram/gram

First Layor

BSS-0 41

BSS-101

BSS-111

BSS-341

BSS-401

BSS -461

BSS-501

BSS-541

\section{Second Layer}

BSS-042A

BSS-102A

BSS-112A

BSS -342

BSS -402

BSS -462

BSS -502

BSS -542

Third Layer

BSS-043A

BSS-103A

BSS -343

BSS -403

BSS -463

BSS -503

BSS -543

\section{Fourth Layer}

BSS-044A

BSS-104A

BSS-113A

BSS-114A

BSS-344

BSS -404

BSS -464

BSS-504

BSS-544

Eiftb Iayex

BSS-045A

BSS-105A

BSS-115A

BSS-345

BSS-405

BSS- 465

BSS -505

BSS-545

\begin{tabular}{|c|c|c|c|}
\hline Inter & 1 (inches) & Modifier & Result \\
\hline 0 & -6 & LT & 0.25 \\
\hline 0 & 6 & LT & 0.25 \\
\hline 0 & -6 & LT & 0.25 \\
\hline 0 & -6 & LT & 0.25 \\
\hline 0 & 8 & LT & 0.25 \\
\hline 0 & 10 & $\mathrm{LT}$ & 0.25 \\
\hline 0 & - & LT & 0.25 \\
\hline 0 & - & LT & 0.25 \\
\hline
\end{tabular}

$$
\begin{array}{r}
6-25 \\
6-16 \\
6-38 \\
6-28 \\
8-20 \\
10-22 \\
6-20 \\
6-24
\end{array}
$$

\section{LT \\ LT$$
\text { LT }
$$ \\ LT \\ LT \\ LT \\ LT \\ LT}

0.25

0.25

0.25

0.25

0.25

0.25

0.25

0.25

$$
\begin{aligned}
& 25-34 \\
& 16-34 \\
& 28-48 \\
& 20-48 \\
& 22-50 \\
& 20-34 \\
& 24-40
\end{aligned}
$$

$$
\begin{aligned}
& \text { LT } \\
& \text { LT } \\
& \text { LT } \\
& \text { LT } \\
& \text { LT } \\
& \text { LT } \\
& \text { LT }
\end{aligned}
$$

0.25

0.25

0.25

0.25

0.25

0.25

0.25

$34-65$
$34=60$
$38-60$
$60-80$
$48-60$
$48=72$
$50=63$
$34=60$
$40-68$

LT

LT

LT

LT

LT

LT

LT

LT

LT

0.25

0.25

0.25

0.25

0.25

0.25

0.25

0.25

0.25

$89-103$
$90-120$
$96-120$
$108-132$
$96-120$
$96-120$
$96-120$
$96-120$

LT

0.25

0.25

0.25

0.25

0.25

0.25

0.25

LT

LT

0.25 


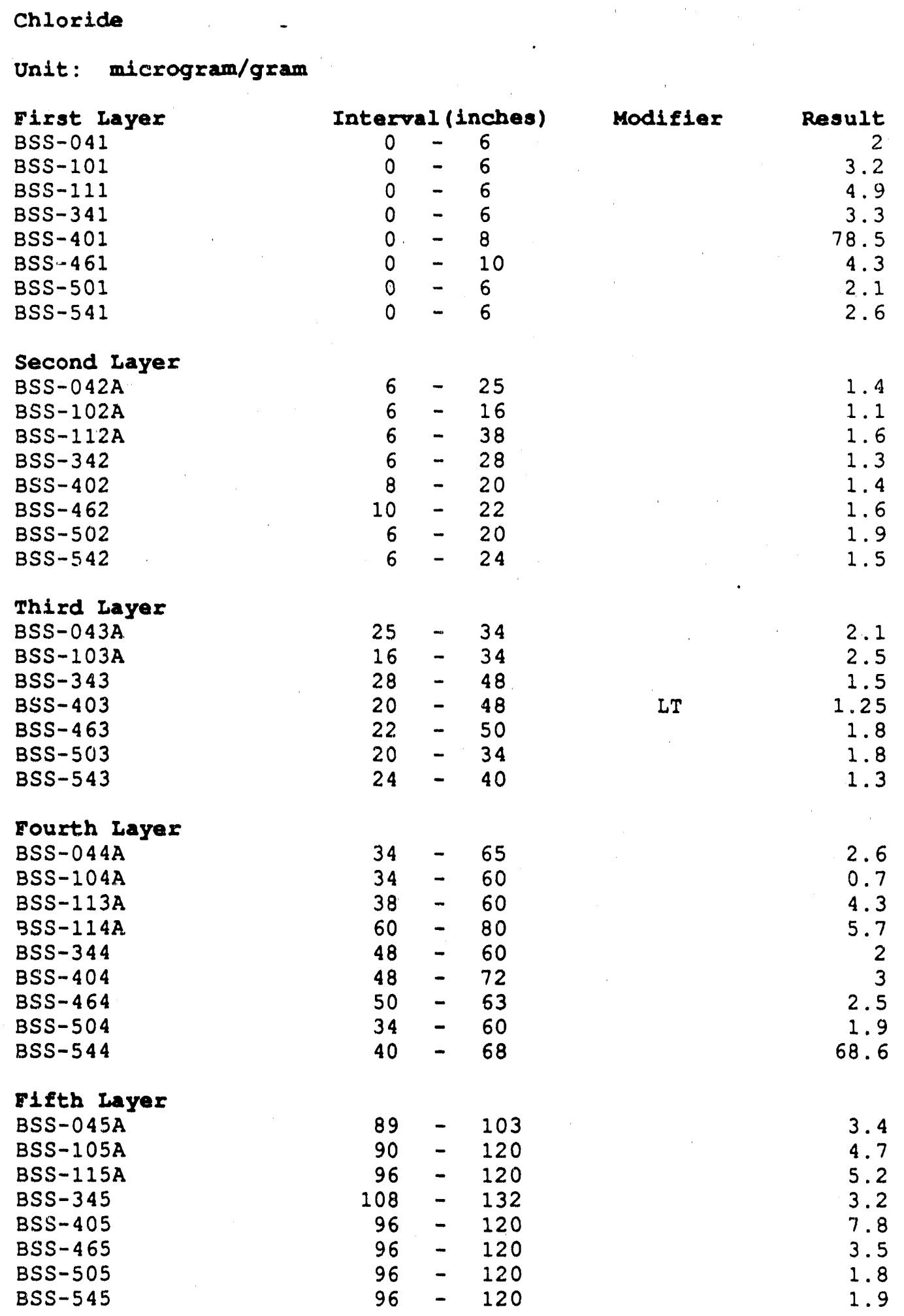




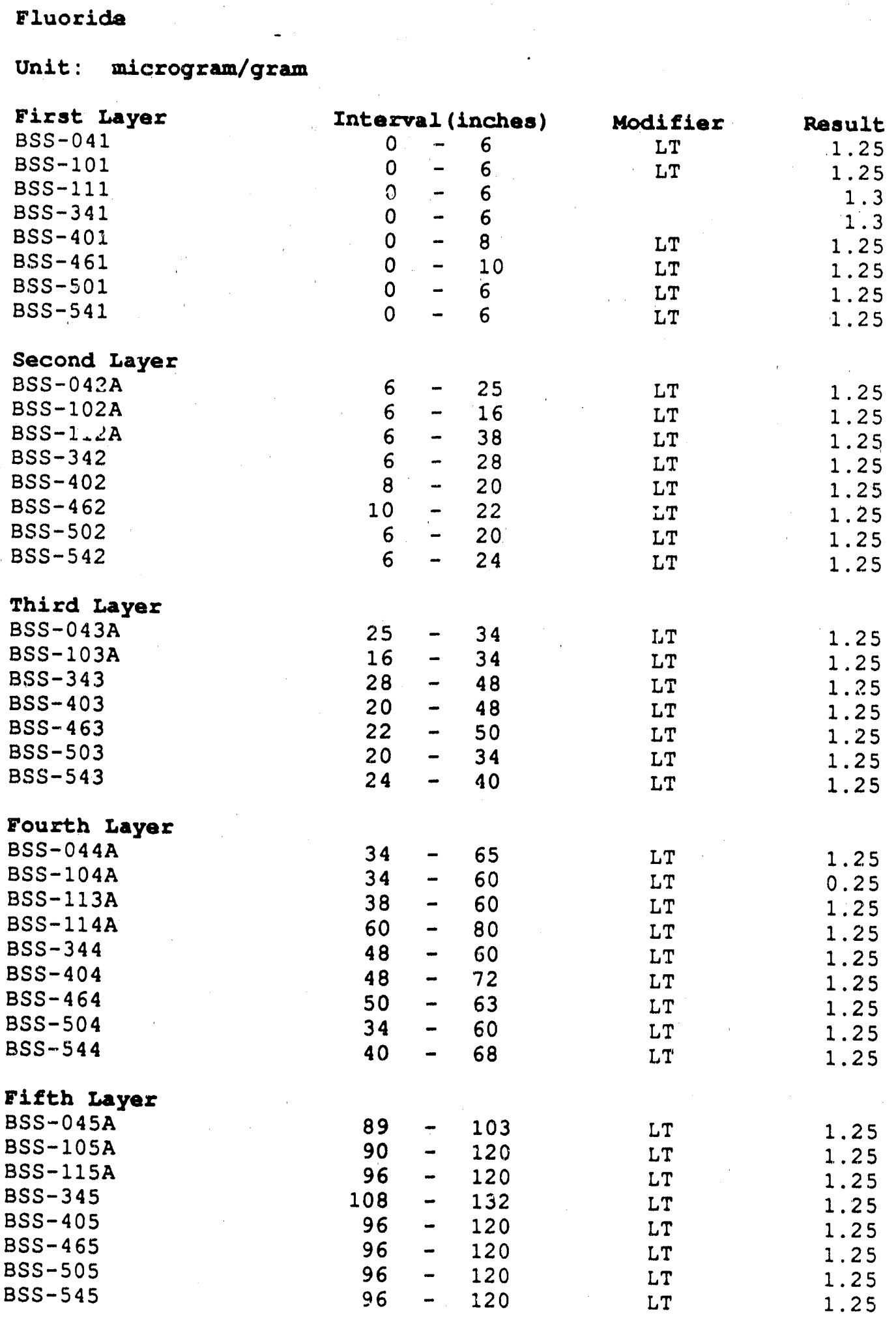




\section{Nitrate}

Unit: microgram/gram

First Layer
BSS -041
BSS -101
BSS -111
BSS -341
BSS -401
BSS -461
$B S S-501$
$B S S-541$

Second Layer BSS-042A BSS-102A BSS-112A BSS -342 BSS- 402 BSS -462 BSS-502 BSS -542

Third Layes
BSS-043A
BSS - 103A
BSS -343
BSS -403
BSS-463
BSS -503
BSS-543

Fourth Layer

BSS-044A

BSS-104A

BSS-113A

BSS-114A

BSS-344

BSS- 404

BSS-464

BSS-504

BSS-544

Iifth Iayer

$$
\begin{aligned}
& \text { BSS-045A } \\
& \text { BSS-105A } \\
& \text { BSS-115A } \\
& \text { BSS-345 } \\
& \text { BSS-405 } \\
& \text { BSS-465 } \\
& \text { BSS-505 } \\
& \text { BSS-545 }
\end{aligned}
$$

Interval (inches)
$0-6$
$0-6$
$0-6$
$0-6$
$0-8$
$0-10$
$0-6$
$0-6$

Modifier

Result

2.1

1. 1

1. 3

1.4

0.67

4. 6

22.5

LT

0.5

$$
6-25
$$

1.7

1. 5

0.99

4.2

1. 7

1. 5

3.6

2. 2

$$
\begin{aligned}
& 25-34 \\
& 16-34 \\
& 28-48 \\
& 20-48 \\
& 22-50 \\
& 20-34 \\
& 24-40
\end{aligned}
$$

0.94

1.4

1. 3

LT

0.5

0.5

2.3

1.4

$34-65$
$34-60$
$38-60$
$60-80$
$48-60$
$48-72$
$50-63$
$34-60$
$40-68$

LT

1.2

0.1

0.79

7

1.8

LT

0.5

LT

0.5

2.5

LT

0.5

$89-103$
$90-120$
$96-120$
$108-132$
$96-120$
$96-120$
$96-120$
$96-120$

0.87

0.67

0.75

1. 1

LT $\quad 0.5$

0.97

LT

0.5

0.88 


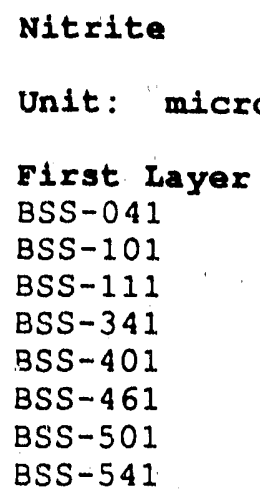

Second Layer<smiles>[14CH3]O[134Sb]</smiles>

BSS-102A

BSS-112A

BSS -342

BSS -402

BSS -462

BSS -502

BSS-542

Third Layer

BSS-043A

BSS-103A

BSS -343

BSS -403

BSS -463

BSS -503

BSS -543

Fourth Layer

BSS-044A

BSS-104A

BSS-113A

BSS-114A

BSS -344

BSS-404

BSS -464

BSS -504

BSS -544

Iifth Layer

BSS-045A

BSS-105A

BSS-115A

BSS-345

BSS -405

BSS- 465

BSS-505

BSS -545

$\begin{array}{ccr}\text { Interval (inches) } & \text { Modifier } & \text { Result } \\ 0-6 & \text { LT } & 1.25 \\ 0-6 & \text { LT } & 1.25 \\ 0-6 & \text { LT } & 1.25 \\ 0-6 & \text { LT } & 1.25 \\ 0-8 & \text { LT } & 1.25 \\ 0-10 & \text { LT } & 1.25 \\ 0-6 & \text { LT } & 1.25 \\ 0-6 & \text { LT } & 1.25\end{array}$

1.25

1.25

1.25

1.25

1.25

1.25

1.25

1.25
1.25

1.25

1.25

1.25

1.25

1.25

1.25

LT

1.25

0.25

1.25

1.25

1.25

1.25

1.25

1.25

1.25

LT

$\begin{array}{rlll}89 & -103 & \text { LT } & 1.25 \\ 90 & -120 & \text { LT } & 1.25 \\ 96-120 & \text { LT } & 1.25 \\ 108-132 & \text { LT } & 1.25 \\ 96-120 & \text { LT } & 1.25 \\ 96-120 & \text { LT } & 1.25 \\ 96-120 & \text { LT } & 1.25 \\ 96-120 & \text { LT } & 1.25\end{array}$




\begin{tabular}{|c|c|c|c|c|}
\hline & Intarts & 1 (inches) & Modifier & Result \\
\hline BSS-041 & 0 & -6 & $L T$ & 5 \\
\hline BSS-101 & 0 & -6 & LT & 5 \\
\hline BSS-111 & 0 & -6 & $\mathrm{LT}$ & 5 \\
\hline BSS -341 & 0 & -6 & LT & 5 \\
\hline BSS-401 & 0 & -8 & & 8 \\
\hline$B S S-461$ & 0 & -10 & LT & 5 \\
\hline BSS-501 & 0 & -6 & & 8.3 \\
\hline BSS-541 & 0 & -6 & LT & 5 \\
\hline Second Layer & & & & \\
\hline$B S S-042 A$ & 6 & 25 & LT & 5 \\
\hline$B S S-102 A$ & 6 & -16 & LT & 5 \\
\hline BSS-112A & 6 & 38 & $\mathrm{LT}$ & 5 \\
\hline BSS-342 & 6 & 28 & LT & 5 \\
\hline BSS-402 & 8 & -20 & LT & 5 \\
\hline BSS -462 & 10 & 22 & LT & 5 \\
\hline BSS -502 & 6 & 20 & & 9.8 \\
\hline BSS - 542 & 6 & $-\quad 24$ & LT & 5 \\
\hline Third Layer & & & & \\
\hline BSS-043A & 25 & 34 & LT & 5 \\
\hline BSS-103A & 16 & $-\quad 34$ & LT & 5 \\
\hline BSS-343 & 28 & -48 & LT & 5 \\
\hline BSS -403 & 20 & 48 & LT & 5 \\
\hline BSS -463 & 22 & 50 & LT & 5 \\
\hline BSS-503 & 20 & $-\quad 34$ & LT & 5 \\
\hline$B S S-543$ & 24 & -40 & LT & 5 \\
\hline Fourth Laye & & & & \\
\hline$B S S-044 A$ & 34 & 65 & LT & 5 \\
\hline BSS-104A & 34 & -60 & LT & 0.25 \\
\hline BSS-113A & 38 & 60 & LT & 5 \\
\hline BSS-114A & 60 & 80 & LT & 1 \\
\hline BSS-344 & 48 & -60 & LT & 5 \\
\hline BSS-404 & 48 & $-\quad 72$ & LT & 5 \\
\hline BSS -464 & 50 & -63 & LT & 5 \\
\hline BSS -504 & 34 & -60 & LT & 5 \\
\hline ESS-544 & 40 & -68 & LT & 5 \\
\hline Eifth Layer & & & & \\
\hline$B S S-045 A$ & 89 & 103 & $L T$ & 5 \\
\hline BSS-105A & 90 & 120 & LT & 5 \\
\hline BSS-115A & 96 & 120 & $L T$ & 5 \\
\hline BSS-345 & 108 & 132 & LT & 5 \\
\hline BSS-405 & 96 & $-\quad 120$ & LT & 5 \\
\hline BSS-465 & 96 & $-\quad 120$ & LT & 5 \\
\hline BSS-505 & 96 & $-\quad 120$ & LT & 5 \\
\hline BSS -545 & 96. & $-\quad 120$ & LT & 5 \\
\hline
\end{tabular}




\section{Sulfate}

Unit: microgram/gram

\section{First Layer}

BSS-041

BSS-101

BSS-111

BSS-341

BSS-401

BSS -461

BSS -501

BSS-541

Second Layer

BSS-042A

BSS-102A

BSS-112A

BSS -342

BSS -402

BSS -462

BSS -502

BSS-542

Third Layer

BSS-043A

BSS-103A

BSS -343

BSS -403

BSS -463

BSS -503

BSS -543

\section{Fourth Layer}

BSS-044A

BSS-104A

BSS-113A

BSS-114A

BSS-344

BSS-404

BSS -464

BSS-504

BSS-544

Eifth Layer

BSS-045A

BSS-105A

BSS-115A

BSS- 345

BSS -405

BSS -465

BSS -505

BSS -545

$\begin{array}{rrr}\text { Interval (inchas) } & \text { Modifier } & \text { Result } \\ 0 & -6 & 5.8 \\ 0 & -6 & 11.4 \\ 0 & -6 & 11.5 \\ 0 & -6 & 12.6 \\ 0 & -8 & 7.9 \\ 0 & -10 & 14.3 \\ 0 & -6 & 9.8 \\ 0 & -6 & 5.9\end{array}$

14.6

15.2

20.7

17.4

20.6

16.5

20.2

14.1

14.2

12.8

18.5

15.3

16.1

14.5

18.1

$34-65$
$34-60$
$38-60$
$60-80$
$48-60$
$48-72$
$50-63$
$34-60$
$40-68$

LT

LT

5

1

2. 8

21.4

6.8

17

12.6

LT

5

$89-103$
$90-120$
$96-120$
$108-132$
$96-120$
$96-120$
$96-120$
$96-120$

LT

LT

LT

LT

LT

LT
5
4.9
5
5
6.8
5
5
5 


\section{Cyanide}

Unit: microgram/gram

First Layer
BSS -131
BSS -132
BSS -201 A
BSS -301
BSS -311
BSS -361
BSS -531
BSS -551
BSS -581

Second Layer

BSS -133

BSS -202 A

BSS -302

BSS -312

BSS -362

BSS $-5 j 2$

BSS -552

BSS -582

\section{Third Layer}

BSS -203A

BSS -303

BSS -313

BSS -363

BSS -533

BSS -553

BSS -583

Interval (Inches)
$0=12$
$12=24$
$0=24$
$0=24$
$0=24$
$0=24$
$0=24$
$0-24$
$0-24$

Modifier
LT
LT
LT
LT
LT
LT
LT
LT
LT

Result

0.25

0.25

0.25

0.25

0.25

0.25

0.25

0.25

0.25

$24-36$
$24-48$
$24-48$
$24-48$
$24-48$
$24-48$
$24-48$
$24-48$

LT

LT

LT

LT

LT

LT

LT

LT

0.25

0.25

0.25

0.25

0.25

0.25

0.25

0.25

$48-72$
$48-72$
$48-72$
$48-72$
$48-72$
$48-72$
$48-72$

LT

LT

LT

IT

LT

LT

LT

0.25

0.25

0.25

0.25

0.25

0.25

0.25 


\section{Chloride}

Unit: microgram/gram

First Layer
BSS -131
BSS -132
BSS -201 A
BSS -301
BSS -311
BSS -361
BSS -531
BSS -551
BSS -581

$\begin{array}{cc}\text { Interval (inches) } & \text { Modifier } \\ 0-12 & \text { LT } \\ 12-24 & \text { LT } \\ 0-24 & \\ 0-24 & \\ 0-24 & \\ 0-24 & \\ 0-24 & \\ 0-24 & \\ 0-24 & \end{array}$

Result

1.25

1.25

3,5

4

2.7

7

5.4

2.4

Second Layer

BSS-133

BSS-202A

BSS -302

BSS -312

BSS -362

BSS -532

BSS -552

BSS -582

$24-36$
$24-48$
$24-48$
$24-48$
$24-48$
$24-48$
$24-48$
$24-48$

LT

1.25

5.4

2. 9

10.2

1.9

10.5

19.7

4.4

Third Layex

BSS-203A

$48-72$
$48-72$
$48-72$
$48-72$
$48-72$
$48-72$
$48-72$

4. 1

3. 5

5.7

2.1

5. 4

3. 3 


\section{Fluoride}

Unit: microgram/gram

\begin{tabular}{|c|c|c|c|c|}
\hline Firat Layer & Inter & 1 (inches) & Modifier & Rasult \\
\hline BSS -131 & 0 & -12 & LT & 1.25 \\
\hline BSS-132 & 12 & 24 & LT & 1.25 \\
\hline$B S S-201 A$ & 0 & $-\quad 24$ & LT & 1.25 \\
\hline BSS-301 & 0 & 24 & LT & 1.25 \\
\hline BSS-311 & 0 & 24 & LT & 1.25 \\
\hline BSS-361 & 0 & -24 & LT & 1.25 \\
\hline BSS-531 & 0 & 24 & LT & 1.25 \\
\hline BSS-551 & 0 & 24 & LT & 1.25 \\
\hline BSS -581 & 0 & $-\quad 24$ & & 1.3 \\
\hline Second Layer & & & & \\
\hline BSS-133 & 24 & 36 & LT & 1.25 \\
\hline$B S S-202 A$ & 24 & 48 & LT & 1.25 \\
\hline BSS-302 & 24 & -48 & LT & 1.25 \\
\hline$B S S-312$ & 24 & -48 & LT & 1.25 \\
\hline BSS -362 & 24 & -48 & LT & 1.25 \\
\hline BSS -532 & 24 & -48 & LT & 1.25 \\
\hline$B S S-552$ & 24 & -48 & LT & 1.25 \\
\hline BSS -582 & 24 & -48 & LT & 1.2 \\
\hline Third Layer & & & & \\
\hline$B S S-203 A$ & 48 & 72 & LT & 1.25 \\
\hline BSS -303 & 48 & 72 & LT & 1.25 \\
\hline BSS-313 & 48 & 72 & LT & 1.2 \\
\hline BSS-363 & 48 & - 72 & LT & 1.2 \\
\hline BSS -533 & 48 & $-\quad 72$ & LT & 1.2 \\
\hline BSS-553 & 48 & -72 & LT & 1.2 \\
\hline BSS -583 & 48 & -72 & LT & 1.25 \\
\hline
\end{tabular}




\begin{tabular}{|c|c|c|c|c|}
\hline BSS-131 & 0 & $-\quad 12$ & & 7.1 \\
\hline BSS-132 & 12 & $-\quad 24$ & $\mathrm{LT}$ & 0.5 \\
\hline BSS-201A & 0 & $-\quad 24$ & LT & 0.5 \\
\hline BSS-301 & 0 & $-\quad 24$ & LT & 0.5 \\
\hline BSS-311 & 0 & $-\quad 24$ & LT & 0.5 \\
\hline BSS-361 & 0 & $-\quad 24$ & & 2.4 \\
\hline BSS-531 & 0 & $-\quad 24$ & & 2.4 \\
\hline BSS-551 & 0 & -24 & & 2.8 \\
\hline BSS-581 & 0 & -24 & LT & 0.5 \\
\hline \multicolumn{5}{|l|}{ Second Layer } \\
\hline BSS -133 & 24 & 36 & LT & 0.5 \\
\hline BSS-202A & 24 & -48 & $\mathrm{LT}$ & 0.5 \\
\hline BSS-302 & 24 & -48 & & 8.9 \\
\hline BSS-312 & 24 & -48 & LT & 0.5 \\
\hline BSS-362 & 24 & -48 & LT & 0.5 \\
\hline BSS-532. & 24 & $-\quad 48$ & & 2.2 \\
\hline BSS-552 & 24 & -48 & & 8.4 \\
\hline BSS-582 & 24 & -48 & & 1.5 \\
\hline \multicolumn{5}{|l|}{ Third Layer } \\
\hline$B S S-203 A$ & 48 & -72 & & 0.93 \\
\hline BSS-303 & 48 & -72 & LT & 0.5 \\
\hline BSS-313 & 48 & $-\quad 72$ & LT & 0.5 \\
\hline BSS -363 & $\therefore 3$ & -72 & & 1.2 \\
\hline BSS -533 & 48 & -72 & & 2 \\
\hline BSS - 553 & 48 & -72 & & 3.2 \\
\hline BSS - 583 & 48 & -72 & & 1.1 \\
\hline
\end{tabular}




\section{Nitrite}

Unit: microgram/gram

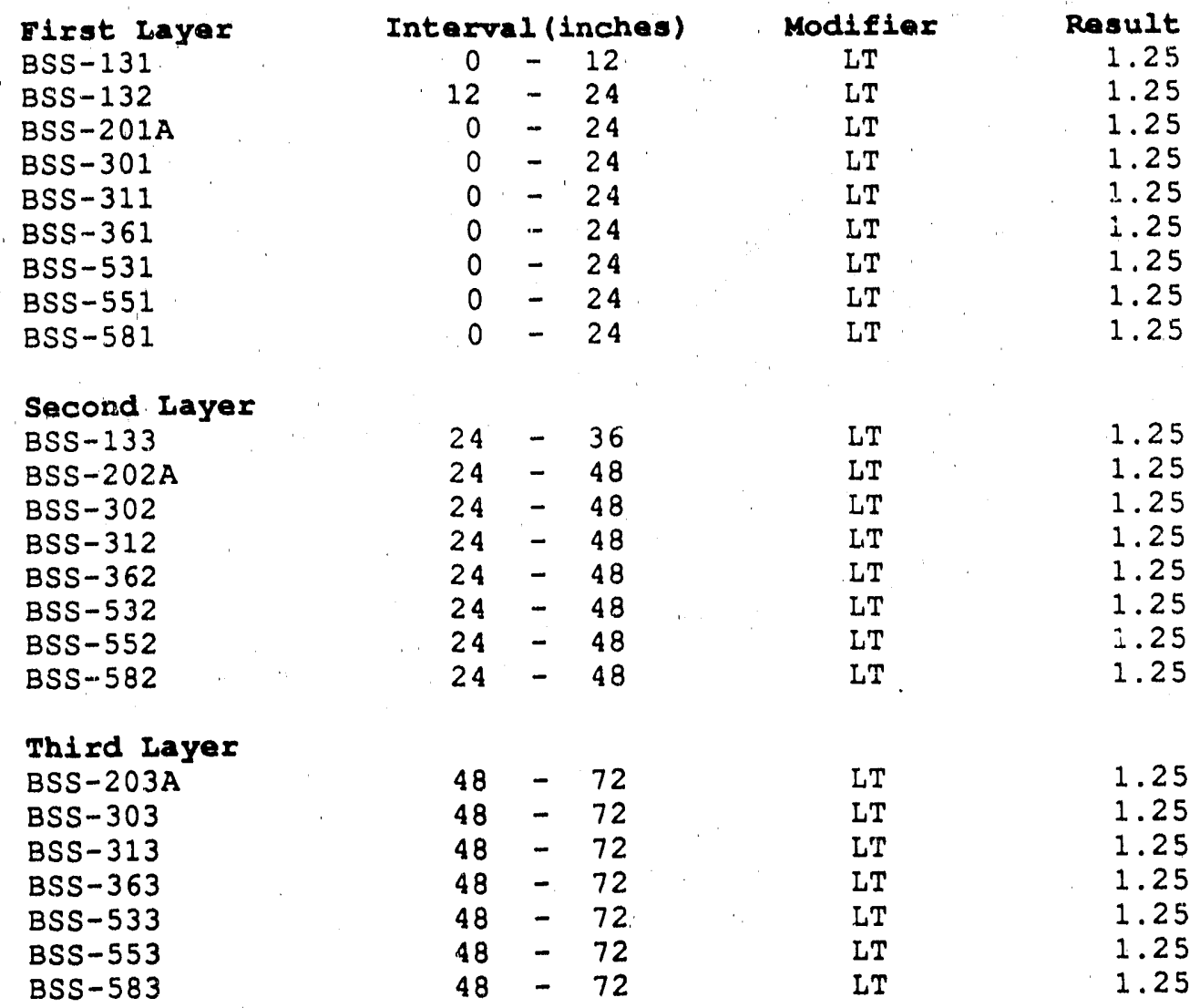


Rhosphate

Unit: microgram/gram

First wayer
BSS-131
BSS-132
BSS-201A
BSS-301
BSS-311
BSS-361
BSS-531
BSS-551
BSS-581

Second Iayex

BSS-133

BSS-202A

BSS-302

BSS-312

BSS-362

BSS- 532

BSS-552

BSS-582

\section{Third Inyer}

BSS-203A

BSS -303

BSS-313

BSS-363

BSS- 533

BSS-553

BSS-583

$\begin{array}{cc}\text { Interval (inches) } & \text { Modifier } \\ 0-12 & \text { LT } \\ 12-24 & \text { LT } \\ 0-24 & \text { LT } \\ 0-24 & \text { LT } \\ 0-24 & \text { LT } \\ 0-24 & \text { LT } \\ 0-24 & \text { LT } \\ 0-24 & \text { LT } \\ 0-24 & \text { LT }\end{array}$

Result

$24-36$
$24-48$
$24-48$
$24-48$
$24-48$
$24-48$
$24-48$
$24-48$

LT

LT

LT

LT

LT

LT

LT

LT

5
5
5
5
5
5
5
5
5

$48-72$
$48-72$
$48-72$
$48-72$
$48-72$
$48-72$
$48-72$

LT

LT

LT

LT

LT

LT

LT

$$
\begin{aligned}
& 5 \\
& 5 \\
& 5 \\
& 5 \\
& 5 \\
& 5 \\
& 5 \\
& 5
\end{aligned}
$$

1
5
5
5
5
5
5 


\begin{tabular}{|c|c|c|c|c|}
\hline \multicolumn{5}{|l|}{ Sulfate } \\
\hline \multicolumn{5}{|c|}{ Unit: microgram/gram } \\
\hline First Layer & \multicolumn{2}{|c|}{ Interval (inches) } & Modifier & Result \\
\hline BSS-131 & 0 & -12 & LT & 5 \\
\hline BSS-132 & 12 & 24 & LT & 5 \\
\hline BSS-201A & 0 & 24 & & 9.7 \\
\hline BSS -301 & 0 & $-\quad 24$ & & 6.2 \\
\hline BSS -311 & 0 & -24 & & 8 \\
\hline BSS -361 & 0 & -24 & LT & 5 \\
\hline BSS-531 & 0 & 24 & & 5 \\
\hline BSS-551 & 0 & 24 & LT & 5 \\
\hline$B S S-581$ & 0 & -24 & & 21.2 \\
\hline \multicolumn{5}{|l|}{ Second Layer } \\
\hline BSS-133 & 24 & -36 & LT & 5 \\
\hline$B S S-202 A$ & 24 & 48 & LT & 5 \\
\hline BSS-302 & 24 & -48 & LT & 5 \\
\hline BSS -312 & 24 & -48 & LT & 5 \\
\hline BSS-362 & 24 & -48 & LT & 5 \\
\hline BSS -532 & 24 & 48 & & 5.3 \\
\hline BSS -552 & 24 & -48 & & 5.3 \\
\hline BSS - 582 & 24 & -48 & & 7.5 \\
\hline \multicolumn{5}{|l|}{ Third Layer } \\
\hline BSS-203A & 48 & & & 2.6 \\
\hline BSS-303 & 48 & -72 & & 11.7 \\
\hline BSS -313 & 48 & -72 & LT & 5 \\
\hline BSS-363 & 48 & -72 & LT & 5 \\
\hline BSS -533 & 48 & -72 & LT & 5 \\
\hline BSS -553 & 48 & 72 & LT & 5 \\
\hline$B S S-583$ & 48 & 72 & LT & 5 \\
\hline
\end{tabular}




\section{Cyanide}

Unit: microgram/gram

First Layer

BSS-121

BSS-241

BSS -471

BSS-591

Second Layer

BSS-122

BSS -242

BSS -472

BSS -592

Third Layer

BSS-123

BSS -243

BSS -473

BSS -593

Fourth Layer

BSS-124

BSS -244

BSS -474

BSS-594

Interval (inches)
$0-23$
$0-22$
$0-12$
$0-23$

Modifier
LT
LT
LT
LT

Result

0.25

0.25

0.25

0.25

$$
\begin{aligned}
& 23-65 \\
& 22-59 \\
& 12-38 \\
& 23-50
\end{aligned}
$$

LT

LT

LT

LT

0.25

0.25

0.25

0.25

$65-82$
$59-74$
$38-72$
$50-72$

LT

LT

LT

LT

0.25

0.25

0.25

0.25

$95-119$
$96-120$
$96-120$
$96-120$

LT

LT

LT

LT

0.25

0.25

0.25

0.25 
Chloride

Unit: microgram/gram

First Layer

BSS-121

BSS-241

BSS -471

BSS-591

\section{Second Layer}

BSS -122

BSS-242

BSS -472

BSS -592

Third Layer

BSS -123

BSS-243

BSS -473

BSS-593

Fourth Layer

BSS- 124

BSS-244

BSS- 474

BSS-594
Interval (inches)

$0-23$

$0-22$

$0-12$

$0-23$

$\begin{array}{cr}\text { Modifier } & \text { Result } \\ & 1.8 \\ \text { LT } & 1.25 \\ & 5.1 \\ & 3.5\end{array}$

$23-65$
$22-59$
$12-38$
$23-50$

LT

1.25

3.7

7.1

1.6

$$
\begin{aligned}
& 65-82 \\
& 59-74 \\
& 38-72 \\
& 50-72
\end{aligned}
$$

1.4

4.1

70.7

1.8

$$
\begin{aligned}
& 95-119 \\
& 96-120 \\
& 96-120 \\
& 96-120
\end{aligned}
$$

1. 6

3. 6

7. 1 


\section{Vaucluse Soil Series}

\section{ruoride}

Unit: microgram/gram

First Layer

BSS-121

BSS -241

BSS-471

BSS- 591

Second Iayer

BSS- 122

BSS -242

BSS -472

BSS -592

Third Layer

BSS- 123

BSS -243

BSS -473

BSS- 593

Fourth Layer

BSS-124

BSS-244

BSS- 474

BSS-594
Interval (inches)
$0-23$
$0-22$
$0-12$
$0-23$

$23-65$

$22-59$

$12-38$

$23-50$

$65-82$

$59-74$

$38-72$

$50-72$

$95-119$

$96-120$

$96-120$

$96-120$
Modifler

LT

LT

LT

LT

LT

LT

LT

LT

LT

LT

LT

LT
Result

1.25

1. 25

1.5

1.25

1.25

1. 25

2.7

1.25

1. 25

1. 25

2.2

1.25

1.25

1.25

1.3

1.25 


\section{Vaucluse Soil Series}

\begin{tabular}{|c|c|c|c|c|}
\hline & daxcon & 1 (1nches) & Modifier & Result \\
\hline $\begin{array}{l}\text { BSS-121 } \\
\text { BSS-241 }\end{array}$ & 0 & 23 & & 3 \\
\hline BSS-241 & 0 & -22 & $\mathrm{LT}$ & 0.5 \\
\hline BSS-471 & 0 & $-\quad 12$ & & 2.4 \\
\hline BSS-591 & 0 & -23 & $\mathrm{LT}$ & 0.5 \\
\hline \multicolumn{5}{|l|}{ Second Layer } \\
\hline BSS-122 & 23 & 65 & LT & 0.5 \\
\hline BSS -242 & 22 & 59 & LT & 0.5 \\
\hline BSS -472 & 12 & 38 & & 1.8 \\
\hline BSS-592 & 23 & 50 & & 2.1 \\
\hline \multicolumn{5}{|l|}{ Third Layer } \\
\hline BSS -123 & 65 & $-\quad 82$ & & 0.59 \\
\hline$B S S-243$ & 59 & -74 & LT & 0.5 \\
\hline BSS -473 & 38 & -72 & & 1.1 \\
\hline BSS-593 & 50 & 72 & & 4.7 \\
\hline \multicolumn{5}{|l|}{ Fourth Layer } \\
\hline BSS-124 & 95 & $-\quad 119$ & LT & 0.5 \\
\hline BSS-244 & 96 & $-\quad 120$ & LT & 0.5 \\
\hline BSS -474 & 96 & 120 & & 1.2 \\
\hline BSS-594 & 96 & -120 & LT & 0.5 \\
\hline
\end{tabular}




\section{Nitrite}

Unit: microgram/gram

Fixst Iayer

BSS- 121

BSS-241

BSS-471

BSS-591

Second Layer

BSS- 122

BSS-242

BSS -472

BSS -592

Third Layer

BSS-123

BSS -243

BSS -473

BSS -593

\section{Eourth Layer}

BSS -124

BSS -244

BSS -474

BSS -594

Interval (inches)
$0-23$
$0-22$
$0-12$
$0-23$

$23-65$
$22-59$
$12-38$
$23-50$

$65-82$
$59-74$
$38-72$
$50-72$

$95-119$

$96-120$

$96-120$

$96-120$

Modifier
LT
LT
LT
LT

Result

1.25

1.25

1.25

1.25

1.25

1.25

1.25

1.25

LT

LT

LT

LT

LT

1.25

1.25

1.25

1. 25

1.25

1.25

1.25

1.25 


\section{Phosphate}

Unit: microgram/gram

First Layer

BSS-121

BSS-241

BSS -471

BSS-591

Second Layer

BSS -122

BSS-242

BSS -472

BSS- 592

Third Layer

BSS- 123

BSS-24.3

BSS -473

BSS -593

Fourth Layer

BSS-124

BSS-244

BSS- 474

BSS-594

Interval (Inches)
$0-23$
$0-22$
$0-12$
$0-23$

$\operatorname{Modifier}$
LT
LT
LT

Result

5

7.5

5

$23-65$
$22-59$
$12-38$
$23-50$

LT

LT

LT

LT

5
5
5
5

$65-82$

$59-74$

$38-72$

$50-72$

LT

LT

LT

LT

$95-119$

$96-120$

$96-120$

$96-120$

LT

LT

LT

LT
5
5
5
5

5
5
5
5 


\begin{tabular}{|c|c|c|c|c|}
\hline First Layer & Interv: & IInches) & Modifier & Rasult \\
\hline$B S S-121$ & 0 & -23 & & 19.7 \\
\hline BSS-241 & 0 & $-\quad 22$ & & 9.5 \\
\hline BSS-471 & 0 & $-\quad 12$ & & 25.1 \\
\hline BSS-591 & 0 & -23 & & 16.3 \\
\hline \multicolumn{5}{|l|}{ Second Layer } \\
\hline BSS-122 & 23 & -65 & LT & 5 \\
\hline BSS -242 & 22 & -59 & LT & 5 \\
\hline BSS -472 & 12 & $-\quad 38$ & LT & 5 \\
\hline BSS-592 & 23 & -50 & & 5.3 \\
\hline \multicolumn{5}{|l|}{ Third Layex } \\
\hline BSS-123 & 65 & $-\quad 82$ & LT & 5 \\
\hline BSS -243 & 59 & -74 & LT & 5 \\
\hline BSS -473 & 38 & -72 & & 21.4 \\
\hline BSS -593 & 50 & -72 & LT & 5 \\
\hline \multicolumn{5}{|l|}{ Fourth Layer } \\
\hline BSS-124 & 95 & $-\quad 119$ & LT & 5 \\
\hline BSS -244 & 96 & $-\quad 120$ & LT & 5 \\
\hline BSS-474 & 96 & $-\quad 120$ & LT & 5 \\
\hline BSS-594 & 96 & -120 & LT & 5 \\
\hline
\end{tabular}


GEOCHEMICAL AND PHYSICAL PROPERTIES OF SOILS AND SHALLOW SEDIMENTS AT THE SAVANNAH RIVER SITE

\author{
Appendix C.3
}

Organic Data Summary 
Total Organic Carbon

Unit: microgram/gram

First Layar
BSS-011
BSS-061
BSS-091
BSS -231
BSS-331
BSS -351
BSS -431
BSS -451

IntervaI (inches)
$0-7$
$0-6$
$0-5$
$0-7$
$0-8$
$0-7$
$0-6$
$0-5$

Result
5870
3800
3080
3435
4669
8842
3556
4785

Second Layer

BSS-0 012

BSS-062

BSS-092A

BSS -232

BSS -332

BSS -352

BSS -432

BSS -452
$10-65$
$6 \div 50$
$5-52$
$7-61$
$8-72$
$7-60$
$6-67$
$5-59$

452

1630

1360

300

4838

857

2130

563

Third Iayer

BSS -0.13

BSS-063

BSS-093A

BSS-233

BSS- 333

BSS -353

BSS -433

BSS-453

\section{Fourth Layer}

BSS-014

BSS-064

BSS-094A

111 - 124

284

$96-120$

322

$96-120$

BSS-2.34

BSS-334

$96-120$

$96-120$

380

270

$96-120$

506

BSS-434

BSS-454

$96-120$

573

860

$96-120$

334 
Total Organic Halogena

Undt: microgram/gram

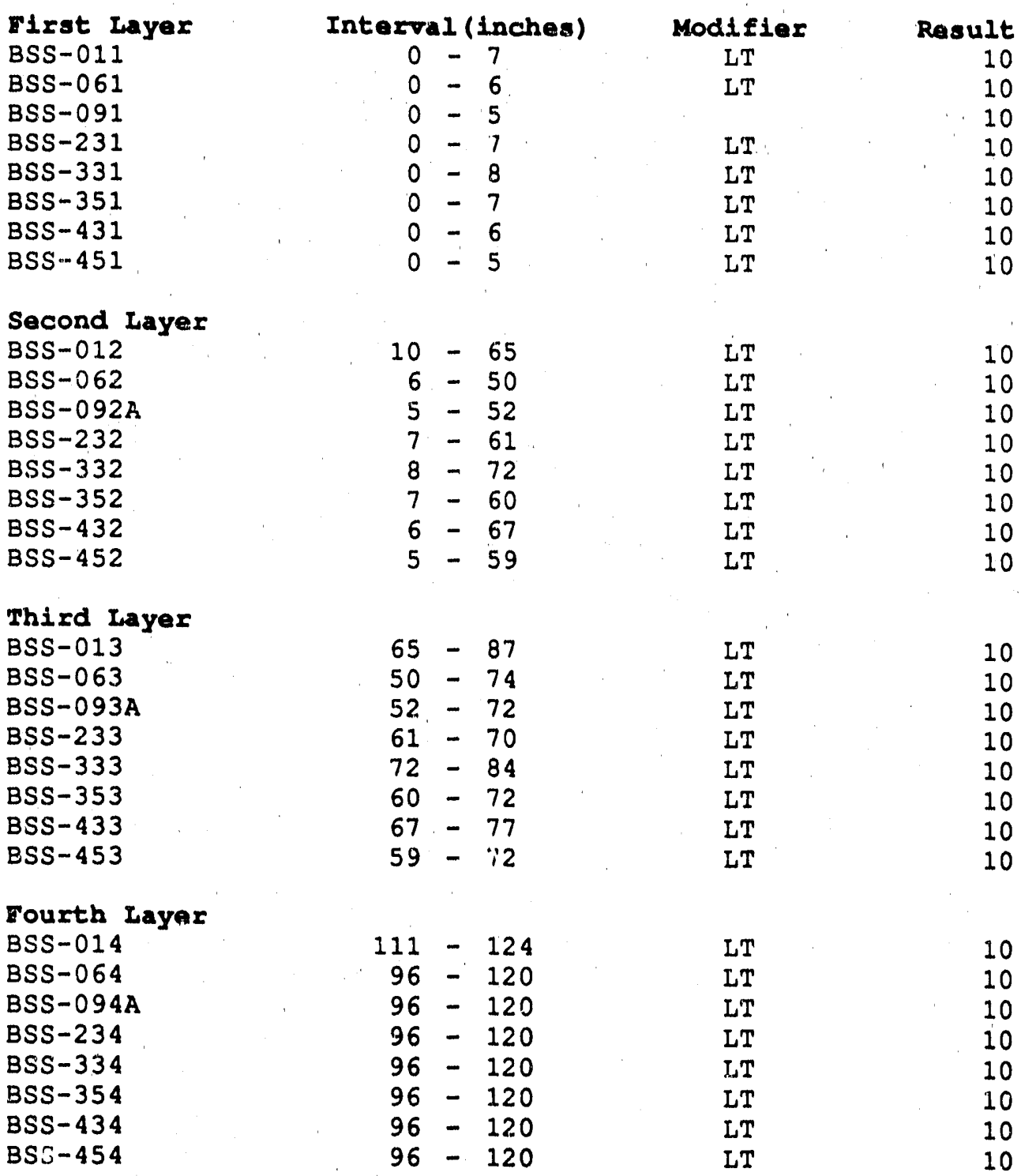


Total Organic Carbon

Unit: microgram/gram

rirst Layer
BSS-031
BSS-071
BSS-081
BSS-211
BSS-321
BSS-421
BSS-441
BSS -491

Second Layer

BSS -032

BSS-072A

BSS-082

BSS $-212 \mathrm{~A}$

BSS -322

BSS -422

BSS -442

BSS -492

\section{Third Layer}

BSS-033

BSS-073A

BSS -083

BSS-213A

BSS -323

BSS -423

BSS -443

BSS -493

Fourth Layex

BSS -034

BSS-074A

BSS -084

BSS -214 A

BSS -324

BSS -424

BSS -444

BSS -494
Interval (inches)

$0=6$
$0=8$
$0=6$
$0=5$
$0=6$
$0=9$
$0=7$
$0-9$

Modiflex

Result

3260

4230

3910

4618

5336

11090

13146

2957

$$
\begin{array}{r}
10-39 \\
8-36 \\
6=36 \\
5=47 \\
6-49 \\
9=45 \\
7=38 \\
9-41
\end{array}
$$

597

540

1270

557

301

936

1172

471

$39-72$
$36-72$
$36-72$
$47-77$
$49-72$
$45=72$
$38=70$
$41-72$

646

400

420

394

1134

545

1330

764

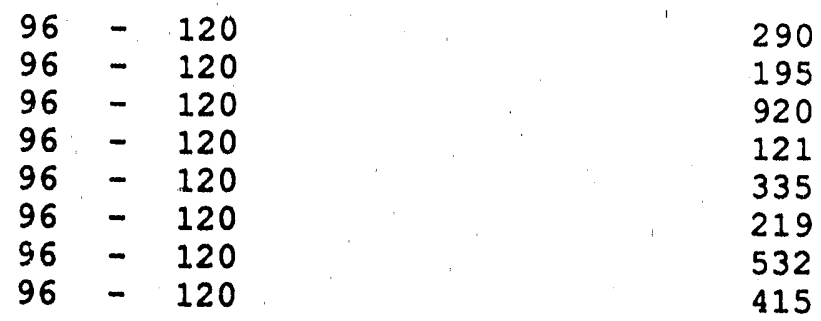


Total Organic Halogens

Unit: microgram/gram

Firat Layer
BSS-031
BSS-071
BSS-081
BSS-211
BSS-321
BSS-421
BSS -441
BSS -491

Second Layex BSS -032

BSS-072A

BSS-082

BSS-212A

BSS -322

BSS -422

BSS -442

BSS -492

Third Layer

BSS-033

BSS-073A

BSS -083

BSS $-213 A$

ESS -323

BSS -423

BSS -443

BSS -493

Fourth Layer

BSS-034

BSS-074A

BSS-084

BSS-214A

BSS- 324

BSS -424

BSS -444

BSS -494

Interval (inchea)
$0-6$
$0-8$
$0-6$
$0-5$
$0-6$
$0-9$
$0-7$
$0-9$

Modifiar

LT

LT

LT

LT

LT

LT

LT

LT

Result

10

10

10

10

10

10

10

10

10

10

$$
10
$$

10

10

$$
10
$$

- 38

- 41

10

$\begin{array}{llll}39-72 & \text { LT } & 10 \\ 36-72 & \text { LT } & 10 \\ 36-72 & \text { LT } & 10 \\ 47-77 & \text { LT } & 10 \\ 49-72 & \text { LT } & 10 \\ 45-72 & \text { LT } & 10 \\ 38-70 & \text { LT } & 10 \\ 41-72 & \text { LT } & 10\end{array}$

$\begin{array}{lll}96-120 & \text { LT } & 10 \\ 96-120 & \text { LT } & 10 \\ 96-120 & \text { LT } & 10 \\ 96-120 & \text { LT } & 10 \\ 96-120 & \text { LT } & 10 \\ 96-120 & \text { LT } & 10 \\ 96-120 & \text { LT } & 10 \\ 96-120 & \text { LT } & 10\end{array}$


Lakeland Soil Series

Total Organic Carbon

Unit: microgram/gram

Pirst Inyer

BSS-021

BSS-221

BSS -411

BSS -481

BSS -511

BSS-521

BSS-561

BSS -571

Second Layer

BSS-022

BSS-222A

BSS -412

BSS -482

BSS -512

BSS -522

BSS -562

BSS -572

Third Iayer

BSS-023

BSS-223A

BSS -413

BSS -483

BSS -513

BSS -523

BSS -563

BSS -573

\begin{tabular}{|c|c|c|c|}
\hline \multicolumn{2}{|c|}{ Interval (inches) } & Modifiex & Result \\
\hline 0 & -8 & & 7770 \\
\hline 0 & -7 & & 2573 \\
\hline 0 & -5 & & 6760 \\
\hline 0 & -4 & & 4092 \\
\hline 0 & -5 & & 908 \\
\hline 0 & -5 & & 8443 \\
\hline 0 & -5 & & 14493 \\
\hline 0 & -9 & & 12426 \\
\hline
\end{tabular}

$$
\begin{aligned}
& 9-84 \\
& 7-80 \\
& 5-80 \\
& 4-80 \\
& 5-80 \\
& 5-80 \\
& 5-80 \\
& 9-80
\end{aligned}
$$

477

220

604

409

4555

436

300

446

$\begin{array}{rr}110-124 & 202 \\ 96-120 & 546 \\ 96=120 & 748 \\ 96=120 & 208 \\ 96=120 & 720 \\ 96=120 & 632 \\ 96=120 & 712 \\ 96-120 & 1646\end{array}$


Total Organic Halogens

Unit: microgram/gram

First Layer

BSS-021

BSS -221

BSS -411

BSS-481

BSS-511

BSS-521

BSS-561

BSS- 571

Second Layer

BSS-022

BSS-222A

BSS -412

BSS -482

BSS -512

BSS -522

BSS -562

BSS -572

Third Layer

BSS-023

BSS-223A

BSS -413

BSS -483

BSS -513

BSS -523

BSS -563

BSS -573

\section{Interval (inches)}

$0-8$
$0-7$

$0-5$

$0-4$

$0-5$

$0-5$

$0-5$

$0-9$

$9-84$

$7-80$

$5-80$

$4-80$

$5-80$

$5-80$

$5-80$

$9-80$

$110-124$

$96-120$

$96-120$

$96-120$

$96-120$

$96-120$

$96-120$

$96-120$

\section{Modifiex}

LT

LT

LT

LT

LT

LT

LT

LT

LT

LT

LT

LT

LT

LT

LT

LT

LT

LT

LT

LT

LT

LT

LT
Result

10

10

10

10

10

17.4

10

10

10

10

10

10

10

10

10

10

10

10

10

10

10

10

10
10 
Total Organic Carbon

Unit: microgram/gram

First Layer
BSS-041
BSS-101
BSS-111
BSS-341
BSS-401
BSS-461
BSS-501
BSS-541

Interral (inches)
$0=6$
$0-6$
$0-6$
$0-6$
$0=8$
$0=10$
$0=6$
$0-6$

Modifier

Result

2830

3219

5480

3661

2499

2725

3693

3430

Second Layer

BSS-042A

BSS-102A

BSS-112A

BSS -342

BSS -402

BSS -462

BSS -502

BSS -542

$$
\begin{array}{r}
6-25 \\
6-16 \\
6-38 \\
6-28 \\
8-20 \\
10-22 \\
6-20 \\
6-24
\end{array}
$$

1230

1313

1840

1870

2084

976

2678

619

Third Layer

BSS-043A

BSS-103A

BSS- 343

BSS -403

BSS -463

BSS- 503

BSS-543

$$
\begin{aligned}
& 25-34 \\
& 16-34 \\
& 28-48 \\
& 20-48 \\
& 22-50 \\
& 20-34 \\
& 24-40
\end{aligned}
$$

967

1150

889

1106

625

865

2615

Fourth Layer

BSS-044A

BSS-104A

BSS-113A

BSS-114A

BSS -344

BSS -404

BSS -464

BS5-504

BSS-544

$34-65$
$34-60$
$38-60$
$60-60$
$48-60$
$48-72$
$50-63$
$34-60$
$40-68$

796

432

900

440

399

643

1034

1107

1272

Iifth Layer

BSS-045A

BSS-105A

BSS-115A

BSS -345

USS -405

BSS -465

BSS-505

BSS -545

$89-103$
$90-120$
$96-120$
$108-132$
$96-120$
$96-120$
$96-120$
$96-120$


Total Organic Halogens

Unit: microgram/gram

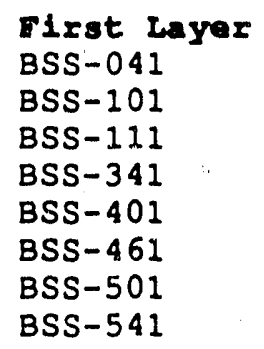

Interval (inches)
$0=6$
$0=6$
$0=6$
$0=6$
$0=8$
$0=10$
$0=6$
$0-6$

Modifier
$L T$
$L T$
$L T$
$L T$
$L T$
$L T$
$L T$
$L T$

Result

10

10

10

10

10

10

10

10

$$
\begin{array}{r}
6-25 \\
6-16 \\
6-38 \\
6-28 \\
8-20 \\
10-22 \\
6-20 \\
6-24
\end{array}
$$

$\begin{array}{ll}\text { LT } & 10 \\ \text { LT } & 10 \\ \text { LT } & 10 \\ \text { LT } & 10 \\ \text { LT } & 10 \\ \text { LT } & 10 \\ \text { LT } & 10 \\ \text { LT } & 10\end{array}$

$$
\begin{aligned}
& 25-34 \\
& 16-34 \\
& 28-48 \\
& 20-48 \\
& 22-50 \\
& 20-34 \\
& 24-40
\end{aligned}
$$

$\begin{array}{ll}\text { LT } & 10 \\ \text { LT } & 10 \\ \text { LT } & 10 \\ \text { LT } & 10 \\ \text { LT } & 10 \\ \text { LT } & 10 \\ \text { LT } & 10\end{array}$

$$
\begin{aligned}
& 34-65 \\
& 34-60 \\
& 38-60 \\
& 60-60 \\
& 48-60 \\
& 48-72 \\
& 50-63 \\
& 34-60 \\
& 40-68
\end{aligned}
$$

$\begin{array}{ll}\text { LT } & 10 \\ \text { LT } & 10 \\ \text { LT } & 10 \\ \text { LT } & 10 \\ \text { LT } & 10 \\ \text { LT } & 10 \\ \text { LT } & 10 \\ \text { LT } & 10 \\ \text { LT } & 10\end{array}$

$89-103$
$90-120$
$96-120$
$108-132$
$96-120$
$96-120$
$96-120$
$96-120$

$\begin{array}{ll}\text { LT } & 10 \\ \text { LT } & 10 \\ \text { LT } & 10 \\ \text { LT } & 10 \\ \text { LT } & 10 \\ \text { LT } & 10 \\ \text { LT } & 10 \\ \text { LT } & 10\end{array}$


Total Organic Carbon

Unit: microgram/gram

First Layer

BSS-131

BSS -132

BSS-201A

BSS -301

BSS -311

BSS -361

BSS -531

BSS -551

BSS -581

Interval (Inches)
$0-12$
$12-24$
$0-24$
$0-24$
$0-24$
$0-24$
$0-24$
$0-24$
$0-24$

Modifier

Rasult

316

192

5260

32

640

1197

386

96

557

Second Layer

BSS -133

BSS-202A

BSS -302

BSS -312

BSS -362

BSS -532

BSS -552

BSS-582

$24-36$
$24-48$
$24-48$
$24-48$
$24-48$
$24-48$
$24-48$
$24-48$

183

490

539

495

692

654

97

440

Third Layer

BSS-203A

BSS -303

BSS -313

BSS -363

BSS -533

BSS -553

BSS-583

$48-72$
$48-72$
$48-72$
$48-72$
$48-72$
$48-72$
$48-72$

236

275

237

43

165 


\begin{tabular}{|c|c|c|c|c|}
\hline First Layex & Interv & 1 (inches) & Modifler & Result \\
\hline BSS-131 & 0 & -12 & LT & 10 \\
\hline BSS-132 & 12 & -24 & LT & 10 \\
\hline BSS-201A & 0 & -24 & LT & 10 \\
\hline BSS-301 & 0 & -24 & LT & 10 \\
\hline BSS-311 & 0 & -24 & LT & 10 \\
\hline BSS-361 & 0 & -24 & LT & 10 \\
\hline BSS-531 & 0 & -24 & LT & 10 \\
\hline BSS-551 & 0 & -24 & LT & 10 \\
\hline BSS-581 & 0 & -24 & LT & 10 \\
\hline \multicolumn{5}{|l|}{ Second Layer } \\
\hline BSS-133 & 24 & -36 & LT & 10 \\
\hline BSS-202A & 24 & -48 & LT & 10 \\
\hline BSS-302 & 24 & -48 & LT & 10 \\
\hline BSS-312 & 24 & -48 & LT & 10 \\
\hline BSS-362 & 24 & -48 & LT & 10 \\
\hline BSS-532 & 24 & 48 & LT & 10 \\
\hline BSS-552 & 24 & -48 & LT & 10 \\
\hline BSS-582 & 24 & $-\quad 48$ & LT & 10 \\
\hline \multicolumn{5}{|l|}{ Third Layer } \\
\hline BSS-203A & 48 & - 72 & LT & 10 \\
\hline BSS-303 & 48 & -72 & LT & 10 \\
\hline BSS -313 & 48 & -72 & LT & 10 \\
\hline BSS -363 & 48 & -72 & LT & 10 \\
\hline BSS -533 & 48 & -72 & LT & 10 \\
\hline BSS-553 & 48 & -72 & LT & 10 \\
\hline BSS-583 & 48 & & $T$ & 10 \\
\hline
\end{tabular}


Total Organic Carbon

Unit: microgram/gram

\begin{tabular}{|c|c|c|c|c|}
\hline $\begin{array}{l}\text { First Layer } \\
\text { BSS-121 } \\
\text { BSS-241 } \\
\text { BSS-471 } \\
\text { BSS-591 }\end{array}$ & $\begin{array}{c}\text { Interv } \\
0 \\
0 \\
0 \\
0\end{array}$ & $\begin{array}{l}1 \text { (Inches) } \\
=\quad 23 \\
-\quad 22 \\
-\quad 12 \\
-\quad 23\end{array}$ & Modifier & $\begin{array}{r}\text { Result } \\
5440 \\
2420 \\
5703 \\
971\end{array}$ \\
\hline \multicolumn{5}{|l|}{ Second Layer } \\
\hline BSS-122 & 23 & 65 & & 263 \\
\hline BSS-242 & 22 & -59 & & 730 \\
\hline BSS -472 & 12 & $-\quad 38$ & & 595 \\
\hline BSS-592 & 23 & -50 & & 6236 \\
\hline \multicolumn{5}{|l|}{ Third Layer } \\
\hline BSS-123 & 65 & -82 & & 487 \\
\hline BSS-243 & 59 & - 74 & & 90 \\
\hline BSS -473 & 38 & -72 & & 243 \\
\hline BSS-593 & 50 & -72 & & 280 \\
\hline \multicolumn{5}{|l|}{ Fourth Layex } \\
\hline BSS-124 & 95 & $-\quad 119$ & & 233 \\
\hline BSS-244 & 96 & -120 & & 79 \\
\hline BSS -474 & 96 & $-\quad 120$ & & 189 \\
\hline BSS-594 & 96 & 120 & & 192 \\
\hline
\end{tabular}


Total Organic Halogens

Unit: microgram/gram

Eirst Layer

BSS-121

BSS-241

BSS -471

BSS -591

Second Layer

BSS -122

BSS -242

BSS -472

BSS -592

Third Layer

BSS- 123

BSS -243

BSS -473

BSS -593

Fourth Layer

BSS-124

BSS -244

BSS- 474

BSS-594

Interval (1nches)
$0-23$
$0-22$
$0-12$
$0-23$

$23-65$

$22-59$

$12-38$

$23-50$

$65-82$
$59-74$
$38-72$
$50-72$

$95-119$

$96-120$

$96-120$

$96-120$

$\begin{array}{cr}\text { Modifier } & \text { Result } \\ \text { LT } & 10 \\ \text { LT } & 10 \\ \text { LT } & 10 \\ \text { LT } & 10\end{array}$

LT

LT

LT

LT

10

10

10

10

LT 10

LT 10

LT 10

LT 10

Lr $\quad 10$

LT 10

LT 10

LT 10 


\title{
GEOCHEMICAL AND PHYSICAL PROPERTIES OF SOILS AND SHALLOW SEDIMENTS AT THE SAVANNAH RIVER SITE
}

\author{
Appendix C.4
}

Radiological Data Summary 


\section{Gross Alpha}

Unit: picocurie/grän

Eirst Layer
BSS-011
BSS -061
BSS -091
BSS -231
BSS -331
BSS -351
BSS -431
BSS -451

Second Layer BSS -012 BSS -062 BSS-092A BSS -232 BSS-332 BSS -352 BSS -432 BSS -452

Third Iayer BSS-0 13 BSS-063 BSS-093A

BSS -233

BSS -333

BSS -353

BSS -433

BSS -453

\section{Fourth Layer}

\begin{abstract}
BSS-014
\end{abstract}
BSS-064

BSS-094A

BSS -234

BSS -334

BSS -354

BSS -434

BSS-454

Interval (inches)
$0-7$
$0-6$
$0-5$
$0-7$
$0-8$
$0-7$
$0-6$
$0-5$

Modifier
LT
LT
LT
$L T$
$L T$
$L T$

Result

9.4

4
6
4
2.9
4
4
4

$10-65$

$6-50$

$5-52$

$7-61$

$8-72$

$7-60$

$6-67$

5. -59

LT

LT

LT

LT

LT

9.9

4
6

4

3.8

5.2

4

$65-87$

$50-74$

$52-72$

$61-70$

$72-84$

$60-72$

$67-77$

$59-72$

LT

8.5

5.2

6

6.1

6.1

6.1

6.1

8.5

$111-124$
$96-120$
$96-120$
$96-120$
$96-120$
$96-120$
$96-120$
$96-120$
10

7.4

LT

IJT

LT

6

4
6.1

5.6

4.1 
Gross Beta

Unit: picocurie/gram

First Layer
BSS -011
BSS-061
BSS-091
BSS -231
BSS -331
BSS -351
BSS -431
BSS -451

Second Iayex

BSS-012

BSS-062

BSS-092A

BSS -232

BSS -332

BSS -352

BSS-432

BSS -452

Third Iayer

BSS-013

BSS -063

BSS-093A

BSS -233

BSS-333

BSS -353

BSS -433

BSS -453

\section{Eourth Iayer}

BSS-014

BSS-064

BSS-094A

BSS-234

BSS-334

BSS-354

BSS -434

BSS-454

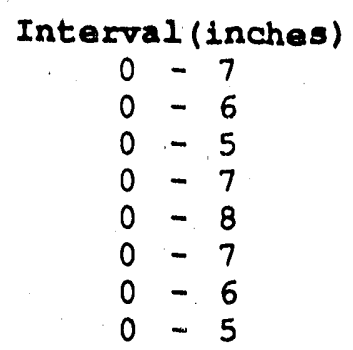

$\begin{array}{cr}\text { Modifiex } & \text { Result } \\ \text { LT } & 5.9 \\ \text { LT } & 6 \\ \text { LT } & 8 \\ \text { LT } & 6 \\ \text { LT } & 5 \\ \text { LT } & 6 \\ & 7 \\ & 10\end{array}$

$10-65$

$6-50$

$5-52$

$7-61$

$8-72$

$7-60$

$6-67$

$5-59$

$$
\begin{aligned}
& 65-87 \\
& 50-74 \\
& 52-72 \\
& 61=70 \\
& 72-84 \\
& 60-72 \\
& 67-77 \\
& 59-72
\end{aligned}
$$

LT

LT

LT

LT

LT

LT

12

6.7

8

6

8

12

7

6

8.8

6.2

7

7
7.3

8.2

12

14

$$
\begin{array}{r}
111-124 \\
96-120 \\
96-120 \\
96-120 \\
96-120 \\
96-120 \\
96-120 \\
96-120
\end{array}
$$

9.3

6.5

LT

LT 
Uranium

Unit: picocurie/gram

irst Layer

BSS-011

BSS-061

BSS-091

BSS -231

BSS -331

BSS- 351

BSS- 431

BSS- 451

\section{Second Iayer}

BSS-012

BSS-062

BSS-092A

BSS -232

BSS -332

BSS-352

BSS- 432

BSS -452

Third Layer

BSS-013

BSS-063

BSS-093A

BSS -233

BSS -333

BSS -353

BSS -433

BSS -453

Eourth Layex

BSS-014

BSS-064

BSS-094A

BSS -234

BSS -334

BSS-354

BSS -434

BSS -454

Interval (inches)
$0-7$
$0-6$
$0-5$
$0-7$
$0-8$
$0-7$
$0-6$
$0-5$

Modifier
LT
LT
LT
LT

$$
\begin{array}{r}
10-65 \\
6-50 \\
5-52 \\
7-61 \\
8-72 \\
7-60 \\
6-67 \\
5-59
\end{array}
$$

$$
\begin{aligned}
& 65-87 \\
& 50-74 \\
& 52-72 \\
& 61-70 \\
& 72-84 \\
& 60-72 \\
& 67-77 \\
& 59-72
\end{aligned}
$$

$$
\begin{array}{r}
111-124 \\
96-120 \\
96-120 \\
96-120 \\
96-120 \\
96-120 \\
96-120 \\
96-120
\end{array}
$$

LT

\section{LT \\ LT \\ LT \\ LT \\ LT \\ LT \\ LT}

LT

LT

LT

LT

LT

Result

1

1

1

1. 3

1

1. 3

$$
\begin{aligned}
& 1 \\
& 1 \\
& 1 \\
& 1 \\
& 1 \\
& 1 \\
& 1 \\
& 1
\end{aligned}
$$

1. 4

1

1

1
1.3

1

1

1. 4

2. 2

LT

LT

LT

1
1
1
1.3
1.1
1.9
1


strontium-90

Unit: picocurie/gram

Elrst Layer

BSS-011

BSS-061

BSS-091

BSS-231

BSS-331

BSS-351

BSS-431

BSS-451

Second Layer

BSS-012

BSS-062

BSS-092A

BSS -232

BSS- 332

BSS-352

BSS -432

BSS -452

Third Layer

BSS-013

BSS-063

BSS-093A

BSS -233

BSS-333

BSS-353

BSS -433

BSS -453

Eourth Layer

BSS-014

BSS-064

BSS-094A

BSS -234

BSS-334

BSS-354

BSS -434

BSS -454

Interval (1nches)
$0-7$
$0-6$
$0-5$
$0-7$
$0-8$
$0-7$
$0-6$
$0-5$

Modifier
LT
LT
LT
LT
LT
LT
LT
LT

Result

$$
\begin{array}{r}
10-65 \\
6-50 \\
5-52 \\
7-61 \\
8-72 \\
7-60 \\
6-67 \\
5-59
\end{array}
$$

$\begin{array}{ll}\text { LT } & 1 \\ \text { LT } & 1 \\ \text { LT } & 1 \\ \text { LT } & 1 \\ \text { LT } & 1 \\ \text { LT } & 1 \\ \text { LT } & 1 \\ \text { LT } & 1\end{array}$

$$
\begin{aligned}
& 65-87 \\
& 50-74 \\
& 52-72 \\
& 61-70 \\
& 72-84 \\
& 60-72 \\
& 67-77 \\
& 59-72
\end{aligned}
$$

$\begin{array}{ll}\text { LT } & 1 \\ \text { LT } & 1 \\ \text { LT } & 1 \\ \text { LT } & 1 \\ \text { LT } & 1 \\ \text { LT } & 1 \\ \text { LT } & 1 \\ \text { LT } & 1\end{array}$

$$
\begin{array}{r}
111-124 \\
96-120 \\
96-120 \\
96-120 \\
96-120 \\
96-120 \\
96-120 \\
96-120
\end{array}
$$

$\begin{array}{ll}\text { LT } & 1 \\ \text { LT } & 1 \\ \text { LT } & 1 \\ \text { LT } & 1 \\ \text { LT } & 1 \\ \text { LT } & 1 \\ \text { LT } & 1 \\ \text { LT } & 1\end{array}$




\section{Gross Alpha}

Unit: picocurie/gram

First rayer
BSS -031
BSS -071
BSS -081
BSS -211
BSS -321
BSS -421
BSS -441
BSS -491

Interval (inches)
$0-6$
$0-8$
$0-6$
$0-5$
$0-6$
$0-9$
$0-7$
$0-9$

$\begin{array}{cr}\text { Modifier } & \text { Result } \\ \text { LT } & 5.4 \\ \text { LT } & 4 \\ \text { LT } & 6 \\ & 4 \\ \text { LT } & 6.9 \\ \text { LT } & 4.9 \\ & 4 \\ & \end{array}$

Second Iayer

BSS -032

BSS-072A

BSS-0 02

BSS-212A

BSS -322

BSS -422

BSS -442

BSS -492

Third Layer

BSS-033

BSS-073A

BSS-083

BSS-213A

BSS- 323

BSS -423

BSS -443

BSS -493

$10-39$
$8-36$
$6-36$
$5-47$
$6-49$
$9-45$
$7-38$
$9-41$

$39-72$
$36-72$
$36-72$
$47-77$
$49-72$
$45-72$
$38-70$
$41-72$

Fourth Layer

BSS-034

BSS-074A

BSS-084

BSS-214A

BSS-324

BSS -424

BSS- 444

BSS -494
$96-120$

$96-120$

$96-120$

$96-120$

$96-120$

$96-120$

$96-120$

$96-120$
4.7

LT $\quad 5.18$

10

6.1

6.7

LT

4

LT

7.2

9

6.9

8.3

LT

8.1

5.3

20

6.7

5.2

7.4

7.3

3.9

14
5 
Gross Beta

Unit: picocurie/gram

Eirst Layer
BSS-031
BSS-07i
BSS-081
BSS -211
BSS-321
BSS-421
BSS -41
BSS -491

\section{Second Layer} BSS-03?

BSS-07?

BSS-08

BSS-212A

BSS- 322

BSS -422

BSS -442

BSS -492

Third Layer

$$
\text { BSS }-033
$$

BSS-073A

BSS-083

BSS-213A

BSS -32.3

BS.5 -423

BSS -443

BSS -493

\section{Fourth Layer}

BSS-034

BSS-074A

BSS-084

BSS-214A

BSS-324

BSS- 424

BSS- 444

BSS -494

Interral (inches)
$0-6$
$0-8$
$0-6$
$0-5$
$0-6$
$0-9$
$0-7$
$0-.9$

$10-39$

$8-36$

$6-36$

$5-47$

$6-49$

$9-45$

$7-38$

$9-41$

$39-72$

$36-72$

$36-72$

$47-77$

4. -72

$45-72$

$38-70$

$41-72$

$\begin{array}{cr}\text { Modifier } & \text { Result } \\ \text { LT } & 6 \\ \text { LT } & 7.6 \\ & 8 \\ \text { LT } & 8.1 \\ & 8.6 \\ \text { LT } & 6 \\ & 9.9 \\ & 6\end{array}$

IT

IT

LT

LT

LT

6

LT

7
15
9.1
12
13
12
15
9.6

$96-120$

16

$96-120$

11

$96-120$

$96-120$

$96-120$

$96-120$

$96-120$

$96-120$ 


\begin{tabular}{|c|c|c|c|c|}
\hline \multicolumn{5}{|l|}{ Uranium } \\
\hline \multicolumn{5}{|c|}{ Unit: picocurie/gram } \\
\hline First Layer & \multicolumn{2}{|c|}{ Interval (inches) } & \multirow{3}{*}{$\underset{\text { LT }}{\operatorname{Modifiex}}$} & \multirow{3}{*}{$\begin{array}{r}\text { Result } \\
1\end{array}$} \\
\hline BSS-031 & 0 & -6 & & \\
\hline BSS-07I & 0 & -8 & & \\
\hline BSS-081 & 0 & -6 & LT & 1 \\
\hline BSS-211 & 0 & -5 & LT & 1 \\
\hline BSS-321 & 0 & -6 & LT & 1 \\
\hline BSS-421 & 0 & -9 & LT & 1 \\
\hline SSS- 441 & 0 & -7 & LT & 1 \\
\hline BSS-491 & 0 & -9 & LT & 1 \\
\hline \multicolumn{5}{|l|}{ Second Layer } \\
\hline BSS-032 & 10 & $-\quad 39$ & LT & 1 \\
\hline BSS-072A & 8 & -36 & LT & 1 \\
\hline BSS-082 & 6 & -36 & LT & 1 \\
\hline BSS-212A & 5 & 47 & LT & 1 \\
\hline BSS-322 & 6 & -49 & LT & 1 \\
\hline BSS-422 & 9 & -45 & & 1.8 \\
\hline BSS-442 & 7 & -33 & LT & 1 \\
\hline BSS -492 & 9 & -41 & LT & 1 \\
\hline \multicolumn{5}{|l|}{ Third Layer } \\
\hline BSS-033 & 39 & 72 & & 1.2 \\
\hline BSS-073A & 36 & 72 & & 1.4 \\
\hline BSS-083 & 36 & 72 & & 1.2 \\
\hline BSS-213A & 47 & 77 & & 1.4 \\
\hline BSS-323 & 49 & -72 & LT & 1 \\
\hline BSS -423 & 45 & 72 & & 1.6 \\
\hline BSS -443 & 38 & -70 & & 1.4 \\
\hline BSS-493 & 41 & -72 & & 1.1 \\
\hline \multicolumn{5}{|l|}{ Eourth Layer } \\
\hline BSS-034 & 96 & $-\quad 120$ & LT & 1 \\
\hline$B S S-074 A$ & 96 & 120 & & 1.2 \\
\hline BSS-084 & 96 & 120 & & 2.4 \\
\hline BSS-214A & 96 & 120 & LT & 1 \\
\hline BSS-324 & 96 & -120 & LT & 1 \\
\hline BSS-424 & 96 & $-\quad 120$ & LT & 1 \\
\hline BSS-444 & 96 & 120 & & 2 \\
\hline BSS-494 & 96 & 120 & LT & 1 \\
\hline
\end{tabular}


Strontium-90

Unit: picocurie/gram

\begin{tabular}{|c|c|c|c|c|}
\hline \multirow{2}{*}{$\begin{array}{l}\text { Eirst Layer } \\
\text { BSS-031 }\end{array}$} & \multicolumn{2}{|c|}{ Interval (Inches) } & \multirow{3}{*}{$\begin{array}{c}\text { Modifiex } \\
\text { LT } \\
\text { LT }\end{array}$} & Result \\
\hline & 0 & -6 & & \\
\hline BSS-071 & 0 & -8 & & 1 \\
\hline BSS-081 & 0 & -6 & LT & 1 \\
\hline BSS-211 & 0 & -5 & $\mathrm{LT}$ & 1 \\
\hline BSS-321 & 0 & -6 & LT & 1 \\
\hline$B S S-421$ & 0 & -9 & LT & 1 \\
\hline$B S S-441$ & 0 & -7 & LT & , \\
\hline BSS-491. & 0 & -9 & LT & 1 \\
\hline \multicolumn{5}{|l|}{ Second Layer } \\
\hline BSS-032 & 10 & 39 & $\mathrm{LT}$ & 1 \\
\hline BSS-072A & 8 & 36 & LT & 1 \\
\hline BSS-082 & 6 & $-\quad 36$ & LT & 1 \\
\hline$B S S-212 A$ & 5 & $-\quad 47$ & LT & 1 \\
\hline BSS-322 & 6 & $-\quad 49$ & LT & 1 \\
\hline BSS-422 & 9 & -45 & LT & J \\
\hline BSS-442 & 7 & $-\quad 38$ & $\mathrm{LT}$ & \\
\hline BSS-492 & 9 & $-\quad 41$ & LT & I \\
\hline \multicolumn{5}{|l|}{ Third Layer } \\
\hline BSS-033 & 39 & 72 & LT & \\
\hline BSS-073A & 36 & -72 & LT & \\
\hline BSS-083 & 36 & -72 & LT & \\
\hline$B S S-213 A$ & 47 & $-\quad 77$ & LT & \\
\hline BSS-323 & 49 & -72 & LT & \\
\hline BSS-423 & 45 & $-\quad 72$ & $\mathrm{LT}$ & \\
\hline BSS-443 & 38 & -70 & LT & \\
\hline BSS -493 & 41 & -72 & LT & \\
\hline \multicolumn{5}{|l|}{ Eourth Layer } \\
\hline BSS-034 & 96 & 120 & LT & \\
\hline$B S S-074 A$ & 96 & $-\quad 120$ & $\mathrm{LT}$ & \\
\hline BSS-084 & 96 & $-\quad 120$ & LT & \\
\hline$B S 5=214$ & 96 & 120 & LT & \\
\hline$B S S-324$ & 96 & 120 & $\mathrm{LT}$ & \\
\hline BSS-424 & 96 & $-\quad 120$ & $\mathrm{LT}$ & \\
\hline RSS- 444 & 96 & $-\quad 120$ & $\mathrm{LT}$ & \\
\hline BSS-494 & 96 & 120 & $\mathrm{LT}$ & \\
\hline
\end{tabular}




\section{Gross Alpha}

Unit: picocurie/gram

First Layer

BSS-021

BSS-221

BSS- 411

BSS -481

BSS-511

BSS -521

BSS -561

BSS -571

\section{Second Layer}

BSS-022

BSS-222A

BSS -412

BSS -482

BSS -512

BSS-522

BSS- 562

BSS-572

Third Iayer

BSS-023

BSS-223A

BSS -413

BSS -483

BSS -513

BSS -523

BSS -563

BSS -573

Interval (inches)
$0-8$
$0-7$
$0-5$
$0-4$
$0-5$
$0-5$
$0-5$
$0-9$

Modifier

$-80$

- 80

-80
$-\quad 80$

-80
$-\quad 80$

- 80

- 80

- 80

$110-124$
$96-120$
$96-120$
$96-120$
$96-120$
$96-120$
$96-120$
$96-120$

LT

LT

LT

LT

LT

LT
LT
LT
LT
LT
LT
LT

4.2

Result

4.9

4

4

3. 8

6.8

5

4

5

4

5

5
4

5.5

6.1

LT

LT

LT

LT

LT

LT

$\begin{array}{lr} & 5.5 \\ \text { LT } & 6.1 \\ \text { LT } & 5 \\ \text { LT } & 5 \\ \text { LT } & 4 \\ \text { LT } & 6 \\ \text { LT } & 5\end{array}$




\begin{tabular}{|c|c|c|c|c|}
\hline & & & Modifier & Result \\
\hline BSS-021 & 0 & -8 & LT & 6 \\
\hline BSS-221 & 0 & -7 & LT & 7 \\
\hline BSS-411 & 0 & -5 & LT & 6 \\
\hline BSS -481 & 0 & - & LT & 6 \\
\hline BSS-511 & 0 & -5 & LT & 6 \\
\hline BSS -521 & 0 & - & & 8.4 \\
\hline BSS -561 & 0 & -5 & LT & 7 \\
\hline BSS-571 & 0 & -9 & & 12 \\
\hline \multicolumn{5}{|l|}{ Second Layer } \\
\hline BSS-022 & 9 & 84 & LT & 6 \\
\hline $\mathrm{BSS}-2 ? 2 \mathrm{~A}$ & 7 & 80 & LT & 6 \\
\hline BSS -412 & 5 & 80 & LT & 6 \\
\hline BSS -482 & 4 & -80 & LT & 6 \\
\hline BSS-512 & 5 & 80 & & 9.7 \\
\hline BSS-522 & 5 & 80 & LT & 7 \\
\hline BSS-562 & 5 & 80 & LT & 7 \\
\hline BSS- 572 & 9 & -80 & & 8.3 \\
\hline \multicolumn{5}{|l|}{ Third Layer } \\
\hline BSS-023 & 110 & 124 & LT & \\
\hline$B S S-223 A$ & 96 & $-\quad 120$ & LT & 6 \\
\hline$B S S-413$ & 96 & 120 & & 6.3 \\
\hline BSS -483 & 96 & 120 & LT & 6 \\
\hline BSS -513 & 96 & $-\quad 120$ & & 13 \\
\hline BSS -523 & 96 & 120 & LT & 7 \\
\hline BSS-563 & 96 & 120 & LT & 7 \\
\hline BSS- 573 & 96 & 120 & & 10 \\
\hline
\end{tabular}




\begin{tabular}{|c|c|c|c|c|}
\hline & & & 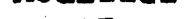 & Result \\
\hline & 0 & 8 & LT & 1 \\
\hline $\begin{array}{l}\text { BSS-221 } \\
\text { BSS-411 }\end{array}$ & 0 & $=7$ & $I T$ & 1.3 \\
\hline BSS -481 & di & -4 & $\begin{array}{l}\text { LI } \\
\text { LT }\end{array}$ & $i$ \\
\hline BSS-511 & 6 & -5 & LT & 1 \\
\hline BSS-521 & 0 & -5 & LT & 1 \\
\hline BSS-561 & 0 & -5 & LT & 1 \\
\hline BSS-571 & 0 & -9 & LT & 1 \\
\hline \multicolumn{5}{|l|}{ Second Layex } \\
\hline$B S S-022$ & 9 & 84 & LT & \\
\hline$B S S-222 A$ & 7 & -80 & LT & 1 \\
\hline BSS-412 & 5 & -80 & LT & 1 \\
\hline BSS -482 & 4 & -80 & LT & 1 \\
\hline BSS -512 & 5 & 80 & LT & 1 \\
\hline BSS-522 & 5 & 80 & LT & 1 \\
\hline BSS -562 & 5 & 80 & LT & 1 \\
\hline BSS-572 & 9 & -80 & LT & \\
\hline \multicolumn{5}{|l|}{ Third Layer } \\
\hline BSS -023 & 110 & 124 & & 1.1 \\
\hline$B S S-223 A$ & 96 & 120 & LT & \\
\hline$B S S-413$ & 96 & 120 & LT & \\
\hline$B S S-483$ & 96 & 120 & LS & 1 \\
\hline BSS-513 & 96 & 120 & & 1.3 \\
\hline BSS-523 & 96 & 120 & LT & \\
\hline BSS -563 & 96 & $-\quad 120$ & LT & 1 \\
\hline BSS -573 & 96 & 120 & LT & \\
\hline
\end{tabular}




\begin{tabular}{|c|c|c|c|c|}
\hline BSS-021 & 0 & -8 & LT & 1 \\
\hline BSS-221 & 0 & -7 & $\mathrm{LT}$ & 1 \\
\hline BSS -411 & 0 & -5 & LT & 1 \\
\hline BSS -481 & 0 & -4 & LT & 1 \\
\hline BSS-511 & 0 & -5 & LT & 1 \\
\hline BSS -521 & 0 & -5 & LT & 1 \\
\hline BSS-561 & 0 & -5 & LT & 1 \\
\hline BSS-571 & 0 & $-\quad 9$ & LT & 1 \\
\hline Second Layer & & & & \\
\hline BSS-022 & 9 & 84 & LT & \\
\hline BSS-222A & 7 & -80 & LT & 1 \\
\hline BSS-412 & 5 & -80 & LT & 1 \\
\hline BSS -482 & 4 & -80 & LT & 1 \\
\hline BSS-512 & 5 & -80 & LT & 1 \\
\hline BSS-522 & 5 & 80 & LT & \\
\hline BSS-562 & 5 & -80 & LT & 1 \\
\hline BSS-572 & 9 & -80 & LT & 1 \\
\hline Third Layer & & & & \\
\hline BSS -023 & 110 & 124 & LT & 1 \\
\hline BSS-223A & 96 & 120 & LT & 1 \\
\hline BSS-413 & 96 & 120 & LT & 1 \\
\hline BSS -483 & 96 & 120 & LT & 1 \\
\hline BSS -513 & 96 & $-\quad 120$ & LT & 1 \\
\hline BSS-523 & 96 & $-\quad 120$ & LT & \\
\hline BSS-563 & 96 & -120 & LT & 1 \\
\hline BSS -573 & 96 & 120 & LT & \\
\hline
\end{tabular}


Gross Alpha

Unit: picocurie/gram

First Layer
BSS-041
BSS-101
BSS-111
BSS -341
BSS -401
BSS -461
BSS -501
BSS -541

Interval (inches)

$0-6$
$0-6$
$0-6$
$0-6$
$0-8$
$0-10$
$0-6$
$0-6$

$\begin{array}{cr}\text { Modifier } & \text { Result } \\ \text { LT } & 10 \\ & 4 \\ \text { LT } & 6.4 \\ \text { LT } & 4.5 \\ \text { LT } & 4 \\ \text { LT } & 5 \\ & 4 \\ & 4\end{array}$

\section{Second Layer}

BSS-042A

BSS-102A

BSS-112A

BSS- 342

BSS -402

BSS-462

BSS -502

BSS -542

$6-25$
$6-16$
$6-38$
$6-28$
$8-20$
$10-22$
$6-20$
$6-24$

5.4

6.8

LT

LT

LT

7.2

4

6

7.2

6.9

Third Layer

BSS-043A

BSS-103A

BSS -343

BSS -403

BSS-463

BSS -503

BSS -543

$25-34$
$16-34$
$28-48$
$20-48$
$22-50$
$20-34$
$24-40$

7.6

7.3

8.7

4.7

LT

6
6.2
8

Fourth Layer

BSS-044A

BSS-104A

BSS-113A

BSS-114A

BSS-344

BSS-404

BSS-464

BSS -504

BSS-544

$34-65$

$34-60$

- 60

$60-80$

8.6

9.3

6.4

$48-60$

$48-72$

$50-63$

$34-60$

$40-68$

LT

6.5

LT

5.4

5.6

11

\section{Fifth Layer}

BSS-045A

BSS-105A

BSS-115A

BSS-345

BSS-405

BSS -465

BSS-505

BSS-545

$89-103$
$90-120$
$96-120$
$108-132$
$96-120$
$96-120$
$96-120$
$96-120$

8.1

5.4

7.6

7.5

LT

5

4.9

6.1 


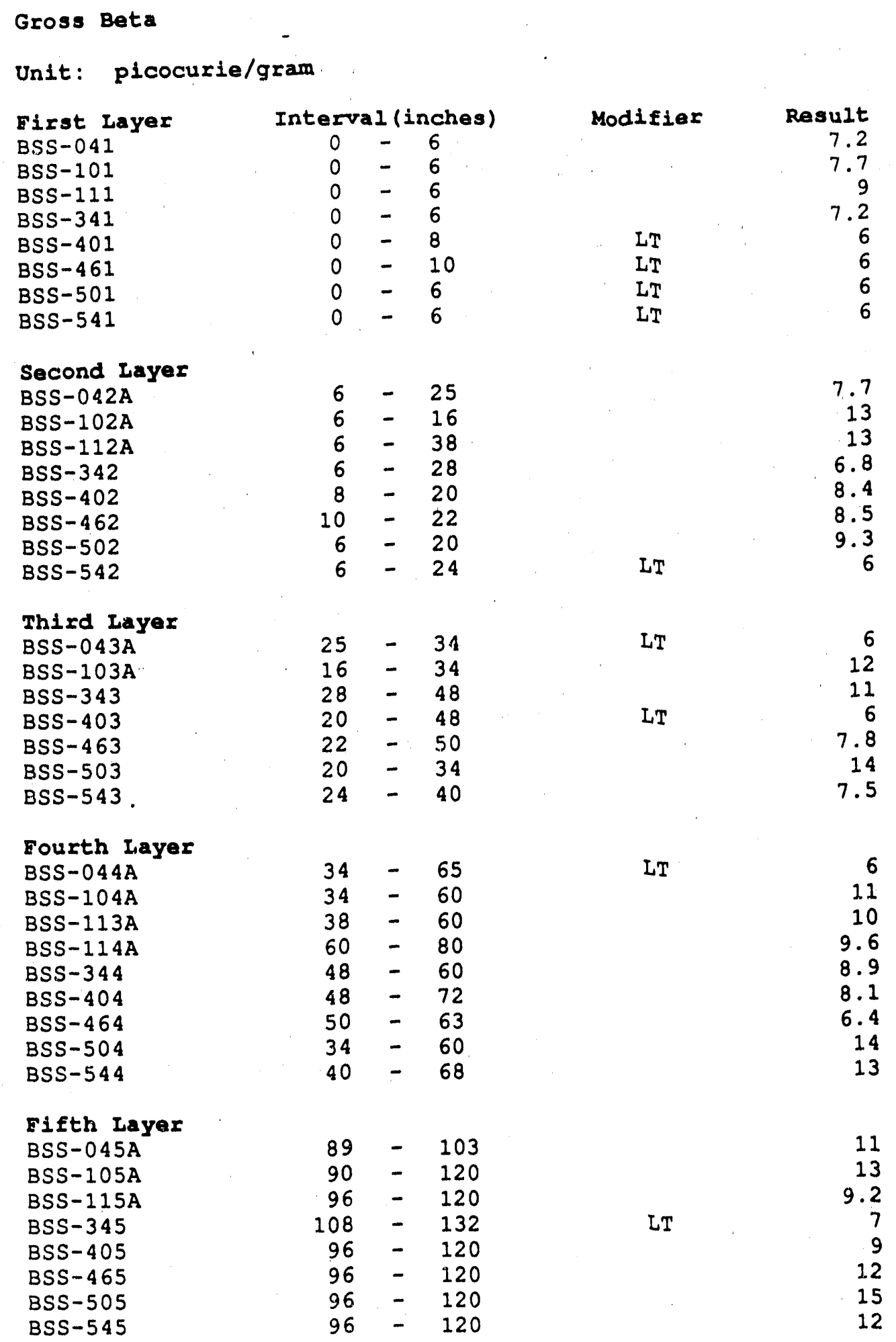


Uranium

Unit: picocurie/gram

Elrst Layar
BSS-041
BSS-101
BSS-111
BSS-341
BSS-401
BSS -461
BSS-501
$B S S-541$

Interval (inches)
$0-6$
$0-6$
$0-6$
$0-6$
$0-8$
$0-10$
$0-6$
$0-6$

$\begin{array}{cr}\text { Modifiex } & \text { Result } \\ \text { LT } & 1 \\ \text { LT } & 1.8 \\ & 1 \\ \text { LT } & 1.4 \\ \text { LT } & 1.2 \\ \text { LT } & 1 \\ & 1\end{array}$

second Layer BSS-042A

BSS-102A

BSS-112A

BSS -342

BSS -402

BSS -462

BSS -502

BSS -542

$$
\begin{array}{r}
6-25 \\
6-16 \\
6-38 \\
6-28 \\
8-20 \\
10-22 \\
6-20 \\
6-24
\end{array}
$$

1.8

1.9

1. 2

1.6

1.4

1.3

1.4

1.3

Third Layer

BSS-043A

BSS-103A

BSS-343

BSS-403

BSS -463

BSS-503

BSS-543

$$
\begin{aligned}
& 25-34 \\
& 16-34 \\
& 28-48 \\
& 20-48 \\
& 22-50 \\
& 20-34 \\
& 24-40
\end{aligned}
$$

LT

$\begin{array}{rr} & 1.3 \\ & 1.8 \\ & 2.6 \\ \text { LT } & 1.1 \\ \text { LT } & 1 \\ & 1 \\ & 1.2\end{array}$

Eourth Iayer

BSS-044A

BSS-104A

BSS-113A

BSS-114A

BSS-344

BSS- 404

BSS -464

BSS-504

BSS-544

$$
\begin{aligned}
& 34-65 \\
& 34-60 \\
& 38-60 \\
& 60-60 \\
& 48-60 \\
& 48-72 \\
& 50-63 \\
& 34-60 \\
& 40-68
\end{aligned}
$$

3.3
1.2
1.7
LT $\quad 1$
1.8

$\quad \begin{aligned} & 1.2 \\ & 1.8 \\ & 1.1\end{aligned}$

\section{Eifth Layer}

BSS-045A

BSS-105A

BSS-115A

BSS-345

BSS-405

BSS -465

BSS- 505

BSS- 545

$89-103$
$90-120$
$96-120$
$108-132$
$96-120$
$96-120$
$96-120$
$96-120$

1.3

1
1
1.2
1
2
1.6
1 
Strontium-90

Unit: picocurie/gram

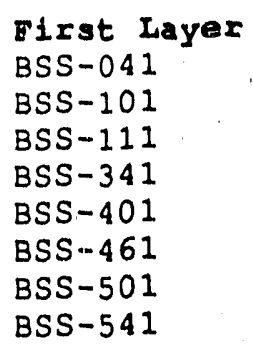

Fourth Layer

BSS-044A

BSS-104A

BSS-113A

BSS-114A

BSS -344

BSS -404

BSS -464

BSS -504

BSS -544

Fifth Jayrar

BSS-045A

BSS-105A

BSS-115A

BSS -345

BSS -405

BSS -465

BSS-505

BSS -545

Interval (inches)
$0-6$
$0-6$
$0-6$
$0-6$
$0-8$
$0-10$
$0-6$
$0-6$

Modifier
LT
LT
LT
LT
LT
LT
LT
LT

Result

1
1
1
1
1
1
1
1

$$
\begin{array}{r}
6-25 \\
6-16 \\
6-38 \\
6-28 \\
8-20 \\
10-22 \\
6-20 \\
6-24
\end{array}
$$

$\begin{array}{ll}\text { LT } & 1 \\ \text { LT } & 1 \\ \text { LT } & 1 \\ \text { LT } & 1 \\ \text { LT } & 1 \\ \text { LT } & 1 \\ \text { LT } & 1 \\ \text { LT } & 1\end{array}$

$25-34$
$16-34$
$28-48$
$20-48$
$22-50$
$20-34$
$24-40$

$\begin{array}{ll}\text { LT } & 1 \\ \text { LT } & 1 \\ \text { LT } & 1 \\ \text { LT } & 1 \\ \text { LT } & 1 \\ \text { LT } & 1 \\ \text { LT } & 1\end{array}$

$34-65$

$34-60$

$38-60$

$60-80$

$48-60$

$48-72$

$50-63$

$34-60$

$40-68$

$89-103$
$90-120$
$96-120$
$108-132$
$96-120$
$96-120$
$96-120$
$96-120$

$\begin{array}{ll}\text { LT } & 1 \\ \text { LT } & 1 \\ \text { LT } & 1 \\ \text { LT } & 1 \\ \text { LT } & 1 \\ \text { LT } & 1 \\ \text { LT } & 1 \\ \text { LT } & 1 \\ \text { LT } & 1\end{array}$

$\begin{array}{ll}\text { LT } & 1 \\ \text { LT } & 1 \\ \text { LT } & 1 \\ \text { LT } & 1 \\ \text { LT } & 1 \\ \text { LT } & 1 \\ \text { LT } & 1 \\ \text { LT } & 1\end{array}$




\section{Gross Alpha}

Unit: picocurie/gram

\begin{tabular}{|c|c|c|c|c|}
\hline $\begin{array}{l}\text { First Layer } \\
\text { BSS }-131\end{array}$ & $\begin{array}{r}\text { Inter } \\
0\end{array}$ & $\begin{array}{l}1 \text { (inches) } \\
-\quad 12\end{array}$ & Modifier & $\begin{array}{r}\text { Rasult } \\
1 \text { ? }\end{array}$ \\
\hline BSS -132 & 12 & -24 & LT & 4 \\
\hline BSS-201A & 0 & -24 & & 11 \\
\hline BSS -301 & 0 & $-\quad 24$ & & 9.8 \\
\hline BSS-311 & 0 & 24 & & 8.4 \\
\hline BSS -361 & 0 & 24 & & 9.7 \\
\hline BSS-531 & 0 & 24 & & 6.2 \\
\hline BSS-551 & 0 & -24 & & 6.3 \\
\hline BSS-581 & 0 & -24 & & 7.7 \\
\hline \multicolumn{5}{|l|}{ Second Layer } \\
\hline BSS-133 & 24 & 36 & & 9.6 \\
\hline BSS-202A & 24 & 48 & & 4.7 \\
\hline BSS-302 & 24 & 48 & & 3.9 \\
\hline BSS-312 & 24 & $-\quad 48$ & & 9 \\
\hline BSS-362 & 24 & -48 & & 5.3 \\
\hline BSS -532 & 24 & -48 & & 11 \\
\hline BSS -552 & 24 & -48 & LT & 4 \\
\hline BSS -582 & 24 & -48 & LT & \\
\hline
\end{tabular}

Third Layer

BSS-203A

$48-72$

6.7

BSS -303

BSS -313

BSS -363

BSS -533

BSS -553

BSS -583 


\section{Gross Beta}

Unit: picocurie/gram

First Layer
BSS-131
BSS-132
BSS-201A
BSS-301
BSS-311
BSS -361
BSS-531
BSS-551
$B S S-581$

Interval (inches)
$0-12$
$12-24$
$0-24$
$0-24$
$0-24$
$0-24$
$0-24$
$0-24$
$0-24$

Result
23
10
12
8.4
6.9
12
10
12
14

Second Layer BSS -133

BSS-202A

BSS- 302

BSS-312

BSS-362

BSS- 532

BSS-552

BSS-582

$$
\begin{aligned}
& 24-36 \\
& 24-48 \\
& 24-48 \\
& 24-48 \\
& 24-48 \\
& 24-48 \\
& 24-48 \\
& 24-48
\end{aligned}
$$

8.8
9.6
10.5
10
7.8
LT 10
7

Third Layer

BSS-203A

BSS -303

BSS -313

BSS -363

BSS-533

BSS-553

BSS -583

$48-72$
$48-72$
$48-72$
$48-72$
$48-72$
$48-72$
$48-72$

$\begin{array}{rr} & 6.4 \\ & 12 \\ & 8.4 \\ & 7.3 \\ \text { LI } & 10 \\ & 9.7\end{array}$




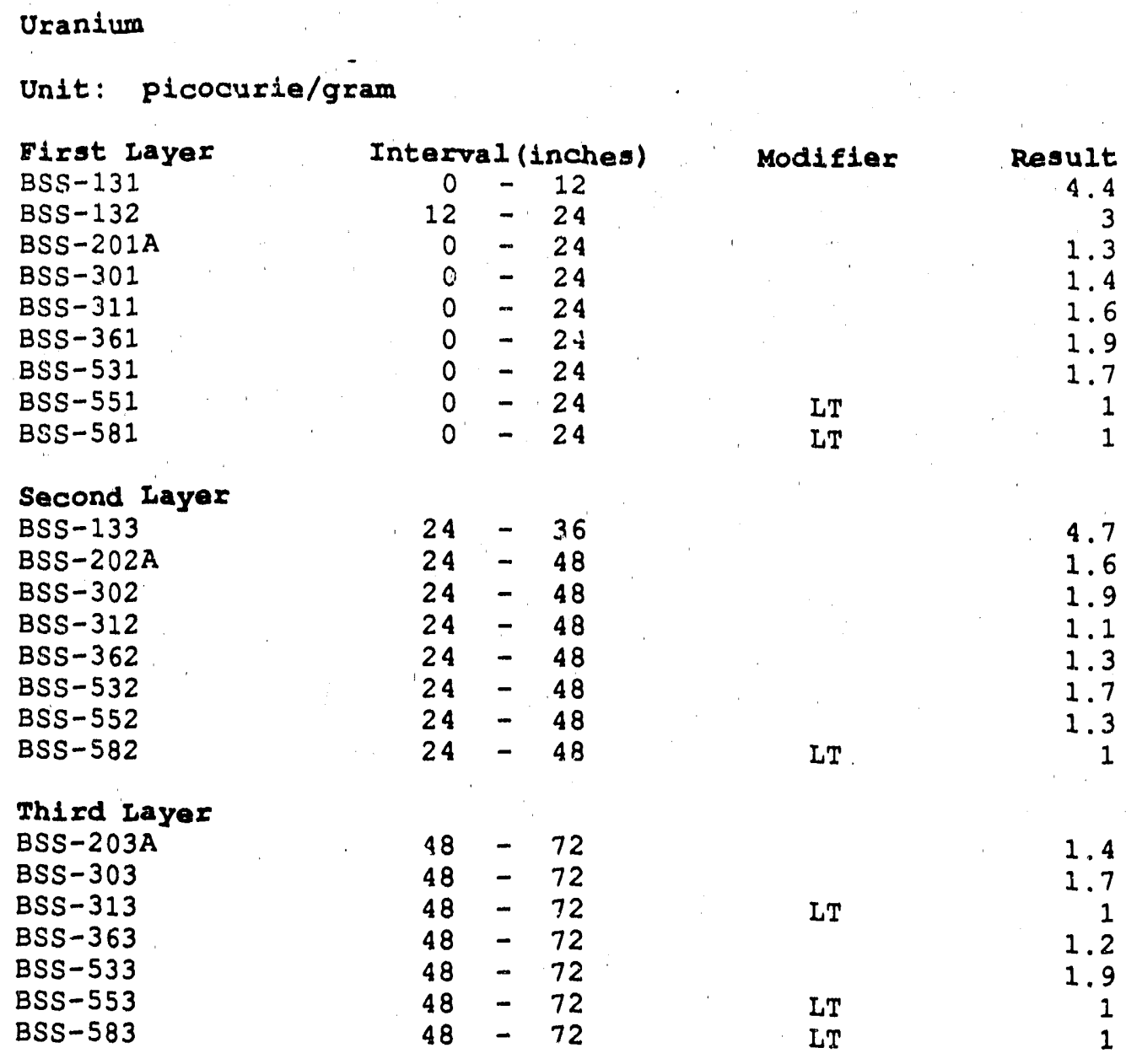


strontium-90

Unit: picocurie/gram

First Layer

BSS-131

BSS -132

BSS - 201A

BSS-301

BSS-311

BSS-361

BSS-531

BSS -551

BSS-581

\section{Second Iayer}

BSS-133

BSS-202A

BSS -302

BSS -312

BSS- 362

BSS-532

BSS -552

BSS- 582

Third Iayer

BSS-203A

BSS -303

BSS-313

BSS- 363

BSS-533

BSS -553

BSS - 583

Interval (inches)
$0-12$
$12-24$
$0-24$
$0-24$
$0-24$
$0-24$
$0-24$
$0-24$
$0-24$

Modifier
LT
LT
LT
$L T$
$L T$
$L T$
$L T$
$L T$
$L T$

Result

1

1

1

1

1

1

1

LT 1

LT 1

LT 1

LT 1

LT 1

LT 1

$\begin{array}{ll}\text { IT } & 1 \\ \text { LT } & 1\end{array}$

$24-48$

$48-72$
$48-72$
$48-72$
$48-72$
$48-72$
$48-72$
$48-72$

LT
LT
LT
LT
LT
LT
LT

1

1

1

1

1

1 


\section{Gross Alpha}

Unit: picocurie/gram

Eirst Layer

BSS-121

BSS-241

BSS-47I

BSS -591

Second Layer

BSS-122

BSS -242

BSS-472

BSS -592

Third Iayex

BSS-123

BSS -243

BSS -473

BSS -593

Fourth Layer

BSS-124

BSS-244

BSS-474

BSS-594

Interval (inches)
$0-23$
$0-22$
$0-12$
$0-23$

\section{Modifier}

$23-65$
$22-59$
$12-38$
$23-50$

$65-82$

$59-74$

$38-72$

$50-72$

$95-119$

$96-120$

$96-120$

$96-120$
LT

Result

7.8

4.4

7.4

7.5

7.8

4.1

5

6.8

4. 3

LT

LT

4

5

5.9

LT

LT

LT

$$
\begin{array}{r}
4 \\
5 \\
4
\end{array}
$$$$
4
$$ 


\section{Gross Beta}

Unit: picocurie/gram

First Inayer

BSS -121

BSS-241

BSS-471

BSS -591

Interval (inches)
$0-23$
$0-22$
$0-12$
$0-23$

Modifier

LT

Result

Second Iayer

BSS-122

BSS -242

BSS -472

BSS-592

Third Iayer

BSS-123

BSS -243

BSS -473

BSS -593

Eourth Layer

BSS-124

BSS-244

BSS -474

BSS-594

$23-65$
$22-59$
$12-38$
$23-50$

$65-82$

$59-74$

$38-72$

$50-72$

$95-119$

$96-120$

$96-120$

$96-120$
LT

LT

LT

6.4

8. 8

7
7.4

9.2

LT

LT
11

7
10

7

14
6

11

12

10

8
7
7
4

7

7 
Uranium

Unit: picocurie/gram

First Layer

BSS-121

BS3m 241

BSS-471

BSS- 591

Interval (inches)
$0-23$
$0-22$
$0-12$
$0-23$

Second Iayer

BSS-122

BSS -242

BSS- 472

BSS -592

Third Layer

BSS- 123

BSS -243

BSS -473

BSS -593

Eourth Iayer

BSS-124

BSS -244

BSS- 474

BSS-594

$23-65$
$22-59$
$12-38$
$23-50$

LT

$65-82$
$59-74$
$38-72$
$50-72$

LT

LT

$95-119$

$96-120$

$96-120$

$96-120$

LT

LT

$\begin{array}{cr}\text { Modifier } & \text { Result } \\ \text { LT } & 1.3 \\ & 1.6 \\ & 2.4\end{array}$

1.1

2.2

1. 1

1.5

1

1

1.1

1.3

1

1. 6 
strontium-90

Unit: picocurie/gram

First Layer

BSS-121

BSS-241

BSS -471

BSS -591

Interval (inches)
$0-23$
$0-22$
$0-12$
$0-23$

Modifier
LT
LT
LT
LT

Result

1

1

1

Second Layer

BSS -122

BSS-242

BSS -472

BSS-592

$23-65$
$22-59$
$12-38$
$23-50$

LT

LT

LT

LT

1

1

Third Iayer

BSS- 123

BSS-243

BSS -473

BSS-593

$65-82$
$59-74$
$38-72$
$50-72$

LT

LI

LT

LT

1

1

Fourth Iayer

BSS-124

BSS-244

BSS- 474

BSS-594

$95-119$
$96-120$
$96-120$
$96-120$

LT

LT

LT

LT

1

1 
GEOCHEMICAL AND PHYSICAL PROPERTIES OF SOILS AND SHALLOW SEDIMENTS AT THE SAVANNAH RIVER SITE

\author{
Appendix C.5
}

Particle Size Data Summary 


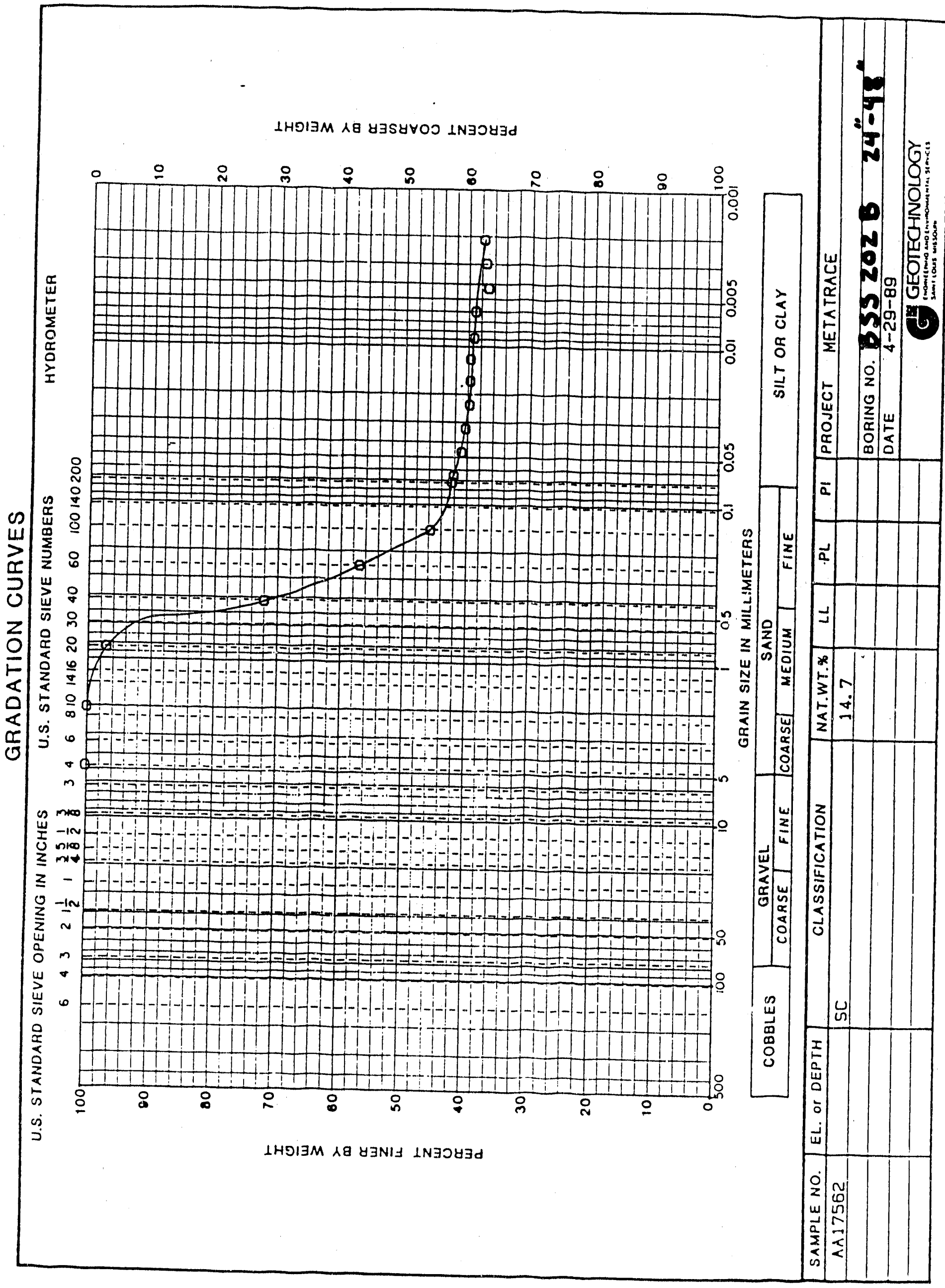




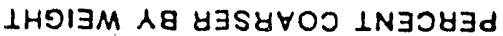

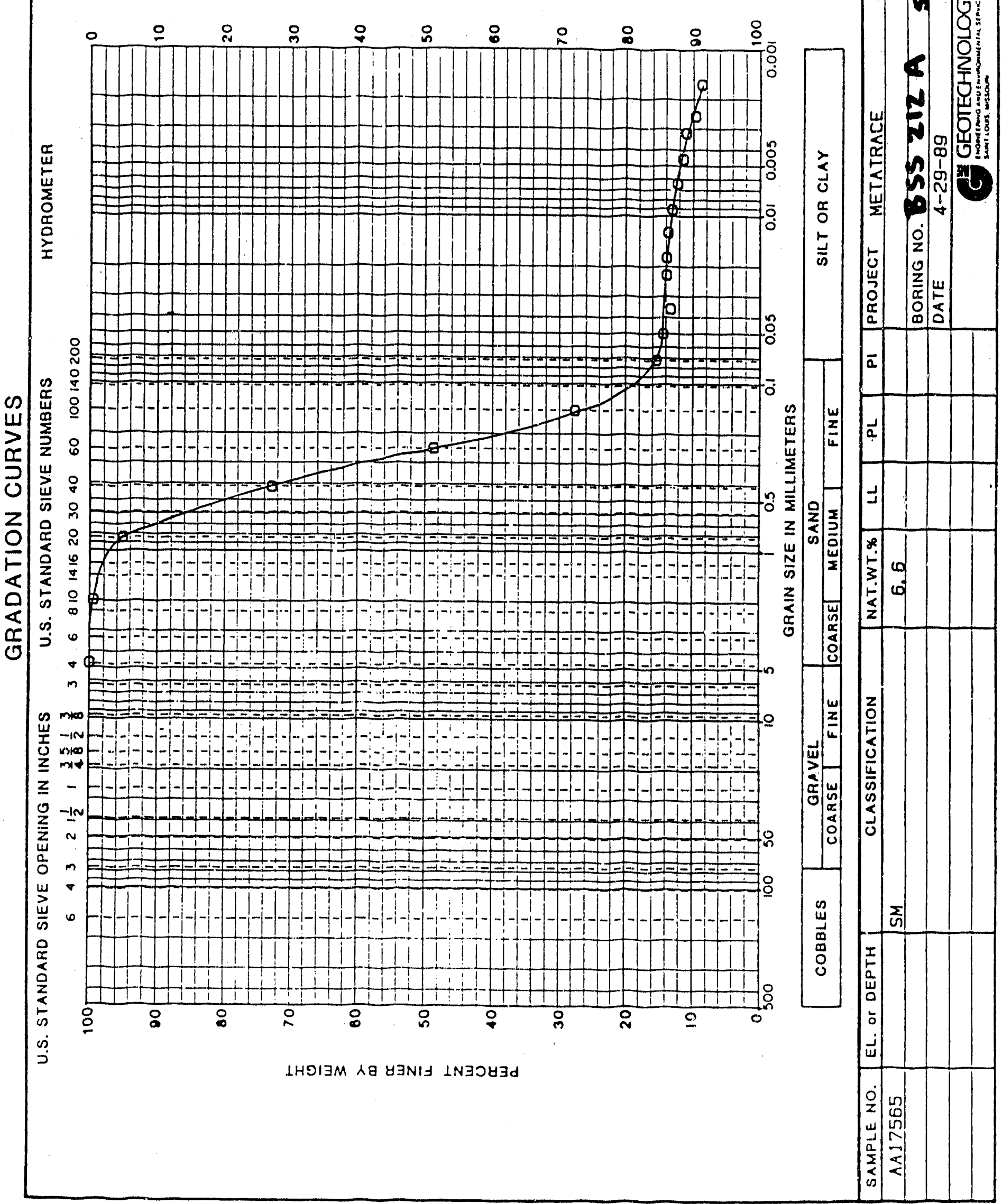


LHOIJM $A$ Y Y SYYOO LNJOUJd

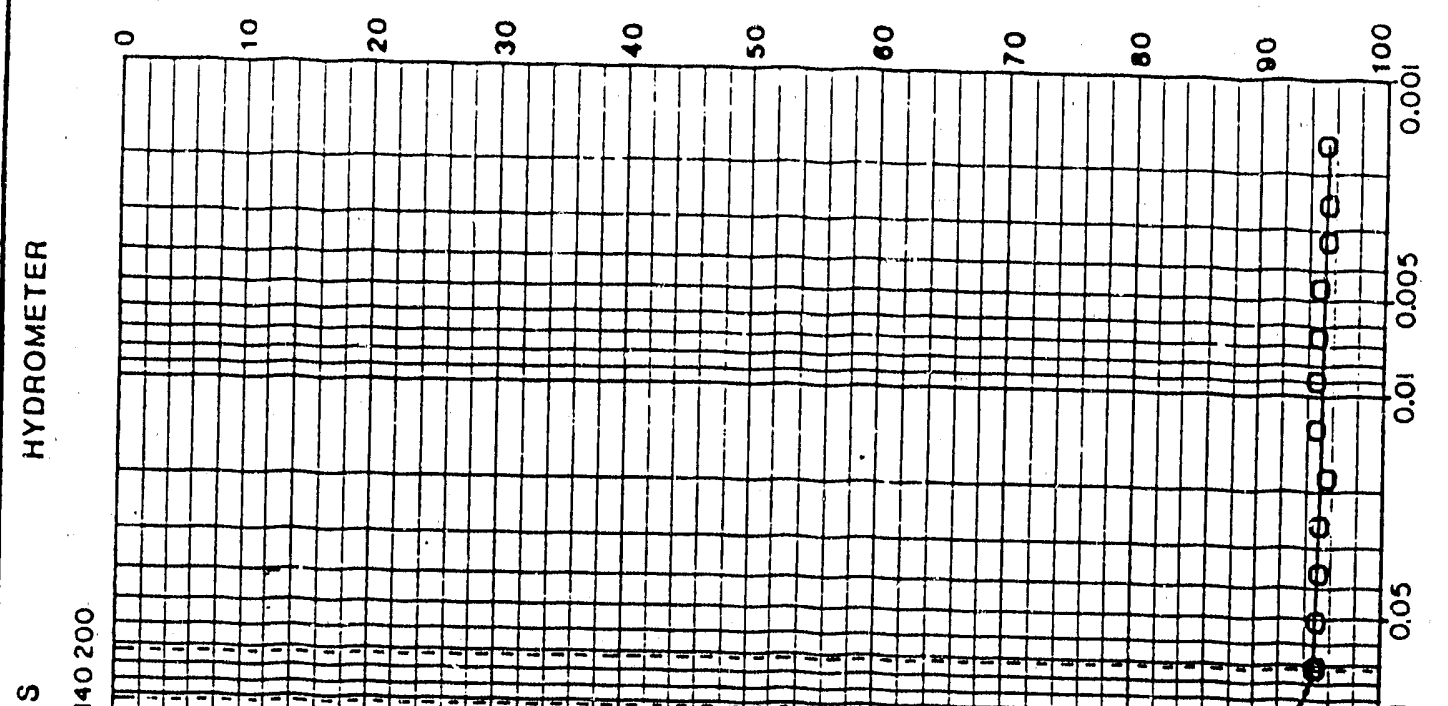

o

$\frac{1}{3}$

展

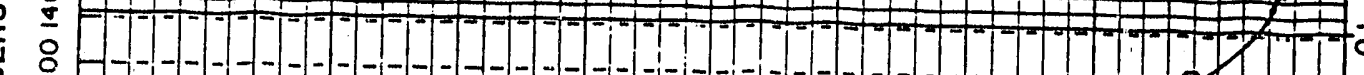

$\sum_{m}^{\infty} 8$

$-$

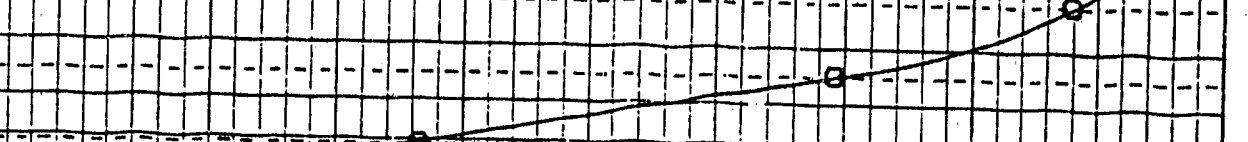

운

莫
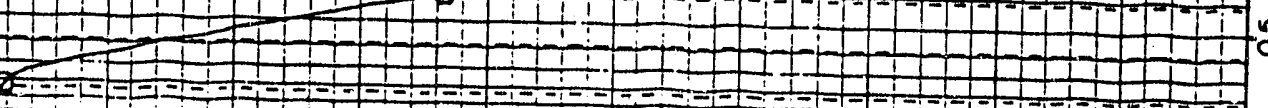

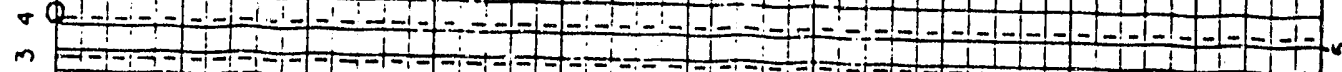
1 -

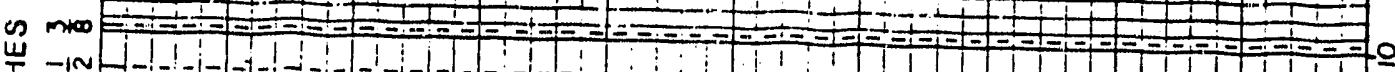

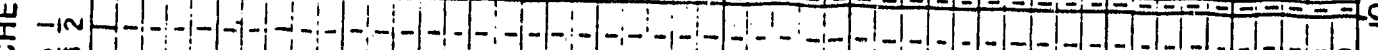

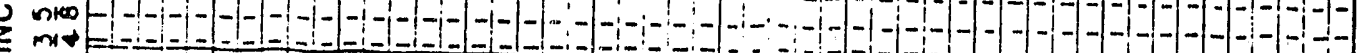

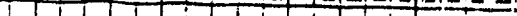

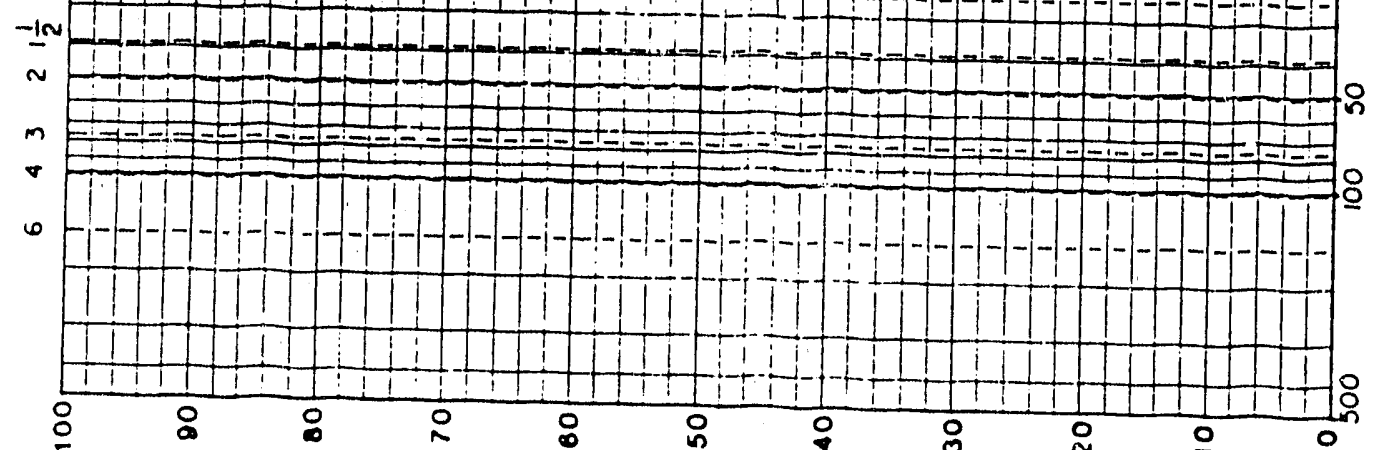

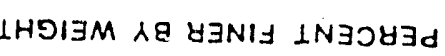

品

$\underline{z}$

$w$

$\frac{N}{6}$

z.

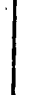

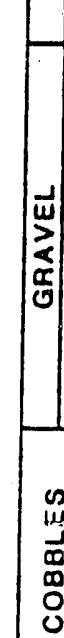

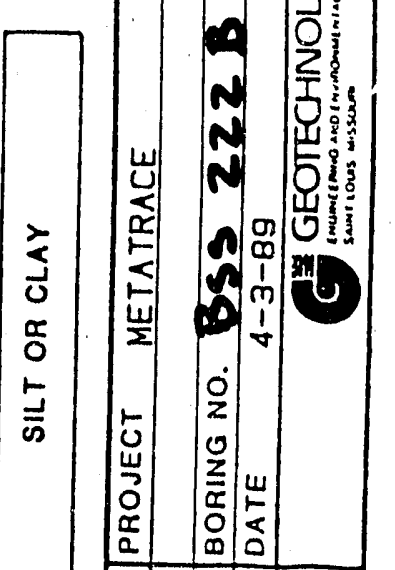

$\vec{a}$
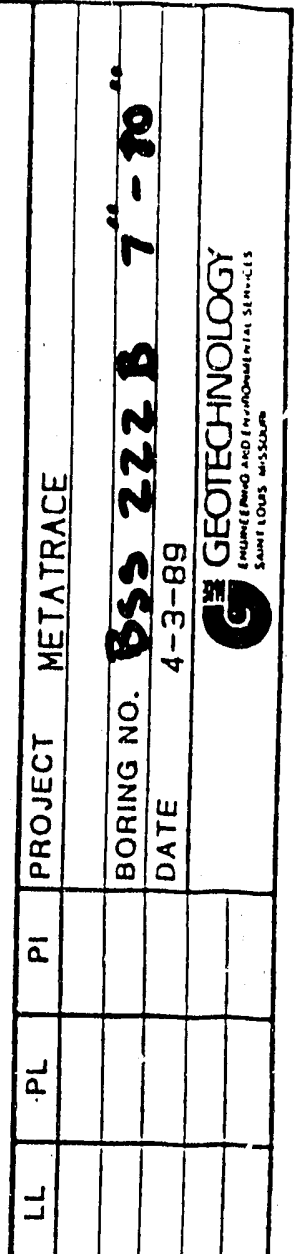

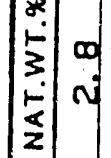

.

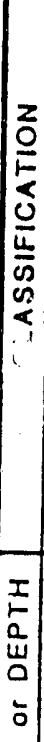

ш

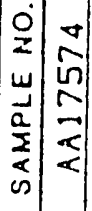




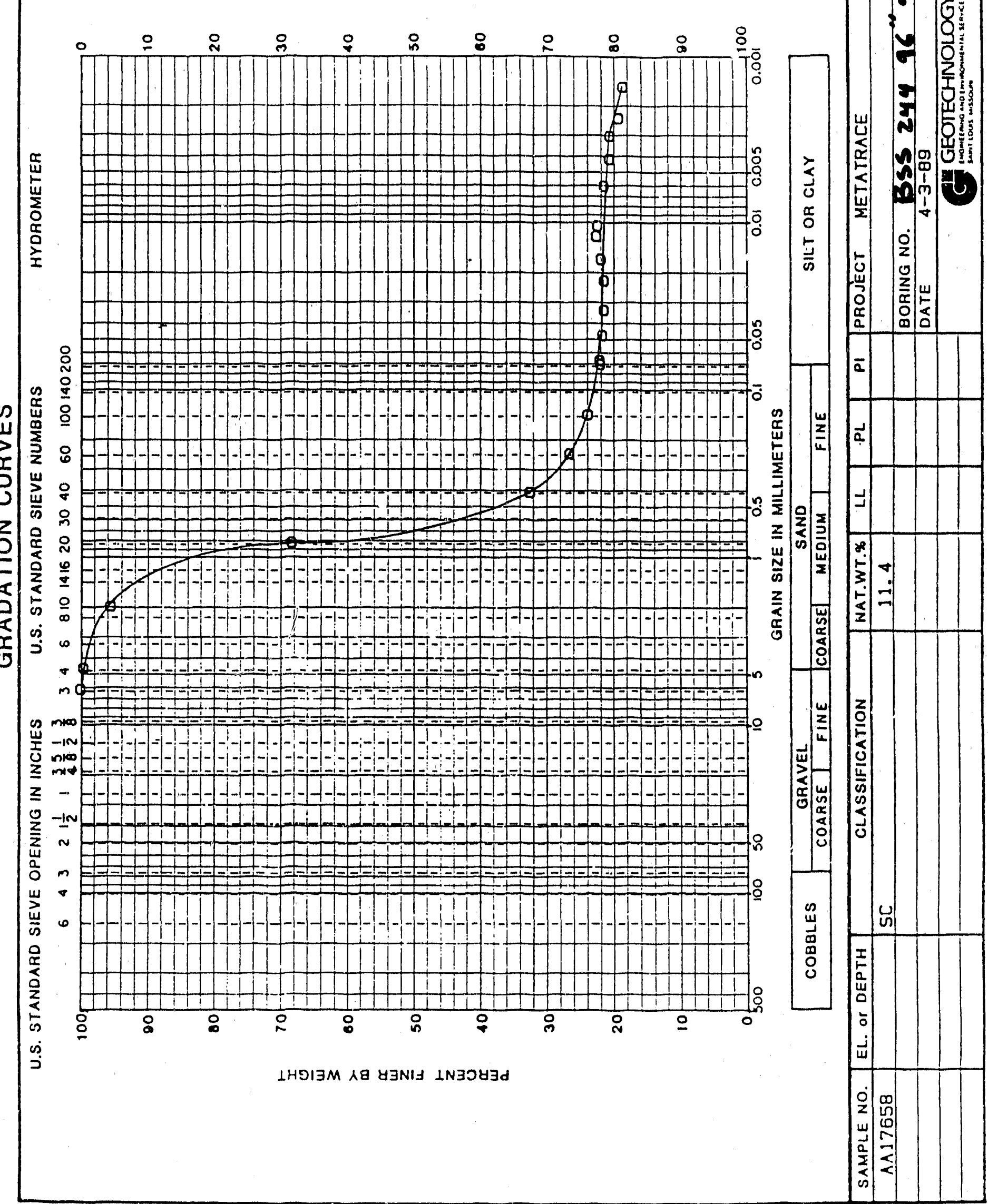


LFIOIJM 18 Y

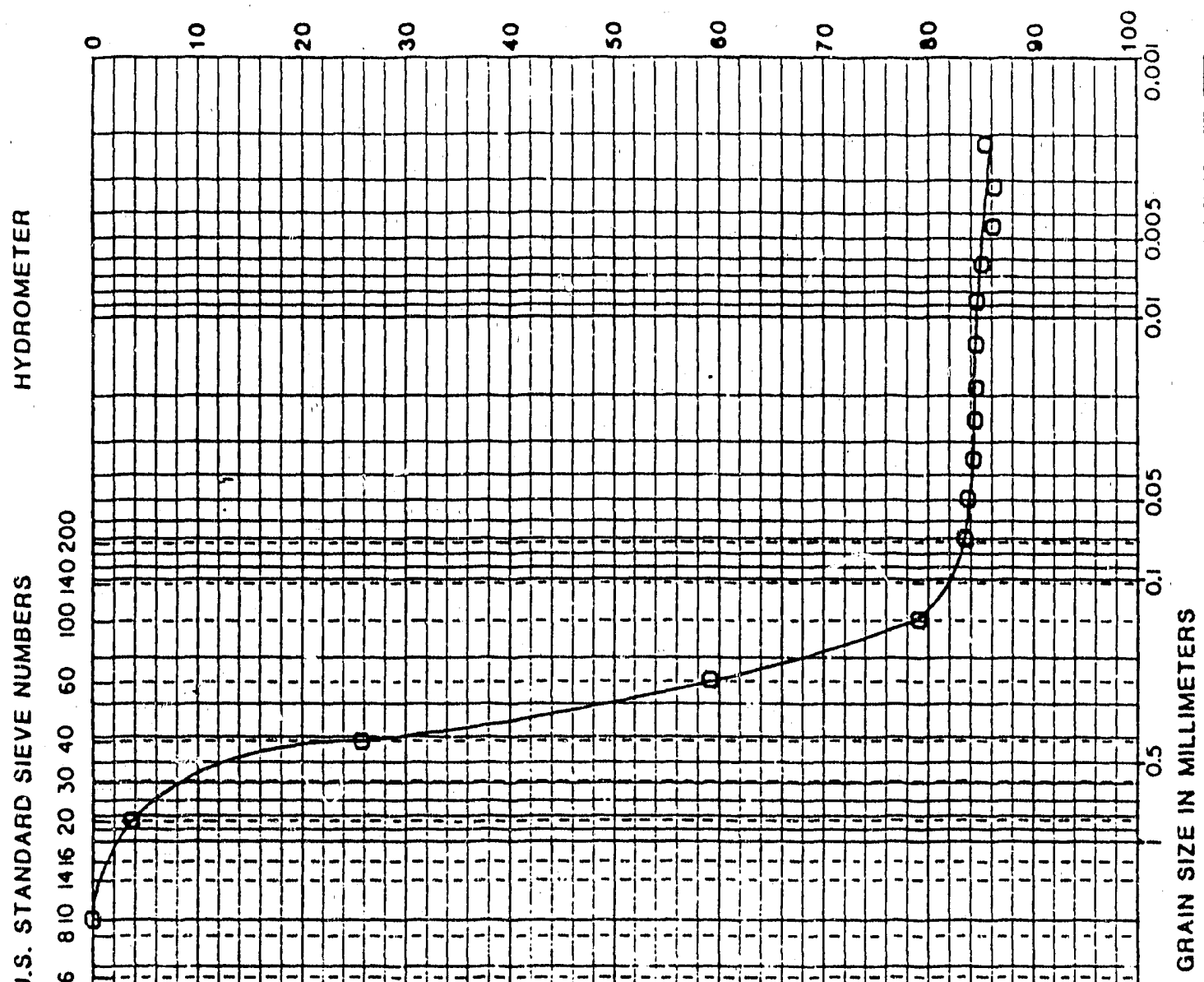

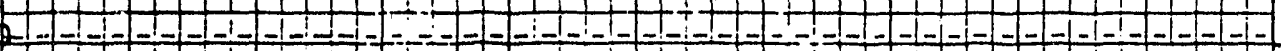
-1-1-1-1-1-1-1-1-1-1-1-2-1-1-1-1-1-1-1-1-1-1-1-1-1-2-1-2-1-1-

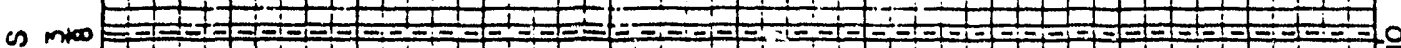

岌

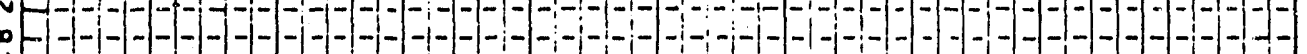

mis

$z-$

O

$\sum_{i=1}^{2}$

m

$\omega *$

$\underset{\underline{m}}{\underline{m}}$

0
2
2
2
0
0
0
0
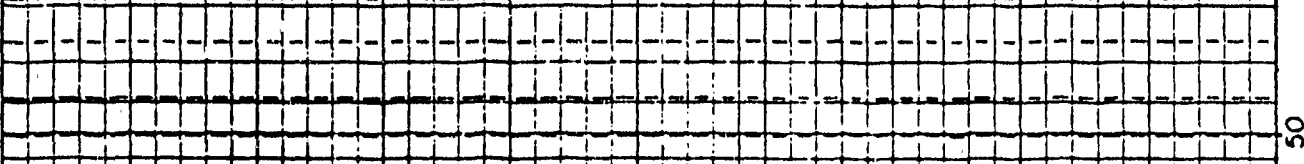

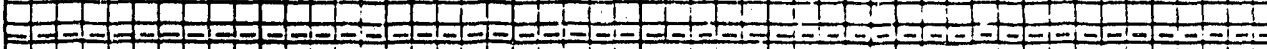

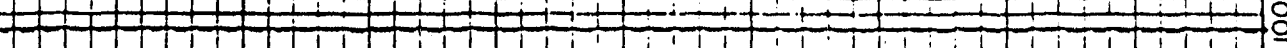




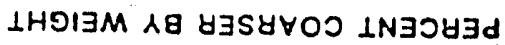
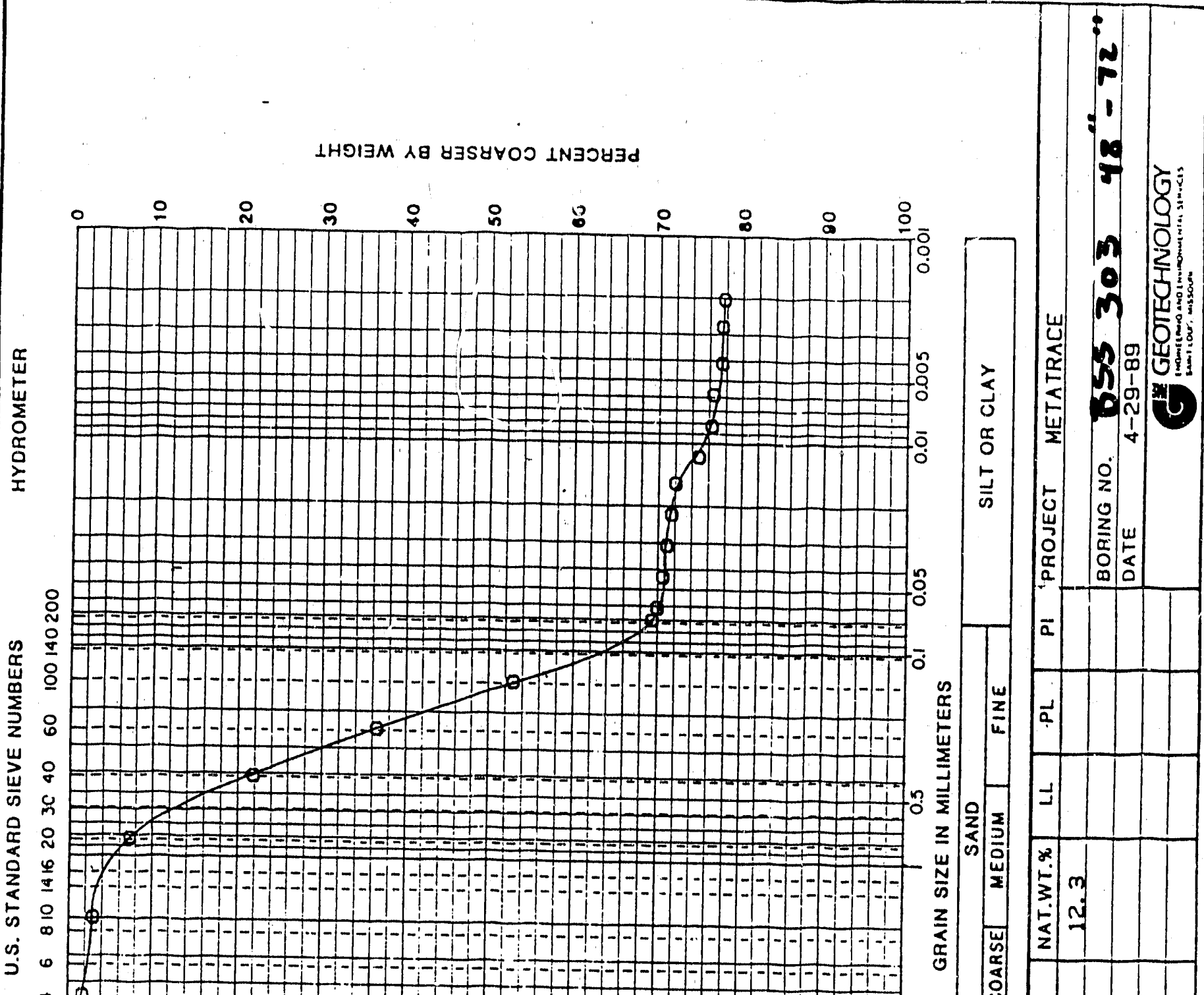

\section{$\infty$
$w$
0
0
0
$z$
0
5
0
$\frac{\pi}{0}$
$\pi$}

$+$

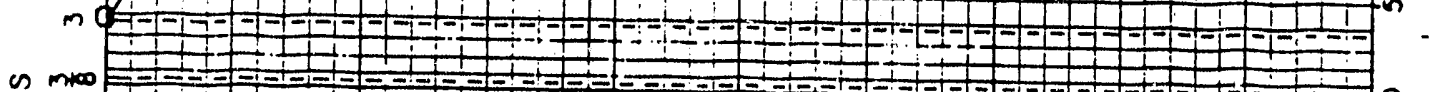

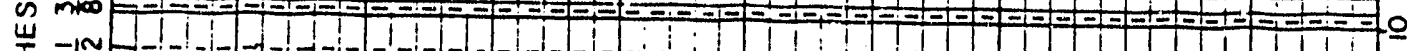

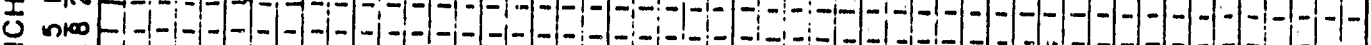
$2 m+=1$ -

$z-1-1-1-1-1-1-1-1-1-1-1-1-1-1-1-1-1-1-1$

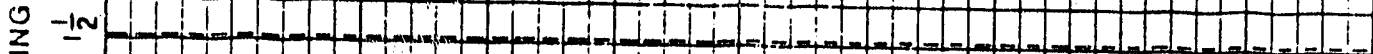

N

$m$

$\checkmark$

$\omega$

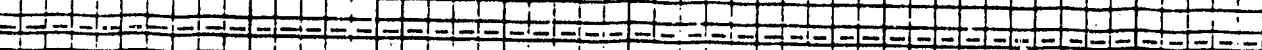

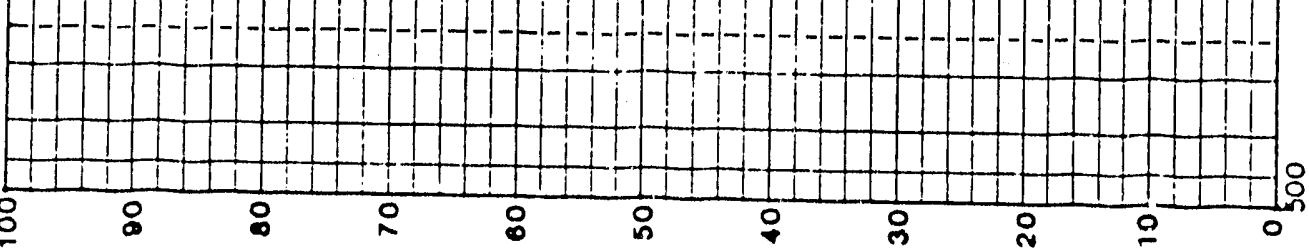

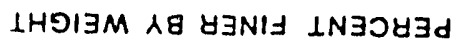




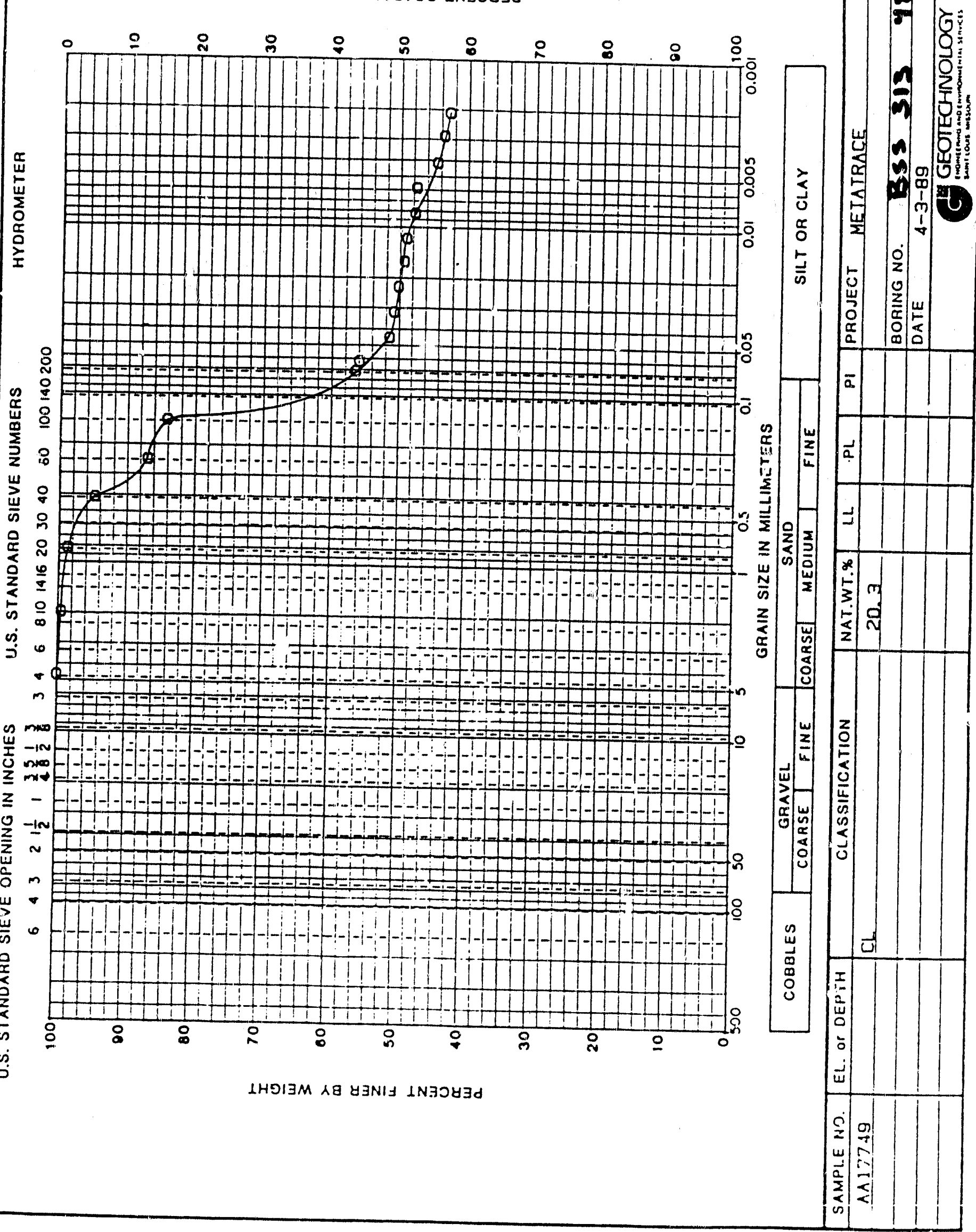


LHOIJM 18 \&

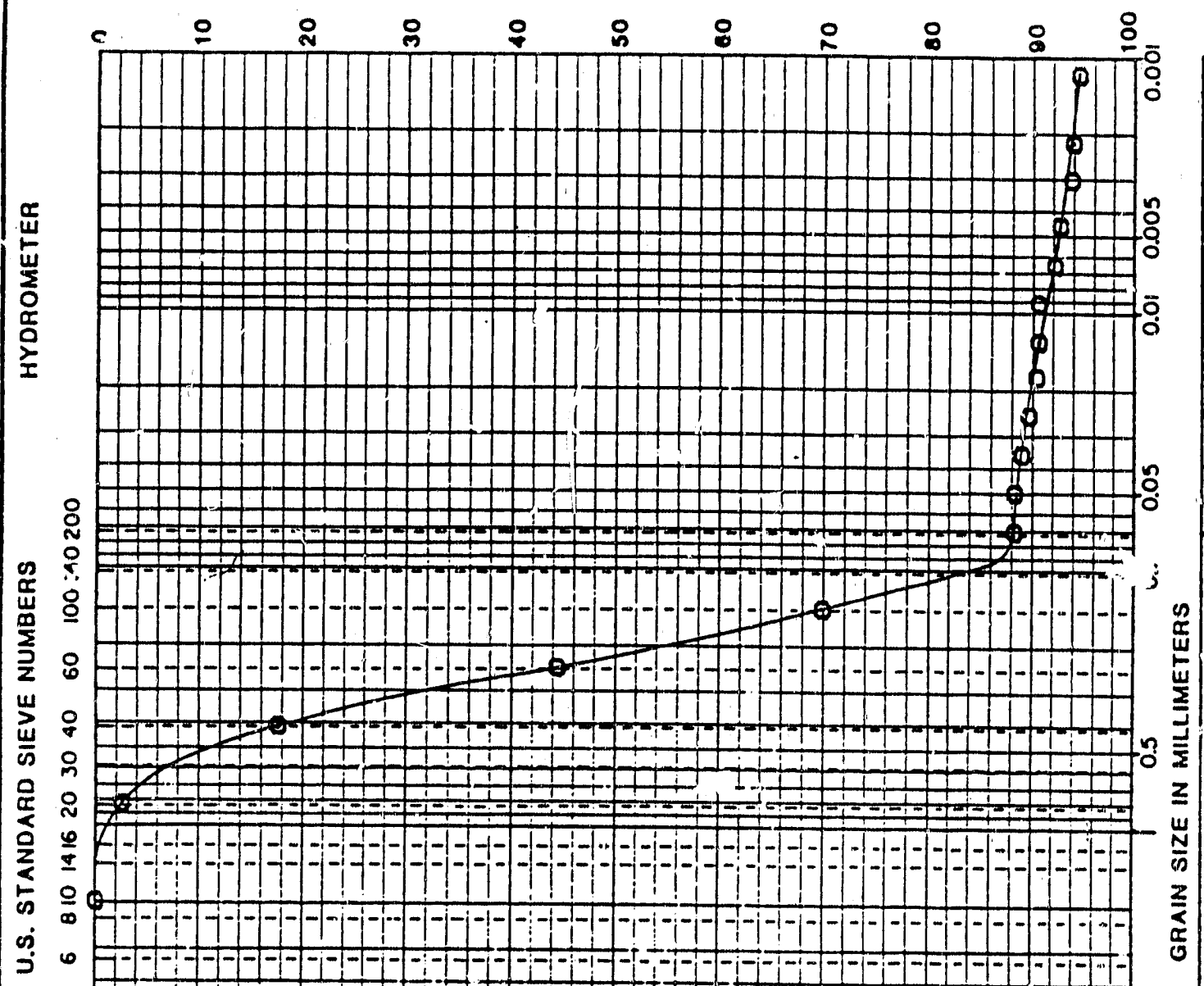

- $1-1-1-2-1-1-1-1-2-1-1-1-1-1-1$

$m$ (1)

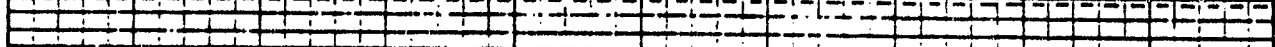

in $\rightarrow 00$ -

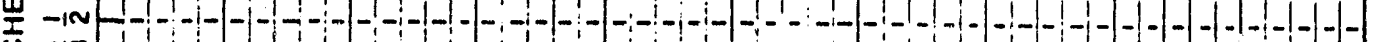

now -

2 -

$-1-1--1-1-1-1-1-1-1---1-1-1-1-1-1-1-1-1-1-1-1-1-1-1-1-1-1-$

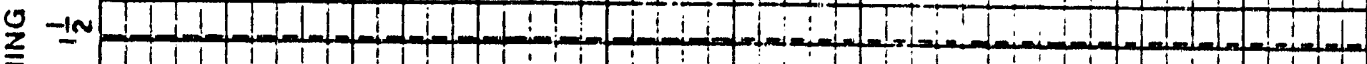

n

$m$

$\circ$

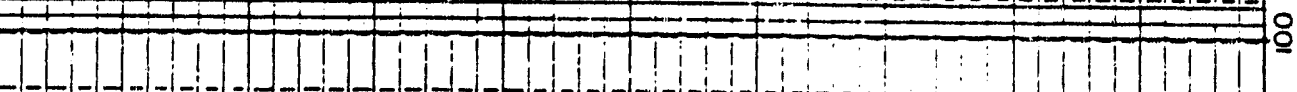

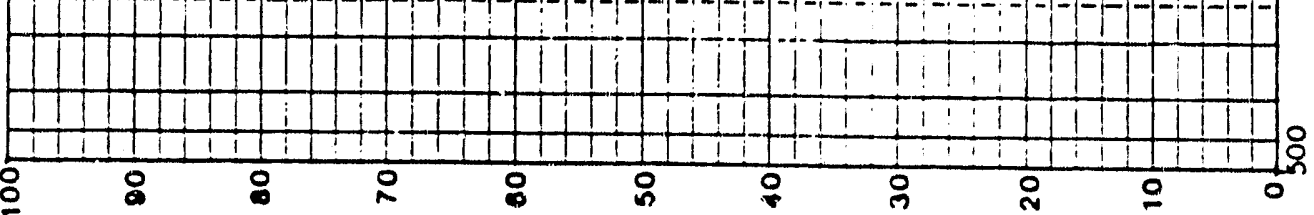



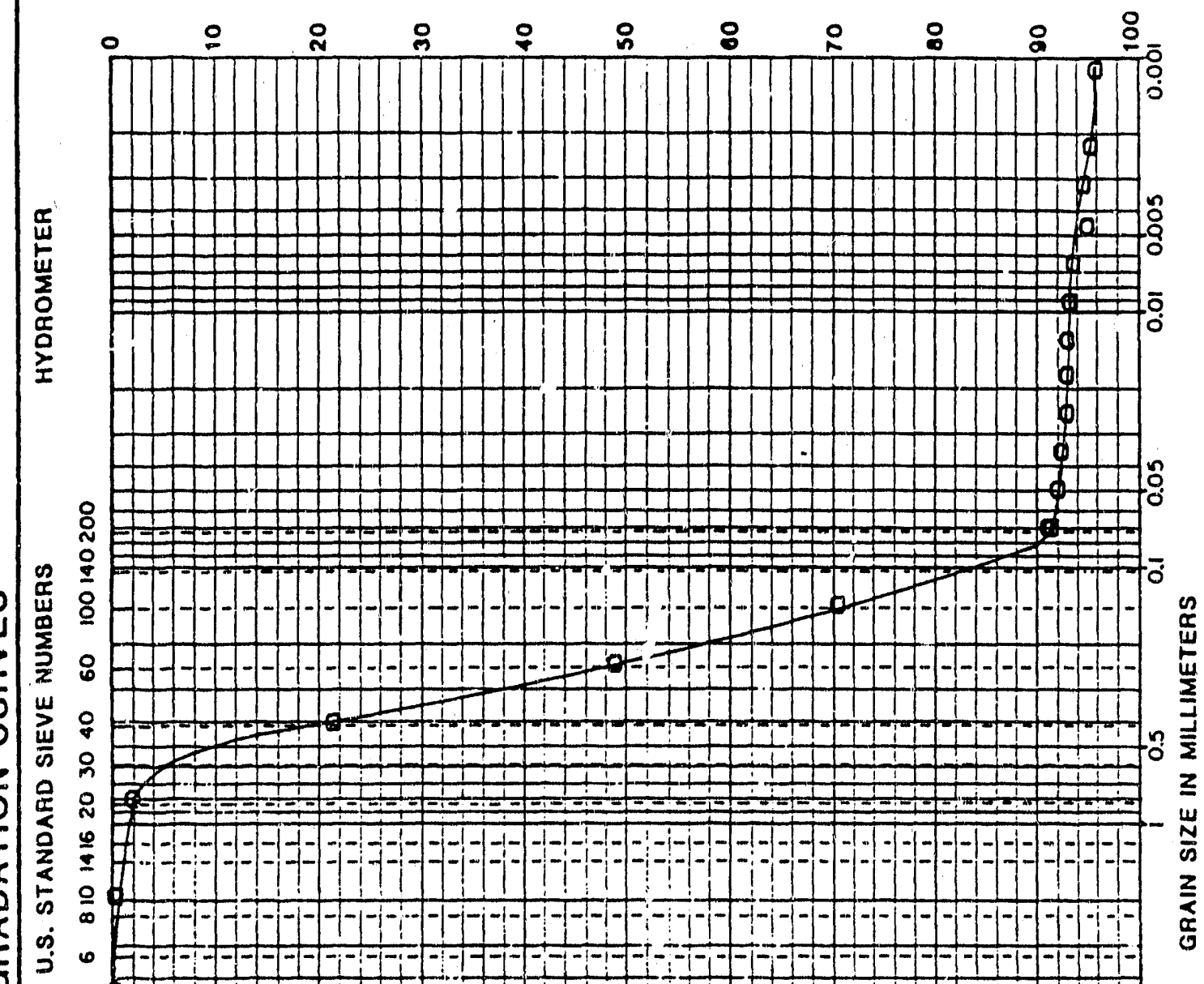

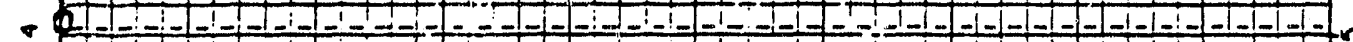

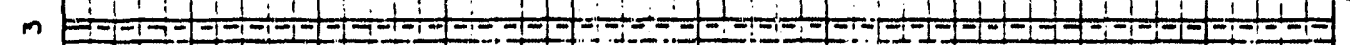

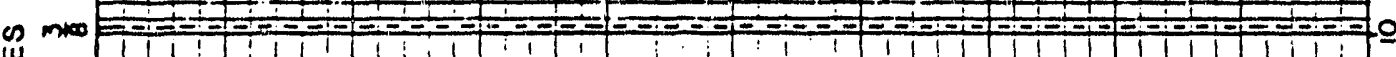

$\frac{m}{J}-10$

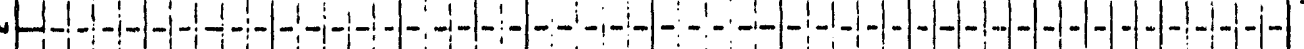

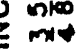

$-1-1-1-1-1-1-1-1+1-1-1+1-1-1,1-1-1-1-1-1-1-1-1-1-1-1-1-1-1-1-$

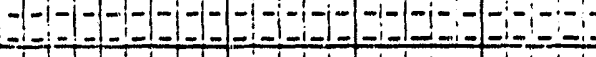

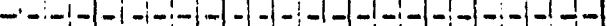

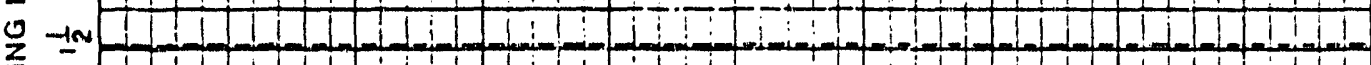

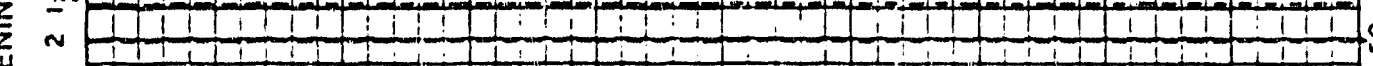

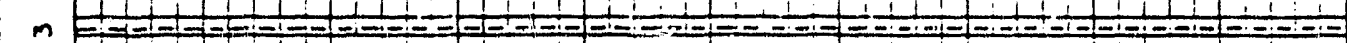

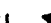

$>$

$\omega$

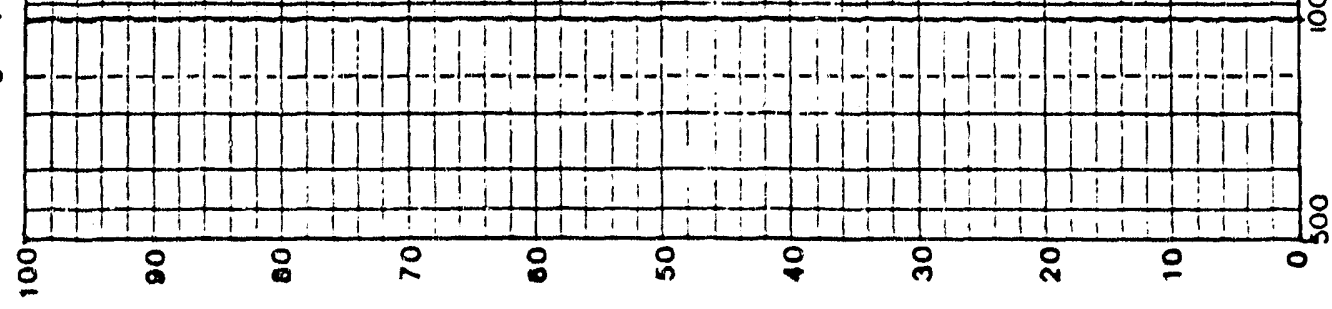




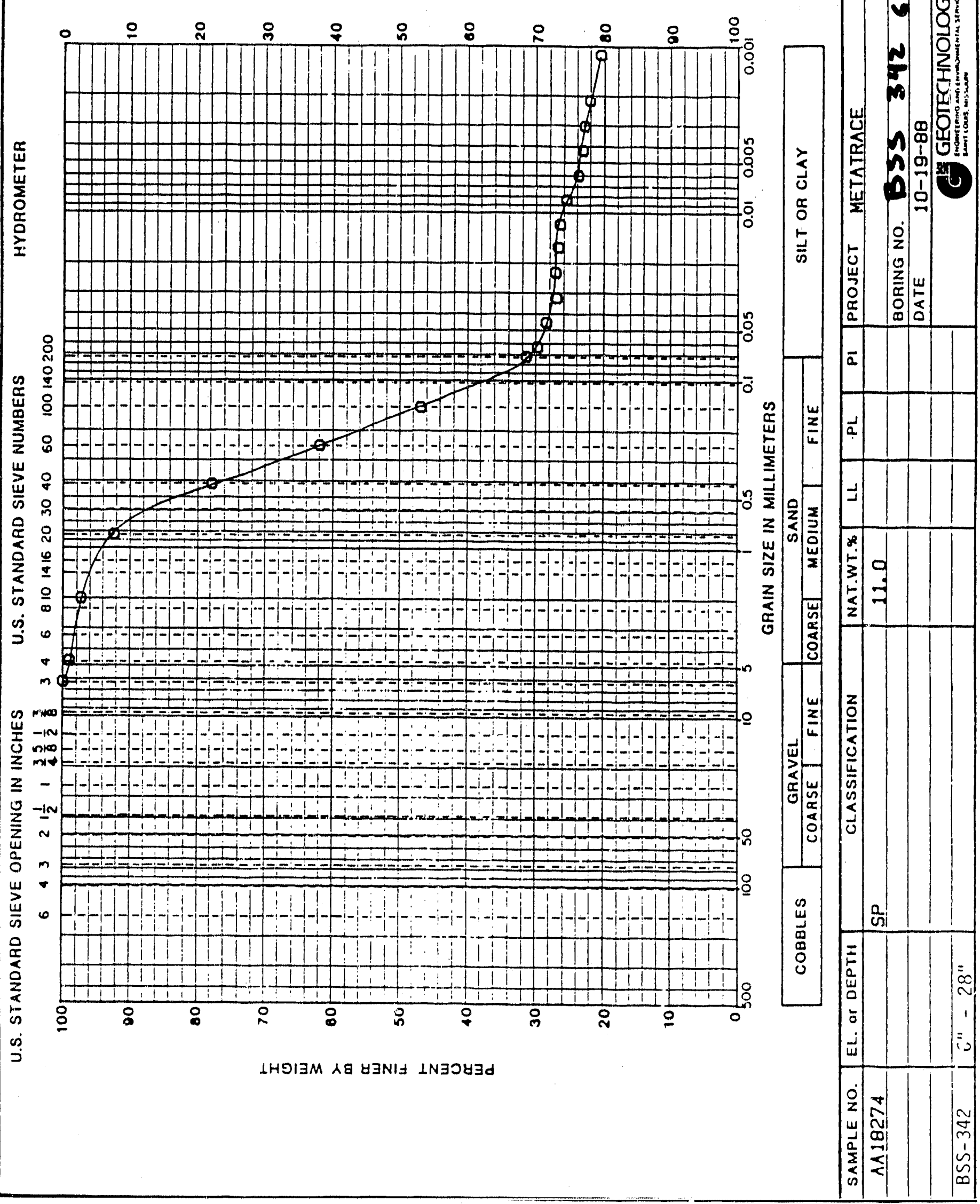




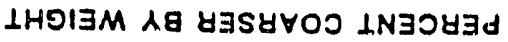

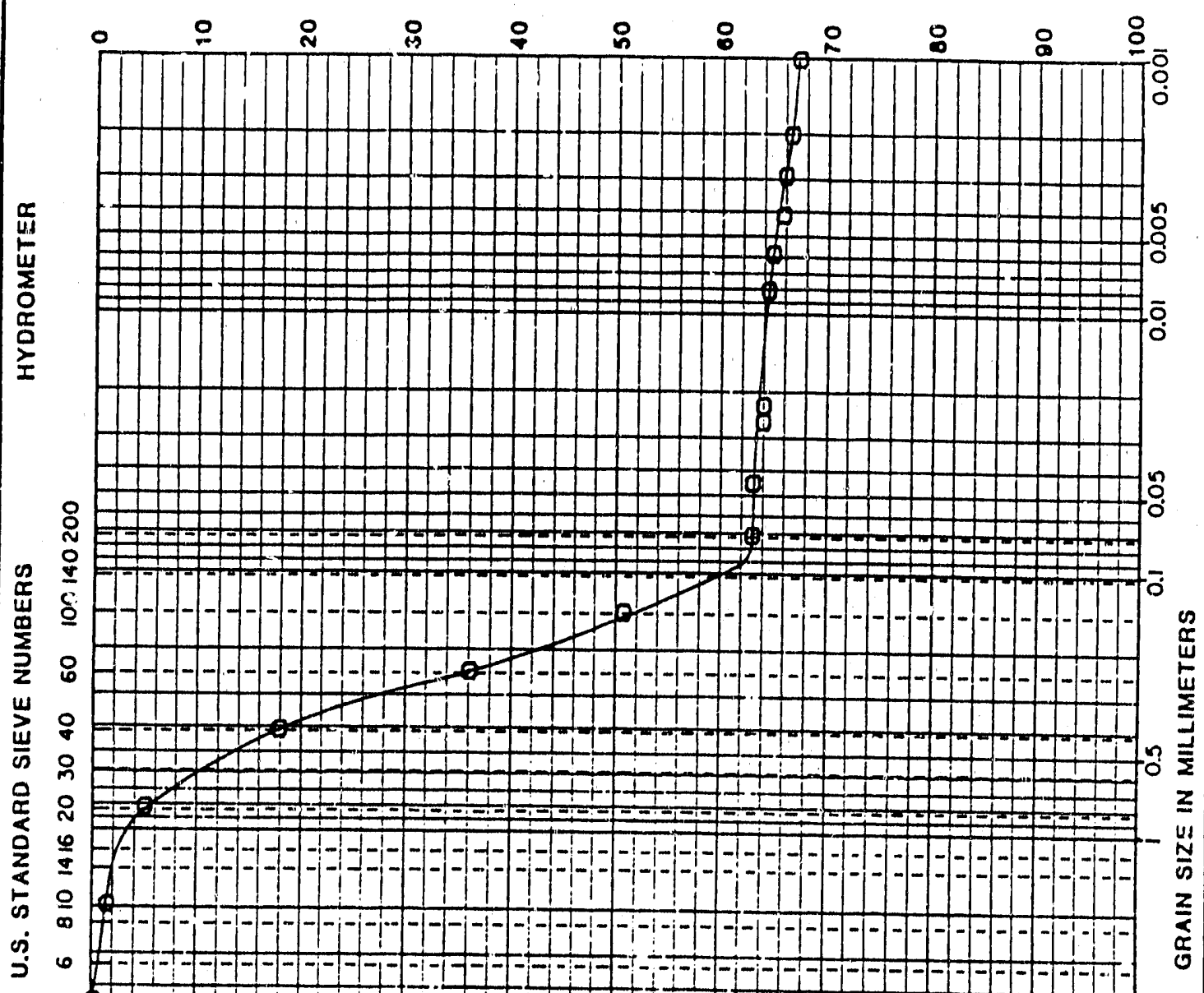

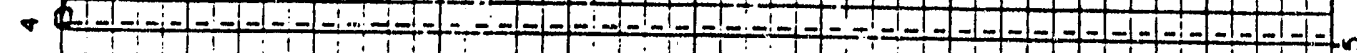

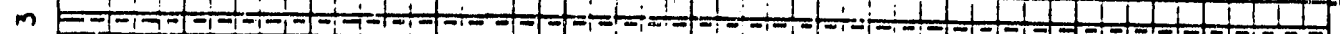

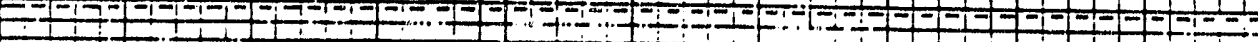

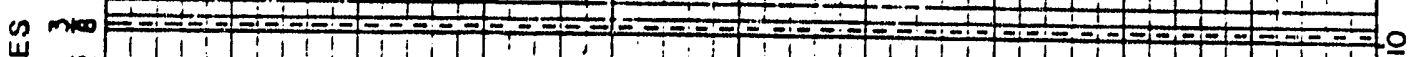

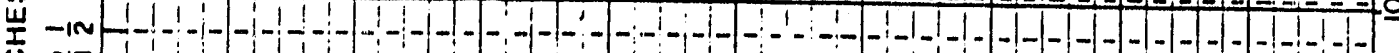

ভ n

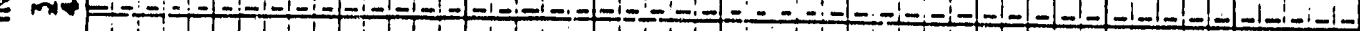

$\geq-1-1-1-1-1-1-1-1-1-1-1-1-1-1,1-1-1-1-1-1-1-1-1-1-1-1-1-1-1-$

こ一

$\sim$ F

$m$

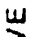

$>$

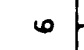

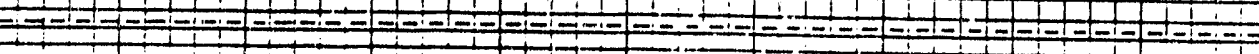

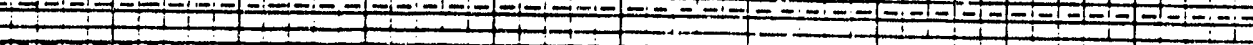

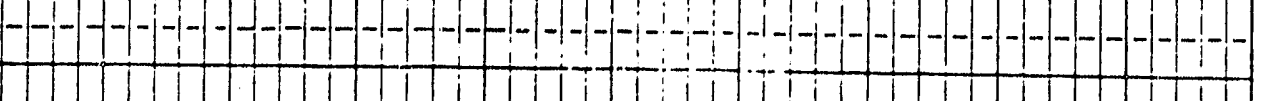

要

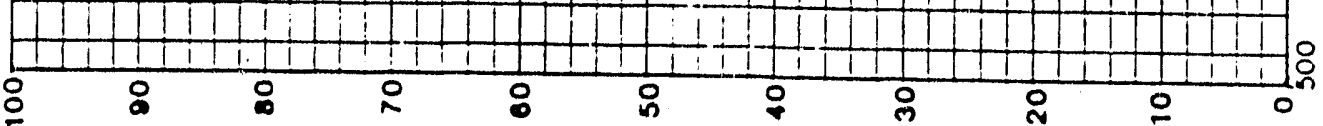




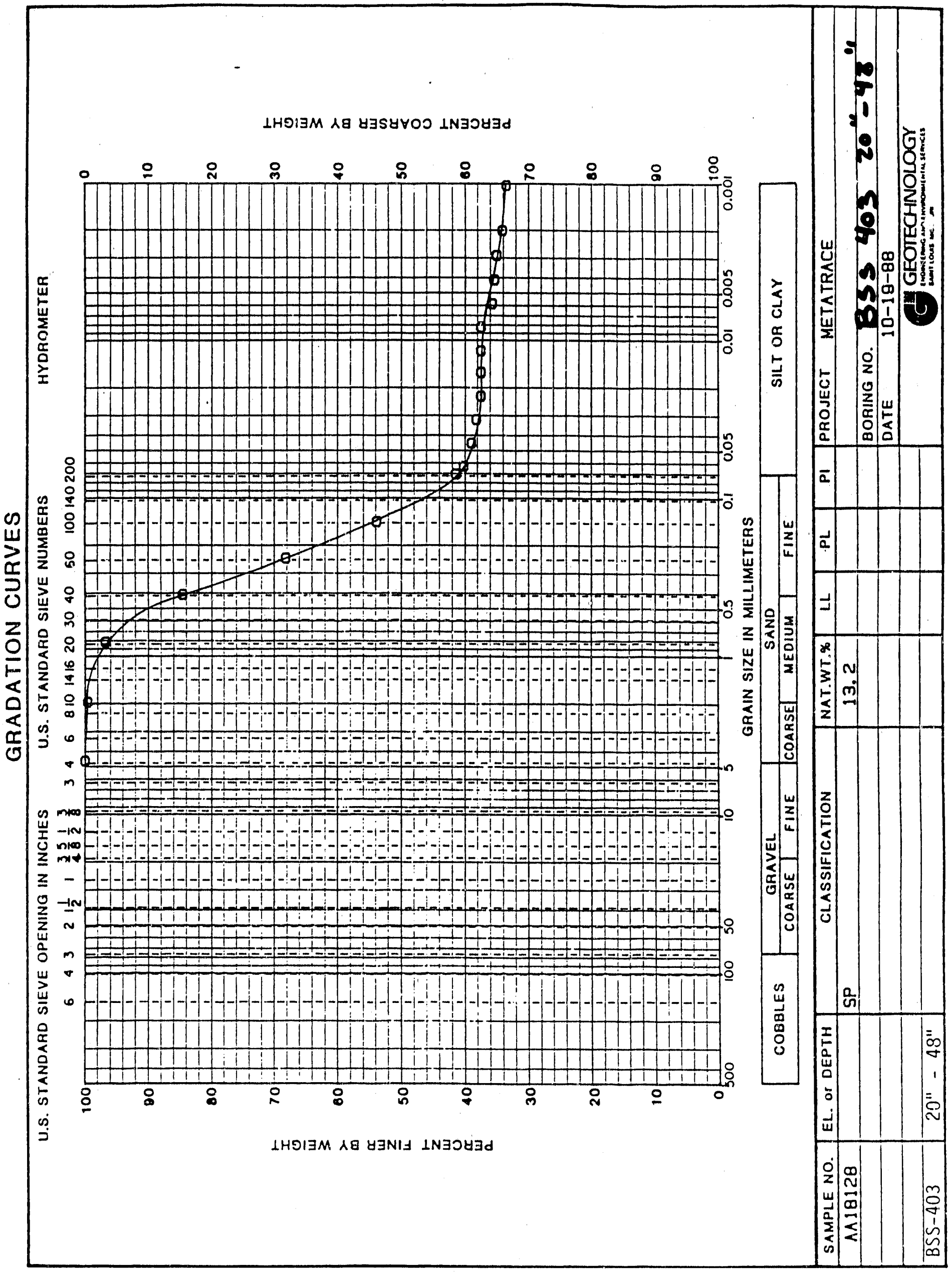




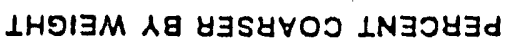

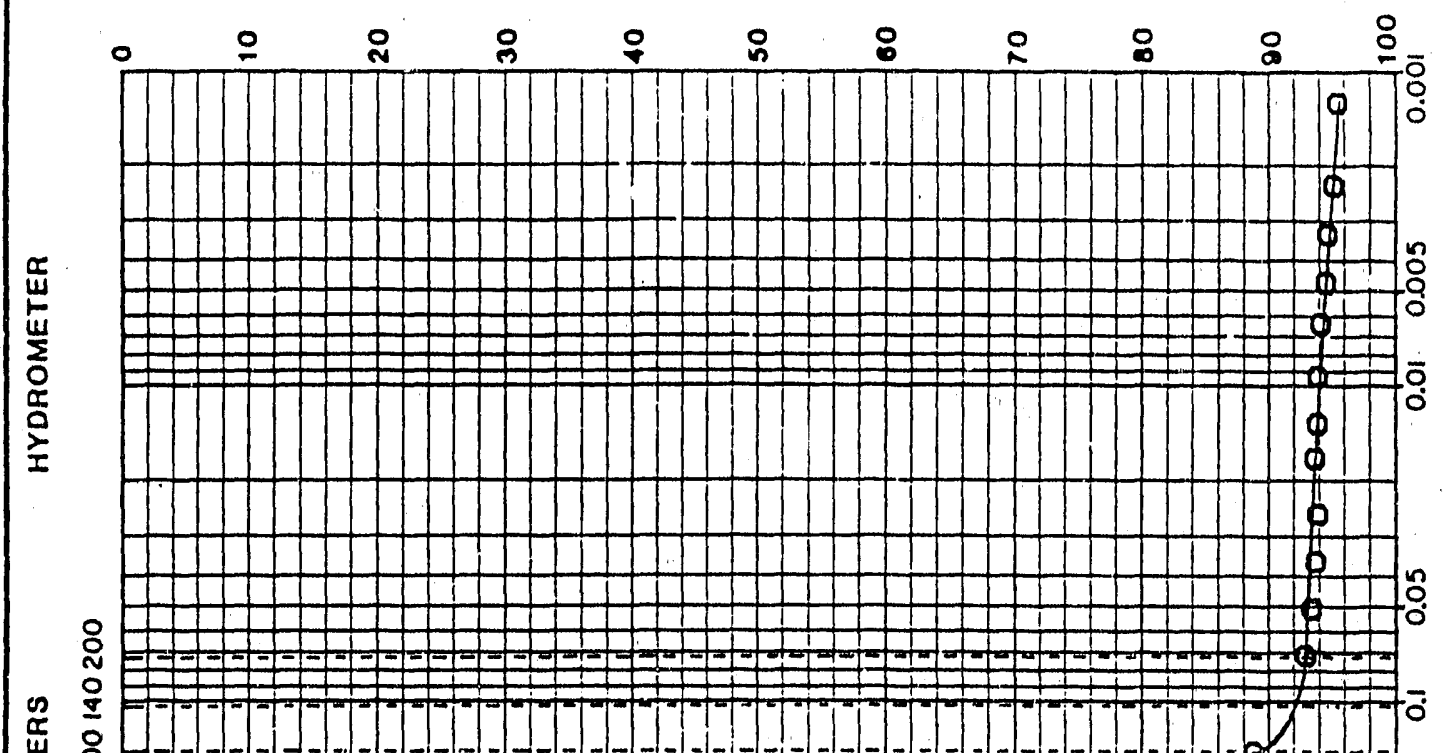

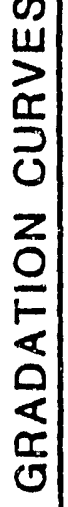

(1)

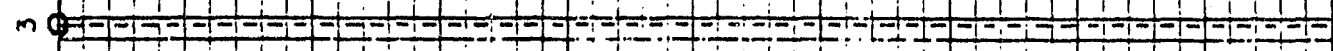

is $\mathrm{m} \times \mathbf{1}+1$

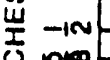

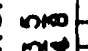

$-1-1-1-1-1-1-1-1-1-10$

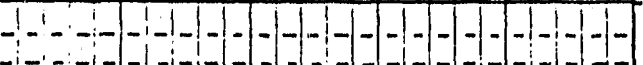

$z-$

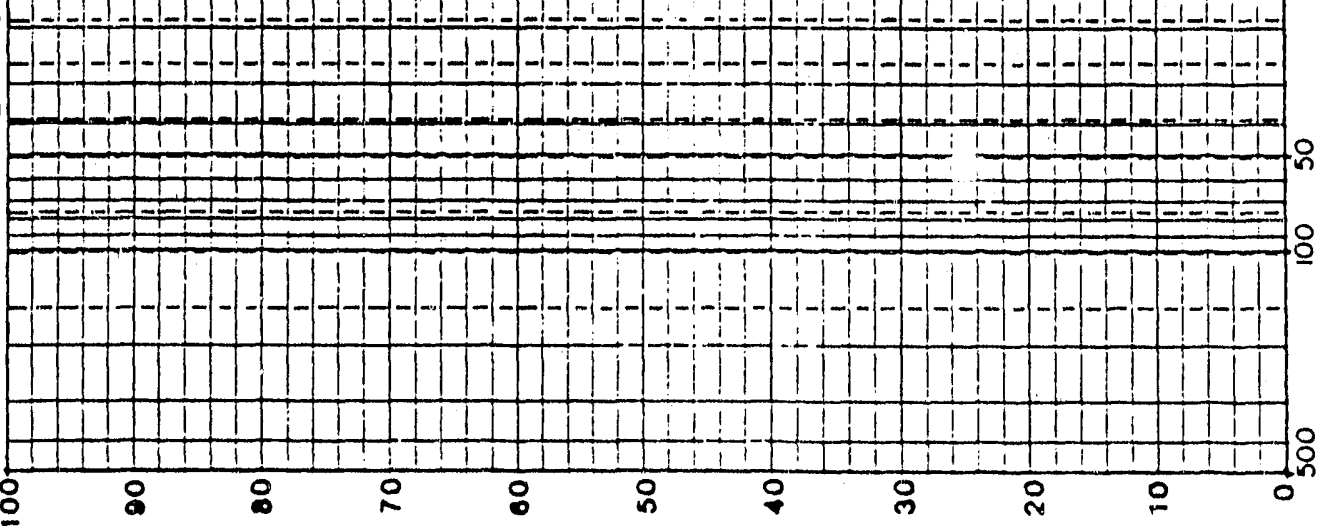

O

N

m

.

0

列

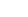

LHOIJNI 18 Y YNNI LNJOYGd 


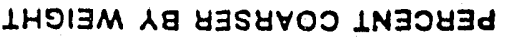

es invos

夏

ine

$\geq$ -

0

$\frac{0}{z}$

絜

品

$\stackrel{m}{\nu}$

䋆

监

웅

نं

j

$\stackrel{8}{\circ}$

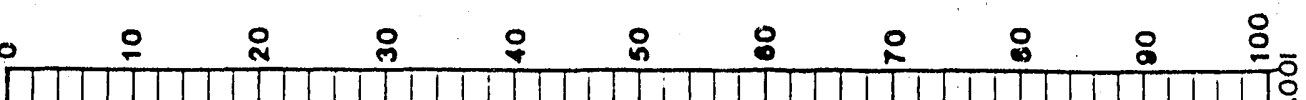

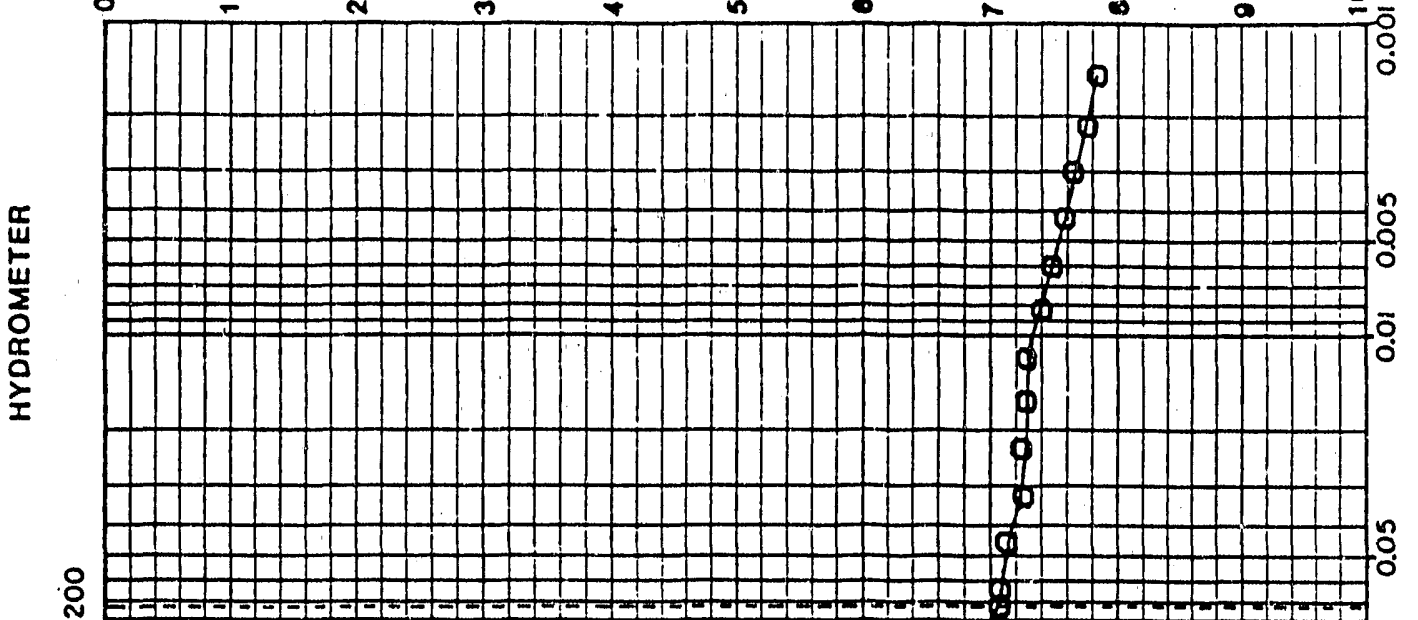

出

需

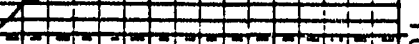

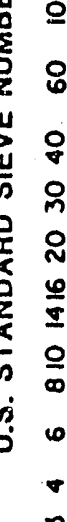

$:$

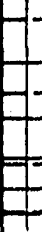

-

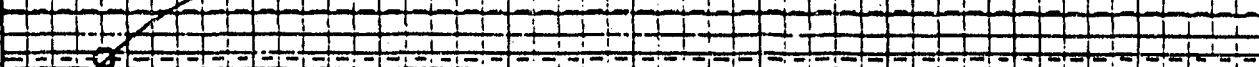

=:

H-

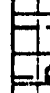

$10-1-1-1-1-1-1-1-1-1-1-1-1-1-2-1-1-1-1-1-1-1-1--1-1-1-1$

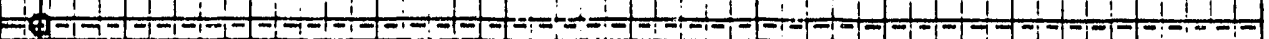

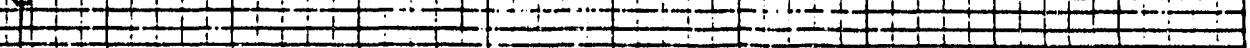

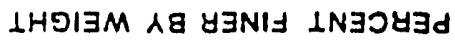

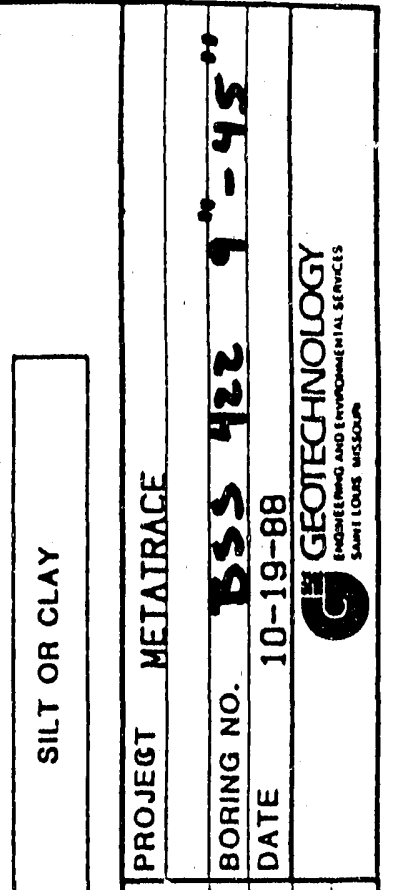

$\bar{a}$

$\frac{1}{4}$

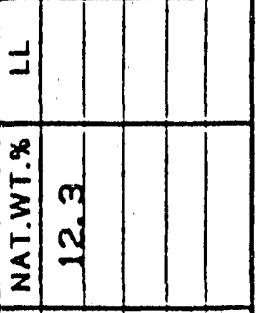

(5)

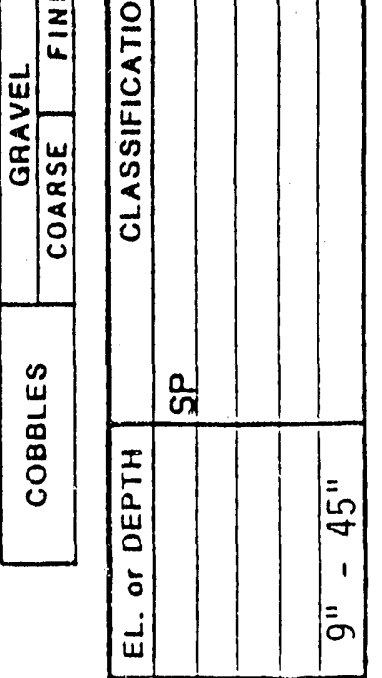

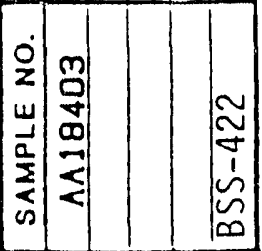




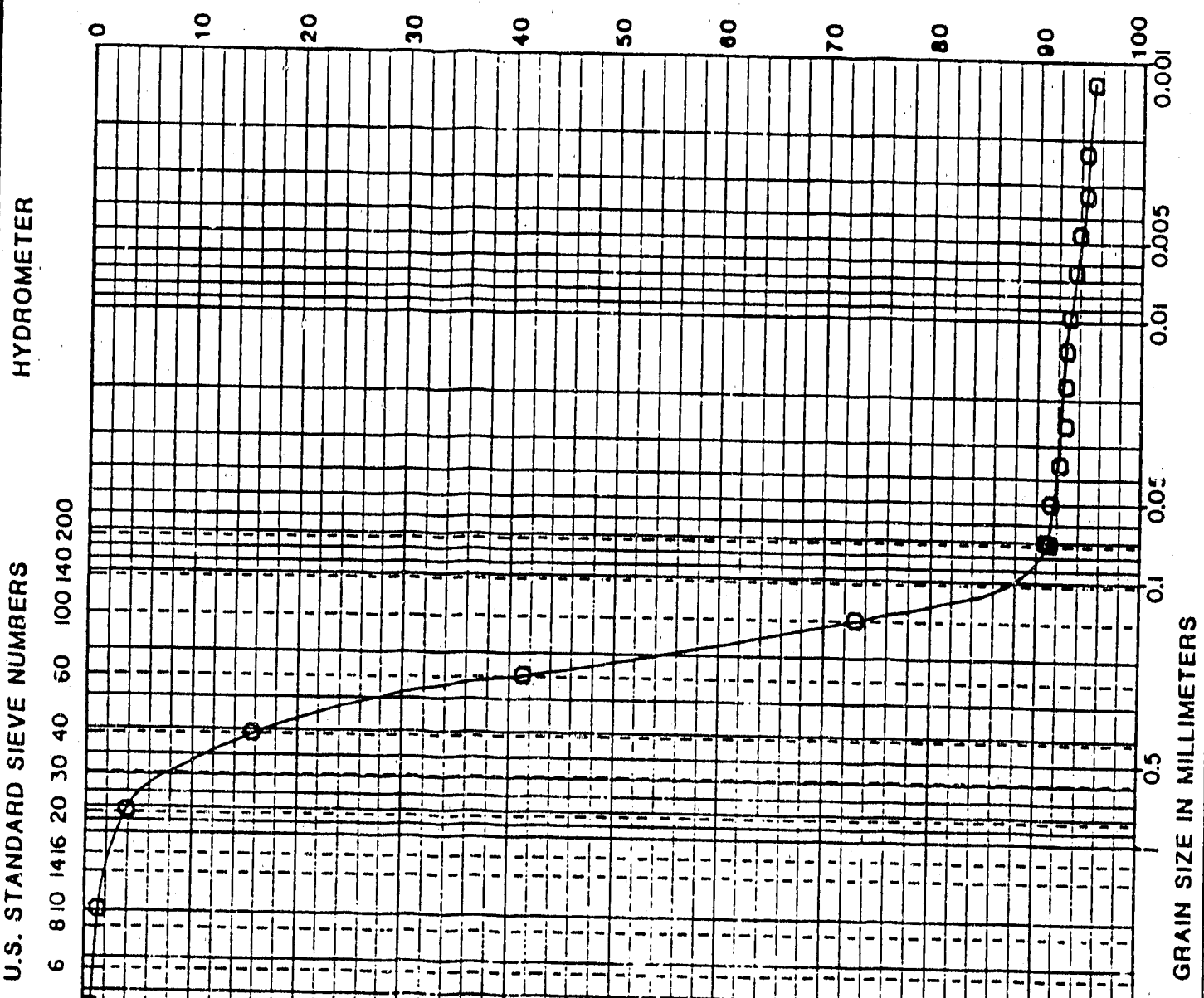

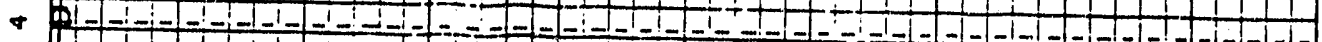

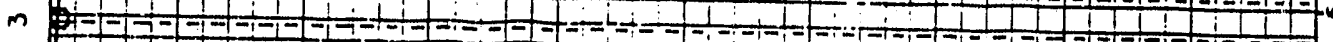

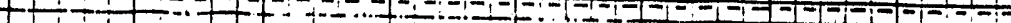

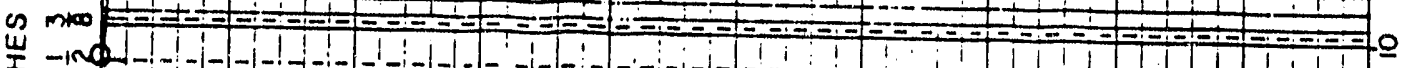

nico

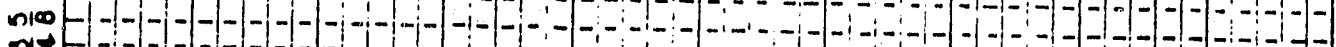

$\leq-$ $-1-1-1-1-1-1-1-1-1-1--1-1-1-1-1-1-1-1-1$

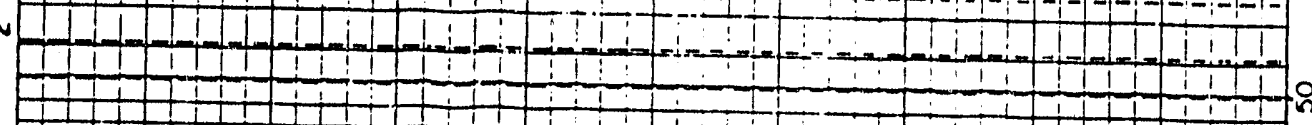

$m$

$m$

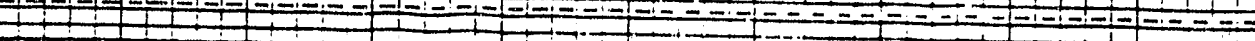

$\omega$

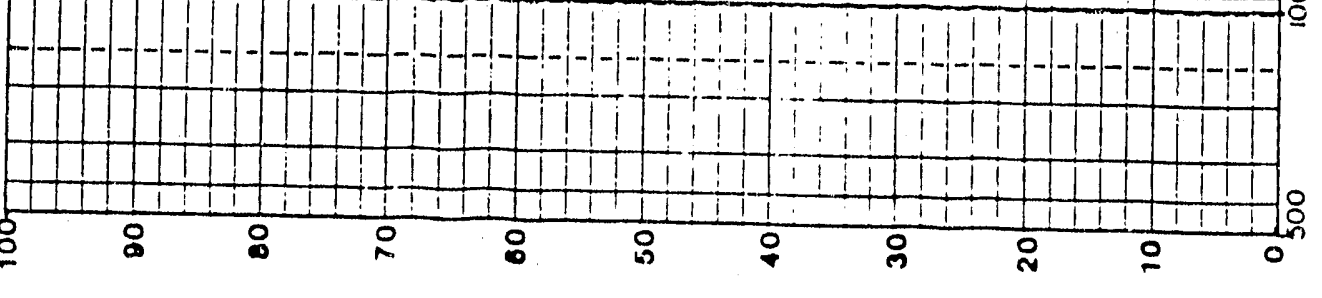

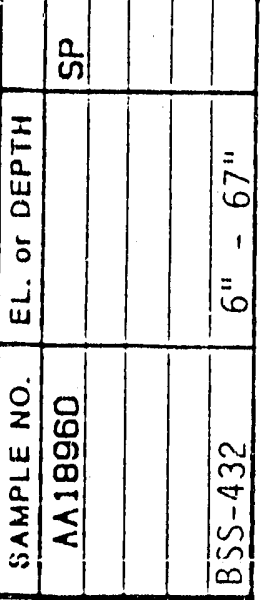


LHOJM Аล $\forall \exists S 4 \forall O O ~ L N \exists J 4 \exists d$

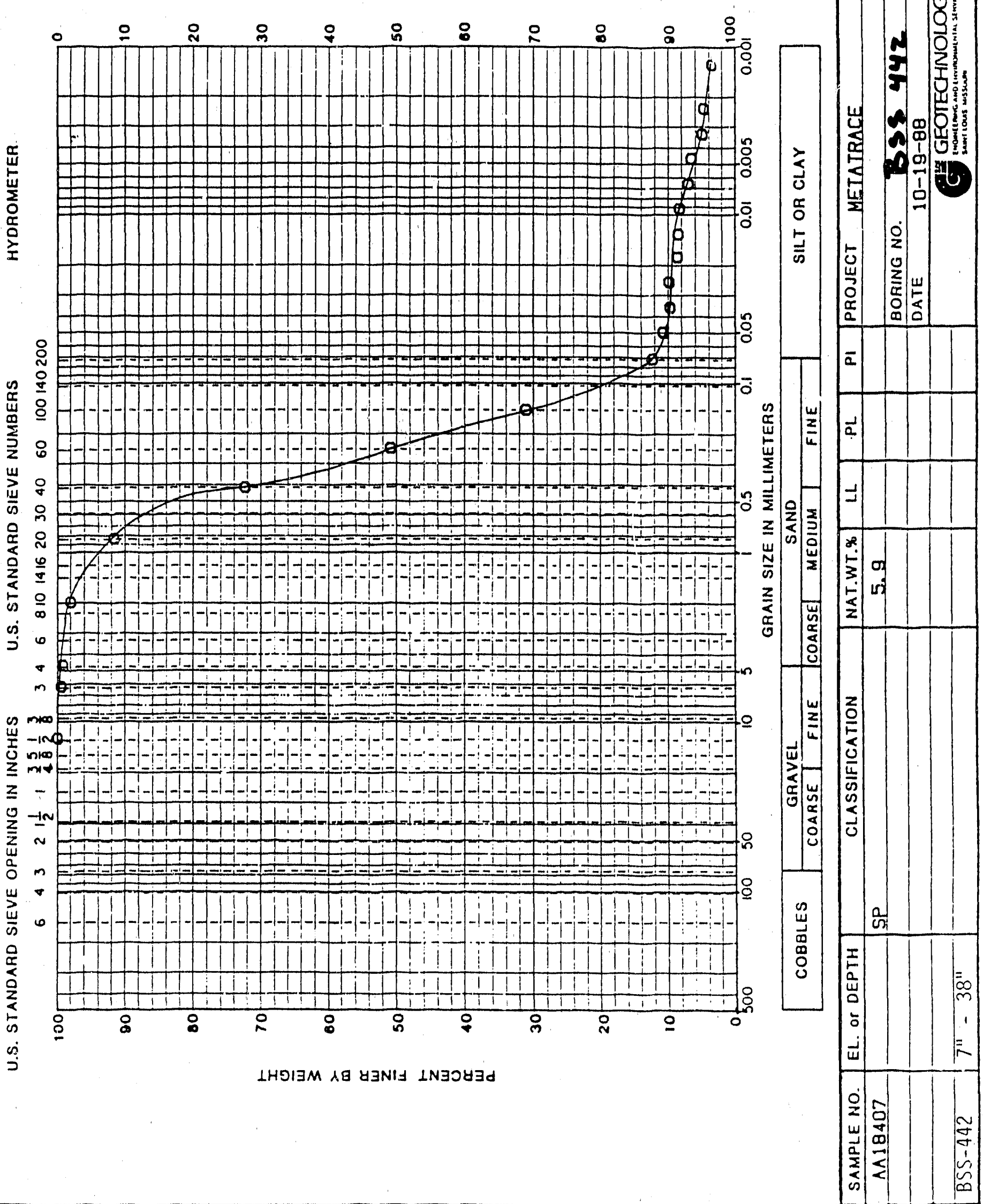


LHOI

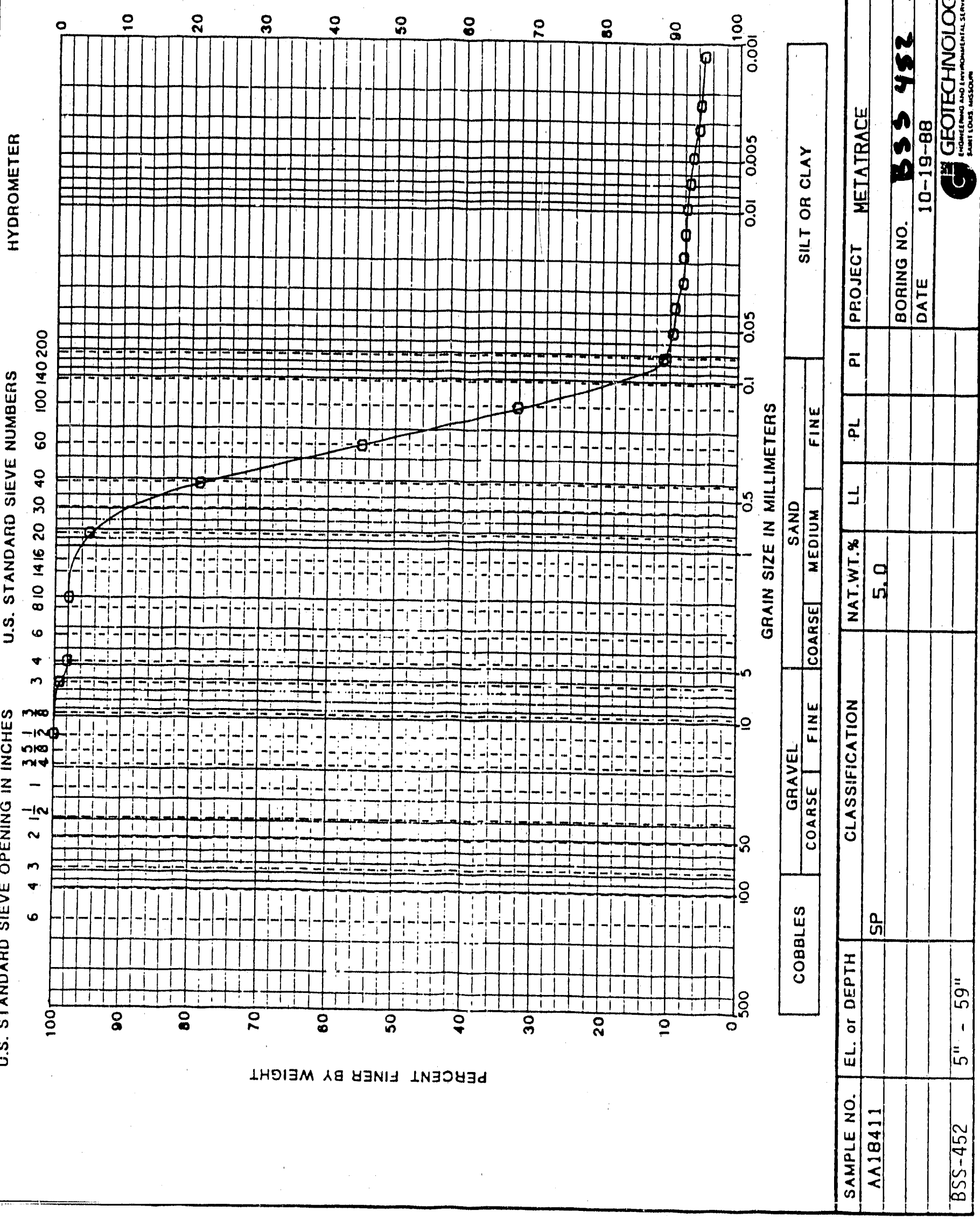




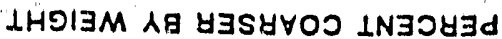

$\infty$
$u$
$z$
5
0
$z$
0
$\overline{5}$
$\square$
$\square$
$\square$
0

:

$\circ \stackrel{2}{2} \stackrel{2}{2}$ $\circ \quad 8$ P 8 8 $\therefore$ $\stackrel{2}{2} \stackrel{\circ}{\circ} \stackrel{\circ}{\circ}$

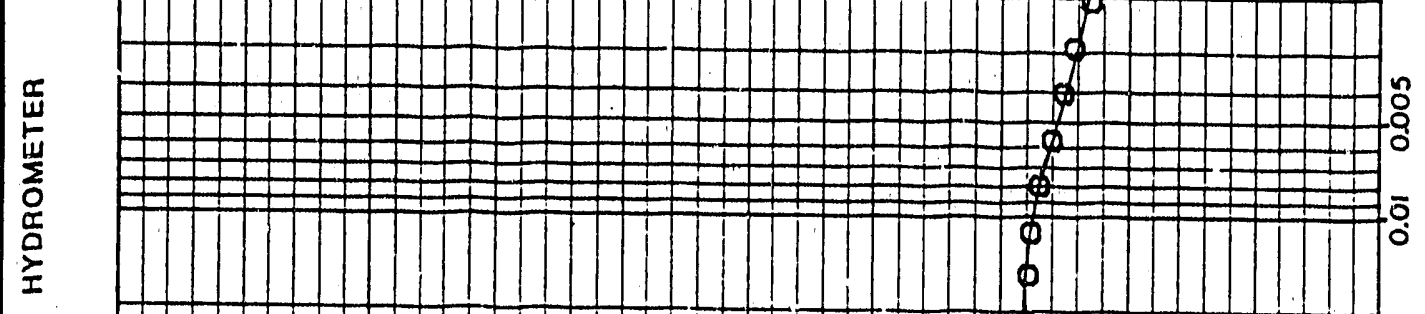
焉

을

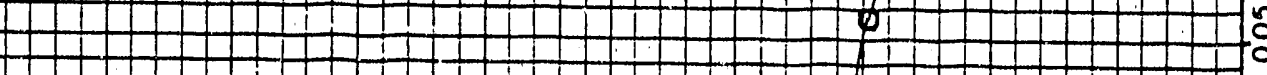

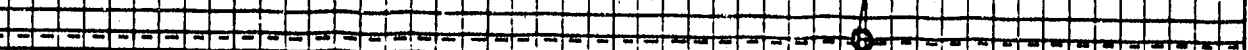

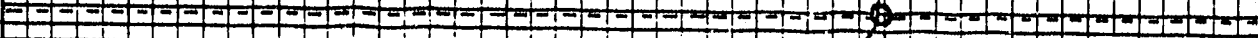

용

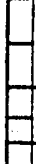
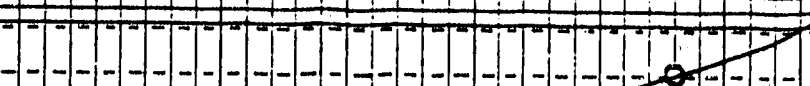

8

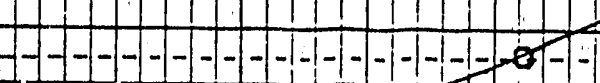

का

o
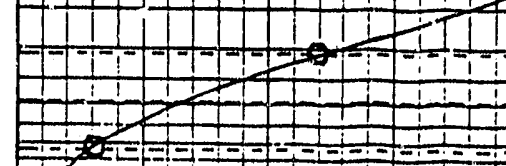

F

- $-1-1-$

$\infty$

$\omega$

$\checkmark$

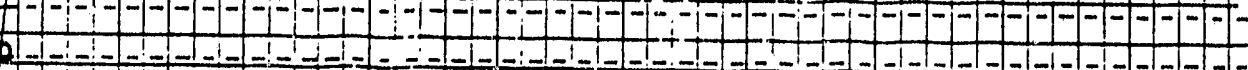

$m$

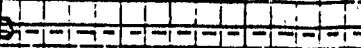

is $1 \times 00101+1$

I

mio - -

-

$-\operatorname{in}$

N

$m$

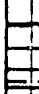

$\checkmark$

$\omega$
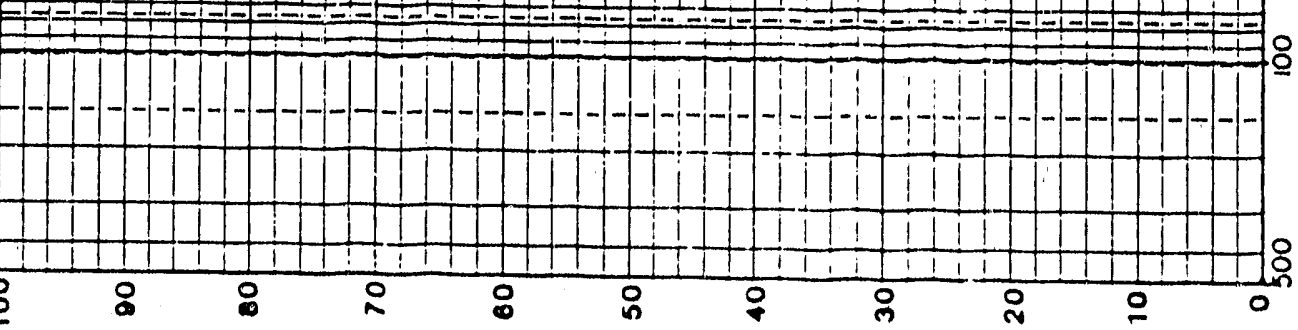

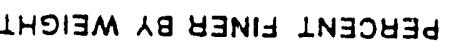

문

$\beth$

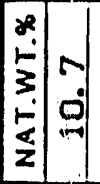
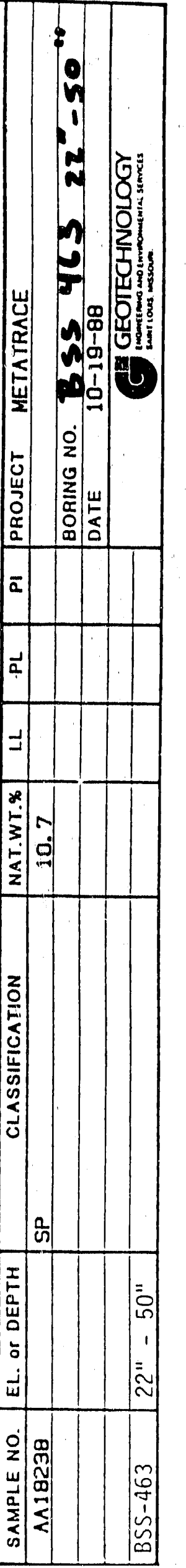


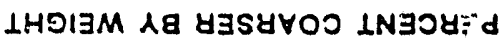

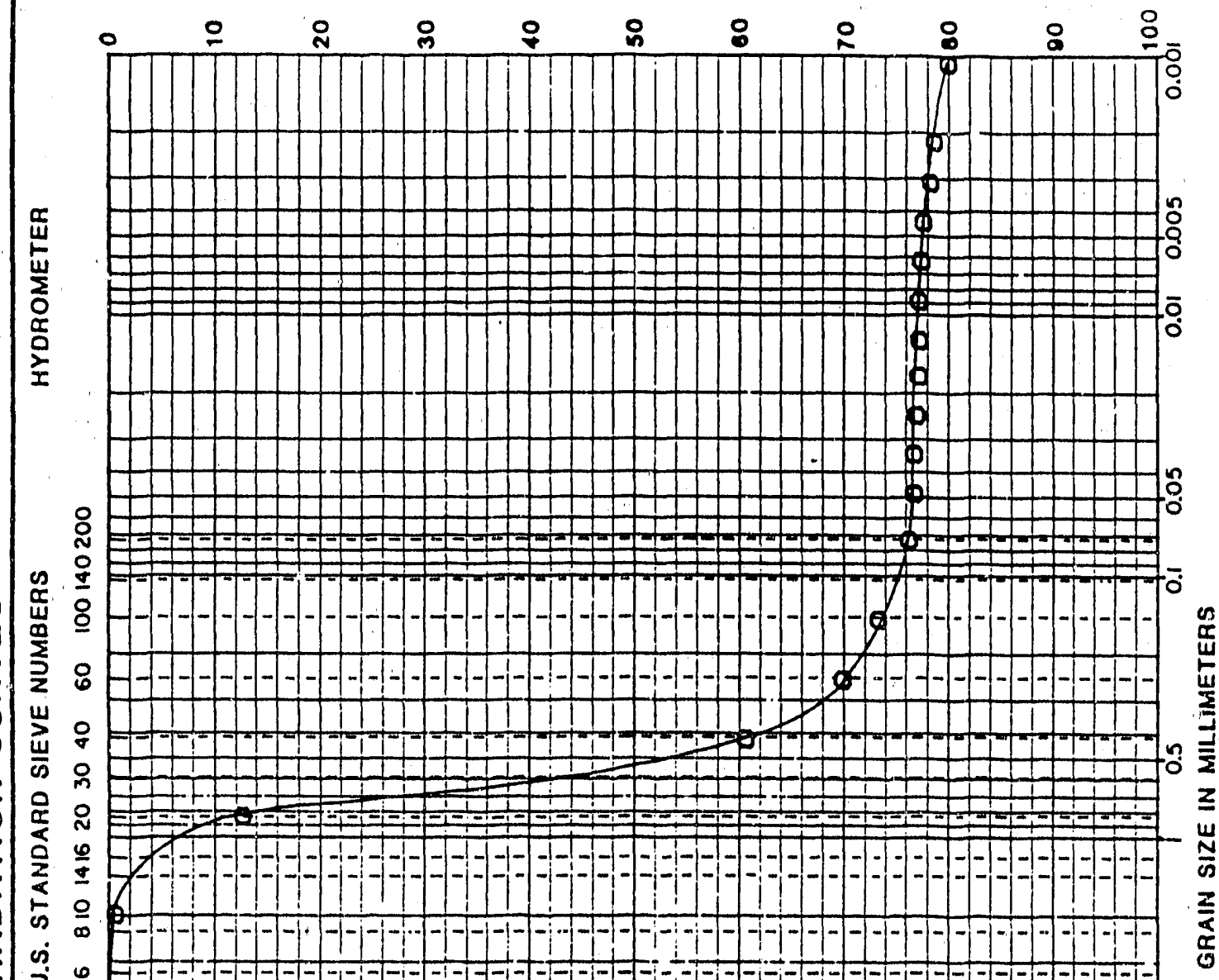

$\circ 6-1-1-1-1-1-1=-1-1-2-1-2-1-1-1-1-1-1-1-1-1$

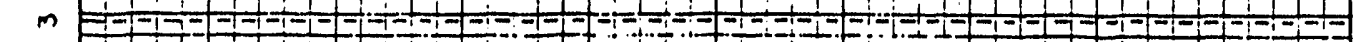

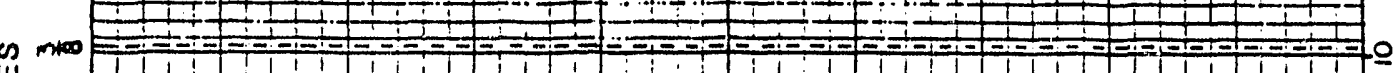

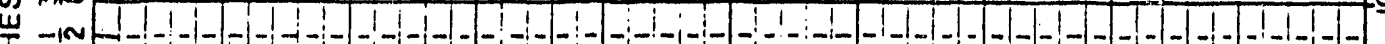

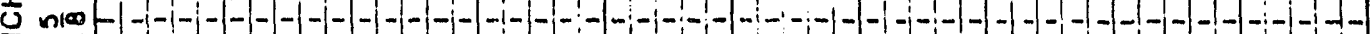

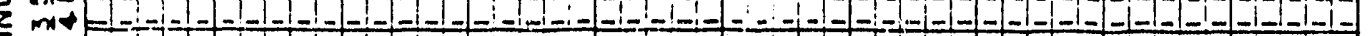

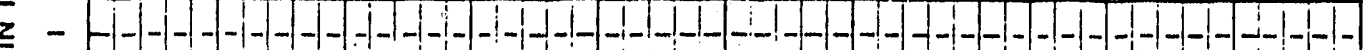

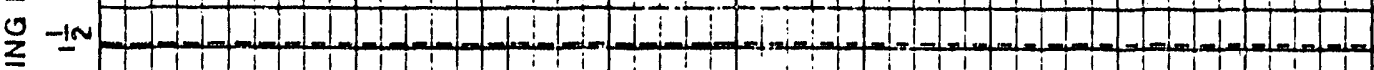

N

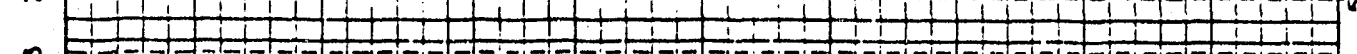

$>$

峞

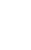

品

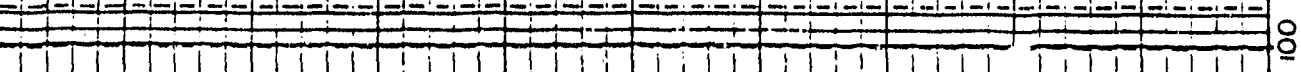

$-$

$---1--1-1--1--1-1-$

$-1-1-1-1-1-1$
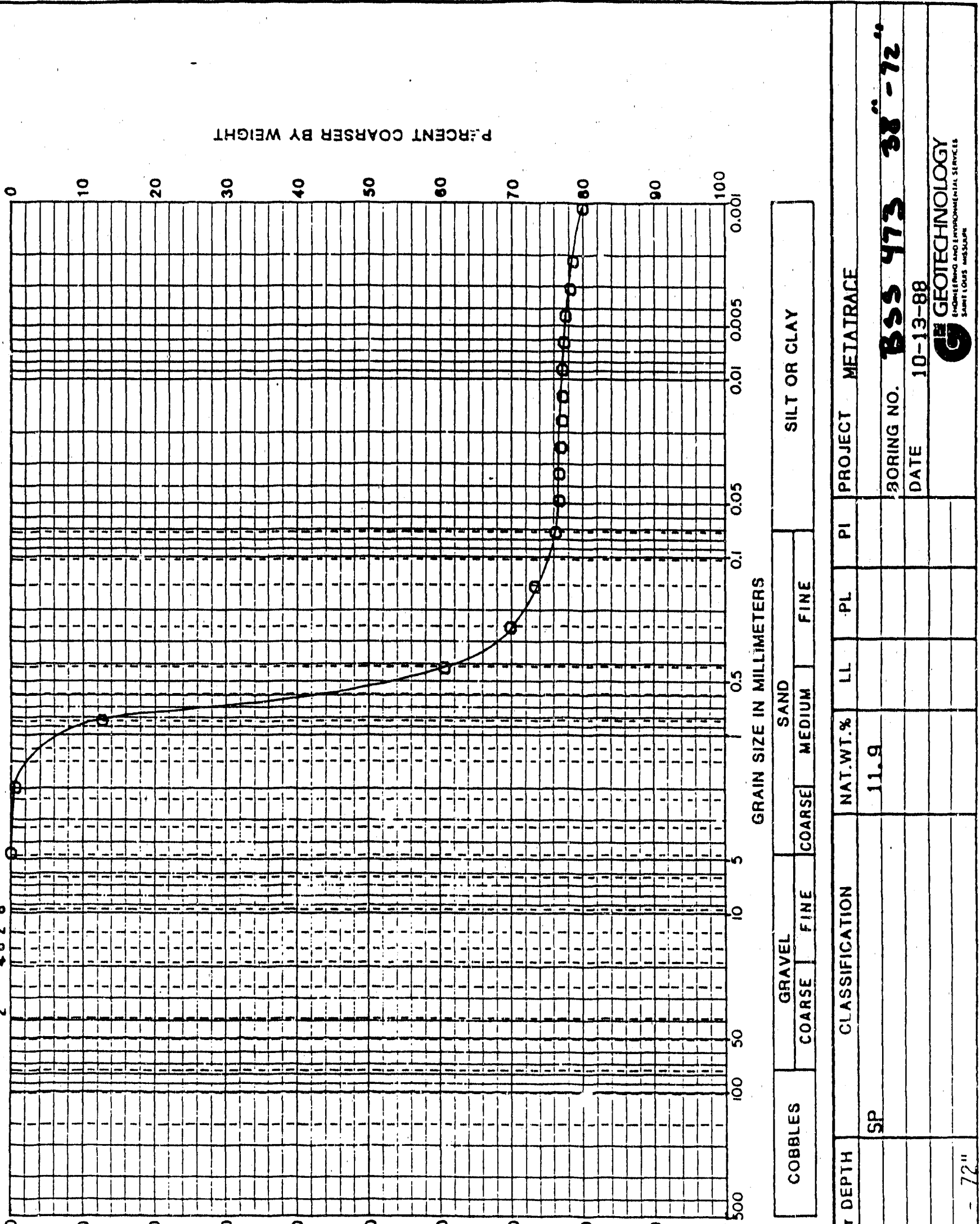

$\bar{a}$

$\frac{1}{4}$

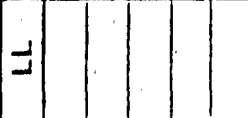

פ

$\stackrel{8}{\circ}$

8

:

:

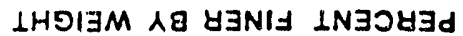
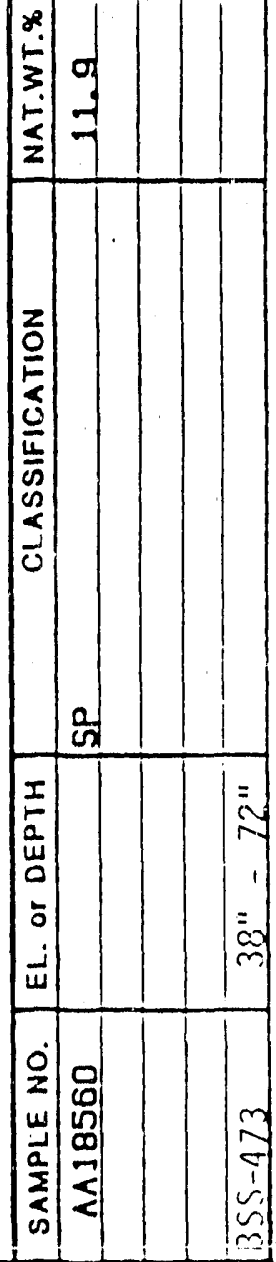


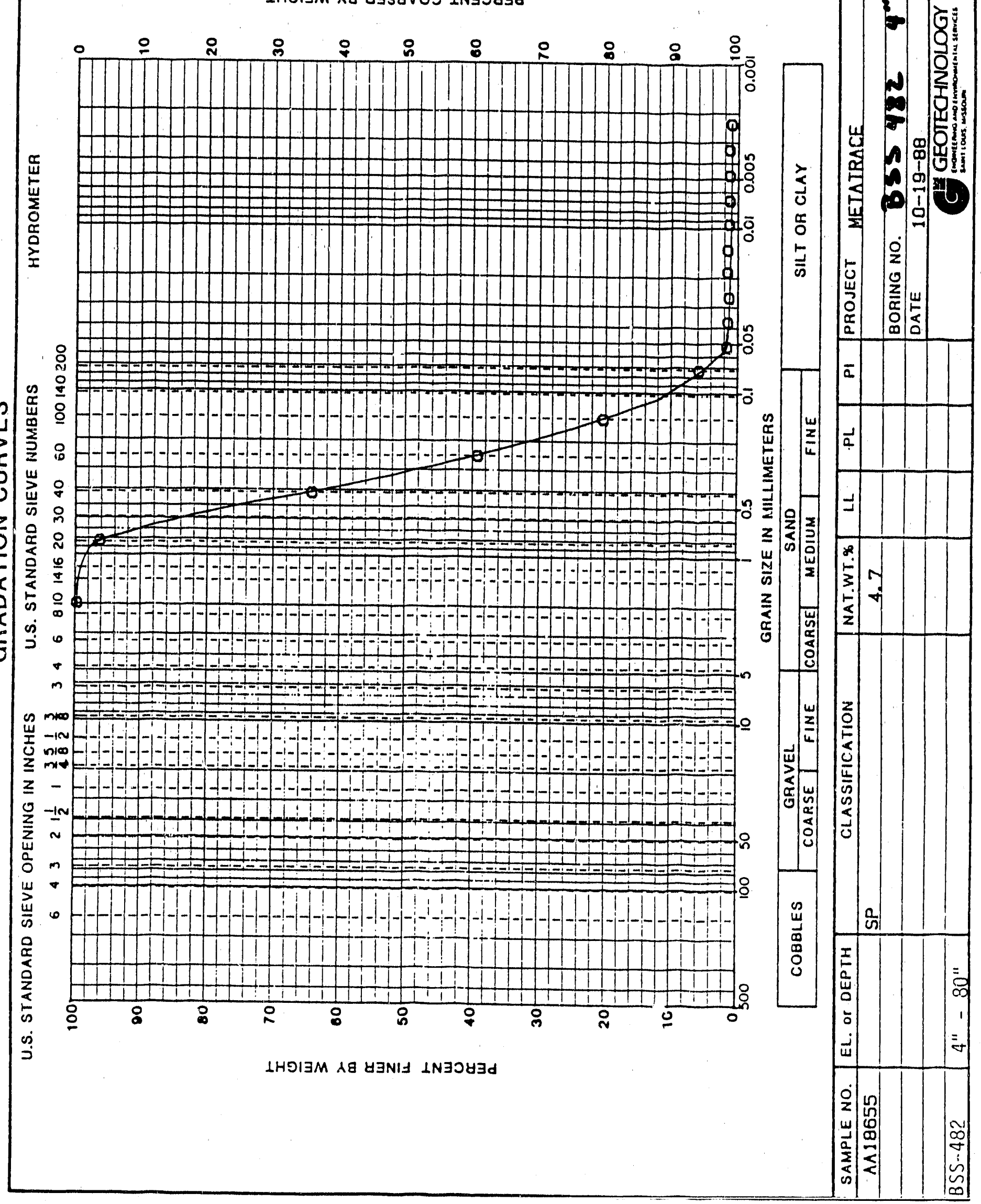



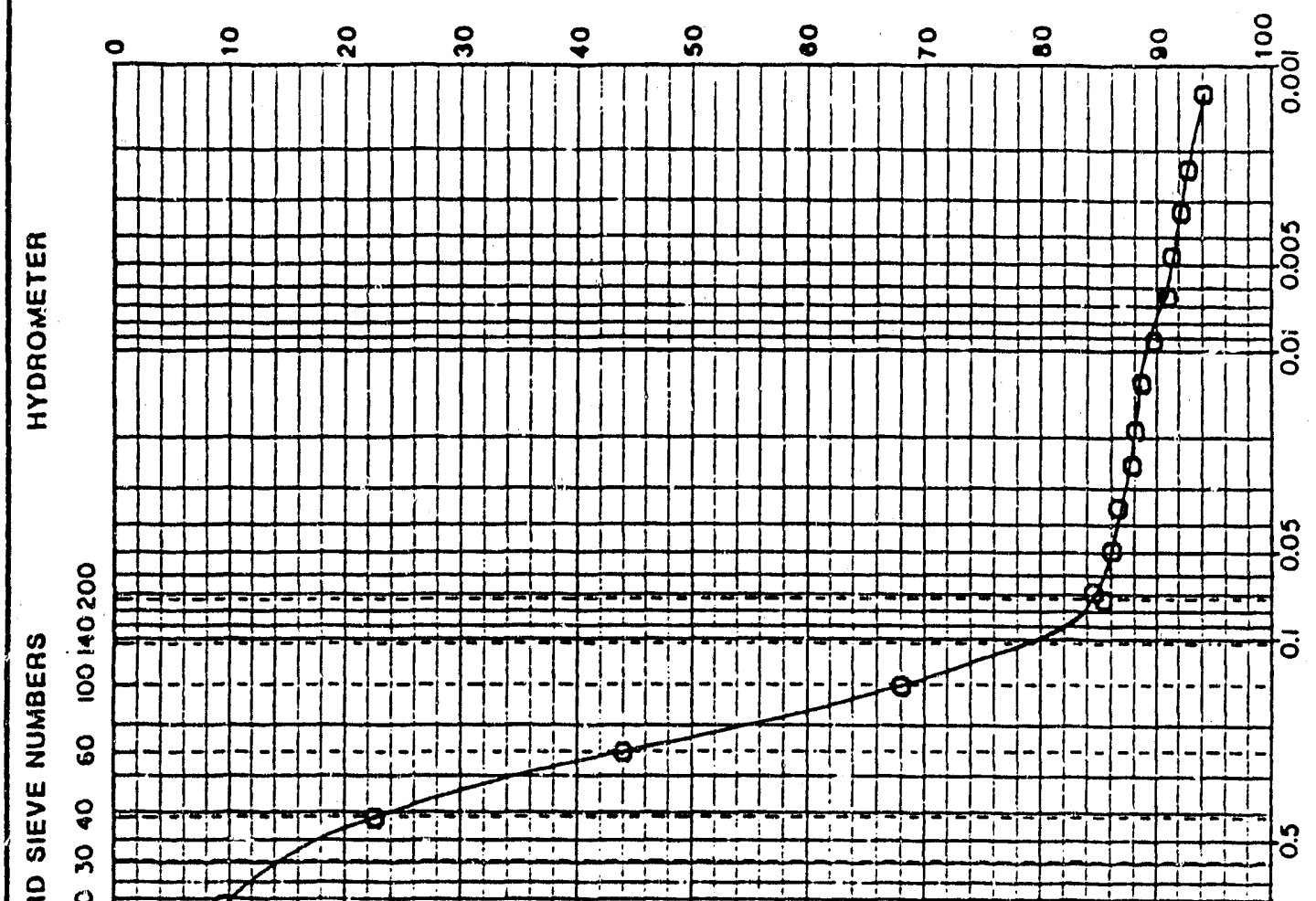

(i)

黑

$-12-$
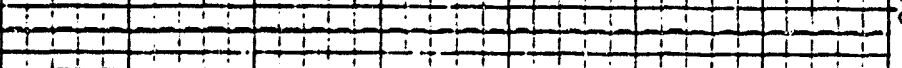

$\frac{0}{\infty}$

$\infty$

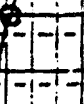

$-1-1-1-1-1-1-1-1$

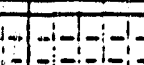

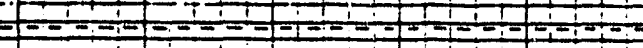

$$
+
$$

$$
m
$$

क ming

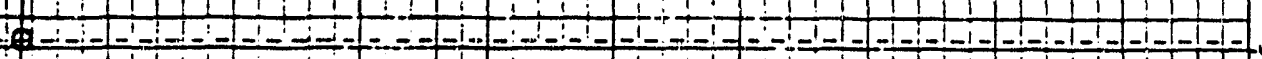

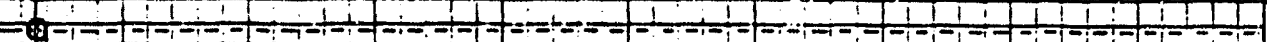

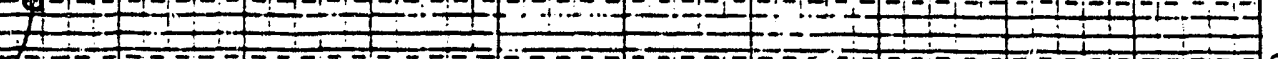

$\lim _{i \infty}$

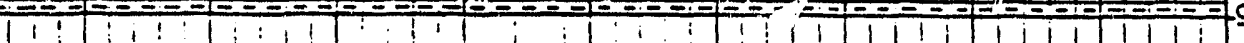

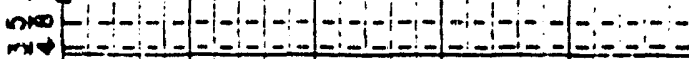

$2-$

0

n

$N$

$m$

$\checkmark$

$\omega$
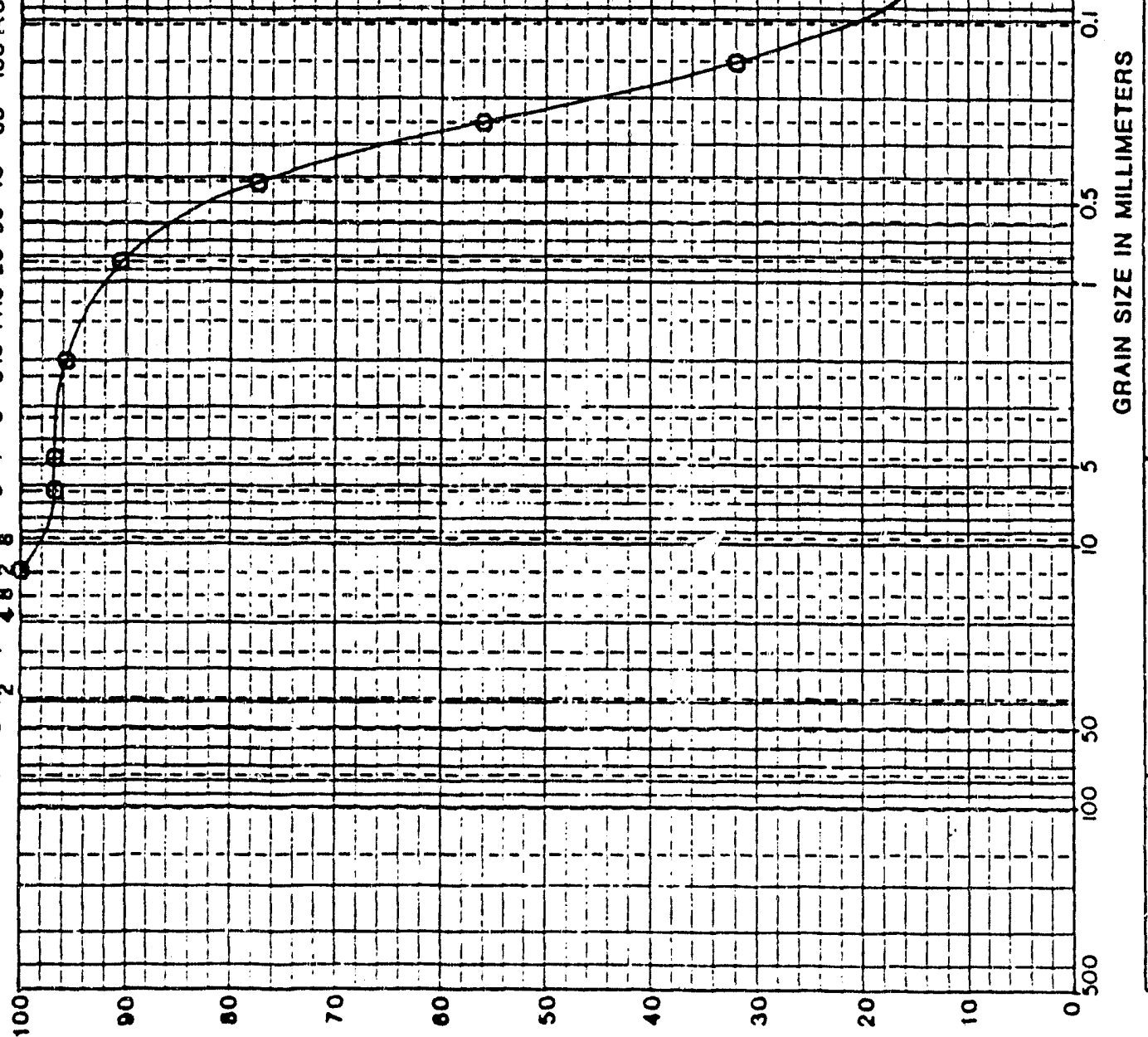

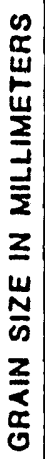

LHOIJM 18 H

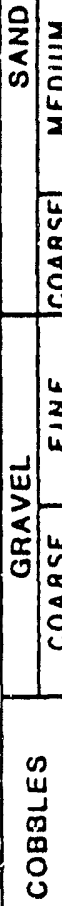

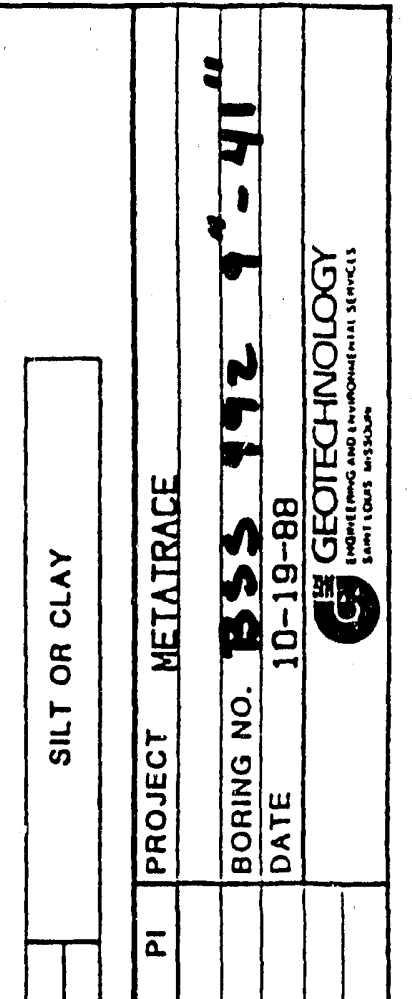

:

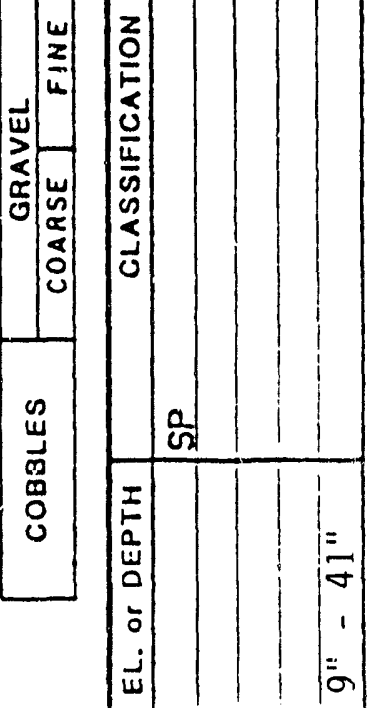

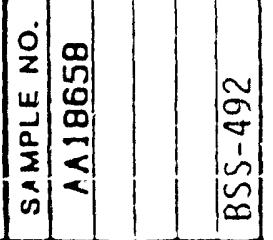



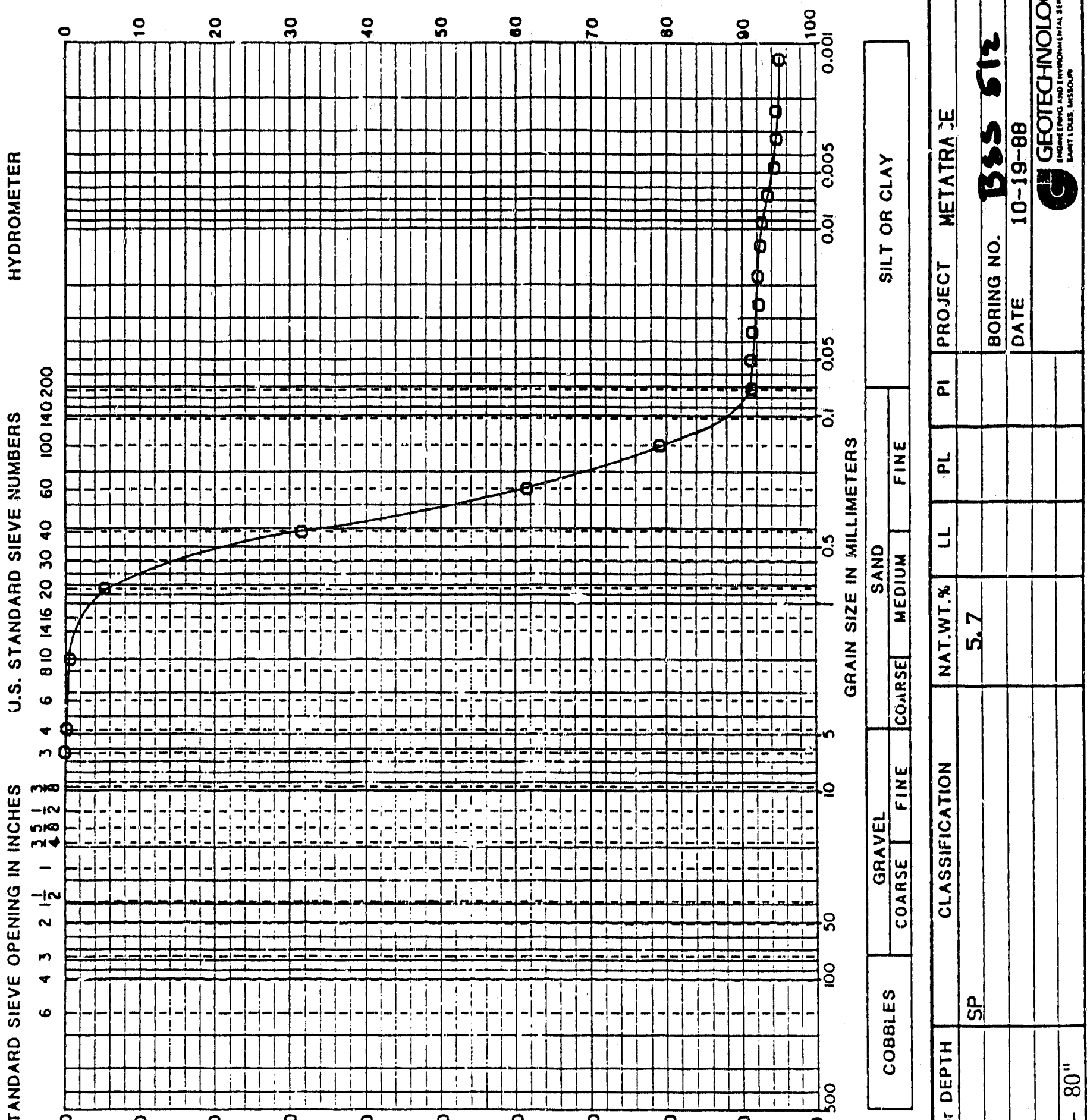

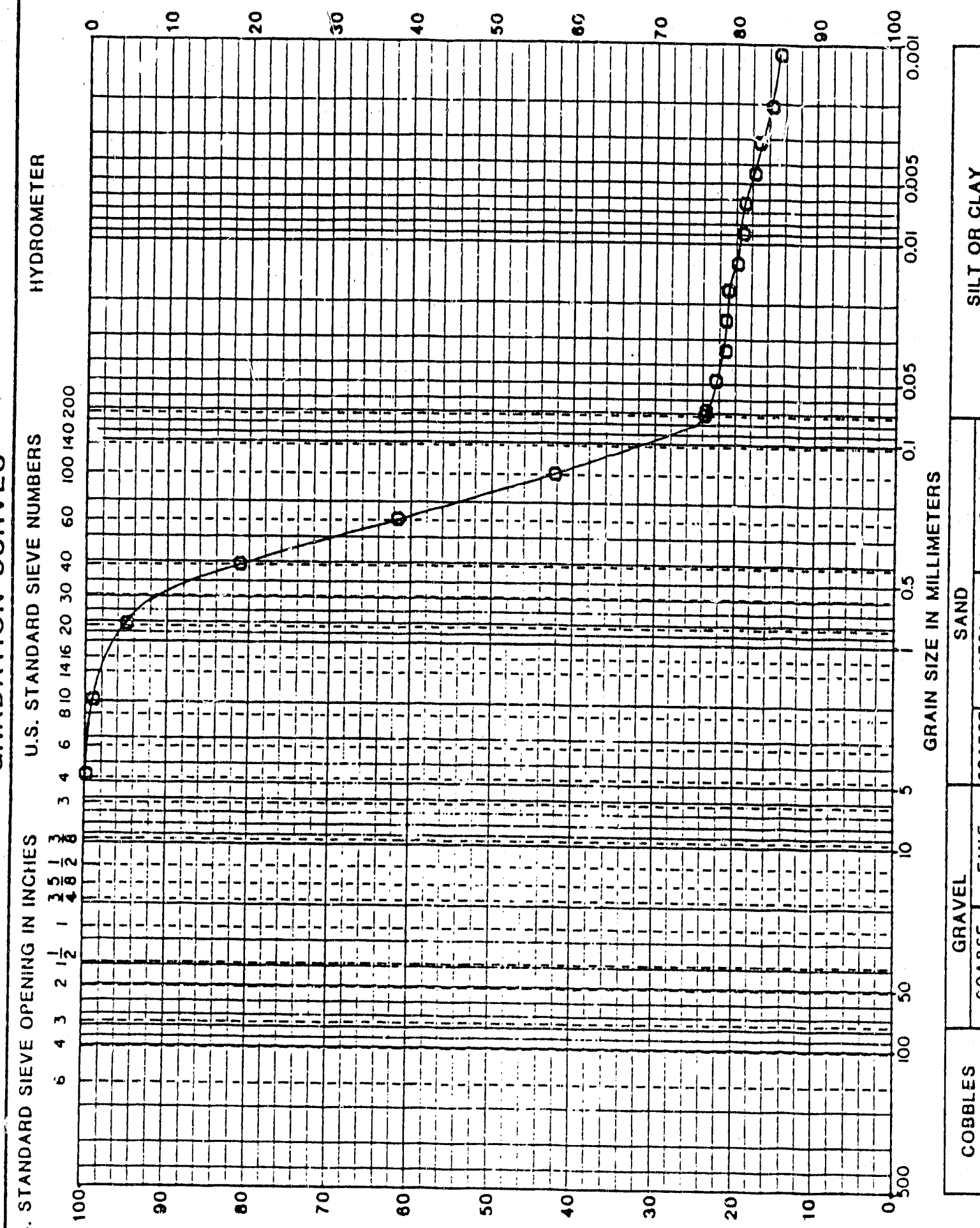

LHOIJM 19 VINIJ LNヨOYZd

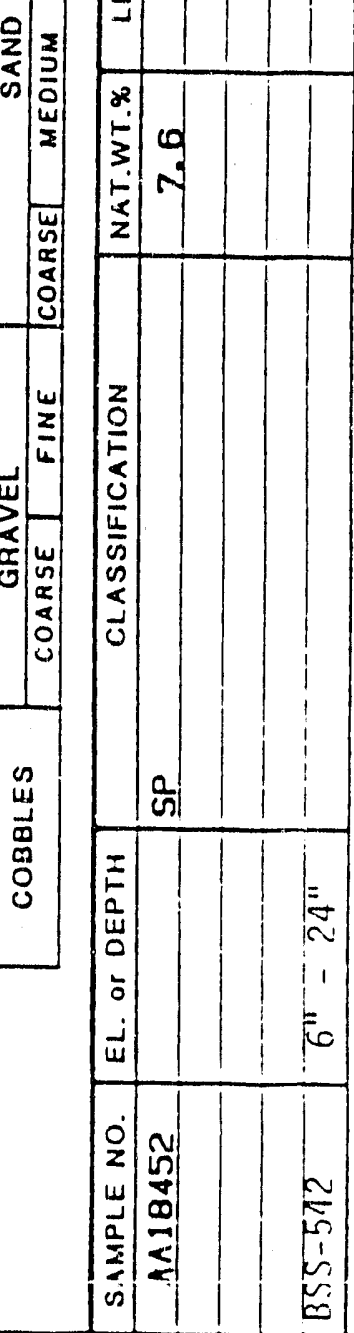




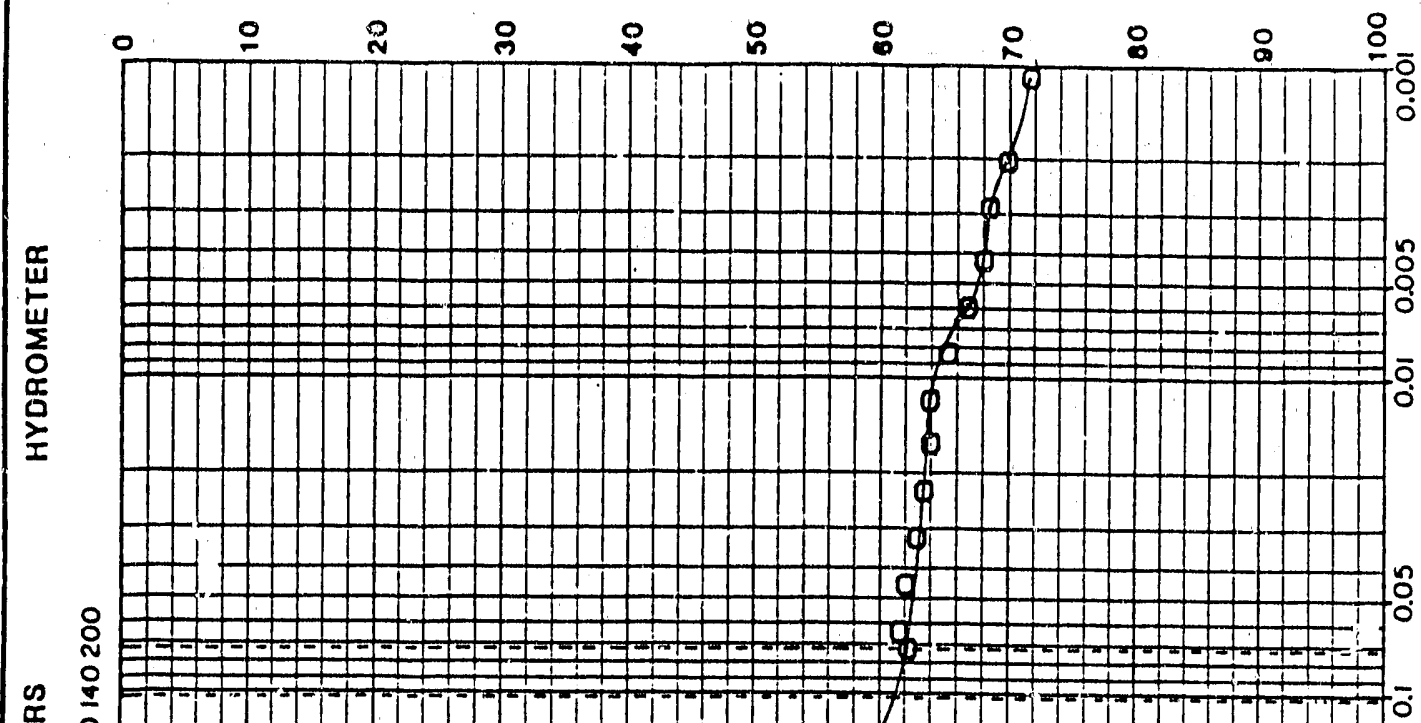

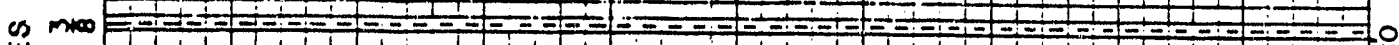

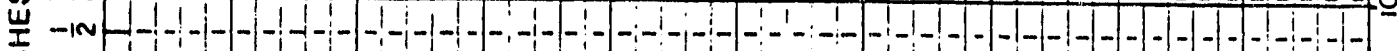

$\frac{1}{U}$ min

$\leq-$

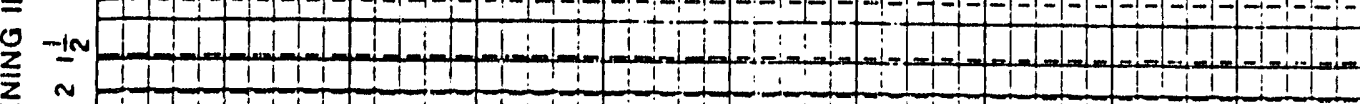

$m$

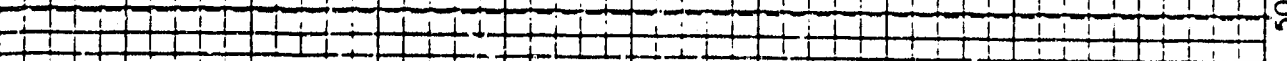

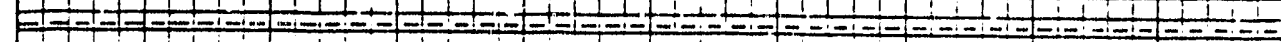

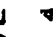

$\circ$

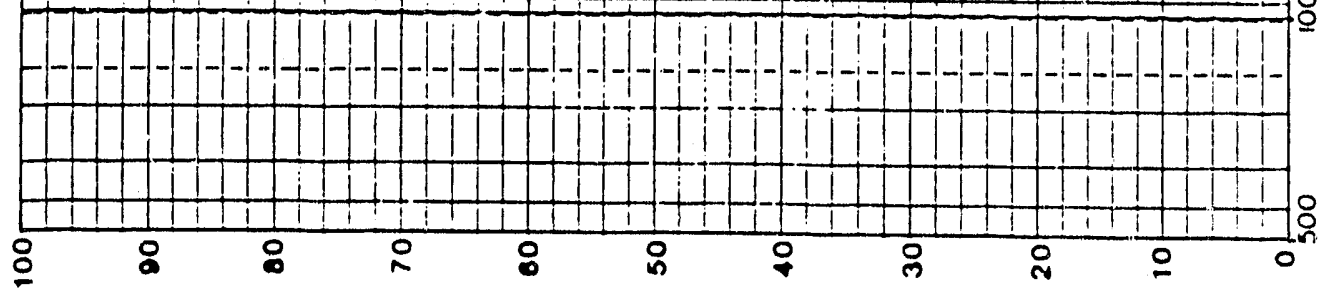


GEOCHEMICAL AND PHYSICAL PROPERTIES OF SOILS AND SHALLOW SEDIMENTS AT THE SAVANNAH RIVER SITE

Appendix C.6

Carbon Stee//Stainless Steel Data Summary 
Carbon/Stainlass Stael Sampler Comparison Data

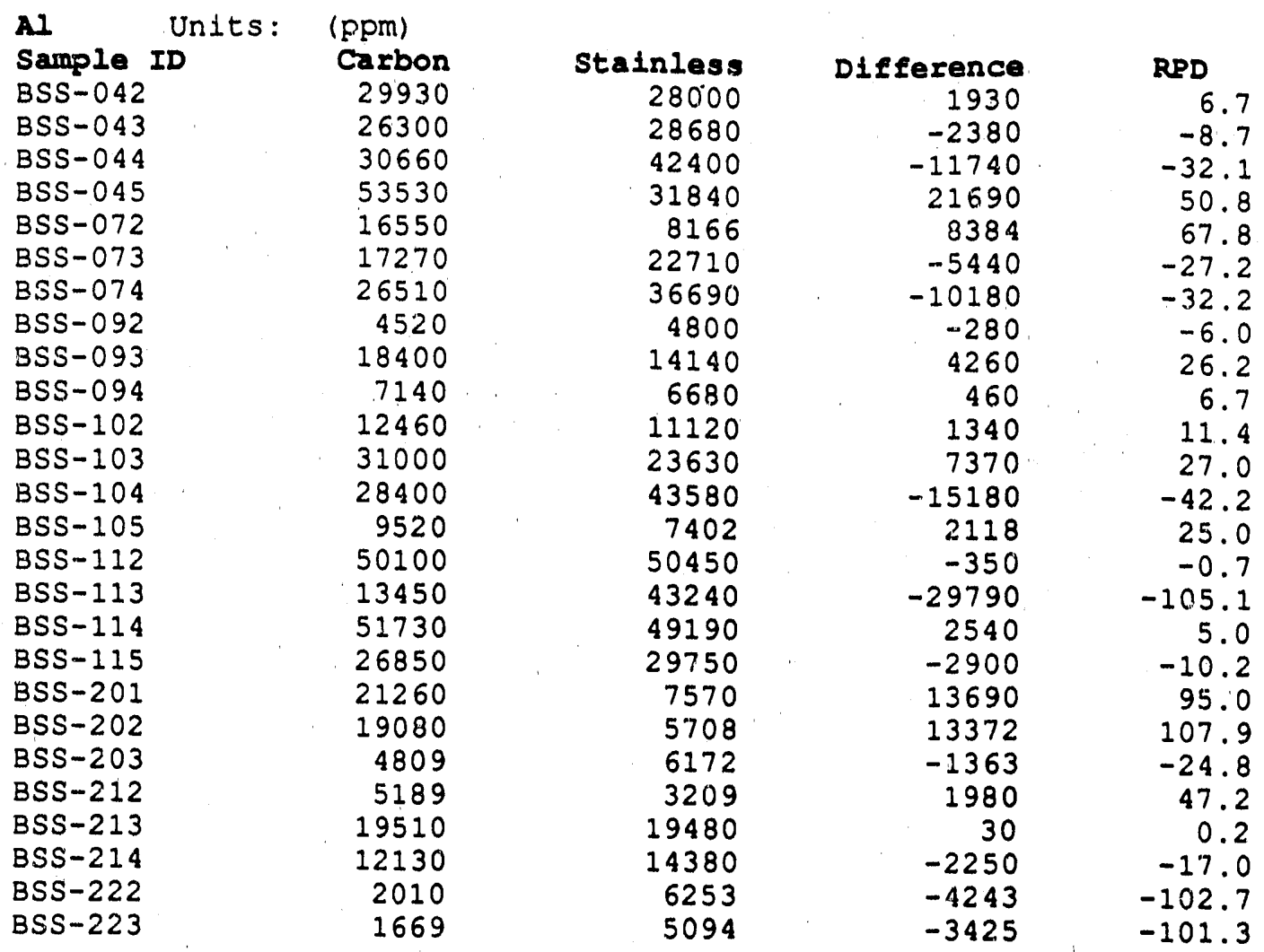

RPD denotes the Relative Percent Difference $R P D=(($ Carbon $-S t a i n l e s s) /($ Carbon+Stainless) ) *200 


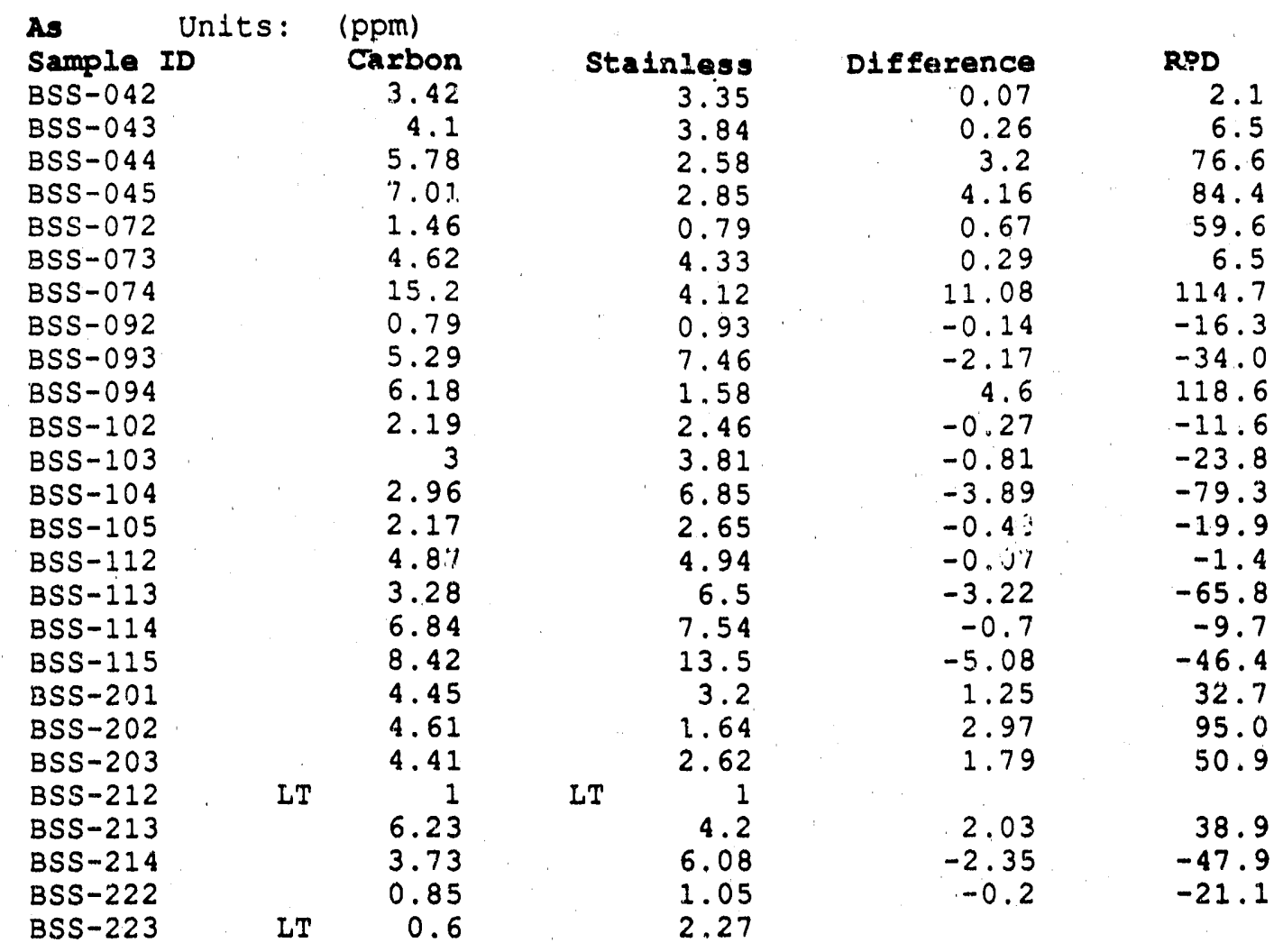

RPD denotes the Relative Percent Difference

$\mathrm{RPD}=(($ Carbon-Stainless $) /($ Carbon + Stainless $)) \star 200$ 
Carbon/Stainless Steal Sampler Comparison Data

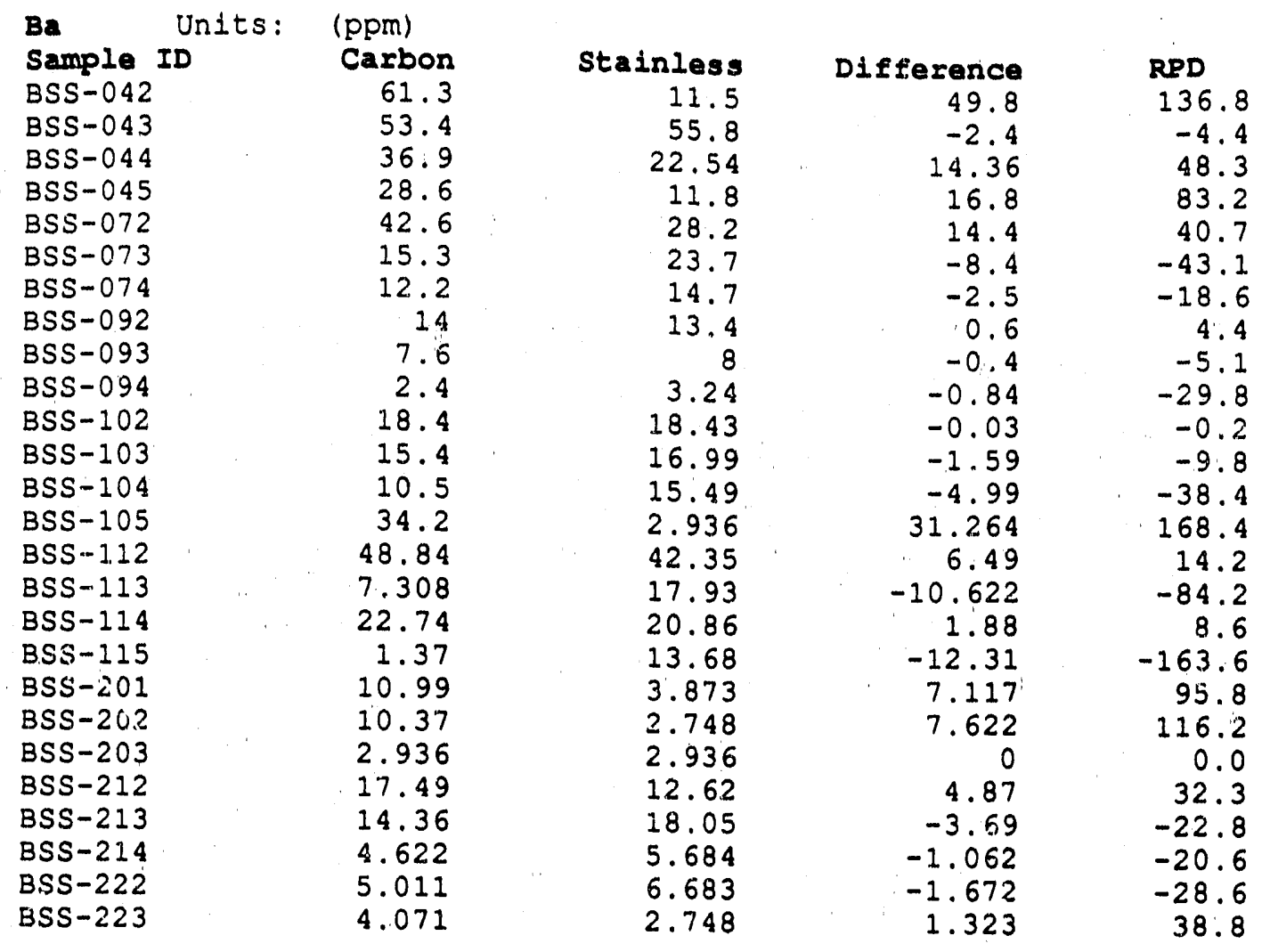

RPD denotes the Relative Percent Difference

$\mathrm{RPD}=(($ Carbon-Stainless $) /($ Carbon+Stainless $)) * 2 v u$ 


\begin{tabular}{|c|c|c|c|c|c|}
\hline Sample ID & & Carbon & Stainless & Difference & RPD \\
\hline BSS-042 & LT & 0.5 & 44.5 & & \\
\hline BSS-043 & LT & 1 & 0.1 & & \\
\hline BSS-044 & LT & 0.5 & 0.1 & & \\
\hline BSS -045 & LT & 0.5 & 0.1 & & \\
\hline BSS -072 & LT & 0.5 & 0.5 & & \\
\hline BSS -073 & LT & 0.5 & 0.5 & & \\
\hline BSS-074 & LT & 0.5 & 0.5 & & \\
\hline BSS-092 & LT & 1 & LT & & \\
\hline BSS-093 & LT & 0.6 & LT & & \\
\hline BSS-094 & LT & 1 & $\mathrm{LT}$ & & \\
\hline BSS -102 & LT & 1 & 0.5304 & & \\
\hline BSS -103 & LT & 1 & 0.5304 & & \\
\hline BSS-104 & LT & 1 & 0.5304 & & \\
\hline BSS -105 & LT & 1 & 0.5304 & & \\
\hline BSS- 112 & & 0.2652 & LT & & \\
\hline BSS-113 & & 0.7956 & 0.663 & 0.1326 & 18.2 \\
\hline BSS-114 & & 0.1326 & 0.5304 & -0.3978 & -120.0 \\
\hline BSS -115 & & 0.5304 & 1.061 & -0.5306 & -66.7 \\
\hline BSS-201 & & 0.5304 & 0.663 & -0.1326 & -22.2 \\
\hline$B S S-202$ & & 0.5304 & 0.7956 & -0.2652 & -40.0 \\
\hline BSS-203 & & 0.5967 & 0.4641 & 0.1326 & 25.0 \\
\hline BSS -212 & & 0.5967 & 0.7293 & -0.1326 & -20.0 \\
\hline BSS -213 & LT & 1 & 0.4641 & & \\
\hline BSS-214 & & 0.5304 & 0.7956 & -0.2652 & -40.0 \\
\hline BSS-222 & LT & 1 & 0.2652 & & \\
\hline BSS-223 & LT & 1 & 0.8619 & & \\
\hline
\end{tabular}


Carbon/Stainless steel Sampler Comparison Data

\begin{tabular}{lrrrr} 
Cr Units: & $\begin{array}{r}\text { (ppm) } \\
\text { Carbon }\end{array}$ & Stainless & Difierance & \multicolumn{1}{c}{ RPD } \\
Sample ID & 16.1 & 35.6 & -19.5 & -75.4 \\
BSS-042 & 18 & 18.8 & -0.8 & -4.3 \\
BSS-043 & 20.7 & 39 & -18.3 & -61.3 \\
BSS-044 & 35.3 & 84.9 & -49.6 & -82.5 \\
BSS-045 & 10.2 & 5.13 & 5.07 & 66.1 \\
BSS-072 & 30.4 & 13.2 & 17.2 & 78.9 \\
BSS-073 & 33.7 & 29.6 & 4.1 & 13.0 \\
BSS-074 & 8.4 & 21.8 & -13.4 & -88.7 \\
BSS-092 & 23.4 & 28.2 & -4.8 & -18.6 \\
BSS-093 & 17.6 & 19 & -1.4 & -7.7 \\
BSS-094 & 9.7 & 9.393 & 0.307 & 3.2 \\
BSS-102 & 27 & 116.1 & -89.1 & -124.5 \\
BSS-103 & 28 & 38.26 & -10.26 & -31.0 \\
BSS-104 & 8.8 & 5.622 & 3.178 & 44.1 \\
BSS-105 & 32.36 & 33.59 & -1.23 & -3.7 \\
BSS-112 & 12.75 & 37.43 & -24.68 & -98.4 \\
BSS-113 & 45.21 & 40.86 & 4.35 & 10.1 \\
BSS-114 & 51.97 & 65.41 & -13.44 & -22.9 \\
BSS-115 & 27.15 & 17.41 & 9.74 & 43.7 \\
BSS-201 & 23.72 & 7.953 & 15.767 & 99.6 \\
BSS-202 & 5.485 & 10.83 & -5.345 & -65.5 \\
BSS-203 & 6.719 & 3.085 & 3.634 & 74.1 \\
BSS-212 & 31.98 & 20.29 & 11.69 & 44.7 \\
BSS-213 & 18.1 & 20.84 & -2.74 & -14.1 \\
BSS-214 & 3.862 & 9.598 & -5.736 & -85.2 \\
BSS-222 & 8.425 & 6.856 & 1.56 .9 & 20.5 \\
BSS-223 & & & &
\end{tabular}

RPD denotes the Relative Percent Difference

$R P D=(($ Carbon - Stainless $) /($ Carbon+Stainless $)) * 200$ 


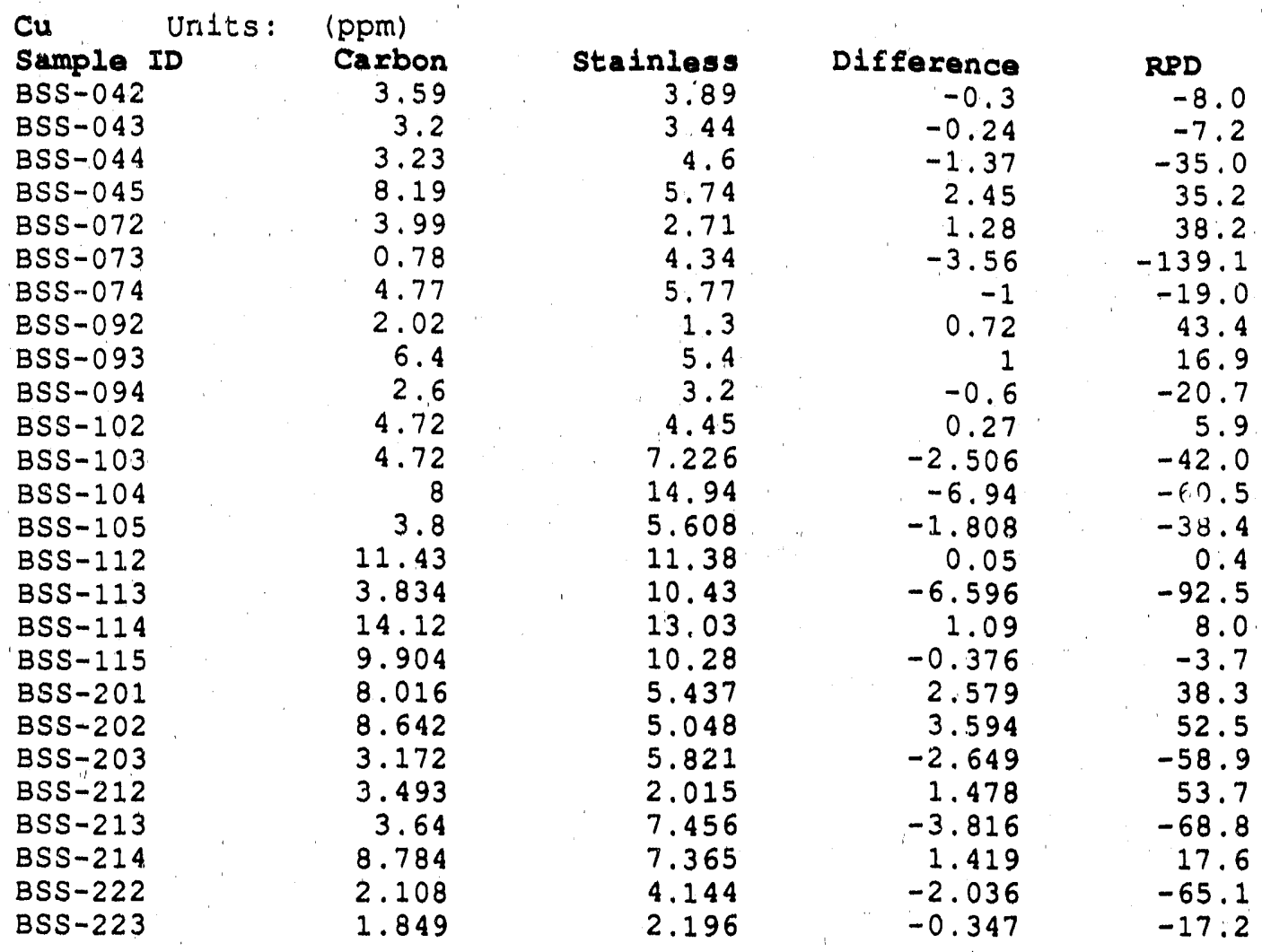

RPD denotes the Relative Rercent Difference $R P D=(($ Carbon-Stainless $) /($ Carbon+Stainless $)) \star 200$ 
Carbon/Stainless Steel Sampler Comparison Data

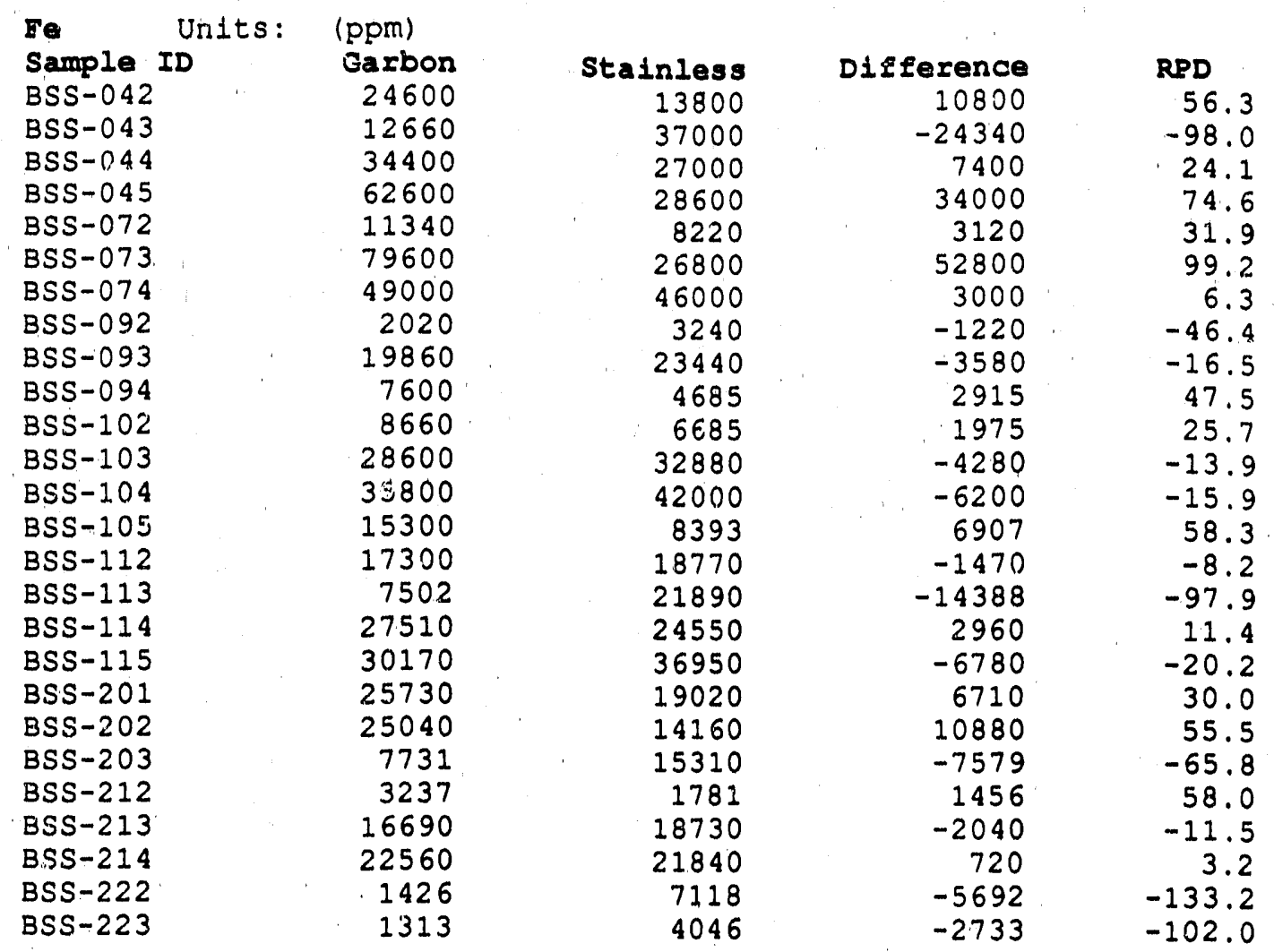

RPD denotes the Relative Percent Difference

$R P D=(($ Carbon-ialnless $) /($ Carbon+Stalnless $)) \star 200$ 
Carbon/Stainless Steel Sampler Comparison Data

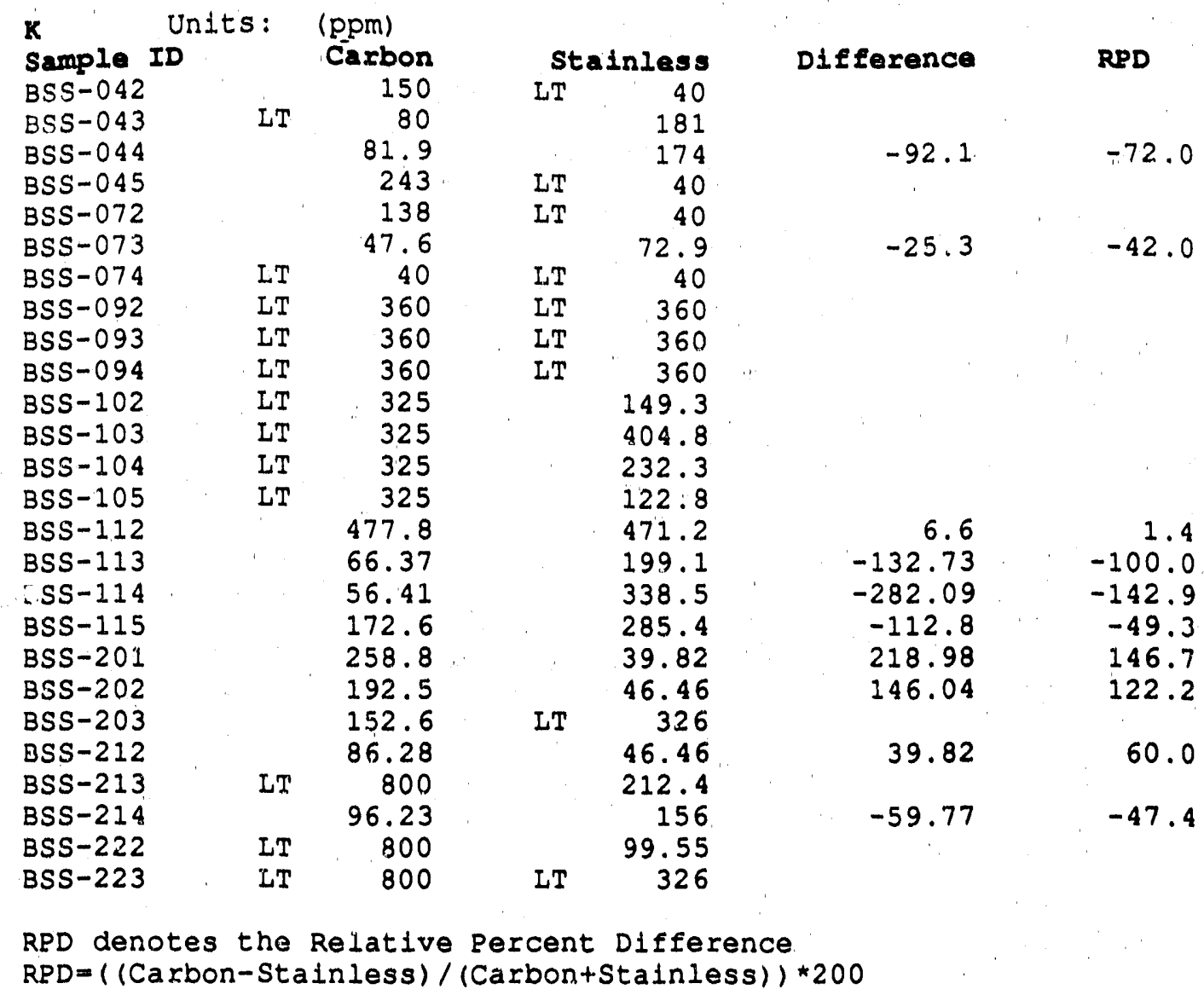


Carbon/Stainless Steel Sampler Comparison Data

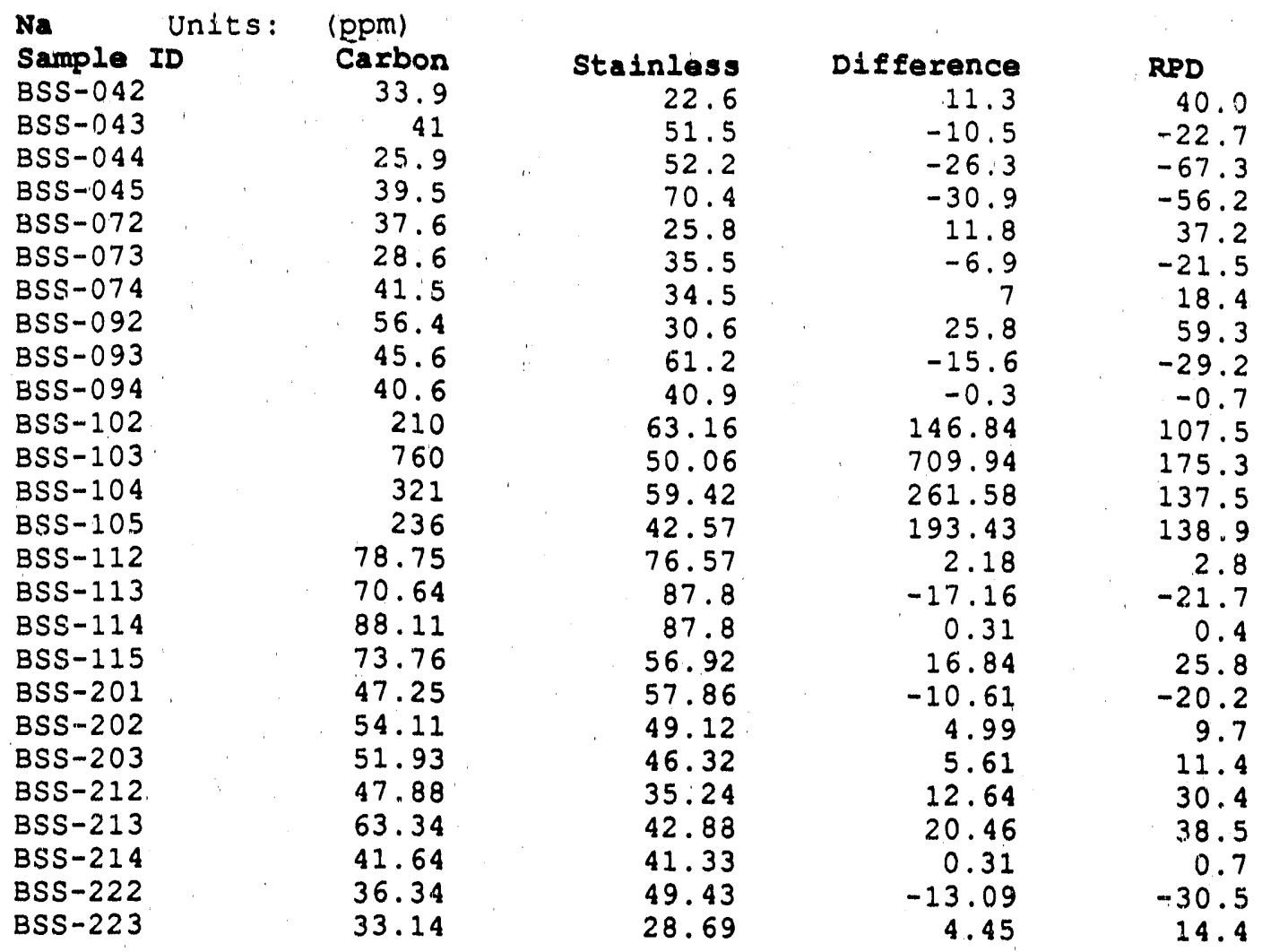

RPD denotes the Relative Percent Difference $\mathrm{RPD}=(($ Carbon-Stainless $) /($ Carbon+Stainless $)) \star 200$ 


\section{Carbon/Stainless steel Sampler Comparison Data}

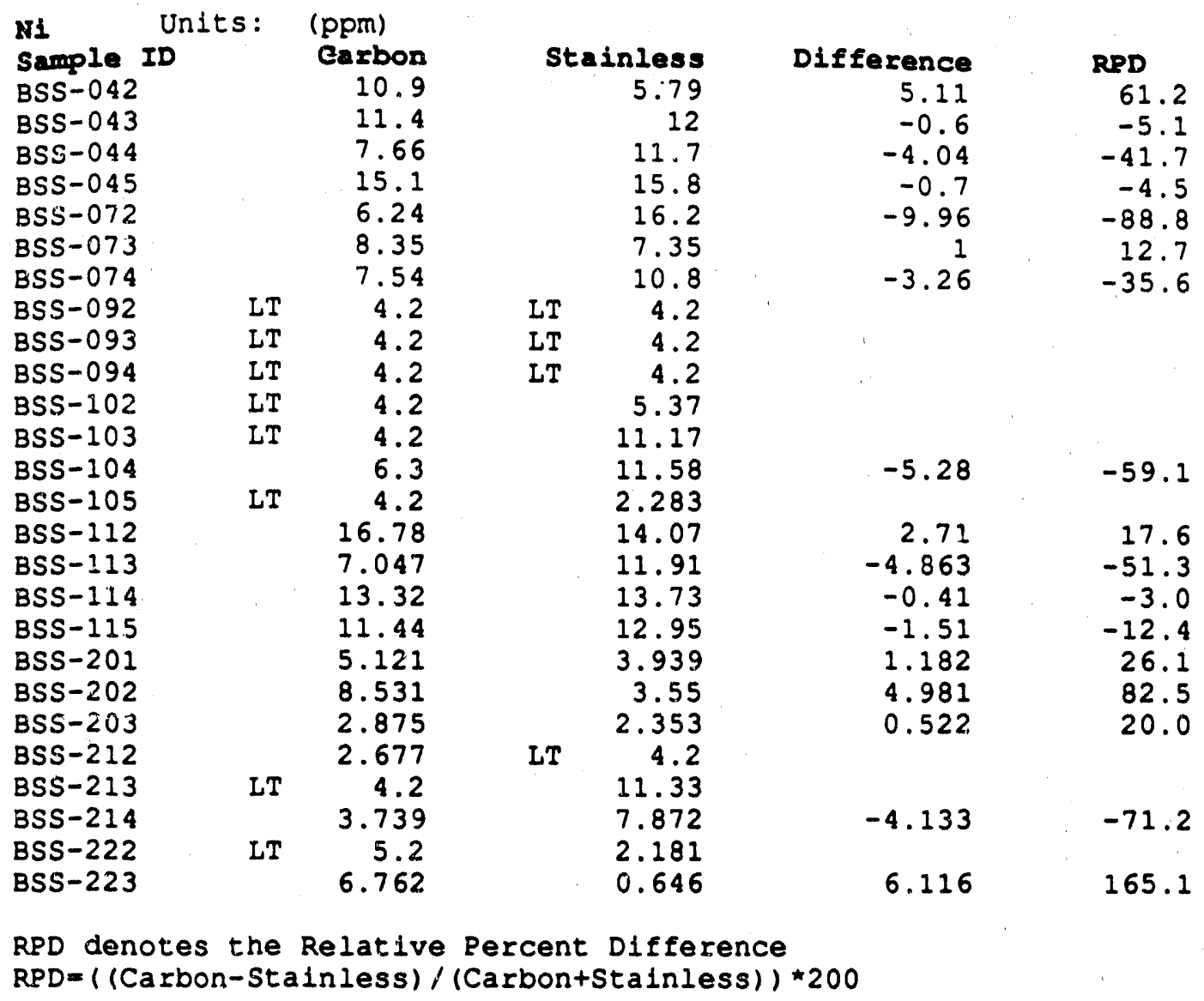




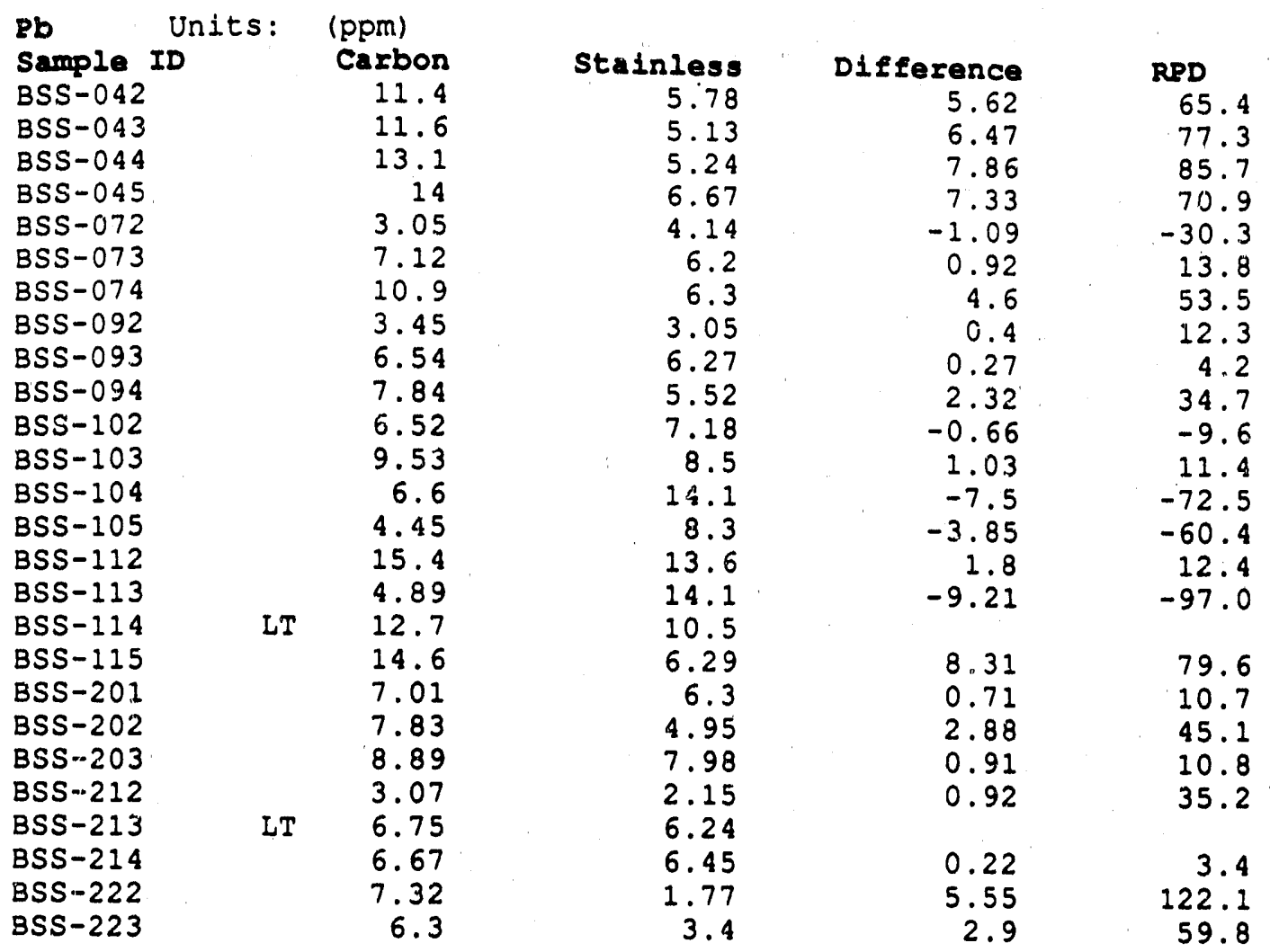

RPD denotes the Relative Percent Difference $R P D=(($ Carbon-Stainless $) /($ Carbon+Stalnless $)) * 200$ 
Carbon/Stainless Steel Sampler Comparison Data

\begin{tabular}{lrrrr} 
Zn Units: & $\begin{array}{r}\text { (ppm) } \\
\text { Carbon }\end{array}$ & Stainless & Difference & \multicolumn{1}{c}{ RPD } \\
Sample ID & 21.3 & 13.9 & 7.4 & 42.0 \\
BSS-042 & 18.4 & 19.3 & -0.9 & -4.8 \\
BSS-043 & 15.6 & 16.9 & -1.3 & -8.0 \\
BSS-044 & 28.6 & 31.5 & -2.9 & -9.7 \\
BSS-045 & 16.8 & 31.8 & -1.5 & -61.7 \\
BSS-072 & 19.6 & 16.5 & 3.1 & 17.2 \\
BSS-073 & 13.7 & 19.6 & -5.9 & -35.4 \\
BSS-074 & 12.6 & 5.8 & 6.8 & 73.9 \\
ESS-092 & 10.5 & 9 & 1.5 & 15.4 \\
BSS-093 & 7.65 & 10.2 & -2.55 & -28.6 \\
BSS-094 & 16.8 & 9.949 & 6.851 & 51.2 \\
BSS-102 & 14.2 & 11.43 & 2.77 & 21.6 \\
BSS-103 & 18 & 14.75 & 3.25 & 19.8 \\
BSS-104 & 11.2 & 9.395 & 1.805 & 17.5 \\
BSS-105 & 28.46 & 26.26 & 2.2 & 8.0 \\
BSS-112 & 23.94 & 18.26 & 5.68 & 26.9 \\
BSS-113 & 33.42 & 19 & 14.42 & 55.0 \\
BSS-114 & 22.37 & 14.62 & 7.75 & 41.9 \\
BSS-115 & 10.51 & 21.05 & -10.54 & -66.8 \\
BSS-201 & 12.05 & 5.062 & 6.988 & 81.7 \\
BSS-202 & 10.46 & 12.24 & -1.78 & -15.7 \\
BSS-203 & 10.76 & 4.444 & 6.316 & 83.1 \\
BSS-212 & 15.82 & 16.19 & -0.37 & -2.3 \\
BSS-213 & 12.67 & 9.093 & 3.577 & 32.9 \\
BSS-214 & 10.11 & 11.16 & -1.05 & -9.9 \\
BSS-222 & 7.72 & 6.467 & 1.253 & 17.7
\end{tabular}

RPD denotes the Relative Percent Difference

$R P D=(($ Carbon-Stainless $) /($ Carbon+stainless $)) \star 200$ 


\title{
GEOCHEMICAL AND PHYSICAL PROPERTIES OF SOILS AND
} SHALLOW SEDIMENTS AT THE SAVANNAH RIVER SITE

\author{
Appendix C.7
}

metaTRACE/Weston Laboratory Comparison Data Summary 
motamRACr/Woston Iaboratory Comparison Data

\begin{tabular}{|c|c|c|c|c|}
\hline Aluminum & (ppm) - & & & \\
\hline $\begin{array}{l}\text { Sarople ID } \\
\text { BSS }-431\end{array}$ & $\begin{array}{r}\text { matatrack } \\
2485.7\end{array}$ & $\begin{array}{l}\text { Weston } \\
2480\end{array}$ & Difference & $\begin{array}{l}\text { RPD } \\
0.2\end{array}$ \\
\hline BSS -432 & 2426.8 & 2210 & 216.8 & 9.4 \\
\hline BSS -433 & 12627 & 19700 & -7073 & -43.8 \\
\hline$B S S-434$ & 10083 & 21600 & -11517 & -72.7 \\
\hline BSS -471 & 6435 & 5220 & 1215 & 20.8 \\
\hline BSS -472 & 8353.8 & 7180 & 1173.8 & 15.1 \\
\hline BSS -473 & 4850.6 & 4550 & 300.6 & 6.4 \\
\hline BSS -474 & 3559.6 & 3680 & -120.4 & -3.3 \\
\hline BSS -481 & 1399.6 & 1510 & -110.4 & -7.6 \\
\hline BSS -482 & 1886 & 2340 & -454 & -21.5 \\
\hline BSS -483 & 3407.2 & 2540 & 867.2 & 29.2 \\
\hline BSS -491 & 2461.2 & 2450 & 11.2 & 0.5 \\
\hline BSS-492 & 4147.8 & $369 n$ & 457.8 & 11.7 \\
\hline BSS -493 & 20492 & 20200 & 292 & 1.4 \\
\hline BSS-494 & 11849 & 10900 & 949 & 8.3 \\
\hline BSS-531 & 6073 & 6550 & -477 & -7.6 \\
\hline BSS -541 & 4418.3 & 2870 & 1548.3 & 42.5 \\
\hline BSS -542 & 13022 & 8010 & 5012 & 47.7 \\
\hline BSS-543 & 21989 & 10400 & 11589 & 71.6 \\
\hline BSS-544 & 25786 & 18000 & 7780 & 35.6 \\
\hline BSS- 545 & 1.9520 & 5910 & 13610 & 107.0 \\
\hline BSS-551 & 4551.6 & 1960 & 2591.6 & 79.6 \\
\hline BSS-552 & 3015.7 & 6400 & -3384.3 & -71.9 \\
\hline BSS-553 & 2899.3 & 5000 & -2100.7 & -53.2 \\
\hline BSS-562 & 1221.4 & 1270 & -48.6 & -3.9 \\
\hline
\end{tabular}

RPD denotes Relative Percent Difference

$R P D=($ (metaTRACE-Weston) $/($ metaTRACE+Weston $)) \star 2 C$ 
matarRACr/Weston Iaboratory Comparison Data

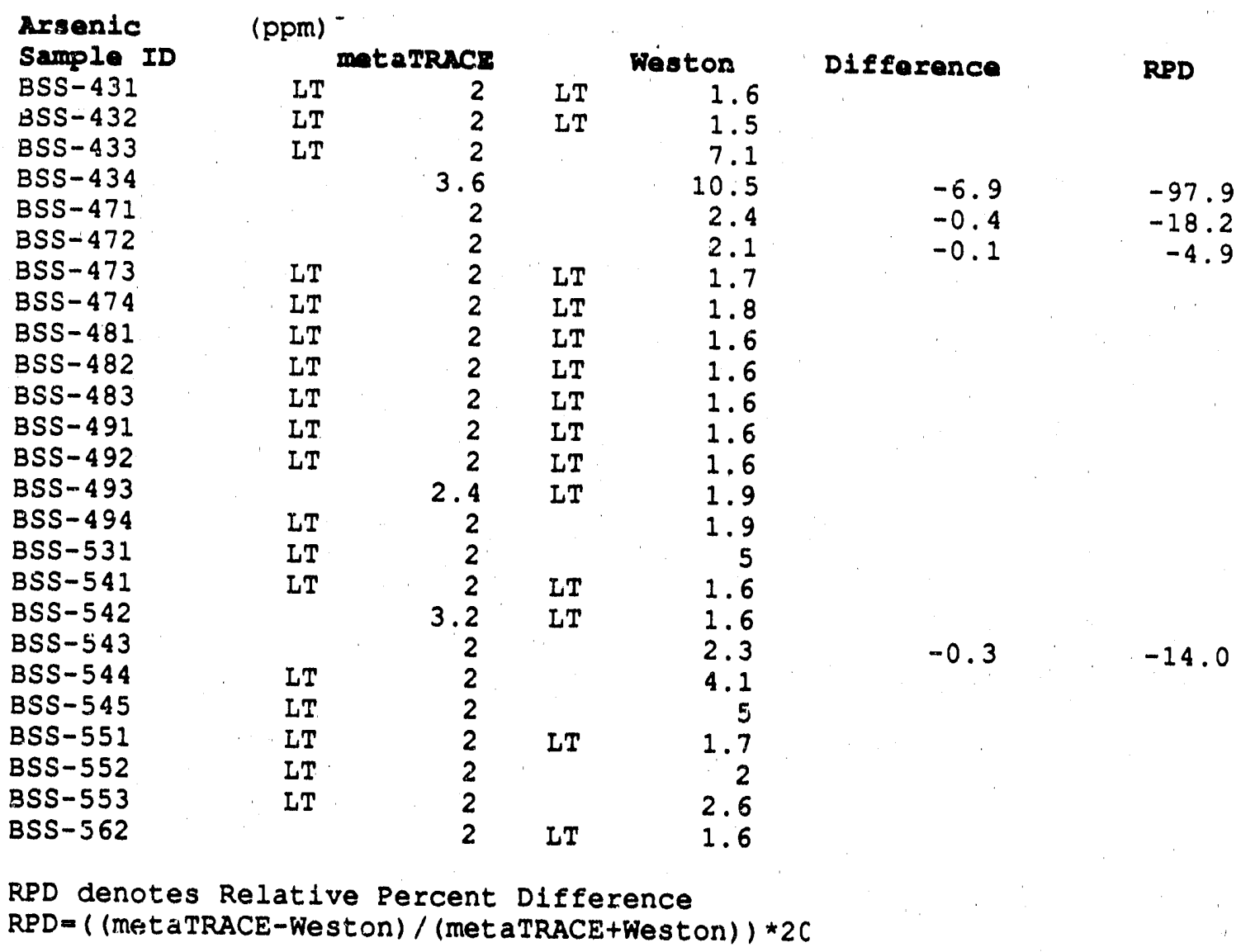


matarruce/Weston Laboratory Comparison Data

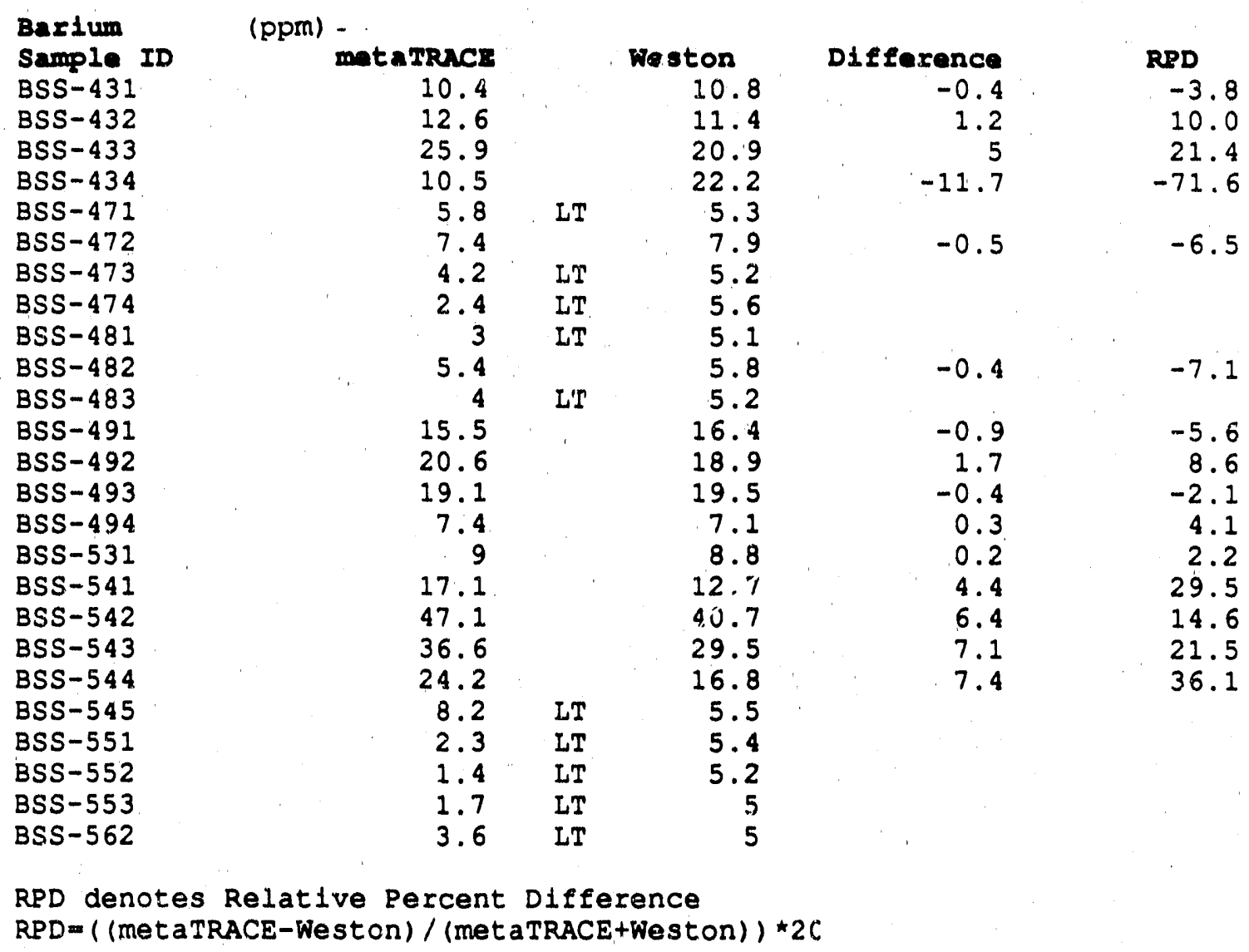


matamRACr/Weston Iaboratory Comparison Data

\begin{tabular}{|c|c|c|c|c|c|c|}
\hline Sample ID & & RACE & & Weston & Difference & RPD \\
\hline BSS- 431 & LT & 0.6 & LT & 0.4 & & \\
\hline BSS-432 & LT & 0.6 & $\mathrm{LT}$ & 0.39 & & \\
\hline BSS -433 & iT & 0.6 & LT & 0.46 & & \\
\hline BSS-434 & LT & 0.6 & & 0.74 & & \\
\hline BSS-471. & & 0.6 & & 0.45 & 0.15 & 28.6 \\
\hline BSS -472 & LT & 0.6 & & 0.68 & & \\
\hline BSS -473 & LT & 0.6 & LT & 0.42 & & \\
\hline BSS -474 & LT & 0.6 & & 0.51 & & \\
\hline BSS -481 & LT & 0.6 & LT & $0.4 i$ & & \\
\hline BSS-482 & LT & 0.6 & LT & 0.41 & 1 & \\
\hline BSS -483 & LT & 0.6 & LT & 0.41 & & \\
\hline BSS-491 & LT & 0.6 & LT & 0.41 & & \\
\hline BSS -492 & LT & 0.6 & LT & 0.41 & & \\
\hline BSS-493 & LT & 0.6 & & 0.72 & & \\
\hline BSS-494 & LT & 0.6 & & 0.51 & & \\
\hline BSS-531 & LT & 0.6 & LT & 0.43 & & \\
\hline BSS-541 & LT & 0.6 & LT & 0.4 & & \\
\hline $\begin{array}{l}B S S-542 \\
B S S-543\end{array}$ & & 0.6 & & 0.41 & 0.19 & 37.6 \\
\hline $\begin{array}{l}\text { BSS-543 } \\
\text { BSS }-544\end{array}$ & LT & $\begin{array}{l}0.6 \\
0.6\end{array}$ & & $\begin{array}{l}0.48 \\
0.61\end{array}$ & & \\
\hline BSS -545 & LT & 0.6 & & $\begin{array}{l}0.61 \\
0.86\end{array}$ & & \\
\hline BSS -551 & LT & 0.6 & LT & 0.43 & & \\
\hline BSS-552 & LT & 0.6 & LT & 0.42 & & \\
\hline BSS-553 & LT & 0.6 & LT & 0.4 & & \\
\hline BSS-562 & LT & 0.6 & LT & 0.4 & & \\
\hline
\end{tabular}




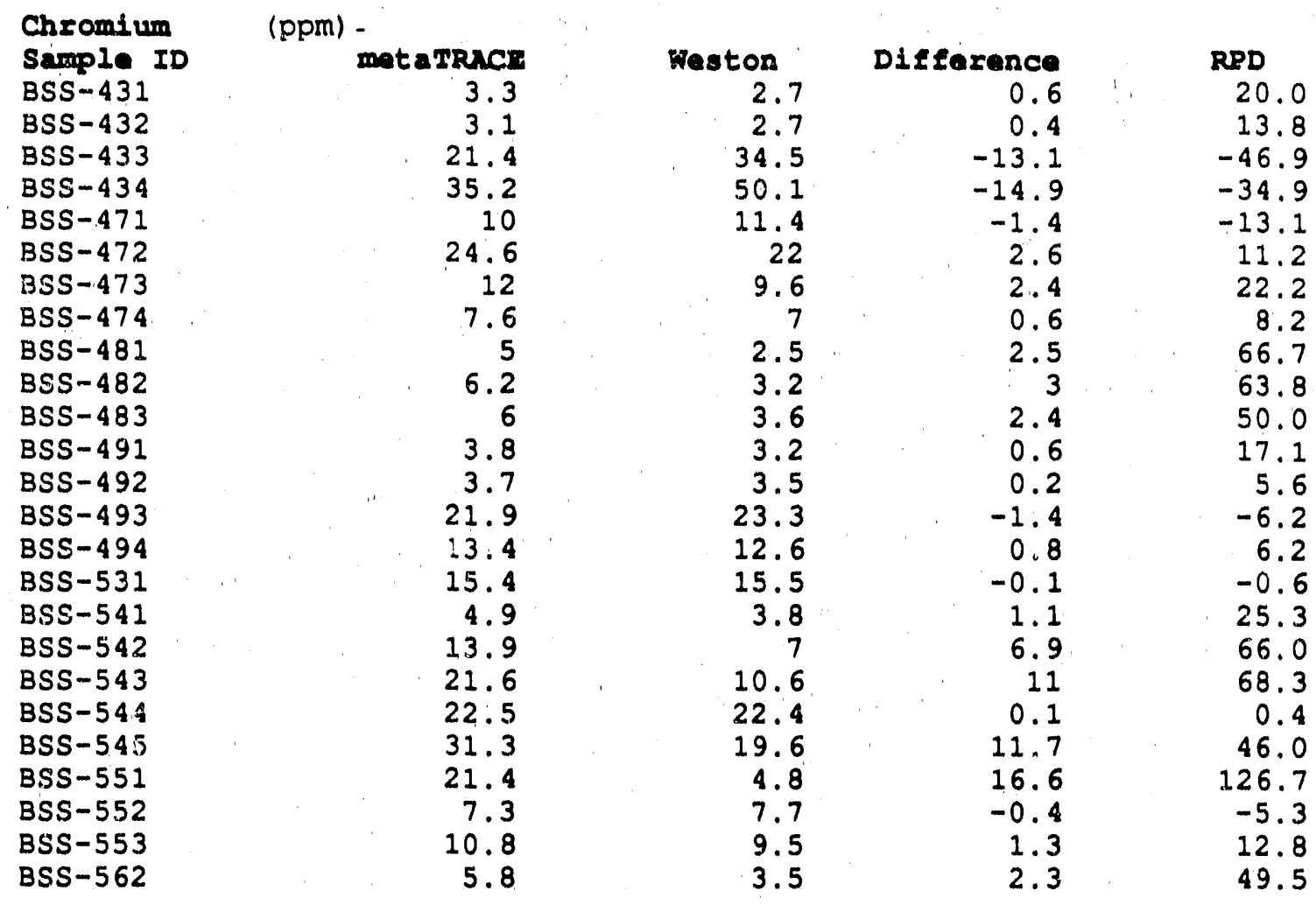

RPD denotes Relative Percent Difference $R P D=($ (metaTRACE-Weston $) /($ metaTRACE+Weston $)) * 2 C$ 
metarRACE/Weston Laboratory Comparison Data

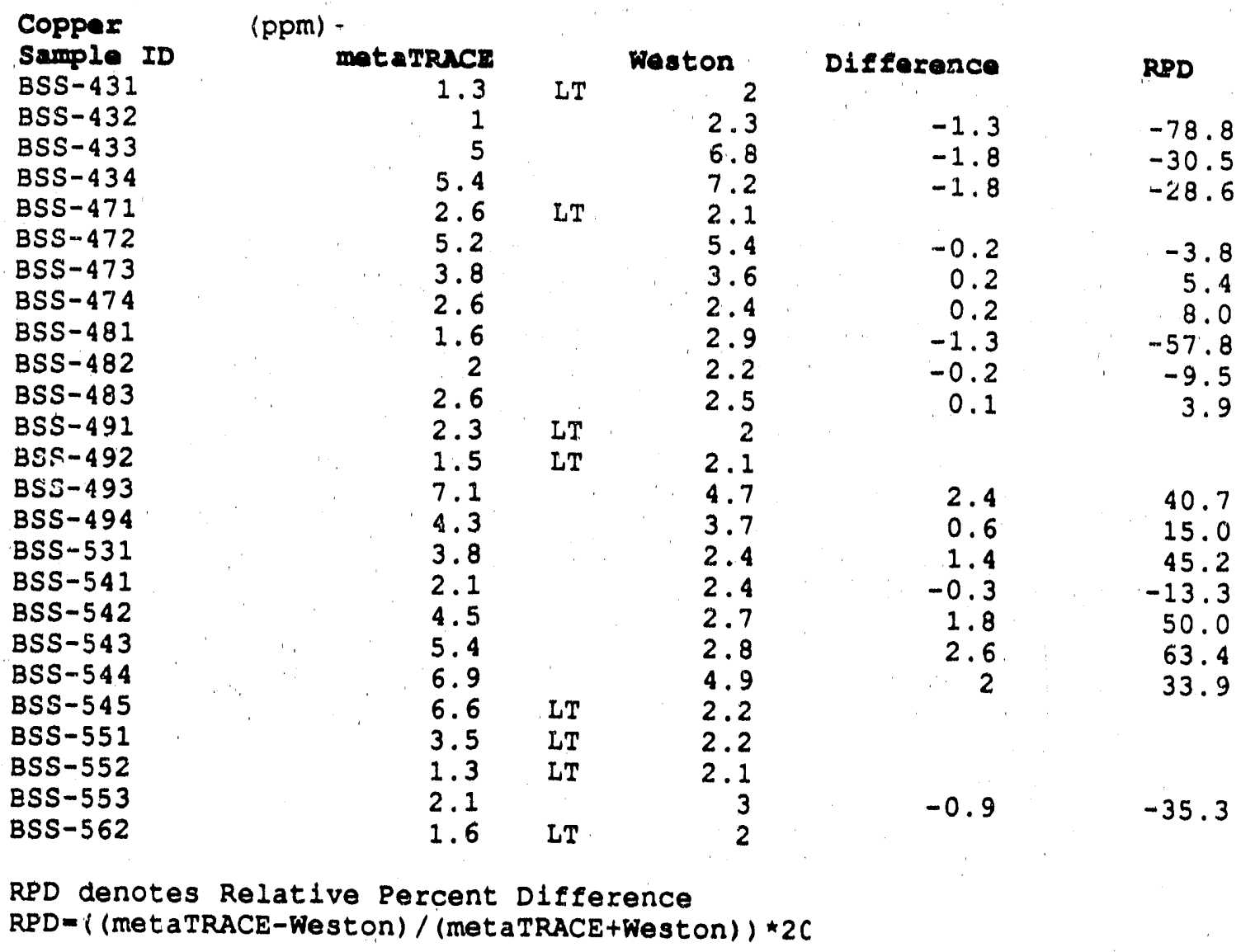




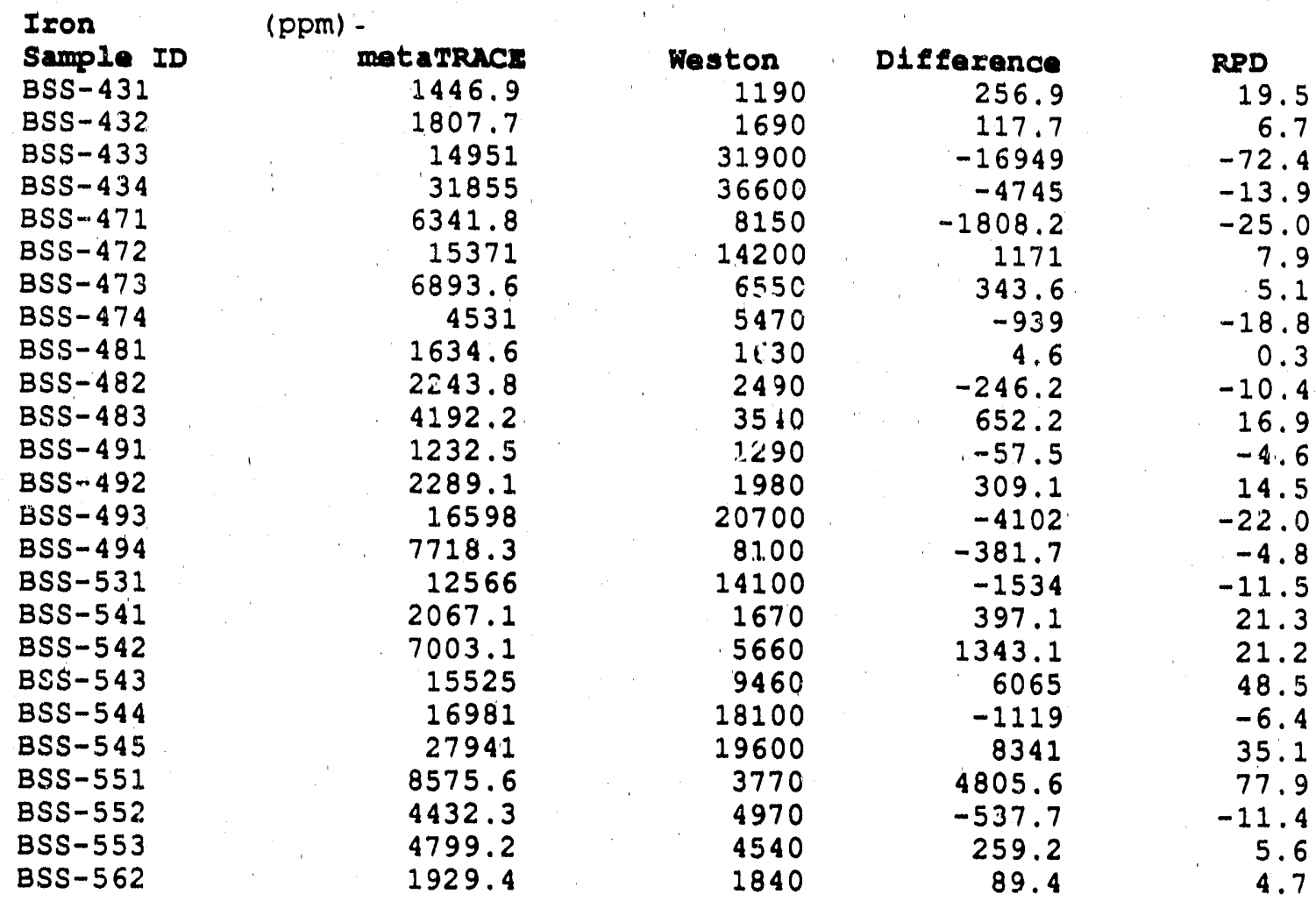

RPD denotes Relative Percent Difference

$\mathrm{RPD}=($ (metaTRACE-Weston) / (metaTRACE+Weston) ) *2C 
matarRACr/waston Laboratory. Comparison Data

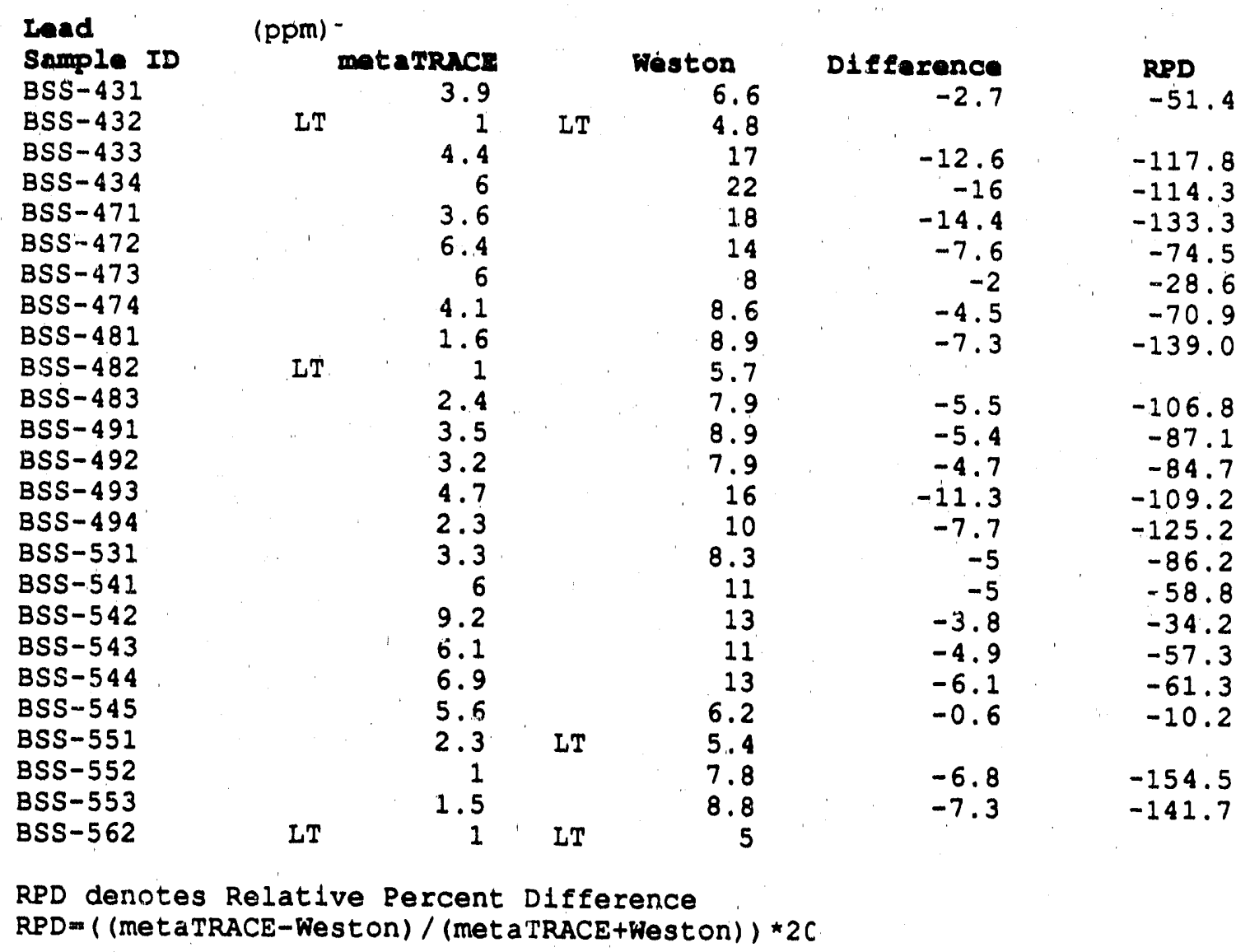


matamrucz/waston Laboratory Comparison Data

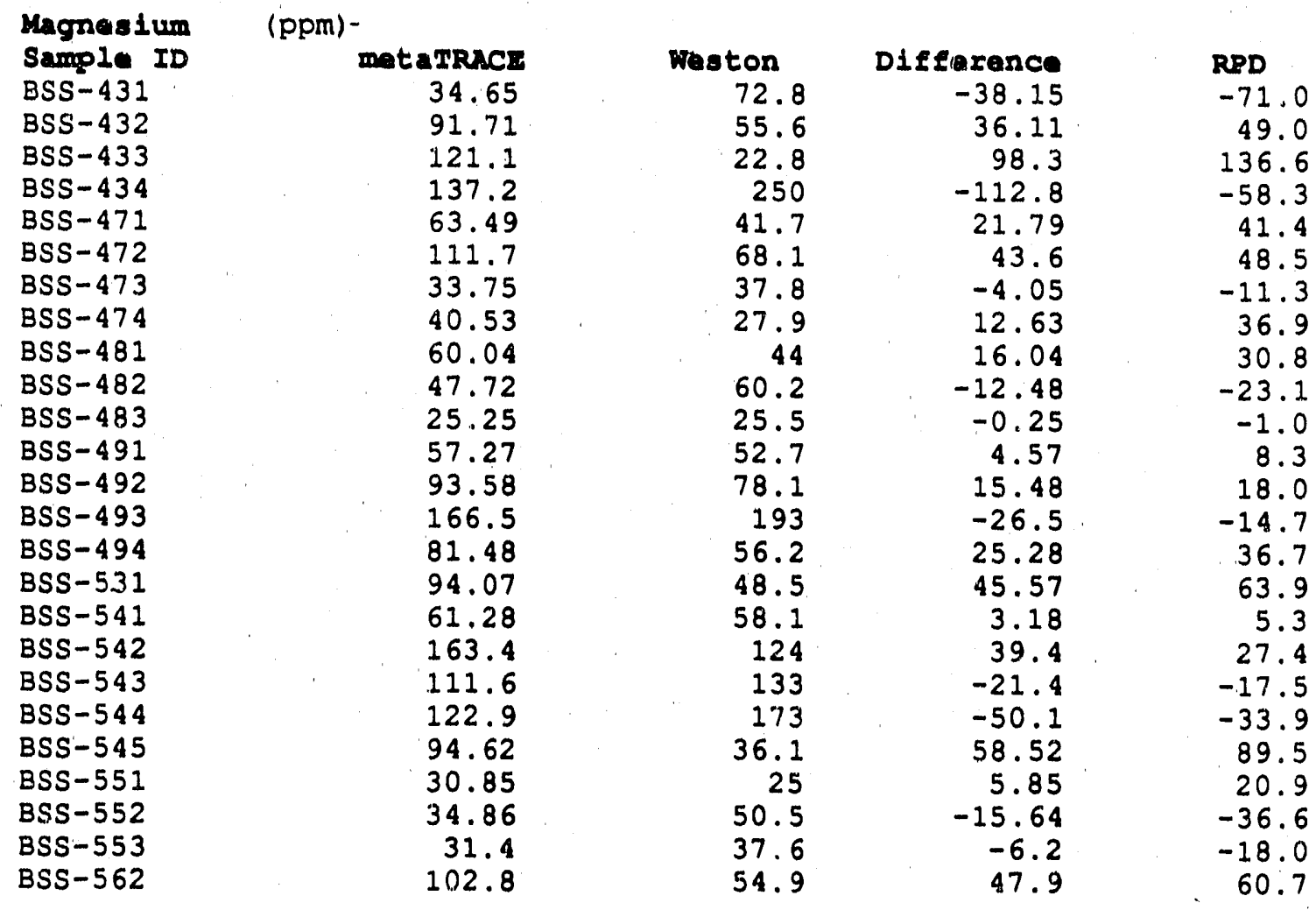

RPD denotes Relative Percent Difference

RPD $=($ (metaTRACE-Weston) / (metaTRACE+Weston) $) \star 2 C$ 
matamRACE/Weston Laboratory Comparison Data

\begin{tabular}{|c|c|c|c|c|}
\hline $\begin{array}{l}\text { Manganese } \\
\text { Sample ID } \\
\text { BSS-431 } \\
\text { BSS-432 } \\
\text { BSS-433 } \\
\text { BSS-434 } \\
\text { BSS-471 } \\
\text { BSS-472 } \\
\text { BSS-473 } \\
\text { BSS-474 } \\
\text { BSS-481 } \\
\text { BSS-482 } \\
\text { BSS-483 } \\
\text { BSS-491 } \\
\text { BSS-492 } \\
\text { BSS-493 } \\
\text { BSS-494 } \\
\text { BSS-531 } \\
\text { BSS-541 } \\
\text { BSS-542 } \\
\text { BSS-543 } \\
\text { BSS-544 } \\
\text { BSS-545 } \\
\text { BSS-551 } \\
\text { BSS-552 } \\
\text { BSS-553 } \\
\text { BSS-562 }\end{array}$ & $\begin{array}{r}\text { opm)- } \\
\text { matacr } \\
32.25 \\
12.64 \\
3.294 \\
3.37 \\
4.519 \\
4.213 \\
8.043 \\
2.528 \\
5.132 \\
6.358 \\
4.673 \\
75.91 \\
13.18 \\
1.6 \\
1.6 \\
7.583 \\
90 \\
125.9 \\
10.88 \\
7.503 \\
3 T \\
\text { LT } \quad 3.6 \\
3.294 \\
3.524 \\
2.834 \\
5.898\end{array}$ & $\begin{array}{r}\text { Weston } \\
34.5 \\
15 \\
13.7 \\
15.2 \\
41.7 \\
34.9 \\
4.8 \\
5.6 \\
6.8 \\
14.5 \\
8 \\
99.7 \\
17.2 \\
7.3 \\
2.8 \\
10.2 \\
115 \\
201 \\
20.4 \\
20.7 \\
8.6 \\
5.6 \\
8.5 \\
6.2 \\
6.2\end{array}$ & $\begin{array}{r}\text { Difference } \\
-2.25 \\
-2.36 \\
-10.406 \\
-11.83 \\
-37.181 \\
-30.687 \\
3.243 \\
-3.072 \\
-1.668 \\
-8.142 \\
-3.327 \\
-23.79 \\
-4.02 \\
\\
-2.617 \\
-25 \\
-75.1 \\
-9.52 \\
-13.197 \\
-5 \\
-2.306 \\
-4.976 \\
-3.366 \\
-0.302\end{array}$ & $\begin{array}{r}\text { RPD } \\
-6.7 \\
-17.1 \\
-122.5 \\
-127.4 \\
-160.9 \\
-156.9 \\
50.5 \\
-75.6 \\
-28.0 \\
-78.1 \\
-52.5 \\
-27.1 \\
-26.5\end{array}$ \\
\hline
\end{tabular}

RPD denotes Relative Percent Difference

$R P D=($ (metaTRACE-Weston $) /($ metaTRACE +Weston $)) * 2 C$ 


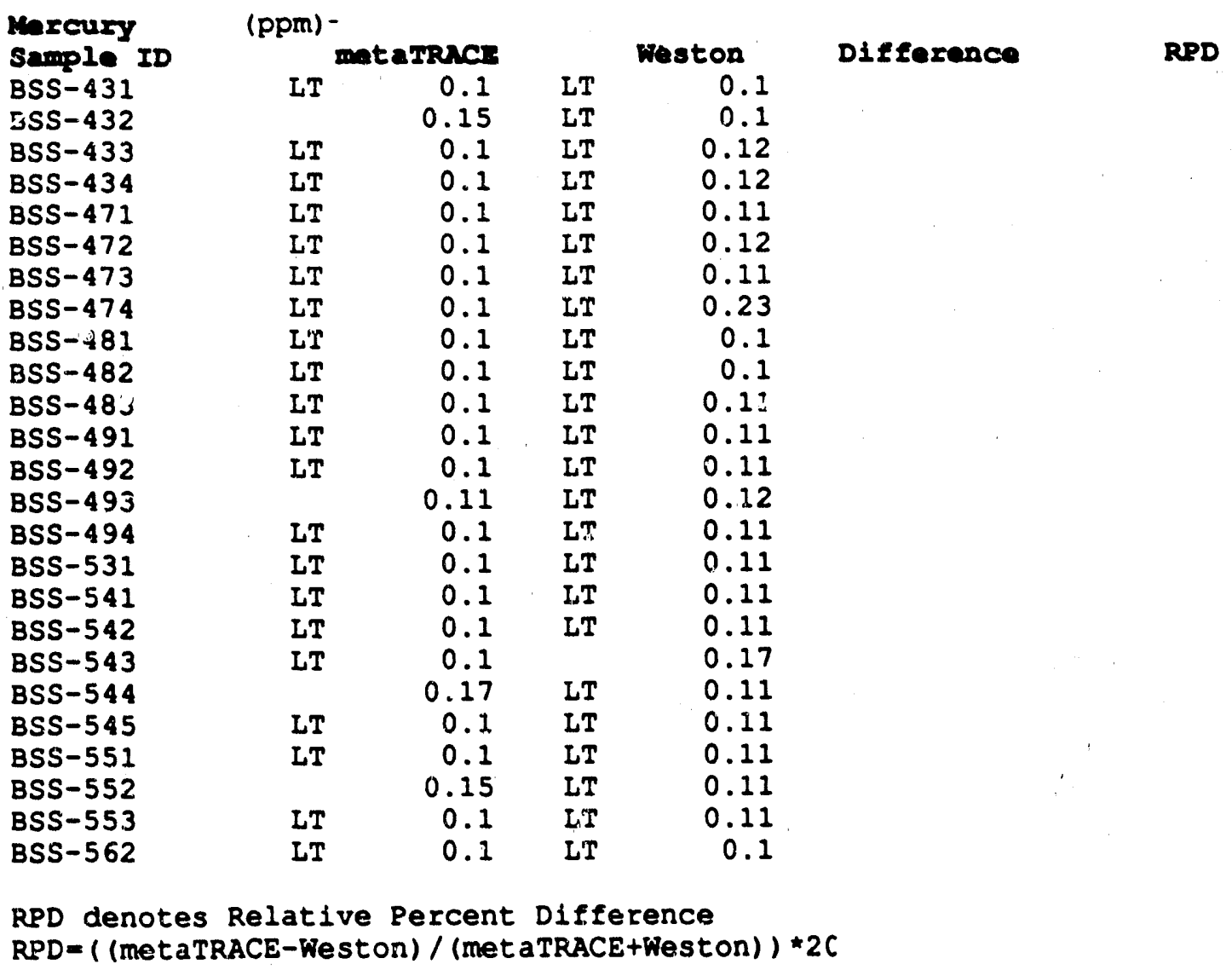


metaIRICE/Weston Laboratory Comparison Data

\begin{tabular}{|c|c|c|c|c|c|c|}
\hline $\begin{array}{l}\text { Nickel } \\
\text { Sample ID }\end{array}$ & (ppm) & ruce & & Weston & & \\
\hline BSS -431 & & 2 & LT & 2 & DIIrerence & RPD \\
\hline BSS -432 & LT & 2 & LT & 1.9 & & \\
\hline BSS -433 & & 3.9 & & 3.8 & 0.1 & 2.6 \\
\hline BSS-434 & LT & 2 & & 5.5 & & \\
\hline BSS-471 & LT & 2 & LT & 2.1 & & \\
\hline BSS -472 & & 3.4 & LT & 2.3 & & \\
\hline BSS -473 & & 3 & IT & 2.1 & & \\
\hline BSS-474 & & 2.6 & LT & 2.2 & & \\
\hline BSS-481 & & 2.8 & LT & 2 & & \\
\hline BSS-482 & & 2.4 & LT & 2 & & \\
\hline BSS-483 & LT & 2 & LT & 2.1 & & \\
\hline BSS- 491 & LT & 2 & LT & 2 & & \\
\hline BSS-492 & LT & 2 & & $2 . \overline{1}$ & & \\
\hline BSS-493 & & 3.7 & & 3 & 0.7 & 20.9 \\
\hline $\begin{array}{l}\text { BSS- } 494 \\
\text { BSS-531 }\end{array}$ & $\begin{array}{l}\text { LT } \\
\text { LT }\end{array}$ & $\begin{array}{l}2 \\
2\end{array}$ & LT & $\begin{array}{l}2.1 \\
2.2\end{array}$ & & \\
\hline BSS-541 & & 2.6 & LT & 2 & & \\
\hline $\begin{array}{l}\text { BSS-5 } 52 \\
\text { BSS-543 }\end{array}$ & & $\begin{array}{l}5.6 \\
5.1\end{array}$ & & 2.3 & 3.3 & 83.5 \\
\hline BSS-544 & & 3.8 & & $\begin{array}{l}2.1 \\
3.5\end{array}$ & $\begin{array}{r}3 \\
0.3\end{array}$ & 83.3 \\
\hline BSS-545 & & 3.8 & LT & 2.2 & & 8.2 \\
\hline BSS-551 & & 2.2 & LT & 2.2 & & \\
\hline BSS -552 & LT & 2 & LT & 2.1 & & \\
\hline BSS-553 & LT & 2 & LT & 2 & & \\
\hline BSS-562 & LT & 2 & LT & 2 & & \\
\hline
\end{tabular}


matamRucE/meston Iaboratory Comparison Data

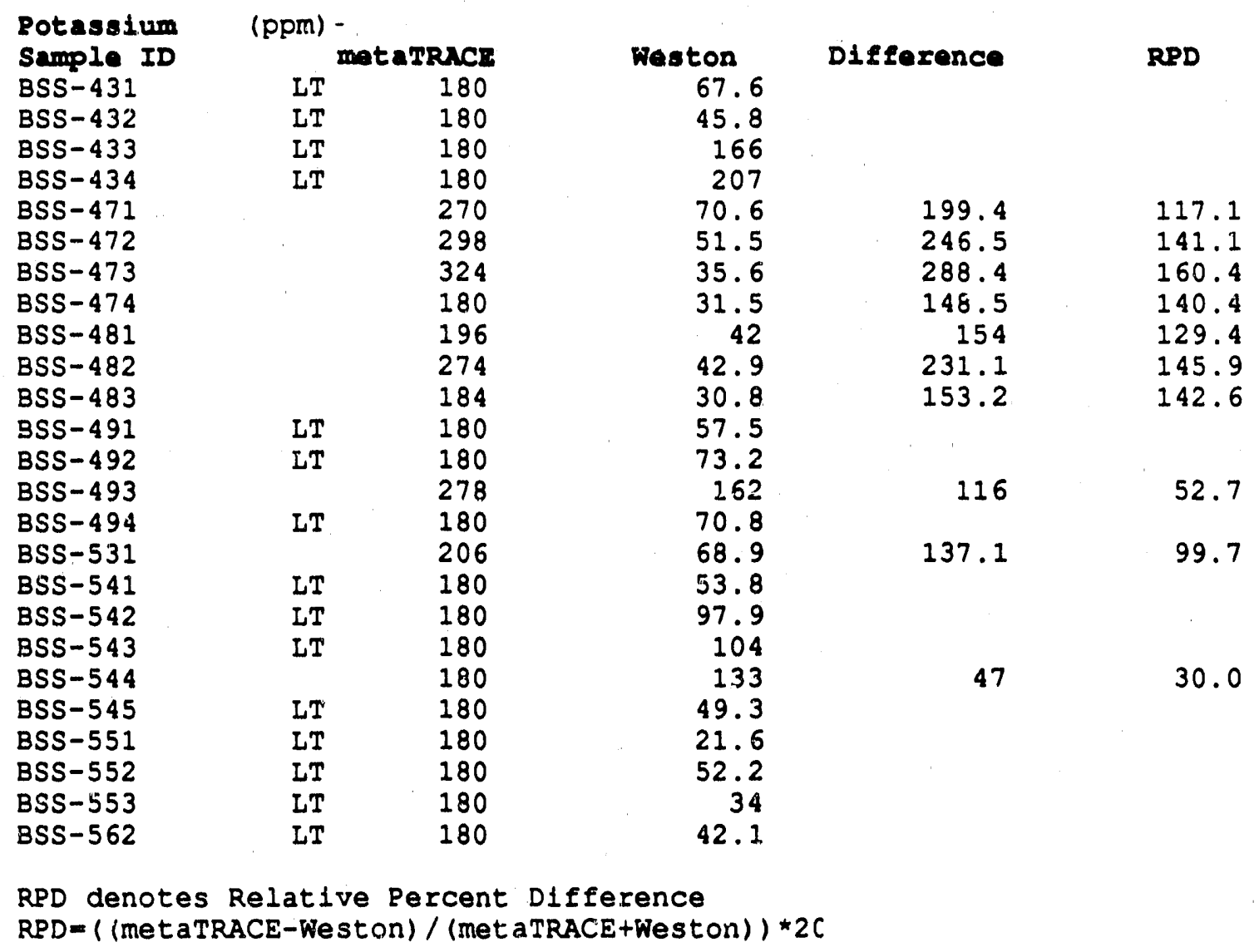


motaTRACE/Weston Laboratory Comparison Data

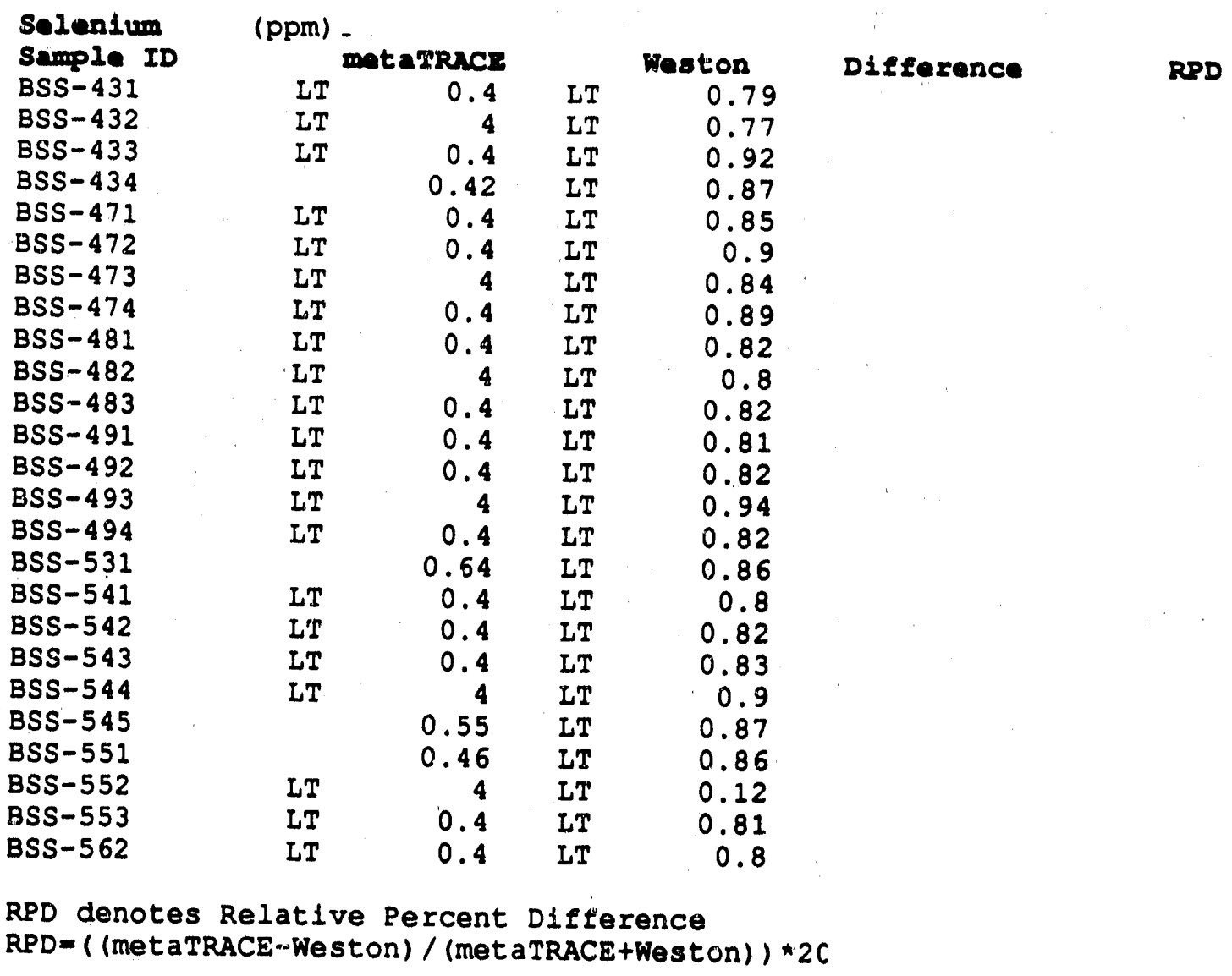


matamRAcr/weston Laboratory Comparison Data

\begin{tabular}{|c|c|c|c|c|c|c|}
\hline $\begin{array}{l}\text { S1lvar } \\
\text { Sample ID }\end{array}$ & (ppm) & RACE & & Weston & Difference & RPD \\
\hline BSS-431 & LT & 1 & LT & 3 & & \\
\hline BSS- 432 & LT & 1 & LT & 2.9 & & \\
\hline BSS -433 & LT & 1 & $\mathrm{LT}$ & 3.4 & & \\
\hline BSS-434 & LT & 1 & LT & 3.3 & & \\
\hline BS5-471 & LT & 1 & & 3.2 & & \\
\hline BSS -472 & LT & 1 & LT & 3.4 & & \\
\hline BSS -473 & LT & 1 & LT & 3.1 & & \\
\hline BSS -474 & LT & 1 & LT & 3.3 & & \\
\hline BSS- 481 & $L \mathbf{T}$ & 1 & LT & 3.1 & & \\
\hline BSS -482 & LT & 1 & LT & 3 & & \\
\hline BSS -483 & LT & 1 & LT & 3.1 & & \\
\hline BSS-491 & LT & 1 & LT & 3 & & \\
\hline BSS-492 & LT & 1 & LT & 3.1 & & \\
\hline BSS -493 & LT & 1 & LT & 3.5 & & \\
\hline BSS-494 & LT & 1 & $\mathrm{LT}$ & 3.1 & & \\
\hline BSS-531 & LT & 1 & LT & 3.3 & & \\
\hline BSS-541 & LT & 1 & LT & 3 & & \\
\hline BSS-542 & LT & 1 & $\mathrm{LT}$ & 3.1 & & \\
\hline BSS-543 & LT & 1 & LT & 3.1 & & \\
\hline BSS-544 & LT & 1 & LT & 3.4 & & \\
\hline BSS -545 & LT & 1 & LT & 3.3 & & \\
\hline BSS -551 & LT & $\overline{1}$ & LT & 3.2 & & \\
\hline BSS -552 & LT & 1 & LT & 3 & & \\
\hline BSS -553 & & $1 . \overline{8}$ & LT & 3 & & \\
\hline BSS-562 & LT & 1 & LT & 3 & & \\
\hline
\end{tabular}


motamRrCE/Meston Laboratory Comparison Data

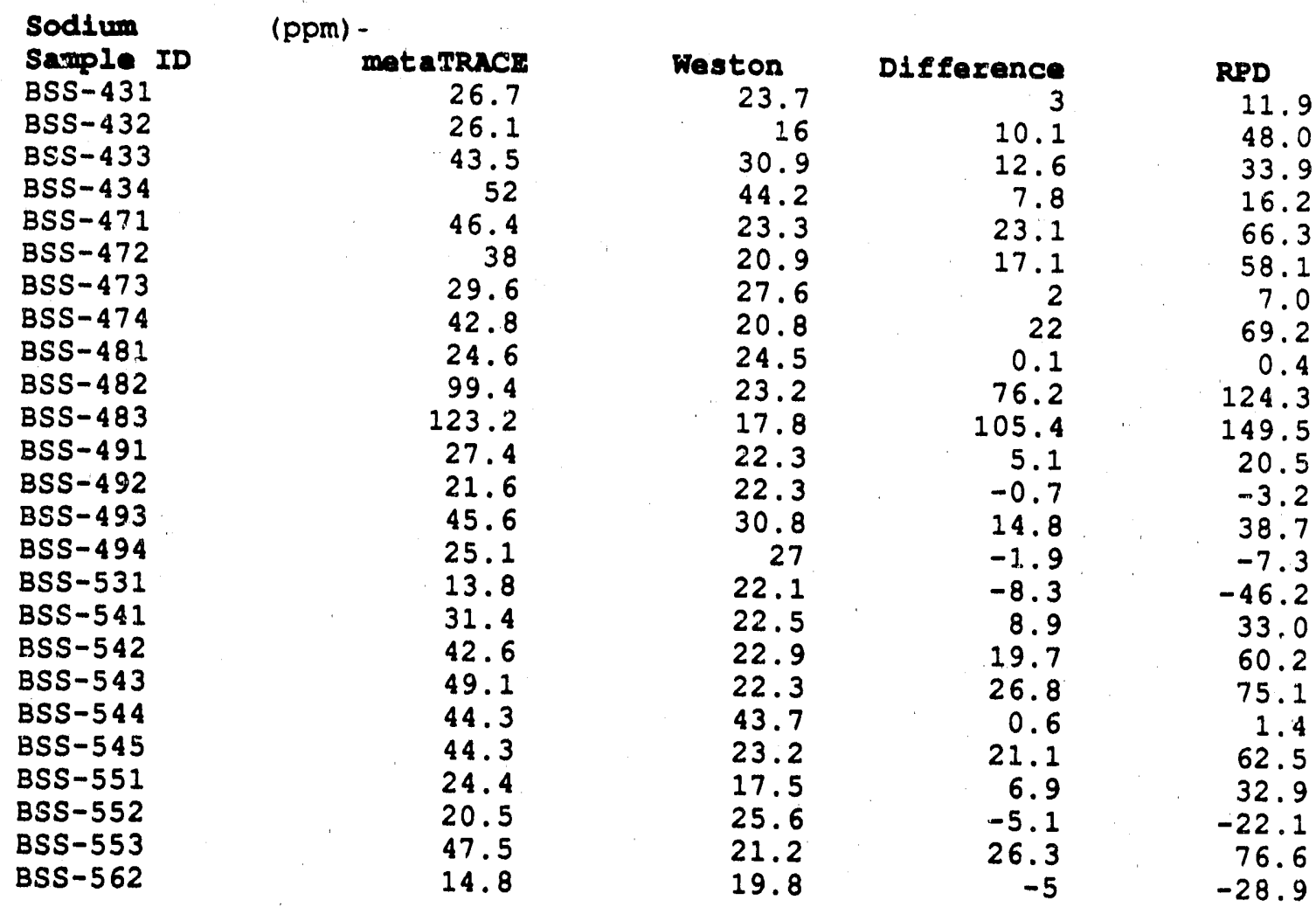

RPD denotes Relative Percent Difference $R P D=($ (metaTRACE-Weston) /(metaTRACE+Weston) ) *2C 
matarRrCE/Weston Laboratory Comparison Data

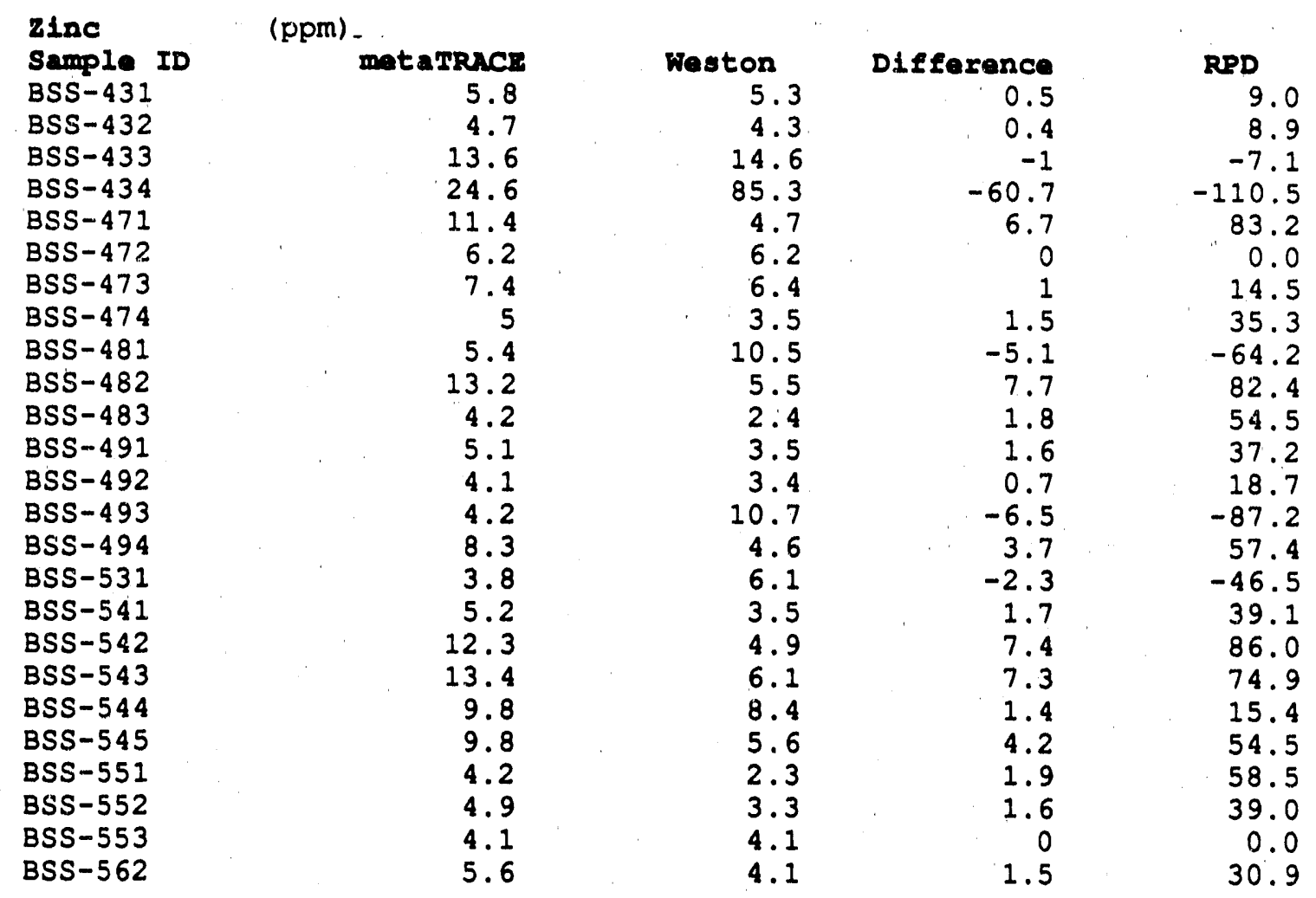

RPD denotes Relative Percent Difference $\mathrm{RPD}=($ (metaTRACE-Weston $) /($ metaTRACE+Weston $)) * 2 C$ 
matarRACr/Weston Laboratory Comparison Data

\begin{tabular}{|c|c|c|c|c|c|c|}
\hline $\begin{array}{l}\text { Iithium } \\
\text { Sample ID }\end{array}$ & (ppm) & exce & & Wiston & Difference & RPD \\
\hline$B S S-431$ & LT & 10 & LT & 2 & & \\
\hline BSS -432 & LT & 10 & LT & 2 & & \\
\hline BSS -433 & L'T & 10 & & 2.7 & & \\
\hline BSS-434 & LT & 10 & LT & 2 & & \\
\hline BSS- 471 & LT & 10 & LT & 2 & & \\
\hline BSS -472 & LT & 10 & LT & 2 & & \\
\hline BSS -473 & LT & 10 & LT & 2.0 & & \\
\hline BSS -474 & LT & 10 & LT & 2 & . & \\
\hline BSS-481 & LT & 10 & LT & 2 & & \\
\hline BSS- 482 & LT & 10 & LT & 2 & & \\
\hline BSS -483 & LT & 10 & $\mathrm{LT}$ & 2 & & \\
\hline BSS-491 & LT & 10 & LT & 2 & & \\
\hline BSS -492 & LT & 10 & LT & 2 & & \\
\hline BSS-493 & LT & 10 & & 6.2 & & \\
\hline & LT & 10 & & 3.5 & & \\
\hline $\begin{array}{l}\text { BSS-531 } \\
\text { BSS-541 }\end{array}$ & $\begin{array}{l}\text { LT } \\
\text { LT }\end{array}$ & $\begin{array}{l}10 \\
10\end{array}$ & LT & $\begin{array}{l}2 \\
2\end{array}$ & & \\
\hline BSS-542 & LT & 10 & LT & 2 & & \\
\hline$B S S-543$ & LT & 10 & & 2.8 & & \\
\hline BSS-544 & LT & 10 & & 2.1 & & \\
\hline BSS-545 & LT & 10 & LT & 2 & & \\
\hline BSS-551 & LT & 10 & LT & 2 & & \\
\hline BSS- 552 & LT & 10 & LT & 2 & & \\
\hline BSS-553 & LT & 10 & LT & 2 & & \\
\hline BSS-562 & LT & 10 & LT & 2 & & \\
\hline
\end{tabular}


matarruce/Waston Laboratory Comparison nata

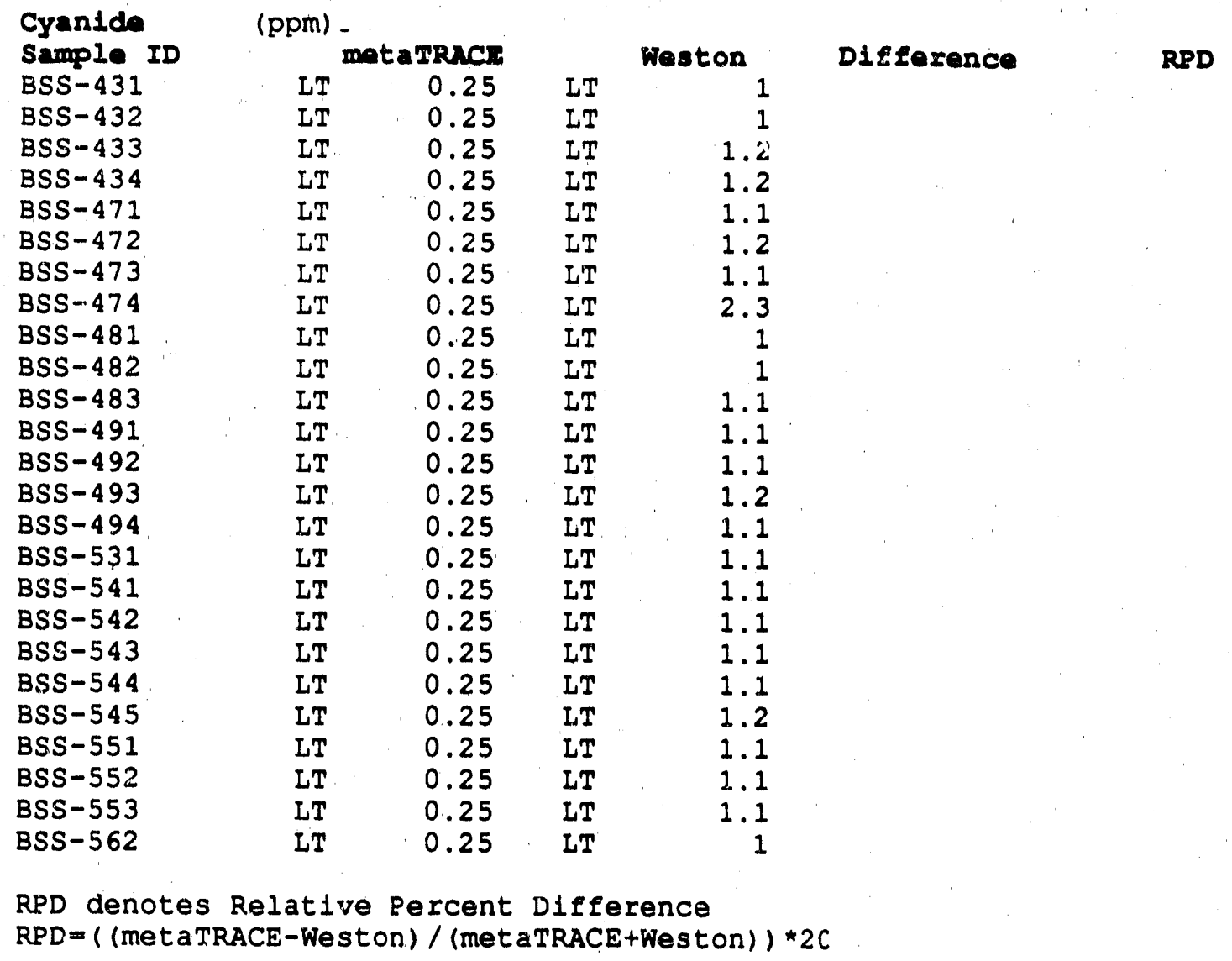


matarRuc:/ioston Laboratory Comparison Data

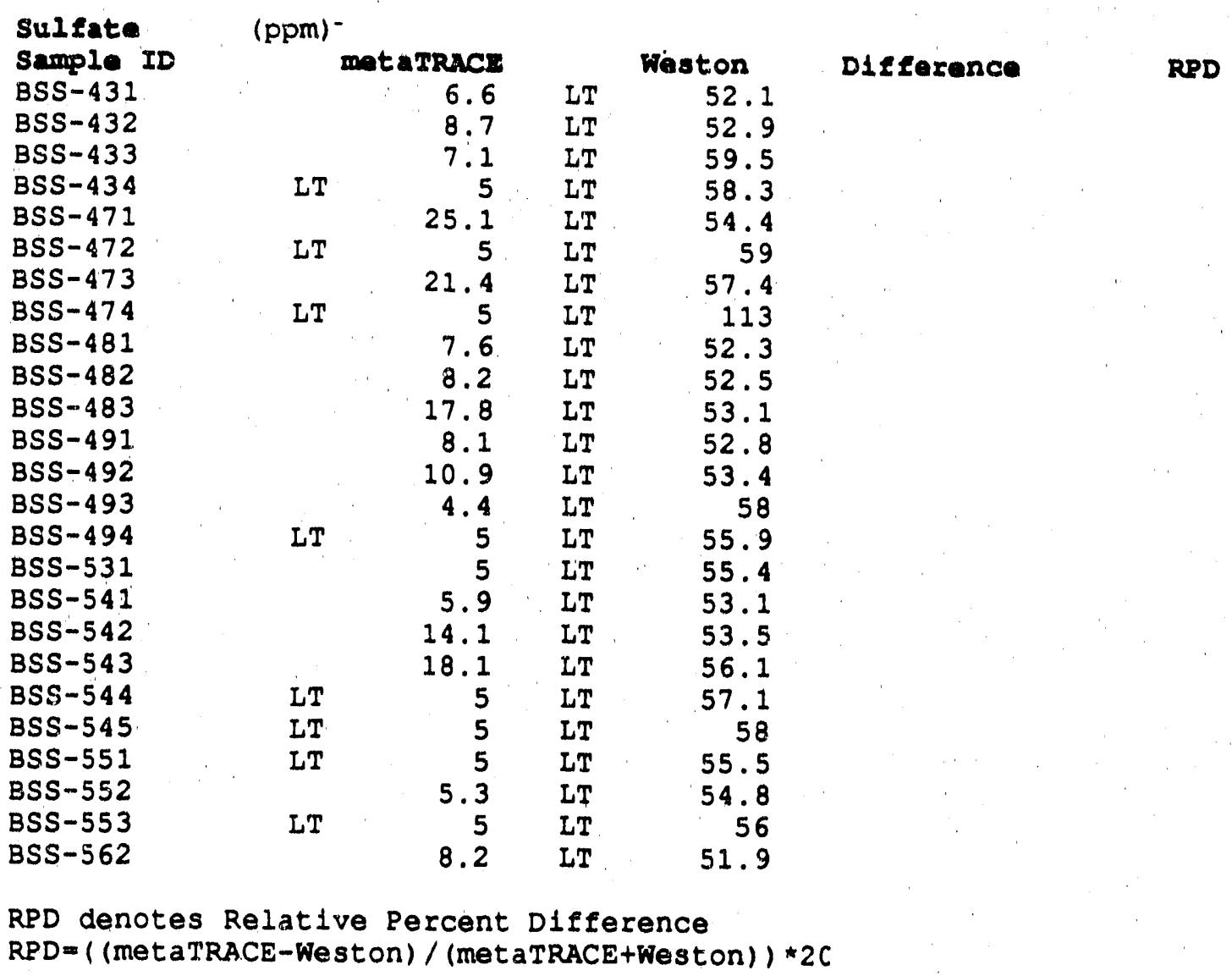


matarRACE/Waston Laboratory Comparison Data

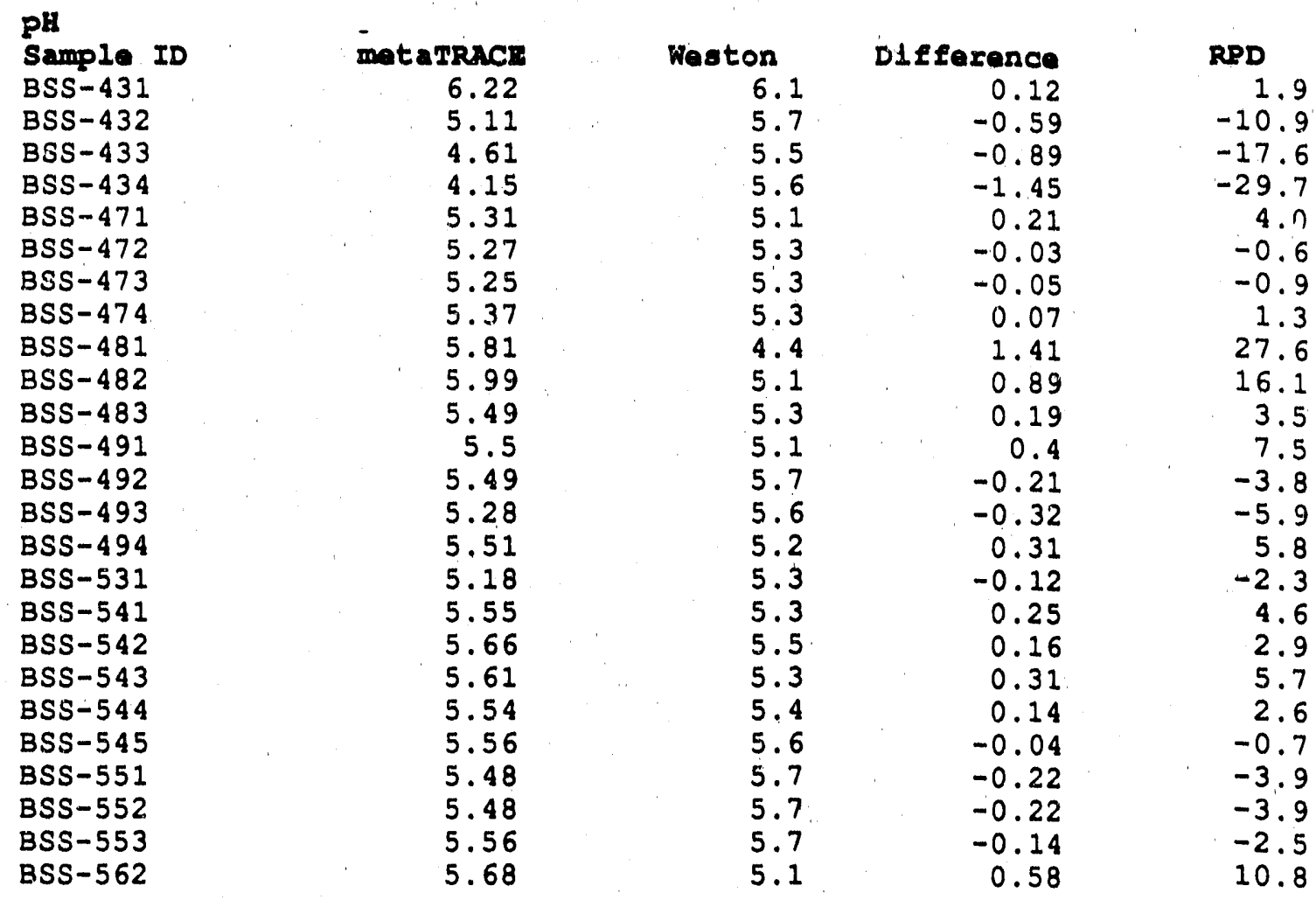

RPD denotes Relative Percent Difference RPD $=($ (metaTRACE-Weston) / (metaTRACE+Weston) $) \star 2 C$ 
matarruce/Waston Iaboratory Comparison Data

Total Organic Carbon

Sampla ID

$B S S-431$

BSS -432

BSS -433

BSS -434

BSS-471

BSS- 472

BSS -473

BSS -474

BSS- 481

BSS-482

BSS -483

BSS-491

BSS -492

BSS-493

BSS -494

BSS-531

BSS- 541

BSS-542

BSS-543

BSS-544

BSS- 545

BSS-551

BSS-552

BSS-553

BSS -562

mataMRnc:

3556

2130

841

860

5703

595

243

189

4092

409

208

2957

471

764

415

386

3430

619

2615

1272

305

96

97

43
300

miston
9090
530
680
1400
2180
1260
460
170
820
330
130
4600
330
990
330
1000
610
740
1080
420
480
240
70
53.4
660

miaton

530

680

1400

2180

460

170

820

330

600

330

990

330

610

740

1080

420

480

70

3.4

660
RPD

$-87.5$

120.3

21.2

$-47.8$

89.4

$-71.7$

$-61.7$

10.6

133.2

21.4

46.2

$-43.5$

35.2

$-25.8$

22.8

$-88.6$

139.6

$-17.8$

83.1

100.7

$-44.6$

$-85.7$

32.3

$-21.6$

$-75.0$

RPD denotes Relative Percent Difference $R P D=($ (metaTRACE-Weston) /(metaTRACE+Weston) $) \star 2 C$ 
Total Organic Halogens

Sample ID

BSS-431

BSS -432

BSS -433

BSS -434

BSS-471

BSS- 472

BSS -473

BSS -474

BSS-481

BSS-482

BSS -483

BSS -491

BSS-492

BSS -493

BSS-494

BSS-531

BSS-541

BSS-542

BSS -543

BSS - 544

BSS -545

BSS-551

BSS-552

BSS -553

BSS-562

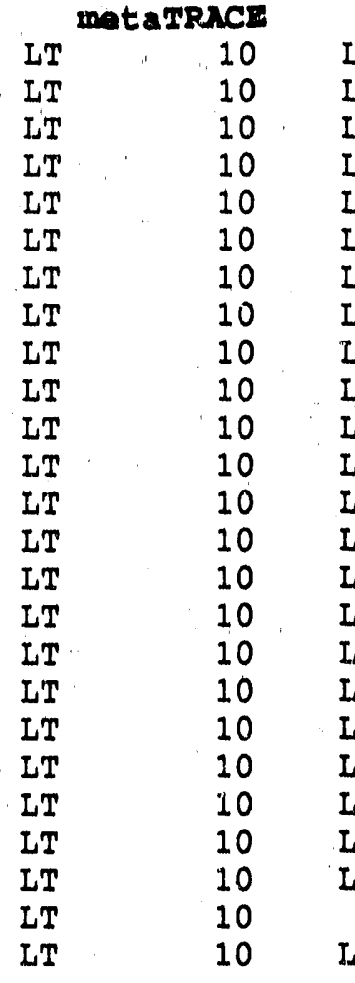

Weston

100

100

100

100

100

100

100

100

100

100

100

100

100

100

100

100

100

100

100

100

100

100

100

140

100

RPD denotes Relative Percent Difference $R P D=($ (metaTRACE-Weston) /(metaTRACE+Weston) ) *2C 

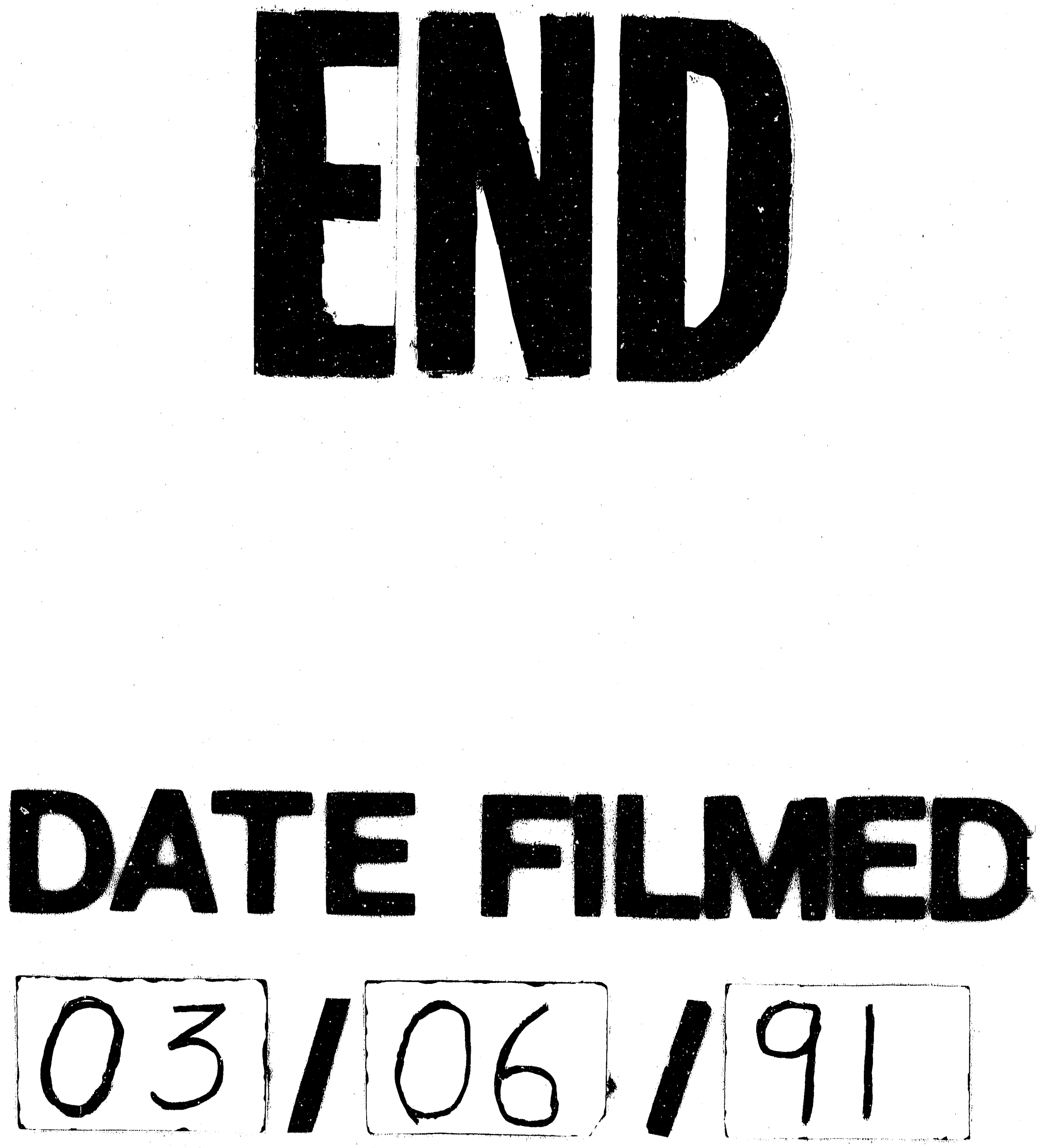